

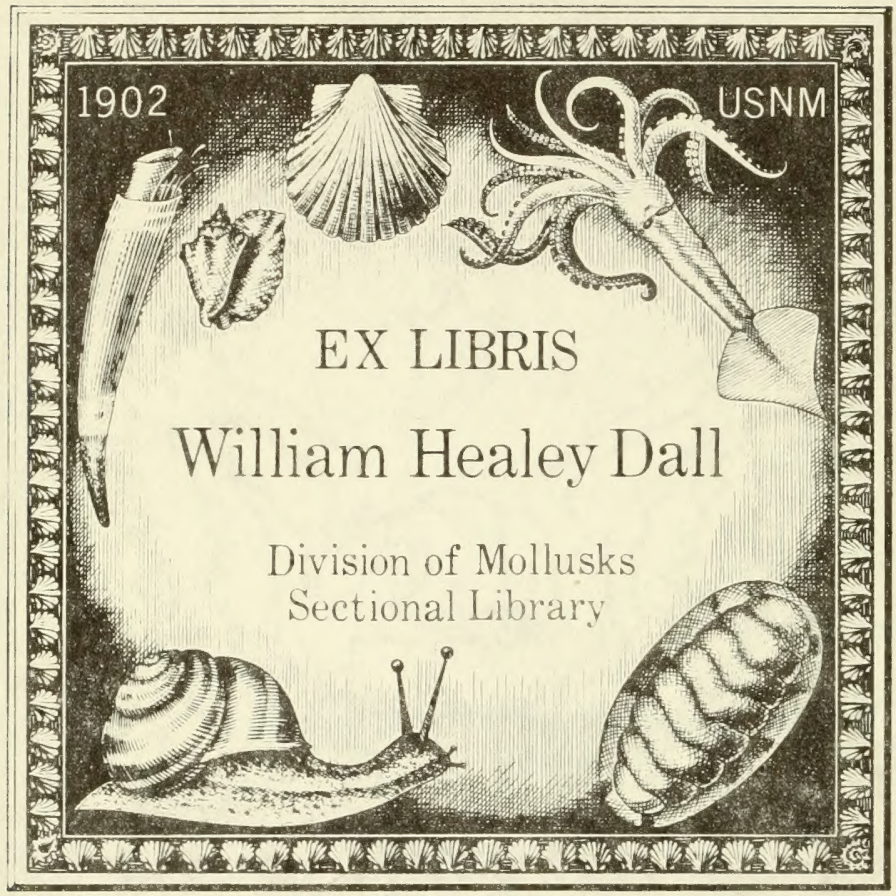



$x+$ 


$$
K-1-i \Delta A C L
$$



DEPARTMENT OF THE INTERIOR

\section{B ULLETIN}

OF THE

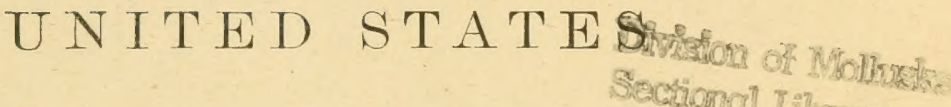 Sigctional Lillenving?}

\section{GEOLOGICAL SURVEY}

No. 24

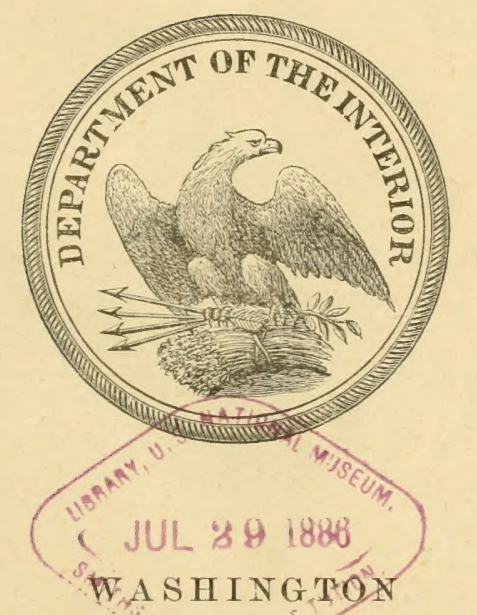

GOVERNMENT, PRENTING OFFICE 1885 

DIy united states geological survey

J. W. POWELL DIRECTOR

\section{I S T}

OI

MARINE MOLLUSCA

COMPRISING THE

QUATERNARY FOSSILS AND RECENT FORMS

FROM

AMERICAN LOCALITIES BETWEEN CAPE HATTERAS AND CAPE ROQUT: INCLUDING THE BERMUDAS

BY

WILLIAM HEALEY DALL

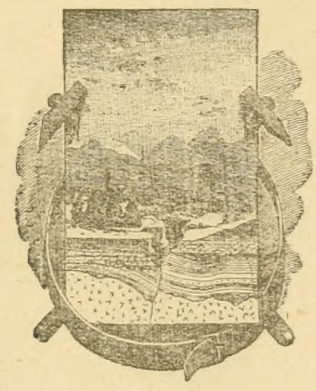

WASHINGTON

GOVERNMENT PRINTING OFEICE 1885 

CONTENTS.

-

Page.

Introductory................................................. 7

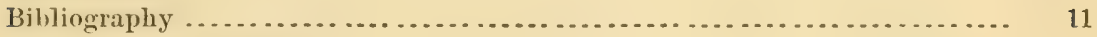

List of marine mollusca . ........................................... 21 



\title{
LIST OF MARINE IIOLLUSCA.
}

\author{
By Williatr Healex Datl.
}

\section{INTRODUCTORY.}

The present list grew out of the needs of the Division of Quaternary Palcontology of the United States Geological Survey. In attempting to sfstematize the collection of quaternary fossils and allied recent forms of the eastern and southern coast of the United States sonthward from Cape IIatteras, it was found, almost at the ontset, that the absence of any general work, list, or discussion of the invertebrate fauna added greatly to the difficulty of identifiention and classificatiou.

A card catalogue was hegun as a means of ready reference. The ntility of this became daily more evident. The researches of collectors on the Florida coast showed that the Antillean famalay at our doors. Forms recent there were found fossil in the so-called post-pliocene of South Carolina. It became necessary to include the Antillean and Caribbean famm, which extends, in a wide sense, to Brazil, on the northern shores of Sonth Americi. The catalogie has gradually grown into its present form, and will continne to grow ; but, for cur own sake, and in the hope of enlisting the attention and facilitating the researches of other students, it mas thought best to print it at its present stage.

The catalogne was preparen, from the original doeuments enumerated in the accompanying biblography, by Mr. Richard Ellsworth Call and Miss A gnes Nicholson, under the writer's direction; to them the eredit and responsibility for such fulluess and acenracy as it may possess chiefls belong. Although many references were added by the writer in person, it has not been practicable for him to personally verify every citation by comparison with the original. He has, however, read the proofs from the beginning, aud in this way has corrected many of the small inacenracies which are almost inevitable in works of this kind. The resulting list is believerl to be fairly accurate. Its scope and mode of construction remain to be stated.

It claims no completeness beyond what inheres in a complete index to the species belonging to the region stated, and specified to so belong in the works referred to in the bibliography herewith. These comprise, however, all the most important conchological joumals from their beginning, and the chief catalognes, local lists, famne, sc., relating to different parts of the region stated. If in any of those journals a spe- 
cies really belonging to the fauna in hand is described without habitat, or with a wrong habitat, it will not be found in this list from that source. Misprints, uuless perfectly obvious, are given as thes stand. There are a number of local lists, such as those of Schramm, Schomburgk, Shuttleworth in the Beruer Mittheilungen, etc., which have not been accessible. There are others omitted all of whose species are cited in om list from several other sources. No attempt has been made to claborate sym. onymy. This is a task belonging to a later stage of the work. The differences of opinion on that subject also stand in the way of a prompt decision until specimens hare been subjected to careful studr. The original localities noted in the works eited from have been preserverl, but no attempt has been marle to give the geographical distribution of each species. This again waits upon the synonymy. No doubt there are many species existing in the region and described in worlis on conchology which are omitted. Others will be found under sereral names in the list. A preliminary study of the fauma indicates that thes specific synouymy is very large. The publication of this l st is desicnerl to assist in improving our knowledge both of the omissions and super. fluities. The deep-sea forms have been included, as there is no doutht a majority of them rise to or originally descended from areas of moderate depth or are found in deposits of fossils. The pelagie forms have been included when they occurred, but have not been sought for ; they belong to no special coast fauna. The Auriculacea and Petroplila have been included as practically as much marine in habit as Litorinas, though julmoniferous. Other pulmonata are generally omitted, though a few fiesh-water forms inadvertently included have been permitted to re. main. To save space, abbreviations have been user. Those relating to the publication in which a species is enumerated are to be found in the bibliograph 5 , each following the title to which it refers, inclosed betwern parentheses. A list of abbreriations used for localities follows the bibliography. A dagger $(\dagger)$ after a title signifies that names iuchuled in that particular work which have already been citerl from more precise evidence are not extracted for use in the List. This applies chiefly to certain catalogues where most of the species are merely rited by name and without locality. In the List an asterisk (or '11. s.') after a name indicates that the species is described at the place cited. Not all the descriptious are thus indicated, however, and in some instances citations of plate and figure are not given with the citation of the page, although a figure exists in the work referred to. It was not practicable to correct or supply all omissions of this chanater in the proofs. Those who have read proof of such matter will be disposed to deal charitably with errors overlooked by the writer in examining more than fhirteen thousand entries. Corrections and additions will be thankfulty received and hereafter acknowledged on a proper occasion.

WASHINGTON, July 1, 1885. 


\section{BIBLTOGRAPHY.}

Adams (Charles B.). Specierum norarum conchyliorum in Jamaica re. pertorum synopsis. (C. B. Ad., Synops.)

In Boston Society of Natural History; Proceedings. Boston, the societร, 1s45. Vol. ii, pp. 1-17, Jan,, 1845. 8 .

Contributions to conchology. New York, H. Ballière, Oct. 1849-Nov. 1852. (Contr. to Conch.)

Vol. i, ir, $258 \mathrm{pp} . \quad 8^{\circ}$. This was published in short, carefully dated parts, the lates of which it seems unnecessary to cite.

Mougraph of Vitrinella, a new genus of new species of Turbinidæ. Amberst, Mass, the author, Feb. 1850. (Mon. Vitr.) 10 Pl. है?

American Journal of Conchology, edited by George W. Tryou, jir. Philadelphia, G. W. Tryon, jr., 1865-1866.

$\approx$ vols. \$lso:

The same. Philadelphia, Conchological Section of the Academy of Natural Sciences, 1867-1872. (Am. Jour. Conch., or A. J.) 5 vols. $8^{\circ}$.

Arango y Molina (Rafael). Contribuciou á la famma malacologica Cubaua. Habana, G. Montiel y Comp., 1878. (Arango, Famua Mal. Cuba.)

Pp. 280, 35. So. This work was first printed in the Anales de la Real Acidemia de Ciencias Mélicas, Físicas y Naturales de la Habana, begiuning in March, 1878; to signature 3, Мау 15, 1878; to siguature 12, January 15, 1879; to signature 14, February 15, 1879; to signature 15 , April 15, 1879 ; to siguature 17 , June 15,1879 ; and the remainder July 15 , 1880 , with a separately paged index.

Beau (Commandant). Catalogne de coquilles recueillies à la Guadalonpe et ses dépendances. Par Mr. Beau, chef de bataillon d'infanterie de la marine. Précédé d'une introduction par M. P[aul] Fischer. Paris, Paul Dupont, 1858. (Beau.)

Pp. 27. 80. Ext. de la Revue Coloniale. 80. Paris, Paul Dupont, Déc., 1857. Title on cover.

Binney (William G.). Bibliography of Yorth American conchology previous to the year 1860 . Washington, the Smithsonian Institution, $1863-1864$.

2 v. Vol. i, viii, 650 pp. ; vol. ii, iv, 298 p). 80. This is Smithsonian Miscellaneous Collections No. 174. From the information contained in it a large number of references have been cited, where bat a fow species were mentioned in a given publication, and referred in the following list to their places of publication. The numerous papers of this sort are not separately eited here, as they would have tended to unduly swell the limits of this bibliography withont any corresponding gain. 
Boston Journal of ITatural Fistory, containing papers and communications read to the Boston Society of Natural History 1834[-]1sen, pub. lished by their direction. Boston, [varions publishers] for the society, 1834-1863. (Bost. Jour. Nat. Hist.)

7 vole. 80.

Bush (Katherine T.). Additions to the shallow-water inollusea of Capo Hatteras, N. C., fredged by the U.S. Fish Commissiou steamer "Albatross" in 1883 and 1884. (Bush, Conn. Ac.)

In Transactious Connectieut Academy of Sciences, New Haven, Conn., vol. vi, pp. 453-480, pl. zlv. 'June, 1885.

Calkins (William W.). Marine shells of Florida.†

Ext. Daveuport Academy of Natural Seiences; Proceedings. Davenport, Iowa, the society, 1878. Vol. ii, pp. 232-252, pl. viii. 80. Extract with bastard title, repeated on cover, pagination of original preserred. Slips with addenda were issued by the author on several occasions. This catalogue is partly a compilation. The new or specially interesting species are quoted by Dail (Hemplill's Shells, $q$. v.) and are cited in the following list from that paper.

Conad (Timothy Abbott). Descriptions of new species of fossil and recent shells and corals.

In Academy of Natural Sciences of Philadelphia; Proceedings, vol. iii, pp. 2327, pl. 1-2, Feb., 1846.

Descriptions of two new genera and new species of recent shells, \&c.

In Acadeny of Natural Sciences of Philadelphia; Proceedings, vol. ir, p. 121, Dec., 1848.

\section{Monograph of the genus Fulgur.}

In Academy of Natural Sciences of Philadephia; Proceedings, vol. vi, pp. 316319, April, 1853.

\section{Notes on shells.}

In Academy of Natural Sciences of Philadelphia; Proceedings, vol. vi, p. 320, April, 1853.

Synopsis of the genus Cassidnla IImmphrey and of a proposed new genus, Athleta.

In Academy of Natural Sciences of Philadelphia; Proceedings, vol. vi, pp. 448449, Dec., 1833.

Notes on shells, with descrintions of three recent and one fossil species.

In Academy of Natural Sciences of Philadelphia; Proceedings, vol. vii, pp. 31-" 23, Mar., 1854.

\section{Description of a new genus of the family Dreissenidæ.}

In Academy of Natural Sciences of Philadelphia; Proceedings, uew series, 1857, p. 167.

Descriptions of new fossil and recent shells of the United States.

In Journal of the Academy of Natural Sciences of Philadelphia, new series, vol. i, part iii, pp. 207-209, 280, pl. xxxix. 
Conrad (Timothy Abbott) - Continued.

Observations on the geology of a part of East Florida, with a catalogue of recent shells of the coast.

In American Journal of Science. New Haren,-B. Silliman and J. D. Dana, 1846. New series, vol. ii, pp. 36-45, 393-398, 1846.

Dall (William Healer). Reports on the results of dredeing, muler the superrision of Alexander $A$ gassiz, in the Gulf of Alexico and in the Caribbean Sea, 185-99, hy the U. S. Coast Snrrey steamer Blalie, Lientenant-Commander Sigsbee, U. S. Y., and C'ommancler J. R. Bartlett, U. S. N., commanding. XV. Preliminary report on the

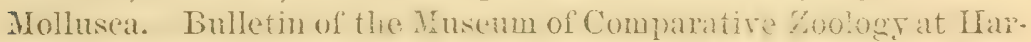
vard College. Cambriclese, for the musenm, July-Decemiex', 1 s\$s (Rep. Blake Moll.)

Vol. ix, No. 2, pp. 33-144. 80. This publication, separately issued as a bulletin, with title on cover, appeared originally in signatures as follows: Pp. 33-4२, July 12, 1831; pp. 49-64, Aug. 12, 1881 ; pp. 65-80, Aug. 25, 1881 ; pp. 81-96, Sept.26, 1881; pp. 97-112, Oct. 31, 1881; pp. 113-128, Nov. 26, 1881 ; pp. 129-144, Dec. 5, 1881.

On a collection of shells sent from Florida by Mr. Henry Hemphill. (Hemphill's Shells.)

In U. S. National Musoum; Proceedings. Washington, the Ninseum, Dec., 1883. Vol. vi, pp. 318-342, pl. x. $8^{\circ}$. The new or specially interesting species signalized by Calkins and Melvill $\left(q . v_{0}\right)$ are enumerated in this article, besides those sent by Hemphill.

D'Orbigny (Aleide Desealines). Histoire plysigne, nolitiqne tat uaturello de l'île de Cuba. Par M. Ramon de la Sagra (\&e.). Mollúsques. Paris, Bertrand, 1853. (Moll. Cuba.)

2vols. $8^{\circ}$. Vols. i, 21 unp., 264 pp.; vol. ii, 2 1. unp., 380 pp. Atlas folio, 11. , xxix pl. u. d. [1842]. This publication, forming one of Sagra's series, but independently issued in tho French language, appeared irregularly as follows: Vol. $i$, signatures 1-14 in 1841, signatures 15-17 and atlas in 1842; rol. ii, signatures 1-7 in 1842, signatures 8-24 in 1847-1853. The two rolumes were issued as a whole in 1853, with the latter date on the title-page. There is an edition in Spanish, conformable with the rest of the Spanish series of the work, which the compiler has nut been able to consult, but which seems to have a widely different pagination, though the plates are the samo.

Dunker (Dr. Wilhelm). Noritates conchologiere. IIollusa marina.

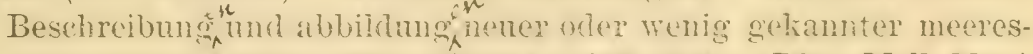
conchylien. Cassel, Then. Fiseher, 1859-1850. (Dhir, Moll. Mar.) $144 \mathrm{pp} .44^{\circ} .45 \mathrm{pl}$.

Folin (Leopoid, Marguis de). On the mallusen of II. M. S. Challenger

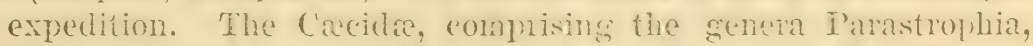
Watsonia, and Chem. With prebitore note by the lier. liobert Boog Watson, B. A., F. R. S. E., F. L. S., \&c. (De Folin, P. Z. S.)

Ext. Zool. Sor. London: Proceclines for 1r-ag, with hasiard title. London, tho society, 1880. Pp. 806-81\%. $8^{\circ}$.

Gould (Dr. Augustus Adrisuir). Descriptious of new grencra aud species of shells. (Gould.)

In Boston Society of Natural History; Proceedings. Boston, tho society, 1862. Vol. viii, pp. 280-284. 80 . 
Gould (Dr. Augnstus Addison)-Continued.

\section{Gld., O.)}

$256 \mathrm{pp} .80$

Guppy (R. J. Lechmere). First sketch of a marine invertebrate fauna of the Gulf of Paria and its neighborhood.t (Gulpy, Paria Faunas)

In Scientific Association of Trinidad; Proccedings. Portofspain, J. Wulff; London, 'Truibner, Dee., 187\%. Vol. ii, part xi, p1). 134-15\%. $8^{\circ}$.

\section{4.)}

On the West Indian Tertiary fossils. $†$ (Guppy, Geol. Mag.,

In Geological Magazine, decade ii, vol. i, Nos. 9 and 10, Sept. and Oct., 1874, pp. 433-454, pl. xvi-xviii. Also a supplement of one page, from the same, Jan., 18\%5. Extras repaginated and issued with the plates and supplementary leaf, with title "West Indian Tertiary fossils" on cover. Total pp. 22. 8०. London, Triibner, 1874.

Higgins (Rev. Пenly II.) [aud Rarratt (Fredrrick P.)]. Free public library, museum, and gallery of art of the borough of Liverpool. Musemu report No. 1. Mollusea of the Argo expedition to the W'st Indies, 18i6. Liverpool, D. Marples \& Co. [1878]. (Marratt, Argo Exp., 1876.)

20 pp. 8०. $1 \mathrm{pl}$. An important contribution to the geographical distribution of mollusea in the West Indies, The species were chiefly identified by Mr. Marratt, who is ou that account cited as authority for their occurrence.

Holmes (Prof. Francis S.). Post-pleiocene fossils of South Carolina. Charleston, S. C., Russell \& Jones, 1858-1860. (Post-pleiocene Fossils S. C.)

VI, 122 pl., xxviii pl. 4\%. See also TuOMEY AND HoLMes. This work was published in 16 parts, of which 98 pages and 14 plates are devoted to invertebrates. The remainder, an account of the vertebrate fossils, are by Dr. Joseph Leidy, and partly belong to the Eocene formation. In neither this nor the Pliocene volume are the unpaginated sheets with plato references counted above as pages.

Jahrbuicher der deutschen malakozoölogischen gesellschaft. Redigirt von. Dr. IV. Kobelt. Frankfurt am Main, Johannes Alt, 1S74-1878. 6 rols. 8\%. Also :

The same. Fraukfurt am Main, Alt \& Neumann, 1879.

1 vol, $8 \circ$. Also :

The same. Frankfurt am Main, Moritz Diesterweg, 1850-1884. (Jahrb.)

5 vols. $8 \circ$.

Jones (T. Matthew, F. L. S.). Contributions to the natural history of the Bermudas. Part I. Mollusea. (Moll. Bermudas

In Nova Scotian Institute of Natural Science; Transactions. Halifax, the society, 1864. Vol. ii, part ii, p1. 14-26. 80.

Journal de Conchyliologie, comprenant l'otude des animanx, des coquilles vivantes et des coquilles fossiles. Publié sous la direction de M. Petit de la Saussaye. Paris, the editor, 1850-1853.

4 vols. $8^{\circ}$. Also: 
Journal de Conchyliologie. Publić sous la direction de MM. Fischer et Bernardi. Paris, Bernardi, 1856 [juillet]-1860 [janvier].

4 rols., $8^{\circ}$ [ending the first series], and 4 vols., $8^{\circ}$ [forming the second series], or 8 vols. 80 . Also:

Publié sous la direction de MM. Crosse et Fischer. Paris, Crosse, 1861-1883.

[Third series] 23 vols. 8०. Also:

Index général et systématique des matì̀res contenues daus les vingt premiers volumes du Jourual de Conchyliologie (\&c.), 1850-1872. Paris, H. Crosse, 1878.

1 vol. viii, $200 \mathrm{pp} .8^{\circ}$. The above series of thirty-five volumes is indicated as Jour. de Conch., or J. C., in the subsequent list, which contains indered the names of all species referred to in any way in the Journal as belonging to the region and systematic groups for which the list was compiled.

[Krebs (Henry)]. The West Indian marine shells, with some remarks. A manuscript printed for circulation between collectors. By * * *. [Kjöbnhavn.] Printed by W. Laubs' widow and Chr. Jörgensen, Nykjöbiug, Falster, 1864. (Krebs.)

3 prel. 1. unp., $137 \mathrm{pp}$. $12 \circ$. The following mentiou of the circumstances attending the printing of this extremely rare, anonymonsly issued, yet scientifically valuable pamphlet oceurs in a letter from the author, dated Dec. 1, 1884: "I beg to inform you that the [above pampllet] was only printed in 20 copies, of which 3 were, according to law, delivered to the public libraries [of Copenhagen], 7 wero lost in transmitting them to St. Thomas, 3 went to the universities of Sweden and Norway, and a few [were] given to friends." "Consequently there are none for sale. My friends tease me that the book is the costliest they know, on account of a copy has been sold in Altona, at auction, for 10 Rd." A copy presented by the author to Mr. Thomas Bland, and given by that gentleman to Hir. Johu H. Redfield, has, with great liberality, been presented by the latter to the library of the U. S. National Musenm. All the speeies in it have been cited under Krebs in the following list.

Remarks on some species of West Indian marine sletls in the museum of Amherst College. (Krebs, Remarks.)

In Lyceum of Natural Histery of Now York; Annals. New York, the society, 1866. Vol, viii, 1866, pp. 394-398. $8^{\circ}$.

Catalogne of marine mollusks collected in the Bahama Islands in November, 1866.† (Krebs, Catalogue.)

In Lycenu of Natnral History of New York; Aunals. New York, the society 1866. Vol, viii, 1866, pp. 4:7-431. 80.

Kurtz (Lieut. John D.). Catalogue of recent marine shells fonnd on the coasts of North and South Carolina. Portland, David Tucher, $1860 . \dagger$ (Kurtz.)

9 pp. $8^{\circ}$.

Fagasin de zoologie. Première année, prenière partie, classe V', mollusques. Planches 1 à 40. Paris, Lequien fils, 1831.

42 l. unp., 40 pl. $8^{\circ}$. Also :

Jomnal destiué à établir une correspondance entre les zoologistes de tous les pays et à leur faciliter les moyens de publier les espèces nourelles ou pen commes qu'ils possèlent, Publié par 
IVagasin de zoologie-Continued.

F. W. Guérin-Méneville (\&c.). Deuxième section. Mollusques et zooplytes. Paris, A. Bertrand, 1831-1839.

2 vols. Vol. i [texte], 206 1. unp. ; vol. ii [plancheo], 119 pl. 80. Also:

Iragasin de zoologie, d'anatomie comparée et de paléontologie: recueil destiné à faciliter aux zoologistes do tous les pays les moyens de publier leurs travax, les espèces nouvelles qu'ils possèdent, et à les tenir surfont au courant des nourelles découvertes et des progrès de la science, pair M. F. E. Guérin.héneville. Denxième section. Mollusques et zoophytes. Anuées 1839 à 1814 . Paris, reuve Bertrand, 1844. (Mag. de Zool., or M. Z.)

2 v. [Texte] 250 1. unp. $8^{\circ}$. [Planches] iv pp., 150 pl. $8^{\circ}$. This publication seems to have been printed with leaves numbered only to correspond with the plates or with the separate articles, which were afterward divided up in sections, each class being bound and sold separately.

Nalakozoologische Blätter. AIs fortsetzung der Zeitschrift für malakozoologie. Derausgegelen von Karl Theodor Menke, in Pyrmont, und Dr. Lonis I'feifer, in Cassel. Cassel, Theoulor Fischer, 1854-1862. 8 vols. $8^{\circ}$. Also:

The same. Herausgegeben von Dr. Louis Pfeiffer, in Cassel. Cassel, Theodor Fischer, 1862-1872.

10 vols. 8 . Also:

The same. Herankgegeben ron Dr. Lunis Pfeiffer, in Cassel, und Dr. Wr. Kobelt, in Schwanheim. Cassel, Theodor Fischer, 18721874.

3 vols. $8^{\circ}$. Also:

The same. Heransgegeben ron Dr. Louis Pfeiffer, in Cassel. Cassel, Theodor Fischer, 1875-1877.

3 vols. 8o. Also:

Dr. Lndwig Pfeiffer's Malakozoologische blätter für 1878. Fortgesetzt von S. Clessin. Cassel, Theodor Fischer, 1878.

1 vol. 8 . Also:

Malakozoologische blätter. Als fortsetzung der Zeitschrift für malakuzoologie. Herausgegeben ron S. Clessin. Treue folge, erster[-sedhiter] band. Catsel, Theodor Fischer, 1879-1893. (Mal. B1., or M. B.)

6 vols. $8^{\circ}$. The earlier volumes of this series carried the date of issue on each siguature. Later volumes are without it, and there is no means of determining the date of issue, which often was not within the year to which the volume ostensibly refers.

Milelvill (Jame: Cosmo, A. M., F. L. S.). List of the mollnsea obtained in Sonth Carolina and Florida, prineipaliy in the ishand of Key West, 1871-1572.

In Journal of Conchology. Leeds, J. Taylor, 1881. Vol. iii, Nos. 5, 6, pp. 155173. 120. The new or especially interesting shells mentioned in this catalogue are quoted by Dall (Homphill's Shells), and here eited from that source. Tho catalogue contains many erroneous identifications, and is therefore not cited in full. 
Mörch (Otto Audreas Lowson). Catalogue of the West Inclia shells in the collection of Dr. C. Mr. Poulsen, Kastanievei 5, Copenhagen. Copenhagen, Bianco Luno, 1878. (Poulsen.)

$16 \mathrm{pp} .80$

Nachrichtsblatt der deutschen malakozoologisehen gesellschaft. Unter mitwirkung von D. F. Heynemann; redigirt von Dr. W. Kobelt. Frankfurt am Main, W. Kuchler, 1869.

1 vol. sm. $8^{\circ}$. Also:

The same. Frankfurt am Main, J. D. Sauerländer, 1870-1871. 2 vols. 80. Also:

The same. Redigirt vou Dr. W. Kobelt. Frankfurt am Main, J. D. Sauerländer, 1872.

1 vol. 80. Also:

The same. Redigirt ron Dr. W. Kíbelt und D. F. Heynemann. Frankfurt am Main, Johannes Alt, 1873.

1 vol. $8^{\circ}$. Also:

- The same. Redigirt von Dr. IV. Kobelt. Frankfurt am Main, Johanues Alt, 1S74-1S77.

5 vols. go. Also :

The same. Frankfurt am Main, Alt \& Neumaun, 1S7S-1S79.

2 rols. 8 . Also:

The same. Fraulifurt an Main, Míoritz Diesterweg, 1SS0-18S4.

(Nachblt.)

5 vols, go.

Pfeiffer (Dr. Lonis). Bericht iiber die ergebnisse meiner reise nach Cuba im winter 1838-9. (Pfeiffer, Archiv fiir naturg. 1839.)

In Wiegmann's Archiv fuir naturgeschichte, 1839, vol. i, pp. 346-358.

- Uebersicht ler in Januar, Februar, und Irïz, 1839, anf Cuba gesammelten molluslen. (Pfeifer, Archiv für naturg. 1S40.)

In same, 1840, vol. i, pp. 250-261.

Ravenel (Dr. Edmund). Catalorne of the recent and fossil shells in the cabinet of the late Ermumi liafenel. Charleston, S. C., Walker, Evans \& Cogswell, 1875.† (Ravenel, Cat.)

68 pp. 120

Foemer (Dr. Ferlinanil). Texas; mit einem naturwissenschaftlicheu anhauge. Bonn, Adolph Marcus, 1849. (Roemer, Texas.)

List in Binney's Bibliography N. Am. Conchology, part ii, pp. 11-12. New species only cited, book not seen.

Stearns (Robert Eitwarls Cari(2)). Descriptions of new species of marino mollusks from the coast of Florida.

Ext. Boston Society of Natural History ; Proceedings, vol. xv, pp. 21-24, Jan. 17, 18\%2. $80^{\circ}, 4 \mathrm{pp}$.

On a new species of Pedipes from Tampa Bay, Florida.

Ext. Boston Society of Natural History; Proceedings, vol. xiii, pp. 108-109, 1869. 1 leaf. $8^{\circ}$. Hoaded "Conchological Momoranda No, 4." 


\section{Stearns (Robert Edwards Carter) - Continued.}

Descriptions of new marine shells from the west coast of Florida.

Ext. Academy of Natural Sciences of Philadelphia ; Proceedings for 1873, pp. $344-347,1873.8^{\circ} .4 \mathrm{pp}$.

Stimpson (Dr. William). Descriptions of new shells.

In Boston Society of Natural History; Proceerlings, iv, pp. 112-114, 1851.

and Kurtz (Lieut. J. D.). Descriptions of new shells.

In Boston Society of Natural History; Proceedings, iv, pp. 114-115, 1851.

Tryon (George Washington, jr.). American marine conchology; or, descriptious of the shells of the Atlantic coast of the United States from Maine to Florida. Philadelphia, the anthor, 1873-1874. (Am. Marine Conch.)

$208 \mathrm{pp} ., 44$ pl. $8^{\circ}$. Issued in six parts, Nov., 1873, to Nov., 1874.

Tuomey (Michael) and Holmes (Francis S.). Pleiocene fossils of South Carolina; containing descriptions and figures of the Polsparia, Echinodermata, aud Mollusca. Charleston, S. C., Russell \& Jones, 1855-1857.t (Pleiocene Fossils S. C.)

1 vol, xvi, $152 \mathrm{pp} ., 32 \mathrm{pl} .4^{\circ}$. Issued in sixteen parts; of which six, of eight pages and two plates each, appeared in 1855 , the remainder with title, etc., in 1856. See also HoLmes (F. S.).

Verrill (Addison E.). List of deep water and surface Mollusca taken off the east coast of the United States by the U. S. Fish Commission steamers Fish Hawk aud Albatross, 1880-1883. (Verrill, List.)

Ext. Connecticut Academy of Sciences; Transactions. New Haven, the society, July, 1884. Vol. vi, pp. 263-290. $8^{\circ}$.

Watson (Rev. Robert Boog). Mollusea of H. M. S. Challenger expedition. Parts I-XX, 1879-1883. Preliminary report to Prof. Sir C. Wyville Thomson (\&c.). (Watson, Lin. Soc. Journ.)

Ext. Linnean Society, Journal. Zoology. London, the society, 1879-1883. Vols. xiv-xvii, 1879-1883. $8{ }^{\circ}$. See also Folin (L. de).

The separate parts appeared as follows:

Part I.-The Journal, vol. xiv, No. 78, pp. 506-507 ; read Nor. 21, 1878; pablished April 23, 1879.

II.-The Journal, vol. xiv, No. 78, pp. 508-529; read Nov. 21, 1878; published April 23, 1879.

III.-The Journal, vol. xir', No. 78, pp. 586-605; read Déc. 5, 1878; published April 23, 18:9. The preceding parts in one cover. Title on cover, and bastard title. $8^{\circ}$. London, Taylor \& Francis, 1879. Original pagination preserved.

IV.-The Journal, vol. xiv, No. 80, pp.694-716 ; read June 5, 1879 ; published Sept. 2, 1879. Covers and bastard title as in the preceding.

V.-The Journal, vol. xv, No. 82, pp. 88-126; read April 15, 1880 ; published July 31,1880 . This and succeeding two parts have no title on cover or elsewhere.

VI.-The Journal, vol. $x v$, No. 84 , pp. 218-230; read April 15, $18=0$; published Nov. 20, 1880. 


\section{Watson (Rev. Robert Boog) - Continued.}

Part VII.-The Jourual, vol. xv, No. 85, pp. 246-274 ; read Dec. 9, 1880 ; published March 25, 1881.

VIII.-The Journal, vol. xv, No. 86, pp. 388-412; read March 3, 1881 ; published Sept. 29, 1881. This part has bastard title, but none on cover.

IX.-The Journal, vol. xv, No. 87, pp. 413-455; read June 2, 1881; published Oct. 4, 1881. This part has no title.

X.-The Journal, vol. $x v$, No. 88, pp. 458-475; read June 16, 1881; published Nov, 3, 1881. No title.

XI.-The Journal, vol.xvi, No. 91, pp. 247-254; $\operatorname{read}$ Dec. 15, 1881; published March 8, 1882. No title.

XIII.-The Journal, vol. xvi, No. 93, pp. 324-343; read Dec. 15, 1881 ; published June 12, 1882. This part has bastard title.

XIII.-The Journal, vol. xvi, No.93, pp.358-372; read March 16, 1881; published June 12, 1882. This part has bastard title.

XIV.-The Journal, vol. xvi, No. 93, pp. 372-392; read March 16, 1882; pullished June 12, 1882. This part has bastard title.

XV.-The Journal, vol.xvi, No. 96, pp. 594-611; read June 15, 1882; published March 10, 1883. This part has bastard title.

XVI.-The Journal, vol. xvii, No. 97 , pp. $26-40$; read Nov. 16,1882 ; published Mareh 24, 1883. 'This part has bastard title.

XVII.-The Journal, vol.xvii, No. 99, pp. 112-130; read March 1, 1883; published July 31, 1883. This part has bastard title.

XVIII.-The Journal, vol. xvii, No. 101, pp. 284-293; read March 15, 1883; published Oct. 20, 1883.

XIX.-The Journal, vol. xvii, No. 101, pp. 319-340 ; read May 3, 1883; published Oct. 20, 1883.

XX.—The Journal, vol. xvii, No. 101, pp. 341-346; read June 21, 1883; publisbed Oct. 20, 1883.

Parts xviii-xx issued in one cover, title on the cover. London, Linnean Society [1883].

Zeitschrift für If́alakozoologie. Heransgegeben von Karl Theodor Menke. Hannover, Hahn, 1844-1845.

2 vols. $8^{c}$. Also:

Heransgegeben ron Karl Theodor Meuke und Dr. Louis Pfeiffer.

Cassel, Theodor Fischer, 1846-1853. (Zt. Mal.)

8 vols. 80 . See also Malakozoologische Blätter.

Bull. 24-2 


\section{ABBREVIATIONS FOR LOCALITIES.}

A. Aspinwall.

Aa. Antigua.

Ag. Anguilla.

Ba. Bahamas.

Bb. Barbados.

Bd. Barbuda.

Ber. Bermudas.

Bz. Brazil.

Ca. Cuba.

Cay. Cayenue.

Coa. Curaçoa.

CS. Caribbean Sea.

Cul. Culebra.

D. Dominica.

Fla. Florida.

G. Guadalupe.

Ga. Georgia.

Gtm. Guatemala.

Hatt. Cape Hatteras.

Hd. Honduras.

Hy. Haỹti.

J. Jamaica.

Lj. Lillienskjold.

M. Martinique.
MG. Marie-Galante.

NO. North Carolina.

NGr. New Granada.

NP. New Providence.

PPl. Porto Plata.

PR. Porto Rico.

SB. St. Bartholomew.

SO. South Carolina.

SCx. St. Croix or Santa Cruz.

SD. Santo Domingo.

SJ. St. John.

SM. Saint Martin.

ST. St. Thomas.

SV. St. Vincent.

Td. Trinidad.

Tort. Tortola.

V. Viéque.

VCz. Vera Cruz.

VD. Van Dyek's Island.

VgI. Virgin Islands.

Vz. Venezuela.

W. Water Island.

Y. Yucatan.

Z. Ziech.

Those names to which no locality is appended are either supposed to be generally spread over the region or are to be taken as from the local. ity for which the local list from which they are cited was intended to enumerate the fauna. A species cited from Beau without locality is supposed to be from Guadalupe; one eited from D'Orbigny or Arango, from Cuba, \&c. No indication of whether the species is fossil or recent is given, and any of them may be expected to be, as many are, found in either condition. 


\section{LIST OF MARINE MOLLUSCA.}

Abra æqualis Say, Am. Marine Conch. 154.

Abra angulata Holm., Post-Pleiocene Fossils S. C., pl. viii, fig. 8. 50.

Abra lioica (Dall) Verrill, List, 1884. 278. CS.

Abra longicallis Sars., Report Blake Moll. 133.

Abra longicallis (Scacchi) Verrill, List, 1884. 278. CS.

Abra (see Syndosmya).

Abralia megalops Verrill, List, 1854. 264. CS. WI.

Acanthopleura picea Chemn., Arango, Famma Mal. Cuba, 1878. 230.

Ca. J. ST. PR. M. G. Ba. (see Chiton).

Acar (see Barbatia) Marrat, Arso Exp., 1876. $\%$.

Achatina (see Odostomia) Arango, Fauna Mal. Cuba, 1878. 161.

Achatina pellucida Pfr., Mal. Bl., iv, 27. G.

Aclis helicteroides Guppy, Geol. Mag., 1874. 437. Td.

Acmra (see Patella).

Acmæa fungus Meusch., Krebs. 75.

Acmæa hamillei Fisch., Beau. 18. G.

Acmæa hamillei Fisch., Jour, de Conch., v, 276. G.

Acmæa hamillei Fisch., Krebs. 75.

Acmæa hamillei Fisch., Mal. Bl., iv, 220. G.

Acmaa leucopleura Gmel., Krebs. 75.

Acmæa melaleuca Gmel., Krebs. 75.

Acmæa melanosticta Gmel., Krebs. 75. ST.

Acmæa puncturata Lam. (sce Patella).

Acmæa pustula Helb., Krebs. 75. Bb. ST. SB. SM. SJ.

Acmæa subrugosa D'Orb., Jahr., ii. 246.

Acroloxus compressus Dkr, Arango, Fauma Mal. Cuba, 1878. 137. Ca. Acroloxus striatulus Dkr., Arango, Fanna Mal. Cuba, 1878. 138* Ca. Actæon caualiculata D'Orb., Krebs. 94.

Actæon cubensis Gabb, Mal. Bl., xxii, 170. Ca. J.

Actæon cubensis Gabb, Poulsen. 8.

Actæou cumingii A. Ad., Mal. Bl. xxii, 169. PR.

Actæon cumingii A. Ad., Poulsen. 8.

Actæon danaida Dall, n. s., Report Blake Moll. 96. CS.

Actæon exiguus Dkr., * Mal. Bl., xxii, 169.

Actæon exiguus Dkr., Poulsen. 8.

Actæon fasciatus Lam., Report Blake Moll. 94. CS.

Actæon floridanus Courad, Am. Jour. Sci., u. s., ii, 396. 1846. Fla. Actæou incisus Dall, u. s., Report Blake MIoll. 95. Cape Sau Antonio.

Yucatan Strait. 
Actæon melampoides Dall, n. s., Report Blaka Moll. 95. CS. Actæon ovulum Pfr., Mal. Bi., xxii, 170. Ca.

Actæon perforatus Dall, n. s., Report Blake Moll. 96. CS. Actron punctatus D'Orb., Dall, Hemphill's shells. 324. Cedar Keys. Actæon punctostriata Stimpson, Kurtz. 9. NC. SC. Actreon splendidula Mörch,* Mial. Bl., xxii, 170. ST. Actæon turritus Watson, Lin. Soc. Journ, 1883, xvii, 286. Cul. Actæonidea ovulum Pfr., Mal. Bl. xxii, 170. Ca. Acus concarus Say, Am. Marine Conch. 45. SC. Acus (Euryta) consentini Phil., Marrat, Argo Exp., 1876. 10. La Guayra.

Acus dislocatus Say, Am. Marine Conch. 45. Acus dislocatus Say, Dall, Hemphill's shells. 325. Cedar Keys. Acus hastata Gmel., Marrat, Argo Exp., 1876. 10. La Guayra, Nassau, Abaco.

Acus protextus Con., Dall, Hemphill's shells. 325. Sarasota Bay, Cedar Keys.

Acus (see Terebra).

Adeorbis adamsi Fisch., Arango, Fauna Mal. Cuba, 1878. 177. G. Adeorbis adamsi Fisch., Hemphill's shells. 335. Cedar Keys. WI. Adeorbis adamsii Fisch., Jour. de Conch., vi, 173. G. Adeorbis adamsii Fisch., ${ }^{*}$ Jour. de Conch., vi, pl. 10, f. 11. 287. G. Adeorbis adamsii Fisch, Poulsen. 13.

Adeorbis beauii Fisch., Jour. de Conch., vi, 173. G.

Adeorbis beauii Fisch., ${ }^{*}$ Jour. de Conch., vi, pl. 10, f. 12. 286. G. Adeorbis beauii Fisch., Poulsen. 13.

Adeorbis eyclostomoides Pfr., Arango, Fanna Mal. Cuba, 1878.' 177. G. Arleorbis inornatum D'Orb., Arango, Fauna Mal. Cuba, 1878. 178. ST. Adeorbis nautiliformis Holm., 11. s., Post.Pleiocene Fussils S. U., pl. xiv, figs. $8,8 a, 8 b .93$.

Adeorbis orbignyi Fisch, Jour. de Conch., vi, 173. Ca.

Adeorbis orbignyi Fisch., Jour. de Couch., vi, 286. Ca.

Adeorbis rerrauxii Fisch., Poulsen. 13.

Admiralis viearius Knorr, Mal. Bl., xxir, 15.

Adrana (see Leda) Marrat, Argo Exp., 1876. 12.

Aeolidiella oceidentalis Bergh, Mal. Bl., xxii, 178.

Ala accipitrina Mart., Arango, Fauna Mal. Cuba, 1878. 195. Ca. Hy. G.

Ala accipitrina Mart., Mal. B1., xxiv, 18.

Ala accipitrina Mart., Moll. Cuba, ii, 119.

Ala papilionis Ch., Mal. Bl., xxiv, 63.

Ala tuberosa, ete. Heb., Mal. Bl., xxiv, 20.

Alaba var. albo-tessellata Mörch, Mal. Bl., xxiii, 57. J.

Alaba incerta D'Orb., Jour de Conch., xxiv, 373. Ca.

Alaba incerta D'Orb., Mal. Bl., xxiii, 57. J.

Alaba melanura C. B. Adams,* Mal. Bl., xxiii, 57. J.

Alaba melanura C. B. Adams, Poulsen. 8. 
Alaba tervaricosa C. B. Adams, Mal. Bl. xxiii, 57. J.

Alaba terraricosa C. B. Adams, Poulsen. 8.

Alata aculeata Humph., Mal. Bl., xxir, 21.

Alata aratrum Humph., Mal. BI., xxiv, 20.

Alata lentiginosa Mart., Moll. Cuba, ii, 123.

Alata lobata, etc., Mal. Bl., xxiv, 19.

Alatus expansus, etc., Mal. Bl., xxiv, 16.

Albula hepatica Bolt., Mal. Bl., xxiv, 59.

Aletes conicus Mörch, Mal. Bl., xxir, 119.

Aletes retifer Mörch, ${ }^{*}$ Mal. B1., xxiv, 121.

Alexia bermudensis Ad., Mal. Bl., i, 151. Ber.

Alexia bermudensis Pfr., Jahrb., vii, 286. Ber.

Allerya krebsii Mörch, Mal. Bl., xxir, 100. ST.

Alvania auberiana D'Orb., Mal. Bl., xxiii, 54. Ca. J.

Alvania caribæa D'Orb., Mal. Bl., xxiii, 54. Ca.

Alvania emaciata Mörch, Mal. Bl., xxiii, 54.

Amalthea effodiens Opr., Jour. de Conch., x, 24.

Amalthea eftodiens Cpr., Mal. Bl., xxiv, 99.

Amalthea panamensis H. \& A. Ad., Jour. de Conch., x, 20.

Ammonia minor Brown, Moll. Cuba, i, 24.

Amuicola auberiana D'Orb., Beau. 17. G.

Amnicola candeana D'Orb., Beau. 17. Pointe-à-Pître.

Amnicola caudeana D'Orb., Jour. de Conch., v, 152. G.

Amnicola corouata Pfr., Arango, Fama Mal. Cuba, 1878. 139. Ca.

Amnicola (Paludina) coronata Pfr., Beau. 17. G.

Amnicola var. coronata Pfr., Beau. 17. G.

Amnicola cristalliua Pfr., Araugo, Fauna Mal. Cuba, 1878. 140. Ca.

Amnicola crystallina Pfr, Beau. 17. G.

Amnicola (Melania) spinifera C. B. Adams, Beau. 17. G.

Amphibulina patula Brug., Marrat, Argo Exp., 1876. 8. St. Kitt's.

Amphiclesma (see Semele) Arango, Fauna Mal. Cuba, 1S78. 246.

Amphidesma bellastriata Courad, Journ. Acad. Nat. Sci., rii, p. 239,

pl. xx, fig. 4, 1837. Mobile, Ala.

Amphidesma cancellata D'Orb., Jour. de Conch., ii, 424.

Amphidesma cancellata D'Orb., Moll. Cuba, ii, 241.

Amphidesma cancellata Sby., Beau. 25. SM,

Amphideswa cancellata Sby., Krebs. 106.

Amphidesma corrugatum Sby., Pfeiffer, Archiv fuir Naturg., 1840. 261.

Ca.

corrvegalusin is Peruve an.

Amphidesma decussata Wood, Beau. 25. G.

Amphidesma deforme Philippi, Roemer, Tex. 452. 1849. Tex. =

Amphidesma deforme Philippi,* Zt. Mal., r, 129. Tex.

$\checkmark$ Amphidesma equalis Say, Kurtz. 3. NC. SC. $=$ of the

Amphidesma jayanum C. B. Adams, Beau. 25. G. = pro

Amphidesma jayanum Ad., Krebs. 106.

Amphidesma jayanum C. B. Adams, Synops., 10, 1845. J. 
$\checkmark$ Amphrdesma obliqua Wood, Beau. ¿5. G. Amphidesma orbiculata Sas, Kuxtz. 3. NC. SC. Amphideswa ornata D'Urb., Jour. de Conch., ii, 424. G. Amphidesma punctatum Say, Kurtz. 3. SC. = Amphidesma purpurascens Lam., Krebs. 100. Amphidesma purpurascens Gmel., Beau. 25. G. Amphidesma radiata Say, Kurtz. 3. = prical?: Amphidesma reticulata D'Orb., Arango, Fauna Mal. Cuba, 1878. 247.

Ca. G. II. Ba. = motua. halle

Amphidesma retienlata D'Orb., Moll. Cuba, ii, 240.

Amphidesma reticulata Linu., Bean. 25. Pointe-à-Pître, \&c. Amphidesma reticulata Sby., Jahrb., ii, 249.

Amphidesma reticulata Sby., Jour. de Conch., iv, 414. G.

Amphidesma subtruncata Rre., Jour, de Conch., ir, 414. G. = ...th-me Amphidesma subtruncata Sby., Bean. 25. G,

Amphidesma transversa Say, Kurtz. $3 .=$ Mliqu e Amphidesma variegata Brug., Jour. de Conch., ii, 424. G. . Cima Amphidesma variegata D'Orb., Arango, Fauna Mal. Cuba, 1878. 247. Ca. Ba.

Amphidesma variegata D'Orb., Moll. Cuba, ii, 239.

Amphidesma variegata Lam., Arango, Fauna Mal. Cuba, 1878. 246. Ca. Ba.

Amphidesma variegata Lam., Beau. 25. G.

Amphidesma variegata Lam., Krebs. 106.

Amphidesma variegata Lam., Moll. Cuba, ii, 239.

Amphidesma variegata Sby., Moll. Cuba, ii, 239.

Anphiperas cauadinensis Mörch, Jahrb., ix, 178.

Ampulīaria ampullacea Born, Moll. Cuba, ii, 3.

Ampullaria chemnitzii Phil., Arango, Fauna Mal. Cuba, 1878. 138. Ca. Ampullaria conica Gray, Arango, Fauna Mal. Cuba, 1878. 138. Ca. Ampullaria conica Pfr., Arango, Fauma Mál. Cuba, 1878. 138. Ca. Ampullaria conica Wood, Arango, Fauna Mal. Cuba, 1878. 138. Ca. Ampullaria cubeusis Arango, Fanna Mal. Cuba, 1878. 139. Ca. Ampullaria effusa Mull., Bean. 17. G.

Ampullaria fasciata Lam., Moll. Cuba, ii, 2.

Ampullaria linnæi Phil., Marrat, Argo Exp., 1876. 15. VCz. Ampullaria reflesa Ptr., Arango, Famna Mal. Cuba, 1878. 139. Ca. Ampullaria retlexa Phil., Arango, Fauna Nal. Cuba, 1878. 139. Ca. Ampullaria reflexa Swains., Arango, Fauna Mal. Cuba, 1875. 139. Ca. Ampullaria teres Pfr., Arango, Fauna Mal. Cuba, 1878. 139. Ca. Ampullaria teres Phil., Araugo, Fauna Mal. Cuba, 1878. 139. Ca. Amussium lucilum Jeffreys, Report Blake Noll. 117. CS. Amussium lucidum Jeffreys, var. marmoratum, Report Blake Moll. 117. Amycla avara Say, Jahrb., ii, 324 .

Anachis (see Columbella).

Anachis acuta Stearus, Dall, Hemphill's shells. 326. Sarasota Bay. 
Anachis acuta Stearns, Proc. Phil. Acad., 1873. 345. Egmont Key, W. Fla.

Anachis arara Say, Dall, Hemphill's shells. 326. Key West.

Anachis nigricans Dall, Hemphill's shells. 326. Panama.

Anachis ostreicola Melvill, Dall, Hemphill's shells. 326. Cedar Keys. Anachis ostreicola Mrelvill, Dall, Hemphill's shells. 342.

Anachis semiplicata Stearns, Dall, Hemphill's shells. 326. Sarasota Bay. Cedar Keys.

Anachis semiplicata Stearns, Proc. Phil. Acad., 1573. 344. W. Fla.

Anachis (Seminella) Stearnsii, Tryon, NIan. r, 179, 1883. Fla.

Anaitis paphia Linn., Mal. Bl., xii, 167.

Anaitis varicosa Sby., Mal. Bl., xii, 172.

Ancillaria glabrata Linn., Arango, Fauna Mal. Cuba, 1878. 193. Ca.

WI.

Ancillaria glabrata Linn., Jahrb. vii, 106.

Ancillaria glabrata Linn., Moll. Cuba, ii, 112.

Ancillaria glabrata Linn., Poulsen. 12.

Aneillaria lienardi Bern., Jahrb., vii, 106.

Ancillaria pinguis Guppy, 11. s., Geol. Mag., 1874. 442. J.

Ancillaria tankervillei Swains., Jahrb., vii, 106. Coa.

Ancillaria tankervillii Swains., Poulsen. 12.

Ancistrosyrinx elegans Dall, n. s., Report Blake Moll. 54. CS.

Ancylus beanii Bourg., Bean. 15. G.

Ancylus chittyi C. B. Adams, Beau. 15. G.

Ancylus compressus Dlkr, Arango. Fauna Mal. Cuba., 1878. 137. Ca. Ancylus excentricus Mor., Arango, Fauna Mal. Cuba., 1878. 138. San

Vicente.

Ancylus havanensis Pfr., Arango, Fauna Mal. Cuba., 1878. 137. Ca.

Havana. San Miguel.

Ancylus obscurus Hald., Beau. 15. G.

Aneylus pallidus Poey., Arango, Fauna Mal. Cuba, 1878. 137. Ca.

Havana. San Miguel.

Ancylus petitianus Bourg., Beau. 15. G.

Ancylus radiatilis Mor., Arango, Fauna Mal. Cuba, 1878. 137. Ca.

Isle de Pinos.

Ancylus radiatus Guild., Araugo, Fauna Mal. Cuba, 1878. 137. Ca.

San Vicente.

Ancylus striatulus Dkr., Arango, Fauna Mal. Cuba, 1878. 138. Ca. Angaria crassa \#olm., n. s., Post-Pleiocene Fossils, S. C. pl. xiv, figs.

$7,7 a, 7 b .92$.

Angeli ala Mart., Moll. Cuba, ii, 119.

Anguinaria rubra Sehum., Mal. Bl., xxiv, 109.

Angulus (see Tellina) Marrat, Argo Exp., 1876. 12.

Anodara (see Area) antiqnata Gray, Krebs. 124.

Anodara notabilis Gray, Krebs. 125.

Anodonta alba Link, Krebs. 119. 
Anomalocardia antiquata Linn., Marrat, Argo Exp., 1876. 13. Santa Marta. (See Arca.)

Anomalocardia flexuosa Linn., Jour. de Conch., vii, 26. MIG.:

Anomalocardia flexuosa Linn., Jour. de Conch., vii, 37.

Anomalocardia flexuosa Linn., Beau. 24. G.

Anozhalocardia flexuosa Linu., Dall, Hemphill's shells. 341. Key

West.

Anomalocardia florirlana Con.,* Am. Jour. Conch. (pl. xiii, fig. 2), r,

108. Fla. (See Arca.)

Anomalocardia membranula Roem.," Mal. Bl., vii, 163. ST.

Anomalocardia nux Sby.,? Marrat, Arge Exp., 1876. 15. Havana.

Anomalocardia pulla Pfr., Mal. Bl., vii, 164. Mataryas, Éba

Anomalocardia secticosta Rre., Am. Jour. Conch., r, 108.

Anomia (see Hyalæa), Arango, Fauna Mal. Cuba, 1578. 150. Antilles.

Anomia ephippium Linn., Arango, Fauna Mal. Cuba, 1878. 273. Ca.

M.

Anomia ephippium Linn., Kurtz. 6. NC. SC.

Anomia glabra Verrill, Am. Marine Conch. 194.

Anomia simplex D'Orb., Arango, Fanna Mal. Cuba, 1878. 273. Ca. M.

Anomia simplex D'Orb., Bean. 21. G.

Anomia simplex D'Orb., Krebs. 137.

Anomia simplex D'Orb., Molì. Cuba, ii, 367.

Anomia simplex D'Orb., Pouisen. 16.

Anomia squamula Liun., Am. Marine Conch. 195.

Anomia tridentata Grmel., Moll. Cuba, i, 72.

Antalis agilis G. O. Sars, Report Blake Moll. 37. (See Dentalium).

Antalis iuversa Desh., Marrat, Argo Exp. 1876. 10. La Guajra.

Anus rugosa? Chem., Arango, Fauna Mal. Cuba, 1878. 250. Ca. G.

Ba.

Anus rugosa Chem., Moll. Cuba, ii, 273.

Aphelodoris antillensis Bergh, Mal. Bl., xxvi, 108.

Aplustrum physis L. et D'Orb., Krebs, p. 92.

Aplysia (see Notarchus), Arango, Fauna Mal. Cuba, 1878. 156. ST.

$\mathrm{SCx}$.

Aplysia cailleti Deshayes, Jour. de Conch., vi, 140. G.

Aplysia fasciata Poiret, Jour. de Conch., xi, 22.

Aplysia nigra, D'Orb., Guppy, Paria Fauna, 1877. 137.

Aplysia parvula, Guild.,* Jour. de Conch., xi, 22. ST.

Aplysia parvula Guild., Mal. Bl., xxii, 176. ST.

Aplysia parvula Guild., Poulsen. 8 .

Aplysia pleii Rang, Moll. Cuba, i, 118.

Aplysia protaa Rang, Beau. 20. Ilet-à-Cochons.

Aplysia protea D'Orb., Arango, Fauna Mal. Cuba, 1878. 155. Ca.

ST. SCx. Ba. M.

Aplysia protea Rang, Arango, Fauna Mal. Cuba, 1878. 155. Ca. ST.

SOx. Ba. M. 
Aplysia protea Rang, Dall, Hemphill's shells. 324. Key West. Aplysia protea Rang, Jour. de Conch., xi, 23. ST.

Aplysia protea Rang, Krebs. 91. ST. NGr.

Aplysia protea Rang, Mal. Bl., xxii, 176. ST.

Aplysia protea Rang, Moll. Cuba, i, 117 .

Aplysia protea Rang, Poulsen. 8 .

Aplysia schrammii Deshayes, Jour. de Conch., vi, 140.

Aporrhais occidentalis Beck, Mal. Bl., xxiv, 23.

Area (see, also, Anodara, Anomalocardia, Argina, and Barbatia).

Arca adlamsi Shuttlew., Arango, Fauna Mal. Cuba, 1878. 263. Ca. G.

Area adamsi Shuttlew., Jour. de Conch., v, 150. G.

Arca adamsi Shuttlew., Krebs. 124. ST. PP.

Arca adamsii Shuttlew., Beau. 22. G.

Area adamsii Shuttlew., Ponlsen. 15.

Area americana D'Orb., Arango, Fauna MIal. Cuba, 1878. 262. Ca. M.

G. Santa Lucia, Ba. ST.

Arca americana D'Orb., Jahrb., ii, 253.

Arca americana D'Orb., Jour. de Conch., ir, 415. G.

Area americana D'Orb., Krebs. 126.

Arca americana D'Orb., Moll. Cubsi, ii, 317.

Arca americana Gray, Am. Marine Conch. 179.

Arca americana Gray, Arango, Fauna Mal. Cuba. 1878. 262. Ca. Fla. Arca americana Gray, Beau. 22. G.

Arca americana Gray, Krebs. 124.

Area americana Gray, Moll. of Bermudas. 25

Area americana Gray, Moll. Cuba, ii, 320.

Area americana Gray, Poulsen. 16.

Area americana Kurtz. 5. NO. SC.

Arca americana Law., Marrat, Argo Exp., 1876. 11. Puerto Cabello.

Area americana Rve., Moll. Cuba, ii, 320.

Area angulata Meusch., Krebs. 126.

Area angulosa Gmel., Krebs. 126.

Arca antiquata Limn., Arango, Fauna Nal. Cuba, 1875. 263. Ca. Ba.

Area antiquata Linn., Krebs. 124. VgI.

Area antiquata Linn., Moll. Cuba, ii, 322.

Arca autiquata Linn., Poulsen. 16.

Area aurienlata Delessert, Moll. Cuba, ii, 321.

Arca auriculata D'Orb., Arango, Fauna Mal. Cuba, 1878. 262. Ca. M. ST.

Arca auriculata Lam., Arango, Fauna MIal. Cuba, 187s. 262. Ca. M. ST.

Area auriculata Lam., Krebs. 124.

Area auriculata Lam., Moll. Cnba, ii, 321.

Arca auriculata Rve., Moll. Cuba, ii, 321.

Arca aurita Brocehi, Report Blake Moll. 118. CS.

Arca barbadensis D'Orb., Arango, Fauna Mal. Cuba, 1878. 261. Ca. J. 
Area barbadensis Petiver, Arango, Fanua Mal. Cuba, 1Sis. 261. Ca. J. Area barbadensis Petiver, Krebs. 124.

Area barbadensis Petiver, Moll. Cuba, ii, 321.

Arca barbata Kurtz. 5. NC. SC.

Area barbata Linn., Arango, Fanna Ma!. Cuba, 1878. 263. Ca.

Area bicops var., Chemn., Arango, Fauna Mal. Cuba, 1878. 262. Ca.

M. G. Bz.

Arca bicops D'Orb., Moll. Cuba, ii, 318.

Area bisuleata Lan., Jahrb., ii, 2\%3. = A Achelingi Brig. (neivea Cl.) Area brasiliana Lam., Arango, Fauna Mal. Cuba, 1878. 262. Ca. M. G. Area brasiliana Lam., Beau. 22. G.

Area brasiliana Lam., Jour. de Conch., v, 151. G.

Area brasiliaua Lam., Krebs. 124.

Area brasiliana Lam., Moll. Cuba, ii, 318.

Area brasiliana Lam., Poulsen. 16.

Area brasiliana Phil., Míoll. Cuba, ii, 318.

Area campechensis Gmel., Arango, Fauna Mal. Cuba, 1878. 262. Ca. Fla.

Arca candida Chemn., Arango, Fauna Mal. Cuba, 1878. 263. Ca. M. Fla. G.

Arca candida ? Chemn., Jour. de Conch., ir, 415. G.

Arca candida Chemn., Moll. Cuba, ii, 319.

Area candida D'Orb., Arango, Fauna Mal. Cuba, 1878. 263. Ca. M.

Fla. G.

Arca candida Gmel., Moll. Cuba, ii, 319.

Area candida Helb., Beau. 22. G.

Area candida Helb., Krebs. 124. ST. Td.

Area candida Helb., Mal. Bl., xvi, 236.

Arca candida Helb., Poulsen. 15.

Area cardiiformis Rre., Moll. Cuba, ii, 318.

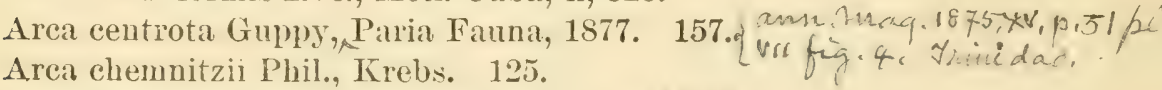

Area chemnitzii Phil., ${ }^{*}$ t. Mal., viii, 50. $\$ ₹ \Im$ l.

Area costata Meusch., Krebs. 127.

A rea decussata Linn., Arango, Fauna Mal. Cuba, 1878. 260. Ca. Santa

Licia. G. Ba.

Arca deshayesii Hanl., Jour. de Conch., vii, 27. G.

Arca deshayesii Hanl., Beau. 22. G.

Area deshayesii Hanl., Krebs. 125.

Arca deshaysii Phil., Krebs. 124.

Area deshajesii Phil., Moll. Cuba, ii, 322.

Arca deshayesii? Rve., Jour. de Conch., ii, 426. G.

Arca deshayesii Rve., Moll. Cuba, ii, 322.

Area divaricata Sby., Krebs. 126.

Arca domingensis Lam., Krebs. 126.

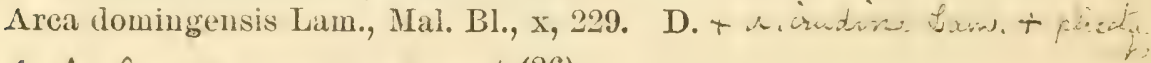

3. de ke. 53, p. 305, zeb.1906 (26) 
Arca domingensis Lam., P'onlsen. 15.

Area dominguensis Lan., Arangzo, Fanua Mal. Cuba, 187S. 263. Ca. Area (Barbatia) dominguensis Lam., Dall, Hemphill's shells. 341.

Key West.

Arca donaciformis ? Hanley, Jour, de Conch., iv, 415. G.

Arca donaciformis Rre., Arango, Fauna Mal. Cuba, 1878. 264. Ca. G. Ba.

Arca donaciformis Rve, Beau. 22. G.

Arca donaciformis Rve., Krebs. 126.

Arca elegans Phil., Zt. Mal. ir, 92. Y.

Area floridana Con., Guppy, Paria Fauna, 1877. 157.

Area fusca Brug., Arango, Fanna inal. Cuba, 1878. 263. Antilles. G. Arca fusca Brug., Krebs. 125.

Area fusca Brug., Moll. Cuba, ii, 319.

Arca fusca ? Chem., Jour. de Conch., ir, 415. G.

- Arca fusca Dorb., drangu, Fanma Mal. Cula, 187s. 263. Antilles. G. Area fusca Lan., Moll. Cuba, ii, 319.

Area glomerula Dall, n. s., Report Blake Moll. 121. CS.

Arar (Barbatial) geradtatal Broul., Dall, Hemphill's shells. 341. Key

West.

Arca gradata Brod., Krebs. 126.

Arca gradata Brod. and Sby., Mal. Bl., x, 229. D.

Arci gradata Brod., Moll. of Bermudas. 20 .

Area granulata Meusch., Krebs. 125.

Area helblingi Chemn., Arango, Fanna Mal. Cuba, 1878. 263. Ca. Arca helblingii Brug., Arango, Fanua Mal. Cuba, 1s78. 263. Ca. M. Fla. Area helblingii Brug., Bean. 22. G.

Arca helblingii Brug., Jour. de Conch., iv, 415. G.

Arca helblingii Brug., Krebs. 124.

Area helblingii Brug., Mal. Bl., xvi, 236.

Area helblingii Brug., Moll. Cuba., ii, 319-20.

Area hemiclemos Dorb., Arango, Fauna Mal. Cuba, 1878. 263. Ca. M.

Area hemidermos Phil., Arango, Fama Mal. Cuba, 1s78. 263. Ca.M. Arca hemidermos Phil., Krebs. 124.

Area hemidermos Phil., Moll. Cuba, ii, 322.

Arca hians T. \& H., n. s., Pleiocene Fossils S. C., pl. xir, figs. 4 and 5. 34.

Arca holmesii Kurtz, Am. Mariue Conch. 179. NC. SC.

Area holmesii Kurtz, u. s., Kurtz. 5. NC. SC.

Area imbricata Brug., Krebs. 126.

Arca improcera Comrad, Am. Journ. Sci., n. s., vol. 1, p. 40t, 1816.

Southern Coast U. S.

Area incongrua Say, Moll. Cuba, ii, 317.

Area incongrua Sìy, Am. Harine Conch. 179.

Arca incongrua Say, Krobs. 125. NGr. 
Arca indica Gmel., Beau. 22. G.

Arca indica Gmel., J.our. de Conch., v, 151. G.

Area indica Gmel., Krebs. 125.

Arca indica Gmel., Poulsen. 16.

Arca jamaicensis Gmel., Arango, Fauna Mal. Cuba, 1878. 263. Ca.M.

Fla. G.

Arca jamaicensis Gmel., Moll. Cuba, ii, 319.

Arca Tabiata Sby., Beau. 22. G.

Area labiata Sby., Krebs. 125.

Arca labiata Sby., Poulsen. 16.

Arca lactea Linn., Beau. 22. G.

Arca lactea Linn., Jour. de Conch., ii, 426. G.

Arca lactea Linn., Krebs. 125.

Area listeri Phil., Arango, Fauna Mal. Cuba, 1878. 263. Ca. Ba Area listeri Phil., Krebs. 125.2 VgI. = Mrea Cancecersia foam Arca listeri Phil., Poulsen. 15.\} + A.cellifera bami.

Area maxtinii Recluz., Dkr. Moll. Mar., pl. xlv, f. 1-4. 133. Brazil.

Arca martinii Recluz., Jahrb., ii, 253.

Arca morliola Linn., Krebs. 129.

Area modiolus Linu., Arango, Fama Mal. Cuba, 1878. 265. Ca. Ba.

Arca mutabilis Rve., Krebs. 126.

Area nivea Ch., Krebs. 125.

Area noæ Kurtz. 5. NC. SC.

Arca noæ Linn., Am. Marine Conch. 178.

Area noæ Limn., Beau. 22. G.

Arca noæ Linn., Jour. de Conch., ii, 426. G.

Area nox Linn., Krebs. 125. VgI.

Area noæ Linn., Mal. Bl., xii, 126. ST.

Area noæ Linn., Moll. of Bermudas. 25.

Area noe D'Orb., Moll. Cuba, ii, 316-18.

Area noe D'Orb., Moll. Cuba, ii, 322.

Arca notabilis Bolt., Krebs. 125.

Area notabilis Bolt. $=$ A. deshayesii Hanl., Poulsen. 16.

Arca occidentalis Phil., Krebs. 125.

Arca occilentalis Phil., Marrat, Argo Exp., 1S76. 11. Puerto Cabello.

Arca occidentalis Phil., Poulsen. 15.

Area oralis Brug., Arango, Fauna Mal. Cuba, 1878. 262. Ca. Fla.

Arca ovata Gmel., Moll. Cuba, ii, 317.

Area ovata Gmel., Krebs. 124.

Arca pectinata Gmel., Krebs. 127.

Area pectunculoides Scacehi, Report Blake Moll. 121. CS.

Arca pectunculoides Scacchi, var. orbiculata, Report Blake Moll. 121.

Arca pectunculus Chem., Moll. Cuba., ii, 313.

Arca pectunculus minor Ch., Krebs. 127.

Area pectunculns Linn., Arango, Famna Mal. Cuba, 1878. 261. M. G.

Arca pectunculus Linn., Moll. Cuba, ii, 313.

see J. de le, 53, is, 303-4, fig. 1, 1906. (7e6.) 
Area pexata D’Orb., Arango, Fauna Mal. Cuba, 1878. 262. Ca. Fla. Arca pexata Gmel., Arango, Fauna Mal. Cuba, 1878். 262. Ca. Fla. Arca pexata Kurtz. 5 .

Arca pexata Phil., Moll. Cuba, ii, 320.

Arca pexata Say, Am. Marine Conch. 179.

Area pexata Say, Alango, Fanna Mal. Cuba, 1878. 262. Ca. Fla. Arca pexata Say, Krebs. 124.

Arca pexata Say, Moll. Cuba, ii, 320.

Arca polycyma Dall, n. s., Report Blake Moll. 122. CS.

Arca ponderosa Say, Am. Jour. Conch., i, 77. Fla.

Arca ponderosa Say, Am. Marine Conch. 178.

Arca ponderosa Say, Poulsen. 16.

Arca retusa Lam., Pfetfier, Archir für naturg., 1840. 260. Ca.

Area rhombea Born, Jahrb., ii, 253. ST.

Arca rhombea Born, Krebs. 125.

Arca rhombea Born, Poulsen. 16.

Area rhombea Brug., Pfeiffer, Archiv für naturg, 1840. 260. Ca.

Area rhombeal rar. Chem., Arango, Fanna Mal. Cuba, 1S7S. 262. Ca.

M. G.

Area rhombea var. Chem., Moll. Cuba, ii, 318.

Area rustica T. \& H., n. s., Pleiocene Fossils S. C., pl. xv, fig. 1, 39.

Area solida Sby., Mal. Bl. x, 229.

Area squamosa Lam., Arango, Fauna Mal. Cuba, 187S. 261. Ca. G. Ba. Area squamosa Lam., Krebs. 126. VgI.

Arca tenera C. B. Adams, Synops., 9, 1545. J.

Arca tetragona Poli, Marrat, Argo Exp., 1876. 17. Nassau.

Area transcersa Say, Am. Marine Conch. 178.

Arca trapezina Lam., Krebs. 124.

Area nubonata Lam., Arango, Famna Mal. Cuba, 1S7S. 261. Ca.

M. G. Santa Lucia. Ba. ST.

Arca umbonata Lam., Beau. 22. G.

Arca umbouata Lam., Dkr. Moll. Mar., 131, pl. xlir, f. こ̌-7. Antilles. Arca nmbonata Lam., Jahrb., ii, 253,

Arca umbonata? Lam., Jour. de Conch., ir, 415. G.

Arca umbonata Lam., Krebs. 126. ST. SCx. T.

Arca umbonata Lam., Mal. B1., ×; 229.

Arca umbonata Lam., Marrat, Argo. Exp., 1876. 6. Aa.

Area umbonata Lam., Ponlsen. 15.

Arca undata Chemn., Moll. Cuba, ii, 314.

Arca unclata Linn., Aiango, Fanua Mal. Cuba, 187S. 260. Ca. Santa

Lncia. G. Ba.

Area uudata Liun., Moll. Cuba, ii, 314.

Area variegata Chemn., Arango, Fanna Mal. Cuba, 187S. 261. Ca. Arca variegata Chemn., Moll. Cuba, ii, 314.

Arca ventricosa Lam., Krebs. 126.

Arca zebra, Kurtz. 5. NO. = occidentalis Ohil, 
Arca zebra Swains., Arango, Fauna Mal. Cuba, 1878. 262. Ca.

Architectonica (see Collonia).

Architectonica gemma Holm., n. s., Post-Pleiocene Fossils S. C., pl. xiv, figs, $6,6 a, 6 b .92$.

Architectonica granulata Isain., Am. Marine Conch. 81.

Architectonica granulata Lam., Marrat, Argo Exp., 1876. 13. Santa Marta.

Architectonica inornata D’Orb., Mal. Bl., xxii, 155. S'T.

Architectonica (Episcynia) inornata D'Urb., Poulsen. 7.

Architectonica krebsii Möreh, Mıl. Bl., xxii, 155. P Pl.

Architectonica krebsii Mörch, Poul:en. 7.

Arehitectonica nobilis Bolt., Mal. 131., ri, 122. VCz.

Architectonica nobilis Bolt., Mal. Bl., xxii, 154. ST:

Architectonica nobilis Bolt., Poulsen. 7.

Architectonica tricarinata Stearns, Proe. B. S. N. H., xv, p. 23, 1872.

Tampa. W. Fla.

Architectonica wroblewskyi Mörch, Mal. Bl., xxii, 154. ST.

Arcinella (see Chama).

Areinella spinosil Schum, Arango, Fanna Mal. Cuba, 1878. 272. Ca. M. G.

Arcinella spinosa Schum., Krebs. 117.

Arcinella spinosa Sehum., Moll. Cuba, ii, 362.

Arcopagia (see Tellina).

Areopagia bimaculata D'Orb., Arango, Fauma MIal. Cuba, 1878. 246.

Ca. Antilles. Fla.

Arcopagia bimaculata D'Orb, Moll. Cuba, ii, 257.

Arcopagia bimaculata Limn., Arango, Fanna Mal. Cuba, 1875. 246.

Ca. Antilles. Fla.

Arcopagia fausta Leach., Krebs. 101.

Argiope (see Megathyris and Cistella).

Argiope antillarum C. \& F., Jour. de Conch., xvii, 116. G.

Argiope antillarum C.\& F., Jour. lle Comeh., xir, pl. viii, fig. 7. 270. G.

Argiope antillarum C. \& F., Jour. de Conch., xiv, 272. G.

Argiope antillarum C. \& F., Report Blake Noll. 103.

Argiope barrettiana Dar., Am. Jour. Conch., iii, 91. J.

Argiope barrettiana Dar., Jour. de Conch., xrii, 116.

Argiope barrettiana Davidson, Report Blake Moll. 103. CS.

Argiope schrammi C. \& F., Jour. de Couch., xvii, 116. G.

Argiope schrammi C. \& F., Jour. de Conch., xis, Il. viii, fig.6. 269,

270. G.

Argiope schrammi C. \& F., Jour. de Conch., xiv, 271. G.

Argiope schrammi U. \& F., Report Blake Moll. 103.

Argiope woodwardiana Dav., Am. Jonr. Conch., iii, 91. J.

Argione woodwarciana Dar., Jour. de Conch., xvii, 116.

Argobuccinum nodosum Chemn., Mal. Bl., xxir, 28.

Argonauta argo D'Orb., Arango, Fanua Mal. Cuba, 1878. 147. WI.

$x$ Conch. He. $P C .118,1833$, includes both the mabit. form an thet
from the Bay goloplel. 
Argonauta argo Fér., Moll. Cuba, i, 24.

Argonauta argo Gmel., Moll. Cuba, i, 24.

Argonauta argo Lam., Moll. Cuba, i, 24.

Argonanta argo Linn., Arango, Famna Mal. Cuba, 1878. 146. WI.

Argonauta argo Linn., Moll. Cuba, i, 28.

Argonauta argo Linn., Verrill, List, 1SS4. 265. CS.

Argonata hians D'Orb., Arango, Fauna Mal. Cuba, 1S7S. 147.

Argonanta bians Sol, Arango, Fauna Mal. Cuba, 1878. 147.

Argonauta hians Sol., Moll. Cuba, i, 28.

Argonauta nitida Lam., Arango, Fauna Mal. Cuba, 187S. 147.

Argonanta nitida Lam., Moll. Cuba, i, 28.

Arģus augustipes Hörch, Jour. de Conch., xi, 32. ST.

Argus angustipes Mörch, MIal. Bl., xxii, 177. ST.

Argus femina Less., Moll. Cuba, ii, 88.

Argus uncleus Gray, Krebs. 134.

Artemis (see Dosinia), Arango, Fauna Mal. Cuba, 1878. 252. Ca. G.

Artemis acetabulum Comrar, Am. Journ. Sci.,n. s., vol. i, 404, 1846. Fla. Artemis concentrica Born, Comral, Proc. Acad. Nat. Sci., ri, 320

1853. Fla. = disoud Rew -

Artemis concentrica Born, Jahrb., ii, 249.

Artemis concentrica Boru, Krebs. 99. SCx. ST.

Artemis discus Reere, Conrad, Proc. Acad. Nat. Sci. Phil., vi, 320, 1853.

S. Coast U. S.

Artemis elegans Conrad, Krebs. 99. ST.

Artemis elegaus Comad, Proc. Acad. Nat. Sci. Phil., vi, 320, 1853. Fla. Artemis radiata Rve., Zt. Mal., x, 81. SF. Cape. Artemis tenuis Recl., Jour. de Conch. iii, pl. x, fig. 1'. 250. G. Artemis tenuis Recl., Jour. de Conch., ir, 415. G.

$\checkmark$ Artemis tenuis Recl., Krebs. 99. ST.

Asaphis coccinca Mart., Jour. de Conch., vii, 140.

Asaphis coccinea Mart.=Sangunolaria rugosa Blr., Poulsen. 14.

Asaplis coceinea Mörch, Jour. de Conch., vii, 140.

Asaphis deflorata Linn., Arango, Fanna Mal. Cuba, 18\%8. “41. Ca. Asaphis deflomata Linn., Marrat, Argo Exp., 1876. 15, 17. Harana.

Nassau. Abaco.

Assimineal auberiana D’Orb., Dall, Hemphill's shells. 33ॅ. Cedar

Keys. Ca.

Assiminea concinna C. B. Adams, Contr. to Conch. 70. J.

Assiminea concimna C. B. Ad., Dall, Hemphill's shells. 335. Key West. Assiminea pupoidea K. \& S. (Rissoa.), Kurtz. 7. SC.

Astarte flabella Conrar, Am. Jouru. Sci., n. s., ii, 393, 1816. Fla.

Astarte flabelia Conrad, Proc. Acad. Nat. Sci. Phila., iii, 24, pl. 1, fig. 3,

184t. Tampa. Eqmait Key

Astarte mactracea Linsley, Kurtz. 4. NC. SC. =

Astarte pfeifferi Phil., Report Blake Moll. 131. = 
Astarte pfeifferi Ph., Zt. Mal., v, 133. Ca. = Cras

Astarte triquetra Conrad, Proc. Acad. Nat. Sci. Phil., iii, 24, pl. 1, fig.

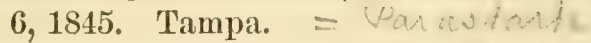

Astræa coralliophora Bolt., Mal. Bl., xxiv, 108.

Astrea lapidifera Bolt., Arango, Fanna Mal. Cuba, 1878. 181. M. WI.

Astræa lapidifera Bolt., Mal. Bl., xxiv, 108.

Astralium brevispinum Lam., Krebs. 82.

Astralium calcar L., Krebs. 82.

Astralium costulatum Lam., Krebs. 8\%. SCx.

Astralium deplanatum Lam., Arango, Fauna Mal. Cuba, 1878. 181. Ca. Ba.

Astralium deplanatum Link, Krebs. 82.

Astralium guadeloupense Crosse,* Jour. de Conch. xiii, pl. 1, figs. 10, 11. 36. G.

Astralium heliacus Phil., Arango, Fauna Mal. Cuba, 187S. 180. Ca.

Astralium longisninum Lam., Marrat, Argo Exp., 1866. 16. Nassau.

Astralium phoebia Bolt., Araugo, Fauna Mal. Cuba, 1878. 181. Ca. Ba.

Astralium phobia Bolt., Krebs. 82. SCx. Tort. NGr. ST.

Astralium phobia Bolt., Krebs' Remarks. 398.

Astralium radians Lam., Krebs. 82. SCX.

Astralium rhodostomum Lam., Krebs. 82. NGr.

Astyris (see Columbella).

Astyris labecula Gid., Otia. 238. Ga. (=Pleurotoma.)

Astyris lunata Say, Dall, Hemphill's shells. 326. Tampa. Cedar Keys. Astyris pura V., Bush, Conn. Ac. vi, 462. Hatt.

Atlanta (sie Heliconoides) Alango, Fama Mal. Cnba, 187s. 154. Atlanta (see Helicophlegma) Araugo, Fauma Mal. Cuba, 1878. 153. Atlanta bulimoides D'Orb., Moll. Cuba, i, 104.

Atlanta inclinata Soul., Bush, Conn. Ac. vi, 469. Hatt.

Atlanta inflata D'Orb., Moll. Cuba, i, 103.

Atlanta keraudrenii D'Orb., Moll. Cuba, i, 99.

Atlanta keraudrenii Rang, Moll. Cuba, i, 99.

Atlanta peronii Blv., Moll. Cuba, i, 102.

Atlanta perouii D’Orb., Arango, Fauna Mal. Cuba, 1878. 154. Ca.

Atlanta peronii Les., D'Orb., Moll. Cuba, i, 102.

Atlanta peronii Lesueur, Arango, Famma Mal. Cuba, 1878. 151. Ca. Atlanta peronii Ponrt, Arango, Fauna Mal. Cuba, 187s. 154. Ca. Atlanta trochiformis D'Orb., Moll. Cuba, i, 104.

Atys (see Bulla).

Atys (?) bathymoplila Dall, n. s., Report Blake Moll. 98. Yucatan Str. Atys caribxa D'Orb., Mal. Bl., xxii, 172. G.

Atys caribiea D'Orb., Poulsen. 8.

Atys guildingii Sby., Am. Jour. Conch, $\nabla, 181$. SV.

Atys guildingii Sby., Krebs. 94. ST. SM.

Atys riiseana Dkr., * Mal. Bl., xxii, 173. S'T.

Atys riiseana Dkr., Poulsen. 8. 
Atys sandersoni Dall., n. s., Report Blake Moll. 99. CS.

Aulacomya (sec Mytilus) Marrat, Argo Exp., 1876. 8.

Aulus lncidus Oken, Krobs. 112.

Aureliella circularis Mörch, Mal. Bl., xxii, 178. ST.

Auricula (see Melampus, Tralia, Alexia).

Auricula biplicata Desh., Am. Jour. Conch., iv, 17.

Auricula cornea Desh., Am. Jour. Conch, iv, 16.

Aurienla dominicensis Fér. Beau. 15. G.

Auricula jaumei Mittré, Am. Jour. Conch, ir, 17.

Auricula muris Mart., Mal. Bl., xxiv, 56.

A uricula nitens Lam., Am. Jour. Conch., iv, 17.

Auricula oliva D'Orb., Am. Jour. Conch., iv, 17.

Auricula ovula D'Orb., Am. Jour. Conch., iv, 17.

Auricula pellucens Mke., Beau. 15. G.

Auris marina Gualt., Moll. Cuba, ii, 37.

Auris pilosa, etc., Mal. Bl., xxir, 34. PR.

Aricula ala-perdicis Rre., Narrat Argo Exp., 1876. 17. Nassau.

Avicula aluco Phil., Zt. Mal., ri, 20. Ca.

Avicula assula Rve., Marrat, Argo Exp., 1876. 19. Abaco.

Avicula atlantica Lam., Krebs. 131.

Avicula atlantica Lam., Am. Marine Conch. 191.

Avicula atlantica Lam., Kurtz. 6. NC.SC.

-A vicula atlantica Lam., Arango Famna, Mal Cuba, 1878. 268. Ca. Ba.

Avicula atlantica Lam., Jahr., ii, 252.

Avicula atlantica Lam., Mal. Bl., x, 231.

A ricula candeaua D’Orb., Arango Fauna Mal. Cuba, 1878. 268. Ca.

- Aricula candeaua D’Orb., Krebs. 131.

-Avicula candeana D’Orb., Moll. Cuba, ii, 343.

-Avicula candeana D'Orb., Poulsen. 16.

Avicula chloris Phil., ${ }^{*}$ t. Mal. viii, 54 . X.

Avicula colymbus Bolt., Arango Fauna Mal., Cuba, 1878. 268. Ca.

Ba.

A vicula colymbus Bolt., Krebs. 131.

A vicula colymbus Bolt., Poulsen. 16.

A ricula communis Lam., Krebs. 131.

A vicula crocata Sw., Krebs. 131.

Avicula guadaloupensis D’Orb., Krebs. 131.

'Avicula guadaloupensis D'Orb., Krebs. 132.

Avicula guadaloupensis D'Orb., Moll., Cuba, ii, 343.

Avicula guadalupensis D’Orb., Arango, Fanna Mal. Cuba, 1878. 26s. G.

Avicula guadalupensis D'Orb., Bean. 21. G.

Avicula guadalupensis D'Orb., Jour de Conch., iv. 415. G.

Aricula heteroptera Lam., Krebs. 131.

A vicula hirundo Chemn., A rango, Fanua Mal. Uuba, 187s. 268. Ca. Ba.

Avicula hirundo Say, Kurtz. 6.

Bull. 24-3 
Avicula hirundo var. nitida, Verrill, List, 1884. 281.

Avicula longisquamosa Dkr., Beau. 21. G.

Avicula longisquamosa Dkr., Jour. de Conch., v, 151. G.

Avícula longisquamosa Dkr., Krebs. 131.

Avicula longisquamosa Dkr., Zt. Mal., ix, 76. Vz.

Avicula macroptera Lam., Beau. 21. G.

Avicula macroptera Lam., Jour. de Conch., ii, 426. G.

Avicula macroptera Lam., Krebs. 132.

Avicula psittacus Phil., Zt. Mal., vi, 21. J.

Avicula pteria Scop., Krebs. 131.

Avicula radiata ? Leach, Jour. de Conch., iv, 415. G.

Avicula radiata Leach, Krebs. 132.

Avicula radiata var. canarina Phil.,* Zt. Mal., vi, 22. X.

Avicula squamulosa D'Orb., Arango, Fauna Mal. Cuba, 1878. 268.

Ca. M. G. Santa Lucia.

Avicula squamulosa D'Orb., Moll. Cuba, ii, 342.

Aricula squamulosa Lam., Arango, Fauna Mal. Cuba, 1878. 268. Ca.

M. G. Santa Lucia.

Avicula squamulosa Lạm., Beau. 21. G.

Avicula squamulosa Lam., Moll. Cuba, ii, 342.

A vicula squamulosa ? Lam., Verrill, List, 1884. 281.

Avicula strix Phil., Zt. Mal., vi, 22.

Axinæa (Pectunculus) pectiniformis Gmel., Marrat., Argo Exp., 1876.

17. Nassau.

Baphia cœrulescens Meusch., Krebs. 112. (See Solecurtus.)

Barbatia (see Area).

Barbatia (Acar) divaricata Sby., Marrat, Argo Exp., 1876. 9. SV.

Barbatia fusca Brug., Marrat, Argo Exp., 1876. 7. Aa. Nassau. Long

Key Island. Abaco.

Barbatia (Acar) gradata Brod., Narrat, Argo Exp., 1876. 7. Aa. Ba.

Barbatia listeri Gray, Krebs. 125.

Basilissa alta Watson, Report Blake Moll. 45. CS.

Basilissa alta Watson, Lin. Soc. Journ. 1879, xiv, 597. Culebra.

Basilissa costulata Watson, Report Blake Moll. 48. Yucatan Str.

Basilissa costulata, Watson, Lin. Soc. Journ., 1879, xiv, 600. Culebra.

- Bela rathbuni Verrill, List, 1884. 266. Cape Hatteras.

Bela subturgida Verrill, List, 1884. 266. Cape Hatteras.

Bela subvitrea Verrill, List, 1884. 266. Cape Hatteras.

Bela undatella Gld., Am. Marine Conch. 50.

Benthodolium abyssorum V. \& S., Verrill, List, 1884. 268. Chesapeake Bay.

Berthella circularis Mörch, Mal. Bl. xxii, 178. ST.

Berthella circularis Mörch, Jour. de Conch., xi, 31. ST.

Berthella quadridens Mörch, Jour. de Conch., xi, 29. ST.

Berthella quadridens Mörch, Mal. Bl., xxii, 178. ST.

Biplex corrugata Perry, Mal. Bl., хxir, 24. 
Bittium (see Cerithiopsis and Cerithium).

Bittium alabastrulum Mörch,* Mal. BI., xxiii, 101. ST.

Bittium alborittatum C. B. Adams,* Mal. Bl., xxiii, 101. J.

Bittium albovittatum C. B. Adams, Poulsen, 9.

Bittium bicolor C. B. Adams, ${ }^{*}$ Mal. Bl., xxiii, 102. J.

Bittium einereoflarum Mörch,* Mal. Bl., xxiii, 102. ST.

Bittium emersoni C. B. Adams,* Mal. Bl., xxiii, 98. SC.

Bittium emersoni C. B. Adams; Poulsen. 9.

Bittium exile Ad., ${ }^{*}$ Mal. Bl., xxiii, 104. J.

Bittium flavum U. B. Adams, Mal. Bl., xxiii, 99. J.

Bittium flavum C. B. Adams, Poulsen. 9.

Bittium galactis Mörch,* Mal. Bl., xxiii, 95. ST.

Bittium gemmulosum C. B. Adlams, ${ }^{*}$ Mal. Bl., xxiii, 102. J.

Bittium gemmulosum C. B. Adams, Poulsen. 9.

Bittium gibberulum U. B. Adams, Jahrb., ii, 244.

Bittium gibberulum Dkr., Mal. Bl., xxiii, 96. G.

Bittium gracile Sby., Mal. Bl., xxiii, 100. ST.

Bittium gracile Sby., Poulsen. 9.

Bittium greenii Ad., Am. Marine Conch. 72.

Bittium greenii C. B.Adams, Dall, Hemphill's shells. 334. Cedar Keys.

Bittium greenii Gould, Mal. Bl., xxiii, 105. J.

Bittium greenii Stimp.,* Mal. Bl., xxiii, 105.

Bittium guaranianum D'Orb., Mal. Bl., xxiii, 95. Bz.

Bittium iota C. B. Adams, ${ }^{*}$ Mal. Bl., xxiii, 97. J.

Bittium iota C. B. Adams, Poulsen. 9.

Bittium latum Ad., Mal. Bl., xxiii, 103. J. G.

Bittium lima Brug.,* Mal. Bl., xxiii, 95. G.

Bittium lima Brug., Poulsen. 9.

Bittium nigrum Totten, Am. Marine Conch. 71.

Bittium nigrum Totten, Dall, Hemphill's shells. 334. Tampa. Cedar

Keys.

Bittium nigrum Totten, Poulsen. 9.

Bittium oryza Mich.,* Mal. Bl., xxiii, 121.

Bittium pallidum Pfr., Mal. Bl., xxiii, 96. Ca.

Bittium pallidum Pfr., Poulsen. 9.

Bittium rugulosum C. B. Adams, ${ }^{*}$ Mal. Bl., xxiii, 100. J.

Bittium rugulosum C. B. Adams, Poulsen. 9.

Bittium subulatum Mtg., Mal. Bl., xxiii, 98. ST.

Bittium subulatum Mtg., Poulsen. 9.

Bittium terebellum C. B. Adams, Mal. Bl., xxiii, 99. J.

Biitium terebellum C. B. Adams, Poulsen. 9.

Bittium terebrale C. B. Adams,* Mal. Bl., xxiii, 99.

Bittium rarium Pfr.,* Mal. Bl, xxiii, 96. Ca.G.

Bittium varium Pfr., Poulsen. 9.

Bittium vicinum Ad., Mal. Bl., xxiii, 101. 
Bittium (?) yucatecanum Dall, 11. s., Report Blake Moll. 90. Yucatan Strait.

Bivonia decussata Chenu, Mal. Bl., xxiv, 120. ST.

Bivonia exserta Dall, n. s., Report Blake Moll. 39.

Blauneria heteroclita Mtg., Beau. 16. G.

Blauneria heteroclita Mtg., Jour. de Conch., v, 152. G.

Blauneria heteroclita Mtg., Mal. Bl., iv, 27. G.

Blauneria heteroclita Mtg., Jahrb., vii, 260. Fla.

Blauneria heteroclita Mtg., Jahrb., vii, 275. J.

Blauneria heteroclita Mtg., Jahrb., vii, 282. G.

Blauneria heteroclita Shuttlew., Jahrb., vii, 266. Ca.

Blauneria pellueida Pfr., Mal. Bl., ii, 85. Ca.

Blauneria pellueida Pfr., Am. Jour. Conch. iv, pl. xviii, fig.13. 10. Fla.

Blauneria pellucida Pfr., Am. Jour. Conch., iv, 18.

Blauneria pellucida Pfr., Beau. 16. G.

Blauneria pellucida Pfr., Jour. de Conch., r, 152, 157. G.

Blauneria pellucida Pfr., Jour. de Conch., xxx, 103.

Blauneria pellucida Pfr., Mal. Bl., i, 152.

Blauneria pellucida Pfr., Mal. Bl., iv, 27. G.

Bolma rugosa Linn., var. Marrat, Argo Exp., 1S76. 12. Tucacas. Santa Marta.

Bornella calcarata Mörch, Jour. de Conch., xi, 36. ST.

Bornella calcarata Mörch, Mal. Bl., xxii, 177. ST.

Botula fusca Gmel., Arango, Fauna Mal. Cuba, 1878. 266. Ca. J. M. SD. G.

Botula fusca Mörch, Krebs. 130.

Bouchardia tulipa Blainv., Jour. de Conch., xiv., 266.

Brachydontes (see Perna) Marrat, Argo Exp., 1876. 19.

Brachydontes modiolus, Swains., Krebs. 129.

Brochina achirona Folin, Am Jour. Conch., iii, 203. Bz.

Brochina someri Folin, Am. Jour. Conch., iii, 203. Bz.

Brochus annulatus Brown, Arango, Fauna Mal. Cuba, 1S78. 233. Ca.

Brochus reticulatus Brown, Arango, Fauna Mal. Cuba, 1878. 233. Ca.

Buccinum (see Crpraecassis) Arango, Fanua Mal. Cuba, 1878. 224. Ca. M. G. Ba.

Buccinum (see Dolium) Arango, Fauma Mal. Cuba, 187S. 225. Ca. Antilles. G.

Bucciuum (see Planaxis) Arango, Fauna Mal. Cuba, 1878. 205. Ca. J. Bb.

Bucciuum (see Semicassis) Arango, Fauna Mal. Cuba, 1878. 223. Ca. Buccinum albescens Dkr.,* Zt. Mal., iii, 170.

Buccinum ambiguum Mtg., Moll. Cuba, ii, 142.

Buccinum ambigum Pfeiffer, Archir füir Naturg., 1840. 257. Ca.

Buccinum angulatum Humph., Mal. Bl., xxiv, 31.

Buceinum antillarum Dkr., Poulsen. 11.

Buccinum antillarum Phil., Zt. Mal., v, 139. Ca. 
Buccinum antoni Dkr.,* Zt, Mal., iv, 61.

Buccinum (Fusus) articulatus Lam., Beau. 10. G.

Buccinum aspretum Rav., n. s., Ravenel. 11. SC.

Buccinum anriculatum Lamarck, Krebs, Catalogue, 428. Ba.

Buccinum auriculus Lam., Arango, Fauna Mal. Cuba, 1578. 214. Ca. Buccinum auritula Liuk, = B. coromandeliaum Lam., Poulsen. 11.

Buccinum bilineatum Gmel., Mal. Bl., xxiv, 37.

Buccinum bilineatum Mtg., Mal. Bl., xxiv, 37.

Buccinum bilingue Sloane, Mal. Bl., xxiv, 19.

Buccinum brevirostre parvum Lister, Mal. Bl. xxiii. 125.

Buccinum brevirostrum List., Moll. Cuba. ii, 112.

Buccinum bulbus Wood, Krebs. 26.

Buccinum canclidissimum, C. B. Adams, Synops., 2, 1845. J.

Buccinum caribæorum D'Orb., Beau. 10. G.

Buccinum caribæorum D'Orb., Krebs. 32.

Buccinum caribæorum D'Orb., Mal. Bl., iv, 27. G.

Buccinum cassideum \&c., Mal. Bl., xxiv, 35.

Buccinum caudatim Gmel., Mal. Bl., xxiv, 33.

Buccinum caudatum Wood, Jahrb., v, 247.

Buccinum cepa, Bolt., Mal. Bl., xxiv, 36.

Buccinum eicatricosum Gron., Mal. Bl., xxiv, 36.

Buccinum cicatricosum Meusch., Mal. Bl., xxiv, 36.

Buccinum cinereum Born, Arango, Fauna Mal. Cuba, 1878.

205.

Ca. G. Santa Lucia.

Baccinum cinereum Boru, Moll. Cuba, ii, 152.

Buccinum cinereum Gmel., Moll. Cuba, ii, 152.

Buccinum cochlidium Chem., Moll. Cuba, ii, 140.

Buccinum concinnum C. B. Adams, Contr. to Conch. 55. J.

Buccinum concinnum Ad., Krebs. 28. S'T.

Buccinum concinnum C. B. Adams, Synops., 2, $1845 . \quad J$.

Buccinum coromandelianum Lam., Mal. Bl., iv, 27. G.

Buccinum coromandelianum Lam., Moll. Cuba, ii, 162.

Buccinum coromandelianum Lam., Arango, Fauna Mal. Cuba, 1878.

214. Ca. Matanzas.

Buccinum coromandelianum var. Lam., Jour. de Conch., ii, 249. G.

Buccium coromantelianum Lam., Jour. de Conch., v, 155. G.

Buccinum coromandelinum Lam., Krebs. 32.

Buccinum costato-tuberculatum Chemn., Mal. Bl., xxiv, 28.

Buccinum costatum Meusch., Mal. Bl., xxiv, 33.

Buccinum cribrarium Kien., Moll Cuba, ii, 135.

Buccinum cribrarium Lam., Arango, Fauna Mal. Cuba, 1878. 198. Ca.

$\mathrm{Ba}$.

Buccinum eribrarium Lam., Moll. Cuba, ii, 135.

Buccinum eribrarium Lam., Zt. Mal., x, 75. SV.

Buccinum decussatum Pennant, Mal. Bl., xxir, 37.

Buccinum dermestoideum Lam., Moll. Cuba, ii, 137. 
Buccinum fastigiatum, \&c., Mal. Bl., xxiv, 33.

Buccinum flammeum Linn., Arango, Fauma Mal. Cuba, 1878. 223. Ca. Ba.

Buccinum flammeum Linn., Moll. Cuba, ii, 180.

Buccinum flexuosum Lam., Pfeiffer, Archir., 1840. 257. Ca.

Buccinum (Tritonidea) floridanum Petit, Jour. de Conch. v, pl. 2, f. 5, 6. 91. Fla.

Buccinum galea Linn., Arango, Fauna Mal. Cuba, 1878. 224. Ca. 'Td. Ba.

Buccinum gibbum Gmel., Mal. Bl., xxiv, 36.

Buccinum glabratum Born, Moll. Cuba, ii, 113.

Buccinum glabratum Brug., Moll. Cuba, ii, 113.

Buccinum glabratum Gmel., Moll. Cuba, ii, 113.

Buccinum glabratum Linn., Arango, Fauna Mal. Cuba, 1878. 193. Ca. Antilles.

Buccinum glabratum Linn., Moll. Cuba, ii, 112.

Buccinum granosum Sol., Mal. Bl., xxiv, 35.

Buccinum granulatum Born, Arango, Fauna Mal. Cuba, 1878. 223. Ca. G. Ba.

Buccinum granulatum Born, Mal. Bl., xxiv, 35.

Buccinum hæmastoma Gmel., Moll. Cuba, ii, 144.

Buccinum hastatum Guel., Arango, Fauna Mal. Cuba, 1878. 205. Ca. G. Ba.

Buccinum inflatum Shaw, Mal. Bl., xxir, 35.

Buccinum janeirense Phil., Zt. Mal., v, 133. Bz.

Buccinum lrvigatum Linn., Arango, Fauna Mal. Cuba, 1878. 199. Ca. Ba.

Buccinum lineatum Da Costa, Arango, Fauna Mal. Cuba, 1878. 205. Ca. Ba.

Buccinum lineatum Da Costa, Mal Bl., xxiii, 124.

Buccinum lineatum Da Costa, Zt. Mal., x, 70. SV.

Buccinum lineatum Dillw., Mal. Bl., xxiii, 124.

Buccinum lineatum Gmel., Mal. Bl., xii, 129.

Buccinum lineatum Gmel., Mal. Bl., xxiii, 137.

Buccinum lineatum Gm., Zt. Mal., x, 72.

Buccinum lineatum Wood, Mal. Bl., xxiii, 124.

Buccinum luteum Hebenstr., Mal. Bl., xxiv, 31.

Buccinum miga Brug., Pfeiffer, Arehiv fuir Naturg., 1840. 257. Ca.

Buccinum mitrula Dkr., Krebs. 28.

Buccinum monile Linn., Krebs. 33.

Buccinum multigranosum Dkr.,* Zt. Mal., iv, 61.

Buccinum musicum List., Moll. Cuba, ii, 123.

Buccinum musicum List., Moll. Cuba, ii, 124.

Buccinum nereidum Humph., Mal. Bl., xxiv, 26.

Buccinum neriteum Linn., Arango, Fauna Mal. Cuba, 1878. 201. Ca.

Buccinum nucleus Brug., Mal. Bl. xxiii, 125. 
Buceinum nucleus Wood, Moll. Cuba, ii, 151.

Buccinum obesun C. B. Adams, Contr. to Couch. 55. J.

Buccinum obesum C. B. Adams, Synops., 2, 1845. J.

Buccinum occellatum Gmel., Araugo, Fauna Mal. Cuba, 1878. 222. Ca. Ba.

Buccinum parvulum Dkr.,* Zt. Mal. iv, 64.

Buccinum parvum, C. B. Adams, Poulsen. 11.

Buccinum parvum integrum, Mal. Bl., xxiii. 134.

Buccinum parvum integrum, \&c., Gualt. Moll. Cuba, i, 203.

Buccinum patulum Gmel., Moll. Ca., ii, 144.

Buccinum patulum Linn., Arango, Fauna Mal. Cuba, 1878. 202. Ca. M. G. Ba.

Bucciuum patulum Linn., Moll Cuba, ii, 144.

Buccinum pediculare Kien., Mal. Bl., xxiii, 124.

Buccinum pediculare Lam., Arango, Fauma Mal. Cuba, 1878. 205. Ca. Ba.

Buccinum pediculare Lan., Mal. Bl., xxiii, 124.

Buccinum pediculare Lam., Zt. Mal., x, 70. SV.

Buccinum pennatum Chem., Poulsen. 11.

Buccinum penuatum Mart., Arango, Fauna Mal. Cuba, 1878. 204. Ca. Baracoa. Ba.

Buccinum pennatum Mart., Moll. Cuba, ii, 146.

Buccinum perdix Linn., Moll. Cuba, ii, 182.

Buccinum plicatum Linn., Mal. Bl., xxiv, 37.

Buccinum plicosum Gould, Am. Jour. Conch., i, 59. Fla.

Buccinum plicosum Mke., Kurtz, 9. NC. SC.

Buccinum plumatum Gmel., Arango, Fauna Mal. Cuba, 1878. 204. Ca. Ba.

Buccinum plumatum Gmel., Beau. 10. G.

Buccinum plumatum Gmel., Moll. Cuba, ii, 146.

Buccinum plumatum Gmel., Krebs, 31.

Buccinum polygonatum Pfr., Archiv für Naturg., 1840. 257. Ca.

Buccinum polygonum Lam., Arango, Fauna Mal. Cuba, 1878. 201. Ca. M. G.

Buccinum polygonum Lam., Moll. Cuba, ii, 140.

Buccinum porcatum Gmel., Zt. Mal., x, 72. SV.

Buccinum pulchellum C. B. Adams, Contr. to Conch. 130. J.

Buccinum pulchellum Ad., Krebs. 33.

Buccinum pulchellum C. B. Adams, Poulsen. 11.

Buccinum pulchellum Blv., Pfeiffer, Archiv für Naturg., 1840. 257. Ca.

Buccinum punctulatum Gmel., Mal. Bl., xxiii, 115.

Bucciuum pusillum Pfi., Arango, Fauna Mal. Cuba, 1878. 200. Ca.

Buccinum pusillum Pfeiffer, Archiv für Naturg., 1840. 257. Ca.

Buccinum pusio Linn., Beau. 10. G.

Buccinum quinquangulare Chemn., Mal. Bl., xxiv, 28.

Buccinum recurvirostrum Gmel., Mal. Bl., xxiv, 36. 
Buccinum recurvirostrum List., Mal. Bl., xxiv, 35.

Buccinum recurvirostum lineatum Da Costa, Mal. Bl., xxiii, 124.

Buccinum reticulatum 'Humph., Mal. Bl., xxiv, 34.

Buccinum striatum Meusch., Mal. Bl., xxiv, 38.

Buccinum strigosum Gmel., Mal. Bl., xxiii, 124.

Buccinum strigosum Gmel., Mal. Bl., xxiii, 125.

Buccinum sturmii Phil., Jahrb., ii, 241. Bz.

Buccinum sturmii Phil., Zt. Mal. v, 135. CS.

Buccinum sublividum List., Mal. Bl., xxiii, 134.

Buccinum sulcatum Meusch., Krebs. 16. SM. SCx. ST.

Buccinum tessellatum Chemn., Arango, Fauna Mal. Cuba, 1878. 224. Ca. G. Ba.

ßJuccinum tessellatum Reeve, Poulsen. 11.

Buccinum testiculus Linn., Moll. Cuba, ii, 181.

Buccinum tinctum Courad, Krebs, catalogue, p. 428. Ba.

Buccinum tinetum Conrad, Poulsen. 11.

Buccinum triangulare Grew, Mal. Bl., xxiv, 31.

Buccinum triticum Sol., Arango, Fauna Mal. Cuba, 1878. 200. Ca.

Buceinum triticum Sol., Krebs., p. 30. ST. SJ. SCx. SM. Ag.

Buccinum tritonis etc., Mal. Bl., xxiv, 26.

Buccinum tritonis Chemn., Mal. Bl., xxiv, 26.

Buccinum tritonis Seba., Mal. Bl., xxiv, 26.

Buccinum tuberosum Linn., Arango, Fauna Mal. Cuba, 1878. 223. Ca. G. Ba.

Buecinum tuberosum Linn., Moll. Cuba, ii, 180.

Buccinum undatum Linu., Moll. Cuba, ii, 139.

Buccinum undulatum Gmel., Mal. Bl., xxiv, 36.

Buccinum variegatum ete., Mal. Bl., xxiv, 29.

Buccinum variegatum Rve., Zt. Mal., x, 73. SV.

Buccinum vibex var. Say, Jour. de Conch., ii, 420. G.

Buccinum viverratum Kien., Zt. Mal., x, 72. SV.

Buccinum zebra Mulf., Mal. Bl., xxiii, 124.

Buccinum zonale Brug., Krebs. 31.

Bufo spadicea Mtf., Mal. Bl., xxiv, 23. G.

Bulimus coniformis Brug., Am. Jour. Conch., iv, 17.

Bulimus dolabratus Brug., Moll. Cuba, i, 228.

Bulinus monile Brug., Am. Jour. Conch., iv, 17.

Bulimus terebellus Brug., Moll. Cuba, ii, 228.

Bulla (see Atys, Acturon, Aplustrum, Bullinula, Cylichna, Philine, Scaphander, Utriculus, ete.).

Bulla (see Cyphoma) Araugo, Fauna Mal. Cuba, 1878. 183. Havana, G. M. Santa Lucia. Ba.

Bulla (see Hyalina) Arango, Fauna Mal. Cuba, 1878. 190. Ca. WI.

Bulla abyssicola Dall, 1. s., Report Blake Moll. 97. Yueatan Str.

Bulla acuminata Brug., Moll. Cuba, i, 126.

Bulla acuta 1'Orb., Araugo, Fauna Mal. Cuba, 1878. 156. Ca. Antilles. 
Bulla acuta D'Orb., Beau. 18. G.

Bulla acuta D'Orb., Krebs. 92.

Bulla acuta 1'Orb., Moll. Cuba, i, pl. iv, figs. 17-20. 126.

Bulla ampulla Brug., Moll. Cuba, i, 121.

Bulla ampulla D'Orb., Beau. 18. G.

Bulla ampulla D'Orb., Krebs. 93.

Bulla ampulla D’Orb., Mal. Bl., xxii, 173. ST.

Bulla ampulla D’Orb., Moll. Cuba, i, 121.

Bulla ampulla Gmel, Moll. Cuba, i, 121.

Bulla ampulla Lam., Moll. Cuba, i, 121.

Bulla ampulla Linn., Jour. de Conch., ii, 426. G.

Bulla ampulla Linn., Moll. Cuba, i, 121.

Bulla ampulla Linn., Moll. Cuba, i, 122.

Bulla ampulla Linn., Moll. Cuba, i, 125.

Bulla amygdala List., Mal. Bl., xxii, 173.

Bulia amygrlalus Limn., Mardat. Argo Exp., 1876. 7. Barbuda. SV.

Bulla amyggdalus List., Marrat. Argo Exp., 1876. 8. D. 'Tucacas. Ha. vana. Nassau.

Bulla angustata Biron, Moll. Cuba, i, 124.

Bulla antillarum D’Orb., Arango, Fauna Mal. Cuba, 1878. 156. Ca.ST.

Bulla antillarum D'Orb., Beau. 18. G.

Bulla antillarum D'Orb., Krebs, p. 92.

Bulla antillarum D'Orb., Mal. Bl., xxii, 175. Ca.

Bulla antillarum D'Orb., Moll. Cuba, i, pl. iv, figs. 9-12. 124.

Bulla auberi D'Orb., Arango, Fauna Mal. Cuba, 1878. 156. Ca. J. ST. M.

Bulla auberi D'Orb., Krebs. 92.

Bulla auberii D' rb., Moll. Cuba, i, pl. iv bis, figs. 5-8. 127.

Bulla bidentata D'Orb., Arango Fauna Mal. Cuba, 1878. 156 Ca. J. ST. M.

Bulla bidentata D'Orb., Beau. 18. G.

Bulla bidentata D'Orb., Krebs. 92. PPI. ST.

Bulla bidentata D'Orb., Mal. Bl., xxii, 171. J.

Bulla bidentata D'Orb., Moll. Cuba, i, pl. iv, figs. 13-16. 125.

Bulla brasiliensis Mart., Arango, Fauna Mal. Cuba, 1878. 183. Ca. G.M. Ba. Santa Lucia.

Bulla brasiliensis Mart., Moll. Cuba, ii, 87.

Bulla canaliculata D'Orb., Arango, Fauna Mal. Cuba, 1878. 157. Ca. G.

Bulla canaliculata D'Orb., Beatr. 18. G.

Bulla canaliculata D'Orb., Jour. de Conch., v, 152. G.

Bulla canaliculata D’Orb., Krebs. 92.

Bulla canaliculata D'Orb., Krebs. 94.

Bulla canaliculata D'Orb., Mal. Bl., xxii, 171. Ca.

Bulla camaliculata D'Orb., Moll. Cuba, i, pl. iv bis, figs. 21-24. 133.

Bulla canaliculata Linu., Amer. Jour. Conch., iii, 182. J.

Bulla canaliculata Say, Kurtz. 7. NC. SO. 
Bulla candeana D'Orb., Beau. 18. G.

Bulla candeana D'Orb., Krebs. 92-94.

Bulla candei D'Orb., Arango, Fauna Mal. Cuba, 1878. 157. Ca. M. ST. J.

Bulla candei D'Orb., Beau. 18. G.

Bulla candei D'Orb., Mal. Bl., xxii, 171 . Ca.

Bulla candei I'Orb., Moll. Cuba, i, pl. iv bis, figs. 1-4. 128.

Bulla caribæa D'Orb, Arango, Fauna Mal. Cuba, 1878. 157. Ca. Ba. J. G. ST. M.

Bulla caribæa D'Orb., Beau. 18. G.

Bulla caribæa D'Orb., Krebs. 92. PPl.

Bulla caribæa D'Orb, Mal. Bl., xxii, 172.

Bulla caribxa D'Orb., Moll. Cuba, i, pl. iv, figs. 21-24. 127.

Bulla cerina Mke.,* Zt. Mal., x, 142. PR.

Bulla cylindirica Helb., Mal. Bl., xxii, 173. PPl.

Bulla (?) eburnea Dall, n. s., Report Blake Moll. 98. CS.

Bulla elegans Mke., Mal. Bl., xxii, 174. SV.

Bulla ferruginea Gmel., Mal. Bl., xxiv, 46.

Bulla ferussaci Q. \& G., Arango, Fauna Mal. Cuba, 1878. 158. Ca.

Bulla ferussaci Q. \& G., Moll. Cuba, i, 133.

Bulla fragilis Lam., Beau. 18. G.

Bulla fragilis Lam., Jour. de Conch., iv., 416. G.

Bulla fragilis Lam., Krebs. 93.

Bulla gibbosa Gmel., Moll. Cuba, ii, 87.

Bulla gibbosa Linn., Moll. Cuba, ii, 87.

Bulla gibbosa Wood, Moll. Cuba, ii, 87 .

Bulla var. globosa, Mal. Bl., xxii, 175 . Ca.

Bulla guadalupensis Sby., Mal. Bl., xxii, 175. Ca.

Bulla guildingii var. Sby., Arango, Fauna, Mal. Cuba, 1878. 157. Ca.

Bulla hydatis, Moll. Cuba, i, 125, 128, 130.

Bulla ibyx Meusch., Krebs. 93. WI.

Bulla ibyx Meusch., Mal. Bl., xxii, 173.

Bulla lajonkairiana Bast., Moll. Cuba, i, 129, 131, 134.

Bulla maculosa Mart., Arango, Fauua Mal. Cuba, 1878. 157. Ca. Ba.

Bulla maculosa Mart., Jahrb. ii, 247.

Bulla maculosa Mart., Krebs., 93. ST. SM. SCx. SJ. T.

Bulla magna Mart., Moll. Cuba, i, 121.

Bulla media Phil., Arango, Fauna Mal. Cuba, 1878. 157. Ca. Ba.

Bulla media Phil., Jahrb., ii, 247.

Bulla media Phil., Krebs. 93.

Bulla media Phil., Mal. Bl., xxii, 173.

Bulla media Phil., ${ }^{*}$ Zt. Mal., iv, 121.

Bulla nebulosa Gld., Marrat, Argo Exp., 1S76. 6. Aa. Santa Marta. Bulla nitidula Dillw., Arango, Famna Mal. Cuba, 1878. 158. Ca.

Bulla nitidula Dillw., Mal. Bl., xxii, 174. SV.

Bulla nitidula Dillw., Moll. Cuba, i, 133. 
Bulla nitidula List., Moll. of Bermudas. 23.

Bulla nitidula Wood, Beau. 18. G.

Bulla nitidula Wood, Jour. de Conch., ii, 426. G.

Bulla nitidula Wood, Moll. Cuba, i, 133.

Bulla nux Mke.,* Zt. Mal., x, 140. Ca.

Bulla oblonga Mart., Mal. Bl., xxir, 53.

Bulla occidentalis A. Ad., Dall. Hemphill's shells. 324. Sarasota Bay.

Bulla olivula A. Ad., Mal. Bl., xxii, 171. Ca.

Bulla olivula C. B. Adams, Beau. 18. G.

Bulla olivula C. B. A. (=canaliculata D'O.), Jour. de Conch.,, , 152. G.

Bulla olivula C. B. Adams, Krebs. 94.

Bulla oryza Totten, Kurtz. '7. NC. SC.

Bulla perstriata Mke., Zt. Mal., x, 138. PR.

Bulla petitii D’Orb., Arango, Fauna Mal. Cuba, 1878. 157. Ca. Ba. Bulla petitii D'Orb., Krebs. 93. ST.

Bulla petitii D'Orb., Mal. Bl., xxii, 174. Ca.

Bulla petitii D'Orb., Moll. Cuba, i, pl. iv bis, figs. 13-16. 130.

Bulla physis D’Orb., Arango, Fauna Mal. Cuba, 1878. 158. Ca. G.

Bulla physis D'Orb., Poulsen. 8.

Bulla physis Gmel., Moll. Cuba, i, 132.

Bulla physis Linn., Beau. 18. G.

Bulla physis Linn., Jour. de Conch., iv, 416. G.

Bulla physis Linn., Krebs. 93. ST.

Bulla physis Linn., Mal. Bl., xxii, 175. Ca.

Bulla physis Linn., Moll. of Bermudas. 23.

Bulla physis Linn., Moll. Cuba, i, 131.

Bulla physis Linn., Zt. Mal., x, 70. SV.

Bulla physis Q. \& G., Araugo, Fauna Mal. Cuba, 187S. 158. Ca. G.

Bulla physis Sby., Moll. Cuba, i, 132.

Bulla punctulata Ad., Moll. of Bermudas. 23.

Bulla pusilla Pfr., Mal. Bl., xxii, 171. Ca.

Bulla pyrum Dillw., Am. Jour. Conch., iii, 150.

Bulla prrum Dillw., Jahrb., ii, 361.

Bulla quojana D'Orb., Moll. Cuba, i, 132.

Bulla recta D’Orb., Arango, Fauna Mal. Cuba, 1878. 157. Ca. G. ST.

Bulla recta D'Orb., Beau. 18. G.

Bulla recta D'Orb., Krebs. 93. S'T.

Bulla recta D'Orb., Moll. Cuba, i, pl. iv bis, figs. 17-20. 131.

Bulla rubiginosa Gould., Mal: Bl, xxii, 174. Bz.

Bulla sagra D'Orb., Araugo, Fauma Nal. Cuba, 1878. 157. M.

Bulla sagra D'Orb., Moll. Cuba, i, pl. iv, figs. 5-8. 123.

Bulla sagræe D'Orb., Krebs. 93.

Bulla secale Wood, Mal. Bl., xxiv, 54.

Bulla secale Wood, Arango, Fauna Mal. Cuba, 1878. 183. Ca. Ba.

Bulla secale Wood, Moll. Cuba, ii, 87.

Bulla semistriata Lam., Moll. Cuba, i, 125 
Bulla semisulcata Phil., Moll. Cuba, i, 130.

Bulla solida Gmel., Beau. 18. G.

Bulla solida Gmel., Krebs. 93.

Bulla solida Rve., Mal. Bl., xxii, 173. Ca.

Bulla solitaria Say, Am. Marine Conch. 102.

Bulla speciosa A. Ad., Mal. Bl., xxii, 172. J.

Bulla spelta Linn., Mal. Bl., xxiv, 53.

Balla striata Brug., Moll. Cuba, i, 127.

Bulla striata Brug., Arango, Fauna Mal, Cuba, 1878. 157. Ca. Ba.

Bulla striata Brug., Beau. 18. G.

Bulla striata Brug., Jour. de Conch., ii, 426. G.

Bulla striata Brug., Krebs. 93.

Bulla striata Brug., Mal. Bl., xxii, 173.

Bulla striata Brug., Moll. Cuba, i, 122.

Bulla striata Lam., Moll. Cuba, i, 123.

Bulla striata Limn., Mal. Bl., xii. 127.

Bulla suecinea Courad, Am. Jour. Sci. n. s., ii, 396. 1846. Fla.

Bulla suceinea Conrad, Dall, Hemphill's shells. 324. Cedar Keys.

Bulla suecinea Courad, Proc. Acad. Nat. Sci. Phil., iii, 26, pl. i, fig. 5. 1845. Tampa.

Bulla sulcata D'Orb., Arango, Fauna Mal. Cuba, 1878. 157. Ca. ST. G.

Bulla sulcata D'Orb., Beau. 18. G.

Bulla sulcata D'Orb., Krebs. 93. PPl.

Bulla suleata D'Orb., Mal. Bl., xxii, 172. ST.

Bulla suleata D'Orb., Moll. Cuba, i, pl. iv bis, figs. 9-12. 129. Ca.

Bulla sulcata Mke., Mal. Bl., xxii, 174. Bz.

Bulla umbilicata Bolt., Krebs. 93.

Bulla umbilicata Bolt., Mal. Bl., xxii, 173.

Bulla umbilico simplici Klein, Moll. Cuba, i, 131.

Bulla undata Brug., Arango, Fauna Mal. Cuba, 1878. 158. Ca.

Bulla undata Brug., Krebs. 93.

Bulla undata Brug., Moll. Cuba, i, 132.

Bulla undata D'Orb., Arango, Fauna Mal. Cuba, 1878. 158. Ca.

Bulla velutina Muill., Moll. Cuba, ii, 36.

Bulla veudryesiana Guppy, n. s., Geol. Mag., 1874. 436. J.

Bulla virescens Moll. Cuba, i, 130.

Bulla virgata Mart., Arango, Fauna Mal. Cuba, 1878. 158. Ca. G.

Bulla viridis Q. \& G., Moll. Cuba, i, 120.

Bullea candeana D'Orb., A rango, Fauna Mal. Cuba, 1878. 156. G. Ca. Bullea candeana D'Orb., Krebs. 94.

Bullea candeana D'Orb., Moll. Cuba, i, pl. iv, figs. 1-4. 119. Ca.

Bullea ibyx Meusch., B. striata D'Orb., Poulsen. 8.

Bullea media (bis) Phil., Mal. Bl., xxii, 173.

Bullea media Phil., Poulsen. 8.

Bullea nux Mke., Mal. Bl., xxii, 174. SV.

Bullea nux Mke., Zt. Mal., $x, 140$. Ca 
Bullea occidentalis A. Ad., Mal. Bl., xxii, 174. SV.

Bullea occidentalis A. Ad., Poulsen. 8.

Bullea perstriata Mke., Mal. Bl., xxii, 173. PR.

Bullea perstriata Mke., Poulsen. 8.

Bullea perstriata Mke., * Zt. Mal., x, 133. PR.

Bullea rubiginosa Gould., Mal. Bl., xxii, 174. Bz.

Bullea solida Gmel., Mal. Bl., xxii, 173. ST.

Bullea solida Gmel., Poulsen. 8.

Bullia acienlata Lam., Krebs. 33.

Bullia (Euryta) aciculata Lam., Poulsen. 12.

Bullina undata Brug., Mal. Bl., xxii, 174. SV.

Bullina undata Brug., Poulsen. 8.

Bullinula elegans Mke., Krebs. 92.

Bullinula nitidula Sol., Krebs. 92.

Bullinula undata Brug., Krebs. 92. ST. SCx. SM.

Bursa (Lampas) affinis Rve., Marrat, Argo Exp., 1876. 12. Tucacas.

Bursa (Lampas) rhodostoma Beck, Marrat, Argo Exp., 1876. 17. Long Key Island.

Bursatella lacinulatus Couth., Mal. Bl. xxii, 176. Bz.

Busyeon aruanum Linn., Arango, Fauna Mal. Cuba, 1878. 216. Ca. Fla.

Busycon candelabrum Lam., Am. Jour. Conch., v, 106.

Busycon carica Linn., Am. Jour. Conch., v, 106.

Busycon carieum Gmel. Jahrb., ii, 360.

Busyeon coaretatum Sby., Am. Jour. Conch., v, 106.

Busycon conradii T. \& H., n. s., Pleiocene Fossils S. C., pl. xxix, fig. 4. 147.

Busycon eliceans Mont., Am. Jour. Conch., $\mathrm{r}, 106$. Fla.

Busycon gibbosum Con., Am. Jour. Conch., iii, 146.

Busycon gibbosum Con., Jahrb., ii, 360. Fla.

Busycon kieneri Phil., Am. Jour. Conch., iii, 143; v, 106.

Busycon perversum Linn., Äm. Jour. Conch., v, 106.

Busycon perversum Linn., Jahrb., ii, 360. Fla.

Busycon perversus Lam., Am. Jour. Conch., i, 61. Fla.

Busycon plagosum Con., Am. Jour. Conch., iii, 150.

Busycon plagosum Con., Jahrb., ii, 361.

Busseon pyrum Lam, Arango, Fama Mal. Cuba, 1878. 216. Ca. Fla.

Busyeon pyrum Say, Am. Jour. Conch., i, 61. Fla.

Busyeon spinosa Con., Jalurb., ii, 360. Fla.

Buşeon striatum Con., Am. Jour. Conch., ii, p. 69, pl. 3, fig. 8.

Busyeum aruanum Linn., Poulsen. 11.

Busyeum perversum Linn., Poulsen. 11.

Bythinella obtusa Lea, Dall, Hemphill's shells: 334 . Key West.

Cabestaua labiosum Wood, Mal. Bl., xxir, 34 .

Cadulus aequalis Dall, n. s., Report Blake Moll. 34. CS.

Cadulus agassizii Dall, n. s., Report Blake Moll. 35. CS. 
Cadulus ampullaceus Watson, Lin. Soc. Jouru., 1879, xiv, 529. Cul.

Cadulus carolinensis Bush, Conn. Ac. vi, 471. Hatt.

Cadulus cucurbitus Dall, n. s., Report Blake Moll. 35. CS.

Cadulus curtus Watson, Lin. Soc. Journ., 1879, xir, 527. Cul.

Cadulus curtus var. congruens Watson, Lin. Soc. Jn., 1879, xiv, 527. Cul. Cadulus exiguus Watson, Lin. Soc. Journ., 1879, xiv, 528. Cul.

Cadulus incisus Bush, Conn. Ac. vi, 471. Hatt.

Cadulus lunulus Dall, u. s., Report Blake Moll. 35. CS.

Cadulus obesus Watson, Report Blake Moll. 36. ST.

Cadulus obesus Watson, Lin. Soc. Journ., 1879, xir, 527. Cul.

Cadulus rastridens Watson, Lin. Soc. Journ., 1879, xiv, 525. Cul.

Cadulus sauridens Watson, Report Blake Moll. 36. Bb. ST.

Cadulus sauridens Watson, Lin. Soc. Journ., 1879, xiv, 525. Cul.

Cadulus rulpidens Watson, Lin. Soc. Journ., 1879, xir, 524. Cul.

Cardulus watsoni Dall, n. s., Report Blake Moll. 34. Yucatan Strait. Caecum annulatum Brown, Arango, Fauna Mal. Cuba, 1878. 233. Ca.

Cæcum breve Folin, Am. Jour. Conch., iii, 203. J.

Cæcum circumvolutum Folin, Am. Jour. Conch., vi, si. A.

Cæcum clava Folin, Am. Jour. Conch., iii, 203. G.

Cæeum cooperi Smith, Bush., Comn. Ac. vi, 463. Hatt.

Cæeum corneum Dkr.,* Jahrb, ii, 244.

Cæcum coronatum Folin, Am. Jour. Conch., iii, 203. J.

Cæcum cuccina Folin, Am. Jour. Conch., vi, 81. VCz.

Cæcum cyclophornm Folin, Poulsen. 10.

Cæcum decussatum Folin, Am. Jour. Conch., vi, 81. Ba.

Cæeum floridanum Stimpson, Proc. Best. Soc. Nat. Hist., iv, 112, 1851.

Cæcum formulosum Folin, Am. Jour. Conch., vi, 81. Ba.

Cæenm gibbesii Stimpson, n. s., Kurtz. 8. SC.

Cæcum infimum Folin, Am. Jour. Conch., vi., 81. A.

Cæcum insigne Folin, Am. Jour. Conch., iii, 203. J.

Cæcum insigne Folin, Poulsen. 10.

Cæcum intricolum Cpr., Poulsen. 11.

Cæecum irregulare Folin, Am. Jour. Conch., iii, 203. (Ponlsen. 11.) Bz.

Cæcum lineicinctum De Folin., P. Z. S., 1879. 808. Cul.

Cæcum nitidum Stimps., Proc. Bost. Soc. Nat. Hist., ir, 112, 1851. Fla.

Cæcum phronimum Folin, Am. Jour. Conch., iii, 203. Ну.

Cæcum phronimum Folin, Poulsen. 11.

Cæcum pulchellum Stimps., Arango, Fauna Mal. Cuba, 233. Ca. Hatt.

Cæcum regulare Cpr., Poulsen. 11.

Cæcum rhyssotitum Folin, Am. Jour. Conch., iii, 203. Bz.

Cæcum striatum Folin, Poulsen. 11.

Cæcum textile Folin, Poulsen. 10.

Cæcum torquatum Folin, Am. Jour. Conch., vi, 81. G.

Cæcum torquatum Folin, Poulsen. 11.

Cæcum veracruzanum Folin, Am. Jour. Conch., vi, s1. VCz.

Cæcum vestitum Folin, Am. Jour. Conch., vi, 81. VCz. 
Calcar (see Trochus).

Calcar cælatus Chem., Krebs. S. 81. ST. Tort.

Calcar cubanus Phil.,* Zt. Mal., v, 104. Ca.

Calear inermis Gmel., Krebs. 81. ST.

Calcar inermis Gmel., Krebs. 82.

Calcar magus Chem., Moll. of Bermudas. 20.

Calcar majus Chem., Moll. Cuba, ii, 72.

Calcar ramosus Meusch., Arango, Fauna Mal. Cuba, 1878. 180. Ca. Ba.

Calcar ramosus Meusch., Krebs. 81. ST. SCx. Tort. G.

Calcar ramosus Meusch., Krebs' Remarks. 394. 'Fla.

Calcar spenglerianus Chem., Krebs. 81. PPl. SM.

Calcar spenglerianus Chem., Krebs' Remarks. 394. J.

Calcar tuber Linn., Krebs. 81. 82. Td. SM. SJ.

Calcar turbinatus, Krebs' Remarks. 394.

Calliostoma (see Trochus, Ziziphinus, etc.).

Calliostoma apicinum Dall, n. s., Report Blake Moll. 46. Bb.

Calliostoma bairdii Verrill \& Smitl, Report Blake Moll. 45. CS.

Calliostoma circumeinctum Dall, n. s., Report Blake Moll. 44. Yuca$\tan$ Strait.

Calliostoma echinatum Dall, n. s., Report Blake Moll. 47. Havana.

Calliostoma psyche Dall, Report Blake Moll. 45. CS.

Calliostoma roseolum Dall, n. s., Report Blake Moll. 45. CS.

Calliostoma sapidnm Dall, n. s., Report Blake Moll. 46, 47. CS.

Calliostoma tiara Watson sp., Report Blake Moll. 45. CS.

Calliostoma yucatecanum Dall, n. s., Report Blake Moll. 47. Yucatan Strait.

Callista (see Venus): =

Callista dione Linn, Marrat, Argo Exp., 1876. 12. Tucacas.

Callista exspinata Rve., Marrat, Argo Exp., 1876. 12. Tucacas.

Callista gigantea Chem., Am. Marine Conch. 160. Fla.

$\checkmark$ Callista gigantea Chem., Mal. Bl., viii, 181.

Callista lubrica Brod., Jahrb., ii, 249.

Callista maculata Linn., Am. Marine Conch. 160. Fla.

Callista maculata Linn,, Mal. Bl., viii, 177.

Vallista purpurata Lam., Jahrb., ii, 249.

Callogaza superba Dall, n. s., Report Blake Moll. 49. CS.

Callogaza watsoni Dall, n. s., Report Blake Moll. 50. Havana.

Calyptra alveolata A. Ad., Marrat, Argo Exp., 1876. 18. Abaco.

Calyptra equestris Linn., Mal. Bl., xxiv, 100.

Calyptra equestris Linn., Marrat, Argo Exp., 1876. 6. Aa.

Calyptra stella Rve., Mal. Bl., xxiv, 101. Hd.

Calyptra uncinata Reeve, Mal. Bl., xxiv, 101. ST.

Calyptræa (see Infundibulum).

Calyptræa aculeata Rve., Mal. Bl., xxiv, 101. Hd.

Calyptrea auriculata Chem., Guppy, Paria Fauna, 1877. 138.

Calyptræa chinensis Guild., Mal. Bl., xxiv, 106. ST. 
Calyptræa dillwynii Gray, Arango, Fanna Mal. Cuba, 1878. 226. Ca. Bb. Calyptræa equestris Lam., Beau. 19. G.

Calyptrea equestris Lam., Mal. Bl., xxiv, 100. Hd.

Calyptræa equestris Lam., Moll. Cuba, ii, 189.

Calyptræa equ, stris Linn., Arango, Fanna Mal. Cuba, 1878. 226. Ca. $\mathrm{Bb}$.

Calyptræa equestris Linn., Mal. Bl., xxiv, 100.

Calyptriea equestris Linn., Poulsen. 10.

Calyptræa stella Rve., Mal. Bl., xxiv, 101. Hd.

Calyptræa striata De Kay, Mal. Bl., xxiv, 102.

Calyptræa striata Reeve, Mal. Bl., xxir, 102.

Calyptræa striata Stimp., Mal. Bl., xxiv, 102.

Calyptræa tubifera Gray, Mal. Bl., xxiv, 101.

Calyptræa umbo Reeve, Mal. Bl., xxir, 101. Hd.

Calyptræa uncinata Reeve, Mal. Bl., xxiv, 101. ST.

Cancellaria (see Phos), Arango, Fauna Mal. Cuba, 1878. 201. Ca. M. Cancellaria brevis Sby., Jour. de Conch., ix, 230. St. Elena.

Cancellaria candeana D'Orb., Arango, Fauna Mal. Cuba, 1878. 201. Ca. G.

Cancellaria candei D'Orb., Krebs. 33.

Cancellaria candei D'Orb., Moll. Cuba, ii, pl. xxi, figs. 23-25. 129.

Cancellaria corrugata Hinds, Jour. de Conch., ix, 240. Guayaquil.

Cancellaria depressa T. \& H., n. s., Pleiocene Fossils S. C., pl. xxviii, fig. 17. 143.

Cancellaria reticulata Lam., Jour. de Conch., ix, 245.

Cancellaria reticulata Lam., Arango, Fauna Mal. Cuba, 1878. 198. Ca.

G. Aa.

Cancellaria reticulata Lam., Beau. 9. MG.

Cancellaria reticulata Lam., Jour. de Conch., iv, 417. G.

Cancellaria reticulata Lam., Jour. de Conch., ix, 237.

Cancellaria reticulata Lam., Moll. Cuba, ii, 128.

Cancellaria reticulata Linn., Am. Marine Conch. 21.

Cancellaria reticulata Linn., Arango, Fanna Mal. Cuba, 1878. 197. Ca.

G. Aa.

Caucellaria reticulata Linn., Krebs. 34. ST. Tort. SM.

Cancellaria reticulata Linn., Poulsen. 13.

Cancellaria rugosa Lam., Jour. de Conch., x, 221.

Cancellaria rugosa Lam., Bean. 9. MG. SM.

Cancellaria rugosa Lam., Jour. de Couch., v, 153. G.

Cancellaria rugosa Lam., Jour. de Conch., xi, 61. G. MG. SM.

Cancellaria rugosa Lam., Krebs. 34. SM. ST. Ag.

Cancellaria rugosa Linn., Poulsen. 13.

Cancellaria scalarina Sby. et Rve., Jour. de Conch., ix, 231.

Cancellaria sealatella Gupps, 11. s., Geol. Mag., 187t. 438. Td.

Cancellaria stimpsonii Calkins, Dall, Hemphill's shells. 342. Cape

Sable, Fla. Proe, Devemp. Acad. Jai. ii p.250, pl, viii, figs, 4-.5,187s

=c. Tenera Phil. 
Cancellaria teuera Phil., Z Zt. Mal., r, 24. Y.

Cancellaria tessellata Sby., Jour. de Conch., ix, 245.

Cancellaria thomasiana Crosse, Jour. de Conch., ix, 231. = Scalsminas Sly

Cancellaria thomasiana Crosse, Jour. de Conch., ix, 245.

Cancellaria renusta T. \& I., n. s., Pleioceno Fossils S. C., pl. xxviii, fig. 18, 144.

Candelabrum cathedralis Dall, Report Blake Moll. 54. CS.

Cantharus (see Pisania, Pollia, Tritonidea).

Cantharus coromandelianus Lam., Dall., Hemphill's shells. 327. Key West.

Cantharus crossus (erosus?) Bolt., Arango, Fauna Mal. Cuba, 1878. 203. Ca. Ba.

Cantharus (Tritonidea) loroisi Petit, Marrat, Argo Exp., 1876. 6. Aa. Havana.

Cantharus (Tritoniclea) proteus Rve., Marrat, Argo Exp., 1876. 14. Havana.

Cantharus (Tritonidea) ringens Rve., Marratt, Argo Exp., 1876. 18. Abaco.

Capsa (see Asaphis).

Capsa (see Iphigenia), Araugo, Fauna Mal. Cnba, 1878. 247. Ca.

Capsa brasiliensis Lam., Jahrb., ii, 249.

Capsa coccinea Mart., Krebs. 105. ST. SJ. SCx. Tort.

Capsa deflorata D'Orb., Arango, Fauna Mal. Cuba, 1878. 241. Ga.

Capsa deflorata D'Orb., Moll. Cuba, ii, 258.

Capsa deflorata Gmel., Bean. 24. G.

Capsa lævigata Lam., Pfeiffer, Archir fuir Naturg., 1840. 261. Ca.

Capsa rugosa Lam., Jour. de Conch., vii, 26. MG.

Capsa rugosa Lam., Jour. de Conch., vii, 27-28. G.

Capsa rugosa Lam., Jour. de Conch., vii, 33.

Capsa rugosa Lam., Beau. 24. G.

Capsa rugosa Lam., Jour. de Conch., vii, 135.

Capsa rugosa Lam., Jour. de Conch., vii, 140.

Capsa rugosa Lam., Krebs. 105.

Capsa spectabilis Hanley, Moll. of Bermudas. 26.

Capulus (see Hippouyx).

Capulus antiquatus Linn., Krebs. 70.

Capulus cernuus S. \& W., Mal. Bl., xxir, 98.

Capulus incurvus Gmel., Krebs. 7\%. ST. SCx. SM. SB. G.

Capulus intortus Blv., Mal. Bl., xxiv, 97. Ca.

Capulus intortus D'Orb., Arango, Fauna Mal. Cuba, 1878. 226. Ca.

Capulus intortus D'Orb., Mal. Bl., xxiv, 97. Bb.

Capulus intortus D'Orb., Moll. Cuba, ii, 186.

Capulus mitrula D'Orb., Mal. Bl., xxiv, 99.

Capulus mitrulus D’Orb., Moll. Cuba, ii, 186.

Capulus pilosa Desh., Krebs. 70.

Bull. 23-4 
Capulus shrevei Con., Am. Jour." Uonch., v, 105.

Capulus subrufa Mart, Krebs. 70. PPl. G.

Capulus subrufus D’Orb., Arango, Fauna Mal. Cuba, 1878. 226. Ca.

Capulus subrufus D'Orb., Mal. Bl., xxiv, 98.

Capulus subrufus D'Orb., Moll. Cuba, ii, 187.

Capulus trigoua Gmel., Krebs. 71.

Cardita (see Venericardia, Lazaria, Trapezium, Cypricardia).

Cardita affinis Shuttlew., Poulsen. 15. = Lof ved y gracues

Cardita conradi Shuttlew., Mal. Bl., iv, 2S. Fla.

Cardita conradi Shuttlew., Jour. de Conch., r, 173. Tampa.

Cardita carditoidea Bly., Krebs. 123. -

Cardita dactylus Brug., Krebs. 123. =

Cardita domingensis D'Orb., Krebs. 123.

Cardita dominguensis D'Orb., Arango, Fauna Mal. Cuba, 1878. 254. Ca. SD.

Cardita dominguensis D’Orb., ${ }^{*}$ Moll. Cuba, ii, 291.

Cardita floridana Comrad, Dall, Hemphill's shells. 341. Sarasota Bay.

Cardita gibbosa Rve., Krebs. 123. =:

Cardita gracilis, Mal. Bl., iv, 28. PR.

Cardita gracilis Shuttlew., Jour. de Conch., r, 173. PR.

Cardita gracilis Shuttlew., Krebs. 123.

Cardita gracilis Shuttlew., Mal. Bl., x, 229.

Cardita incrassata Cour., Jour. de Conch., r, 173. Tampa.

Cardita minima Sby., Guppy, Geol. Mag., 1874. 450. Td.

Cardita ovata Ad., Krebs. 123. = Venced joygnerín vin

Cardita ovata C. B. Adams, Synops., 9, 1845. J.

Cardita pectunculus Brug., Guppy, Paria Fauna, 1S77. 153.

Cardita tridentata Say, Am. Marine Conch. 167: SC.

Carditamera floridana Con., Am. Journ. Sci., n. s., ii., 393. 1846. Fla.

$\checkmark$ Cardium xolicum Born, Zt. MLal., x, S0. SV.

Cardium angulatum D'Orb. Arango, Fauna Mal. Cuba, 1878. 257. Ca. M. ST. G. Ba. = subelingatum siy.

$\checkmark$ Cardium angulatum Lam., Arango, Fauna Mal. Cuba, 1878. 257.

Ca. M. ST. G. Ba.

$\checkmark$ Cardium angulatum Lam., Moll. Cuba, ii, 306.

Cardium antillarum D’Orb., Arango, Fauna Mal. Cuba, 1878. 258.

Ca. G. M. J.

Cardium antillarum D'Orb., Beau. 23. G.

Cardium antillarum D’Orb., Krebs. 115.

Cardium antillarum D'Orb., Moll. Cuba, ii, pl.xxvii, figs. 53-55. 309.

Cardium antillarum 1)'Orb.=C. graniferum Brod., Poulsen. 15.

JCardium bicolor Mart., Arango, Fauna Mal. Cuba, 1878. 251. Ca.

Ba. = Venus cancellatä $\not$

Vardium bicolor Mart., Moll. Cuba, ii, 271.

Cardium bullatum jun?, Jour. de Conch., iv, 415. G. = spinatum mass 
$\checkmark$ Cardium bullatum D'Orb., Arango, Fauna Mal. Cuba, 1878. 259. Ca.

II. G. Santa Lucia, Ba.

Cardium bullatum Lam., Jour. de Conch., iv, 415. G.

Cardium bullatum Lam., Moll. Cuba, ii, 308.

Cardium bullatum Linn., Beau. 23. G.

Cardium bullatum Linn., Mal. Bl., xii, 126. D. M.

Cardium bullatum Rre., Moll. Cuba, ii, 308. G.

Cardium eitrinum Chem., Arango, Fauna Mal. Cuba, 1878. 257. Ca.

M. G. Santa Lucia. Ba. = serratum L .

Cardium citrinum Chem., Krebs. 115. ST. Tort. Td. SCx.

$\checkmark$ Cardium citrinum Chem., Poulsen. 15.

Cardium citrinum Wood, Report Blake Moll. 131. CS.

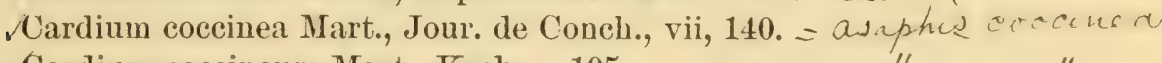

Cardium coccineum Mart., Krebs. 105.

$\checkmark$ Cardium erista-galli Mart., Arango, Fauna Mal. Cuba, 1878. 272. Ca.

II. G. = chavia arcinclln

$\checkmark$ Cardium erista-galli Mart., Moll. Cuba, ii, 362.

Cardium eygnorum Desh., Moll. of Bermudas. 25. cusfralia :n pecies

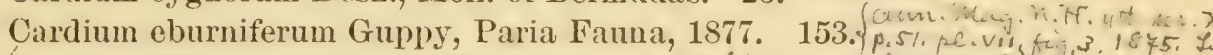

Cardium efflorata D'Orb., Krebs. 105. =asaphi

Cardium egmontianum Shuttlew, Mal. Bl., iv, 28. Fla. $=c . i 20 \mathrm{en}$ dic $Z$

Cardium egmontianum Shuttlew., Jour. de Conch., v, 172. Tampa.

Cardium graniferum B. \& S., Arango, Fauna Mal. Cuba, 1878. 258.

Cardium graniferum Brod., Krebs. 115.

Cardium haitense Sby., Gupps, Paria Fauna, 1877. 153. also Miorecue

$\checkmark$ Cardium hiatus Meusch., Krebs. 115. $=\complement_{1}$ deratum.

Cardium isocardia Chem., Moll. Cuba, ii, 307.

. Cardium isocardia D'Orb., Arango, Fauna Mal. Cuba, 1878. 258. Ca.

M. Santa Lueia.

Cardium isocardia Gmel., Moll. Cuba, ii, 307.

Cardium isocardia Linn., Am. Mariue Conch. 173.

Cardium isocardia Linn., Arango, Fauna Mal. Cuba, 1878. 258. Ca.

M. Santa Lncia.

Cardium isocardia Linn., Beau. 23. G.

Cardinm isocardia Linn., Jour. de Conch, ii, 425. G.

Cardium isocardia Linn., Krebs. 115. ST. SCx. SJ. Tort. Td.

Cardium isocardia Linn., Mal. BI., x, 226. Ca.

Cardium (Isocardia) isocardia Linn., Marrat, Argo Exp., 1876. 13.

Santa Marta. Point Savanilla.

Cardium isocardia Linn., Moll. Cuba, ii, 307.

Cardium isocardia Linn., Poulsen. 15.

Cardium isocardia Rve., Moll. Cuba, ii, 307.

Cardium lævigatum, Kurtz, 5. SC.

Cardium lævigatum Chem., Moll. Cuba, ii, 306.

Cardium lievigatum D'Orb., Arango, Fanna Mal. Cuba, 1878. 258. Ca.

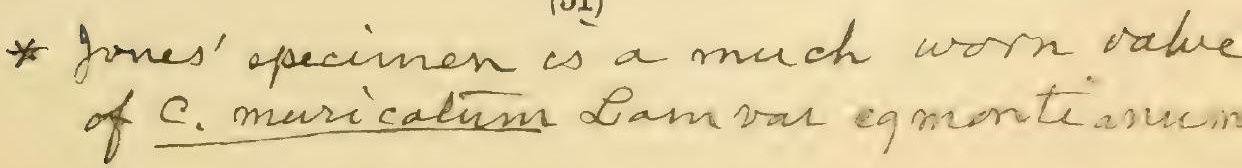


Cardinm lævigatum Gmel., Moll. Cuba, ii, 302. = serratum

Carlium lævigatum Lam., Arango, Fauna Mal. Cuba, 1878. 258. Ca. M. G. Santa Lucia. Ba.

Cardiuın lævigatum Lam., Beau. 23. G.

Cardium lævigatum Lam., Jour de Conch., ii, 425. G.

Cardium lævigatum Lam., Moll. Cuba, ii, 304.

Cardium lævigatum Liun, Arango, Fauna Mal. Cuba, 1878. 258. Ca.

Cardium lævigatum Linn., Beau. 23. G.

Carlium levigatum Linn., Krebs. 116. ST. Tort. VgI. SCx.

Cardium lævigatum Linn., Moll. Cuba, ii, 306.

Cardium lævigatum Linn. (Reeve.), Poulsen. 15.

Cardium lævigatum Rve., Moll. Cuba, ii, 306.

Cardium leucostomum Born, Krebs. 116.

Cardium leucostomum Born, Poulsen. 15.

Cardium lineatum Gmel., Krebs. 115. = serratum

;rum $\checkmark$ Cardium maculatum Gmel., A rango, Fauna Mal. Cuba, 1878. 258. Ca.

Cardium magnum Born, Kurtz. 5. NC. and SC.

Cardium magnum Born, Am. Marine Conch. 174.

Cardium magnum Born, Arango, Fauna Mal. Cuba, 1878. 258. Ca.

Cardium marmoreum, Lam., Krebs. 116. = leucastomum

Cardium medium Chem., Moll. Cuba, ii, 307.

Cardium mediun D'Orb., Arango, Fauna Mal. Cuba, 1878. 258. Ca.

M. Santa Lucia. Ba.

Cardium medium Gmel., Moll. Cuba, ii, 307.

Cardium medium Lam., Pfeiffer, A rehiv für Naturg., 1840. 260. Ca. Cardium medium Linn., Arango, Fauna Mal. Cuba, 1878. 258. Ca. M.

Santa Lucia. Ba.

Cardium medium Linn., Beau. 23. G.

Cardium medium Linn., Jour. de Conch., ii, 425. G.

Cardium medium Linn, Krebs. 116. ST. SCx. SJ. Tort.

Cardium medium Linn., Moll. Cuba, ii. 307.

Cardium medium Linn., Poulsen. 15.

Cardium medium Linne, Report Blake Moll. 132.

Cardium medium Rve., Moll. Cuba, ii, 307.

Cardium medium Wood, Moll. Cuba, ii, 307.

Cardium mortoni Say, Dall, Hemphill's shells. 341.

Cardium muricatum Chem., Moll. Cuba., ii, 305.

Cardium muricatum D'Orb., Arango, Fauna Mal. Cuba, 1878. 259. Ca.

M. G. J. Ba.

Cardium muricatum D'Orb., Moll. Cuba, ii, 305.

Cardium muricatum Lam., Moll. Cuba, ii, 305.

Cardium muricatum Linn., Am. Marine Conch. 174.

Cardium muricatum Linn., Araugo, Fauna Mal. Cuba, 1878. 259. Ca. M. G. J. Ba.

Cardium muricatum Linn., Beau. 23. G.

Cardium muricatum Linn., Jahrb., ii, 249. 
'Cardium muricatum Linn., Jour, de Conch., ii, 425. G.

$\checkmark$ Cardium muricatum Linn., Krebs. 116. VgI.

$\checkmark$ Cardium muricatum Linn., Mal. Bl., x, 229.

$\checkmark$ Cardium (Trachyeardiuı) muricatum Liun., Marrat, Argo Exp., 1876.

6. Aa. Tucacas.

$\checkmark$ Cardium muricatum Limn., Moll. Cuba, ii, 305.

Cardium muricatum Linn., Poulsen. 15.

Cardium oviputamen Rve., Krebs. 116. - serratum

Cardium papillosum Poli, Mal. Bl., viii, 37. not intellern

Cardium pectinatum Linn., Zt. Mal., x, 80. SV. if rican crast

Cardiun (Fuivia) peramabilis Dall, n. s., Report Blake Moll. 132. CS.

Cardium petitianum D'Orb., Arango, Fauna Mal. Cuba, 1878. 259. Ca.

G. Ba. = semisulcatum Gray.

Cardium petitianum D'Orb., Beau. 23. G.

Cardium petitianum D'Orb., Jour, de Conch., iv., 415. G.

Cardium petitiauum D'Orb., Krebs. 116. ST. SJ. T.

Cardium petitianum D'Orb., Moll. Cuba, ii, pl. xxvii, figs. 50-52. 309.

¿Cardium pictum Dkr., Mal. Bl., riii, 37. probably = papilhum prli, European,

Cardium pinnulatum Cour., Bush, Conn. Ac. vi, 477. Hatt.

Cardium prista Val., Arango, Fauna Mal. Cuba, 1878. 258. Ca.-sesratum

Cardium pristis Val., Krebs. 116.

Cardium ringiculum D’Orb., Arango, Fauna Mal. Cuba, 1878. 259.

Carlium ringiculum Rve., Moll. Cuba, ii, 305. = semisulcatum iray-

Cardium ringiculum Sby., Arango, Fauna Mal. Cuba, 1878. 259. San

Vicente.

Cardium ringiculum Sby., Krebs. 116.

Cardium ringiculum Sby., Moll. Cuba, ii, 305. SV.

Cardium ringieulum Sby.=C. petitianum D'Orb., Poulsen. 15.

Cardium serratum Chem., Beau. 23. G.

rCardium serratum Chem., Krebs. 115.

Cardium serratum D'Orb., Moll. Cuba, ii, 304.

Cardium serratum Lam., Kurtz. 5. SC.

Cardium serratum Linn., Arango, Fauna, Mal. Cuba, 1878. 258. Ca.

Cardium serratum Linn., Moll. of Bermudas. 25.

Cardium serratum Linn., Moll. Cuba, ii, 304.

Cardium serratum Linn., Report Blake Moll. 131. CS.

Cardium serratum Reeve, Report Blake Moll. 131. CS.

Cardium soleniforme Brug., Krebs. 117. = Jpinosem rnens ch.

Cardium spinosum Meusch., Arango, Fauna Mal. Cuba, 1878. 259.

Ca. M. G. Santa Lucia. Ba.

Cardium spinosum Meusch., Krebs. 116. Td. NGr.

Cardium spinosum Meusch., Poulsen. 15.

$\checkmark$ Cardium subelongatum Rve., Arango, Fauna Mal. Cuba, 1878. 257.

Ca. M. ST. G. Ba.

Cardium subelongatum Rve., Moll. Cuba, ii, 306. 
¿ardium subelougatum Sby., Arango, Fauna Mal. Cuba, 1878. 257. Ca. M. ST. G. Ba.

\Cardium subelongatum Sby., Beau. 23. G.

Cardium subelongatum Sby., Jour. de Conch., iv, 415. G.

Cardium subelongatum Sby., Krebs. 117. SCx.

Cardium (Trachycardium) subelongatum Sby., Marrat, Argo Exp., 1876.

13. Santa Marta.

$\checkmark$ Cardium subelongatum Sby., Moll. Cuba, ii, 306.

Cardium tigrinum Mart., Krebs. 97. = Venus maculata.

Cardium trigonium Mart., Arango, Fauna Mal. Cuba, 1878. 250. Ca.

G. M. J. Santa Lucia, Ba. = denus maculata

$\checkmark$ Cardium trigynum Mart., Moll. Cuba, ii, 269. "

$\checkmark$ Cardium triste Linn., Krebs. 115. = derratum

Cardium ventricosum Brug., Arango, Fauna Mal. Cuba, 1878. 258. Ca.

Cardium ventricosum Brug., Mal. B1., x, 229. = magnum Born

Cardium venustum Dkr., ${ }^{*}$ Mal. Bl., viii, $37 .=$ media $\mathcal{L}^{\text {. }}$

Careliopsis styliformis Mörch,* Mal. Bl., xxii, 169. ST.

Carina holothurianum Rumph., Jour. de Conch., viii, 277. J.

$\checkmark$ Caryatis varians Wood, Jahrb., ii, 249.

Cassidaria cingulata Lam., Krebs. 24.

Cassidaria cingulata Lam., Mal. Bl., xxir, 33.

Cassidaria? coronadoi Crosse, Arango, Fauna Mal. Cuba, 1878. 224. Ca., Matanzas.

Cassidaria lævigata Sby., Mal. Bl., xxiv, 41. J.

Cassidaria oniscus Zt. Mal., ii, 135.

Cassidaria oniseus, Lam., Mal. Bl., xxiv, 40.

Cassidaria oniscus, Lam., Mal. Bl., xxiv, 41.

Cassidaria oniseus Lam., Moll. Cuba, ii, 131.

Cassidaria striata Desh., Mal. Bl., xxir, 41. J.

Cassidaria striata Kien., Mal. Bl., xxiv, 41. J.

Cassi aria striata Lam., Mal. Bl., xxiv, 41. J.

Cassidaria sublævigata Guppy, Mal. Bl., xxiv, 41. J.

Cassidaria vexillum Kien., Moll. Cuba, ii, 147.

Cassidea grandis Humph., Mal. Bl., xxiv, 37.

Cassidea granulosa Brug., Arango, Fauna Mal. Cuba, 1878. 223. Ca. G. Ba.

Cassidea granulosa Brug., Mal. Bl., xxir, 35. Bz.

Cassidea granulosa Brug., Moll. Cuba, ii, 181.

Cassidea inflata Shaw, Mal. Bl., xxiv, 35. Bz.

Cassidea oniseus Brug., Mal. Bl., xxiv, 40.

Cassidea oniseus Brug., Moll. Cuba, ii, 131.

Cassidea testiculus Linu., Marrat, Argo Exp., 1876. 7. Barbuda. NP. Cassidula (see Hemifusus).

Cassidula corona Gmelin, Conrad, Proc. Acad. Nat. Sci., Phil., vi, 449. 1853. Fla. 
Oassidulus carolinensis T. \& H., n. s., Pleiocene fossils S. C., pl. xxx, fig. 1. 147.

Cassidulus melongena Linn., Jahrb., ii, 355.

Cassidulus melongena Limu., Marrat, Argo Exp., 1876. 11. Puerto Cabello, Tucacas.

Cassidulus morio Linn., Poulseu. 11.

Cassidulus patula Brod. (?), Jahrb., ii, 355.

Cassis (see Cassidaria) Araugo, Fauma Mal. Cuba, 1878. 224. Ca. Matanzas.

Cassis (see Semicassis) Arango, Fauna Mal. Cuba, 1878. 223. Ca. G. Ba.

Cassis abbreviata Kien. Mal. Bl., xxiv, 36. G.

Cassis abbreviata Lam., Beau. 8. G.

Cassis abbreviata Lam., Krebs. 34.

Cassis abbreviata Lam., Mal. Bl., xxir, 36. G.

Cassis abbreviata Lam., Poulsen. 10.

Cassis alba Perry, Mal. Bl., xxiv, 38.

Cassis bisulcata Wag., Krebs. 34.

Cassis cameo Stm., Am. Marine Conch. 42.

Cassis cameo Stm., Mal. Bl., xxiv, 37.

Cassis cicatricosa Meusch., Krebs. 34. Td. SOx.

Cassis cicatricosa Meusch., Mal. Bl., xxiv, 36.

Cassis cicatricosa Meusch., Poulsen. 10.

Cassis elathrata, \&c., Mal. Bl., xxiv, 39.

Cassis cornuta Linn., Beau. 8. G.

Cassis cornuta Limn., Krebs. 34.

Cassis coronadoi Crosse, Am. Jour. Conch., iii, 203. Ca.

Cassis coronadoi Crosse, Jahrb., iii, 82.

Cassis coronadoi Crosse,* Jour. de Conch. xv, pl. v, vi, fig. 1, 64. Ca. Cassis coronadoi Crosse, Mal. Bl., xxiv, 35. Ca.

Cassis crumena Brug., Krebs. 35.

Cassis crumena Lam., Mal. Bl., xii, 128. ST.

Cassis crumena Lam., Zt. Mal., x, 77. SV.

Cassis crumena Linn. (?), Mal. Bl., x, 225. ST.

Cassis fasciatim flammea, Mal. Bl., xxiv, 39.

Cassis flammea Kurtz. 9. NO. SC.

Cassis flammea D’Orb., Arango, Fauna Mal. Cuba, 187S. 223. Ca. Ba.

Cassis flammea Lam., Moll. Cuba, ii, 180.

Cassis flammea Linn., Arango, Fauma Mal. Cuba, 1878. 22:3. Ca. Ba.

Cassis flammea Linn., Beau. S. G.

Cassis flammea Linn., Krebs. 34. Bb. Ag. Tort.

Cassis flammea Linn., Mal. Bl., xxiv, 37, 38.

Cassis flammea Limu., Marrat, Argo Exp., 1876. 16. Nassau.

Cassis flammea Iinn., Poulsen. 10.

Cassis gibba Gmel., Krebs. 34. SM. 
Cassiis gibba Gmel., Mal. Bl., xxiv, 36.

Cassis gibba Gmel., Poulsen. 10.

Cassis globulus Bolt., Krebs. 34.

Cassis globulus, Bolt., Mal. Bl., xxiv. 36 .

Cassis granulata Born, Mal. Bl., xxiv, 35. Bz.

Cassis granulata Borm, = C. ventricosa, Mart., Poulsen. 10.

Cassis granulatum Born, Krebs. 34.

Cassis granulosa Brug., Am. Marine Conch. 43.

Cassis granulosa Brug., Beau. S. G.

Cassis granulosa Brug., Jahrb., iii, 79.

Cassis granulosa D’Orb., Arango, Fauna Mal. Cuba, 1878. 224. Ca.

G. Ba.

Cassis granulosa D’Orb., Moll. Cuba, ii, 181.

Cassis granulosa Kien., Krebs. 34.

Cassis granulosa Lam., Krebs. 34.

Cassis granulosa Lam., Mal. Bl., xxiv, 35. Bz.

Cassis granulosa Lam., Moll. Cuba, ii, 181.

Cassis granulosus Brug., Jour. de Conch., iv, 417. G.

Cassis intlata Shaw, Arango, Fauna Mal. Cuba, 1878. 223. Ca. G. Ba.

Cassis inflata Shaw, Jahrb., iii, 79.

Cassis inflata Shaw, Mal. Bl., xxiv, 35.

Cassis labiata Chem., Beau. 8. G.

Cassis labiata Chem., Krebs. 34.

Cassis lactea Kien., Beau. 8. G.

Cassis lactea Kieu., Krebs. 34.

Cassis lactea Kien., Mal. Bl., xxiv. 37.

Cassis lactea Kien., Mal. Bl., xxiv, 37. S'T.

Cassis lactea Kien., Poulsen. 10.

Cassis lactea Mörch, Mal. Bl., xxir, 37. S'T.

Cassis lrevigata Menke, Mal. Bl., xxiv, 36.

Cassis madagaseariensis Chenu, Mal. Bl., xxiv, 37.

Cassis madagascariensis Kieu., Mal. Bl., xxiv, 37.

Cassis madagascariensis Lam., Arango, Fanna Mal. Cuba, 1878. 223.

Ca. Ba.

Cassis madagascariensis Lam., Krebs. 35. ST. SCx.

Cassis madagascariensis Lam., Mal. Bl., xxiv, 37.

Cassis madagascarieusis Lam., Marrat, Argo. Exp., 1876. 16. NP.

Cassis madagascariensis Lam., Poulsen. 10.

Cassis malum Bolt., Mal. Bl., xxiv, 36. Wla.

Cassis minuta Mke., Mal. Bl., xxiv, 37. ST.

Cassis penita Meusch., Mal. Bl., xxir, 34. PR.

Cassis reclusa Guppy, и. s., Geol. Mag., 1874. 442. J.

Cassis recurvirostris Gmel., Krebs. 34.

Cassis rotundata Perry, Mal. Bl., xxiv, 38. J.

Cassis, Seba, Mal. Bl., xxir, 38. J.

Cassis sulcosa? Jahrb., iii, 79. 
Cassis suleosa Kien., Moll. Cuba, ii, 182.

Cassis sulcosa Kien., Mal. Bl., xxiv, 36.

Cassis sulcosa? Lam., Kurtz. 9. NO. SO.

Cassis tessellata Pfr., Mal. Bl., xxiv, 35.

Cassis tessellatum Chem., Beau. 8. G.

Cassis testiculus D'Urb., Arango, Mal. Cuba, 1878. 224. Ca. M. G. Ba.

Cassis testiculus D'Orb., Moll. Cuba, ii, 181.

Cassis testiculus Lam., Moll. Cuba, ii, 181.

Cassis testiculus Linn., Beau. 8. G.

Cassis testiculus Linn., Jahrb., iii, 78.

Cassis testiculus Linn., Jour. de Conch., iv, 417. G.

Cassis testiculus Linn., Krebs. 35. SCx. Tort. SB. Bb. Td.

Cassis testiculus Linn., Mal. Bl., xxiv, 39.

Cassis testiculus Linn., Poulsen. 10.

Cassis tuberosa D’Orb., Arango, Fauna Mal. Cuba, 1878. 223. Ca. G. Ba.

Cassis tulıerosa Kien., Mal. Bl. xxiv, 38.

Cassis tuberosa Lam., Beau. S. G.

Cassis tuberosa Lam., Moll. Cuba, ii, 180.

Cassis tuberosa Linn., Arango, Fauna Mal. Cuba, 1878. 223. Ca. G. Ba. Cassis tuberosa Linn., Jahrb., iii, 77.

Cassis tuberosa Linn., Krebs. 35. Ag. SJ. Tort. SM. Bb.

Cassis tuberosa Linn., Mal. Bl., xxiv, 38.

Cassis tuberosa Limn., Marrat, Argo Exp., 1876. 16. Nassau.

Cassis tuberosa Linn., Poulsen 10.

Cassis tuberosus Linn,, Jour. de Conch., iv, 417. G.

Cassis undulata Humph., Mal. Bl., xxiv, 38. Ca.

Cassis ventricosa Mart., Krebs. 34. ST.

Uassis ventricosa Mart., Mal. Bl., xxir, 35.

Cassis ventricosa Mörch, Mal. Bl., xxiv, 35.

Catinus (see Sigaretus).

Catinus maculatus Say, Am. Marine Conch. 61.

Catinus perspectivus Say, Am. Marine Conch. 61.

Cavolina (see Hyalæa).

Cavolina natans Abilı., Arango, Fauna Mal. Cuba, 1878. 150. WI.

Cavolina natans Abild., Moll. Cuba, i, 72.

Carolina tuomeyi Holm., 11. s., Post.Pleiocene Fossils S. C., pl.ix, figs. 8 to 8 e. 60 .

Cerithidea ambigua C. B. Adams,* Mal. Bl., xxiii, 91. J.

Cerithidea ambigua C. B. Adams, Marrat, Argo Exp., 1876. 7. Barbuda.

Cerithidea ambigua C. B. Adams, Poulsen. S.

Cerithidea costata Da Costa, Arango, Fauma Mal. Cuba, 1878. $20 \%$. Ca. J. ST. Ba.

Cerithidea costata Da Costa, Poulsen. 8.

- Cerithidea costata Lam., Marrat, Argo Exp., 1876. 7. Barbuda.

Cerithidea costata Wood, Dall, Hemphill's shells. 334.

Cerithidea costata Wood, ${ }^{*}$ Mal. Bl., xxiii, 90. ST. 
Cerithidea crassilabrum Ad., Dall, Hemphill's shells. 334. CedarKeys. Cerithidea hegewischii Phil.,* Mal. Bl., xxiii, 89.

Cerithidea iostoma P'fr., Arango, Fauna Mal. Cuba, 1878. 209. Ca.

Cerithidea lavalleana D'Orb., ${ }^{*}$ Mal. Bl., xxiii, 89. Ca.

Cerithidea minor Mörch, Mal. Bl., xxiii, 92. Ca.

Cerithidea pliculosa Mke., ${ }^{*}$ Mal. Bl., xxiii, 85. J.

Cerithidea pupoidea Mörch, Mal. Bl., xxïi, 93. J.

Cerithidea salmacidum Morelet, Poulsen. 8.

Cerithidea scalariformis Say, Dall, Hemphill's shells. 334. Cedar Keys.

Cerithidea scalariformis Say,* Mal. Bl. xxiii, 87. Fla.

Cerithidea scalariformis Say, Poulsen. 8.

Cerithidea tenuis Pfr., Arango, Fauna Mal. Cuba, 1878. 209. Ca.

Cerithidea tenuis Pfr., Dall, Hemphill's shells. 334.

Cerithidea tenuis Pfr.,* Mal. Bl., xxiii, 87. Ca.

Cerithidea tenuis Pfr., Poulsen. 8.

Cerithidea tenuis Rve. or Sby., Mal. Bl., xxiii, 88.

Cerithidea turrita Stearns, Dall, Hemphill's shells. 334. Key West.

Cerithidea turrita Stearns,* Mal. Bl., xxiii, 92-93.

Cerithidea turrita Stearns,* Proc. B. S. N. H. xv, 1872. Tampa. W. Fla.

Cerithidea varicosa Sby., Poulsen. 8.

Cerithidea varicosa Sby., Mal. Bl., xxiii, 88. Ca. J.

Cerithiopsis (see Bittium, Cerithium).

Cerithiopsis (?) crystallina Dall., n. s., Report Blake Moll. 89. Bb.

Cerithiopsis emersonii C. B. Adlams, Dall, Hemphill's shells. 333. Cedar Keys.

Cerithiopsis emersonii Stimpson, Kurtz. S. SC.

Cerithiopsis funiformis C. B. Adams,* Mal. Bl., xxiii, 104. J.

Cerithiopsis fusiformis C. B. Adams, Poulsen. 9.

Cerithiopsis gemmulosa Ad., Mal. Bl., xxiii, 103.

Cerithiopsis greenii C. B. Adams,* Mal. Bl., xxiii, 105.

Cerithiopsis greenii C. B. Adams, Poulsen. 9.

Cerithiopsis neglectus C. B. Adams, Mal. Bl., xxiii, 105. J.

Cerithiopsis pulchellum C. B. Adams, ${ }^{*}$ Mal. Bl., xxiii, 105. J.

Cerithiopsis punctatum Linn., Guppy, Paria Fauna, 1877. 139.

Cerithiopsis (?) sigsbeana Dall, n. s., Report Blake Moll. 87. CS.

Cerithiopsis terebellum Stimpson, Kurtz. 8. NC. SC.

Cerithiopsis terebellum Stimpson, Mal. Bl., xxiii, 99.

Cerithiopsis terebralis Ads., Dall, Hemphill's shells. 333. Key West.

Tampa. Cedar Keys.

Cerithiopsis tuberculata Mont., Dall, Hemphill's shells. 333. Key West.

Cerithium (see Cerithiopsis, Bittium, Pyrazus, Triforis, Cerithidea).

Cerithium aberrans C. B. Adams,* Mal. Bl., xxiii, 120. J.

Cerithium aberrans C. B. Adams, Poulsen. 9.

Cerithium alabastrulum Morch, ${ }^{*}$ Mal. Bl., xxiii, 121. ST. 
Cerithium alabastrum Morch, ${ }^{*}$ Mal. Bl., xxiii,120. ST.

Cerithium albidum d. Ad., Arango, Fauna Mal. ('nba, 187s. 206. Ca.

Cerithium albovittatum C. B. Adams, Contr. to Conch. 122. J.

Cerithium albovittatum Ad., Krebs. 47.

Cerithium albovittatum C. B. Adams, Krebs' Remarks. 394.

Cerithium albovittatum C. B. Adams, Mal. Bl., xxiii, 102. J.

Cerithium algieola Ad., Arango, Fanua Mal. Cuba, 1878. 206. Ca. Ba.

Cerithium algicola Ad., Krebs. 47. ST. Bb.

Cerithinm algicola C. B. Adams, * Mal. Bl., xxiii, 114, 115. ST. J.

Cerithium algicola C. B. Adams, Poulsen. 9.

Cerithium algicola C. B. Adams, Synops, 5, 1845. J.

Cerithium ambiguum C. B. Adams, Arango, Fauna Mal. Cuba, 1878. 209. Ca. S. S'T. Ba.

Cerithium ambiguum C. B. Adams, Beau. 11. G.

Cerithium ambiguum Ad., Krebs. 47.

Cerithium ambiguum C. B. Adams, Krebs' Remarks. 394.

Cerithium ambiguum C. B. Adams, Mal. Bl., xxiii,91. J.

Cerithium ambiguum C. B. Adams, Mal. Bl., xxiii,91. G.

Cerithium ambiguum F. \& H., Mal. Bl., xxiii, 92.

Cerithium ambiguum C. B. Adams, Synops., 4, 1845. J.

Cerithium angustum Anton, Mal. Bl., xxiii, 112.

Cerithium antillarum Dkr., * Mal. Bl., xxiii, 112. J., \&c.

Cerithium antillarum Dkr., Poulsen. 9.

Cerithium atratum Born, Bean. 11. G.

Cerithium atratum Born, Jahrb., ii, 244.

Cerithium atratum Born, Jour. de Conch., ii, 428. G.

Cerithium atratum Born, Krebs. 47. ST.

Cerithium atratum Born, Mal. Bl., x, 225.

Cerithium atratum Born, Mal. BI., xxiii, 113.

Cerithium atratum Born, Poulsen. 9.

Cerithium atratum Brug., Mal. Bl., xxiii, 113.

Cerithium atratum Kien., Mal. Bl., xxiii, 113.

Cerithium atratum Lam., Mal. BI., xxiii, 113.

Cerithium atratum Wood, Mal. Bl., xxiii, 113.

Cerithium bermudæ Sby., Am. Jour. Conch., ii, 286. Ber.

Cerithium bermudx Sby., * Mal. Bl., xxiii, 118. Ber.

Ceritbium bicolor Ad., Krebs. 47.

Cerithium bicolor C. B. Adams, Krebs' Remarks. 394. WI.

Cerithium bicolor C. B. Adams, Mal. Bl., xxiii, 102. J.

Cerithium bicolor C. B. Adams, Synops. 5, 1845. J.

Cerithium (Triforis) bigemma Watson, Report Blake Moll. 81. ST.

Cerithium cancellatum H. C. Lea, Mal. Bl., xxiii, 123.

Cerithium caudatum Sby., Jour. de Conch., $\nabla, 153 . \quad$ G.

Cerithium caudatum Sby.,* Mal. Bl., xxiii, 113, 1I4. G.

Cerithium caudatum Sby., Marrat, Argo Exp., 1876. 6. Aa., Puerto Cabello. 
Cerithium clathratum Mke., Mal. Bl., xxiii, 93. Fla.

Cerithium elıvulus H. C. Lea, Conrad, Am. Journ. Sci., n. s., 1, 405, 1846. Tampa Bay.

Cerithium clavulus H. C. Lea, Mal. Bl., xxiii, 123, Fla.

Cerithium columellare D'Orb., Arango, Fauna Mal. Cuba, 1878. 207. Ca. G. J. SD. S'T.

Cerithium columellare D'Orb., Beau. 11. G.

Cerithium columellare D'Orb., Krebs. 47.

Cerithium columellare D'Orb., Mal. Bl., xxiii, 96. Ca.

Cerithium columellare D'Orb., Moll. Cuba, ii, 155.

Cerithium eostata Da Costa, Krebs' Remarks. 394.

Cerithium costatum Brown, Mal. Bl., xxiii, 90.

Cerithium costatum Da Costa, Arango, Fauna Mal. Cuba, 1878. 209. Ca. J. ST. Ba.

Cerithium costatum Sby., Mal. B1., xxiii, 91.

Cerithium costatum Wood, Beau. 11. G.

Cerithium costatum Wood, Jour. de Conch., $\nabla, 153 . \quad$ G.

Cerithium costatum Wood, Krebs. 47.

Cerithium crassilabrum Krauss, Mal. Bl., xxiii, 121.

Cerithium dealbatum C. B. Adams, Contr. to Conch. 117. J.

Cerithium dealbatum Ad., Mal. Bl., xxiii, 110. J.

Cerithium decoratum Ad., Arango, Fauna Mal. Cuba, 1878. 206. Ca.

Cerithium decoratum C. B. Adams, Contr. to Conch. 117. J.

Cerithium decoratum Ad., Mal. Bl., xxiii, 110. J.

Cerithium dislocatum Holmes, Mal. Bl, xxiii, 122. Fla.

Cerithium dislocatum Say, Mal. Bl., xxiii, 122. Fla.

Cerithium dissimile Yoldi, Krebs. 48.

Cerithium eburneum Brug., Arango, Fauna Mal. Cuba, 1878. 206. Ca. G. M. Ba.

Cerithium eburneum Brug., Krebs. 48. ST.

Cerithium eburneum Brug., Mal. Bl., xxiii, 114.

Cerithium eburneum Brug., Marrat, Argo Exp., 1876. 16. Nassau.

Long Key Island. Athol Island.

Cerithium eburneum Brug., Moll. Cuba, ii, 154.

Cerithium eburneum Brug., Poulsen. 9.

Cerithium eburneum D'Orb., Arango, Fauna Mal. Cuba, 1878. 206. Ca. G. M. Ba.

Cerithium eburneum D'Orb., Mal. Bl., xxiii, 114. G.

Cerithium eburneum Kien., Mal. Bl., xxiii, 114. Ca.

Cerithium eburneum Lam., Mal. Bl., xxiii, 114.

Cerithium eburneum Lam., Moll. Cuba, ii, 154.

Cerithium eburneum P. \& M., Mal. Bl., xxiii, 114.

Cerithium eburneum Sby., Mal. Bl., xxiii, 114, 1i5. G.

Cerithium elegans Blv., Mal. Bl., xxiii, 98.

Cerithium elegans F. \& H., Mal. Bl., xxiii, 98.

Cerithium emersoni Ad., Mal. Bl., xxiii, 98. 
Cerithium emersonii C. B. Adams, Beau. 11. G.

Cerithium emersonii C. B. Adaus, Jour. de Couch., v, 153. G.

Cerithium emersonii C. B. Adams, Krebs. 50.

Cerithium emersonii C. B. Adams, Krebs' Remarks. 394.

Cerithium emersonii Gould, Mal. Bl., xxiii, 98.

Cerithium eriense Kien., Beau. 11. G.

Cerithium eriense Kien., Jour. de Conch., ii, 428. G.

Cerithium eriense Kien., Krebs. 48.

Cerithium eriense Val., Arango, Fauna Mal. Cuba, 1878. 208. Ca. Fla. J. Ba.

Cerithium eriense Val., Mal. Bl., xxiii, 94.

Cerithium eriense Val., Mal. Bl., xxiii, 117.

Cerithium eriense Val., Marrat, Argo Exp., 1876. 14. Point Savanilla.

Cerithium eriense Val., Moll. of Bermudas. 18.

Cerithium exiguum C. B. Adams, Contr. to Conch. 118. J.

Cerithium exignum C. B. Adams, Mal. Bl., xxiii, 107. J.

Cerithium exile C. B. Adams, Contr. to Conch. 120. J.

Cerithium exile Ad., Krebs. 48.

Cerithium exile Ad., Mal. Bl., xxiii, 104. J.

Cerithium ferruginea Say, Zt. Mal., viii, 67.

Cerithium ferrugineum Say, Am. Marine Conch. 71.

Cerithium ferrugineum Say, Arango, Fauna Mal. Cuba, 1878. 206.

Ca. Ba. Fla. J.

Cerithium ferrugineum Say, Beau. 11. G.

Cerithium ferrugineum Say, Dall, Hemphill's shells. 333. Sarasota

Bay. Key West.

Cerithium ferrugineum Say, Krebs. 48.

Cerithium ferrugineum Say, Krebs' Remarks. 395.

Cerithium ferrugineum Say, Mal. Bl., xxiii, 116-7. Fla.

Cerithium ferrugineum Say, Marrat. Argo Exp., 1876 18. Abaco.

Cerithium ferrugineum Say, Poulsen. 9.

Cerithium ferrugineum Sby., Mal. Bl., xxiii, 116.

Cerithium ferrugineum Sby., Mal. Bl., xxiii, 118. J.

Cerithium flavum? Ad., Arango, Fauna Mal. Cuba, 1878. 207. Ca.J.

Cerithium flavum C. B. Adams, Contr. to Conch. 122. J.

Cerithium flavum Ad., Krebs. 48.

Cerithium flavum C. B. Adams, Mal. Bl., xxiii, 100. J.

Cerithium floridanum Mörch,* Mal. Bl., xxiii, 114. Fla.

Cerithium fusiforme C. B. Adams, Mal. Bl., xxiii, 105. J.

Cerithium fusiforme C. B. Adams, Contr. to Conch. 120. J.

Cerithium fusiforme Ad., Krebs. 48.

Cerithium fusiforme C. B. Adams, Krebs' Remarks. 394.

Cerithium gallopaginis Sby., Mal. Bl., xxiii, 120. ·Bb.?

Cerithium gemmulosum Ad., Arango, Fauna Mal. Cuba, 1878. 207. Ca. J.

Cerithium gemmulosum O. B. Adams, Contr. to Conch. 120. J. 
Cerithium gemmulosum C. B. Adams, Jahbr., ii, 244.

Cerithium gemmulosum Ad., Krebs. 48. ST.

Cerithium gemmulosum C. B. Adams, Krebs' Remarks. 394.

Cerithium gemmulostum C. B. Adams, Mal. Bl., xxiii. 103.

Cerithium gemmulosum Sby., Mal. Bl., xxiii. 103.

Cerithium gibberulum Ad., Arango, Fauna Mal. Cuba, 1878. 207. Ca. G. J. SD. ST.

Cerithium gibberulum C. B. Adams, Beau. 11. G.

Cerithium gibberulum C. B. Adams, Jour. de Conch., v, 153. G.

Cerithium gibberulum Ad., Krebs. 48. PPI. S'T. SJ. SM.

Cerithium gibberulum O. B. Adams, Mal. Bl., xxiii, 96. J.

Cerithium gibberulum C. B. Adams, Synops., p. 5. $1845 . \quad$ J.

Cerithium gibberulum Sby., Mal. Bl., xxiii, 96 . G.

Cerithium gracile Sby., Mal. Bl., xxiii, 100. ST.

Cerithium graciliforme Sby.?* Mal. Bl., xxiii, 115. NP.

Cerithium graeiliforme Sby., Marrat, Argo Exp., 1876. 16. Nassau.

Cerithium graciliformis Sby., Poulsen. 9.

Cerithium greenii C. B. Adams, * Mal. Bl., xxiii, 105.

Cerithium guaranianum D'Orb., Mal. Bl., xxiii, 95. Bz.

Cerithium guinaicum Phil., Araugo, Fauna Mal. Cuba, 187S. 207. Ca.

Cerithium hegewischii Phil., Mal. Bl., xxiii, 89.

Cerithium helicoide C. B. Adams, Mal. Bl., xxiii, 89.

Cerithium heteroelites Pot. \& Mich., Mal. Bl., xxiii, 93. Fla.

Cerithium (Triforis) inflatum Watson, Report Blake Moll. 81. CS.

Cerithium intermedium C. B Adams, Contr. to Conch. 119. J.

Cerithium intermedium Ad., Mal. Bl., xxiii, 109. J.

Cerithium interruptum Mke., Krebs. 48. PPl. ST. SJ. SM.

Cerithium interruptum Mke., Mal. Bl., xxiii, 99.

Cerithium iostoma Rve., Mal. Bl., xxiii, 89.

Cerithium iostoma Sby., Mal. Bl., xxiii, 89.

Cerithium iota Ad., Krebs. 48.

Cerithium iota C. B. Adams, Krebs' Remarks. 394.

Cerithium iota C. B. Adams, Synops. p..5, 1845. J.

Cerithium jota C. B. Adams, * Mal. Bl., xxiii, 97. J.

Cerithium lafondii Kien., Mal. Bl. xxiii, 91.

Cerithium lafondii Mich., Arango, Fauna Mal. Cuba, 1878. 209. Ca.

J. ST. Ba.

Cerithium lafondii Mich., Krebs. 49.

Cerithium lafondii Mich., Krebs' Remarks. 394.

Cerithium lafondii Mich., Mal. Bl., xxiii, 91.

Cerithium latreillei Payr., Jour de Conch., ii, 428. G.

Cerithium latum C. B. Adams, Contr. to Conch. 122. J.

Cerithium latum Ad., Mal. Bl., xxiii, 104.

Cerithium lavalleanum D’Orb., Arango, Fanna Mal. Cuba, 1878. 209. Ca.

Cerithium lavalleanum D’Orb., Krebs. 49. 
Cerithium lavalleanum D'Orb., Mal. Bl., xxiii, 89.

Cerithium lavalleamum D'Orb., Moll. Cuba, ii, pl. xxiii, fig. 16. 156.

Cerithium lima Brug., Araugo, Fauna Mal. Cuba, 1878. 207. Ca. M. G.

Cerithium lima Brug., Beau. 11. G.

Cerithium lima Brug., Jour. de Conch., ii, 428. G.

Cerithium lima Brug., Krebs. 49.

Cerithium lima Brug., Mal. Bl., xxiii, 95.

Cerithium lima Brug., Moll. Cúba, ii, 154.

Cerithium líma D’Orb., Arango, Fauna Mal. Cuba, 1878. 207. Ca. M. G.

Cerithium lima D'Orb., Mal. Bl., xxiii, 95.

Cerithium lima Lam., Mal. Bl., xxiii, 95.

Cerithium lima Lam., Moll. Cuba, ii, 154.

Cerithium litteratum Born, Arango, Fauna Mal. Cuba, 187S. 207. Ca. G. M. ST. Ba.

Cerithium litteratum Born, Beau. 11. G.

Cerithium litteratum Born,* Mal. Bl., xxiii, 111. Ca. G.

Cerithium litteratum Born, Marrat, Argo Exp., 1876. 7. Barbuda,

Tucacas, Sauta Marta, Nassau, Long Key Islaud, Athol Island.

Cerithium litteratum Born, Moll. of Bermudas. 18.

Cerithium litteratum Born, Poulsen. 9.

Cerithium litteratum Brug., Jour. de Conch., ii, 428. G.

Cerithium litteratum Brug., Krebs. 49. ST. Tort. SM. Bb. Td.

Cerithium litteratum Brug., Krebs. 50.

Cerithium litteratum Brug., Mal. Bl., xxiii, 111.

Cerithium litteratum Brug., Moll. Cuba, ii, 153.

Cerithium litteratum D'Orb., Arango, Fauna Mal. Cuba, 1878. 207.

Ca. G. M. S'T. Ba.

Cerithium litteratum var. parra Kien., Mal. Bl., xxiii, 115.

Cerithium litteratum Lam., Mal. BI., xxiii, 111.

Cerithium litteratum Lam., Moll. Cuba, ii, 153.

Cerithium litteratum Sby., Mal. Bl., xxiii, 112.

Cerithium luctuosum Mke., Mal. Bl., xxiii, 119, 120. Ca.

Cerithium lutosum Mke., ${ }^{*}$ Mal. Bl., xxiii, 119. Ca.

Cerithium Iutosum Mke., Poulsen. 9.

Cerithium marocianum Brug., Moll. Cuba, ii, 155.

Cerithium megasoma C. B. Arlams, Contr. to Couch. 119. J.

Cerithium megasoma Ad., Krebs. 49.

Cerithium megasoma C. B. Adams, Krebs' Remarks. 395.

Cerithium megasoma Ad., Mal. Bl., xxiii, 119. ST.

Cerithium megasoma C. B. Adams, * Mal. Bl., xxiii, 122. J.

Cerithium melanura C. B. Adams, Contr. to Conch. 117. J.

Cerithium melanmra C. B. Adams, Mal. Bl., xxiii, 108. J.

Cerithium minimum Gmel., Krebs. 49, 50.

Cerithium minimum Gmel., Krebs' Remarks. 395.

Cerithium minutum Brown, Mal. Bl., xxiii, 92. J.

Cerithium mirabile C. B. Adams, Contr. to Conch. 118. J. 
Cerithium mirabile Ad., Krebs. 49.

Cerithium mirabile C. B. Adams, Mal. Bl., xxiii, 110. ST.

Cerithium modestum C. B. Arlams, Contr. to Conch. 117. J.

Cerithium modestum Ad., Mal. Bl., xxiii, 108.

Cerithium muscarum D'Orb., Arango, Fauna Mal. Cuba, 1878. 208. Ca. Fla.

Cerithium muscarum Say, Arango, Fauna Mal. Cuba, 1878. 208. Ca. Fla.

Cerithium muscarum Say, Dall, Hemphill's shells. 333. Sarasota Bay.

Cerithium muscarum Say, Krebs. 49.

Cerithium muscarum Say, Mal. Bl., xxiii, 111.

Cerithium musearum Say, Moll. Cuba, ii, 157.

Cerithium muscarum Say, Mal. Bl. xxiii, 111.

Cerithium mutabile Ad., Krebs. 49.

Cerithium mutabile C. B. Adams, ${ }^{*}$ Mal. Bl., xxiii, 119. J.

Cerithium mutabile C. B. Adams, Poulsen. 9.

Cerithium mutabile var. C. rawsoni Krebs, Poulsen. 9.

Cerithium mutabile C. B. Adams, Synops. 5. 1845. J.

Cerithium mutabile Sby., Mal. Bl., xxiii, 119. J.

Cerithium nanum C. B. Adams, Contr. to Conch. 117. J.

Cerithium nanum Ad., Mal. Bl., xxiii, 107. J.

Cerithium neglectum C. B. Adams, Mal. Bl. xxiii, 105. J.

Cerithium nigrescens Mke., Moll. of Bermudas. 18.

Cerithium nigrescens Mke., Arango, Fauna Mal. Cuba, 1878. 208. Ca. Fla. J. Ba.

Cerithium nigrescens Mke., Dall, Hemphill's shells. 333. Sarasota Bay.

Cerithium nigrescens Mke, Jour. de Conch., ii, 428. G.

Cerithium nigreseens Mke., Krebs. 49,50. ST. SM. NGr.

Cerithinm nigrescens Mke., Krebs' Remarks. 395.

Cerithium nigrescens Mke., Mal. Bl. xxiii, 94.

Cerithium nigrocinctum Ad., Mal. Bl., xxiii, 106.

Cerithium nigrocinetum Gould, Mal. Bl. xxiii, 106.

Cerithium nigrum Phil., Mal. Bl., xxiii, 118. J.

Cerithium nivosum Say, var. Arango, Fauna Mal. Cuba, 1878. 208. Ca. Cerithium notatum Mke., Mal. Bl., xxiii, 111.

Cerithium novæ Hiberniæ A. Adams, Thes. Conchyl., p. 857, pl. 180, figs. $84,85,1847 .=$ C. eburneus Say. Fla.

Cerithium ornatum Desh., Mal. Bl., xxiii, 109.

Cerithium ornatum Dkr., Krebs. 50.

Cerithium oryza Mörch," Mal. Bl., xxiii, 121. ST.

Cerithium oryza Mörch, Poulsen. 9.

Cerithium pallidum Pfr., Araugo, Fauna Mal. Cuba, 1878. 207. Ca.

G. J. SD. ST.

Cerithium pallidum Pfr., Mal. Bl., xxiii, 96. Ca.

Cerithium pallidum Pfeiffer, Archiv fiir Naturg., 1840. 257. Ca. 
Cerithium peloritanum Kien., Mal. Bl., xxiii, 94.

Cerithium perrersum Lam., var. dextra? Pfeiffer, Archiv fuir Taturg., 1840. 257. Ca.

Cerithium petitii Kien., Arango, Fanna Mal. Cuba, 1878. 209. Ca. J. ST. Ba.

Cerithium petitii Kien., Krebs. 50. PPl. SM.

Cerithium petitii Kien., Krebs' Remarks. 394.

Cerithium petitii Kien., Mal. Bl., xxiii, 91. G.

Cerithium pliculosum Mke., Mal. Bl., xxiii, SS. J.

Cerithium pliculosum var. Mke., Mal. Bl., xxiii, 91. G.

Cerithium præustum Reeluz, Mal. Bl., xxiii, 57. J.

Cerithium protextum Con., Mal. Bl., xxin, 121. (Terebra). Fla.

Curithium protextum Con., Proc. Acarl. Nat. Sci. Phil., iii, 26, 1845. Tampa.

Cerithium pulchellum C. B. Adams, Contr. to Conch. 121. J.

Cerithium pulchellum Ad., Mal. Bl., xxiii, 106. J.

Cerithium pulchellum Sby.,* Mal. Bl., xxiii, 106. J.

Cerithium pulicarium Phil.,* Mal. Bl., xxiii, 115, 116. Y.

Cerithium pulicarium Phil., Zt. Mal., v, 20. Y.

C'erithium punctatum? Alango, Famma Mal. Cuba, 1878. 205. Ca. G.

Cerithum punctatum Lam., Pfr., Arehir. füir Naturg., 1510. 256. Ca.

Cerithium punctatum Linn., Beau. 11. G.

Cerithium punctatum Linn., Jour. de Conch., $\nabla, 153$. G.

Cerithium punctatum Linn., Krebs. 50. ST. PPl.

Cerithium punctatum Linn., Krebs' Remarks. 39t. ST.

Cerithium punctatum bis, Linn., Mal. Bl, xxiii, 9S, 99.

Cerithium pusillum Pfr., Mal. Bl., xxiii, 108.

Cerithium pusillum Pfeiffer, Archir füir Naturg., 1S40. 256. Ca.

Cerithium rarimaculatum Sby., Mal. Bl., xxiii, 121.

Cerithium rawsoni Krebs., Mal. Bl., xxiii, 120. Bb. (\%)

Cerithium reticulatum Mont., Arango, Fauna Mal. Cuba, 1878. 208. Ca.

Cerithium rissoide Sby., Am. Jour. Conch., ii, 286. ST.

Cerithium rissoide Sby., Mal. Bl., xxiii, 118. ST.

Cerithinm rugulosum C. B. Adams, Contr. to Conch. 121. J.

Cerithium rugulosum Ad., Krebs. 50. ST. SJ. SM.

Cerithium rugulosum C. B. Adams, Krebs' Remarks. 395.

Cerithium rugulosum C. B. Adams, Mal. Bl., xxii, 159. V.

Cerithium ruguloșum C. B. Adams, Mal. Bl., xxiii, 100. J.

Cerithium rugulosum Ad., Mal. Bl., xxiii, 101.

Cerithinm rugulosum Sby., Mal. Bl., xxiii, 95. ST.

Cerithium sagræ D'Orb., Arango, Fauna Mal. Cuba, 1878. 208. Ca.

Cerithium sagræ D’Orb., Krebs. 50.

Cerithiurn sagræ D’Orb.,* Mal. Bl., xxiii, 119. Ca.

Cerithium sagræ D'Orb., Moll. Cuba, ii, pl. xxiii, figs. 17-19, 156.

Cerithium sagræ D’Orb., Poulseu. 9.

Bull. 24-5 
Cerithium sayi Menke, Mal. Bl., xxiii, 97. J.

Cerithium sayi Sby., Mal. Bl., xxiii, 97. J.

Cerithium scabrum Olivi, Jour. de Conch., ii, 428. G.

Cerithium scabrum Olivi, Mal. Bl., xxiii, 95.

Cerithium scalariforme Say, Krebs. 50.

Cerithium scalariforme Sby., Mal. Bl., xxiii, 87.

Cerithium semifcrrugineum Kien., Mal. Bl., xxii, 112. ST.

Cerithium semiferrugineum Lam., Araugo, Fauna Mal. Cuba, 1878. 208. Ca.

Cerithium semiferrugineum Lam., Beau. 11. G.

Cerithium semiferrugineum Lam., Krebs. 50. ST. SM.

Cerithium semiferrugineum Lam., Mal. B1., xxiii, 112. ST.

Cerithium semiferrugineum Lam., Mal. Bl., xxiii, 114. ST.

Cerithium semiferrugineum Lam., Poulsen. 9.

Cerithium semiferrugineum P. \& M., Mal. Bl., xxiii, 112. ST.

Cerithium septemstriatum Say, Arango, Fauna Mal. Cuba, 1878. 208.

Ca. Fla. J. Ba.

Cerithium septemstriatum Say, Dall, Hemphill's shells. 333. Key

West.

Cerithium septemstriatum Say, bis. Krebs. 50.

Cerithium septemstriatum Są, Krebs’ Remarks. 395.

Cerithium septemstriatum Say, Mal. Bl., xxiii, 93. Fla.

Cerithium septemstriatum Say, Mal. Bl., xxiii, 102. J.

Cerithium servile C. B. Adams, Arango, Fauna Mal. Cuba, 1878. 209.

Ca. J. ST. Ba.

Cerithium servile C. B. Adams, Beau. 11. G.

Cerithium servile C. B. Adams, Krebs. 47.

Cerithium servile C. B. Adams, Krebs' Remarks. 394.

Cerithium spadiceum Phil.,* Zt. Mal. viii, 67. Y.

Cerithium stercus-muscarum Kien., Mal. Bl., xxiii, 111.

Cerithium stercus-muscarum Saly, Arango, Fauna Mal. Cuba, 1878. 208.

Ca.

Cerithium stereus-muscarum Say, Mal. Bl., xxiii, 111. Fla.

Cerithium stereus-muscarum Say, Mal. Bl., xxiii, 117.

Cerithium stercus-musearum Say, Poulsen. 9.

Cerithium striatissimum Sby., Arango, Fauna Mal. Cuba, 1878. 208. Ca.

Cerithium subulatum Montf., Beau. 11. G.

Cerithium subulatum Montf., Jour. de Conch., v, 153. G.

Cerithium subulatum Montf., Krebs. 50.

Curithium terebellum Ad., Arango, Fauna Mal. Cuba, 1878. 209. Ca. Ba.

Cerithium terebellum Ad., Krebs. 50. ST. SM. PPl.

Cerithium terebellum Ad., Mal. Bl., xxiii, 99.

Cerithium thomasiæ Sby., Am. Jour. Conch., ii, 286. ST. 
Cerithium thomasia Sby., Dall, Hemphill's shells. 333. Key West. Antilles.

Cerithium thomasiæ Sby.,* Mal., Bl. xxiii, 118. S'T.

Cerithium thomasia Sby., Poulsen. 9.

Cerithium trilineatum Phil., Mal. Bl., xxiii, 99.

Cerithium tuberculatum Sby., Arango, Fauma Mal. Cuba, 1878. 208. Ca.

Cerithium tuberculatum Sby., Mal. Bl., xxiii, 112. ST.

Cerithium turris Chemu., Mal. Bl., xxiii, 108, 109. J.

Cerithinn turris-thoms D'Orb., Arangu, Fanua Mal. Cuba, 1878. 209. Ca. G.

Cerithium turris-thomæ D'Orb., MIoll. Cuba, ii, pl. xxiii, figs. 10-12, 155.

Cerithium turris-thomæ D'Orb., Report Blake Moll. 81.

Cerithium umbonatum Sby., Mal. Bl, xxiii, 112. J.

Cerithium uncinatum Gm., Mal. Bl., xxiii, 113.

Cerithium uncinatum Sby., Mal. Bl., xxiii, 113.

Certhium variabile C. B. Adams, Arango, Fauna Mal. Cuba, 1878. 206. Ca. Ba. Fla. J.

Cerithium variabile Ad., Krebs. 50.

Cerit!ium variabile C. B. Adams, Krebs' Remarks. 394.

Cerithium variabile C. B. Adams,* Mal. Bl., xxiii, 117. J.

Cerithium variabile Ad.,* Mal. Bl., xxiii, 118. J.

Cerithinm rariabile C. B. Adams, Marrat, Argo Exp., 1876.16. Nassau.

Cerithium variabile C. B. Adams, Poulsen. 9.

Cerithium variabile C. B. Adams, Synops., 5, 1845. J.

Cerithium varicosum Sby., Mal. Bl., xxiii, 88.

Cerithium varium Pfr., Mal. Bl., xxiii, 96.

Cerithium varium Pfr., Arehiv fuir Naturg., 1840. 256. Ca.

Cerithium versicolor C. B. Arlams, Arango, Fauna Mal. Cuba, 1878. 206. Ca. Ba. Fla. J.

Cerithium versicolor C. B. Adams, Beau. 11. G.

Cerithium versicolor C. B. Adlams, Contr. to Conch. 119. J.

Cerithium versicolor Ad., Krebs. 50.

Cerithium versicolor Ad.,* Mal. Bl., xxiii, 116,117. J.

Cerithium versicolor C. B. Adams, Poulsen. 9.

Cerithium viciuum U. B. Adams, Contr. to Conch. 122. J.

Cerithium vicinum Ad., Krebs. 51.

Cerithium vicinum C. B. Adams, Krebs' Remarks. 395.

Cerithium vicinum Ad., Mal. Bl., xxiii, 101.

Cerithium vulgatum Lam., Pfeiffer, Archiv fiir Naturg., 1840. 256. Ca.

Cerithium zonale Brug., Krebs. 49.

Cerithium zonale Brug., Krebs' Remarks. 395.

Cerithium zonale Brug., Mal. Bl., xxiii, 94. J.

Cerithium zonale Desh., Mal. Bl., xxiii, 94. J. 
Chactopleura (see Chiton).

Chatoplenra apiculata Say, Dall, Hemphill's shells. 337. Florida Keys. Chateplenra janeirensis Gray, Guplyy, Paria Fauma, 1577. 139.

Chama (see Arcinella).

Chama appressa Reeve, Jahrb., ii, 249.

Chawa arcinella Chem., Mioll. Cuba, ii, 362.

Chama arcinella D'Crb., Araugo, Fauna Mal. Cuba, 1878. 272. Ca. M. G.

Chama arcinella Linn., Am. Marine Conch. 177.

Chama alcinella Linn., Arango, Fanna Mal. Cuba, 1878. 272. Ca. II. G.

Chama arcinella Linn., Bean. 23. G.

Chama arcinella Linn., Krebs. 117. S'T. SCx. Tort.

Chama arcinella Linn., Moll. Cuba, ii, 362.

Chama arcinella Linn., Poulsen. 15.

Chama bicornis Linu., Krebs. 117.

Chama congregata Conr., Bush, Conn. Ac. vi, 478. Hatt.

Chama coralliophaga Chem., Krebs. 123.

Chama ferruginea Rve, Ponlsen. 15.

Chama ferruginea Rve., Krebs. 117. ST. SJ. Tort. Td.

Chama florida Lam., Jour. de Conch., vii, 30.

Chama florida Lam., Arango, Fuma Mal. Cuba, 1878. 272. Ca. Ba.

Chama florida Lam., Beau. 23. G.

Chama florida Lam., Krebs. 117. ST. SCx. SB.

Chama florida Lam., Poulsen. 15.

Chama foliacea Cxmel., Arango, Famua Mal. Cuba, 187S. 272. Ca.

Chama frondosa Brod., Beau. 23. G.

Chama frondosa Brod., Krebs. 117.

Chama gryphoides Linn., Pfeifter, Arehiv für Naturg., 1840. 260. Ca. Chawa imbricata Lam., Araugo, Fauna Mal. Cuba, 1878. 272. Ca. Ba.

Chama imbricata Lam., Krebs. 117.

Chama involuta Guppy, n. s., Geol. Mag., 1874. 444. J.

Chama lamellosa Guel., Pfeiffer, Archiv für Naturg., 1840. 260. Ca. Chama lazarus Kurtz. $6 . \quad$ SC.

Chama lazarus Lam., Moll. Cuba, ii, 363.

Chama lazarus Linn., Jour. de Conch., ii, 426. G.

Chama lingua-felis Rre., Moll. of Bermudas. 25.

Chama macerophyila Chem., Beau. 23. G.

Chama macerophylla Chem., Moll. Cuba, ii, 363.

Chama macerophylla Chem. . sulphurea and purpurascens, Poulsen. 15.

Chama macrophylla Chem. Am., Marine Conch. 177.

Chama macroplyylla Chem., Arango, Famua Mal. Cuba, 1878. 272. Ca. Ba.

Chama macrophylla Chem., Jour, de Conch., ii, 426. G. 
Chama macrophylla Chem., Narrat, Argo Exp., 1576. 7. La., Mavama. C'hama macrophylla D’Orb., Arango, Famma Mal. Cuba, 1S78. 272. Ca. Ba.

Chama macrophylla Linn., Moll. of Bermudas. 25.

Chama macroplylla Chem., Krebs. 117. VgI.

Chama persica Meusch., Krebs. 97.

Chama radians Lam., Jour. de Conch., vii, 30.

Chama radians Lam., Beau. 23. G.

Chama radians Lam., Krebs. 118.

Chama reflexa Rre., Marrat, Argo Exp. 1876. 17. Nassau.

(hama ruderalis Lam., Guppy, Paria Fauna, 1877. 153.

Chama sarda Rre., Jour. de Conch., vii, 30.

(Thama sarda lire., Arango, Fanua Mal. Cuba, 1575. 272. Ca. G. Ba. Chama sarda Rre., Beau. 23. G.

Chama sarda Rre., Jour. de Conch., r, 151. G.

Chama sarda Rve., Krebs. 118. S'1. SB. SJ. Td.

Clıama sarda Rre., Poulsen. 15.

Chama sinuosa Brod., Jour. de Couch., vii, 357. ST.

Chama sinuosa Brod., Poulsen. 15.

Chama sordida Rve., Krebs, 118.

Chama tigerina Mart., Krebs. 121.

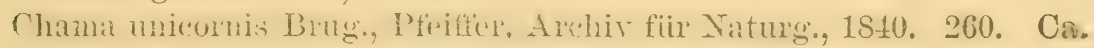
Chama variaus Dkr., Arango, Fauna Mal. Cuba, 1S7S. 272. Ca.

Chama variegata Rve., Kírebs, 118.

Chama venosa Rve., Jour. de Conch., vii, 30.

Chama renosa Rre., Beau. 23. G.

Chama renosa Rre., Jour. de Conch., r, 151. G.

Chama renosa Rve., Krebs. 118.

Chama vexillum pacis, Mal. Bl., xir, 116.

Chelyconus (see Conus) Marrat, Argo Exp, 1876. 8.

Chemnitzia (see Turbonilla).

Chemnitzia americana D'Orb., Mal. Bl., xxii, 160. Bz.

Chemnitzia babylonia C. B. Adams, Poulsen. S.

Chemnitzia babylonia C. B. Adlams, Synops., 1845. 6. J.

Chemnitzia babylonica C. B. Adams, Mal. Bl., xxii, 165. J.

Chemnitzia bisuturalis ". Stimpson, Kurtz. S. SC.

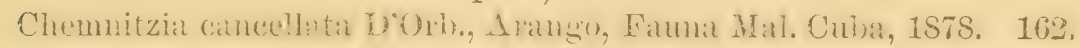

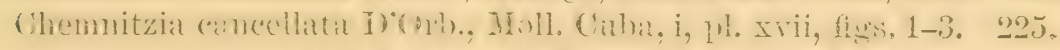

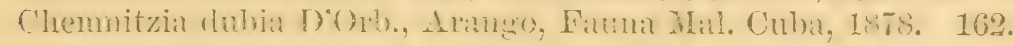

Chemnitzia? dubia D'Orb., Beau. 17. G.

Chemnitzia duhia D’Orb., Mal. Bl., xxiii, 57.

Chemnitzia dubia D'Orb., Noll. Cuba, i, pl. xvii, figs. 1-6. 226.

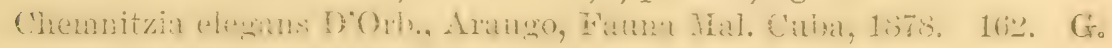

Chemnitzia elegans D'Orb., Beau. 17. G.

Chemnitzia elegans D'Orb, Mal. Bl., xxii, 160. ST.

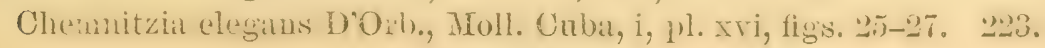


Chemnitzia elegans D'Orb., Poulsen. 7.

Chemnitzia equalis? Kurtz. 8. SC.

Chemnitzia (Ebusa) erythroselera Hörch, Mal. Bl., xxii, 160. ST.

Chemnitzia exilis C. B. Adams, Contr. to Conch. 74. J.

Chemnitzia exilis C. B. Adams, Krebs' Remariss. 395.

Chemnitzia exilis C. B. Adams, Mal. Bl., xxii, 162. J.

Chemnitzia fasciata D'Orb., Mal. Bl., xxii, 164. Bz.

Chemnitzia flavocineta U. B. Adams, Contr. to Conch. 74. J.

Chemnitzia flarocincta C. B. Adlams, Krebs' Remarks. 395.

Chemnitzia flarocincta C. B. Adams, Mal. Bl., xxii, 163. J.

Chemnitzia flarocincta C. B. Adams, Poulsen. S.

Chemnitzia impressa Say (Turritella), Kurtz. S. NC. SC.

Chemuitzia interrupta Stimpson, Kurtz. 8, NC. SC.

Chemnitzia (Elusa) krebsii Mörch, Mal. Bl., xxii, 159. ST.

Chemnitzia lrrebsii Mörch, Poulsen. 7.

Chemnitzia levigata D'Orh., Araugo, Fauma Kü. Cuba, 187s. 162. Ca.

Chemnitzia lavigata D’Orh., Moll. Cuba, i, 1). xvii, figs. 7-9. 227.

Chemnitzia lævis O. B. Adams, Krebs' Remarks. 395.

Chemnitzia latior C. B. Adams, Contr. to Conch. 72. J.

Chemnitzia leris C. B. Adams, Contr. to Conch. 73. J.

Chemnitzia levis C. B. Adams, Mal. Bl., xxii, 161. J.

Chemnitzia levis C. B. Adams, Poulsen. 8.

Chemnitzia modesta D'Orb., Arango, Fauna Mal. Cuba, 1878. 162. J.

Chemnitzia modesta D'Orb., Mal. Bl., xxii, 161. J.

Chemnitzia modesta D'Orb., Moll. Cuba, i, pl. xvi, figs. 22-24. 222.

Chemnitzia multicostata C. B. Adams, Contr. to Conch. 74. J.

Chemuitzia multicostata C. B. Adlams, Krebs' Remarks. 395.

Chemnitzia multicostata C. B. Arlams, Mal. Bl., xxii, 164. J.

Chemnitzia obeliscus C. B. Adams, Contr. to Concb. 72. J.

Chemnitzia obeliscus C. B. Adams, Krebs' Remarks. 395.

Chemnitzia obeliscus C. B. Adams, Mal. Bl, xxii, 163. J.

Chemnitzia obeliscus C. B. Adams, Poulsen. 8.

Chemnitzia ornata D'Orb., Arango, Fauna \Ial. Cuba, 1878. 162. Ca. M. G. S'T.

Chemnitzia ornata D'Orb., Beau. 17. G.

Chemnitzia ornata D'Orb., Mal. Bl., xxii, 160. G. SM. J.

Chemnitzia ornata D'Orb., Moll. Cuba, i, pl. xvi, figs. 18-21. 221.

Chemnitzia pulchella D’Orb., Arango, F'anna Mirl. Cuba, 1878. 163. Ca. M. G. ST.

Chemnitzia pulchella D'Orb., Beau. 17. G.

Chemnitzia pulchella D'Orb., Mal. Bl., xxii, 161. G. S'T.

Chemnitzia pulchella W’Orb., Moll. Cuba, i, pl. xvi, figs. 14-17. 220.

Chemnitzia pulchella D'Orb., Poulsen. S.

Chemnitzia puucta C. B. Adams, Contr. to Conch. 72. J.

Chemnitzia puncta C. B. Adams, Krebs' Remarks. 395.

Chemnitzia puncta C. B. Adams, Mal. Bl., xxii, 162. J. 
Chemnitzia puncta C. B. Adams, Poulsen. 8.

Chemnitzia pupoides D'Orb., Arango, Fauna Mal. Cuba, 1878, 163. Havana. G.

Chemnitzia pupoides D'Orb., Beau. 17. G.

Chemnitzia pupoides D'Orb., Jour. de Conch., v, 152. G.

Chemnitzia pupoides D'Orb., Mal. Bl., xxii, 164. Ca. et al.

Chemuitzia pupoides D'Orb., Moll. Cuba, i, pl. xvi, figs. 32-36. 224.

Chemnitzia pupoides D'Orb., Poulsen. 8.

Chemnitzia pusilla C. B. Adams, Contr. to Conch. 74 . J.

Chemnitzia pusilla C. B. Adams, Mal. Bl., xxii, 161. J.

Chemnitzia pusilla C. B. Adams, Poulsen. S.

Chemnitzia reticulata C. B. Adans, Contr. to Conch. $75 . \quad \mathrm{J}$.

Chemnitzia reticulata C. B. Adaws, Mal. Bl., xxii. 164. J.

Chemnitzia reticulata C.B. Adams, Poulsen, 8.

Chemnitzia risei Mörch, Mal.Bl., xxii, 165. ST.

Chemnitzia rufa D'Orb., Moll. Cuba, i, 222.

Chemuitzia seminuda Stimpson, Kurtz. 8. NC. SC.

Chemuitzia simplex D9Ob., Aramgo, Fama Mal. C'nba, 1878. 163.

Ohemnitzia simplex D'Orb., Mal. Bl., xxii, 165. J.

Chemnitzia simplex D’Orb., Moll. Cuba, i, pl. xri, figs. 2S-31. 224.

Chemnitzia spirata K. \& Stm., Kurtz. 8. NO.

Chemnitzia spirata K. \& Stm., Proc. Bust. Soc. Nat. Hist., iv, 151. NC.

Chemnitzia substriata C. B. Adáms, Contr. to Conch. 73. J.

Chemnitzia substriata C. B. Adams, Mal. Bl., xxii, 162. J.

Chemnitzia substriata C. B. Adams, Poulsen. S.

Chemnitzia subulata C.B. Adams, Contr. to Conch. $73 . \quad J$.

Clemnitzia subulata C. B. Adams, Krebs' Remarks. 395.

Chemnitzia subulata O. B. Adams, Mal. Bl., xxii, 163. J.

Chemnitzia subulata C. B. Adams, Poulsen. 8.

Chemnitzia textilis Kurtz, n. S., Kurtz. 8. SC.

Chemnitzia turris D’Orb., Araugo, Fauna Mal. Cuba, 1878. 163.

Chemnitzia turris D'Orb., Jahrb., ii, 243.

Chemnitzia turris D'Orb., Mal. Bl., xxii, 162. ST.

Chemnitzia turris D'Orb., Moll. Cuba, i, pl. xvi, figs. 10-13. 219.

Chemnitzia turris D'Orb., Poulsen. S.

Chemuitzia turritella Ad., Arango, Famna Mal. Cuba, 1878. 279. Ca.

Chemnitzia turritella Pfr., Arango, Fauua Mal. Cuba, 1878. 279. Ca.

Chicoreus (see Murex) Marrat, Argo Exp., 1876. 9.

Chimæra pernula Poli, Krebs. 131.

Chione (see Venus, Uallista, Dione).

$\checkmark$ Chione albida Hanley, Mal. Bl., ix, 67. J.

Chione alboradiata Sby., Mal. Bl., xiv, 125.

$\checkmark$ Chione alveata Conr., Bush, Conn. Ac. vi, 477. Hatt.

rChione beauii Recluz, Mal. Bl., xiv, 54.

Chione cancellata Chemn., Mal. B!., xiv, 93.

Chione cancellata Linu., Marrat, Ar(g) Lxp., 1876. 12. Tucacas. 
Chione cardioides Lam., Mal. Bl., xir, 94. Qaciti

Chione cingenda Dillw., Am. Marine Conch. 159.

Chione vingulata Lam., Mal. Bl., xis, 119. = puble jow?

Vhione crenulata Chemn., Mal. Bl., xiv, 41.

Chione dysera Limm., Mal. Bl., xiv, 45.

Chione granulata Gmel., Mal. Bl. xir, 93.

Chione (Timoclea) granulata Gmel., Marrat, Argo Exp., 1876. 12. Tucacas.

Chione paphia Linu., Krebs. 97.

Chione (Circomphalus) paphia Limn., Marrat, Argo Exp., 1876. 13. Santa Marta. Havana.

Chione parva Sb5., Mal. Bl., xiv, 60. Fla.

( Chione pygmæa Lam., Mal. Bl., xir, 60.

Chione pygmaa Lam., Marrat, Argo Exp., 1876. 19. Abaco.

$\checkmark$ Chione rugosa Chemn., Mal. Bl., xiv, 116.

Chione subrostrata Lam., Mal. Bl., xiv, 47.

Chione trapezoidalis Kurtz, Am. Marine Conch. 160.

Chiton (see, also, under subgenera).

Chiton (sce Ischnochiton) Arango, Famma Nal. Cuba, 1878. 231. Ca. Bb. J.

Chiton (see Lophyrus) Arango, Fauna Mal. Cuba, 1878. 231-232. Ca. J. ST. PR. SCx: G.

Chiton acutiliratus Pre., Araugo, Famna Mal. Cuba, 1878. 231. Ca. Bb. J.

Chiton acutiliratus Rve., Beau. 20. G.

Chiton acutiliratus Rve., Krebs. 89.

Chiton (Ischnochiton) acutiliratus Rre., Poulsen. 14.

Chiton apiculatus Say, Am. Marine Conch. 97.

Chiton (Chætopleura) apparata Cpr., Poulsen. 14.

Chiton apparata = rufocostata, Guppy, Paria Fauna, 1877. 139.

Chiton asper Shuttlew., Mal. Bl., iv, 28.

Chiton asper Shuttlew., Bean. 20. G.

Chiton (Chretopleura) asper Shuttlew., Jour. de Conch., v. 169. G.

Chiton asper Shuttlew., Krebs. 89.

Chitou assimilis Rve., Beau. 20. G.

Chiton assimilis Rre., Jour. de Conch., ii, 426. G.

Chiton assimilis Rre., Krebs. 89. ST.

Chiton (Acanthochites) astriger Krr., Poulsen. 14.

Chiron bistriatus IVoul, Arango, Fama Mal. Cuba, 1878. 332. Ca. J. S'T. PR. SCx.

Chiton blauneri Shuttlem., Mal. Bl. iv, 28. PR.

Chiton (Acanthuplenar) blatueri Shuttlew, Jour. de Conch., r, 170. PR.

Chiton candisatus Shuttler, MLal. Bl., iv, 28. G.

Chiton canclisatus Shuttlew., Beau. 20. (T.

Chiton (Chetopleura) candisatus Shuttlew., Jour. de Conch., r, 168. G. 
Chiton candisatus Shuttlew., Krebs. 89.

Chiton (Ischnochiton) caribæorum Cpr., Poulsen. 14.

Chiton (Ischnochiton) caribæortum Cpr., var. janaicensis Cpr., Poulsen. 14.

Chiton chemnitzii Pfr., Arango, Famna Mal. Cuba, 1S7S. 231. Ca.J. ST. PR. SCx.

Chiton costatus C. B. Allams, Araugo, Fauna Mal. Cuba, 187S. 231. Ca. J. ST. PR.

Chiton costatus Ad., Krebs. $\$ 9$.

Chiton costatus C. B. Aclams, Synops., 8, 1845. J.

Chiton erythronotus C. B. Adams, Krebs. 89.

Chiton erythronotus C. B. Adams, Beau. 20. G.

Chiton erythronotus C. B. Adams, Syuops., 9, 1845. J.

Chiton excaratus Gray, Arango, Fanna Mal. Cuba, 187S. 231. Ca. J. ST. PR.

Chiton excavatus Gray, Beau. 20. G.

Chiton excavatus Gray, Krebs. 91.

Chiton fasciatus Wood, Jorir. de Conch., $\nabla, 155$. G.

Chiton fasciatus Wood, Mal. Bl., iv, 27. G.

Chiton foreolatus Rive, Arango, Fauma Mal. Cuba, 1S78. 231. Ca. J. ST. PR.

Chiton foveolatus Sby., Beau. 20. G.

Chiton foveolatus Sby., Krebs. 91.

Chiton (Ischnochiton) funiculatus Cpr., Poulsen. 14.

Chiton graunlatus DOrb., Axumg, Fauna Mal. Cuba, 1S78. 231. Ca. J. ST. PR. M. G. Ba.

Chiton gramulatus Gurel., Arango, Famma Mal. Cuba, 187S. 231. Ca. J. ST. PR. M. G. Ba.

Chiton granulatus Gmel., Krebs. S9.

Chiton granulatus Gmel., Moll. Cuba, ii, 200.

Chiton guildingi Rve., Guppy, Paria Fauna, 1877. 139.

Chiton inca D'Orb., Jahrb., ii, 247.

Chiton isabellei D'Orb., Jahrb., ii, 247.

Chiton lævis Rve., Krebs. 90.

Chiton (Stenoplax) limaciformis Sb5., v. sanguinea Krr., Ponlsen. 14. Chiton marmoratus Chem., Jour. de Conch., $\nabla, 151$. G.

Chiton marmoratus Chem., Krebs. 89.

Chiton marmoratus Chem., Poulsen. 14.

Chiton marmonatus Gmel., Gums, Paria Fauna, 1877. 139.

Chiton marmoratus Rve., Beau. 20. G.

Chiton marmoratus Rre., Krebs. 89.

Chiton marmoreus, var., Mal. Bl., ir, 27. G.

Chiton mammoreu: Uhom., Arango, Famn Mal. Cuba, 1878. 232. Ca. J. ST. SCx. G.

Chiton marmoreus Chem., Beau. 20. G.

Chiton marmoreus var., Chem., Jomr. de Conch., ii, 126. G. 
Chiton marmoreus var., Chem., Jour. de Conch., v, 153. G. Chiton marmoreus Chem., Krebs. 89.

Chiton marmoreus Rve., Arango, Fauna Mal. Cuba, 1878. 231. Ca. J. ST. PR. SCx.

Chiton marmoreus Rve., Krebs. 90.

Chiton multicostatus C. B. Arlams, Arango, Fauna Mal. Cuba, 1878. 230. Oa. Bb. J.

Chiton multicostatus Ad., Krebs. 89.

Chiton multicostatus C. B. Adams, Synops., 8, 1845. J.

Chiton occidentalis Rve., Mal. B1., iv, 27. G.

Chiton oceidentalis Rre., Araugo, Fauma Mal. Cuba, 1878. 231. Ca. J. ST. PR. M. G. Ba.

Chiton occidentalis Rve., Bean. 20. G.

Chiton occidentalis Rve., Jour. de Conch., iv, 416. G.

Chiton occidentalis Rve., Jour. de Conch., v. 155. G.

Chiton oceidentalis Rve., Krebs. 90.

Chiton papillosus Ad., Krebs. 39.

Chiton papillosus C. B. Adams, Synops., 9, 1845 . J.

Chiton pectinatus Sby., Beau. 20. G

Chiton pectinatus Sby., Jour. de Conch., $\nabla, 151$. G.

Chiton pectinatus Sbj., Krebs. 89.

Chiton piceus Chem., Arango, Fauna Mal. Cuba, 1878. 230. Ca. J. ST. PR. M. G. Ba.

Chiton piceus Gmel., Beau. 20. G.

Chiton piceus Grmel., Dall, Hemphill's shells. 337. Florida Keys.

Chiton piceus Gmel., Jour. de Conch., iv, 416. G.

Chiton piceus Gmel., Krebs. 90. NGr. Ber. ST.

Chiton piceus Gmel., Moll. Cuba, ii, 200.

Chiton punctatus Linn., Krebs. 89. SCx. NGr.

Chiton purpurescens C. B. Adams, Beau. 20. G.

Chiton purpurascens C. B. Adams, Jomr. de Conch., r, 151. G.

Chiton purpurascens Ad., Krebs. 90.

Chiton purpuraseens C. B. Adams, Synops., 9, 1845. J.

Chiton rugosus Gray, Krebs. 90.

Cliton (Acauthopleura) salamander Spgl., Poulsen. 14.

Chiton salamandra Spgl., Krebs. 90. NGr.

Chiton scaber-variegatus Ch., Krebs. 90.

Chiton schrammi Shuttlew, Mal. Bl., ir, 28. G.

Ohiton schrammi Shuttlew., Jour. de Conch., v, 151. G.

Chiton (Tonicia) schrammi Shuttlew., Jour. de Conch. (pl. 6, fig. 9), v,

171. G.

Chiton schrammii Shuttler, Beau, 20. G.

Chiton schrammii Shuttlew, Krebs. 90.

Chitou segmentatus Rve., Guppy, Paria Fauna, 1877. 139.

Chiton similis, Jour. de Conch., $\nabla, 155$. G.

Chiton similis, Mal. Bl., iv, 27. G. 
Chiton (Phakellopleura) spiculosa Gray, Jour. de Conch., v, 151. G. Ohiton (Acanthochites) spiculosa Krr., Poulsen. 14.

Chiton spiculosus Gray, Beau. 20. G.

Chiton spiculosus Gray, Krebs. 90.

Chiton squalidus Ad., Krebs. 90.

Chiton squalidus C. B. Adams, Synops., 8, 1845. J.

Chiton squamosus Born, Mal. Bl., iv, 27. G.

Chiton squamosus Born, Krebs. 90.

Chitou squamosus Chem., Arango, Fauna Mal. Cuba, 1878. 231. Ca. J. S'T. PR.

Chiton squamosus Linn., Arango, Fauna Mal. Cuba, 1878. 282. Ca. J. ST. PR. SCx.

Chiton squamosus Linn., Beau. 20. G.

Chiton squamosus Linn., Jour. de Conch., ii, 426. G.

Chiton squamosizs Linn., Jour. de Conch., v, 155. G.

Chiton squamosus Linn., Krebs. 90. NGr.

Chiton squamosus Linn., Moll. of Bermudas. 21.

Chiton squamosus Tinn., Poulsen. 14.

Chiton squamosus-denticulatus Chem., Krebs. 90.

Chiton squamulosus C. B. Adams, Bean. 20. G.

Chiton squamulosus C. B. Adams, Jour. de Conch., v, 151. G.

Chiton squamulosus C. B. Adams, Krebs. 90,

Chiton squamulosus C. B. Adams, Synops., 8, 1845. .J.

Chiton strigatus Shuttlew., Krebs. 90.

Chiton (Chitonellus) strigatus Shuttlew., Poulsen. 14.

Chiton tessellatns Wood, Arango, Fanma Mal. Cnba, 1S78. 232. Ca. J.

ST. PR. SUx.

Chiton tuberculatus Linn., Krebs. 90.

Chiton undatus Spgl., Krebs. 90.

Chiton undatus Spgl, Poulsen. 14.

Chiton viridis Spgl., Krebs. 90.

Chiton viridis Wood, Arango, Fauna Mal. Cuba, 1878. 231. Ca. J. ST. PR.

Chiton viridis Wood, Poulsen. 14.

Chitonella strigatus Shuttlew., Krebs. 90.

Chitonellus lævis Rve., Beau. 20. G.

Chitonellus strigatus Shuttlew., Beau. 20. G.

Chlamys (see Pecten) Marrat, Argo Exp., 1876. 19.

Chlamys corallinus Chem., Krebs. 134.

Chlamys ornatum Bolt., Krebs. 134.

Choristodon (sce Petricola).

Choristodon robusta Jonas, Mal. Bl., ir, 27. G.

Choristodon robusta Jonas, Jour. de Conch., v, 155. G.

Choristodon typica Jonas, Krebs. 108.

Choristodon typieum Jonas, Arango, Fauna Mal. Cuba, 1878. 248. Ca. Ba. 
Choristodon typieum Jonas, * Zt. Mal., i, 185. ST.

Chromodoris gonatophora Bergh, Mal. Bl., xxvi, 102.

Chromodoris mörchii Bergh, Mal. Bl., xxri, 98, 99.

Chromodoris picturata Bergh, Mal. Bl., xxii, 177. ST.

Chromodoris pieturata Mörch, Mal. Bl., xxii, 177. ST.

Chrysallida, sp. nor., Mal. BI., xxii, 167. ST.

Chrysallida, n. sp., Poulsen. 8.

Chrysallida curtina Gld., Otia. 237. SC.

Cikota eecidentalis Browne, Krebs. 125. = 0owot

Cibota unbonata Brown, Krebs. 126.

Cingula (see Rissoa, Littorinella).

Cingula concinna Ad., Krebs. 53.

Ciugula concinna C. B. Adams, Mal. Bl., xxiii, 55. J.

Cingula (?) coniea C. B. Adams, Contr. to Conch. 70. J.

Cingula conica Ad., Krebs. 53.

Cingula conica C. B. Adams, Mal. B1., sxiii, 55. J.

Cingula modesta Gould, Mal. Bl., xxiii, 56.

Cingula modesta H. C. Lea, Mal. Bl., xxiii, 56.

Cingula robusta H. C. Lea, Mal. Bl., xxiii, 56.

Cingula sandersoni Verrill, List, 1884. 269. Cape Hatteras.

Cingula (?) solida C. B. Adams, Contr. to Conch. 70. J.

Cingula solida Ad., Krebs. 53.

Cingula solida C. B. Arlams, * Mal. B1., xxiii, 55. J.

Cingula syngenes Verrill, List, 1884. 269. Cape Hatteras.

Cingula turriculus Gould, Mal. Bl., xxiii, 56.

Cingula tumienlus. II. C. Lea, Boston Jomr. Sat. Hist. v, pl. 24, fig. 6, 289. SO.

Cingula turriculus H. C. Lea, Mal. Bl., xxiii, 56. SC.

Cingulina earinata Mörcl, Mal. Bl., xxiii, 53. (Ponlsen, 8.) ST.

Cinnlia semistriata D'Orb., Krebs. 71.

Circomplalus (see Chione), Mlarrat, Argo. Exp., 1876. 13.

Cistella (see Argiope, Megathyris).

Cistella antillarum C. \& F., Am. Jour. Conch., vi, 146. G.

Cistella barrettiana Dav., Am. Jour. Conch., vi, 146. J.

Cistella barrettiana Dar., Report Blake MIoll. 103. CS.

Cistella barrettiana, var. rubrotineta, Report Blake Moll. 104.

Cistella barrettiana (?), var. selmrammi, Report Blake Moll. 104.

Cistella lutea. Dall, Report Blake MIoll. 103. OS.

Cistella (barrettiana rar.) lutea Dall, Report Blake Moll. 103.

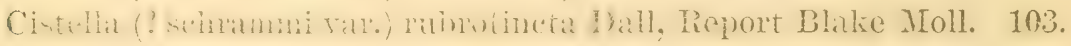

Cistella schrammi C. \& F., Am. Jour. Conch., vi, 146. G.

Cistella woodwardiana Dar., Am. Jonr. Conch., ri, 146. J.

Cithna olivacea Ver., Bush, Conn. Ac. ri, 463. Hatt.

Cladopoda arenaria Quoy, Mlarmat, Argo Exp., 1876. 18. Abaco.

Clathrus (see Scala), Marrat, Argo Exp., 1876. 13.

Clathurella (see Pleurotoma). 
Clathurella jervettii Stearus, Dall, Hemphill's shells. 3:9. Cedar Keys.

Clathurella jerrettii* Stearns, Proc. Phil. Acad., 1573. 316. Tampa, W. Fla.

Cleodora (see Creseis), Arango, Fauna Mal. Cuba, 1878. 152. Ca.Antilles.

Cleodora (see Curieria), Arango, Fanna IJal. Cuba, 187S. 152. Ca. Antilles.

Cleodora aciculata D'Orb., Moll. Cuba, i, SS.

Cleodora caudata Blv., Moll. Cuba, i, 84 .

Cleodora cuspiclata Bose, Arango, Fauna Mal. Cuba, 187S. 151. Ca. Antilles.

Cleodora cuspidata D'Orb., Arango, Fauma Mal. Cuba, 187s. 151. Ca. Antilles.

Cleodora cuspidata D'Orb., Moll. Cuba, i, 82 .

Cleodora cuspidata Q. \& G., Moll. Cuba, i, 82.

Cleodora cuspidata Rang, Moll. Cuba, i, 81, 82.

Oleodora lamartinieri D'Orb., Moll. Cuba, i, 84.

Cleodora lanceolata Rang, Moll. Cuba, i, 84 .

Cleodora lessonii Rang, Lesson, Moll. Cuba, i, 82.

Cleodora lessonii Iang, Arango, Fanua Mal. Cuba, 1878. 151. Ca. Antilles.

Cleodora lessonii Rang, Moll. Cuba, i, 82.

Cleortora obtusa Q. \& G., Mioll. Cuba, i, 89, 90.

Cleorlora pyramidata Blv., Moll. Cuba, i, 84 .

Cleodora 15ramidata Brown, Arango, Fauna Mal. Cuba, 1878. 151. Ca. Antilles.

Cleodora pyramidata Brown, Moll. Cuba, i, 83.

Cleodora psramillata D'Orb., Araugo, Fanna Nal. Cuba, 1878. 151. Ca. Antilles.

Cleodora pyramidata D'Orb., Moll. Cuba, i, 84.

Cleodora pyramidata Rang, Moll. Cuba, i, 84 .

Cleodora quadrispinosa Less., Moll. Cuba, i, 82.

Cleodlora quadrispinosa Rang., Arango, Fanna Mal. Cuba, 1878. 151.

Ca. Antilles.

Cleodora quadrispinosa Rang, Moll. Cuba, i, 82.

Cleodora subula D'Orb., Moll. Cuba, i, 85.

Cleodora subula Q \& G., Moll. Cuba, i, 85.

Cleodora virgula D'Orb., Moll. Uuba, i, 86 .

Clio (sec Cleodora), Arango, Fauna Mal. Cuba, 1878. 151. Ca. Antilles.

Clio candata Brown, Arango, Fauna Mal. Cuba, 1878. 151. Ca. Antilles.

Clio caudata Brown, Moll. Cuba, i, 83.

Clio caudata Gmel., Moll. Uuba, i, 83.

Clio pyramidata Brown, Moll. Cuba, i, 83.

Clio pyramidata Gmel., Moll. Cuba, i, 83. 
[BULL. 2A.

Clypidella (see Fissurella).

Clypidella fascicularis Lam., Marrat, Argo Exp., 1876. 16. Nassau.

Long Key Island. Abaco.

Clypidella fascicularis Sby., Krebs. .87.

Cocculina beauii Dall, Proc. U.S. Nat. Mus., April, 18\&e. 403. Bho

M. Granada.

Cocculina leptalea Verrill, List, 1SS4. 271.

Cocculina rathbuni Dall, Pros. U. \$. Kat. Mus., April, 1882, 402. Bb. M.

Cochlea (see Cassis, Natica, Nerita).

Cochlea (see Pachypoma), Araugo, Fauna Mal. Cuba, 1878. 179. Ca.

M. G. Santa Lucia. Ba.

Cochlea calata Klein, Moll. Cuba, ii, 74.

Cochlea canaliculata, \&c., Mal. Bl., xxir, 34. PR.

Cochlea cassidiformis, \&c., Mal. Bl., xxiv, 38.

Cochlea cassidiformis Gualt., Mal. Bl., xxiv, 39.

Cochlea eburnea, \&c., Mal. Bl., ххіт, 53.

Cochlea fasciata Seba, Moll. Cuba, ii, 2.

Cochlea indica, \&e., Mal. Bl., xxri, 38.

Cochlea leviter, \&c., List., Mal. Bl., xriii, 137.

Cochlea margaritica Seba, Moll. Cuba, ii, 75.

Cochlea marina, \&c., Mal. Bl., xxiii, 130.

Cochlea mariua, Mal. Bl., xxiii, 134.

Cochlea marina D'Argv., Mal. Bl., xxir, 63.

Cochlea marina Gere., Mal. Bl., xxiv, 63.

Cochlea striata, \&c., Mal. Bl., xxiv, 38. Ca.

Cochlea sublivida, \&c., List., Mal. Bl., xxiii, 137.

Cochlea sulcata Bolt., Mal. Bl., xxiv, 61.

Cochlea umbilicata Kirch., Mal. Bl., xxir, 63.

Cochlea verruculata Petiver, Mal. Bl., xxiii, 134.

Cochliolepis parasiticus Stimp., Am. Marine Conch. 86. SO.

Cochlis (see Natica, Nacea).

Cochlis canrena Bolt., Mal. Bl., xxiv, 63.

Cochlis lacernula D'Orb., Mal. Bl., xxiv, 65. M.

Cochlis limacina Jouss:, Mal. Bl., xxiv, 64.

Cochlis lirida Pfr., Mal. Bl., xxiv, 64. Ca.

Cochlis pennata Ch., Mal. Bl., xxiv, 64.

Cochlis plicata Bolt., Mal. Bl. xxiv, 62.

Cochlis proxima C. B. Adams, Mal. Bl. xxir, 65.

Cochlis rufilabris Reeve, Mal. Bl. xxiv, 65.

Cochlis sagraiana D'Orb., Mal. Bl., xxiv, 66. Ca.

Cochlodesma leanum Conthouy, Kurtz. 3. NC.

Cochlolepas (see Hipponyx, Capulus).

Cochlolepas antiquatus Liun., Arango, Fauna Mal. Cuba, 1878. 225. Ca. G. Ba.

Cochlolepras alimsoi Dkr., Arango, Fauna Mal. Cuba, 1878. 225. Ca. 
Cochlolepas imbricata Glä, Mirrat, Argo Exp., 1876. 18. Abaco.

Cochlolepas incurva Gmel., Arango, Fauna Mal. Cuba, 1878. 226. Ca. Cochlolepas subrufus List., Arango, Fauna Mal. Cuba, 1878. 226. Ca. Codakia (see Lucina) Marrat, Argo Exp., 1876. 8.

Uodakia jamaicensisvScop., Krebs. 119.

Codakia pennsylvanica:Seop., Krebs. 120.

Codakia tigerina Scop., Krebs. 121.

Collonia (Turbo) indntus Watson, Watson Lin. Soc. Journ., 1879, xiv, 715. Cul.

Columbella (see Alia, Amycla, Lmachis, Astyris, Nitidella).

Columbella (see Pleurotoma) Arango, Fauma Mal. Cuba, 1S78. 219. Ca. J.

Columbella aciculata Lam., Jour. de Conch., ii, 429. G.

Columbella adansoni Mke., Zt. Mal., $x, 74$. SV.

Columbella (Astyris?) amphissella Dall, u. s., Report Blake Moll. 91. Yucatan Strait.

Columbella argus D'Orb., Arango, Fauna Mal. Cuba, 1878. 198. G. Columbella argus D'Orb., Bean. 10. G.

Columbella argus D'Orb., Krebs. 28.

Columbella argus D'Orb., Moll. Cuba, ii, 138.

Columbella atomella Ducl., Krebs. 28.

Columbella avara Say, Am. Marine Conch. 37.

Columbella avara Say, Jahrb., ii, 243.

Columbella avara Say, Poulsen. 12.

Columbella barbadensis D'Orb., Krebs. 28.

Columbella barbadensis D’Orb., Moll. Cuba, ii, 133.

Columbella barbadensis Pet., Araugo, Fauna Mall. Cuba, 1S78. 198. Antilles.

Columbella broderipii Sby., Beau. 10. G.

Columbella broderipii Sby., Krebs. 28.

Columbeila broderipii Sby., Krebs' Remarks. 397.

Columbella catenata Sby., Arango, Fauna Mal. Cuba, 187S. 198. Ca. Ba.

Columbella catenata Sby., Beau. 10. G.

Columbella catenata Sby.. Jour. de Conch., ji, 429. G.

Columbella catenata Sby., Krebs. 28. ST. SJ. SM.

Columbella catenata Sby., Krebs' Remarks. 395.

Columbella catenata Sby., Poulsen. 12.

Columbella ciucta Mitg.= C. atomella Ducl., Poulsen. 12.

Columbella concinna Sloj., Arango, Fauna Mal. Cuba, 1878. 198. Ca. $\mathrm{Ba}$.

Columbella concinna Sby., Krebs. 29. Td. ST. SCx. SM. NGr.

Columbella concinna Sby., Moll. Cuba, ii, 134.

Columbella corniculata Ad., Krebs. 28.

Columbella costulata C. B. Adams, Arango, Fauna Mal. Cuba, 187S.

199. Ca. J. G. 
[BULL. 24.

Columbella costulata C. B. Adams, Contr. to Conch. 58. J.

Columbella costulata C. B. Adams, Krebs' Remarks. 395. J.

Columbella cribraria, Am. Jour. Conch., i, 194. Ber.

Columbella cribraria D’Orb., Arango, Famma Nal. Cuba, 1878. $19 \mathrm{~S}$. Ca. Ba.

Columbella cribraria D'Orb., Moll. Cuba, ii, 135.

Columbella cribraria D'Orb., Moll. Cuba, ii, 138.

Columbella cribraria Lam., Arango, Fama Mal. Cuba, 187S. 198. Ca. Ba.

Columbella cribraria Lam., Beau. 10. G.

Columbella cribraria Lám., Jour. de Conch., ii, 429. G.

Columbella cribraria Lam., Mal. Bl., х, 225. J.

Colnubella (Mitrella) cribraria Lam., Marrat, Argo Exp., 1876. 9. SV. Abaco.

Columbella cribraria Sby., Mal. 131., xii, 130.

Columbella cribraria Sby., Moll. of Bermudas. 17.

Columbella cribraria Sby., Zt. Mal., x, 75. SV.

Columbella decipiens (:. B. Adams, Contr. to Conch. 55. J. =ohese

Columbella decipiens Ad., Krebs. 28.

Columbella dermestoides Kien., Krebs. 28.

Columbella dermestoides Lam., Beau. 10. G.

Columbella dermestoides Lam., Jour de Conch., ii, 429. G.

Columbella dichroa Sby., Kreus. 28.

Columbella dichroa Sby., c. v. C. argus D’Orb., Poulsen. 12.

Columbella dormitor Sby., Contr. to Conch. 5̃̃.

Columbella dormitor Sby., Krebs. 28.

Columbella dormitor Sby, Krebs, Remarks. 395.

Columbella dormitor Sby., Poulsen. 12.

Columbella duclosiana Araugo, Fatma Mal. Cuba, 1878. 200. Ca.

Columbella duclosiana D'Orb., Moll. C'uba, ii, pl. xxi, figs. 31-33. 136.

Columbella (Astyris) duclosiana D'Orb., Rep. Blake Moll. 91.

Columbella duclosiana D'urb., Krebs. 28.

Columbella fastigata Kien., Krebs. 28.

Columbella fenestrata C. B. Adams, Contr. to Couch. 57. J.

Columbella fenestrata Ad., Krebs. 28. ST.

Columbella fusiformis D’Orb., Arango, Fauna Mal. Cuba, 1878. 198. Ca. M. J.

Columbella fusiformis D'Orb., Krebs. 28.

Columbella fusiformis D'Orb., Moll. Culba, ii, pl. xxi, figs. 37-39. 136.

Columbella fusilormis D'Orb., Poulsen. 12.

Columbella guildingii Sby., Arango, Fauna Mal. Cuba, 1878. 199. Ca. Columbella guildingii Sby., Krebs. 29.

Columbella gruildingii Sby.=C. hotessieri D'Orb., Poulsen. 12.

Columbella rar. guttata Sby., Beau. 10. G.

Columbella guttata Sby., Jour. de Conch., ii, 429. G.

Columbella guttata Sby., Zt. Mal., x, 75. SV. 
Columbella hotessieri D’Orb., Arango, Famna Mal. Cuba, 1878. 199. Ca.

Columbella hotessieri D'Orb., Jour. de Conch., iv, 418. G.

Columbella hotessieri D'Orb., Krebs. 29.

Columbella hotessieri D'Orb., Moll. Cuba, ii, 138.

Columbella hotessieriana 1) Orb., Moll. Cuba, pl. xxi, figs. 37-39.

Columbella Lotessicriana DOrb., Arango, Fauna Mal. Cuba, 1878. 199. Ca.

Columbella hotessieriana D'Orb., Report Blake Moll. 91. WI.

Columbella hotessierii D'Urb., Beau. 10. G.

Columbella iontha Rav., An. Marine Conch. 40, 41.

Columbella jaspidea Sby., Am. Mariue Conch. ${ }^{40 .}$ WI.

Columbella lactea ? Kien., Arango, Mal. Cuba, 1878. 199. Ca. Ba.

Columbella lactea Sby., Krebs. '29.

Columbella lactea Sby., Poulsen. 12.

Columbella lævigata Lam., Krebs. 29.

Columbella lævigata Lam., Poulsen. 12.

Columbella liprigata Linn, Arango, Fauna Mal. Cuba, 1878. 199. Ca. $\mathrm{Ba}$.

Columbella lrevigata Linu., Beau. 10. G.

Columbella lavigata Limn., Jour. de Conch., ii, 429. G.

Columbella lavigata Limn., Mal. Bl., x, 225.

Colmubella (Nitidelia) levigata Linu., Marrat, Argo Exp., 1876. 11. La Guayra.

Columbella lævigata Mle., Mal. Bl., xii, 130.

Columbella lafresnayi F. \& B., Mal. Bl., iv, 222. MG.

Columbella lafresnayi F. \& B., Beau. 10. MG.

Columbella lathesnayi T. \& B., Jour. de Conch., v., pl. 12, figs. 4, 5, 357. MG.

Columbella lafresuayi $\mathrm{H}$. \& B., Krebs. 29.

Columbella lunata Say, Am. Marine Conch. 38, 41.

Columbella lunata Say, Poulsen. 12.

Columbella lunata Sby., Kurtz. 9. NC. SC.

Columbella Iyrata Sby., Jahrb., ii, 243.

Columbella mendicaria Lam., Jour. de Conch., xi, 84.

Columbella mereatoria Linn., Jour. de Conch., vii, 255, 256.

Colombella mereatoria DoOrb., Arango, Fauna Mal. Cuba, 187S. 199. Oa. Antilles.

Columbella mercatoria Lam., Moll. Cuba, ii, 132.

Columbella mercatoria Linn., Am. Marine Conch. 41.

Columbella nercatoria Linn., Arango, Fauna Mal. Cuba, 187s. 199. Ca. Antilles.

Columbella mercatoria Linn,, Beau. 10. G.

Columbella mereatoria Linn., Jour. de Conch., ii, 429. G.

Columbella mereatoria L., Krebs. 29. ST. SB. SM. SCx. Ag. Bb.

Columbella mercatoria Limn., Marrat, Argo Exp., 1576. 6. Aa. Bar-

Bull. 24-6 
buda. D. Havana. Nassau. Point Savanilla. Long Key Island. Abaco.

Columbella mercatoria Linn., Moll. of Bermudas. 17.

Columbella mercatoria Linn., Poulsen. 12.

Columbella monilifera Sby., Contr. to Conch. 55.

Columbella monilifera Sby., Krebs. 30.

Columbella nitida D'Orb., Arango, Fauna Mal. Cuba, 1878. 199. Ca. Antilles.

Columbella nitida Lam., Arango, Fauna Mal. Cuba, 1878. 199. Ca. Antilles:

Columbella nitida Lam., Beau. 10. G.

Columbella nitida Lam., Jour. de Conch, ii, 429. G.

Columbella nitida Lam., Krebs. 29.

Columbella (Nitidella) nitida Lam., Marrat, Argo Exp.. 1876. 8. Barbuda. SV. Nassan. Abaco.

Columbella nitida Lam., Moll. Cuba, ii, 134.

Columbella nitida Lam., c. v. major, Poulsen. 12.

Columbella niticlula Sby., Arango, Fauna Mal. Cuba, 1878. 199. Ca. Antilles.

Columbella nitidula Sby., Moll. Cuba, ii, 134.

Columbella nivea Rav., Am. Marine Conch. 40. SC.

Columbella nivea Sby., Ponlsen. 12.

Columbella obesa Ad., Arango, Fanna Mal. Cuba, 1878. 199. Ca. J. G.

Columbella obesa C. B. Adams, Arango, Fauna Mal. Cuba, 1878. 199. Ca. J. G.

Columbella obesa C. B. Adams, Beau. 10. G.

Columbella obesa C. B. Adams, Contr. to Conch. 55.

Columbella obesa C. B. Adams, Jour. de Conch., v, 153. G.

Columbella obesa, Ad., Krebs. 29. ST. NGr.

Columbella obesa C. B. Adams, Krebs' Remarks. 395.

Columbella obesa C. B. Adams, Poulsen. 12.

Columbella ocellatil D'Orb., Arango, Faana Mal. Cuba, 1s78. 198. Ca. Ba. G.

Columbella ocellata D'Orb., Krebs. 30.

Columbella ocellata D’Orb., Moll. Cuba, ii, 134.

Columbella ocellata Gmel., Beau. 10. G.

Columbella ocellata Gmel., Jonr. de Conch, vii, 258.

Columbella ocellata Gmel., Krebs. 30. ST. SJ. SB. Ag. SM.

Columbella ocellata Gmel.=C. cribraria Sby., Poulsen. 12.

Columbella oruata Rav., Am. Marine Conch. 42.

Columbella ornata Ravenel. 15. SC.

Columbella ovulata D’Orb., Arango, Fauna Mal. Cuba, 1878. 199. Ca. PR. Ba.

Columbella ovulata Duclos, Moll. Cuba, ii, 133.

Columbella ovulata Lam., Arango, Fauna Mal. Cuba, 1878. 199. Ca. PR. Ba. 
Columbella ovulata Lam., Beau. 10. G.

Columbella ovulata Lam., Krebs. 30. SM. ST. SJ. SCx. SB. Ag.

Columbella ovulata Lam., Moll. Cuba, ii, 133.

Columbella ovulata Lam., Poulsen. 12.

Columbella ovuloides C. B. Arlams, Contr. to Conch. 53. Ba.

Columbella ovuloides Ad., Krebs. 30.

Columbella parvula Phil., Krebs. 30. ST. SJ. SM.

Columbella peculiaris Guppy, Geol. Mag., 1874. 441. Td.

Columbella peleei Kien., = O. rudis Sby., Krebs. 30.

Columbella petraci Duclos, Poulsen. 12.

Columbella phasianus Jour. de Conch., vii, 255.

Columbella pulchella Kien., Arango, Fauna Mal. Cuba, 1878. 200. Havana.

Columbella pulchella Kien., Beau. 10. G.

Columbella pulchella Kien., Jour. de Conch., ii, 42\%. G.

Columbella pulchella Kien., Krebs. 30.

Columbella pulchella Kien., Poulsen. 12.

Columbella pulchella Sby., Am. Marine Conch. 40. WI.

Columbella purpurascens C. B. Adams, Contr. to Conch. 55. J.

Columbella purpurascens Ad., Krebs. 28.

Columbella purpurascens C. B. Adams, Krebs' Remarks. 395.

Columbella purpurascens C. B. Adlams, Synops., 2. 1845. J.

Columbella pusilla Pfr., Arango, Fauna Mal. Cuba, 1875. 200. Ca.

Columbella pusilla Pfr., = C. duclosiana Sby., Poulsen. 12.

Columbella pusilla Sby., Krebs. 30.

Columbella reticulata D'Orb., Arango, Fauna Mal. Cuba, 1878. 198. Antilles.

Columbella reticulata Lam., Arango, Fauna Mal. Cuba, 1878. 198. Antilles.

Columbella reticulata Lam., Moll. Cuba, ii, 133.

Columbella rudis Sby., Krebs. 30.

Columbella rufa Mke.,* Zt. Mal., x, 75. SV.

Columbella rustica Linn., Dall, Hemphill's shells. 326. Cedar Keys.

Columbella rustica Linn., Mal. Bl., xii, 129.

Columbella sagra D'Orb., Arango, Fanna Mal. Cuba, 1878. 200. G.

Columbella sagra D'Orb., Beau. 10. G.

Columbella sagra D'Orb., Jour. de Conch., iv, 418. G.

Columbella sagra D'Orb., Krebs. 30.

Columbella sagra D'Orb., Moll. Cuba, ii, pl. xxi, figs. 2S-30. 137. G.

Columbella saint-pairiana Caillet, Am. Jour. Conch., i, 186. MG.

Columbella saint-pairiana Caillet,* J. de C., xii, pl. xi, fig. 4, 279. MG.

Columbella saint-pairiana Caillet, Poulsen. 12.

Columbella scalaris Sby., Jour. de Conch., ii, 429. G.

Columbella schrammi Petit, Jour. de Conch., iv, 418. G.

Columbella schrammii Petit, Beau, 10. G. 
Columbella schrammii Petit, Krebs. 31.

Columbella similis Rav., Am. Marine Concl. 39.

Columbella similis Ravenel. 15. SC.

Columbella spirantha Rav., Ravenel. 15. SC.

Columbella spizantha Rav., Am. Marine Conch. 41. SO.

Columbella striata Duclos, Arango, Fauna Mal. Cuba, 1878. 200. Ca.

Columbella (Pyrene) stricta Watson, Lin. Soc. Jn., 1852, xvi, 340. Cul.

Columbella (Pyrene) strix Watson, Lin. Soc. Journ., 1882, xvi, 338. Cul.

Columbella (Pyrene) strix, var. subacta, Watson, Lin. Soc. Jouru., 1882, xvi, 339. Sombrero.

Columbella subcostata Ad., Krebs. 28, 31. ST. SJ. SM. SB. A.

Columbella subeostata C. B. Adans, Krebs' Remarks. 395.

Columbella subcostulata C. B. Adams, Synops., 2, 1845. J.

Columbella terpsichore Leathes, Krebs. 31.

Columbella terpsichore Leathes, Poulsen. 12.

Columbella translirata Rav., Am. Marine Conch. 39, 41.

Columbella translirata Ravenel. 15. SC.

Columbella tuberosa, Jour. de Conch., vii, 255.

Columbella variabilis Schum., Jour. de Conch., ii, 429. G.

Columbella (Astyris) verrilli Dall, n. s., Report Blake Moll. 91. CS.

Concha lævis Aldr., Mal. Bl., xxiv, 23.

Concha semiaurita Chem., (Perna) Arango, Fanna Mal.Cuba, 1878, 269. Ca. M. Ba.

Conclia semiaurita Chem., Krebs. 132.

Concha semiaurita Chem., Moll. Cuba, ii, 346, 347.

Concha veneris List., (Cypræa) Moll. Cuba, ii, 88, 91, 93.

Concha reneris Pet., Moll. Cuba, ii, 88, 91, 93.

Concha veneris Sloane, Moll. Cuba, ii, 88, 91.

Concha veneris umbilicata List., Moll. Cuba, i, 121.

Conchylium albo-nigro, \&c., Mal. Bl., xхiv, 19.

Conchylium maximum Browne, Mal. Bl., xxiv, 15.

Conopleura cancellata D'Orb., Krebs. 15.

Conus Africanus Kien., Zt. Mal., x, 78. SV.

Conus anaglypticus Crosse, Jahrb., i, 280. Vz.

Couns anaglypticus Crosse,* Jour. de Couch. xiii, pl. xi, f. S-8a., 314.

WI.

Conus arausiacus Chem., Moll. Cuba, ii, 114.

Conus arausiacus var. Gmel., Moll. Cuba, ii, 114.

Couns archetypus Crosse, Jahrb. i, 263.

Conus armillatus C. B. Adams, Contr. to Conch. 59. J.

Conus armillatus Ad., Krebs. 3. ST.

Conus aurautiacus Chem., Arango, Fauna Mal. Cuba, 1878. 193. Ca. Ba.

Conns aurantiacus IIwass, Arango, Fauna Mal. Cuba, 1878. 193. Ca. Conus aurantius Hwass, Krebs. 3.

Conus aurantius Hwass, Krebs' Remarks. 395. 
Conus badius Kien., Jahrb., i, 258.

Conus brticus Rve., Krebs. 3.

Conus barbadensis Hwass, Jahrb., i, 250. Bb.

Conus barbadensis Hwass, Krebs. 3. G., SCx., VD.

Conus barbadensis Hwass, Krebs. 5 .

Conus biliosus Bolt., Krebs. 6.

Conus breviusculus Sby., Jahrb., i, 245.

Conus cardinalis Hwass, Jahrb., i, 252. Vz.

Conus cardinalis Hwass, Poulsen. 13.

Conus castus Rve., Krebs. 3. ST.

Conus cedonulli Hwass, Jahrb., i, 252. Vz.

Conus cedonulli Lam., Pfeiffer, Archir für Naturg., 1840. 258. Ca.

Conus cedonulli Linn., Krebs. 3. ST. SCx. Tort.

Conus centurio Born, Jahrb., i, 255. Vz.

Couns centurio Born, Krebs. 3.

Conus centurio Born, Poulsen. 13.

Conus characteristicus Chem., Krebs. 3.

Conus cidaris, Kien., Jahrb., i, 252.

Conus cleryi Rve., Jahrb., i, 256. Vz.

Conus columba Brug., Jour. de Conch., iv, 419. G.

Couus columba Hrass, Beau. 7. G.

Conus columba Hwass, Jahrb., i, 273.

Conus columba Hwass, Krebs. 4.

Conus (Chelfconus) columba IIrass, Marrat, Argo Exp., 1876. 8. Barbuda.

Conus columba Hwass, Poulsen. 13.

Conus columba Kien., Arango, Fauna Mal. Cuba, 1873. 193. Ca. G. Conus concolor Sby. (C. testudinarius Auct.), Poulsen. 13.

Conus consobrinus Sby., Guppy, Geol. MIag., 1874. 439. Ј. Пу.

Conus cretacens Kien., Arango, Fama Mal. Cnba, 1878. 194. Ca. Ba.

Conus cretacens Kien., Jahrb., i, 280. Vz.

Conus cretaceus Kien., Krebs' Remarks. 395. J.

Conus cretaceus Kien., Krebs. 4.

Conus daucus Brug., Arango, Fauma Mal. Cuba, 187s. 193. Ca. Ba.

Conus daucus Brug., Jour. de Conch., ii, 430. G.

Conus daueus Brug., Moll. Cuba, ii, 114.

Conus daueus Lam., Moll. Cuba, ii, 114.

Conus daucus Linn., Bean. 7. G.

Conus daueus Linn., Krebs. 4. SM. Tort. VD.

Conus daucus Linn., Poulsen. 13.

Conus dancus var. luteus Krebs. 4.

Conus delessertii Recluz, Am. Jour. Conch., iv, 195. CS. Hatt.

Conus duvali Bern., Jahrb., i, 273.

Conus duvali Bern., Jour. de Conch. x, pl. xiii, f. 3, 404. G.

Conus echinulatus Kien., Bean. 7. G.

Conus echinulatus Kien., Jahrb., i, 280. 
Conus echinulatus Kien., Krebs. 4.

Conus elventinus Ducl., Jahrb., i, 280. Vz.

Conus emarginatus Rve., Jalırb., i, 257. Vz.

Conus flammeus Lam., Jahrb., i, 245.

Conus flavescens Gray, Poulsen. 13. Ba.

Conus floridanus Gabb, Dall, Hemphill's shells. 329.

Conus floridanus Gabb, ${ }^{*}$ Am. Jour. Conch, iv, pl. 15, f. 4, 195. Fla.

Conus floridensis Sby., Am. Jour. Conch, vii, 205. Fla.

Conus floridensis Sby:, Jahrb., i, 257. Fla.

Conus fumigatus Hwass, Krebs. 4.

Conus granulatus Gmel., Jahrb., i, 282.

Conus gramulatus Limn., Arango, Fauna Mal. Cuba, 1878. 194. Ca.

Baracoa. G. Ba.

Conus granulatus Linn., Beau. 7. G.

Conus granulatus Linn., Jour. de Conch., iv, 419. G.

Conus granulatus Linn., Krebs. 4.

Conus granulatus Limn., Poulsen. 13.

Conus granulatus Mart., Araugo, Fauna Mal. Cuba, 187S. 194. Ca. Ba.

Conus granulatus Mart., Krebs. 7.

Conus granulosus Bolt., Krebs. 7.

Conus gubba Kien.? Krebs. 6.

Conus hieroglyphicus Duclos, Jahrb. i, 284.

Conus japonicus Hwass, Dall, Hemphill's shells. 342.

Conus lentiginosus Rve., Jahrb., i, 257. Bz.

Conus leoninus Chem.. Jahrb., i, 245.

Conus leoninus Hwass, Beau. 7. G.

Conus leoninus Hwass, Krebs. 4.

Conus leoninus Hwass, Poulsen. 13.

Conus leneostictus Gmel., Arango, Fauna Mal. Cuba, 1878. 194. Ca.

Havana. Matanzas. M. Ba. Santa Lucia. G.

Conus leucostictus Hwass, Beau. 7. G.

Conus leucostictus Hwass, Krebs. 3.

Conus leucostictus Hwass, Krebs. 5. SCx. SM. Td.

Conus leucostictus Hwass, Krebs' Remarks. 395.

Conus leucostictus Hwass, Poulsen. 13.

Conus lictorr A. Bois., Poulsen. 13.

Conus litteratus Knorr, Moll. Cuba, ii, 117.

Conus lorenzianus Chem., Jahrb., i, 256.

Conus lubeckanus Bern., Jahrb., i, 252.

Conus lubeckiaums Bernardi,* Jour. de Conch. ix, pl. 6, f. 7-8, 169. G. Conus maculiferus Brod., Krebs. 5.

Conus maculiferus Sby., Arango, Famna Mal. Cuba, 1878. 194. Ca.

Havana. Matanzas. M. Ba. Santa Lucia. G.

Conus maeuliferus Sby., Beau 7. G.

Conus maculiferus Sby., Krebs' Remarks. 395. J.

Conus magellanicus Hwass, Jahrb., i, 252. G. 
Conus martinianus Rve., Krebs. 6.

Conus mazei Desh., Jahrb., i, 258. M.

Conus mazei Desh.,* Jour. de Conch. xxii, pl. i, f. 1, 61, 66. TVI.

Conus melvillii Sby. jr., Dall, Hemphill's shells. 342. Key West.

Conus mercator Linu., Jahrb., i, 266.

Conus mercator Linn., Krebs. 5. ST.

Conus mindanus Krebs. 6.

Conus mindauus Hwass, Arango, Fauna Mal. Cuba, 1878. 194. Ca. Ba.

Conus mindanus Hwass, Jahrb., i, 280. Vz.

Conus mindanus Hwass, Krebs. 3,5. ST. SB. Ag. SM.

Conus mindanus Hwass, Krebs' Remarks. 395.

Conus mindanus Hwass, Poulsen. 13.

Conus minutus Rve., Jahrb., i, 256. SV.

Conus minutus Rve., Krebs. 5.

Conus mus Brug., Arango, Fauna Mal. Cuba, 1875. 194. Ca. WI.

Conus mus Brug. Jour. de Conch., ii. 430. G.

Conus mus Brug. Moll. Cuba, ii. 116.

Conus mus D’Orb. Arango. Fauna Mal. Cuba, 1878. 19t. Ca. WI.

Conus mus Hwass., Beau. 7. G.

Conus mus Hwass, Jahrb., i, 248.

Conus mus Hwass, Krebs. 5, 6. NGr. ST. SCx. SJ. SB. SM.

Conus (Stephanoconus) mus Hwass, Marrat, Argo Exp., 1S76. 9. SV.

Point Savanilla. Havana. VCz. Nassan. Abaco.

Conus mus Hwass, Poulsen. 13.

Conus mus Lam., Moll. Cuba, ii, 116.

Conus mus Linn., Moll. of Bermundas. 17.

Conus mus Wood, Moll. Cuba, ii, 116.

Conus muscosus Lam., Jahrb., i, 245. Fla.

Conus musicus Hwass., Krebs. 5.

Conus mutabilis Chem., Jahrb., i, 258.

Conus narcissus Lam., Jahrb., i, 263.

Uonus nebulosus Brug., Jour. "de Conch., iv, 419. G.

Conus nebulosus Brug., Moll. Cuba, ii, 115.

Conus nebulosus Lam., Moll. Cuba, ii, 115.

Conus nebulosus Sol., Arango, Fauna Mal. Cuba, 1S78. 194. Ca.

Havana. Matanzas. M. Ba. Santa Lucia. G.

Conus nebulosus Sol., Beau. 7. G.

Conus nebulosus Sol., Dall, Hemphill's shells. 342.

Conus nebulosus Sol., Jahrb., i, 253. Vz.

Conus nebulosus Sol., Krebs. 5.

Conus (Stephanocouus) nebulosus Sol., Marrat, Argo Exp., 1876. 16.

Nassau.

Conus nebulosus Sol., Moll. Cuba, ii, 115.

Conus nivosus Linn., Mal. B1., xii, 128.

Conus nodiferus Kien., Beau. 7. G.

Conus nodiferus Kien., Jahrb., i, 280. 
Conus nodiferus Kien., Krebs. 6.

Conus pealei Green, Jahrb., i, 256. Fla.

Conus piperatus Dillw., Krebs. 6.

Conus portoricanus Hwass, Jahrb., i, 275.

Conus proteus Brug., Moll. Cuba, ii, 117.

Conus proteus D'Orb., Arango, Fauna Mal. Cuba, 1878. 194. Ca. SD. ST. Ba.

Conus proteus Hwass, Arango, Fauna Mal. Cuba, 1878. 194. Ca. SD. ST. Ba.

Conus proteus Hwass, Bean. 7. G.

Conus proteus Hwass, Jahrb., i, 245. Fla.

Conus proteus Hwass, Krebs. 6.

Conus proteus Lam., Moll. Cuba, ii, 117.

Conus prototypus Guppy, Geol. Mag., 1874. 439. Td.

Conus psendo-thomæ Chem., Beau. 7. G.

Conus psendo-thomæ Chem., Krebs. 6.

Conus puertoricanus Hwass, Krebs. 6.

Conus punctatus Hwass, Krebs. 6.

Conus purpuraseens Brod., Beau. 7. G.

Conus purpurascens Brod., Krebs. 6.

Conus pusio Brug., Krebs. 5.

Conus pusio Brug., Krebs. 6.

Conus pusio Brug., Krebs.' Remarks. 395.

Conus pusio Lam., Arango, Fauna Mal. Cuba, 187S. 194. Ca. Ba.

Conus pygmæus Rre., Jahrb., i, 273.

Conus pygmæus Rve., Krebs. 6. ST. Tort.

Conus pygmæus Rve., Poulsen. 13. Conch fág 260.9.2.5. 1843

Conus radiatus Gmel., Krebs. 6.

Conus recognitus Guppy, Geol. Mag., 1874. 439. J. Нy.

Conus roseus Lam., Jahrb., i, 249.

Conus rosens Lam., Krebs. 6. ST.

Conus rudis Chem., Jahrb., i, 265.

Conus speciosissimus A. Ad., Jahrb., i, 252.

Conus spurius Gmel., Arango. Fauna Mal. Cuba, 187s. 194. Ca. SD. S'í. Ba.

Conus spurius Gmel, Beau. 7. G.

Conus spurius Gmel., Jahrb., i, 245.

Conus spurius Gmel., Krebs. 6. Tort. ST. SC. SJ.

Conus spurius Gmel., Poulsen. 13.

Conus spurius Rve., Krebs. 4.

Conus stearnsii Con., Am. Jour. Conch. v, pl. x, fig. 1, 104. Fla.

Couzs stearusii Con., Dall, Hemphill's shells. 329. Sarasota Bay.

Conus strictieus A. Ad., Jahrb., i, 280.

Conus testudinarius Mal. Bl., xii, 128.

Conus testudinarius Mart., Beau. 7. G.

Conus testudinarius Mart., Krebs. 7. 
Conus varius Linn., Krebs. 7. ST. Z.

Conus verrucosus Hwass, Arango, Fauna Mal. Cuba, 1878. 194. Ca. Ba.

Conus verrucosus Hwass, Jahrb., i, 280.

Conus verrucosus Hwass, Krebs. 7.

Conus (Stephanoconus) rerrucosus Hwass, Marrat, Argo Exp., 1876.

16. Nassau. Abaco.

Conus rerrucosus Hwass, Poulsen. 13.

Conus verrucosus Hwass, Jahrb., i, 282.

Conus villepini Bern., Jahrb., i, 257. G.

Conus villepinii Fisch. \& Bern., Beau. 7. MG.

Conus villepinii F. \& B., Jour. de Conch. v, pl. ix, fig. 12, 292. MG.

Conus villepinii F. \& B., Krebs. 7.

Conus vittatus ? Lam., Arango, Fauna Mal. Cuba, 1878. 194. Ca. Havana. Baracoa.

Coralliophaga dactylus Brug., Poulsen. 15.

Coralliophila (see Rhizochilus), Marrat. Argo Exp., 1876. 6.

Coralliophila (?) plicata Linn., Arango, Fanua Mal. Cuba, 18is. 203. Ca. Ba.

Corbis magna Cuv., Krebs. 122.

Corbula æquivalvis Phil., Arango, Fanna Tal. Cuba, 1878. 252. Ca.

Havana. + cubaniana Orb. +? Knoxiana Ads.

Corbula barrattiana C. B. Adams, Contr. to Conch. 237. J.

Corbula barrattiana Ad., Krebs. 109.

Corbula barrattiana C. B. Adlams, Report Blake Moll. 114.

Corbula blandiana C. B. Adams, Contr. to Conch, 234. J.

Corbula blandiana Ad., Krebs. 109.

Corbula caribæa D'Orb., Arango, Fauna Mal. Cuba, 1878. 253. Ca. SD.

Corbula caribæa D'Orb., Krebs. 109, 110. ST.

Corbula caribra D'Orb., Moll. Cuba, ii, pl. xxvii figs. 5-8,284. SD.

Corbula caribæa D'Orb., Poulsen. 15.

Corbula chittyana C. B. Adams, Contr. to Conch. 238. J.

Corbula chittiana Ad., Krebs. 109.

Corbula contracta Say, Am. Marine Conch. 141.

Corbula costellata Desh., Report Blake Moll. 110.

Corbula crassa Hinds, Marrat, Argo Exp., 1876. 13. Tueacas.

Corbula cnbaniana D'Orb., Arango, Fauna Mal. Cuba, 1878. 252. Ca.

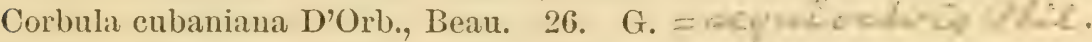

Corbula cubaniana D'Orb., Krebs. 109.

Corbula enbaniaua D'Orb., Marrat, Argo Exp., 1876. 13. Tucacas.

Corbula cubaniaua D'Orb., Moll. Cuba, ii, pl. xxvi, tigs. 51-5.1, 283. Ca.

Corbula cubaniana D'Orb., Poulsen. 15.

Corbula cubaniana D'Orb., Report Blake Moll. 114.

Corbula cymella Dall, n. s., Report Blake Moll. 115.

Corbula dietziana C. B. Adams, Contr. to Conch. 235. J.

Corbula dietziana Ad., Krebs. 110. 
Corbula dietziana C. B. Adams, Report Blake Moll. 114.

Corbula disparilis D'Orb., Arango, Fauna Mal. Cuba, 1878. 253. Ca. SD.

Corbula disparilis D'Orb., Beaı. 26. G.

Corbula disparilis D'Orb., Krebs. 110.

Corbula disparilis D'Orb.,* Moll. Cuba, ii, pl. xxvii, figs. 1-4,283. G.

Corbula disparilis D'Orb., Poulsen. 15.

Corbula disparilis D'Orb., Report Blake Moll. 115. CS.

Corbula duplex Ad., Krebs. 110.

Corbula elliptica Recl., Krebs. 110.

Corbula equivalvis Phil., Contr. to Conch. 233. Ca.

Corbula fulva C. B. Adams, Contr. to Conch. 240.

Corbula kjæriana C. B. Adams, Contr. to Conch. 237. ST. J.

Corbula kjæriana Ad., Krebs. 110. S'T.

Corbula kjæriana C. B. Adams, Poulsen. 15.

Corbula knoxiana C. B. Adams, Contr. to Conch. 238. J.

Corbula knoxiana Ad., Krebs. 110.

Corbula krebsiana C. B. Adams, Contr. to Conch. 234. Kingston.

Corbula krebsiana Ad., Krebs. 110. ST.

Corbula krebsiana C. B. Adams, Poulsen. 15.

Corbula lavalleana D’Orb., Arango, Fauna Mal. Cuba, 1878. 253. Ca. G. M. J.

Corbula lavalleana D'Orb., Beau. 26. G.

Corbula lavalleana D'Orb., Jour. de Conch., v, 150. G.

Corbula lavalleana D'Orb., Krebs. 110. ST.

Corbula lavalleana D’Orb., Moll. Cuba, ii, pl. xxvii, figs. 9-12, 284. G. Corbula limatula Conrad, Proc. Acad. Nat. Sci., Phil., iii, 25, pl. i, fig. 2, 1845. Fla.

Corbula nasuta Rve, Arango, Fauna Mal. Cuba, 1878. 253. Ca. На. vana. J.

Corbula nasuta Sby., Arango, Fauna Mal. Cuba, 1878. 253. Сa. Нavana. J.

Corbula nasuta Sby., Jahrb., ii, 248.

Corbula newtoniana, C. B. Adams, Contr. to Conch. 240. ST. Tort. Corbula newtoniana Ad., Krebs. 110.

Corbula nitens Turt., Krebs. 110. ST. Tort.

Corbula uuciformis Sby., Marrat, Argo Exp., 1876. 6. Aa.

Corbula operculata Phil., Contr. to Conch. 233. ST. Tort.

Corbula operculata Phil:, Krebs. 110. S'T.

Corbula operculata Phil.,* Zt. Mal., v, 13. ST.

Corbula quadrata D'Orb., Arango, Fauna Mal. Cuba, 1878. 253. Antilles. G.

Corbula quadrata Hinds, Arango, Fauma Mal. Cuba, 1878. 253. Antilles. G.

Corbula quadrata Hinds, Contr. to Conch. 239. ST.

Corbula quadrata Hinds, Krebs. 111. ST. 
Corbula quadrata Hinds, Moll. Cuba, ii, 283.

Corbula quadrata live., Arango, Fauna Mal. Cuba, 1878. 253. Antilles. G.

Corbula quadrata Reeve, Moll. Cuba, ii, 282.

Corbula radiata Bolt., Krebs. 105.

Corbula rosea Bolt., Arango, Fanna Mal. Cuba, 1878. 241. Ca.

Corbula swiftiana C. B. Adams, Arango, Fauna Mal. Cuba, 1878. 253.

Ca. Havana. J.

Corbula swiftiana C. B. Adams, Contr. to Conch. 236. ST. J.

Corbula swiftiana Ad. Krebs. 111.

Corbuia swiftiana C. B. Adams, Report Blake Moll. 114.

Corbula vieta Guppy, Geol. Mag., 1S7t. 449. Td. J. Нy.

Cornu hammonis Mart., Moll. Cuba, i, 64:

Coroua mexicana Chem., Jahrb., ii, 355. Fla.

Costellaria rawsoni Mörch,* Jour. de Couch., xxiv, 373. Bb.

Cranchia cardioptera D'Urb., Moll. Cuba, i, 54.

Cranchia cardioptera Fér., Moll. Cuba, i, 53.

Cranchia scabra D'Orb., Arango, Fauna Mal. Cuba, 1878. 147. Antilles.

Cranchia scabra D'Orb., Moll. Cuba, i, 32.

Cranchia scabra Leach, Arango, Fauva Mal. Cuba, 187s. 147. Antilles.

Cranchia scabra Leach, Moll. Uuba, i, 32.

Cranchia scabra Owen, Moll. Cuba, i, 32.

Crania pourtalesii Dall, Report Blake Moll. 104.

Crania (? anomala $v$ ir.) pourtalesii Dall, Report Blake Moll. 104.

Crassatella (see Grouldia).

Crassatella antillarum Rve., Krebs. 122.

Crassatella antillarum Rve., Mal. Bl., vii, 199.

Crassatella antillarum Rre., Mal. Bl. x, 229.

Crassatella antillarum (?), Reeve, Report Blake Moll. 131.

Crassatella antillarum Rve., Zt. Mal., i, sS.

Crassatella gibbesii T. \& H., n. s., Pleiocene Fossils S. C., pl. xx, figs. 9 and 10.74 .

Crassatella guadalupensis D'Orb., Arango, Fauna Mal. Cuba, 1878. 254. Ca. G. M. ST.

Crassatella guadalupensis D'Orb., Beau. 22. Pointe-à-Pître.

Crassatella guadalupensis D'Orb., Krebs. 122. VgI.

Crassatella guadalupensis D'Orb., Mal. Bl., vii, 199. G.

Crassatella guadalupensis D'Orb., * Moll. Cuba, ii, pl. xxvii, figs. 24, 25. 289. ST.

Crassatella guadelupensis D’Orb., Report Blake Moll. 131.

Crassatella (Eriphyla) lumulata Con., Dall, Hemphill's shells. 340. Sarasota Island.

Crassatella martinicensis D’Orb., Arango, Fauna Mal. Cuba, 1878. 254. M. J. SD.

Crassatella martinicensis D'Orb., Krebs. 122. VgI. 
Crassatella martinicensis D'Orb., * Moll. Cuba, ii, pl. xxvii, figs. 21-23. 288. M. J.

Crassatella martiuicensis D'Orb., Report Blake Moll. 131.

Crassatella (Eriphyla) parva C. B. Arlams, Report Blake Moll. 131.

Crassatella rostrata Lam., Krebs. 122.

Crassinella martinicensis D’Orb., Guppy, Paria Fauna, 1877. 151.

Crassispira (see 1)rillia), Marrat, Argo Exp., 1876. 11.

Cremides (see Fissurella), Marrat, Argo 1xp., 1876. 6.

Crenella decussata Mont., Report Blake Moll. 116.

Crenella decussata Sars, Report Blake Moll. 116.

Crenella ? divaricata Phil., Araugo, Fauna Nal. Cuba, 1878. 266. Oa.

Crenella lateralis Say, Am. Mariue Conch. 190.

Crenella viator D’Orb., Guppy, Paria Fauma, 1877. 154.

Crepidula (see Crypta, Tanacus).

Crepidula acnleata Chem., Arango, Fauma Mal. Cuba, 1878. 226. Ca. $\mathrm{Ba}$.

Crepidula aculeata Chem., Jahrb., ii, 245.

Crepidula aculeata Chem., Krebs. 69. ST. SJ. SCx.

Crepidula aculeata D’Orb., Arango, Fauma Mal. Cuba, 1878. 226. Ca. Ba.

Crepidula aculeata D'Orb., Moll. Cuba, ii, 191.

Crepidula aculeata Gmel., Am. Marine Conch. 94.

Crepidula aculeata Lam., Bean. 19. G.

Crepidula aculeata Lam., Mal. Bl., xxiv, 102. Fla.

Crepidula aculeata Lam., Moll. Cuba, ii, 191.

Crepidula aplysioides Reeve, Mal. Bl., xxiv, 103. Bz.

Crepidula convexa Say, Kurtz. 7. NC. SC.

Crepidula depressa Say, Mal. Bl., xxiv, 106.

Crepidula fornicata Ad., Mal. Bl., xxiv, 104.

Crepidula fornicata DeKay, Mal. Bl., xxiv, 104.

Crepidula fornicata Gould, Mal. Bl., xxiv, 104.

Crepidula fornicata Lam., Arango, Fauma Mal. Cuba, 1878. 226. Ca.

Crepidula fornicata Lam., Krebs. 70.

Crepidula fornicata Lam., Mal. Bl., xxir, 104.

Crepidula fornicata Lam., Moll. Cuba, ii, 192.

Crepidula fornicata Linn., Am. Marine Conch. 93.

Crepidula fornicata Limu., Moll. Cuba, ii, 192.

Crepidula fornicata Rve., Mal. Bl., xxiv, 104.

Crepidula fornicata Say, Mal. Bl., xxiv, 103.

Crepidula fornicata Say, Mal. Bl., xxiv, 106.

Crepidula glauca DeKay, Mal. Bl., xxiv, 104.

Crepidula glauea Rve, Mal. Bl., xxiv, 104.

Crepidula glauca Say, Mal. Bl., хxir, 104.

Crepidula hepatica Desh., var., Arango, Fauna Mal. Cuba. 1878. 227. Ca. 
Crepidula hepatica Desh., Mal. Bl., xxiv, 105.

Crepidula intorta DeKay, Mal. Bl., xxir, 107.

Crepidula intorta? var. Say,* Mal. Bl., xxiv, 107.

Crepidula listeri var., Jonas, Arango, Fauna Mal. Cuba, 1878. 227. Ca.

Crepidula maculosa Conrad, Mal. Bl., xxiv, 105. Fla.

Crepidula maculosa Courad, Proc. Acad. Nat. Sei. Phil., 1545, iii, 26.

Tampa.

Crepidula nautiloides Less, Mal.Bl., xxiv, 104.

Crepidula nivea Ad., Arango, Fauna Mal. Cuba, 1875. 227. Ca.

Crepidula plana Say, Arango, Fauma Mal. Cuba, 1878. 227. Ca.

Crepidula plana Say, Krebs. 70.

Crepidula plana Say, Mal. Bl., xxiv, 106.

Crepidula porcellana Lam. Aranggo, Fanua Mal. Cuba, 1s78. 227. Ca.

Crepidula porcellana Pfr., Mal. Bl., xxiv, 103.

Crepidula protea D'Orb., Arango, Fanua Mal. Cuba, 1S7S. 227. Ca.

Crepidula protea D'Orb., Beau. 19. G.

Crepidula protea $\mathrm{D}^{\prime} \mathrm{Orb}$, Krebs. 70. ST. PPl.

Crepidula protea D'Orb., Mal. B1., xxiv, 105. Ca.

Crepidula protea D'Orb., Mal. Bl., xxiv, 106.

Crepidula protea D'Orb., Moll. Cuba, ii, pl. xxir, figs. 30-33. 193.

Crepidula protea D'Orb., Report Blake Moll. 80.

Crepidula riisei Dkr., Krebs. 70.

Crepidula riisei Dkr., Mal. Bl., xxir, 106.

Crepidula sinnosa Turt., Mal. Bl., xxir, 105. Ca.

Crepidula squamosa Mke., Mal. Bl., xxiv, 107.

Crepidula unguiformis Lam., Am. Marine Conch. 94.

Crepidula unguiformis Lam., Beau. 19. G.

Crepidula unguiformis Lam., Dall, Templill's shells. 335. Sarasota Bay.

Crepidula unguiformis Lam., Jour. de Conch., r, 151. G.

Crepidula unguiformis Lam., Krebs. 70.

Crepidula unguiformis Lam., Moll. Cuba, ii, 193.

Crepidula unguiformis Stimp., Mal. Bl., хxir, 106.

Crepidula variegata 1)kr., Araugo, Fauna Mal. Caba, 157s. 227. Ca.

Crepipatella (see Crypta) Marrat, Argo Exp., 1876. 6.

Crepipatella aculeata Chem., Jahrb., ii, 245.

Crepitacella cepula Guppy, Geol. Mag., 1874. 442. Cumana. J.

Creseis aciculata J'Orb., Arango. Famna Mal. Cuba, 1sis. 152. ITL.

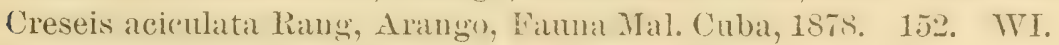

Creseis aciculata Rang, Moll. Cuba, i, SS.

Creseis clava Rang, Araugo, Famna Mal. Cuba, 1875. 152. Antilles.

Creseis clava Rang, Moll. Cuba, i, 88.

Creseis corniformis D’Orb., Arango, Fama Mal. Cuba, 15.8. 15ّ. Ca. Antilles.

Creseis corniformis D'Orb., Moll. Cuba, i, 87. 
Creseis spinifera Rang, Arango, Fauna Mal. Cuba, 1878. 152. Ca. Antilles.

Creseis spinifera Q. \& G., Moll. Cuba, i, 85.

Creseis spinifera Rang, Moll. Cuba, i, 85.

Creseis striata D'Orb., Arango, Fanna Mal. Cuba, 1878. 152. Antilles. Creseis striata Rang, Arango, Fauna Mal. Cuba, 1875. 152. Antilles. Creseis striata Rang, Moll. Cuba, i, 87.

Creseis subula D'Orb., Arango, Famna Mal. Cuba, 1878. 152. Ca. Antilles.

Creseis subula D'Orb., Moll. Cuba, i, 85.

Creseis subula Q. \& G., Arango, l'amna Mal. Cuba, 1878. 152. WI. Creseis virgula D'Orb., Aranggo, Famna Ma1. Cuba, 1878. 152. Antilles. Creseis virgula Rang, Arango, Fama Mal. C'nba, 1878. 152. Antilles. Creseis virgula Rang, Moll. Cuba, i, 86 .

Cricostoma striatum Klein, Mal. Bl., xxiii, 130.

Cricostoma striatum Klein, Moll. Cuba, ii, 57.

Crucibulum auriculatum Chem., bis. Krebs. 69. SCx. SB. Ag. Bb.

Crucibulum auriculatum Chem., Mal. Bl., xxiv, 101.

Crucibulum auriculatum Chem., Mal. Bl., xxir, 102.

Crucibulum auriculatum Chem., Poulsen. 10.

Crucibulum piliferum Guppy, Geol. Mag., 1874. 443. Td.

Crucibulum planatum Schum., Mal. Bl., xxiv, 102.

Crucibulum radiata Brod., Krebs. 69. ST. T. SCx.

Crucibulum striatum Stimp., Mal. Bl., xxiv, 102.

Crucibulum subsutum Guppy, Geol. Mag., 1874. 443. 'Td.

Crypta (see Crepirlula).

Crypta aculeata Chem., Jahrb., ii, 245.

Crypta (Crepipatella) aculeata Chem., Marrat, Argo Exp., 1876. 6.

Aa. Havana.

Crypta aculeata Gmel., Mal. Bl., xxiv, 102. Fla.

Crypta aculeata, Gmel., Poulsen. 10.

Crypta aplysioides Rve.,* Mal. Bl., xxiv, 103. Bz.

Crypta aplysioides Rve., Poulsen. 10.

Crypta convexa Say, Mal. Bl., xxiv, 104.

Crypta fornicata Linn., Mal. Bl., xxiv, 103.

Crypta glauca Say, Mal. Bl., xxir, 104.

Crypta maculosa Con., Mal. Bl., xxiv, 105. Fla.

Crypta nautarum Humph., Mal. Bl., xxiv, 103.

Crypta nautarum Humph. = Crep. fornicata Rve., Poulsen. 10.

Crypta navicula Dkr., Mal. Bl., xxiv, 105.

Crypta nivea C. B. Adams, Jahrb., ii, 245.

Crypta plana Say, Jahrb., ii, 245.

Crypta plana Say, Mal. Bl., xxiv, 106.

Crypta protea D'Orb., * Mal. Bl., xxir, 105. Ca.

Crypta protea D’Orb., Poulsen. 10.

Crypta riisei Dkr.,* Mal. Bl., xxiv, 105. 
Cryptodon conradi Rve, Arango, Fauna Mal. Cuba, 1878. 240. Ca. Cryptodon obesus (?) Verrill, Report Blake Moll. 136. WI. Hatt. $\checkmark$ Cryptogramma (see Venus).

Cryptogramma flexuosa Linn., Jahrb., ii, 249.

Cryptogramma flexuosa Linn., Mal., Bl., xiv, 21.

- Cryptogramma membranula Röm., Mal. Bl., xiv, 23. ST.

Cryptogramma puella Pfr., Mal. Bl., xiv, 23. Ca.

Cryptogramma rostrata Sby., Mal. Bl., xiv, 25. Fla.

Cryptospira (see Marginella) Marrat, Argo Exp. 1876.12.

Cryptostoma (see Sigaretus).

Cryptostoma carolinum Cuv., Mal. Bl., xxiv, 56.

Cryptostoma leachii Blv., Arango, Fauna Mal. Cuba, 1878. 172. Ca.

Santa Lucia.

Cryptostoma leachii Blv., Mal. Bl., xxiv, 56.

Cryptostoma leachii Blv., Moll. Cuba, ii, 37.

Ctenoides (see Lima).

Ctenoides (sce Radula) Marrat, Argo Exp., 1876. 6.

Ctenoides scabra Klein, Krebs. 133.

Ctenoides tenera Klein, Krebs. 133.

Cultellus bidentatus Speng., Mal. Bl., x, 230 (see Tagelus).

Cultellus plebeius Sol., Mal. Bl., x, 230.

Cuma diadema Lam., Marrat, Argo Exp., 1876. 6. Aa. D. Nassau.

Cumingia (see Lavignon) Arango, Fauna Mal. Cuba, 1878. 245. Ca.

Cumingia antillarum D'Orb., Beau. 25. G.

Cumingia antillarum D'Orb., Krebs. 107. ST.

Cumingia lineata D'Orb., Krebs. 107.

Cumingia petitiana D'Orb, Beau. 25. G.

Cumingia petitiana D'Orb., Krebs. 107.

Oumingia sinuata A. Ad., Krebs. 107.

Cumingia sinuata A. Ad., Poulsen. 15.

Cumingia tellinoides Con., Am. Marine Conch. 155.

Cuvieria columella D'Orb., Moll. Cuba, i, 89.

Cuvieria columella Rang, Arango, Fauna Mal. Cuba, 1878, 153. WI. Cuvieria columella Rang, Moll. Cuba, i, 89.

Cuvieria obtusa D'Orb., Arango, Fauna Mal. Cuba, 1878. 153. WI. Cuvieria obtusa D'Orb., Moll. Cuba, i, 89, 90.

Cuvieria obtusa Q. \& G., Arango, Fauna Mal. Cuba, 1878. 152. WI. Cyclas (see Lucina), Marrat, Argo. Exp., 1876. 19.

Cyclas maritima D’Orb., Krebs. 107.

Cyclas maritima D’Orb., Moll. Cuba, ii, pl. xxvi, figs. 47-50, 280. Ca.

Cyclas quadrisulcata D’Orb., Krebs. 120.

Cyclina tenuis Recl., Beau. 24. G.

Cyclina tenuis Recl., Krebs. 99.

Cyclostoma (see Truncatella).

Cyclostoma truncatulum Drap., Moll. Uuba, ii, 5.

Oyclostoma truncatulum Lam., Moll. Cuba, ii, 5 . 
Cyclostoma truncatulum Mich., Moll. Cuba, ii, 6.

Cyclostrema schrammii Fisch., Jour. de Conch. vi, 173. G.

Cyclostrema schrammi Fisch., ${ }^{*}$ Jour. de Conch. vi, pl. x, fig. 10, 287. G.

Cyclotrema diaphana D'Orb., Poulsen. 13.

Cylichua coelata Bush, Conn. Ac. vi, pl. xlv, fig. 15. 468. Hatt.

Cylichna discus Watson, Lin. Soc. Journ., 1883, xvii, 319. Culebra.

Cylichna krebsii Mörch,* Mal. Bl., xxii, 172. SM.

Cylichna krebsii Mörch, Poulsen. 8.

Cylichna ovum-lacerti Guppy, Geol. Mag., 1874. 437. Td.

Cylichna (V'olvula) paupercula Watson, Lin. Soc. Jouru., 1853, xvii, 325.

Culebra.

Cylichnella bidentata D’Orb., Mal. Bl., xxii, 171. J.

Cylichnella bidentata D'Orb., Poulsen. 8 .

Cylindrobulla beanii Fisch., Beau. 18. G.

Cylindrobulla beanii Fisch., Jour. de Conch. v, pl. viii, fig. 8, 9, 275. G.

Cylindrobulla beauii Fisch., Mal. Bl., iv, 220. G.

Cyliudrobulla beauii Fisch., Mal. Bl., xxii, 175. ST. G.

Cylindrobulla beauii Fisch., Poulsen. 8.

Cyllene chrysostoma Meusch., Jahrb., ir, 297.

Cymatium femorale Linu., Jahrb., $\nabla, 249$.

Cyphoma (see Orulum, Volva).

Cyphoma dorsata Boit., Mal. Bl. xxiv, 53.

Cyphoma emarginata Sby., Arango, Fauna Mal. Cuba, 1878. 183. Ca.

Cyphoma gibbosa Bolt., Mal. Bl., xxiv, 53.

Cyphoma giboosa Linu., Arango, Fauna Mal. Cuba, 1878. 183. Ha-

vana. G. M[. Santa Lucia. Ba.

Cyphoma gibbosa Linu., Marrat. Argo Exp., 1876. 16. Nassau. Long Key Island.

Cyploma var. rostrata, Mal. Bl. xxiv, 53.

Cypriea (sce Trivia) Arango, Fauna Mal. Cuba, 1878. 185. Ca. G. Ba. Cyprea acicularis Gmel., Arango, Fauna Mal. Cuba, 1878. 184. Ca. Ba.

Cyprea acicularis Gmel., Mal. Bl., xxiv, 48. = apuce

Uypraa acicularis Gmel., Moll. Cuba, ii, 90.

Cypræa acicularis Gmel., Zt. Mal., x, 79. SV.

Crprea approximaus Beck, Mal. B1., xxir, 50.

Cypræa armaudina Duclos, Mal. Bl., xxiv, 49. =

Cypræa armandina Duclos, Araugo, Fanna Mal. Cuba, 1878. 186. Ca. G. Ba.

Cyproa armandina Duclos, Beau. 7. G.

Cypræa armandina Duclcs, Krebs. 42.

Cypræa auberiana Jouss., Jahrb., viii, 151. $\quad \nabla C z .=$ auhryana

Cyprea aubryana Jouss., Mal. Bl., xxiv, 47. G. = bicnlewa

Cyprea aubryana Jouss., Am. Jour. Conch., vi, 83. G.

Cypræa aubryana Sby., Mal. Bl., xxiv, 47. G.

Cypræa autumualis Perry, Arango, Fauna Mal. Cuba, 1878. 184. Ca. 
Cypræa autumnalis Perry, Mal. Bl, xxiv, $46 .=$ mus.

Cyprea bicallosa Gray, Beau. 7. MG.

Cypræa bicallosa Gray, Jahrb., viii, 151. SV.

Cyprea bicallosa Gray, Krebs. 41.

Cyprea bicallosa Gray, Mal. Bl., xxir, 47. G.

Cypræa bicallosa Sby., Mal. Bl., xxiv, 48. MG.

Cypraa bifasciata Gmel., Arango, Fauna Mal. Cuba, 1878. 184. Ca.

M. G. Santa Lucia. Ba. = exastherne

Cypræa bifasciata Gmel., Mal. Bl., xxiv, 45.

Cyprea candidula Gask., Mal. Bl., xxiv, 50. = Zrivia

Cyprea candidula Rve., Mal. Bl., xxiv, 50.

Cypræa carnea Gray, Krebs. $41 .=7$ = Fre

Cypræa cerviua Kien., Jahrb., viii, 134. = eervas

Cypræa cervina Lam., Arango, Fauna Mal. Cuba, 1878. 184. Ca.

Cypræa cervina Lam., Krebs. 41.

Cypræa cerrina Lam., Mal. Bl., xxiv, 44.

Cypraa cervus Linn., Arango, Fauna Mal. Cuba, 1878. 184. Ca.

Cspræa cervus Limu., Nachblt., 1877. 59.

Cypræa cervus Linn., Krebs. 41.

Cypræa cervus Iinn., Mal. Bl., xxiv, 44.

Cypræa cervus Linn., Moll. of Bermudas. 17.

Cypræa cervus Sloy., Jalurb, viii, 134.

Cypraca cinerea D'Orb., Arango, Fauna Mal. Cuba, 1878. 185. Ca. Ba. Cypraca cinerea Gmel., Arango, Fauna Mal. Cuba, 1878. 185. Ca. Ba. Cypræa cinerea Gmel., Beau. 7. G.

Cypræa cinerea Gmel., Jahrb., viii, 135.

Cypræa cinerea Gmel., Jour. de Conch, ii, 430. G.

Cypræa cinerea Gmel., Mal. Bl., xxiv, 46.

Cypræa cinerea Gmel.. Mal. Bl., xxiv, 47.' J.

Cyprea ciucrea (rmel., Marrat, Argo Exp., 1876. 8. Barbuda. SV.

\section{Nassau.}

Cypræa cinerea Gmel., Moll. Cuba, ii, 91.

Cypræa cinerea Gmel., Poulsen. 10.

Cypræa cinerea Lam., Moll. Cuba, ii, 91.

Cypræa cinerea Linn., Moll. of Bermudas. 17.

Cypræa cinerea Mart., Krebs. 42.

Cspræa cinerea Wood, Moll. Cuba, ii, 91.

Cyprea clara Gask., Jahrb., viii, 135. = eimerc

Oypræa conoidea Scop., Mal. Bl., xxiv, 40. = Onjova

Cypræa conoidea Scop., Jalırb., v, 238.

Cypræa conoidea Scop., Mial. Bl., xxiv, 39.

Cyprea cornea Gray, Beau. 7. G. = erz wew

Cypræa cornea Gray, Jour. de Conch., iv, 419, G.

Cjpræa (Trivia) cornea Gray, Jour. de Conch., v, 156. G.

Bull. 24- -7 
Cyprea dubia Gmel., Arango, Fauna Mal. Cuba, 1878. 184. Ca. M. G. Santa Lucia. Ba. = exareines -

Cypræa dubia Gmel., Mal. Bl., xxiv, 45.

‘Cypræa exanthema D’Orb., Arango, Fauna Mal. Cuba, 1878. 184. Ca. M. G. Santa Lucia. Ba.

Cypræa exanthema Gmel., Moll. Cuba, ii, 88.

Cypræa exanthema Lam., Moll. Cuba, ii, 88.

Cyprea exanthema Linn., Arango, Fanna Mal. Cuba, 1878. 184. Oa.

M. G. Santa Lucia. Ba.

Cypræa exanthema Linn., Beau. 7. G.

Cypræa exanthema Linn., Jahrb., viii, 134.

Cypræa exanthema Linn., Jour. de Conch., iv, 418. G.

Cypræa exanthema Linn., Krebs. 41. ST. SM. SB.

Cypræa exanthema Linn., Mal. Bl., xxir, 45.

Cyprea exanthema Limn, Marrat, Argo Exp., 1876. 8. Barbuda.

Cypræa exanthema Linn., Moll. Cuba, ii, 88.

Cypræa exanthema Linn., Poulsen. 10.

Cypræa exanthema Wood, Moll. Cuba, ii, 89.

$\checkmark$ Cypræa flaveola Desh., Mal. Bl., xxir, 48.

Cypræa flaveola var. Gask., Mal. Bl., xxiv, 48. Fla.

Cypræa flaveola Lam., Jahrb., viii, 150.

Cypræa flaveola Lam., Mal. Bl., xxiv, 48.

Cypræa flaveola Lam., Moll. Cuba, ii, 90.

Cypræa flaveola Lam., Zt. Mal., x, 78。 SV.

Cypræa flareola Linn., Arango, Fama Mal. Cuba, 1878. 184. Ca. Ba.

Oypræa flaveola Linn., Jour. de Conch., ii, 430. G.

Cypræa flaveola Rve., Beau. 7. G.

Cypræa flaveola Rve., Krebs. 42.

Cypræa flaveola var. alba Sby., Mal. Bl., xxiv, 48.

? Cypræa fragiliodes Meusch., Mal. Bl., xxiv, 47.

/ Cypræa (Trivia) globosa Gray, Jour. de Conch., v, 153. G.

Cypræa globosa Sby., Beau. 7. G.

Cypræa globosa Sby., Krebs. 41.

Cyprea isabella Lim., Marrat, Aigo Exp., 1876. 12. Tucacas. - emen Cypræa labrolineata Gask., Araugo, Fauna Mal. Cuba, 1878. 184. Ca.

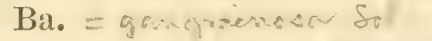

Cypræa lencopsis Shaw, Mal. Bl., xxiv, 45.

Cypræa lota Born, Krebs. 42. = spurc.

Cypræa lota Born, Mal. Bl., xxiv, 48.

Cypræa maxima Chemn., Mal. Bl., xxiv, 44.

Cypræa meleagris Bolt., Mal. Bl., xxiv, 45.

Cypræa moneta Gmel., Moll. Cuba, ii, 92. ....

Cypræa moneta Lam., Moll. Cuba, ii, 92.

Cypræa moneta Linn., Moll. Cuba, ii, 92.

Cypræa mus D'Orb., Axango, Fauna Mal. Cuba, 1878. 184. Ca.

Cypræa mus Gmel., Moll. Cuba, ii, \$\%. 
Cypræa mus Lam., Moll. Cuba, ii, 89.

Cypræa mus Linn., Arango, Fauna Mal. Cuba, 1878. 184. Ca.

Cypræa mus Linn., Jahrb., viii, 141. Vz.

Cypræa mus Linn., Krebs. 41.

Cypræa mus Linn., Mal. Bl., xxiv, 45.

Cypræa mus Linn., Moll. Cuba, ii, 89.

Cypræa mus Linn., Poulsen. 10.

Cypræa mus Wood, Moll. Cuba, ii, 89.

Cypræa nebulosa Kien., Mal. Bl., xxiv, 47. = Apsen

Cypræa nivea D’Orb., Arango, Fauna Mal. Cuba, 1878. 185. Ca.

G. Ba.

Oypræa nivea Gray, Beau. 7. G. = J゙niea

Cypræa nivea Gray, Jour. de Conch., iv, 419. G.

Cypræa nivea Gray, Krebs. 41.

Cypræa nivea Gray, Mal. Bl., xxiv, 50.

Cypræa nivea Gray, Moll. Cuba, ii, 94.

Cypræa oculata Gmel., Arango, Fauna Mal. Cuba, 1878. 184. Ca.

Cypræa orulata Meusch., Mal. Bl., xxiv, 4 .

Cypræa olorina Duclos, Mal. Bl., xxiv, 50.

Cypræa pediculus D’Urb., Arango, Famna Mal. Cuba, 1878. 185. Ca.

Cypræa pediculus Gmel., Moll. Cuba, ii, 93. = Jrivia

Cypræa pediculus Gray, Moll. Cuba, ii, 93.

Cypræa pediculus Lam., Moll. Cuba, ii, 93.

Cypræa pediculus Linn., Araugo, Fauna Mal. Cuba, 1878. 185. Ca.

Cypraea pediculus Linn., Beau. 7. G.

Cypræa pediculus Linn., Jour, de Conch., ii, 430. G.

Cypræa pediculus Limn., Krebs. 41. ST. Tort. SJ. SB. SM. SCx.

Cypræa (Trivia) pediculus Linn., Marrat, Argo Exp., 1876. 6. Aa.

Cypræa pediculus Limn., Moll. Cuba, ii, 93.

- Cyprea picta Gray, Jahrb., viii, 145.

Cyprea pieta Gray, Zt. Mal., x, 78. SV.

Cypræa pilula Kien., Beau. 7. G. - Trivi.

Cypræa (Trivia) pilula Kien., Jour. de Conch., v, 153. G.

Cypræa pilula Kien., Krebs. 41. S'T. SCx. SJ. SB. Ag.

Cypræa pilula Kien., Mal. Bl., xxiv, 50. Ca.

Cypræa pisum Meusch., Mal. Bl., xxir. 49. Fla.

Cypræa plumbea Gmel., Araugo, Famma NaI. Cuba, 1878, 184. Ca. M.

G. Santa Lucia. Ba. $=E_{x} a$

Cypræa plumbea Ġmel., Mal. Bl., xxir, 45.

Cypræa pullata Owen, Mal. Bl., xxiv, $49 .=$ Firicín

Cypræa var. pumilio, Mal. Bl., xxiv, 45.

Cypræa var. pyriformis Sby., Mal. Bl., xxiv, 45.

Cypræa quadripunctata D'Orb., Arango, Fanua Mal. Cuba, 1878. 185.

Cyprea quadripunctata Gray, Arango, Fauma Mal. Cuba, 187s. 185.

Cypræa quadripusetata Gray, Beau. 7. G.

Cypræa quadripunctata Gray, Jour. de Conch., ii, 430, G. 
Cypræa quadripunctata Gray, Krebs. 42. Ag. Bb. Td.J.

Cyprea quadripunctata Gray, Moll. of Bermudas, 17.

Cypræa quadripunctata Gray, Moll. Cuba, ii, 94.

Cypræa quadripunctata Reeve, Mal. Bl., xxiv, 49. Ca.

Cypræa quadripunctata Sby., Moll. Cuba, ii, 94.

Crprea rattus Lam., Jour. de Conch., iv, 418. G.

Crpræa rosea Auct., Arango, Fauna Mal. Cuba, 1878. 185. Ca. Antilles.

Cypræa rosea Kien., Beau. 7. G. 'trivia

Cypræa rosea Kien., Jour. de Conch., ir, 419. G.

Cspræa (Trivia) rosea Kiener, Jour. de Conch., v, 156. G.

Cypræa rosea Kien., Krebs. 41.

Cyprea rotuuda Kien., Araugo, Fauna Mal. Cuba, 1878. 185. Ca. WI.

Cyprea rotunda Kien., Beau. 7. G. Tivira

Crprea rotunda Kien., Jour. de Conch., ii, 430. G.

Cyprea rotunda Kien., Krebs. 42.

Cyprea rotunda Kien., Mal. Bl., xxiv, 49. Bb.

Cypræa seabriuscula (Gray) Kien., Arango, Fauna Mal. Cuba, 1878.

185. Ca. G. Ba.

Cypræa scabriuscula Kien., Mal. Bl., xxiv, 50.

C5prea sordida Lam., Arango, Fauna Mal. Cuba, 1878. 185. Ca. Ba.

Cypræa sordida Lam., Beau. 7. G. = exivere.

Cypræa sordida Lam., Jahrb., viii, 135.

Cypræa sordida Lam., Jour. de Conch., ii, 430. G.

Cypræa sordida Lam., Krebs. 42.

Cypræa sordida Lam., Mal. Bl., xxiv, 47. J.

Cypræa sordida Lam., Moll. Cuba, ii, 91.

Cyprea spurea D’Orb., Arango, Mal. Cuba, 1878. 184. Ca. Ba.

Cypræa spurea D’Orb., Moll. Cuba, ii, 90.

Cyprea spurea Gmel., Arango, Fauna Mal. Cuba, 1878. 184. Ca. Ba.

Cyprea spurea Gmel., Moll. Cuba, ii, 90.

Ospræa spurea Linn., Beau. 7. G.

Cypræa spurea Linn., Jahrb., viii, 150.

Cypræa spurca Limn., Krebs. 42. ST. SCx. SM. Bb.

Cypræa spurca Linn., Mal. Bl., xxiv, 48.

Cyprea spurca Linn., Moll. Cuba, ii, 90.

Cypræa spurca Linn., Pouisen. 10.

Cspræa spurea Linn., Zt. Mal. x, 78. SV.

Cyprea stercoraria Linn., Beau. 7. G.

Cspræa stercoraria Linn., Krebs. 42.

Cyprea subrostrata Gray, Arango, Fauna Mal. Cuba, 1878. 185. Ca.

G. Ba. = Jrweor

Cspræa subrostrata Gray, Beau. 7. G.

Cyprea subrostrata Gray, Jour. de Conch., iv, 418. G.

Cyprea subrostrata Gray, Krebs. 42. Ag. ST. SJ. SCx. SB. Bb.

Cypræa subrostrata var., Krebs, Mal. Bl., xxir, 50. Ca.

Cypræa subrostrata Rre., Mal. Bl., xxiv, 50. Bb. 
Cyprea suecincta Gmel., Arango, Fauna Mal.Cuba, 1878. 185. Ca. Ba. Cypræa succincta Linw., Arango, Fauna Mal.Cuba, 1878. 185. Ca. Ba. Csprea suecincta Linn., Krebs. 4\%. SD. ST. SB. SM. Tort. Ag. Bb. Cypræa succincta Linn., Mal. Bl., xxir, 47. J. = eine

Cyprea suffusa Gray, Beau. 7. G.

Cypræa suffusa Gray, Jour. de Conch., ir, 41S. G.

Cypræa suffusa Gray, Krebs. 42. Ag. ST. SB. SCx. SM. Bb.

Cyprea sulcata Dillw., Arango, Fauna Mal. Cuba, 187S. 185. Ca. Antilles. Frivin

Cypræa sulcata Dillw., Jour. de Conch., ii, 430. G.

Cypræa sulcata Dillw., Mal. Bi., xxir, 49. Fla.

Cylriea sulcata WVood, Moll. Cuba, ii, 93.

Cypræa surinamensis Mörch, Mal. Bl., xxiv, 47. - Cercerare a

Cypræa surinamensis Perry, Mal. Bl., xxiv, 47.

Cypræa translucens Gmel., Arango, Fauna Mal. Cuba, 187S. 185. Ca.

$\mathrm{Ba}$. = einerea.

Cypræa translucens Gmel., Mal. Bl., xxiv, 47. J. =

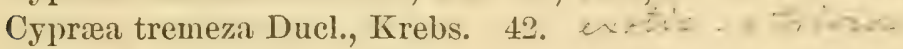

Cypræa tremeza Ducl., Mag. de Zool., 1833, pl. xxг.

Cypræa vanelli Humpl., Mal. Bl, xxiv, 46.

Cypræa zebra Linn., Arango, Fauna Mal. Cuba, 187s. 184. Ca. M. G.

Santa Lucia. Ba. = exa. Lia.....

Cypræa zebra Linn., Mal. Bl., xxiv, 45.

Cypræcassis testiculus Liun., Arango, Famna Mal. Cuba, 1878. 224.

Ca. M. G. Ba.

$\checkmark$ Cypricardia coralliophaga, Moll. Cuba, ii, 266.

Oypricardia coralliophaga Gmel., Beau. 23. G.

Cypricardia coralliophaga Gmel., Jour. de Conch., ii, 425. G.

Cypricardia gracilis Shuttlew., Beau. 23. G. = coralio phaga a.c.

Cypricardia gracilis Shuttlew., Jour. de Conch., v, 150. G.

Cypricardia gracilis Shuttlew., Krebs. 123.

Cypricardia hornbeckiana D’Orb., Arango, Fauna Mal. Cuba, 1878. 24S. Ca. ST. = eonlliophaga val,

Cypricardia horubeckiana D'Orb., Krebs. 123.

Cypricardia hornbeckiana D'Orb., Moll. Cuba, ii, pl. xxvi, figs. 33-34, 266.

Cypricardia nodulosa Mighels, Proc. Bost. Soc. Nat. Hist., 1844, i,

188. Key West. - Carditamera fluridana Comad.

Cyrachæa scabra Leach, Krebs. 121.

Cyrena carolinensis Lam., Dall, Hemphill's shells. 339. Key Wrest.

Cyrena colorata Prime, Am. Jour. Conch., iii, si. NP.

Cyrena floridana, Am. Jour. Conch., v, 107. Fla.

Cyrena tloridana Conrad, Am. Jour. Conch., r, 227. Fla.

Cyrena floridaua Courarl, Dall, Hemphill's shells. 338. Sarasota Bay. Cyrena floxidana Conrad, Proc. Acad. Nat. Sci. Phil., iii, 23, p]. 1, fig.

1, 1845. Tampa Bay. 
Cyrena protexta Conrar,,* Am. Jour. Conch., v, pl. 12, f. 3, 107. Fla. Cyrena protexta Conrad, Am. Jour. Conch., v, 227. Fla.

Cyrena protexta Conrad, Dall, Hemphill's shells. 338. Sarasota Bay. Cyrenella americana Shuttlew., Mal: Bl., i, 170. PR:

Cyrtopletira exilis Tryon,* Am. Jour. Conch., v, pl, 14, f. 2, 170. SCx. Cytherea (see Callista).

Cytherea (see Venus) Arango, Fauna Mal. Cuba, 1878. 249. 'Ca. Cytherea affinis Gmel., Poulsen. 15. : ?ent x. in

Cytherea albida Gmel., Mal Bl., ix, 67. J.

Cytherea albida Gray, Krebs. 95. NGr.

Cytherea auberiana, D'Orb., Poulsen. 15.

Cytherea? bella? Kurtz. 4. SC.

Cytherea cardilla Lam., Mal. B1., xiv, 95.

Cytherea circinata Born, Beau. 24. G.

Cytherea circinata Born, Poulsen. 15.

Cytherea circinata Dillw., Jour. de Conch., iv, 414. G.

Cstherea concentrica Lam., Krebs. 98.

Cstherea concentrica Lam., Krebs. 99.

Cytherea concentrica Lain., Moll. Cuba, ii, 270.

Cytherea concentrica Lam., Moll. Cuba., ii, 274.

Cytherea concentrica Phil., Moll. Cuba, ii, 274.

Cytherea (Transenuella) conradiua Dall, n. s., Dall, Hemphill's shells.

340. Cedar Keys.

Cytherea convexa Say, Kurtz. 4. NO.

Cytherea corbicula Desh., Mal. Bl., viii, 24. = Livela maclizri.. -

Cytherea corbicula Gmel., Jour. de Conch., ii, 425. G.

Cytherea corbicula Gmel., Krebs. 97.

Cytherea corbicula Lam., Moll. Cuba, ii, 276.

Cytherea dione Lam., Moll. Cuba, ii, 275.

Cytherea dione Linn., Beau. 24. G.

Cytherea dione Linn., Jour. de Conch., ii, 425.

Cytherea dione Linn., Mal. Bl., x, 231.

Cytherea dione Linn., Poulsen. 15.

Cytherea elevata Courad, Am. Journ. Sci, n. s. 1, 404. 1846. Gulf of Mexico.

Cytherea flexuosa Lam., Mioll. Cuba., ii, 271.

Cytherea flexuosa Lam., Poulsen. 15.

Cytherea Hexuosa Linu., Jour. de Conch., ii, 425. G.

Cytherea fragilis Roem.,* Mal. Bl., vii, 160.

Cytherea gigantea Kurtz. 4. NC.

Cytherea gigantea Guel., Arango, Tauma Mal. Cuba, 1878. 251. Ca. Cytherea guineensis Lam., Arango, Fauma Mal. Cuba, 1878. 249. Ca. Cytherea guineensis Lam., Krebs. 95. = civenti.

Cytherea guineensis Lam., Moll. Cuba, ii, 275.

Cytherea hebriea Lam., Poulsen. 1\%.

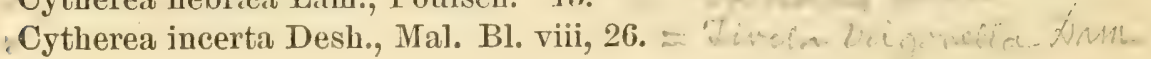


Cytherea kingii Desh., var.? Mal. Bl. ix, 67. J.

$\checkmark$ Cytherea kingii Gray, Jour, de Conch., v, 150. G.

Vytherea kingii Gray, Jour. de Conch., v, 155. G.

Cytherea kingii Gray, Mal. Bl., iv, 27. G.

Cytherea kingii Gray, Mal. B1., vii, 150. ST.

Cytherea lata Lam., Krebs. 95. = o f f

Cytherea lavacrum Bolt., Krebs. 96. =

Cytherea lunularis Lam., Jahrb. ii, 249.

Cytherea lunularis Lam., Mal. Bl., xiv, 21.

$\checkmark$ Cytherea mactroides Born, Mal. Bl., viii, 25. $=A_{c}:$

Cytherea mactroides Born, Mal. Bl., x, 231.

Cytherea mactroides Born, Poulsen. 15.

$\checkmark$ Cștherea maculata Lam., Moll. Cuba, ii, 269.

Cytherea maculata Linn., Beau. 24。 G.

$\checkmark$ Cytherea maculata Linn., Poulsen. 15.

Cytherea maculata Mart., Mal. Bl., xxiv, 121.

Cytherea minuta Koch, Poulsen. 15.

Cytherea modesta Phil., Jonr. de Conch., r, 155. G.

Cytherea modesta Phil., Mal. Bl., iv, 27. G.

Cytherea munda Roem., Mal. I3l., vii, 150. ST.

Cytherea munda Roem., Mal. BI., ix, 81. ST.

Cytherea occulta Say, Kurtz. 4.

Cytherea patagonica Phil., Arango, Fauma Mal. Cuba, IS7S. 252. Ca.

Cytherea patagonica Phil., Krebs. 99.

Cytherea patagonica Phil., Moll. Cuba, ii, 270.

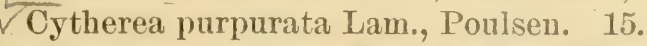

Cytherea rubigiuosa Phil., Arango, Fauna Mal. Cuba, 187S. 251. Ca.

M. Fla.

Cytherea rubiginosa Phil., Beau. 24. G.

Cytherea rubiginosa Phil., Krebs. 97.

Cytherea rubiginosa Plil., Mal. Bl., ix, 74.

Cytherea rubiginosa Phil., Moll. Cuba, ii, 272.

Cytherea tenuis Recluz, Mal. Bl., vii, 161. G. = $\mathrm{C}_{\mathrm{L}}$

Cytherea tigeriua Lam., Krebs. 121.

Cytherea tigerrina Lam., Moll. Cuba, ii, 297.

Cytherea trigonella Lam., Amango, Fauna Mal. Cuba, 187S. 251. Ca.

Oytherea trigonella Lam., Poulsen. 15.

Cytherea trigonella Phil., Arango, Fauua Mal. Cuba, 1878. 250. Ca.

G. Ba.

Cytherea trigonella Phil., Moll. Cuba, ii, 276.

$\checkmark$ Cytherea tripla Linn., Poulsen. $15 .=$

Cytherea umbonella Lam., Dkr., Moll. Mar. xiii, f. 4-6. 42. 1858.

ST. WI.

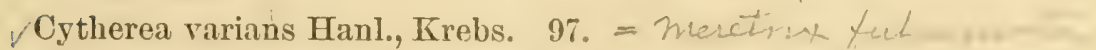

Cytherea varians Hanl., Mal. Bl., ix, 74.

Cytherea varians Phil., Beau. 24. G. 
Cytherea varians Phil., Krebs. $97 .=$

Cytherea varians Wood, Jahrb., ii, 249.

Dactylidia mutica Say, Marrat, Argo. Exp., 1876. 11. La Guayra.

Dactylina (see Pholas).

Dactylina (Gitocentrum) eampechensis Gmel., Am. Marine Conch. 126.

Dactylina campechensis Gmel., Axango, Fauna Mal. Cuba, ${ }^{\circ} 878.235$.

Ca.

Dactylus (see Oliva).

Dactylus (Strephona) oblonga Marrat, Argo. Exp., 1876. 13. Santa Marta.

Dactylus (Strephona) olivaceus Meusch., Marrat, Argo Exp., 1876. 8.

D. Tucacas. Santa Marta. Nassau.

Dactylus (Strephona) reticularis Lam., Marrat, Argo Exp., 1s76. 8.

Barbuda.

Dactylus (Porphyria) seripta Lam., Marrat, Argo Exp., 1876. 12.

Tucacas.

Daphne occidentalis Poli, Krebs. 125.

Daphnella (see Pleurotoma).

Daphnella (Pleurotoma) decorata Ad., Krebs. 9.

Daphnella decorata Ad., Krebs. 12.

Daphnella lymnxiformis Kien., Arango, Fauna Mal. Cuba, 1878. 221.

Ca. Havana. Ba. G.

Daphuella lymnæformis Kien., Krebs. 12. Ag. VD. W. ST. SJ. SB. SM.

Daphnoderma squamosa Mörch, Krebs. 126.

Decapoda myopsidæ D'Orb., Moll. Cuba, i, 30.

Delphinula radiata Kien., Arango, Fauna Mal. Cuba, 1878. 179. Baracoa. Matanzas.

Delphinula radiata Kien., Beau. 13. G.

Delphinula radiata Kien., Jour. de Conch., ii, 428. G.

Delphinula radiata Kien., Krebs. 80.

Delphinula tuberculosa D'Orb., Ârango, Fauna Mal. Cuba, 1878. 179.

J. ST.

Delphinula tuberculosa D'Orb., Krebs. 80.

Delphinula tuberculosa D'Orb., Krebs. 85.

Delphinula tuberculosa D'Orb., Moll. Cuba, ii, pl. xix, figs. 28-30. 69.

Dendrodoris erucis Oersted, Jour. de Conch., xi, 33. ST.

Dendrodoris crucis Oersted, Mal. Bl.,xxii, 177. ST.

Dens sanguineus Chem., Moll. Cuba, ii, 49.

Dentalium (see Cadulus, Siphodentalium, Artalis).

Dentalium agile M. Sars., Report Blake Moll. 37.

Dentalium antillarum D’Orb., Arango, Fanma Mal. Cuba, 1878. 232.

Ca. Havana. ST. G.

Dentalium antillarum D'Orb., Beau. 18. G.

Dentalium antillarum D'Orb., Jour. de Conch., v, 151. 'G.

Dentalium antillarum D'Orb., Krebs. 91. 
Dentalium antillarum D'Orb., Moll. Cuba, ii, pl. xxr, figs. 10-13. 202.

Dentalium antillarum D'Orb., Poulsen. 14.

Dentalium antillarum D'Orb., Report Blake Moll. 37. Yucatan Strait. Barbados.

Dentalium capillosum Jeffreys, Watson, Lin. Soc. Journ., 1879, siv, 508. Gulf of Mexico, Cul.

Dentalium ceras Watson, Report Blake Moll. 37.

Dentalium ceratum Dall, n. s., Report Blake Moll. 38. W. Fla.

Dentalium cireumcinctum Watson, Lin. Soc. Jouru., 1S79, xir, 513. Sombrero.

Dentalium coarctatum Lam., Conrad, Am. Journ. Sci., n. s., i., 405. 1846. Gulf of Mexico.

Dentalium compressum Watson, Report Blake Moll. 3S.

Dentalium compressum Watson, Lin. Soc. Journ., 1879, xir, 516. Cul. Dentalium didymum Watson, Report Blake Moll. 38. WI.

Dentalium didymum Watson, Lin. Soc. Jouru., 1879, xiv, 517. Cul.

Dentalium disparile D’Orb., Arango, Fauna Mal. Cuba, 1878. 232. Ca. M. G.

Dentalium disparile D'Orb., Beau. 18. G.

Dentalium disparile D'Orb., Jour. de Conch., $\nabla, 151$. G.

Dentalium disparile $\mathrm{D}^{\prime} \mathrm{Or} \mathbf{b}$., Krebs. 91.

Dentalinm disparile D'Orb., Moll. Cuba, ii, pl. xxv, figs. 14-17. 202.

Dentalium disparile D'Orb., Poulsen. 14.

Dentalium disparile D'Orb., Report Blake Moll. 37. Yucatan Strait. Barbados.

Dentalium domingense D'Orb., Krebs. 91.

Dentalium dominguense D'Orb., Arango, Fauna Mal. Cuba, 1878. 232. Ca. M. SD. ST.

Dentalium dominguense D’Orb., Moll. Cuba, ii, pl. xxr, figs. 7-9. 201. Dentalium dominguense D'Orb., Poulsen. 14.

Dentalium eboreum Conrad, Am. Journ. Sci., n. s., ii, 396. 1S46. Fla. Dentalium eboreum Conrad, Proc. Acad. Nat. Sci. Phil., iii, 27. 1845. S. Fla.

Dentalium ensiculus Jeffreys, Watson, Lin. Soc. Journ., 1879. xir, 518. Sombrero.

Dentalium leptum Bush, Conn. Ac., vi, pl. xlr, figs. 18, 18a. 470. Hatt.

Dentalium modicellum Kurtz. 6. SC.

Dentalium ophiodon Dall, n. s., Report Blake Moll. 38.

Dentalium perlongum Dall, Report Blake Moll.36. Yucatan Strait.

Dentalium pliocenum Tumey \& Holmes, Am. Marine Conch. 97. SC. Dentalium pleiocenum T. \& H., n. s., Pleiocene fossils SC., pl. xxr, fig: 2. 105 .

Dentalium pseudo-hexagonum Desh., Marrat, Argo Exp., 1876. 12. Tucacas.

Dentalium semistriatum Gould, Arango, Fauna Mal. Cuba, 1878. 233. Ca. G. 
Dentalium semistriatum Guild., Beau. 18. G.

Dentalium semistriatum Guild., Jour. de Conch., $\nabla, 151$. G.

Dentalium semistriatum Guild., Krebs. 91.

Dentalium semistriatum Guild., Poulsen. 14.

Dentalium sericatum Dall, n. s., Report Blake Moll. 37. Yucatan Strait.

Dentalium sigsbeanum Dall, n. s., Report Blake Moll. 38. Yuca$\tan$ Strait.

Dentalium texasianum Philippi, Roemer, Texas, 454, 1849. Texas.

Dentalium texasianum Phil.,* Zt. Mal., v, 144. Texas.

Diala, sp. nov., Poulsen. 8.

Diala concinna C. B. Adams, Mal. B1., xxiii, 55. J.

Diala concinna C. B. Adams, Poulsen. 8.

Dione (see Cytherea, Venus).

Dione circinata Born, Krebs. 95.

Dione circinata Born, Mal. Bl., x, 32.

Dione dioue Gray, Krebs. 96. NGr. PR.

Dione dione Linn., Mal. Bl., x, 27.

Dione dione Linn., Mal. Bl., x, 231.

Dione purpurata Lam., Jahrb., ii, 249.

Dione veneris Hanley, Mal. Bl., x, 27.

Diplodonta (see Lucina).

Diplodonta brasiliensis Phit., Guppy, Paria Fauna, 1877. 151.

Diplodonta candeana, DOrb., Guppr, Paria Fauna, 1877. 151.

Diplodonta pilula Dall, n. s., Report Blake Moll. 136. Uewre un y

Diplodonta semiaspera, Mal. Bl., x, 228. Dec.1863, name only.

Diplodonta semiaspera Phil., Arango, Fauna Mal. Cuba, 1878. 257.

Diplodonta semiaspera Phil., Krebs. 122. Ca. Ba.

Diplodonta semiaspera Phil., Poulsen. 15.

Diplodonta turgida V. \& S., Bush, Conn. Ac. vi. 479. Hatt.-

Diplodonta renezuclensis Dliro, Report Blake Moll. 136. $=p \cdot-\mathrm{h}_{\mathrm{h}} \mathrm{f}$,
Discina (see Discinisca, Orbicula).

Diseina antillarum D’Orb., Jour. de Conclí, xiv, 266.

Discina antillarum D'Orb., Jour. de Couch., xir, 273. Ca. M. J.

Discina antillarum, D'Orb., Jour. de Conch., xvii, 116.

Discina cumingii, Brod., Jahrb., ii, 254.

Discina radiata, Dkr., Jahrb., ii, 254.

Discina striata Brod., Jahrb., ii, 254.

Discina striata Sby., Mal. B1., x, 231. J.

Discina strigata Brod., Jahrb., ii, 254.

Discinisca autillarum D'Orb., Am. Jour. Conch., vii, 77. Ca. M.

Discodoris erueis Oersted, Jahrb., iv, 61.

Discodoris modesta Bergh, Jahrb., ir, 61.

Discodoris muta Bergh, Jahrb., iv, 61.

Diseodoris notha Bergh, Jahrb., iv, 61.

Dispothæa striata Say, Mal. Bl., xxiv, 102. 
Distortio (see Persona, Tritonium).

Distorsio acuta Perry, Jahrb., v, 370.

Distorsio acuta Perry, Mal. Bl., xxiv, 34.

Distorsio cancellata Roissy, Nachblt., 1877. 59.

Distorsio cancellina Desh., Marrat. Argo Exp., 1876. 12. Tucacas.

Distorsio clathrata Lam., Nachblt., 1877. 59.

Distorsio clathrata Lam., Mal. Bl., xxiv, 34.

Distorsio clathrata Lam., Poulsen. 10.

Distortio muricina Bolt., Araugo, Fauna Mal. Cuba, 1878. 213. Ca. G. Ba.

Distorsio reticulata Bolt., Mal. Bl., xxiv, 34. G.

Distorsio reticulata Mart., Mal. Bl., xxiv, 34.

Ditrupa dentalium Guppy, Geol. Mag., 1875. J.

Dolabrifera asciferus Rang, Beau. 20. Fleur-d'Epée.

Dolabrifera ascifera Rang, Jour. de Conch., xi, 23. ST.

Dolabrifera ascifera Rang, Mal. Bl., xxii, 176. ST. et al.

Dolabrifera ascifera Rang, Poulsen. 8.

Dolium antillarum Mörch, Mal. Bl., xxiv, 41. ST.

Dolium album Conrad, Proc. Acad. Nat. Sci. Phil., vii, 31, 1854.

Dolium antillarum Mörch, Poulsen. 10.

Dolium bairdii Verrill \& Smith, Verrill, List, 1854. 268.

Dolium crenulatum Phil.,* Zt. Mal., ii, 148.

Dolium galea Krebs, Mal. B1., xxiv, 42. J.

Dolium galea Linn., Am. Marine Conch. 43.

Dolium galea Linn., Arango, Fauna Mal. Cuba, 1878. 224. Ca.Td.

Ba.

Dolium galea Linn., Krebs. 35 .

Dolium minor Mörch, Nachblt., 1877. 59.

Dolium pennatum Mal. Bl., xxir, 42.

Dolium penuatum Mart., Krebs. 3ॅ. SJ. Tort. SM. SB. Bb.

Dolium pennatum Mart., Mal. Bl., xxiv, 42.

Dolium pennatum Mörch, Jahrb., ii, 265.

Dolium pennatum Mörch, Mal. Bl., xxiv, 42.

Dolium perdix D’Orb., Arango, Famua Mal. Cuba, 1878. 225. Ca. Antilles. G.

Dolium perdix D'Orb., Moll. Cuba, ii, 182.

Dolium perdix Lam., Moll. Cuba, ii, 182.

Dolium perdix Linn., Am. Marine Conch. 43. WI.

Dolium perdix Linn., Arango, Fauna Mal. Cuba, 1S78. 225. Ca. Antilles. G.

Dolium perdix Linn., Beau. 8. G.

Dolium perdix Linn., Jour, de Conch., iv, 418. G.

Dolium perdix Linn., Mal. Bl., xxiv, 42.

Dolium perdix Linn., Marrat, Argo Exp., 1876. S. Barbuda. Nassau. Dolium perdix Linn., Moll. of Bermudas. 17.

Dolium perdix Linn., var. occidentalis, Poulsen. 10. 
Dolium perdix var., Mörch, Mal. Bl., xxiv, 43. Bz.

Dolium plumatum Green, Jahrb., ii, 265.

Dolium rostratum Mart., Jahrb., v, 247.

Dolium rostratum Mart., Mal. Bl., xxiv, 33.

Dolium striatum D'Arg., Moll. Cuba, i, 131.

Donacillit rosea D'Orb., Arango, Fauna Mal. Cuba, 1878. 246. M.

Donacilla rosea D'Orb., Krebs. 105.

Donacilla rosea D'Orb., Moll. Cuba, ii, pl. xxv, figs. 39-41. 238.

Donacina brasiliensis Lam., Jahrb., ii, 249. . th f. w : a tr.

Donax angustatus Sby., Am. Jour. Conch., iii, 200. = D. forts, Ltm

Donax assimilis Hanley, Poulsen. 15. = Pan a.1. a A p i

Donax cayauensis Lam., Moll. Cuba, ii, 258. 260. $=d-86 d . t u$

Donax cacennensis D'Orb., Arango, Fauna Mal. Cuba, 1878. 247. Antilles.

Douax cayeunensis Lam., Arango, Fauna Mal. Cuba, 1878. 247. Antilles.

Donax cayenrensis Lam., Krebs. 99.

Donax creuulatus Don., Arango, Fanna Mal. Cuba, 1878. 247. Ca. Ba. = denticulatus $\mathrm{A}$

Donax denticulata Limn., Jour. de Conch., vii, 26. MG.

Donax denticulata Linn., Jour. de Conch., vii, 35, 36, 37. G.

Donax denticulata Linn., Beau. 25. Mg. G.

Donax denticulata Linn., Jour. de Conch., ii, 425. G.

Donax denticulata Linn., Krebs. 99. S.T. SB. Td. T.

; Donax denticulata Linn., Poulsen. 15.

Douax denticulatus Linn., Arango, Fauna Mal. Cuba, 1878. 247. Ca. Ba.

Donax denticulatus Liun., Marrat, Argo Exp., 1878. 13. Tucacas. Santa Marta.

Donax fabagelloides Guppy, Geol. Mag., 1874. 443. Td.

Donax fossor Say, Am. Jour. Conch., iii, 200.

Donax hanleyanus Phil., Jahrb., ii, 248. Fere

Douax martinicensis Lam., Zt. Mal., i, 88.

Donax martinicensis Lam., Arango, Fauna Mal. Cuba, 1878. 244. Ca. J. M. G.

Donax martinicensis Lam., Krebs. 99.

Donax martinicensis Lam., Krebs. 103.

Donax martinicensis Lam., Moll. Cuba, ii, 243.

Donax (Heterodonax) ovuliuus Desh., Marrat, Argo Exp., 1876. 13, Santa Marta.

$\checkmark$ Donax parrula Phil.,* Zt. Mal., v, 146. Fla. =? foaser

Donax protracta Conrad, Journ. Acad. Nat. Sci. Phil., n. s., i, 208, 280.

pl. xxxix, fig. 8, 1849. St. Joseph's Bay, Fla.

Donax pulchellis Hanl., Guppý, Paria Fauna, 1877. 148.

Donax punctatus Chemn., Arango, Fauna Mal. Cuba, 1878. 247. Ca.

Ba. $=$ denteculatices

D)

previsianded (Reel)

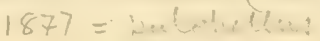


Donax roemeri Philippi, Roemer, Texas. 452. 1849. Texas.

Donax roemeri Phil., * Zt. Mal., v, 147. Texas.

Donax rugosa Chem., Moll. Cuba, ii, 260.

Donax rugosa D'Orb., Arango, Fauna Mal. Cuba, 1878. 247. Antilles.

Donax rugosa D'Orb., Krebs. 99.

Donax rugosa D’Orb., Moll. Cuba, ii, 26c.

Donax rugosa Lam., Moll. Cuba, ii, 261.

Donax rugosa Linn., Arango, Fauna Mal. Cuba, 1878. 247. Antilles.

Donax rugosa Linn., Moll. Cuba, ii, 260.

Donax striata Linn., Poulsen. 15. = africat $\%$.

Donax striatus Lin1., Guppy, Paria Fauna, 1877. 148.

Donax texasiana Phil., ${ }^{*}$ Zt. Mal., ir, 77.

Donax truncatus Da Costa, Arango, Fauna Mal. Cuba, 1878. 247. Ca.

Ba.

Donax tumidla Philippi, Roemer, Texas. 453. 1849. Texas:

Donax tumida Phil., Zt. Mal., v, 147. Texas.

Donax variabilis Say, Am. Marine Conch. 154.

. Donax variabilis Say, Dall, Hemphill's shells. 338. Sarasota Bay. Fernandina.

Donax variabilis Say, Poulsen. 15.

Doridium gemmulatum Mörch, Jour. de Conch., xi, 25. ST.

Doridium gemmatum Mörch, Mal. Bl., xxii, 175. ST.

Doriopsis krebsii Bergh, Jahrb., vi, 44. ST.

Doris (see Glaucus) Arango, Fauna Mal. Cuba, 1878. 154. Ca. Antilles.

Doris angustipes Mörch, Jour. 'de Conch., xi, 32. ST.

Doris angustipes Mörch, Mal. Bl., xxii, 177. ST.

Doris atropos Bergh,* Jahrb., vi, 49. Bz.

Doris crucis Oersted, Jour. de Couch., xi, 33. ST.

Doris crucis Oersted, Mal. Bl., xxii, 177. ST.

Doris krebsii Mörch, Jahrb., vi, 44. ST.

Doris krebsii Mörch, Jour: de Conch., xi, 34. ST.

Doris radiata Gmel., Moll. Cuba, i, 109.

Dosina concentrica Born, Krebs. 99. = Dosenter

Dosina listeri Gray, Krebs. 97. = cy huc wh

Dosina tenuis Recluz, Krebs. 99. = cir Lir arum

Dosinia concentrica Born, Arango, Fanna Mal. Cuba, 1878. 251. Ca.

Dosinia concentrica Born, Jahrb., ii, 249.

Dosinia concentrica Born, Mral. B1, x, 231.

Dosinia concentrica Born, Poulsen. 15 .

Dosinia concentrica Gmel., Beau. 24. G.

Dosinia eyclas Roemer, Am. Jour. Conch., vii, 211. Bz. Mal

Dosinia discus Rve., Am. Marine Conch. 161.

Dosinia discus Rve., Poulsen. 15.

Dosinia floridana Con., * Am. Jour. Conch. ii, pl. 15, f. 4, 280. Fla. - 
Dosinia kraussii Roemer, Mal. Bl., viii, 213.

Dosinia obovata Comt., Bush, Conn. Ac. vi, 477. Hatt.

Dosinia philippii D'Orb., Guppy, Paria Fauma, 1877. 151.

Dosinia tenuis Recluz, Arango, Fauna Mal. Cuba, 1878. 252. Ca. G.

Dosinia tenuis Rechz,* Jour. de Conch. iii, pl. x, f. 1, 1', 250. G.

Dosinia tenuis Recluz, Jonr. de Conch., r. 155. G.

Dosinia tenuis Recluz, Mal. B1., ir, 27. G.

Dosinia tenuis Recluz, Poulsen. 15.

Draconis pileus Mart., Mal. Bl., xxiv, 100.

Dreissena domingensis Recl., Jour. de Conch. iii, pl. x, f. 8 255. SD.

Dreissena gundlachi Dkr., Arango, Fauna Mal. Cuba, 1878. 265. Ca.

Dreissena leucophæata Conrad, Am. Marine Conch. 190.

Dreissena leucophæatus Conrad, Proc. Acad. Nat. Sci. Phil., vi, 320, 1853. Va.

Dreissena pfeifieri Dlir., Arango, Fauna Mal. Cuba, 1878. 265. Ca.

Dreissena riisei Krebs. 130. PPI.

Drillia (see Pleurotoma).

Drillia affinis Gray, Marrat, Argo Exp., 1876. 12. Tucacas.

Drillia albomaculata D'Orb., Dall, Hemphill's shells. 328. Sarasota Bay.

Drillia elozantha Rav., Am. Marine Conch. 48. SC.

Drillia (Crassispira) fucata Rve., Marrat, Argo Exp., 1876. 16. Nassau. Athol Island.

Drillia leneocyma Dall, n. s., Hemphill's shells. 328. Key West.

Drillia limonitella Dall, n. s., Hemphill's shells. 329. Cedar Keys. Drillia ostrearum Stearns, Dall, Hemphill's shells. 328. Key West. Drillia ostrearum Stearns, Proc. B. S. N. H., xv, 22, 1872. Tampa. W. Fla.

Drillia pallida Sby., Marrat, Argo Exp., 1S76. 12. Tucacas.

Drillia thea Dall, n. s., Hemphill's shells. 328. Sarasota Bay. Drillia vexillum Rve. ?, Marratt, Argo Exp., 1876. 8. Barbuda. Dunkeria (see Rissoa).

Dunkeria canceliata D'Orb., Mal. Bl., xxii, 168. Ca. ST.

Dunkeria cancellata D'Orb., Poulsen. 8.

Dunkeria faleifera Watson, Lin. Soe. Journ., 18\&0, xv, 250. Ber.

Dunkeria gemmulosa C. B. Adams, Mal. Bl., xxii, 168. J. Fla.

Dunkeria gemmulosa C. B. Adams, Poulsen. 8.

Dunkeria suturalis Gld., Otia. 237. SC.

Eburna see Ancillaria).

Eburna flavida Schum., Jahrb., vii, 106.

Eburna glabrata Lam., Jahrb., vii, 106.

Eburna glabrata Lam., Moll Cuba, ii, 113.

Eburna glabrata Sby., Moll. Cuba, ii, 113.

Eburnea balteata Sby., Krebs. 36.

Eburnea glabrata Lam., Krebs. 36.

Eburnea tankervillii Sw., Krebs. 36. 
Ellobium barbadense Bolt., Am. Jour. Conch., iv, 17.

Elusa erythrosclera Mörch, Mal. Bl., xxii, 160. ST.

Elusa krebsii- Mörch, Mal. Bl., xxii, 159. ST.

Elyria crispata Oersted, Jour. de Conch., xi., 40. SCx.

Elyria schiadura Oersted, Jour. de Conch, xi, 40. SCx.

Elysia crispata Oersted, Mal. Bl., xxii, 180.

Emarginula clausa D'Orb., Arango, Fama Mal. Cuba, 1878. 229. Ca.

Emarginula clausa D’Orb., Jour. de Conch., v, 151. G.

Emarginula clausa D'Orb., Moll. Cuba, ii, 1)]. xxir, figs. 34-36. 194.

Emarginula depressa Blv., Jour. de Conch., v, 151. G.

Emarginula depressa Blv., Krebs. 86.

Emarginula (Subemarginula) depressa Blv., Marrat, Argo Exp., 1876.

6. Aa. SV. Long Key Island. Abaco.

Emarginula laqueare Gray, Arango, Fauna Mal. Cuba, 1878. 229. Ca.

Emargiuula listeri Ant., Arango, Fauna Mal. Cuba, 1s7s. 229. Ca.

Emarginula listeri Ant., Krebs. 86.

Emarginula notata Linn., Krebs. . 85.

Emarginula octoradiata Gmel., Arango, Famna Mal. Cuba, 1878. 229 Ca.

Emarginula octoradiata Gmel., Krebs. 86.

Emarginula (Subemarginula) octoradiata Gmel., Marrat, Argo Exp., 1876. 13. Santa Marta.

Emarginula octoradiata Gmel. c. v. octocostata Wagu., Poulsen. 13.

Emarginula (Subemarginula) polygonata Ad., MIarrat, Argo Exp., 1876.

18. Abaco.

Emarginula rollandi Fisch., Report Blake Aloll. 7 .

Emarginula rollandii Fisch., Beau. 19. G.

Emarginula rollandii Fisch., Jour. de Conch., r, pl. xii, fig. 10. 356. G.

Emarginula rollandii Fisch., Krebs. 86.

Emarginula rollandii Fisch., Poulsen. 13.

Emarginula tricarinata Sby., Arango, Fauna Mal. Cuba, 1878. 229. Ca.

Emarginula tricarinata Sby., Krebs. 86.

Emarginula (see also Subemarginula and Zeidora).

Enæta guildingi H. \& A. Ads., Jour. de Conch., xir, 115. SV.

Enæta guildingi Sby., Jahırb., vi, 178.

Engina sehrammi Crosse,* Jour. de Conch., xi, pl. i, fig. 7. 82. G.

Ensina ferruginosa Rve., Poulsen. 12.

Ensina granata Koch, Poulsen. 12.

Ensina rosea Rve., Poulsen. 12.

Ensina turbinella Kien., Poulsen. 12.

Ensis (see Solen).

Ensis americanus Gld., Am. Marine Conch. 142.

Epidromus (see Triton).

Epidromus (see Tritonium), Marrat, Argo Exp., 1876. 16.

Epidromus arachnoides Klein, Mal. Bl., xxiv, 25.

Epidromus canadensis Klein, Mal. I3l., xxir, 23. 
Episcynia inornata D'Orb., Mal. Bl., xxii, 155. ST.

Epitonium (see Turritella).

Epitoniun marmoreum Bolt., Mal. Bl., xxiii, 127.

Epitonium squamatum Bolt., Mal. Bl., xxiii, 128.

$\checkmark$ Erato eypræoides U. B. Arlams, Krebs' Remarks. 395 . J.

Erato eypræoides C. B. Adams, Mal. Bl., xxiv, 51.

Erato cypræoides Krebs, Mal. Bl, xxiv, 51.

Erato cypræoides C. B. Adlams, Synops., i, 1845. J.

Erato hæmatita Rve, Jahrb., vii. 46. D. = marginella

Erato hematina Rve., Am. Jour. Conch., ii, 160. PR.

Erato maugerix Gray, Arango, Fauna Mal. Cuba, 1878. 186. Ca.

Erato mangeriæ Gray, Beau. 7. G.

Erato maugeriæ Gray, Jahrb., vii. 107.

Erato maugeriæ Gray, Jour. de Conch., ii, 56.

Erato mangeriæ Gray, Krebs. 42. S'T. SB.

Erato mangeriæ Gray, Mal. Bl., xxiv, 51. ST. G.

Erato maugerix Gray, Poulsen. 10.

Erato vitellinus Hiurls, Marrat, Argo Exp., 1876. 19. Abaco-?-

Ervilia (see Corbula).

Ervilia concentrica Gld., $\Lambda$ m. Marine Conch. 156. NC.

Frvilia concentrica Gld., Otia. 239. NC.

Ervilia nitens Jonas, Poulsen. 15.

Ervilia nitens MIont., Marrat, Argo Exp., 1876. 8. Barbuda.

Ervilia nitens Turton, Beau. 25. G.

Ervilia nitens Turton, Jour. de Conch., ii, 425. G.

Ervilia nitens Turton, Krebs. 110.

Erycina deshayesi Recluz, Moll. Cuba, ii, 302.

Eryeina donacina Recluz, Moll. Cuba, ii, 302.

Erycina longicallis Phil., Report Blake Moll. 133.

Ethalia anomala D'Orb., Report Blake Moll. 52. Yucatan Strait. Lthalia multistriata Verrill, List., 1884. 270. Cape Hatteras.

Ethalia semistriata D'Orb., Poulsen. 13.

Eucharis (see Corbula).

Eucharis elliptica Recl., Beau. 26. SM.

Eucharis elliptica Recl., Jour. de Conch., i, 168. G.

Eucharis elliptica Recl., Jour. de Conch., ii, 424. G.

Eucharis elliptica Recl., Jour. de Conch., viii, 26. G.

Eucharis quadrata Hds., Jour. de Conch., viii, 26. G.

Eucharis quadrata Hds., Poulsen. 15.

Eucharis quadrata Recl., Beau. 26. SM.

Eucharis quadrata Recl., Jour. de Conch., i, 168. G.

Encharis quadrata Recl., Jour. de Conch., ii, 424. G.

Eucharis quadrata Recl., Krebs. 111.

Euciroa elegantissima Dall, Report Blake Moll. 106.

Furdesia floridana Pourtalès, Report Blake Moll. 103.

Ėulima (see Rissoina) Arango, Fauna Mal. Cuba, 1878. 168. J. 
Eulima affinis C. B. Adams, Contr. to Conch. 110. J.

Eulima affinis Ad., Krebs. 73.

Eulima affinis C. B. Adams, Mal. Bl., xxii, 183. J.

Eulima arcuata Ad., Arango, Fauna Mal. Cuba, 1878. 163. Ca. Ba. J.

Eulima arcuata C. B. Adams, Contr. to Conch. 110. J.

Eulima arcuata Ad., Krebs. 73.

Eulima areuata C. B. Adams, Krebs' Remarks. 395.

Eulima arcuata C. B. Adams, ${ }^{*}$ Mal. Bl., xxii, 182. J.

Eulima bicarinata D'Orb., Beau. 17. G.

Eulima bifasciata D'Orb., Arango, Fauna Mal. Cuba, 1878. 163. G. ST.

Eulima bifasciata D'Orb., Beau. 17. G.

Eulima bifasciata D'Orb., Krebs. 74.

Eulima bifasciata D'Orb., Mal. Bl., xxii, 181. G.

Eulima bifasciata D'Orb., Moll. Cuba, i, pl. xvi, figs. 1-3, 216.

Eulima bifasciata D'Orb., Arango, Fauna Mal. Cuba, 1878. 163. G. ST. Eulima breviuscula Dkr., Jahrb., ii, 243.

Eulima chascanon Watson, Lin. Soc. Jonrn., 1883, xrii, 114. Cul.

Eulima channax Watson, Lin. Soc. Journ., 1883, xvii, 114. Cul.

Eulima chydæa Watson, Lin. Soc. Journ., 1883, xvii, 124. Cul.

Eulima conica C. B. Adams, Contr. to Conch. 110. J.

Eulima conica Ad., Krebs. 74.

Eulima conica C. B. Adams, ${ }^{*}$ Mal. Bl., xxii, 183. J.

Eulima conoidea Kurtz \& Stm., Am. Marine Conch. 70.

Eulima conoidea Kurtz \& Stm., Dall, Hemphill's shells. 330. Cedar Keys.

Eulima conoidea Kurtz \& Stm., Kurtz. 8. N. and SC.

Eulima conoidea Kurtz \& Stm., Proc. Bost. Soc. Nat. Hist., iv, 115, 1851. NC. SC.

Eulima cylindrata Watson, Lin. Soc. Journ., 1883, xvii, 125. Cul.

Eulima eburnea Mühlf., Poulsen. 8.

Eulima eburnea Mühlf., Mal. Bl., xxii, 181. ST.

Eulima fasciata Watson, Lin. Sor. Journ., 1883, xvii, 113. Cul.

Eulima fulvocineta Ad., Araugo, Fauna Mal. Cuba, 1878. 163. G. ST.

Eulima fulvorincta C. B. Adams, Contr. to Conch. 111. J.

Eulima fulvocincta C. B. Adams, Krebs. 74.

Eulima fulvocincta C. B. Adams, Mal. Bl., xxii, 180. J.

Eulima gomphus Watson, Lin. Soc. Jour., 1883, xvii, 125. Cul.

Eulima gracilis C. B. Adams, Contr. to Conch. 110. J.

Eulima gracilis Ad., Krebs. 74.

Eulima gracilis O. B. Adams,* Mal. Bl., xxii, 182。J.

Eulima gracillima Sby., Am. Jour. Conch., ii, 286. Gtm.

Eulima gracillima Sby.,* Mal. Bl., xxii, 182. Gtm.

Eulima guildingii A. Ad., ${ }^{*}$ Mal. Bl., xxii, 182。 SV.

Bull. 24-_ 8 
Eulima (Leiostraca ?) hemphillii Dall, w. s., Dall, Hemphill's shells. 330. Cedar Keys.

Eulima hians Watson, Watson. Lin. Soc. Jour., 1883, srii, 115. Cul. Eulima hyalina Watson, Watson. Lin. Soc. Jour., 1883, xvii, 126. Cul. Eulima incerta D'Orb., Jour. de Conch., xxiv, 373. Ca.

Eulima incerta D'Orb., Krebs. 54.

Eulima incerta D'Orb., Krebs. 74.

Enlima incerta D'Orb., Krebs' Remarks. 397.

Eulima incerta D'Orb., Mal. Bl., xxiii, 58. J.

Eulima incerta D'Orb., Moll. Cuba, i, pl. xvi, figs. 7-9, 218.

Eulima jamaicensis Ad., Araugo, Fauma Mal. Cuba, 1878. 163. C. Ba. J.

Eulima jamaicensis Ad., Krebs. 74. SM. SB. ST. SJ.

Eulima jamaicensis C. B. Adams, Mal. Bl., xxii, 181. J.

Eulima jamaicensis C. B. Adams, Poulsen. 8.

Eulima jamaicensis C. B. Adams, Synops., 6. 1845. J.

Eulima nutaus Mühlf.,* Mal. BI., xxii, 182. ST.

Enlima oleacea Kurtz \& Stm., Am. Marine Conch. 69. NO.

Eulima oleacea Kurtz \& Stm., Kurtz. 8. NO. SO.

Enlima onychina Folin, Mal. Bl., xxii, 181. G.

Eulima psila Watson, Lin. Soc. Jour., 1883, xvii, 112. Cul.

Lulima subcarinata D'Orb., Arango, Famua Mal. Cuba, 1878. 163. G. ST.

Tulima subcarinata D'Orb., Krebs. 74.

Wulima subcarinata D'Orb., Mal. Bl., xxii, 181. G.

Q

Eulima vitrea (?) Ad., Arango, Fauna Mal. Cuba, 1878. 163. G. ST.

Fulimella (Menestho?) lissa Verrill, List, 1884. 272. Cape Hatteras.

Euomphalus bisulcatum Sby. (?) Krebs. 62.

Eupleura tampaensis Con., Am. Jour. Conch., v, 106. Fla.

Ertpleura caudata Say, Dall, Hemphill's shells. 326. Cedar Keys.

Euryta (see Acus), Marrat, Argo Ixp., 1876. 10.

Eutropia (Tricolia) sp? Marrat, Argo Exp., 1876. 11. La Guayra.

Wxogyra angustata Lam., Jahrb., ii, 253.

Fasciolaria aurantiaca Lam., Jahrb., ii, 363. Bz.

Fasciolaria concolor Chem., Jahrb., ii, 362.

Fasciolaria crocata Phil., Jahrb., ii, 363.

Tasciolaria crocata Phil., ${ }^{*}$ Zt. Mal., r, 25. Y.

Fasciolaria disloeatus Lam., Conral, Am. Jour. Sci. n. s., ii, 397, 1846. Fla.

Fasciolaria distans Lam., Am. Marine Conch 21.

Fasciolaria distans Lam., Jabrb. ii, 362.

Fasciolaria distans Lam., Moll. of Bermudas. 16.

Fasciolaria distans Lam., Poulsen. 11.

Fisciolaria gigantea D'Orb., Arango, Fauna Mal. Cuba, 1878. 216. Ca. Ba. 
Fasciolaria gigantea D'Orb., Krebs. 15.

Fasciolaria gigantea D'Orb., Moll. Cuba, ii, 169.

Fasciolaria gigantea Kieu., Am. Mariue Conch.

20.

Fasciolaria purpurea Jonas, Jahrb., ii, 363.

Fasciolaria rugosa Dkr., Jahrb., ii, 362.

Fasciolaria textilis Guppy, n. s., Geol. Mag., 1874. 440. J.

Fasciolaria trapezium Lam, Courad., Am. Jour. Sci. n. s., ii, 397, 1846. Fla.

Fasciolaria tulipa var. Dkr., Arango, Fanna Mal. Cuba, 1878. 217. Ca. G. M. ST. Ba.

Fasciolaria tulipa Lam., Krelos. 15. NGr. ST. SJ. Tort. Td. SM.

Fasciolaria tulipa Lam., Moll. Cuba, ii, 168.

Fasciolaria tulipa Limn., Am. Marine Conch. 21.

Fasciolaria tulipa Linn., Alango, Fauna Mal. Cuba, 1878. 217. Ca.G. M. ST. Ba.

Fasciolaria tulipa Lim., Beau. 9. Guadeloupe. Les Saintes. MG. SM. Fasciolaria tulipa Linn., Jahrb. ii, 362.

Fasciolaria tulipa Linn., Jour. de Conch, ii, 428. Gr.

Fasciolaria tulipa Lim., Marrat, Argo Exp. 1876. 16. Nassau.

Fasciolaria tulipa Linn., Poulsen. 11.

Fasciolaria tuomeyi Uolmes, Pleiocene fossils S. C., pl. xxx, fig. 10. 152.

Fastigiella poulsenii Mörch, Poulsen. 9.

Fastigiella poulseni Mörch, *Jour. de Conch., xxv, 207. Eleuthera. Ba.

Fastigiella poulseni Mörch, Mal. Bl. xxiv, 141. Ba.

Fenella dubia D'Orb., Mal. Bl., xxiii, 56. J. G.

Fenella dubia D'Orb., Poulsen. 8.

Fenella elougata Watson, Lin. Soc. Jouru., 1850, xv. 249. Cul.

Ficula gracilis Petit, Mal. Bl., xxir. 43. Fla.

Ficula gracilis Phil., Jahrb., ii, 359.

Ficula gracilis Phil.,* Zt., Mal. v, 97. Fla. Y.

Ficula gracilis Sby., Mal. Bl., xxiv, 43. Fla.

Ficus cancellata Klein, Mal. Bl., xxiv, 44.

Ficus gracilis Sby., Jour. de Conch., iii. 149.

Ficus papyraceis Say, Am. Marine Conch., 21.

Ficus pellucidus Desh., Jour. de Conch. v, pl. 6, f. 1. 2, 184. 1856.

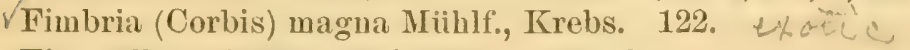

Fissurella agis Rre., Mal. Bl., iv, 27. G.

Fissurella ægis Rve, Jour. de Conch., iv, 416. G.

Fissurella agis Petit, Jour. de Conch., v, 155. G.

Fissurella albastrites Rve, Zt. Mal., x, 79. SV.

Fissurella alteruata Say, Am. Marine Conch. 91.

Fissurella alteruata Say, Beau. 19. G.

Fissurella alternata siy, Dall., Hemphill's shells. 336. Cedar Keys. Fissurella alternata Say, Jour. de Conch., v, 151. G.

Fissurella alternata Say, Jour. de Conch., v, 155. G.

Fissurella alternata Say, Jour. de Conch., r, 235. 
Fissurella alternata Say, Krebs. 86.

Fissurella alternata Say, Mal. Bl., iv, 27. G.

Fissurella antillarum D'Orb., Arango, Fauna Mal. Cuba, 1878. 227. Ca.

Fissurella antillarum D'Orb., Krebs. 86.

Fissurella antillarum D'Orb., Moll. Cuba, ii, pl. xxiv, figs. 40-42. 198.

Fissurella arcuata Sby., Poulsen. 14.

Fissurella atricapilla Dillw., Krebs. 86.

Fissurella atricapilla Dillw.=F. viridula Lam., Poulsen. 13.

Fissurella balanoides Rve., Beau. 19. G.

Fissurella balanoides Rve., Jour. de Conch., $\nabla, 151$. G.

Fissurella balanoides Rve., Krebs. 88 .

Fissurella barbadensis Lam., Jour. de Conch. vi, 119. Bb.

Fissurella barbadensis Lam., Krebs. 88.

Fissurella barbadeusis D'Orb., Arango, Fauna Mal. Cuba, 1878. 227. Ua. G.

Fissurella barbadensis Gmel., Arango, Fauna Mal. Cuba, 1878. 227. Ca. G.

Fissurella barbadensis Gmel., Beau. 19. G.

Fissurella barbadensis Gmel., Jahrb., ii, 246.

Fissurella barbadensis var. Gmel., Jour, de Conch ii, 426. G.

Fissurella barbadensis Gmel., Jour. de Conch., v, 235.

Fissurella barbadeusis Gmel, Krebs. 86. Ag. ST. SCx. PPl. J. SM. Fissurella barbadensis Gmel., c. v., Poulsen. 13.

Fissurella barbadensis Lam., Jour. de Conch., v, 151. G.

Fissurella barbadensis Lam., Moll. of Bermudas. 21.

Fissurella barbadensis Lam., Moll. Cuba, ii, 196.

Fissurella bicolor jun., C. B. Adams, Beat. 19. G.

Fissurella cancellata Sol., Arango, Fauna Mal. Cuba, 1878. 228. Ca. Ba.

Fissurella cancellata Sol., Beau. 19. G.

Fissurella cancellata Sol., Krebs. 86. ST. SM. SB.

Fissurella cancellata Sol., Poulsen, 13.

Fissurella cayennensis Lam., Beau. 19. G.

Fissurella cayeunensis var. Lam., Jour. de Conch. ii, 426. G.

Fissurella eayenuensis Lam., Poulsen. 13.

Fissurella (Cremides) clathrata Rve., Marrat, Argo Exp., 1876. 18. Abaco.

Fissurella (Cremides) clathratula Rve., Marrat, Argo Exp., 1876. 6. Aa. Fissurella costaria Desh., Pfeiffer, Archiv. für naturg., 1840. 259. Ca. Fissurella dysonii Rve., Krebs. 87.

Fissurella (Lucapina) dysoni Rve., Marratt, Argo Exp., 1876. 6. Aa. Fissurella dysonii Rve., Beau. 19. G.

Fissurella dysonii Rve., Jour. de Conch., v, 235.

Fissurella elevata Dkr., Arango, Fauna Mal. Cuba, 1878. 228. Ca.

Fissurella elongata Ad., Krebs. 87. 
Fissurella elongata (?) Phil., Arango, Fauma Mal. Cuba, 1878. 228. Ca. G.

Fissurella elongata Phil., Poulsen. 13.

Fissurella elongata Rve., Beau. 19. G.

Fissurella elongata Rve., Jour. de Conch., v, 151. G.

Fissurella elongata Rve., Jour. de Conch., $\nabla, 155$. G.

Fissurella elongata Rve., Jour. de Conch., v, 234.

Fissurella elongata Rve., Krebs. 87. ST. SM. SB.

Fissurella elongata Rre., Mal. Bl., iv, 27. G.

Fissurella elongata C. B. Adams, Synops. 8. 1845. J.

Fissurella fascicularis Lam., Arango, Fauna Mal. Cuba, 1878. 228 Ca.

Fissurella fascicularis Lam., Krebs, 87.

Fissurella fascicularis Lam., Poulsen. 14.

Fissurella firmata Rve., Moll. of Bermudas. 21.

Fissurella gemmata Rve., Poulsen. 14.

Fissurella gemmulata Rve., Arango, Fauna Mal. Cuba, 1878. 228. Ca. G. Ba.

Fissurella gemmulata Rve., Beau. 19. G.

Fissurella gemmulata Rve., Jour. de Conch., v, 151. G.

Fissurella gemmulata Rve., Jour. de Conch., v, 235.

Fissurella gemmulata Rre., Krebs, p. 87. ST. SCx. SB. Ag. PPl. SM. Fissurella gibba Phil., Arango, Fama Mal. Cuba, 1878. 228. Ca.

Fissurella gibberula Lam., Mal. Bl., iv, 27. G.

Fissurella gibberula? Lam., Jour. Conch., iv, 416. G.

Fissurella gibberula Petit, Jour. de Conch., v, 155. G.

Fissurella græca Linn., Moll. Cuba, ii, 197.

Fissurella græeca Lam., Pfeiffer, Archiv für naturg. 1840. 259. Ca. Fissurella græca Linn., Krebs. 87. ST. SJ. SM. PPI.

Fissurella hondurasensis Rve., Marrat, Argo. Exp., 1876. 15. $\nabla C z$. Nassau.

Fissurella jamaicensis Gmel., Beau. 19. G.

Fissurella jamaicensis Gmel., Krebs. 87.

Fissurella larva Rve, Beau. 19. G.

Fissurella larva Rve., Jour. de Conch., iv, 416. G.

Fissurella larva Rve, Krebs. 87.

Fissurella listeri D'Orb., Arango, Fauna Mal. Cuba, 1878. 228 Ca.

Fissurella listeri D'Orb., Beau. 19. G.

Fissurella listeri D'Orb., Jour. de Conch., ii, 426.

Fissurella listeri D'Orb., Krebs. 87.

Fissurella (Lucapina) listeri D'Orb., Marrat, Argo Exp., 1876. 7. Bd. Fissurella listeri D'Orb., Moll. Cuba, ii, pl. xxiv, figs. 37-39. 197.

Fissurella listeri D'Orb., Poulsen. 13.

Fissurella minuta Lam., Arango, Fauna Mal. Cuba, 1878. 228. Ca. G. Ba.

Fissurella minuta Sby., Beau., 19. G.

Fissurella minuta Sby., Krebs. 87. 
Fissurella mus ? Rve., Jour. de Conch., iv, 416. G.

Fissurella nimbosa Lam., Krebs. 87. NGr.

Fissurella nimbosa Lam., Mal. Bl., xii, 127.

Fissurella nimbosa Lam., Moll. Cuba, ii, 195.

Fissurella nimbosa Lam., Poulsen. 13.

Fissurella nimbosa Linn., Beau. 19. G.

Fissurella nimbosa Linn., Jour. de Conch., ii, 426. G.

Fissurella nodosa Born, Arango, Fanna Mal. Cuba, 1878. 228. Ca.

Havana. Ba.

Fissurella nodosa Born, Beau. 19. G.

Fissurella nodosa Boru, Dall, Hewphill's shells. 336. Key West.

Fissurella nodosa Born, Jour. de Conch., ii, 426. G.

Fissurella nodosa Born, Krebs. 87. S'T. SCx. PR. PPl.

Fissurella (Cremides) nodosa Born, Marrat, Argo Exp., 1876. 6. Aa. Abaco.

Fissurella nodosa Born, Poulsen. 13.

Fissurella nodosa Lam., Moll. Cuba, ii, 197.

Fissurella pustula Linn., Jour. de Conch., vi, 118, 119.

Fissurella pustula Chem., Arango, Fauna Mal. Cuba, 1878. 229. Ca. Fla. G. Ba.

Fissurella pustula D'Orb., Moll. Cuba, ii, 195.

Fissurella pustula Lam., Jour. de Conch, iv, 416. G.

Fissurella pustula Linn., Beau. 19. G.

Fissurella pustula Limn., Krebs. S8. ST. SCx. SM. Ag. PPl.

Fissurella pustula Linn., Poulsen. 14.

Fissurella radiata Lam., Beau. 19. G.

Fissurella radiata Lam., Guppr, Paria Fauna, 1877. 146.

Fissurella radiata Lam., Jour. de Conch., v, 151. G.

Fissurella radiata Lam., Krebs. 86.

Fissurella radiata Lam., Krebs. 88:

Fissurella reticulata Say, Krebs. 88.

Fissurella riiseana Dkr., Arango, Fauna Mal. Cuba, 1878. 229. Ca.

Fissurella rosea Lam., Krebs. 88.

Fissurella rosea Lam., Moll. Cuba, ii, 196.

Fissurella (Cremides) rugosa Sby., Marrat, Argo Exp., 1876. 6. Aa. Abaco.

Fissurella rüppellii Sby., Poulsen. 14.

Fissurella sayennensis Iam., (=eayennensis ?) Krebs. 87.

Fissurella sehrammii Fisch., * Jour. de Conch. vi, pl. xi, figs. 5, 6, 383. G.

Fissurella sowerbyi Guild., Beau. 19. G.

Fissurella sowerbyi Guild., Krebs. 86.

Fissurella spinosa Gmel., Beau. 19. G.

Fissurella spinosa Gmel., Krebs. 87.

Fissurella tanneri Verrill, List, 1884. 271. Hatt.

Fissurella viridula Lam., Beau. 19. G. 
Fissurella viridula Lam., Jour. de Conch., iv, 416. G.

Fissurella viridula Lam., Krebs. 86.

Fissurella viridula Lam., Krebs. 88.

Fistulana rupestris Bose, Arango, Fama Mal. Cuba, 1878. 237. Ca. G.

Fistulana rupestris Bosc, Krebs. 114.

Fluxina brunnea Dall, n. S., Report Blake Moll. 52. WI.

Foraminella sowerbyi Guill., Arango, Fauna Mal. Cuba, 1878. 228. Ca. Ba.

Fossar (Narica) sulcata D'Urb., Beau. 13. G.

Fossar sulcatus D'Orb., Beau. 13. G.

Fossarus anomalus C. B. Adams, Jour. de Conch., xii, 256. J.

Fossarus anomalus Ad., Mal. Bl., xxiv, 96. J.

Fossarus fischcri Mörch,* Mal. Bl., xxiv, 96. S'T.

Fossarus fischeri Mörch, Poulsen. 10.

Fossarus orbignyi Fisch., Jour. de Conch., xii, 250. St. Lucia. Ca. G. J. Fossarus orbignyi Fiseh., Mal. Bl., xxir, 95. G.

Fossarus orbignyi Fisch., Ponlsen. 10.

Fossarus sulcatus D’Orb., Jour, de Conch., v, 152. G.

Fossarus sulcatus D'Orb., Krebs. 58.

Fossarus sulcatus Fisch., Jour, dle Conch., xii, 256. J.

Fossarus sulcatus Fisch., Mal. B1., xxir, 95. G.

Fragum (see Hemieardia) Marrat, Argo Exp., 1876. 13.

Fragum medium Bolt., Krebs. 116.

Fulgur (see Busycon).

Fulgur coarctatus Sby., Am. Jour. Conch., iii, 143. Fla.

Fulgur coarctatus Sby., Ain. Jour. Conch., iii, 146. Fla.

Fulgur gibbosum Conrat, Proc. Acad. Nat. Sei. Phil., vi, 319. 1853.

Campeachy.

Fulgur perversus Liuu., Am. Jonr. Conch., iii, 143, 144. Fla. Y.

Fulgur perversus Linn., Am. Jour. Conch., iii, 146.

Fulgur pyruloides Say, Am. Jour. Conch., iii, 150.

Fulgur pyruloides Say, Jour. de Conch., iii, 145. Fla.

Fulgur pyrum Dillmyn, Comad, Proc. Acad. Nat. Sci. Phil., vi, 319 . 1853. South Fla.

Fulvia (see Papyridea), Marrat, Argo 1Exp., 1876, 17.

Fusus (see Hemifusus, Pisania, Tritonidea).

Fusus ansatus Gmel., Krebs. 15.

Fusus articulatus Lam., Arango, Fauna Mal. Cuba, 1878. 204. Сa.

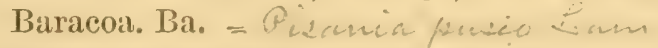

Fusus articulatus Lam., Jahrb., ix, 19.

Fusus articulatus Lam., Jour. de Conch., ii, 429. G

Fusus articulatus Lam., Krebs. 31.

Fusus articulatus Lam., Moll. Cuba, ii, 146.

Fusus bullata Holm., n. s., Post Pleiocene Fossils S. C., pl. xi, figs. 10, 10a. 69. 
Fusus cinereus Say, Am. Jour. Conch., i, 58. Fla.

Fusus closter Phil., Jahrb., ix, 9.

Fusus conus Holm., n. s., Post Pleiocene Fossils, S. C., pl. xi, figs. 7, 7a, 8, 8a. 69.

Fusus corona Lam., Jour. de Conch., iii, 64.

Fusus corona Lam., Jour. de Conch., iii, 65.

Fusus corona Lam., Mal. Bl., iii, 190.

Fusus coronatus Lam., Arango, Fauna Mal. Cuba, 1878. 216. Ca. M. Fusus coronatus Lam., Jour. de Conch., ii, 428. G. Fusus coronatus Lam., Krebs. 18.

Fusus coronatus Lam., Moll. Cuba, ii, 166.

Fusus couei Petit, Jahrb., ix, 9.

Fusus conei Petit, Jour. de Conch., iv, pl. viii, fig. 1. 249.

Fusus cutaceus Linn., Krebs. 24. = Tiesi.

- Tusus distans Lam., Krebs.* 15.

Thusus perrugatus Conrad,* Am. Journ. Sci., n. s., ii, 397, 1846. Manatee R., Fla.

Fusus filiformis Holm., n. s., Post Pleiocene Fossils S. C., pl, xi, fig 9. 69.

Fusus floridanus Con., Poulsen. 11.

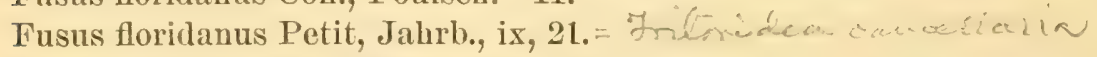

Fusus gradatus Rve., Krebs. 15. ST.

Fusus hartvigi Shuttlew., Mal. Bl., iv, 28. ST. - wacew

Fusus hartvigii Shuttlew., Jahrb., ix, 11.

Fusus hartvigii Shuttlew., Jour. de Conch., v, 171, Tortugas. ST.

Fusus lamellosus (Trophon?), Arango, Fauna Mal. Cuba, 1878. 215. Ca. Fusus lineatus Chem., Krebs. 15. = matritim

Fusus lineatus Chem. = F. ansatus Gmel., Poulsen. 11.

Jusus maroccanus Chem., Jahrb., is, 17.

Fusus minor Holm., n. s., Post Pleiocene Fossils S. C., pl. xi, figs, 6, 6a. 68.

Fusus morio var. Brug., Jour. de Conch., ii, 429. G.

Fusus morio Brug., Moll. Cuba, ii, 166.

Fusus morio D’Orb., Arango, Fauna Mal. Cuba, 1878. 216. Ca. M.

Fusus morio D'Orb., Moll. Cuba, ii, 166.

Fusus morio Lam., Jour. de ('onch., vii, 60.

Fusus morio Lam., Moll. Cub.ı, ii, 166.

Fusus morio Linn., Arango, Famna Mal. Cuba, 1878. 216. Ca. M.

Fusus multangulus Phil., Jahrb., ix, 17.

Fusus multangulus Phil., Zt. Mal., v, 25. Y.

Fusus muricoides Ad., Krebs. 15.

Fusus muricoides C. B. Adams, Krebs' Remarks. 396.

Fusus muricoides C. B. Adams, Synops., 3, 1845. J.

Fusus nitens C. B. Adams, Contr. to Conch. 60. J.

Fusus nitens Ad., Krebs. 15.

Fusus nitens C. B. Adams, Krebs' Remarks. $396 . \quad J$. 
Fusus paeteli Dkr., Jahrb., ix, 11. =

* Fusus petitii Dkr. Poulsen. 11.

Fisus plumatus Gmel., Jour. de Conch., $\mathrm{ii}, 429$. G. C wertur

Fusus pulchellus Pfeiffer, Archir fïir naturg. 1840. 258. Ca.

Fusus pusillus Pfeiffer, Archiv fiir naturg. 1840. 258. Ca.

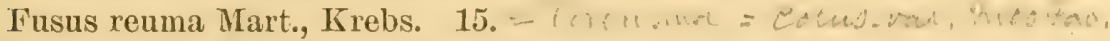

Fusus rudis Holm., 11. s., Post-Pleiocene Fossils S. C., pl. xi, figs. 11,

11a. 70 .

Fusus schrammi Crosse, Jahrb., ix, 14.

Fusus schrammi Crosse, *Jour. de Conch. xiii, pl. i, fig. 9. 31. G.

Fusus sinistralis Lam., Jahrb., ix, 17.

Fusus tenuiliratus Dkr., Poulsen. 11.

Gadinia afra Gray, Guppy, Geol. Mag., 1874. 449. Td.

Gadinia carinata Dall, *Am. Jour. Conch. vi, pl. iv, figs. 12, 13. 18. A.

Gadinia mammillaris Linn., Moll. of Bermudas. 21.

$\sqrt{ }$ Gafrarium magnum Bolt., Krebs. 122. Gyatic.

Galea striata Klein, Mal. Bl., xxiv, 42.

Galea striata Klein, Moll. Cuba, ii, 128.

Galerus (sce. Infundibulum, Trochita).

Galerus candeanus D'Orb., Dall, Hemphill's shells. 335. Sarasota

Island. Ca.

Galerus candeanus D'Orb., Mal. Bl. xxiv, 106. Ca.

Galerus candeanus D'Orb., Poulseu. 10.

Galerus parvulus Dkr., Jahrb., ii, 244.

Galerus parvulus Dkr., Mal. Bl., xxir, 107.

Gastrochaema (see Rocellaria) Arango, Fauna Mal. Cuba, 1878. 237.

Ca. G.

Gastrochaena (see Spengleria) Arango, Fauna Mal. Cuba, 1878. 237. Ca. ST.

Gastrochaena americaua Stimpson, n. s., Kurtz. 3. SC.

Gastrochaena callosa Phil., Arango, Fauna Mal. Cuba, 1878. 237. Ca. S'T.

Gastrochaena callosa Phil., Krebs. 114.

Gastrorhaena chemnitziana D'Orb., Arango, Fauna Mal. Cuba, 1878. 237. Ca. ST.

Gastrochaena chemnitziana D'Orb., Beau. 27. G.

Gastrochaena chemnitziana D'Orb., Krebs. 115.

Gastrochaena chemnitziana D'Orb., Moll. Cuba, ii, pl. xxv, figs. 29-30. 229.

Gastrochaena cuneata Rve., Poulsen. 14.

Gastrochaena cuneiformis D'Orb., Arango, Fauna Mal. Cuba, 1878. 237. Ca. G.

Gastrochaena cuneiformis Liam., Beau. 27. G.

Gastrochaena cuneiformis Lam., Jour de Conch., iv, 413. G.

Gastrochaena cuneiformis Lam., Moll. Cuba, ii, 228.

Gastrochaena cuneiformis Phil.. Krebs. 114.

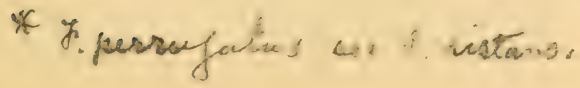


Gastrochaena cuneiformis Speng., Moll. Cuba, ii, 228.

Gastrochaena hians Chem., Krebs. 114. ST. SCx.

Gastrochaena hians Chem., Poulsen. 14.

Gastrochaena ovata Sby., Krebs. 114.

Gastrochaena ovata Sby., Mal. Bl., x, 228.

Gastrochaena rostrata Spgl., Krebs. 114.

Gastrochaena rostrata Spgl., Poulsen. 14.

Gastrochaena truncata Sby., Arango, Fauna Mal. Cuba, 1878. 237. Ca.

Gastrochaena truneata Sby., Krebs. 114.

Gastrochaena truncata Sby., Mal. Bl., x, 228.

Gemma gemma Totten, Am. Marine Consh. 159. NO.

Gibbula (see Trochus).

Gibbula dentata Linn., Marrat, Argo Exp., 1876. 16. Nassan. Long Key Island. Athol Island. Abaco.

Glaucus atlanticus Blum., Moll. Cuba, i, 110.

Glancus atlanticus Forster, Arango, Fauna Mal. Cuba, 1878. 155. Ca. Antilles.

Glaucus atlanticus Forster, Moll. Cuba, i, 110.

Glaucus atlanticus Less., Moll. Cuba, i, 110.

Glanens anstralis Peron, Arango, Fauna Mal. Cnba, 1878. 155. Ca. Antilles.

Glaucus australis Peron, Moll. Cuba, i, 110.

Glaucus boscii Lesson, Arango, Fauna Mal. Cuba, 1878. 155. Ca. Antilles.

Glaucus boscii Less., Moll. Cuba, i, 110.

Glaucus colymbus Poli, Krebs. 131.

Glancus forsteri Lam., Arango, Fauna Mal. Cuba, 1878. 155. Ca. Antilles.

Glaucus forsterii Lam., Moll. Cuba, i, 110.

Glaucus forsterii Q. \& G., Moll. Cuba, i, 110.

Glancus hexapterigius Cuv., Arango, Fauna Mal. Cuba, 1878. 155. Ua. Antilles.

Glaucus hexapterigius Cuv., Moll. Cuba, i, 110.

Glancus hexapterigius Rang, Moll. Cuba, i, 110.

Glaueus pacifieus Lesson, Arango, Fauna Mal. Cuba, 1878. 155. Ca. Antilles.

Glancus pacificus Lesson, Moll. Cuba, i, 110.

Glancus peronii Lesson, Arango, Fauna Mal. Cuba, 1878. 155. Ca. Antilles.

Glancus peronii Lesson, Moll. Cuba, i, 110.

Glauens radiatus D'Orb., Arango, Fauna Mal. Cuba, 1878. 155. Ca. Antilles.

Glaucus radiatus D'Orb., Moll. Cuba, i, pl. iii, 109, 110.

Glaucus radiatus Gmel., Arango, Fauna Mal. Cuba, 1878. 154. Ca. Antilles. 
Glaucus tetrapterigins Less., Moll. Cuba, i, 110.

Glaucus tetrapterigius Rayg, Moll. Cuba, i, 110.

Globulus (see Ethalia, Rotella, Modulus, Vitrinella).

Globulus anomalus D'Orb., Krebs. $\$ 3$.

Globulus carinatus D’Orb., Krebs. $\$ 3$.

Globulus diaphanus D’Orb., Krebs. $\$ 3$.

Globulus marinus Seba, Mal. Bl., xxiii, 130.

Globulus semistriatus D'Urb., Krebs. 83:

Globulus strictus D'Orb., Krebs. 83.

Glottidia (see Lingula).

Glottidia antillarum Rve., Am. Jour: Conch., vi, 159.

Glotticlia audebarti Brod., Am. Jom. Couch., vi, 159.

\section{M.}

Glottidia peramidata Stm., Am. Jour. Conch., vi, 158. NC.

Glottidia pyramidata Stm., Am. Marine Conch. 200.

Glottidia pyrauidata Stm., Dall, Hemphill's shells. 34:. Cedar Keys. Glycimeris (I'anopaea) bitruncata Comrad, Proc. Acad. Nat. Sci. Phil., Smads. 303. Ft. Macon. Florida?

Guathodon cuneatum Con., Jour. de Conch., i, 73. Fla.

Gnathodon cuneatus Gray, Jour. de Conch., iv, 164. La.

Gnathodon flexnosa ? Con., Jour. de Conch., iv, 16t, 165. Fla.

Gnathodon rostratum Petit., *Jour. de Conch. iv, pl. vi, figs. 1, 2, 3. 8t, 164, 167. Fla.

Goniodoris picturata Mörch, Jonr. de Conch., xi, 35. ST.

Goniodoris picturata Mörch, Mal. Bl., xxii, 177. ST.

Goniodoris picturata Mörch, Mal. Bl., xxri, 98.

Gouldia (see Circe, Crassatella).

Gouldia cerina C. B. Adams, Poulsen. 15.

Gouldia cerina C. B. Adams, Report Blake Moll. 130.

Gouldia cubaniana D'Orb., Report Blake Moll. 130.

Gouldia fastigiata Gld., Am. Mariue Conch. 166. NO.

Gouldia fastigiata Gld., Otia. 239. NC.

Gouldia fastigiata Gld., Report Blake Moll. 131.

Gonldia (Crassatella) guadalupensis D’Orb., Jour. de Conch., v, 156. G.

Gouldia guadalupensis D'Orb., Mal. Bl., x, 229.

Gouldia guadalupensis D'Orb., Poulsen. 15.

Gouldia mactracea Linsley, Am. Marine Conch. 165.

Grouldia (Crassatella) martinicensis D'Orb., Jour. de Couch., v, 156. G.

Gouldia martinicensis D'Urb., Poulsen. 15.

Gouldia parva C. B. Adams, Jour. de Conch., v, 156. G.

Gouldia parva C. B. Adams, Report Blake Moll. 131.

Gundlachia (see Poeyia).

Gundlachia adelosia Bourg., Alaugo, Fauna Mal. Cuba, 1878. 138.

Ca. San Miguel.

Gundlachia ancyliformis Pfr., Arango, Fanna Mal. Cuba, 1878. 138.

Ca. San Miguel.

Gundlachia poeşi Bourg., Arango, Fanna Mal. Cuba, 1S7s. 138. Ca. 
Gutturnium (see Tritonium), Marrat, Argo Exp., 1876. 6.

Haliotis (Padollus) pourtalesii Dall, n. s., Report Blake Moll. 79. Fla. Haminea (see Bulla).

Haminea auberi D’Orb., Mal. Bl., xxii, 175. Ca.

Haminea cerina Mke., Mal. Bl., xxii, 175. ST. PR.

Haminea cerina Mke. = B. antillarum D’Orb. Poulsen. 8.

Haminea cerina Mke.,* Zt. Mal., x, 142. PR.

Haminea glabra A. Ad., Mal. Bl., xxii, 174. Ca.

Haminea guadalupensis Sby., Am. Jour. Conch., iv, 283. G.

Haminea guildingii Sws., Mal. Bl., xxii, 174. ST.

Haminea guildingii Sws., Poulsen. 8.

Haminea petiti D'Orb., Mal. Bl., xxii, 174. Ca.

Haminea petiti D’Orb. =H. glabra A. Ads., Poulsen. 8.

Hanleyia tropicalis Dall, Hemphill's shells. 337.

Hanleyia tropicalis Dall, n. s., Report Blake Moll. 53. Fla.

Haustator (see Turritella) Marrat, Argo Exp., 1876. 12.

Heliacus (see Solarium, Torinia).

Heliacus cylindraceum Chem., Arango, Fauna Mal. Cuba, 1878. I'/8. Ca. G.

Heliacus heberti D’Orb., Arango, Fauna Mal. Cuba, 1878. 179. Ca. G.

Heliacus heberti D’Orb., Mal. Bl, xxii, 156.

Heliacus heberti D'Orb., Moll. Cuba, ii, 68.

Heliacus herbertii D'Orb., Krebs. 63.

Heliconoides bulimoides D’Orb., Araugo, Fauna Mal. Cuba,1878. 154. Antilles.

Heliconoides bulimoides D'Orb., Moll. Cuba, i, 104.

Heliconoides inflata D’Orb., Arango, Fauna Mal. Cuba, 1878. 154. Ca. Antilles.

Heliconoides inflata D'Orb., Moll. Cuba, i, 103.

Heliconoides trochiformis D’Orb., Arango, Fauna Mal. Caba, 1878. 154. Ca.

Heliconoides trochiformis D'Orb. Moll. Cuba, i, 104.

Melicophlegma candei D’Orb., Arango, Fauna Mal. Cuba. 1878. 153. Antilles.

Helicophlegma candei D'Orb., Moll. Cuba, i, pl. ii, figs. 15-17, 100.

Helicophlegma keraadreni D’Orb., Arango, Fauna Mal. Cuba. 1878. 153. Antilles.

Helicophlegma kerandreni Rang, Arango, Fauna Mal. Cuba, 1878. 153. Antilles.

Helicophlegma keraudrenii, D’Orb., Moll. Cuba, i, 99.

Helix (see Adeorbis, Littorina, Pyramidella, Janthina, Dolium).

Helix (see Ampullaria), Arango, Fauna Mal. Cuba, 1878. 138. Ca.

Helix ampullacea Born, Moll. Cuba, ii, 2.

Helix ampullacea Chem., Arango, Fauna Mal. Cuba, 1878. 138. Ca.

Helix ampullacea Chem., Moll. Cuba, ii, 2.

Helix ampullacea Gmel., Moll. Cuba, ii, 2. 
Helix ampullacea Linn., Moll. Cuba, ji, 3.

Helix corallina Chem., Mal. Bl., xxii, 184. ST.

Helix cyclostomoides Pfr., Arango, Fauna Mal. Cuba, 1878. 177. G.

Helix decussata Mtg., Mal. Bl., xxiii, 47.

Helix dolabrata Müll., Moll. Cuba, i, 228.

Helix eburnea Mühlf., Mal. Bl., xxii, 181. ST.

Helix haliotoidea Gmel., Moll. Cuba, ii, 37, 8.

Helix haliotoidea Linn., Arango, Fauna Mal. Cuba, 1878. 172. Ca. Santa Lucia.

Helix janthina Born, Moll. Cuba, ii, 81.

Helix janthina Gmel., Moll. Cuba, ii, 81.

Helix mespilum Mühlf., Mal. Bl. xxiii, 141.

Helix scabra Chem., Moll. Cuba, i, 213.

Helix scabra Gmel., Moll. Cuba, i, 213.

Helix scabra Linn., Moll. Cuba, i, 212.

Helix scabra Wood, Moll. Cuba, i, 213.

Helix subcylindrica Mtg., Moll. Cuba, ii, 5 .

Helix sulphurea Ad., Mal. Bl., xxiv, 43.

Helix terebella Miill., Arango, Fauna Mal. Cuba, 1878. 161. Ca. G. Ba.

Helix terebella Müll., Moll. Cuba. i, 228.

Hemicardia (see Cardium).

Hemicardia (Fragum) media Linn., Marrat, Argo Expr. 1876. 13. SM. Abaco.

Hemicardium medium Sws., Krebs. 116.

Hemifusus (see Fusus).

Hemifusus corona var. $\beta$, estephomenos Melrill, Dall, Hemphill's shells. 342.

Hemifusus morio Linn., Moll. of Bermudas. 16.

Hermæa viridis Desh., Jour. de Conch., xi, 37. ST. G.

Hermæa viridis Desh., Mal. Bl., xxii, 180. ST.

Heromorpha antillensis Bergh, Mal. Bl. xxii, 178. ST.

Heterodonax (see Tellina).

Heterodonax (sce Donax), Marrat, Argo Exp., 1876. 13.

Heterodonax bimaculata Linn., c. var., Poulsen. 15.

Heterodonax sexradiata Mörch, Krebs. 100.

Hima (see Nassa), Marrat, Argo Exp., 1876. 6.

Hippagus novemeostatus Ads. \& Rve., Report Blake Moll. 105. CS.

Hippocastanum fasciatum Mart., A rango, Fauna Mal. Cuba, 1878. 203. Ca. H. Ba.

Hippocastanum fasciatum Mart., Krebs. 27. ST.

Hipponyx (see Capulus, Pileopsis).

Hipponyx antiquata Linn., Beau. 19. G.

Hipponyx antiquatus Linn., Dall. Hemphill's shells. 337. Key West.

Hipponyx antiquatus Linn., Jour. de Conch., v, 151. G.

Hipponyx antiquatus Linn., Jour. de Conch., x, 20. 
Hipponyx antiquatus Linn., Mal. Bl., xxiv, 98. G.

Hipponyx antiquatus Linn., Poulsen. 10.

Hipponyx antiquatus Mke., Mal. Bl. xii, 127.

Hipponyx antiquatus Mlke., Zt. Mal., x, 79. SV.

Hipponyx barbatus Cpr., Jour. de Conch., x, 23.

Hipponyx barbatus var. Cpr., Mal. Bl., xxiv, 98. ST.

Hipponyx bullii T. \& H., n. s., Pleiocene Fossils S. C., pl. xxv, fig. 13. 112.

Hipponyx cernuus Gmel., Mal. B1., xxiv, 97.

Hipponyx cernuus Gray.=Capulus subrufus D'Orb., Poulsen. 10.

Hipponyx costellatus Cpr., Jour. de Conch., x, 23.

Hipponyx effodiens Cpr., Jour. de Conch., x, 24.

Hipponyx effodiens Cpr., Mal. B1., xxiv, 99.

Hipponyx effodiens Cpr., Poulsen. 10.

Hipponyx militaris Linn., Mal. Bl., xxiv, 97.

Hipponyx militaris Limn.=Capulus intortus I'O., Poulsen. 10.

Hipponyx mitrula Def., Jour. de Conch., x, 20.

Hipponyx mitrula Def., Moll. Cuba, ii, 186.

Hipponyx mitrula Sby., Zt. Mal, x, 79. SV.

Hipponyx panamensis C. B. A., Jour. de Conch., $x, 20$.

Hipponyx pilosa Desh, Beau. 19. G.

Hipponyx radiatus Gray, Zt. Mal., x, 79. SV.

Hipponyx subrufus Mart, Mal. Bl., xxiv, 98.

Hipponyx trigona Mörch, Mal. Bl., xxiv, 98. ST.

Hipponyx trigonus Gmel., Mal. Bl., xxiv, 98. ST.

Hipponyx trigonus Gmel., Poulsen. 10.

Hipponyx tuberculatus Cpr., Jour. de Conch., x, 23.

Hipponyx tubereulatus Cpr., Mal. Bl., xxiv, 98. Ca.

Hipponyx tubereulatus Cpr., Mal. Bl., xxiv, 99.

Hormomya (see Mytilus).

Hormomya exustus Mörch, Krebs. 128.

Hyalæa (see Cavolina).

Hyalæa (see Cleodora) Arango, Fauna Mal. Cuba, 1878. 151. Ca. Antilles.

Hyalæa (see Creseis) Arango, Fauna Mal. Cuba, 1878. 152.

Hyalæa aciculata D'Orb., Moll. Cuba, i, 88.

Hyalæa caudata Roissy, Moll. Cuba, i, 83.

Hyalia cornea Roissy, Arango, Fauna Mal. Cuba, 1878. 150. Antilles.

Hyalæa cornea Roissy, Moll. Cuba, i, 72.

Hyalæa corniformis D'Orb., Moll. Cuba, i, 87.

Hyalæa cuspidata Blv., Moll. Cuba, i, 82.

Hyalæa cuspirlata Bose, Moll. Cuba, i, 82.

Hyalæa cuspidata D'Orb., Moll. Cuba, i, 82.

Hyalæa cuspidata Lam., Moll. Cuba, i, 82.

Hyalea flava D'Orb., Arango, Fauma Mal. Cuba, 1878. 149. 
Hyalad forskalii Lesueur, Arango, Fauna Mal. Cuba, 1878. 150. An. tilles.

Hyalæa forskalii Lesueur, Moll. Ouba, i, 72.

Hyalæa gibbosa (Rang) D'Orb., Arango, Fauna Mal. Cuba, 187S. 149. Autilles, Ca.

Hyalxa gibbosa D’Orb., Arango, Fauma Mal. C'uba, 1878. 150. Antilles. Ca.

Hyalæa gibbosa D'Orb., Moll. Cuba, i, pl. ii, figs. 1-4. 74.

Hyalæa gibbosa Rang, Moll. Cuba, i, 74 .

Hyalæa intlexa Blr., Moll. Cuba, 1, 79.

Hyalaa inflexa, D’Orb., Arango, Fauna Mal. Cuba, 1878. 150. An. tilles.

Hyalaa inflexa D'Orb., Moll. Cuba, i, 79.

Hyalaea inflexa Lesueur, Arango, Fama Mal. Cuba, 1878. 150. Antilles.

Hyalaa inflexa Lesueur, Moll. Cuba, i, 79. Hatt. Ca.

Myalea labiata D'Orb., Arango, Famna Mal. Cuba, 187s. 150. Antilles. Ca.

Hyalae labiata D'Orb., Moll. Cuba, i, pl. ii, figs. 9-12. 78.

Hyalaa lanceolata Lesueur, Arango, Fauna Mal. Cuba, 1878. 151. Ca. Autilles.

Hralæa Ianceolata Lesneur, Moll. Cuba, i, 84.

Hyalaa limbata D’Orb., Arango, Fauna Mal. Cuba, 187S. 150. Autilles.

Hyalæa limbata D'Orb., Moll. Cuba, i, pl. ii, figs. 5-8. 77.

Hyalaa longirostra D’Orb., Araugo, Fauna Mal. Cuba, 1878. 150. Antilles.

Hyalæa lougirostra D'Orb., Moll. Cuba, i, 76.

Hyaliea longirostra Lesueur, Arango, Fauna Mal. Cuba, 1S7S. 150. Antilles.

Hyalæa lougirostra Lesueur, Moll. Cuba, i, 76. Hatt. Ca.

Hyalæa papilionacea Bory St. Vinc., Araugo, Fauna Mal. Cuba, 1878. 150. Antilles.

Hyalæa papilionacea Bory St. Vinc., Moll. Cuba, i, 72.

Hyalea pyramidata Bose, Moll. Cuba, i, S3.

Hyalaa pyramidata D'Orb., Moll. Cuba, i, 84.

Hyalæa pyramidata P. \& L., Moll. Cuba, i, 84.

Hyalsea pyramidata Roissy, Moll. Cuba, i, 83.

Hyalaca quadridentata D'Orb., Alango, Fauna Mal. Cuba, 1878. 150. Antilles.

Iyalaea quadridentata D'Orb., Noll. Cuba, i, 75, 81. Hatt. Car. Hyaliea qualridentata Lesueur, Arango, Fauna Mal. C'uba, 1878. 150. Antilles.

Hyalæa striata D'Orb., Moll. Uuba, i, 87.

Hyalæa subula, D’Orb., Moll. Cuba, i, 85.

Hyalæa tridentata Bose, Moll. Cuba, i, 72. 
Hyalæa tridentata D'Orb., Arango, Fauna Mal. Cuba, 1878. 151. Antilles.

Hyalra tridentata D'Orb., Moll. Cuba, i, 72.

Hyalæa tridentata Gmel., Arango, Fauna Mal. Cuba, 1878. 150. Antilles.

Hyalæa tridentata Gmel., Moll. Cuba, i, 72.

Hyalæa tridentata Lam., Beau. 20. G.

Hyalæa tridentata Lam., Moll. Cuba, i, 72. Hatt. Ca.

Hyalæa trispinosa. D'Orb., Arango, Fauna Mal. Cuba, 1878. 151. Antilles. Ca.

Hyalæa trispinosa D'Orb., Moll. Cuba, i, 80.

Hyalæa trispinosa Guérin, Moll. Cuba, i, 80.

Пyalæa trispinosa Lesueur, Arango, Fauna Mal. Cuba, 1878. 151. Antilles. Ca.

Hyalæa trispinosa Lesueur, Moll. Cuba, i, 80.

Hyalæa trispinosa Pourt., Arango, Fauna Mal. Cuba, 1878. 151. Antilles. Ca.

Hyalæa uncinata D'Orb., Arango, Fauna Mal. Cuba, 1878. 151. Antilles. Ca.

Hyalea uncinata D'Orb, Moll. Cuba, i, 73. Hatt. Ca.

Hyalæa uncinata Pourt., Arango, Fauna Mal. Cuba, 1878. 151. Antilles. Ca.

Hyalæa uncinata Rang, Arango, Fauna Mal. Cuba, 1878. 151. Antilles. Ca.

Hyalæa uncinata Rang, Moll. Cuba, i, 73.

Hyaliea (Diacria) vendryesiana Guppy, Geol. Mag., 1874. 435. J. Hyalæa virgula D'Orb., Moll. Cuba, i, s6.

Hyalina (see Marginella).

IIyaliua (sce Volvaria), Arango, Fauna Mal. Cuba, 1878. 190. Ca. Ber. G. Ba.

Hyalina albolineata D'Orb., Arango, Fauna Mal. Cuba, 1878. 189. Ca. Havana. Antilles. J. G.

Hyalina gracilis Ad., Arango, Fanna Mal. Cuba, 1878. 189. Ca.J. Hyaliua lactea Kien., Arango, Fauna Mal. Cuba, 1878. 190. Ca. Hyalina pallida Linn., Arango, Fauna Mal. Cuba, 1878. 190. Ua. Antilles.

Hyalina subtriplicata D'Orb., Arango, Fauna Mal. Cuba, 1878. 190. Ca. Antilles.

Hydatina (see Bulla).

Hydatina filosa Schum., Krebs. 92.

Hydatina filosa Schum., Zt. Mal., x, 70. SV.

Hydrobia (see Amnicola, Bythinella).

Hydrobia monroënsis (Franenfeld) Dall, Proc. U. S. Nat. Mus. 1885, pl 17, fig. 9. 256. Fla.

Пydrobia wetherbyi Dall, Proc. U. S. Nat. Mus. 1885, pl. 17, fig. 10. 258. Fla. 
Ianthina (see Janthina).

Ianthina communis Lam., Moll. of Bermuda. 20.

Tanthina exigua Lam., Krebs. 65.

Ianthina fragilis Ad., Krebs. 65.

Ianthina globosa Sby., Krebs. 65.

Ianthina globosa Swains., Moll. of Bermudas. 21.

Ianthina planispira Rve., Krebs. 65. ST. SJ.

Ianthina prolongata Bir., Krebs. 66.

Ianthina umbilicata D'Orb., Krebs. 66.

Idotea perforata Schum., Krebs. 122.

Ilyanassa obsoleta Say, Dall, Шemphill's shells. 325. St. Angustine. Imperator (see Calcar, 'Trochus).

Imperator calcar Linn., Moll. of Bermudas. 20.

Infuudibulum (see Galerus).

Infundibulum candeanum D'Orb., Arango, Fama, Mal. Cuba, 18is. 226. Ca.

Infundibulum candeanum D'Orb., Nal. Bl., xxiv, 106. J.

Infำudibulum candeanum I' Orb., Moll. Cuba, ii, pl. xxir, figs. 2\$-29. 190.

Infundibulum depressum Say, Am. Marine Conch. 93.

Iphigenia (see Capsa, Donax).

Iphigenia brasiliensis Lam., Arango, Fauna Mal. Cuba, 1878. 247. Ca. Iphigenia brasiliensis Lam., Jahrb., ii, 249.

lphigenia braziliensis Lam., Mal. Bl., xvi, 151. Ca. PR.

Iphigenia brasiliensis Lam., Poulsen. 15.

Iphigenia intermedia Shuttlew., Poulsen. 15.

Iphigenia levigata Schum., Comrad. Am. Marine Conch., 68, pl. xvii,

fig. 1, 1831. Fla.

Iphigenia media Shuttlew., Mal. Bl., iv, 28. PR.

Iphigenia media Shuttlew., Jour. de Conch., v, 174. PR.

Iphigenia media Shuttlew., Mal. Bl., xri, 152. Ca. PR.

Isapis anomala C. B. Adams, ${ }^{*}$ Mal. Bl., xxiv, 96. J.

Isapis anomala H. \& A. Ad., Jomr. de Conch., xii, 256. J.

Isapis anomala Chenu, Mal. Bl., xxiv, 96. J.

Ischnochiton (see Chiton).

Ischuochiton multicostatus C. B. Ad., Dall, Hemphill's shells. 337. Key West.

Ischnochiton pectimatus Sloy., Arango, Fama, Mal. Cuba, 157s. 22:1. Ca. Bb. J.

Ischnochiton pectinatns Sby., Dall, Memphill's shells. 337. Key West. Isocardia (see Cardium) Marrat, Argo Exp., 1876. 13.

Isognomon (see Perma).

Isognomou alatum Gurel., Arango, Fauna Mal. Cuba, 1878. 269. Ciı, II. Ba.

Isognomon chemnitzianum D'Orb., Arango, Fauna Mal. Cuba, 1878. 269. C. M. SCx.

Bull. $24-9$ 
Isognomon constellatum Con., Arango, Fanna Mal. Cuba,1S78. 269. Ca. Isognomon flexuosum Sby.; Mal. Bl., x, 229.

Isognomon perna Linn., Arango, Fauma Mal. Cuba, 1878. 269. Ca. M. Ba.

Isognomon perna Linn., Mal. Bl., x, 225.

Isognomon serratula Rre., Marrat, Argo Exp., 1876. 7. Aa. SY.

Point Saranilla. Nassau. Abaco.

Isognomon serratum Rre., Marrat, Argo Exp., 1876. 15. Havana.

Isognomon vulsella Lam., Mal. Bl., x, 229.

Isognomon vulsella Lam., Mal. Bl., x, 231.

Isognomum alatum Gmel., Poulsen. 16.

Isognomum perna Linn., Poulsen. 16.

Isognomum vulsella Lam., Poulsen. 16.

Jamaica wartshell Petiver, Mal. Bl., xxiii, 134.

Janira (see Pecten).

Janira affinis T. \& H., n. s., Pleiocene Fossils S. C., pl. viii, figs. 5 and × 6.26.

J.Janira maxima Sehum., Moll. Cuba, ii, 355.

Janira ziczac Schum., Krebs. 134.

Janira zigzag Linn., MIoll. Cuba, ii, 355.

Janira zigzag D'Orb., Arango, Fanna Míal. Cuba, 1878. 270. Ca. G. M. Santa Lucia., SD. ST. Ba.

Janira zigzag D’Orb., Moll. Cuba, ii, 356.

Janthina (see Ianthina).

Janthina bicolor Da Costa, Arango, Fauna Mal. Cuba, 1878. 182. Ca. Janthina bicolor Da Costa, Moll. Cuba, ii, 81.

Janthina bicolor Lam., Beau. 11. G.

Janthina bicolor Less., Moll. Cuba, ii, 81 .

Janthina bicolor Menke, Jahrb., ii, 247.

Janthina bicolor Menke, Moll. Cuba, ii, 81.

Janthina bicolor Phil., Moll. Cuba, ii, 81.

Janthina communis Da Costa, Moil. Cuba, ii, 83.

Janthina communis Lam., Arango, Fauna Mal. Cuba, 1878. 182. Ca.

Janthina communis Lam., Jahrb., ii, 247.

Janthiua communis Lam., Moll. Cuba, ii, 81 .

Janthina communis Payr., Moll. Cuba, ii, 81 .

Janthina exigua Blv., Moll. Cuba, ii, 84 .

Janthina exigua D'Orb., Moll. Cuba, ii, 84.

Janthina exigua Lam., Arango, Fanna Mal. Unba, 1878. 182. Antilles.

Janthina exigua Lam., Beau. 11. G.

Janthina exigua Lam., Marrat, Argo Exp., 1876. 15. $\quad \nabla C z$.

Janthina exigua Lam., Moll. Cuba, ii, 84.

Janthina exigua Less., Moll. Cuba, ii, 84.

Janthina exigua Q. \& G., Moll. Cuba, ii, 84.

Janthina exigua Sby., Moll. Cuba, ii, 84 .

Janthina fragilis D'Orb., Arango, Fauna Mal. Cuba, 1878. 182. Ca. 
Janthina fragilis D:Orb., Moll. Cuba, ii, 81.

Janthina fragilis Guérin, Jour. de Conch., viii, 277. J.

Janthina fragilis Lam., Arango, Fauna Mal. Cuba, 1878. 182. Ca.

Janthina fragilis Lam., Moll. Cuba, ii, 80.

Janthina fragilis Lam., Moll. Cuba, ii, 81.

Janthina fragilis Less., Moll. Cuba, ii, 81.

Janthina fragilis Q. \& G., Moll. Cuba, ii, 81.

Janthina fragilis Sby., Moll. Cuba, ii, 81.

Janthina fragilis Sws., Moll. Cuba, ii, 81.

Janthina globosa Srs., Arango, Fama Mal. Cuba, 1878. 182. Ca.

Janthina globosa Sws., Marrat, Argo Exp., 1876. 15. VCz.

Janthina globosa Sws., Moll. Cuba, ii, 83.

Janthina grandis Browne, Jour. de Conch, viii, 277. J.

Janthina nitens Mke., Arango, Fanna Mal. Cuba, 18is. 182. Ca.

Janthiua nitens Mke., Moll. Cuba, ii, 83.

Janthina nitens Phil., Moll. Cuba, ii, 83.

Jañthina penicephala Péron, Moll. Cuba, ii, 81.

Janthina prolougata Blv., Arango, Fauna Mal. Cuba, 1878. 182. Ca.

Janthina prolongata Blv., Noll. Cuba, ii, \$3.

Janthina prolongata D'Orb., Arango, Fauna Mal. Cuba, 1S78. 183. Ca.

Janthina prolongata D'Orb., Moll. Cuba, ii, 83.

Janthina prolongata Less., Moll. Cuba, ii, 83.

Janthina prolongata Payr., Moll. Cuba, ii, 83.

Janthina umbilicata D'Orb., Arango, Fauna Mal. Cnba, 1878. 183. Antilles.

Janthina umbilicata D'Orb., Moll. Cuba, ii, pl. xx, figs. 22-23. 85.

Janthina violacea Bolt., Jour. de Conch., viii, 277. J.

Labiosa (see Mactra).

Labiosa eyprina Gray, Arango, Fanna Mal. Cuba, 1sis. 240. Ca., Havana.

Lachesis monilifera Sby., Poulsen. 13.

Lævicardium (see Cardium, Liocardium).

Lævicardium mortoni Con., Am. Marine Conch. 175.

Lævicardium pictum Rav., Am. Marine Conch. 176. SC.

Lævicardium serratum Linn., Am. Marine Conch. 175.

Læricardium serratum Limn., Marrat, Argo Exp., 1S76. 12. Tucacas. Athol Island.

Lævicardium serratum Linn., var., Marrat, Argo Exp., 1876. 17. Nas. sall.

Lxricardium vitellinum Rve., Marrat, Argo Exp., 1876. 12. Tucacas. Lambis (see Strombus).

Lambis curruca Bolt., Mal. Bl., xxir, 19.

Lampanella (see Cerithium, Pyrazus).

Lampanella eriensis Val., Mal. B1., xxiii, 94.

Lampanella minimus Gmel.,* Mal. Bl., xxiii, 93. Fla. 
Lampanella nigrescens Mke.," Mal. B1., xxiii, 94. Ca.

Lampanella zonalis Brug., Mal. Bl., xxiii, 94. J.

Lampania septemstriata Say. Mal. Bl., xxiii, 93. Fla.

Lampania septemstriata Sby., Mal. Bl., xxiii, 94.

Lampas (see Bursa), Marrat, Argo Exp., 18i6. 12.

Lampusia (see Triton, Tritonium).

Lampusia antillarum, D'Orb., Guppy, Paria Fanna, 1877. 142.

Lampusia martiniana D'Orb., Gupey, Paria Fama, 1877. 142.

Lampusia suceincta Lam., Guppy, Paria Fanna, 1877. 142.

Latirus (see Tritonidea).

Latirus angulifera Lam., Marrat, Argo Exp., 1476. 14. Point Savanilla.

Latirus carrohuesonicus Sby.jr., \& Melvill, Dall, Пemphill's shells. 342. Key West.

Latirus infundibulum Gmel, Marrat, Argo Exp., 1876. 13. Santa

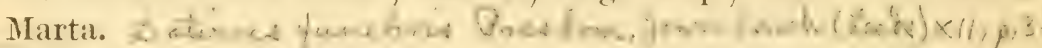

Laviguon (sce Cumingia, Lntricola, Lutraria, Listera, Scrobicularia).

Larignon antillarum D’Orb., Arango, Fanna Mal. Cuba, 1878. 245. Ca. G.

Lavignon antillarum D'Orb., Moll. Cuba, ii, pl. xxr, figs. 36-38, 236. Larignon lineata D'Orb., Arango, Fanna Mal. Cuba, 1878. 245. Ca. Lavignon lineata D’Orb., Moll. Cuba, ii, 235.

Lavignon lineata Lanu., Arango, Fanna Mal. Cuba, 1878. 245. Ca. Lavignon mutica Sby., Arango, Fama Mial. Cuba, 187s. 245. Ca. SD. G.

Lavignon petitiana D'Orb., Arango, Fanna Mal. Cuba, 1878. 246. Ca. SD. G.

Lavignon petitiana D'Orb., Jour. de Conch., iv, 414. G.

Iarignon petitiana D'Orb., Moll. Cnba, ii, pl. xxr, figs. 33-33, 2236.

Leda acuminata Jeffreys, Report Blake Moll. 124.

Leda (Junonia) aenminata Seguenza, Report Blake Moll. 124.

Leda acuta Con., Am. Marine Conch. 182. NO.

Leela acuta (Conrad) Verrill, List, 280.

Leda bisulcata Guppy, Geol. Mag., 1875. J.

Leda bushiana Verrill, List, 1884. 280. Cape Hatteras.

Leda carpenteri Dall, n. s., Report Blake Moll. 125.

Ledla clara Guppy, Geol. Mag., 1875. J.

Lerlat (Neilonella) corpulenta Dall, n. s., Report Blake Moll. 125.

Leda crenifera Sby., Jahrb., ii, 253.

Leda dilatata Phil., Report Blake Moll. 125.

Lerta eborea Conrar, Dall, Hemphill's shells. 341. Cedar Kę̧s.

Leda elenensis Sby., Marrat, Argo Exp., 1876. 12. Tueacas.

Leda illecta Guppy, Geol. Mag., 1875. Td.

Leda incognita Guppy, Geol. Mag., 1875. Td.

Leda intermedia Sars, Report Blake Moll. 127.

Ledla jamaicensis D'Orb., Arango. Fauna Mal. Cuba, 1S7S. 260. Antilles. 
Leda jamaiceusis D'Orb., Moll. Cuba, ii, pl. xxvi, figs. :30-32. 263. J. Leda jamaicensis D'Orb., Report Blake Moll. 124.

Leda jeftreysi Hidalgo, Report Blake Moll. 124.

Leda jeftreysi Hidalgo, var. quadraugulus, Report Blake Moll. 120.

Leda (Lembulus) lamellicostata Segueuza, Iieport Blake Moll. 124.

Leda lata Jetrireys, Report Blake Moll. 124.

Leda (Yoldia) limatula Say, Am. Marine Conch. 183. NC.

Leda messaneusis Seguenza, Report Blake Moll. 124.

Leda packeri Forbes, Guppy, Geol. Mag., 1875. Bb.

Leda perlepida Guppy. Geol. Mag., 1875. Td.

Leda solida Dall, n. s., Report Blake Moll. 126.

Leda (Adrana) tellinoides Wood, Marrat, Argo Exp., 1576. 12. Tucacas.

Leda unca Gld., Am. Marine Conch. 183. NC.

Leda unca Gld., Otia. 239. NO.

Leda unea Gld., Report Blake Moll. 124.

Leda ventricosa Hinds, Marrat, Argo Exp., 1876. 13. Tucacas.

Leda vitrea D’Orb., Arango, Fauna Mal. Cuba, 187. 260. M. SD.

Leda vitrea D’Orb., Moll. Cuba, ii, pl. xxri, figs. 27-29. 262. MI.J.

Leda vitrea D’Orb., Report Blake Moll. 126.

Leda vitrea (?) D’Orb., var. cerata, Report Blake MIoll. 126.

Leguminaria Horidaua Comrad, Proc. Acad. Nat. Sci. Phil., ir, 121, $18 t 8$.

Tampa.

Leguminaria lucida Sehum., Krebs. 112.

Leiostraca (see Eulima).

Leiostraca bifasciata D'Orb., Mal. Bl., xxii, 181. G.

Leiostraca bifasciata D'Orb., Poulsen. 8.

Leiostraca clavata Guppy, Geol. Mag., 1874. 437. Td.

Leiostraca fulvocineta C. B. Adams, Mal. Bl., xxii, 180. J.

Leiostraca fulvocincta C. B. Adams, Poulsen. S.

Leiostraca onjchina Folin, Mal. Bl., xxii, 181. G.

Lentilaria tigeriua Schum., Krebs. 121. = C A d cece

Lepas alba Mart., Mal. Bl., xxiv, 98. G.

Lepas alba Mart., Moll. Cuba, ii, 186.

Leptoconus (see Conus).

Leptoconus tlavescens Gray, Marrat, Argo Exp., 1876. 16. Nassau. Abaco.

Leptocouns proteus Brug., Marrat, Argo Exp., 1876. 12. Tucacas.

Leptoconus (Chelyconus) pusio Brug., Marrat, Argo Exp., 1876. 13.

Santa Marta.

Leptoconus (Chelyconus) pusio Lam., Narrat, Argo Esp)., 1876. 12. Tucacas.

Lepton fabagella Con., Kurtz. 4.

Lepton lepidum Say, Am. Marine Conch. 172. SO.

Lepton longipes Stm., n. S., Kurtz. 4. SC.

Lepton longipes Stm., Am. Marine Conch. 172. SC. 
Lepton placunoideum Cpr., Mal. Bl., x, 230.

Leptothyra (see Collonia, Turbo).

Leptothyra (induta var.?) albida Dall, n. s., Report Blake Moll. 48.

Yucatan Strait. CS.

Lepus marinus Rond., Moll. Cuba, i, 116.

Leuconia (see Melampus, Sayella).

Leuconia hemphillii Dall, n. s., Dall, Hemphill's shells. 323. Cerlar Keys.

Leuconia occidentalis Pfr., Mal. Bl., ii, 85. Ca.

Lenconia occidentalis Pfr., Jahrb., vii, 266. Ca.

Leuconia occidentalis Pfr., Mal. BJ., i, 152. Ca.

Leuconia oceidentalis Pfr., Mal. Bl., i, 155. Ca.

Leuconia sayi Kiister, Am. Jour. Conch., iv, pl. xviii, fig. 12. 10.

Leuconia sayi Kiister, Am. Jour. Conch., iv, 18.

Leuconıa sayi Kiister, Mal. Bl., i, 152.

Leuconia succinea Pfr., Mal. Bl., ii, 85. Ca.

Leuconia succinea Pfr., Jahrb., vii, 266. Ca.

Leuconia succinea Pfr., Mal. Bl., i, 152. Ca.

Leuconia succinea Pfr., Mal. Bl., i, 156. Ca.

Lencozonia angularis Rre., Jahrb., iii, 18.

Leucozonia brasiliana D'Orb., Jahrb., ii, 242.

Leucozonia cingulifera Lam., Dall, Hemphill's shells. 327. Key West.

Leucozonia cingulifera Lam., Jahrb., iii. 18.

Leucozonia knorrii Desh., Dall, Hemphill's shells. 327.

Lencozonia kuorrii Desh., Marrat, Argo Exp., 1876. 18. Abaco.

Leucozonia nassa Gmel., Jahrb., iii, 18.

Leucozonia nassa Gmel, Marrat, Argo Exp., 1876. 6. Aa.

Leucozonia occellata Gmel., Jahrb., iii, 18.

Lencozonia riiseana, Dkr., Jahrb., iii, 1 .

Ligula profundissima Forbes, Report Blake Moll. 133.

Lima (see Limaria, Limatula, Limopsis, Ctenoides, Radủla).

Lima? n. s., Kurtz. 6. SC.

Lima aspera Chem., Krebs. 133.

Lima aspera Chem., Moll. Cuba, ii, 336.

Lima bullata Born, Krebs. 132.

Lima bullata Chem., Jour de Conch., ii, 426. G.

Lima caribaa D'Orb., Arango, Fauna Mal. Cuba, 1Sis. 267. Ca. G. Lima caribæa D'Orb., Krebs. 133.

Lima caribce D'Orb., Moll. Cuba, ii, pl. xxviii, figs. 17-19. 337. Ca.

Lima caribæa D'Orb., Poulsen. 16.

Lima caribsea D'Orb., Report Blake Moll. 118.

Lima cubaniana D'Orb., Arango, Fauna Mal. Cuba, 1878. 267. Ca.

Lima cubaniana D'Orb., Krebs. 133.

Lima cubaniana D’Orb., Moll. Cuba, ii, pl. xxviii, 20-22. 337. Ca. .

Lima fragilis Lam., Krebs. 133.

Lima fragilis Mont., Jour de Conch., ii, 426. G. 
Lima fragilis Sby., Moll. of Bermudas. 24.

Lima glacialis G. \& L., Krebs. 133.

Lima glacialis, Gmel., Arango, Fauna Mal. Cuba, 1878. 267. Ca. M. G. Santa Lucia. Ba.

Lima glacialis Lam., Beau. 21. G.

Lima glacialis Lam., Jour. de Conch., iv, 416. G.

Lima glacialis Lam., Moll. Cuba, ii, 336.

Lima inflata Lam., Krebs. 133. ST. SM. SB.

Lima multicostata Sby., Krebs. 133.

Lima ovata (Wood) Jelfreys, Report Blake Moll. 118.

Lima pellucida Ad., Krebs. 133.

Lima pellucida O. B. Adams, Poulsen. 16.

Iima pellucida C. B. Adams, Proc. Bost. Soc. Nat. Hist., ii, 102. 1846. J.

Lima scabra Born, Arango, Fauna Mal. Cuba, 1s78. 267. Ca. M. G. Santa Lucia. Ba.

Lima scabra Born, Beau. 21. G.

Lima scabra Born, Jeur. de Conch., iv, 416. G.

Lima scabra Born, Krebs. 133.

Lima scabra Born, Poulsen. 16.

Lima scabra Dillwyn, Am. Marine Conch. 197.

Lima scabra D'Orb., Arango, Fauma Mal. Cuba, 1878. 267. Ca. M. G. Santa Lucia. Ba.

Lima scabra Sby., Moll. Cuba, ii, 336.

Lima squamosa Lam., Am. Marine Conch. 197.

Lima squamosa Lam., Arango, Fauna Mal. Cuba, 1878. 267. Ca. G.

Lima squamosa Lam., Beau. 21. G.

Lima squamosa Lam., Jour. de Conch., iv, 416. G.

Lima squamosa Lam., Krebs. 133.

Lima squamosa Lam., Moll. Uuba, ii, 33\%.

Lima tonera Chem., Dall, Hemphill's shells. 341. Cedar Keys. WI. Lima tenera Chem., Krebs. 133. VgI.

Lima tenera Chem., Poulsen. 16.

Limaria asperula Lam., Arango, Fauna Mal. Cubn, 187S. 267. Ca. M.

G. Santa Lucia. Ba.

Limaria asperula Link, Krebs. 133.

Limaria glacialis Lam., Krebs. 133.

Limatula bullata Wood, Krobs. 132.

Limatula ovata Jeffreys, Report Blake Moll. 118.

Ijimuæa cubensis Pfr.,Arango, Wauna Mal. Cuba, 1878. 135. Ca. PR. ST.

Limnae francisca Poey, Arango, Fauna Mal. Cuba, 1873. 135. Ca.

Limurea umbilicata Ads., Arango, Famna Mal. Cuba, 187S. 135. Ca. PR. ST.

Limopsis antillensis Dall, n. s., Report Blake Moll. 119.

Limopsis aurita Brocchi, Report Blake Moll. 118. 
Limopsis aurita Jeffreys, Report Blake Moll. 118.

Limopsis borealis Woodward, Report Blake Moll. 119.

Limopsis eristata Jefireys, Report Blake Moll. 119.

Limopsis minuta Phil., Report Blake Moll. 119.

Limopsis tenella Jeffreys, Report Blake Moll. 118.

Linatella (see 'Triton, Tritonium).

Linatella poulsenii Mörch, Mal. Bl., xxiv, 33. ST.

Linatella rostratum Mart, Mal. Bl., xxiv, 33.

Linatella tranquebaricum Lam., Mal. B1., xxiv, 32.

Lingula (see Glottidia).

Lingula antillarum Rve., Jour. de Conch., xiv, 266.

Lingula antillarum Rve., Jour. de Conch., xiv, 273. M.

Lingula antillarum Rve., Jour. de Conch., xvii, 116.

Lingula pyramidata Stm., Am. Jour. Conch., vi, 158. Fla.

Lingula pyramidata Stm., Jour. de Conch., xvii, 116.

Liocardium (see Cardium, Laevicardium).

Liocardium citrinum Sws., Krebs. 115.

Liotia cruentata Miihlf., Krebs. 80.

Liotia cruentata Miihlf., Ponlsen. 13.

Liotia riisei Dkr., Poulsen. 13.

Liotia tubereulosa D'Orb., Poulsen. 13.

Lithoarea candida Gray, Krebs. 124.

Lithodomus (see Lithophaga, Lithophagus).

Lithodomus autillarum D'Orb., Arango, Finna Mal. Cuba, 187S. 267. Ca. M. G.

Lithodomus antillarum D'Orb., Bean. 22. G.

Lithodomus antillarum D’Orb., Jour. de Coneh., iv, 415. G.

Lithodomus antillarum D’Orb. Moll., Cuba, ii, pl. xxviii, figs. 12-13. 33:. G. Ca.

Lithodomus appendiculatus Phil., Beau. 22. G.

Lithodomus appendiculatus Phil., Jour. de Conch., iv, 415. G.

Lithodomus bipenniferus Guppy, Paria Fauma, 1877. 154.

Lithodomus bisulcatus D'Orb., Arango, Fauna Mal. Cuba, 187s. 266. Ca. G. M. SD. J.

Lithodomus bisulcatus D')rb., Beau. 22. G.

Lithodomus bisulcatus D’Orb., Jahrb., ii, 252.

Litholomus bisuleatus D'Or'b., Jour. de Conch., iv, 415 . G.

Lithodomus bisulcatus D'Orb.* Noll. Cubi, ii, pl. xxrii, figs. 11-16. 333. J. M.

Lithodomus caudigerus, Lam. Moll. Cuba, ii, 333.

Lithodomus candigerus Lam., Arango, Fimua Mal. Cuba, 1578. 267. Ca.

Lithodomus cinnamomeus Chem.; Bear. 22. G.

Lithodomus cinnamomeus Chem., Jour. de Conch., $5,151$. G.

Lithodomus cinnamomens D'Onb., Alango, Fama Mal. Cuba, 1878. 266. Ca.J. M. SD. G. 
Lithodomus cinnamomeus D'Orb., Moll. Cuba, ii, 333.

Lithodomus corrugatus Phil., Jour. de Conch., iv, 415. G.

Lithodomus corrugatus Phil., Arango, Fauna Mal. Cuba, 157s. 267. Ca. M. G.

Lithodomus dactylus Cuv., Krebs. 130.

Lithodomus dactylus Sby., I'feiffer, Arehiv fiir naturg., 1S40. 260. Ca. Lithodomus forficatus Rav., Am. Marine Conch., 188. SC.

Lithorlomus niger D'Orb., Moll. Cuba, ii, pl. xxviii, figs. 10-11. 331. Lithodomus niger List., Arango, Fauna Mal. Cuba, 1S78. 267. Ca. II. SD. Santa Lucia. G.

Lithodomus niger List., Beau. 22. G.

Lithodomus niger List., Jour. de Conch., iv, 415. G.

Lithodomus (Botula) semen Lam., Beau. 22. G.

Lithodomus semen Lam., Jour. de Conch., iv, 415. G.

Lithophaga appendiculata Phil., Jahrb., ii, 252.

Lithophaga appendiculata Phil., Poulsen. 16.

Lithophaga caribæa Phil., Poulsen. 16.

Lithophaga caudigera Lam., Poulsen. 16.

Lithophaga cinnamomea Lam., Poulsen. 16.

Lithophaga crenulata Dkr., Zt. Mal., v, 180.

Lithophaga dactylus Sby, Poulsen. 16.

Lithophaga divaricata Phil., Poulsen. 16.

Lithophaga fusea Gmel., Poulsen. 16.

Lithophaga lithoglypha Menseh., Marrat, Argo Exp., 1876. 6. Aa.

Lithophaga nasuta Phil., Marrat, Argo Exp., 1876. 6. Aa.

Lithophaga nigra D'Orb., Poulsen. 16.

Lithophaga opifex Say, Jahrb., ii, 252.

Lithophagus antillarum D'Orb., Krebs. 130.

Lithophagus antillarum Phil., Krebs. 130.

Lithophagus antillarum Phil.,* Zt. Mal., ir, 116. ST.

Lithophagus aristatus Sol., Mal. B1., x, 229.

Lithophagus bisulcatus D’Orb., Krebs. 129.

Lithophagus caribæa Phil.,* Zt. Mal., iv., 116.

Lithophagus caribæus Phil., Krebs. 130.

Lithophagus caudigerus Lam., Nal. B!., x, 229.

Lithophagus cinnamomeus Chem., Mal. B1., x, 229. Ca.

Lithophagus corrugata Phil., Krebs. 130.

Lithophagus dactylus Sby., Krebs. 130.

Lithophagus fuscus Gmel., Krebs. 130. ST. SCx. Tort.

Lithoplagus niger Lister, Krebs. 130.

Lithopoma (see Trochus) Marrat, Argo Exp., 1876. 6.

Lithopoma tuber Linn., Arango, Fanua Mal. Cuba, 18is. 180. Ca. Mr. SCx. Ba.

Lithopoma tuber L., var. spinosa, Marrat, Argo. Exp., 1876. 1.. Wavaua.

Litiopa atlantica Rang, Moll. of Bermudas. 19. 
Litiopa bombix Kien., Moll. Cuba, ii, 149.

Litiopa bombyx Rang, Verrill, List, 1884. 269.

Litiopa carinata Pfr., Archiv für naturg., 1840. 255. Ca.

Litiopa effusa C. B. Adams, Contr. to Conch. 71. J.

Litiopa effusa Ad., Krebs. 52.

Litiopa maculata Rang, Arango, Fanna Mal. Cuba, 1878. 204. Ca.

Litiopa maculata Rang, Krebs. 52. ST.

Litiopa maculata Rang, Moll Cuba, ii, 149.

Litiopa melanostoma Rang, Arango, Fauna Mal. Cuba, 1878. 204. Ca.

Litiopa melanostoma Rang, Moll Cuba, ii, 149.

Litiopa nitidula Pfr., Archiv für naturg., 1840. 255. Ca.

Litiopa obesa C. B. Ads., Contr. to Conch. 71. J.

Litiopa obesa Adams, Krebs. 52. ST.

Litiopa striata Pfr., Arango, Fauna Mal. Cuba, 187s. 204. Ca.

Litiopa striata Pfr., Krebs. 52.

Litiopa striata Pfr., Archiv für naturg., 1840. 255. Ca.

Litiopa ventrosa Pfr., Archiv für naturg., 1840. 255. Ca.

Littorina (see Melaraphe, Tectarius).

Littorina adamsii Rve., Mal. Bl., xxiii, 142.

Littoriua angulifera Desh., Mal. Bl., xxiii, 137.

Littoriua angulifera Lam., Krebs. 58.

Tittorina angulifera Lam., Mal. Bl., xxiii, 137.

Littorina angulifera Lam., Poulsen. 9.

Littorina angulifera Sby., Mal. Bl., xxiii, 137. J.

Littorina angustior Mörch, Jahrb., x, 220. Ca.

Littorina angustior Mörch, Mal. Bl., xxiii, 139.

Littorina angustior Mörch, Poulsen. 9.

Littorina antoni Phil., Jahrb., x, 227.

Littorina antoni Phil., Mal. Bl., xxiii, 132.

Littorina antonii Phil., Krebs. 59.

Littorina arenica Nutt. Phil.,* Zt. Mal.. ii, 166. (Fayal ?) SV.

Littoriua carinata D'Orb., Araugo, Famna Mal. Cuba, 187S. 158. Ca. Ba.

Littorina carinata D'Orb., Jahrb, ii, 244.

Littorina carinata D'Orb., Jahrb., x, 220.

Littorina carinata D'Orb., Krebs. 59.

Littorina carinata D'Orb.* Mal. Bl. xxiii, 139. M.

Littorina (Melaraphe) carinata D'Orb., Marrat, Argo Exp., 1876. 18. Abaco.

Littorina carinata D'Orb., Moll. Cuba, i, pl. xr, figs. 1-4. 209.

Littorina carinata D'Orb., Poulsen. 9.

Littoriua carinata Phil., Mal. B1., xxiii, 139. M.

Littorina carinata Reeve, bis, Jahrb., x, 220. ST.

Littorina carinata Sby., Mal. Bl., xxiii, 139. Ca. 
Littorina (Melaraphe) carinifera D'Orb., Marrat, Argo Exp., 18i6. 9. SV. Santa Marta. Athol Isl.

Littorina (Melaraphe) carinitera Mke, var., Marrat, Argo Exp., 1876. 16. Nassaut.

Littorina cingulata Pfr., Mal. Bl.. xxiii, 138.

Littorina colnmellaris D'Orb., Arango, Fanna Mal. Cuba, 1878. 158. Ca. G.

Littorina columellaris D'Orb., Beau. 12. G.

Littorina columellaris D'Orb., Jahrb., x, 217. Fla.

Littorina columellaris D'Orb., Jour. de Conch., iv, 417. G.

Littorina columellaris D'Orb., Krebs. 59.

Littorina columellaris D'Orb., Mal. Bl., xxiii, 135, 136. M.

Littorina (Melaraphe) columellaris D'Oıb., Marrat, Argo Exp., 1876. 12.

Tucacas. VCz. Nassau.

Littorina columellaris D'Orb., Moll. Cuba, i, pl. xr, figs. 18-20. 213.

Littorina columellaris D'Orb., Poulsen. 9.

Littorina columellaris Phil., Mal. Bl., xxiii, 136.

Littorina cubaua Wein., Jahrb., $x, 220$. Ca.

Littoriva dilatata Arl., Moll. of Bermudas. 19.

Littorina dilatata D'Orb., Am. Marine Conch. 79.

Littorina dilatata D'Orb., Arango, Fauna Mal. Cuba, 1878. 158. Ca. G.

Littoriua dilatata D'Orb., Beau. 12. G.

Littorina dilatata D'Orb., Jour. de Conch., r, 152. G.

Littorina dilatata D'Orb., Krebs. 59.

Littorina dilatata D'Orb., bis, Mal. Bl., xxiii, 133, 134. Ca.

Littorina dilatata D’Orb., Moll. Cuba, i, pl. xiv, figs. 20-23. 207.

Littorina dilatata D'Orb., Poulsen. 9.

Littorina dilatata Phil., Mal. Bl., xxiii, 134. Ca.

Littorina dispar Linu., Mal. Bl., xxiii, 143.

Littoriua d'orbigniana Phil., Krebs. 61.

Littorina d'orbigniana Phil., Mal. Bl., xxiii, 138.

Littorina flava Brod., Jahrb., ii, 244.

Littoriua flava Brod., Jalırb., x, 217. Fla. Bz.

Littorina flava Brod., Krebs. 59.

Littorina flava Brod., Mal. Bl., xxiii, 136.

Littorina flava Brod., Poulsen. 9.

Littorina flava D'Orb., Mal. Bl., xxiii, 136.

Littorina flava King, Guppy, Paria Fauna, 1877. 140.

Littorina flava Phil., Mal. Bl., xxiii, 136.

Littorina floccuta Beck, Jahr., x, 220.

Littorina floceosa Beck, Mal. Bl., xxiii, 138.

Littorina flocesa Beck, Poulsen. 9.

1.ittorina fusca Pfr., Arango, Fauua Mal. Cuba, 1878. 159. Ca. Ba. Littorina fusca Pfr., Jahrb., x, 219. J.

Littorina fusca Pfr., Krebs. 60. 
Littorina fusca Pfr., Mal. Bl., xxiii, 141.

Littorina fusca Pfr., Pfeiffer Archiv fiir naturg., 1840. 254. Ca.

Littoriua fusea Sby, Mal. Bl., xxiii, 141.

Littorina glaucoeineta Beck, Mal. Bl., xxiii, 138.

Littorina globosa Dkr., Zt. Mal., x, 70. SV.

Littorina gundlachi Phil., Jahrb., x, 227.

Littorina gundlachi Phil., Krebs. 59.

Littorina gundlachi Phil.,* Mal. Bl., xxiii, 142.

Littorina gundlachi Phil.,* Zt. Mal., r, 150. Ca.

Littorina guttata Phil., Beau. 12. G.

Littorina guttata Phil., Jahrb., x, 221. G.

Littorina guttata Phil., Jour. de Conch., v, 153. G.

Littorina guttata Phil., Krebs. 59. ST. SJ. PPl. NGr. SM.

Littorina guttata Phil., Mal. B1., xxiii, 141.

Littorina (Melaraphe) guttata Phil., Marrat, Argo Exp., 1876. 14. Ca. Littorina hiclalgoi Arango, Fauna Mal. Cuba, 18ъS. 159. Havana.

Littorina irrorata Say, Jour. de Conch., iv, 417。 G.

Littoriua irrorata DeKay, Mal. Bl., xxiii, 135.

Littorina irrorata Gray, Mal. Bl., xxiii, 135.

Littorina irrorata var. Pet., Krebs. 59.

Littoriua irrorata Phil., Mal. Bl., xxiii, 135.

Littorina irrorata Say, Am. Marine Conch. 80.

Littorina irrorata Say, Jahrb., x, 217. Fla. Bz.

Littorina irrorata var. Say, Jour. de Conch., ii, 428. G.

Littorina irrorata Say, Mal. Bl., xxiii, 135. Fla.

Littorina irrorata Say, Poulsen. 9.

Littorina jamaicensis C. B. Adams, Contr. to Conch. 71. J.

Littorina jamaicensis C. B. Adams, Jahrb., x, 220.

Littorina jamaicensis Ad., Krebs. 60.

Littorina jamaicensis C. B. Adams, Mal. Bl., xxiii, 139, 140. J.

Littorina jamaicensis C. B. Adams, Poulsen. 9.

Littorina lamellosa Mont., Mal. B1., xxir, 96. ST.

Littorina lineata D'Orb., Arango, Fauna Mal. Cuba, 1878. 159. Ca. M. Ba.

Littorina lineata D'Orb., Beau. 12. G.

Littorina lineata D'Orb., Jahrb., x, 220. Ca.

Littorina lineata D'Orb., Jour. de Conch., ii, 428. G.

Littorina lineata D'Orb., Krebs. 60. ST.

Eittorina lineata D'Orb., Mal. Bl., xxiii, 138.

Littorina lineata D'Orb., Mal. B1., xxiii, 139.

Littorina lineata D'Orb., Moll. Cuba, i, pl. xiv, figs. 24-27. 208.

Littorina lineata Lam., Arango, Fama Mal. Guba, 187s. 159. Ca. II Bir.

Littorina lineata Lam., Mal. Bl., xxiii, 137.

Littorina lineata Lam., Poulsen. 9.

Littorina lineata Phil., Mal. Bl., xxiii; 139. Ca. 
Littorina lineolata D'Orb.,** Mal. Bl., xxiii, 13S. Bz.

Littorina lunata Gould, Mal. Bl., xxiii, 142.

Littorina Iunata H. C. Lea, Mal. Bl., xxiii, 142.

Littorina mauitiana l'hil., Moll. of Bermudas.

Littorina mauritiana Phil., Jahrb., x, 220. Ca.

Littorina meleagris Beck, Mal. Bl., xxiii, $1+1$.

Littorina meleagris Beck = Phasianella punctata Pfr., Poulsen. 9.

Littorina mespilum Modeer, Krebs. 60.

Littorina mespilum Mihlf., Arango, Famma Mal. Cuba, 187S. 159. Ca.

Ba.

Littorina mespilum Miihlf., Jahrb., x, 219. J.

Littorina mespilum Miihlf., * Mal. B1., xxiii, 141.

Littorina mespilum Miihlf.=fusca I'fr., P'oulsen. 9.

Littorina mespilum Phil., Mal. Bl., xxiii, 142.

Littorina minima Gray, Hal. Bl., xxiii, 141. G.

Littorina minima Gray, Poulsen. 9.

Littorina minima Wood, Beau. 12. G.

Littorina minima Wood, Jahrb., $\mathrm{x}, 221$. G.

Littorima minima Wood, Jour. de Conch., r, 153. G.

Littorina minima Wood, Krebs. 60. SM.

Littorina modulosa Pfr., Mal. Bl., xxiii, 132.J.

Littorina muricata Linn., Am. Jour. Conch., vii, 2, 3. SD.

Littorina muricata Ad., Moll. of Bermudas. 18.

Littorina muricata Desh., Mal. Bl., xxiii, 134. J.

Littoriua muricata D’Orb., Arango, Fauna Mal. Cuba, 187s. 159. Ca.

Ba.

Littorina muricata D'Orb., Mal. Bl., xxiii, $13 \pm . \quad$ J.

Littorina mmicata D'Orb., Moll. Cuba, i, pl. xir. figs. 9-10. 203.

Littoriua muricata Fér., Pfeiffer, Arehiv fiir naturg., 1840. 25t. Ca.

Littorina muricata Linn., Araugo, Fanna Mal. Cuba, 1S78. 159. Ca.

Ba.

Littorina muricata Linn., Beau. 12. G.

Littorina muricata Liun., Jahrb., x, 226.

Littorina muricata Linn., Jour. de Conch., ii, 428. G.

Littorina muricata Linn., Krebs. 60. SM. ST.

Littorina muricata Linn., Mal. BI., xxiii, 134.

Littorina muricata Liun., Poulsen. 9.

Littorina uaticoides D’Orb., Araugo, Fauma Mal. Cuba, 1878. 159. Ca.

$\mathrm{Ba}$.

Littorina naticoides D’Orb., Jahrb., x, 219. Ca.

Littorina naticoides D' Orb., Krebs. 60.

Littorina naticoides D'Orb., Mal. Bl., xxiii, 142.

Littorina naticoides 1'Orb, Moll. Cıba, i, pl. xr, figs. 21-23. 214.

Littorina nebulosa Lam., Alango, Famna Mal. Cuba, 18бS. 159. Ca.

Littorina nebulosa Lam., Mal. Bl. xxiii, 136.

Littoriua nebulosa Lam., Poulsen. 9. 
Littorina nebulosa Phil., Mal. Bl., xxiii, 136.

Littorina nebulosa Sby., Mal. Bl., xxiii, 136.

Littorina nodulosa D'Orb., Arango, Fauna Mal. C'uba, 1878. 160. Ca. M. J. Santa Lucia. G. Ba.

Littorina nodulosa D'Orb., Beau. 12. G.

Littorina nodulosa D'Orb., Jahrb., x, 225. Fla.

Littorina nodulosa D'Orb., Jour. de Conch., ir, 417. G.

Littorina nodulosa D'Orb., Jour. de Conch., v, 152. G.

Littorina nodulosa D'Orb., Krebs. 61.

Littorina nodulosa D’Orb., Moll. Cuba, i, pl. xiv, figs. 11-14. 205.

Littorina nodulosa Gmel., Krebs. 60.

Littorina nodulosa Pfr., Arango, Fauna Mal. Cuba, 1878. 160. Ca. G. Littorina nodulosa Pfr., Jahrb., x, 227.

Littorina nodulosa Phil., Beau. 12. G.

Littorina nodulosa Phil., Jour. de Conch., v, 152. G.

Littorina nodulosa Phil. bis, Krebs. 61.

Littorina-nodulosa Phil., Mal. Bl., xxiii, 133. J.

Littorina nodulosa Wood, Arango, Fauna Mal. Cuba, 1878. 159. Ca. G.

Littorina orbignyana Phil., Beau. 12. G.

Littorina orbignyana Phil., Jour. de Conch., $\nabla, 153$. G.

Littoriua pfeifferiana Weink., Jahrb., x, 227.

Littorina punctata Pfr., Jahrb., x, 221. G.

Littorina punctata Pfr., Krebs. 59.

Littorina pusilla Phil., Mal. Bl., xxiii, 140.

Littorina pusilla Phil., Poulsen. 9.

Littorina pusilla var., or n. sp. ? Mal. Bl., xxiii, 140. Bz.

Littorina riisei Mörch, Jahrb., x, 220.

Littorina riisei Mörch, Mal. Bl., xxiii, 140. Ca.

Littorina sayi D'Orb., Jahrb., x, 217. Fla.

Littorina sayi Phil.,* Mal. Bl., xxiii, 135. Fla.

Littorina sayi Rve., Jahrb., x, 217. Fla. Bz.

Littorina scabra Anton, Jahrb., x, 227.

Littorina seabra Anton, Mal. Bl., xxiii, 132.

Littorina scabra D’Orb., Araugo, Famna Mal. Cuba, 1878. 160. Ca. M. G. J.

Littorina scabra D'Orb., Krebs. 58. ST. SJ. SCx. PR. J.

Littorina seabra D'Orb., Mal. Bl., xxiii, 137.

Littorina scabra Lam., Arango, Fauna Mal. Cuba, 1878. 160. Ca. M. G. J.

Littorina scabra Linn., Beau. 12. G.

Littorina scabra Linn., Jour. de Conch., ii, 428. G.

Littorina scabra Linn., Krebs. 61.

Littorina seabra Linn., Moll. of Bermudas. 19.

Littorina seabra Linn., Moll. Cuba, i, pl. xr, figs. 15-17. 212.

Littorina scabra Pfr., Archiv füir naturg., 1840. 254. Ca.

Littorina scabra Pfr., Mal. Bl., xxiii, 137. J. 
Littorina tessellata Phil., Arango, Fauna Mal. Cuba, 1878. 160. Ca. G. Littorina tessellata Plil., Beau. 12. G.

Littorina tessellata Phil., Jahrb., x, 221. Ca.

Littorina tessellata Phil., Jour. de Conch., v, 153. G.

Littorina tessellata Phil., Krebs. 61.

Littorina tessellata Phil., Mal. Bl., xxiii, 137.

Littorina thiarella Anton, Jahrb., x, 225. Fla.

Littorina thiarella Anton, Mall Bl., xxiii, 133. Ca.

Littorina tigrina C. B. Adams, Mal. BI., xxiii, 137.

Littorina tigrina D'Orb., Arango, Fauna Mal. Cuba, 1878. 160. Ca. Littorina tigrina D'Orb., Beau. 12. Ga.

Littorina tigrina D'Orb., Krebs. 61.

Littorina tigrina D'Orb., Mal. Bl., xxiii, 136.

Littorina tigrina D'Orb., Moll. Cuba, i, pl. xv, figs. 9-11. 211.

Littorina tigriua D'Orb., Arango, Fauna Mal. Cuba, 1878. 160. Ca.

Littorina tigrina Phil., Mal. Bl., sxiii, 136.

Littorina trochiformis Dillw., Arango, Fauna Mal. Cuba, 1878. 160.

Ca. M. J. Santa Lucia. G. Ba.

Littorina trochiformis Dillw:, Beau. 12. G.

Littorina trochiformis Dillw., Jour. de Conch.; $\nabla, 152$. G.

Littorina trochiformis Dillw., Krebs. 61. SM.

Littorina trochiformis Dillw., Mal. Bl., xxiii, 133.

Littorina trochiformis Phik, Jahrb., x, 225. Fla.

Littorina trochoides Grily, Mal. Bl., xxiii, 132.

Littorina tuberculata D'Ork., Arango, Fanna Hal. Cuba, 1878. 160.

Ca. G.

Littorina tuberculata D'Orb., Beau. 12. G.

Littorina tuberculata D'Orb., Jahrb., x, 227.

Littorina tuberculata D'Orb., Jour. de Conch., $\nabla, 152$. G.

Littorina tuberculata D'Orb., Krebs. 61. PPI.

Littorina tuberculata D'Orb., Mal. Bl., xxiii, 133. Ca.

Littorina tuberenlata D'Orb., Moll. Cuba, i, pl. xiv, figs. 15̃-19. 206.

Littorina tuberculata Gray, Jahrb., $x, 227$.

Littorina tuberculata Gray, Mal. Bl., xxiii, 132.

Littorina tuberculata Mke., Mral. Bl., xxiii, 133.

Littorina tuberculata Mlke., Pfeiffer, Archiv fiir naturg., 1840. 254.

Ca.

Littorina tuberculata Mke, Poulsen. 9.

Littorina tuberculata Phil., Mal. Bl., xxiii, 133. J.

Littorina undulata D'Orb., A rango, Fanna Mal. Cuba, 1878. 160. Ca. G.

Littorina undulata D'Orb., Jahrb., x, 221. Ca.

Littorina undulata D'Orb., Krebs. 61.

Littorina undulata D'Orb., Mal. Bl., xxiii, 137.

Littorina undulata D'Orb., Moll. Cuba, i, pl. xr, figs. 12-14, 212. 
Littoriua (Melarnphe) undulata Lam., Marrat, Argo Exp., 1876. 11. Puerto Cabello.

Littorina ziczac var. C. B. Adams, Mal. Bl., xxiii, 138.

Littoriua ziezac $\Lambda \mathrm{ngl.,}$ Mal. Bl., xxiii, 139.

Littorina ziczac Auct., Jahrb., x, 220. ST.

Littorina ziczac Chem., Bean. 12. G.

Littorina ziczac Chem., Jour. de Conch., ii, 428. G.

Littorina ziczac Chem., Krebs. 61. ST.

Littorina (Melaraphe) ziczac ('hem., Marrat, Argo Exp., 1876. 9. SV. Tucacas. Long Key Island.

Littorina ziczac Chem., Poulsen. 9.

Littorina ziczac Desh., Mal. Bl., xxiii, 138.

Littorina ziczac D'Orb., Mal. Bl., xxiii, 138.

Littoriua ziczac Pfr., Mal. Bl., xxiii, 138.

Littorina ziczac var. Pfr., Mal. Bl., xxiii, 138.

Littorina ziczac Phil., Mal. Bl., x xiii, 138.

Littorina zigzac Chem., Mal. Bl., xxiii, 137.

Littorina zigzag Chem., Arango, Fauua Mal. Cuba, 1878. 159.

Littorina zigzag Chem., Arango, Fauna Mal. Cuba, 187S. 160. Ca. II. Ba.

Littorina zigzag Chem., Jahrb., x, 220.

Littorina zigzag Dorb., Araugo, Fauma Mal. Cuba, 1878, 160. Ca. M. Ba.

Littorina zigzag D’Orb., Moll. Cuba, i, pl. xv, figs. 5-8. 210.

Littorina zigzag Lam., Moll. of Bermudas. 19.

Lituus (see Spirula).

Lituus minor Brown, Arango, Fauna Mral. Cuba, 1575. 149. Ca. Ba.

Lituus minor Brown, Moll. Cuba, i, $6 t$.

Livona (see Turbo) Marrat, Argeo Exp., 1876. 6.

Lisona pica Gmel., Mal. Bl, xxir, 116. ST.

Livona pica Linn., Marrat., Argo Exp., 1876. S. D. Tucacas. Point

Savanilla. Nassau. Long Key Island.

Lirona pica Linn., var. Marrat, Argo Exp., 1876. 18. Abaco.

Lobaria (see Sanguinolaria).

Lobaria rosacea Schum., Krebs. 104.

Lobiger souverbii Fisch., Beau. 20. G.

Lobiger souverbii Fisch.* Jour. de Conch., r, pl. i, f. 7-10. 273. G.

Lobiger souverbii Fisch., Jour. de Conch., xi, 48. G.

Lobiger souverbii Fisch., Mal. Bl. iv, 220. G.

Lobiger sowerbii Fisch., Krebs. 91.

Lobiger sowerbyi Fisch., Mal. Bl., xxii, 179. G.

Loligo (see Ommastrephes) Aravgo, Fauna Mal. Cubn, 1878. 279. Autilles.

Loligo (sce Onychotenthis) Arango, Fanna Mal. Cuba, 1878. 278. Gulf of Mexico. 
Loligo (see Sepiotenthis), Arango, Fauma Mal. Cuba, 1878. 148. Ca. M.

Loligo angulatus Fér., Moll. Cuba, i, 46.

Loligo auctorum Lau., Moll. Cuba, i, 58.

Loligo bartlingii Blv., Moll. Cuba, i, 46.

Loligo bartlingii Fér., Moll. Cuba, i, 46.

Loligo bartliugii Lesueur, Araugo, Fauna Mal. Cuba, 1S7S. 278. An. tilles.

Loligo bartlingii Les., Moll. Cuba, j, 46.

Loligo bartramii Blv., Moll. Cuba, i, 59.

Loligo bartramii Fér., Moll. Cuba, i, 59.

Loligo bartramii Les., Moll. Cuba, i, 59.

Loligo bergii Blv., Moll. Cuba, i, 46.

Loligo bergii Fér., Moll. Cuba, i, 46.

Loligo brasiliensis Blv., Araugo, Fauma Mal. Cuba, 187S. 148. Ca. Loligo brasiliensis Blv., Moll. Cuba, i, 38 .

Loligo brasiliensis D'Orb., Arango, Fanna Mal. (Muba, 1s:8. 148. Ca. Loligo brasiliensis D'Urb., Moll. Cuba, i, 39-41.

Loligo brasiliensis Fér., Moll. Cuba, i, 35-42.

Loligo brevipiuna Lesueur, Kurtz. 9. SC.

Loligo brevis, Moll. Cuba, i, 41-2.

Loligo caraibra Blv., Moll. Cuba, i, 57.

Loligo caraibæa Fér., Moll. Cuba, i, э7.

Loligo cardioptera Blv., Moll. Cuba, i, 53.

Loligo cardioptera Peron, Moll. Cuba, i, 53.

Loligo eranchii Blv., Moll. Cuba, i, 32.

Loligo eranchii Fér., Moll. Cuba, i, 32.

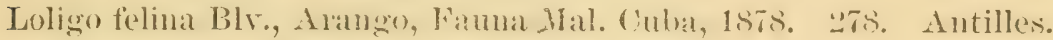

Loligo feliua Blv., Moll. Uuba, i, 46.

Loligo plei Blv., Arango, Fauna Mal. Cuba, 1878. 148. Antilles. M.

Loligo plei Blr., Moll. Cuba, i, 4. .

Loligo plei D'Orb., Arango, Fauna Mal. Cuba, 1878. 14. WV. M.

Loligo plei Fér., Moll. Caba, i, 42.

Loligo poeyanus Fér., Arango, Fauna Ma!. Cuba, 187s. 148. Ca.

Loligo poeyanus Fér., MLll. Cuba, i, 39-42.

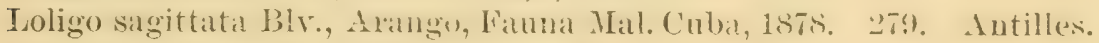

Loligo sagittata Blv., Moli. Cuba, i, 59.

Loligo sepioiclea Blr., Moll. Cuba, i, 37-8.

Loligo sepioidea Blv., Moll. Cuba: i, 35.

Loligo uncinata Q. E (x., Alango, Fauna Mal. Cuba, 1s7s. 278. Antilles.

Loligo uncinata Q. \& G., Moll. Cuba, i, 46.

Loligo vulgaris, Moll. Cnba, i, 40-1.

Longebrus (see Pyramidella, Obeliscus).

Longchrus caudidus Meusch., Mal. Bl., xxii, 158. ST.

Bull. 24-—10 
Lophyrus (sec Chiton).

Lophyrus assimilis Ree, Marrat, Argo Exp., 1876. 8. D. Abaco.

Lophyrus excavatus Chem., Arango, Fama Mal. Cuba, 1878. 231. Ca. J. ST. PR.

Lophyrus fasciatus Wood, Arango, Fauna Mal. Cuba, 1878. 231. Ca. J. ST. PR. SCx.

Lophyrus foveolatus Poli., Marrat, Argo Exp., 1876. 18. Abaco.

Lophyrus marmoratus Chem., Arango, Fauna Mal. Cuba, 1878. 232. Ca. J. S'T. SCx. G.

Lophyrus marmoratus Chem., Miarrat, Argo Exp., 1876. 8. D.

Lophyrus marmoratus Poli, Krebs. 89.

Lophyrus squamosus Limn., Arango, Fanua Mal. Cuba, 1878. 232. Ca., J., ST., PR., SCx.

Lophyrus squamosus Linn., Marrat, Argo Exp., 1876. 8. D. SV. Tucacas. A baco.

Loripes (see Lueina).

Loripes chrysostoma'Meuseh., Moll. of Bermudas. 25.

Loripes chrysostoma Meusch., Araugo, Fauna Mal. Cuba, 1878. 257. Ca. M. Fla.

Loripes ehrysostoma Poli, Krebs. 119.

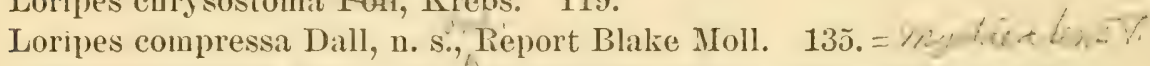
Loripes edeutula Linn., Dall, Hemphill's shells. 338. Sarasota Bay. Loripes ieteriea Rve., Report Blake Moll. 135.

Loripes philippiana Rve., Krebs. 119. = д̈ecin ๔

Lotorium lotor Mtf., Mal. Bl., xxiv, 31.

Lottia (see Acmæa, Tectura, Patella).

Lottia antillarum Sby.; Krebs. 75.

Lucapina (see Fissurella) Marrat, Argo Exp., 1576. 6.

Lueapina cancel!ata Sol., Marrat, Argo Exp., 1576. 9. SV. VCz. Nassau. Long Key Island. Abaco.

Lucapina elegaus Gray, Arango, Fauna Mal. Cuba, 1878. 228. Ca. Ba. Lneapina fasciata Pfr., Dall, Ilemphill's shells. 336. Key West.

Lucapina minuta Sby., Marrat, Argo Exp., 1876. 18. Abaco.

Lucapina suffusa Rre., Marrat, Argo Exp., 1876. 18. Abaco.

Lucapina viridula Lam., Marrat, Argo Exp., 1876. 16. Nassau. Long Key Island. Abaco.

Lueina (see Anodonta, Codakia, Cyclas, Diplodonta, Lentilaria, Loripes, Myrtaea).

Lucina (see Loripes) Arango, Fauna Mal. Cuba, 1878. 257. Ca. Havana. M. Fla.

Lucina americana C. B. Adams, Contr. to Conch. 243. WI. Lucina americana Ad., Krebs. 118.

Lucina anatellinoides Rve., Contr. to Conch. 243. IVI.

Lucina antillarum Rve., Arango, Fauma Mal. Cuba, 1878. 255. Ca. ST. G. J. = crets Pert.

Lucina antillarum Rve., Beau. 23. G. 
Lucina antillarum Rre., Contr. to Conch. 243. WI.

Lucina antillarum Rve., Jour. de Conch., ii, 425. G.

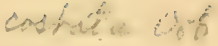

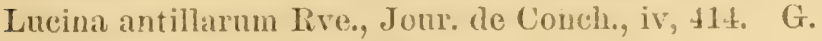

Lucina antillarum Rve., Krebs. 119.

Lucina antillarum Rve, Report Blake Moll. 136.

Lucina aurantia'Desh., Beau. 23. G.

Lucina aurantia/Desh., Coutr. to Conch. 244. WI.

Lncina aurantiandesh., Jour. de Conch., v, 150. G.

Lucina aurantiaDesh., Krebs. 118. ST. SCx.

Lucina aurantia Desh., Marrat, Argo Lxpo, 1Sï6. 17. Nassau. Abaco. Lucina aurantiarDesh., Poulsen. 15.

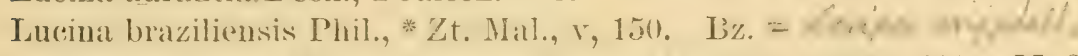

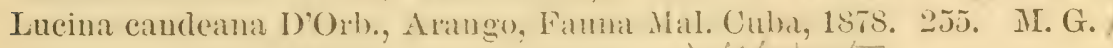

Lucina candeana D'Orb., Beatr. 23. G. Dipls de wh

Lucina candeana D'Orb., Jour. de Conch., iv, 414. G.

Lucina caudeana D'Orb., Krebs. 118.

Lucinal candeallia 1) Or.b., 1loll. Cuba, ii, pl. xxvii, figs. 13-15. 299.

Lucinar caurleana D'Orb., Poulsen. 15.

Lucina caribra D'Orb., Beau. 23. (3.

Lucina caribæa D'Orb., Jounr. de Conch., v, 151. G.

Lucina caribæa D'Orb., Krebs. 118.

Luciua carnaria Lam., Krebs. 100. = Strailla

Luciua earnaria Lam., Moll. Cuba, ii, 244.

Lucina chemnitzii Philo, Contr. to Conch. 244. IVI.

Lucina chemnitzii Phil., Krebs. 121.

Lucina ehemnitzii Phil., Zt. 11a1., r, 151. Y.

Lucina chrysostomanIreusch., Krebs. 119. S'T. SCx. Tort. PR.

Lucina chrysostomaneusch., Poulsen. 15.

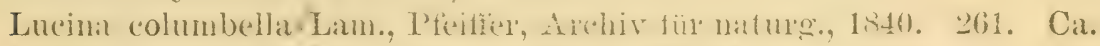

Lucina commutata/hil, Arango, Fuma Mal. Cuba. 18\%s. 236. Ca.

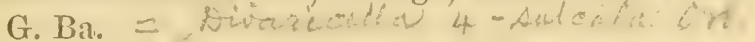

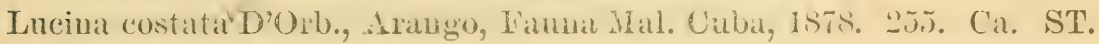
G. J.

Lucina costata'D'Orb., Beall. 2.3. G.

Lucina costatal:D'Orb., Jour'. de Concl., iv, 414. G.

Lucina costata/D'Orb., Krebs. 119. ST. SCx.

Lucina costata D'Orb., Krebs. 120.

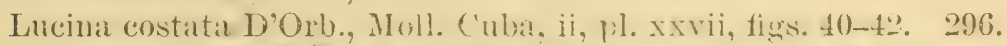

Lucinal costata dwan. = L. antillartun live., I'oulsen, 15.

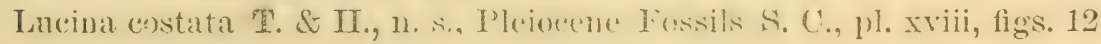
and 13. 60.

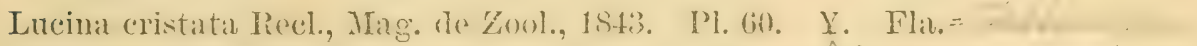
Lucina dentata Wood, Am. Marine Conch. 169. = divaziecía d, Lucina dentata Wond, Brau. 23. G.

Lucina deutata Wood, Krebs. 120.

Lucinu dentata Wood, Poulsen. $1 \tilde{5}$. 
Lncina digitalis Lam., Krebs. 119. = 6 friades

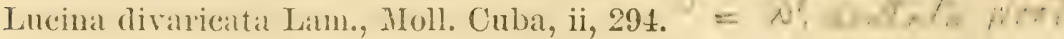

Lncina diraricata Chem., Arango, launa Mal. Cuba, 187s. 256. Ca.

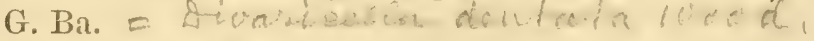

Lncina divaricatavGmel., Jour. de Conch., ii, 425. G.

Lucinat divaricata Lam., Jour. de Conch., ix, 318. Fla.

Lueina divaricatarLam., Jour. de Conch., ix, 333.

Lncina divaricata Linu, Beau. 23. G.

Lucina (Cyclas) divaricata Linn., MIarrat, Argo Exp., 1876. 19. Abaco.

Lncina divaricata Rre., Contr. to Conch. 244.

Lucina divaricata Rve., Krebs. 121.

Lucina edentula Arango, Fanna Mal. Cuba, 1878. 257 Ca. Havana.

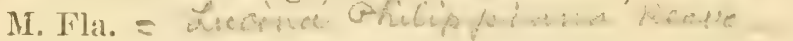

Lucina edentula Linn., Jour. de Conch., vi, 139.

Lucina edentula Limn., Jour. de Conch., vii, 26. MG.

Lucina edentula Linn., Jour. de Conch, vii, 34, 35. G.

Lucina edentulat Linn., Jour. de Conch., ix, 318. G.

Lucina edeutula Linn., Jour. de Conch., ix, 333.

Luciua elentula:Phil., * Zt. Mal., ii, 180.

Lucina edentula Chem., Bean. 23, G.

Incina ertentula Dourb. Arango, Fanna MIal. Cuba, 1878. 257. Ca.

Havana. M. Fla.

Lucina edentulavLam., Moll. Cuba, ii, 399.

Lucina edentulakinn., Am. Marine Conch 169.

Lucina edentular (Venus) Linn., Contr. to Conch. 244. WI.

Lucina edentularLinn., Jour. de Conch., ii, 425. G.

Lucina edeutularLim., Zt, Mal., ii, 179.

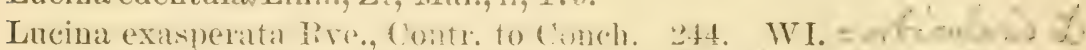

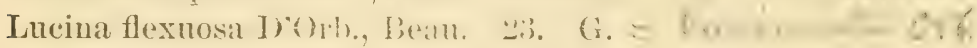

Lucina flexuosa D'Orto, Krebs. 119.

Lucina florilana/Con, Poulsey. 15.

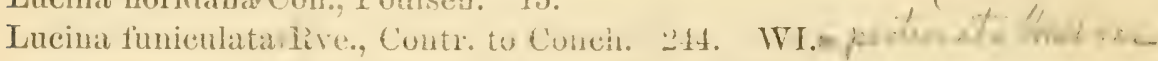

Lueina funienlatallere, Report Blake IInll. 136.

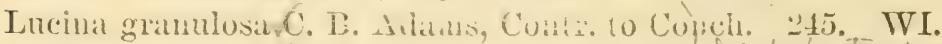

Lucina granulosal C. B. Allams, Krebs. 119. Diplodmta Demeaspera

Lueina granulosa C. B. Adaus, Poulsen. 15.

Lucina granulosa C. 13. Adams, syopos. 9. 1845. J.

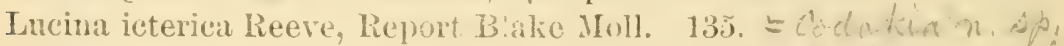

Lucina imbricatulir O B. Adims. Bean. 23. G. E peaten ven, fonn

Lucina imbricatula C. B. Adams, Coutx. to Conch. 245. WI.

Lacina imbricatula Ads., Jom, de Conch., ir, 114 . G.

Lneina imbricatula C. B. Adams, Krebs. 120.

Incina imbricatula O. B. Adams, Synops. 10. 1845. J.

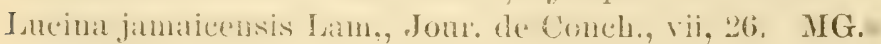

Lucina jamaicensis Lam., Jour. de Conch., vii, 34. G. J.

Lucina jamaicensis Lam., Jour. de Couch., vii, 35. G. J. 
Lucina jamaicensis Lam., Jour. de Conch, ix, 31s. .J. Cx.

Lucina jamaicensis Lam., Jour. de Conch., iv, 319, 321.

Lucina jamaicensis Lan., Jour. de Conch., ix, 333.

Lucina jamaicensis Lam., Zt. Mal., ii, 180. J.

Lucina jamaicensis Chem., Beau. 23. G.

Lncina jamaicensis (Venus) Chem., Contr. to Conch. 2t5. WI.

Lncina jamaicensis Chem., Jour. de Conch., ii, 425. G.

Lucina jamaicensis D'Orb., Moll. Cuba, ii, 29)

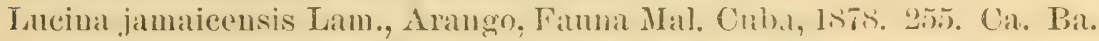

Lucina jamaicensis Lam., Moll. Cuba, ii, 294.

Lucina jamaicensis Spgl., Jahrb., ii, 250.

Lucina jamaicensis Spgl., Krebs. 119. ST. SCx. ST. Tort.

Lucina jamaicensis Spgl., Poulsen. 15.

Lucina janeirensis Rve., Contr. to Oonch. 245. WT. N.t.

Lucina janeiroensis Rre., Krebs. 119. ST.

Lueina kiawahensis Holm., n. s., P’ost-PJoineme Fosils s. C., pl. vi, fig. 5. 2!).

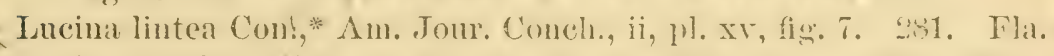

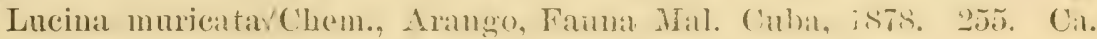
M. J. S'T. G.

Lucina muricatarchem., Beau. ๓3. G.

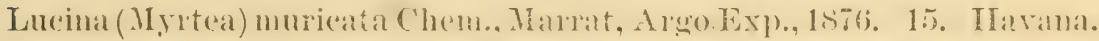
Lucina muricata D'Orb.' Arango, Famua Mal. Cuba, 187S. 25ธ̃. Ga. M. J. ST. G.

Lucina muricata'D'Orb., Nioll. Cuba, ii, 295.

Lucina muricata'Linn., Jour. de Conch., iv, 414. G.

Luciua muricata Spgl.=L. scobinata Recl., Poulsen. 15.

Lucina nassula Compad, Proce Acad. Nat. Sil. Phil., ii, 24, 1St5̃. Tampa. $= \pm$, Linter Cenche 1866 ,

Lucina nasuta Cou., Gunpy, Paria Fauna, 1877. 150.

Lucina obliqua Rve., Krebs. $120 .=p+4 a$.

Lucina occidentalis Rre., Contr. to Conch. 245. $=$

Lucina occidentalis live., IKrebs. 120.

Lucina occidentalis Reeve, Report Blake Moll. 136.

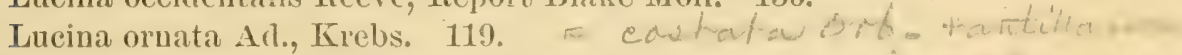

Lucina orstedti-Mörch, Poulsen. 15.

Lucina patagonica, Moll. Cuba, ii, 302.

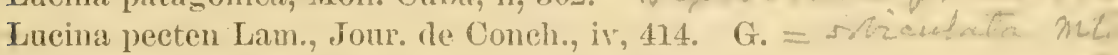

Lucina pecten Lam., Jour. de Couch, ix, 318.

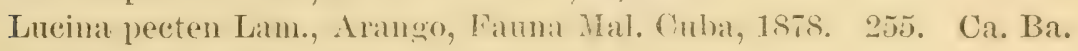

Lucina pecten Lam., Beau. 23. G.

Lucina pecten Lam., Jour. de Conch., ii, 425. G.

Lucina pecten Lam., Krebs. 120. S1. Tort.

Lucina (Codakia) pecten Lam., Marrat, Argo Exp., 18:6. 12. Tucaeas. Nassau.

Lucina pecten Lam., Poulsen. 15.

y te Ti a li (149) 
Incina pecten Petit, Contr. to Conch. 245. = osfiecelata $71 \mathrm{~kJ}$ -

Luciua pecten Rire, Uontr. to Conch. 245.

Lncina pecten Rre., Krebs. 120.

Lucina pecten Rve., Report Blake Moll. 136.

Lucina pectinatave. B. Arams, Contr. to Conch. 245. WI.

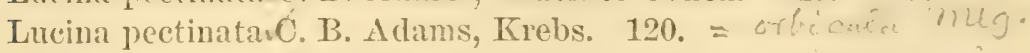

Lucina pectiuellac. B. Arlams, Contr. to Conch. 246. WI.

Lucina pennsylvanica Linu., Jour, de Coneh., vii, 26, 33, 34. MG.

Lucina peunsylranica Linn., Jour. de Conch., ix, 31s. J. G.

Luciua penusylvanica Linu, Jour. de Conch., ix, 319, 321, 333.

Lnciua pennsylvaniea Linn., Krebs. 21. S'I. Tort. SM.

Lncina pennsylvauica Linn., Zt. Mal., ii, 180.

Lucina pennsylvanica D'Urb., Arango, Famna Mal. Cuba, 1878. 255.

Ca. ST. M. Ba.

Lucina pennsylvanicaL Lam., Moll. Cuba, ii, 300.

Lucina pennsylvanica Linn., Arango, Fanna Mal. Cuba, 1875. 255. Ca. ST. M. Bir.

Lueina pennsylvanicalLimn., Beau. 23. G.

Luciua penusylranich (Ventas) Liun., Contr. to Conch. 246. WI.

Lueina penusylvanicatLiun, Jour. de Cuba, ii, 425. G.

Lucina pennsplrauica: Linu., Krebs. 120. ST. SOx. ST. Tort.

Lucina pennsylvanica Linu., Poulsen. 15.

Lucina pilula C. B. Arams, Contr. to Conch. 246. WI. Dims zirelle Lucina pilula Ad., Krebs. 120. ST.

Lncina pisum, ?Jome, de Conch., iv, 414. G.

Lucina pulchellal O. B. Adams, sirnops. 10. 1845. J.

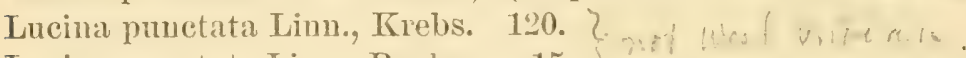

Lucina punctata Linn., Poulsen. 15.

Lucina pusilla Gla., Am. Narine Conch. 169. NC. Nopecruc

Lucina pusilla Gid., Otia. 239. NC.

Lucina qualrisulcata Dorb., Alango, Famua Mal. Cuba, 1878. 256. Ca.

G. Ba.

Lucina quadrisulcatav'Orb., Beau. 23. G.

Lucina quadrisuleata D'Orb., Jour. de Conch., ir, 41.. G.

Incina quadrisuleata DOHh., Kinebs. 120. VgI.

Lucina quahisnleata HOrb., Noll. Cnba, ii, pl. xxvii, figs. 34-36. 294.

Lucina quadrisuleata'D'Orb., Poulsen. 15.

Lucina radula? Kurtz. 4. NC. SC. = dileo.

Lucina retieulata Lam., Moll. Cuba, ii, 241. $=216$

Lucina reticulata Lam., Krebs. 106.

Lucina scabra Lam., ? Arango, Fanna Mal. Cuba, 1875. 255. Ca. M.

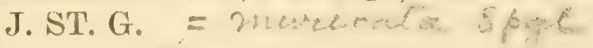

Lucina seabra Lam., Beau. 23. G.

Lucina scabrarLam., Jour. de Conch., iv, 414. G.

Lucina seabratLam., Krebs. 121. 
Lucina (Myrtæa) scabra Lam., Marrat, Argo Exp., 1876. 7. Aa. Barbuda.

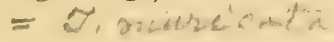

Lueina scabra Lam., Moll. Cuba, ii, 298.

Luciua scabra Rre., Contr. to Conch. 247. WI.

Lueina schrammi Crosse, Jour. de Conch., xxiv.

166. G.

Lueina schrammi Crosse,* Jour. de Conch. xxri, pl. x, fig. 6. 328. G. Lucina scobiuata Recl., Arango, Fauna Mlal. Cuba, 1878. 256. Ca. G.

Lucina scobinata Recl., Beau. 23. G. = mubtenta b/gt,

Lucina scobinata Recl.," Jour. de Conch. iii, pl. x, fig. 6, 6'. 252. G.

Lucina scobiuata Recl., Jour. de Conch., ir, 414. G.

Luciua scobinata Recl., Krebs. 121.

Lucına semireflecta/D'Urb., Krebs. $121 .=$

Lutina semireticulata D'Orb., Moll. Cuba, ii, 302.

Lucina semireticulata D'Orb., Arango, Fanna Mal. Cuba, 1878. 256. Ca.

Luciua semireticulata D'Orb., Krebs. 119.

Lucina semireticulata D'Orb., Moll. Cuba, ii, 297.

Lucina serrata D'Orb., Arango, Fanua Mal. Cuba, 1878. 256. Ca.

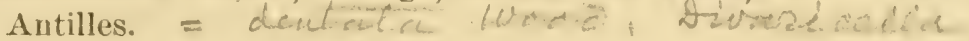

Lucina serratarD'Orb., Beau. 23. G.

Lueina serrata D'Orb., Contr. to Conch. 244.

Lucina serrata D'Orb., Krebs. 121.

Lucina serrata D'Orb., Moll. Cuba, ii, pl. xxrii, figs. 37-39. 295.

Lucina soror. C. B. Adams, Contr. to Conch. 247. WI.

Lncina squamosa Lam., Jour. de Conch., iv, 414. G. = or thicul atca

Lueina squamosa Lam., Poulsen. 15.

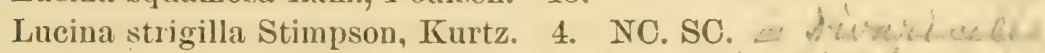

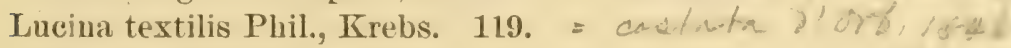

Lucina tigerina Lam., Am. Marine Conch. 155. = orízel

Lucina tigerina Lam., Jour. de Conch,, vii, 26. M. G.

Lucina tigerina Lam., Jour. de Conch., vii, 35. G.

Lucina tigerina Lam., Jour. de Conch., ix, 318.

Lucina tigerina Lam., Jour. de Conch., ix, 332.

Lucina tigerina Lam., Moll. Cuba, ii, 298.

Lucina tigerina Desh., Moll. Cuba, ii, 297.

Lucina tigerina D'Orb., Moll. of Bermudas. 25.

Lucina tigerina Lam., Contr. to Conch. 244.

Lucina tigerina Linu., Am. Marine Conch. 169.

Lucina tigerina Linn., Arango, Fanna Mal. Cuba, 1878. 256. Ca. M.

G. ST. Ba.

Lucina tigerina Linn., Beau. 23. G.

Lucina tigerina (Venus) Linn., Contr. to Conch. 247. WI.

Lueina tigerina Linn., Dall, Hemphill's shells. 338. Key West.

Lucina tigerina Limn., Jour. de Conch., ii, 4:5. G.

Luciua tigerina Linn., Krebs. 121. PR. SJ. ST. SCx. Td.

Lucina tigerina Linn., Mal. Bl., x, 228. 
Lucina (Codakia) tigerina Linn., Marrat, Argo Exp., 1876. 8. Barbuda. Tucacas. = orfecculecen

Lueina tigerina Linn., Poulsen. 15.

Lucina trisinuata D'Orb., A rango, Fauna Mal. Cuba, 1878. 256. M. G.

Lucina trisinuata D'Orb., Beau. 23. G. = Jhy redern

Lucina trisinuata D'Orb., Krebs. 121.

Lucina trisinuata D'Orb., Moll. Cuba, ii, pl. xxvii, figs. 46-49. 300.

Lueina trisuleata Conrad, Am. Journ. Sei., n. s., i, 404. 1846. Fla.

Lucina vilardiboana D'Orb., Moll. Cuba, ii, 299. Bz.

Lucina virgo Rre., Arango, Fauna Mal. Cuba, 18.8. 256. Ca.

Lucina virgo Rve., Beau. 23. G.

Lucina virgo Rve., Contr. to Conch., 247. WI.

Lucina virgo Rve., Krebs. 121. VgI.

Lucinopsis (see Artemis, Cyclina, Dosinia).

Lueinopsis gundlachi Dkr., Arango, Fauna Mal, Cuba, 1578, 252. Ca.

Havana. G. = Lit - Retics

Lucinopsis kroyeri Phil., Poulsen. 15. =

Lucinopsis tenuis Petit, Mal. Bl., iv, 27. G.

Lucinopsis tenuis Petit, Jour. de Conch., r, 155. G.

Lucinopsis tenuis Petit, Krebs. 99.

Lucinopsis tenuis Recl., Guppy, Paria Fauna, 1877. 151.

Lucinopsis tenuis 'Tryon, Arango, Fama MIal. Cuba, 187s. 252. G. Ca.

Lunatia heros Dall, Hemphill's shells. 342.

Lunatus vittatus Humpl., Mal. Bl., xxir, 63.

Luponia (see Cyprea).

Luponia flaveola Rbts., Mal. Bl., xxiv, 48.

Luponia spurca Linn., Marrat, Argo Exp., 1876. 16. Nassau.

Lutraria (see Mactra, Raëta).

Lutraria canalienlata Say, Kurtz. 4. NC. SC.

Lutraria lineata Say, Moll. Cuba, ii, 235.

Lutraria lineata Say = L. papyracea Lam., Poulsen. 14.

Lutraria papyracea Lam., Arango, Fama Mal. Cuba, 1878. 245. Ca. Lutraria papyracea Lam., Moll. Cuba. ii, 235.

Lyonsia (see Magdala, Osteodesma).

Lyousia avicularis Stimpson, n. s., Kurtz. 3. NC.

Lyonsia beana D'Orb., Jour. de Conch., iv, 414. G.

Lyonsia beana D'Orb., Moll. Cuba, ii, pl. xxv, figs, 26-28. 225. TI.

Lyonsia beauiana D'Orb., Poulsen. 15.

Lyonsia beanii D'Orb., Arango, Fama Mal. ('nba, 1868. 240. Antilles. G.

Lyonsia bulla Dall. n. S., Report Blake Moll. 107. CS.

Lfonsia floridana Conrad, Jomrn. Acarl. Nat. Sici. 1'hil., n. s., i, 208, 1849. Fla.

Lyonsia floridana Conrad, Proc. Acad. Nat. Sei. Phil., iv, 121, $18 t 8$. Tampa.

Lyonsia hyalina Conrad, Am. Marine Conch. 134. 
Lyonsia hyalina var. floridana Conrad, Dall. Hemphill's shells. 338.

Sarasota Bay. Cedar Keys.

Lyonsia plicata Gray, Mal. Bl., x, 230.

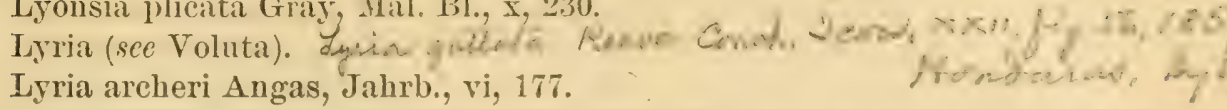

Lyria areheri Angas, Jour. de Conch., xiv, 113.

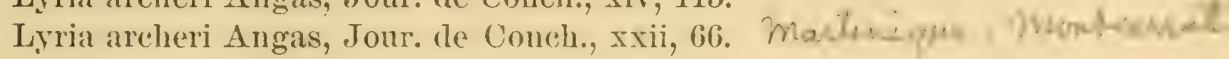

L ria beaui F. \& B., Jahrb., vi, 177.

Lyria beaui Fisch., Jour. de Conch., xiv, 112. MG.

Iyria cumingi Brod., Jour. de Conch., xiv, 114. = Westrfond the

Lyria guildingi Sby., Jour. de Conch., xir, 11\%. SV.

Macba (see Solecurtus, Solen, Cultellas).

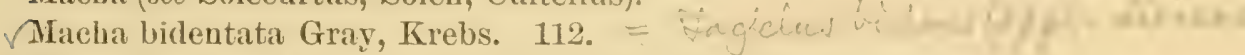

Macha cumingiana Dkr., P. Z. S., 1861. 425. Hatt.

Macha sanctæ-marthe Chem., Arango, Fanua Mal. Cuba, 18is. 239.

Antilles. Ca. G.

Machæra (see Solen, Solecurtus, Siliqua).

Machæra bidens (Solecurtus) Chem., Beau.

Machæ:a fragilis Mont., Beau. 26. G. =

Machara lucida Gould, Krebs. 112.

Macoma (see T'llina).

Macoma balthica Linn., Am. Marine Couch. 151.

Macoma constrieta Brug., Jahrb., ii, 248.

Macoma proxima Gray, Dall, Hemphill's sliells. 338.

Macoma tenuis Da Costa, Am. Marine Conch. 152. SC.

Macrodon (see Arca).

Macrodon acperula Dall, n. s., Report Blake Moll. 120.

Mactra (sce Cumingia, Lutraria, Raibal).

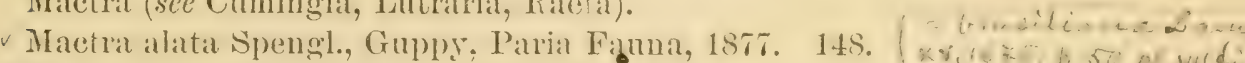

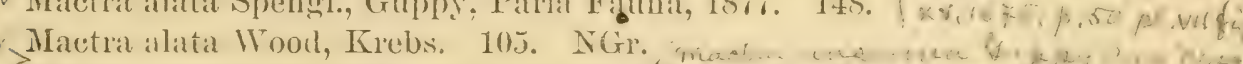

Hactra arctata Comrad, Journ. Acald. Xat. Sici. Phil, vi, 25i, pl. xi,

fig. 1, Mar., 1831. Mass. to Fla

Nactra aretata Conrad, Moll. Cuba, ii, 238.

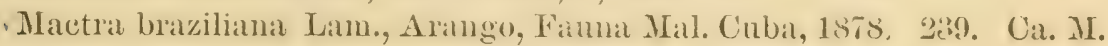

G. Ba.

Mactra brasiliana Lam., Beau. 26. G.

$\checkmark$ Mactra brasiliana Lam., Jalurb., ii, 248.

Mactra brasiliana Lam., Jour. de Conch., iv, 414. G.

$\checkmark$ Mactra brasiliana Kurtz. 4. SC.

Mactra carinata Lam., Krebs. 105.

- Mactra donacia Lam., Moll. Cuba, ii, 2238.

*. Mactra donaciformis Gray, Krebs. 10\%.

Mactra donaciformis Gray, Ponken. 14.

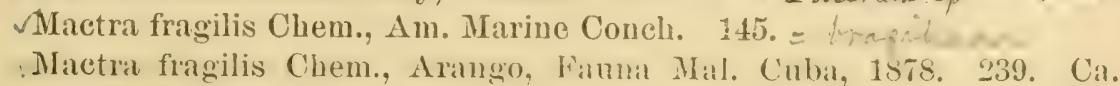

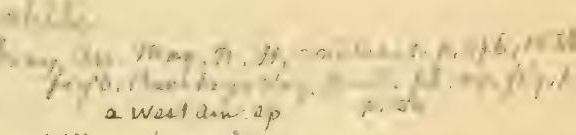

M. G. Ba.

= gradalompansis Detit. 
Mactra framilis Chem., Dall, Hemphill's shells. 338. Cedar Keys.

Mactra fragilis Chem., Jahrb., ii, 248.

Mactra fragilis Chem., Mal. Bl., x, 228.

Mactra fragilis Chem., Moll. Cuba, ii, 222.

Mactra fragilis Chem.=M. brasiliana Lam., Poulsen. 14.

Mactra fragilis D'Orb., Arango, Fauna Mal. Cuba, 1878. 239. Ca. M. G. Ba.

Mactra fragilis D'Orb., Moll. Cuba, ii, 222.

Mactra fragilis Gmel., Moll. Cuba, ii, 222.

Mactra guadalupeusis Recl., Beau. 26. Pointe à-Pìtre.

Ma tra guadalupensis Recl., Jour. de Conch., iii, pl. x., figs. 4, 4'. 249. G.

Mactra guadalnpensis Recl., Jour. de Conch., ir, 414. G.

Mactra guadalupensis Recl., Krebs. 105.

Mactra lateralis Sar Am, Marine Conch. 145. = Baldia mect acen? Mic̈tra oblonga Say, Araugo, Fauna Mal. Cuba, 1878. 239. Ca. Mí.

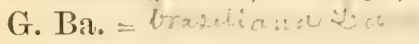

Mactra oblonga Say, Jahrb., ii, 248.

Mactra oralina Lam., Arango, Fauna Mal. Cuba, 1878. 239. Ca. M. G. Ba.

Mactra ovalina Lam., Moll. Cuba, ii, 222. = trasilian a fide Cón. Nactra ovalis Kurtz. 4.

Mactra ovalis Gld., Am. Marine Conch. 145. NC.

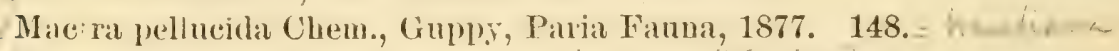
Mactra ponderosa Phil., Kurtz. 4. NO.= polécisima

Mactra Raveneli Conrad, Am. Marine Conch., 64, 1831. S. coast U. S. Mactrat rostrata Phil., Poulsen. 14.

Mactra rostrata PLil., Roemer, Texas, 452, 1849. Tex.

Mactra rostrata Phil., Zt. Mal., $v, 152$. Tex. = ri.luterabis Say.

Mactra sayii Gray, Chatrlw. Mag. N. Hist., i, 373, 1837. Fla. = ...

Mactra similis Say, Poulsen. 14.

Maetra solidissima Chem., Am. Marine Conch. 144.

Mactra striatula Linn., Krebs. 105.

Nactra tellinoides Conrad, Journ. Acad. Nat. Sci. Phil., 258, pl. xi, figs. 2, 3, April, 1831. Fla.

Mactra tumida Chem., Guppy, Paria Fauna, 187๘. 148.

Mactra turgida Gmel., Guppy, Geol. Mag., 1874. 449. Td.

Magdala (Lyousia) beaniana D'Orb., Krebs. 109.

Malleus (Avicula) candeana D'Orb., Beau. 21. G.

Malleus caudeana D'Orb., Krebs. 131.

Mallens candeanus D'Orb., Beau. 21. G.

Mumilla beruardii Recluz, Mal. Bl., xxiv, 58 .

Mamma (see Natica), Marrat, Argo Exp., 1876. S.

Mamma mamilla Linu., Marrat, Argo Exp., 1876. 18. Abaco.

Mangelia (see Plenrotoma),

Mangelia albovittata C. B. Adams, Contr. to Conch. 55. J. 
Mangelia angulata Rve., Krebs. 13. ST.

Mangelia antillarum Rve., Krebs. 13.

Mangelia astricta Rve., Krebs. 14. ST.

Mangelia badia Rve., Krebs. 13. ST.

Mangelia badia Rre., Krebs. 14.

Mangelia balteata Rre., Krebs. 13, 14. ST. SMI. SB. Ag.

Mangelia biconica C. B. Adams, Contr. to Conch. 65. J.

Mangelia biconica C. B. Adams, var, alba, Contr. to Conch. 65. J.

Mangelia biconiea U. B. Adams, Krebs. 13.

Mangelia biconica var. alba, C. B. Adams, Krebs. 13.

Mangeha brevis C. B. Adams, Contr. to Conch. 66. J.

Mangelia brevis C. B. Adams, Krebs. 13.

Mangelia candidissima C. B. Adams, Contr. to Conch. 55. J.

Maugelia candidissima C. B. Adams, Krebs. 13.

Maugelia candidissima O. B. Adams, Krebs' Remarks. 396.

Maugelia cerina Iínty \& Stimpson, Dall, Hemphill's shells. 328.

Sarasota Bay. Cedar Keys.

Maugelia ceroplasta Bush, Conn. Ac. vi, 458. Hatt.

Mangelia erassicostata C. B. Adams, Contr. to Coneh. 66. J.

Mangelia crassicostata C. B. Adams, Krebs. 13.

Mangelia densestriata O. B. Adams, Conti. to Conch. 65. J.

Mangelia densestriata U. B. Adams, Krebs. 14.

Mangelia dubia (Pleurotoma) C. B. Adams, Contr. to Conch. 55. J.

Mangelia eplamilla Bush, Conn. Ac. vi, 45i, pl. xlv, fig. 4. Hatt.

Mangelia eritima Bush, Conu. Ac. vi, 456. Hatt.

Mangelia filiformis Holmes, Am. Marine Conch. 52. SC.

Mangelia fusca (Plenrotoma) U. B. Adams, Contr. to Conch. 55. J.

Mangelia glypta Bush, Conm. Ac. vi, 462, pl. xlv, fig. 5. Hatt.

Mangelia gracilis Mont., Jahrb., ii, 241.

Mangelia horubecki Rve, Krebs. 14. ST.

Mangelia labecula Gld., Am. Marine Conch. 52.

Mangelia labecula Gld., Otia. 238. Ga.

Mangelia lanceolata C. B. Adams, Contr. to Conch. 66. J.

Mangelia lanceolata C. B. Adams, Krebs. 14.

Mangelia luteo-fasciata Rve., Krebs. 14.

Mangelia melanitica Dall, val. oxia Bus!, Comn. Ac. vi, 459, pl. xls, fig. 3. Hatt.

Mangelia microplenra Guppy, Geol. Mag., 1874. 439. Tג.

Mangelia multilineata C. B. Adams, Contr. to Conch. 55. J.

Mangelia multilineata C. B. Adams, Krebs. 14.

Mangelia muricoides C. B. Adams, Kreos. 14.

Mangelia mmicoides U. B. Adams, Contr, to Conch. 65. J.

Mangelia occidentalis Rvo., Krebs. 14. ST.

Mangelia oxytata Bush, Coun. Ac. vi, pl. xlv, fig. 1. f60. Hatt.

Mangelia psila Bush, Comn. Ac. vi, pl. xlv, fig. 2, 45. Hatt.

Mangelia quadrata Rve., Krebs. 9. 
Mangelia quadrata Rve., Krebs. 14.

Mangelia rubella Kurtz \& Stm., Am. Marine Conch. 51. NC.

Mangelia rubella Kurtz \& Stm., Kurt\%. 9. NC.

Mangelia rubella Kintz \& Stum. Proc. Bost. Soe. Nat. Hist., iv. 115, 1851.

NC. SC.

Mangelia sculpta Kurtz. 9. NC.

Mangelia stellita Stearns, Dall, Hemplill's shells. 32s. Fep West.

Mangelia stellata Stearms, Proe. B. S. X. II., xr, 22, 1872. West Fla.

Maugelia tincta Rre., Krebs. 15.

Mangelia trilineata C. 13. Adams, Arango, Fama Mal., ('uba, 1878. 221. Ca.

Mangelia trilineata C. B. Adiams, Contr. to Conch. 55. J.

Mangelia triticea Rre., Krehs. 15. Ag.

Mangelia vicina C. B. Adams, Coutr. to Conch. 66. J.

Mangelia ricina C. B. Adams, Krebs' Remarks. 396.

Mangilia (sec Mangelia).

Margarita (see Trochus).

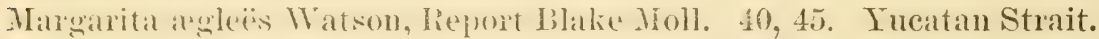
Margarita agleës Watson, Liu. Soc. Journ., 1879, xly, 704. Cnl.

Margarita asperrima Dall, n. s., Report Blake Moll. 40. CS.

Margarita biearinata Kurtz, 1. s., Kurtz. 7. SU.

Margarita (1rochus) daratus Watson, Lin. Soc. Journ., 1879, xiv, 705.

Cul.

Margarita (?) euspira I)all, n. s., Report Blake Moll. 44. CS.

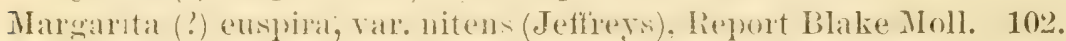

Margarita filogyra Dall, n. s., Report Blake Moll. 42. Ca.

Margarita (Tureicuia) imperalis Dall, 11. s., livport Blalke Moll. 42. Ca. Margatita infuntibulum Watson, Lin. Sox. Jonm., 1s79, xiv, 707. Ber. Margarita iris Dall, n. s., Report Blalie Moll. 43. CS.

Margarita laemella Dall, n. s., Report Blake Moll. 102. CS.

Margarita lamellosa Verrill \& Smith, Report Blake Mīoll. 40.

Margarita lissocona Dall, 11. s., Report Blake Moll. 41. CS.

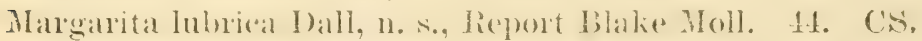

Margarita maculata Dall, n. s., Report Blake NIoll. 43: CS.

Margarita (Trochus) pompholugotus Watson, Lin. Soc. Journ., 1879, xiv, 702. Cul.

Margarita (Thoehms) rhysus Watson, Lin. Soc. Jomn., 1S79, xiv, 706. Somlirero.

Margarita scabriuscula Dall, n. s, Report Blake Moll. 41. CS.

Margarita scintillans Watson, Lin. Soc. Ionun., 1879, xiv, 712. Cul. Ber. Margarita reuustus Phil., Zt. Mal., r, 126.

Margaritifera radiata Browne, Krebs. 132.

Margaritifera radiata Leach, c. v. crocata Sw., \& v. guadalupensis D'Orb., Poulsen. 16.

Margaritiphora (see Avicula). 
Margaritiphora maiata Leach, Mal. Bl., x, 231.

Marginella (sce Closia, Persicula, Volvarina, Volutella).

Margiuella (see Iyalina), Alango, Fauna Mal, Cuba, 187s. 189. Ca.

Havana. Antilles. J. G.

Marginella (see Hyaliua), Anango, Tanua Mal. Cuba, 1sis. 190. Ca.

Marginella (see Persicula), Arango, Famma Mal. C'uba, 1sis. 186, 187.

Ca. Havana. Ba. 'Tort. Hd.

Marginella abbreviata C. B. Adaus, Coutr. to Conch. 56. J.

Marginella abbreviata C. B. Adams, Jahrb., vii, 62.

Marginella abbreviata C. B. Adams, Jour. de Conch., ii, 54. J.

Marginella affinis Rve., Am. Jour. Conch., i, 27\%. ST.

Marginella affinis Rve., Jahrb., vii, 62.

Marginella alabaster Rve., Am. Jour. Conch., v, 94. Ca.?

Marginella alabaster Rive., Arango, Fanua Mal. Cuba, 1sis, 18s. Ca. Coa.

Marginella alba C. B. Adams, Contr. to Conch. 56. J.

Marginella alba C. B. Adams, Jahrb., vii, 50. Ca. J.

Marginella albida C. B. Adams, Krebs' Remarks. 396.

Margiuella albilabris Courad, Am. Journ. Sci, 11. s., ii, 397. 1846. Fla.

Marginella albilabris Connad, P'oc. Acad. Nat. Sei. Phil, „26. 1545.

Tampa.

Marginella albolineata D’Orb., Jahrb., vii, 49. Ca.

Marginella albolineata D'Orb., Krebs. 43.

Marginella albolineata IYOrb., Moll. C'uba, ii, jl. xx, fi心s. 27-29. 99.

Marginella albolineata D'Orb. = Mr. varia Sby., Poulsen. 12.

Marginella amabilis Redf., Anu. N. Y. Lус., r, 225. 185̌.. Yueatan.

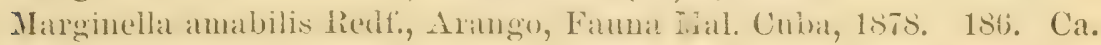

Ba. Incatan.

Marginella amabilis Redf., JaLrb., vii, 58.

Marginella amabilis Redf. $=$ M. oblonga Sws. var.,? Punlsen. 11.

Marginella amygdala Kien., Ponlsen. 11.

Marginella angustata Sby., Am. Jour. Conch., r, 91. Bz.?

Marginella autiqua Redield, Anm. N. Y. Lyc., г, 226. 1852. Va.

Marginella apieina Mke., Am. Mariue Conch., 54. WI.

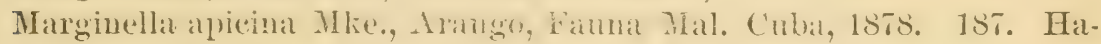

rana. Autilles. Fia.

Marginella apicina Mke., Jahrb., vii, כ̃9.

Marginella apicina Mke., Jour. de Conetr., ii, 53. Fla.

Marginella apiciua Mke., Krebs. 43. ST.

Marginella apicina Mke., Krebs' Remarlis. 396.

Marginella apiciua Mke.= M. conoirlalis, Kien., Poulsen. 11.

Marginella (Glabella) aureocincta Stearns, Proc. B. S. N. H., xv, 22,

1872. Long Kej. W. Fla.

Mrarginella arena (Val.) Kien., Arangूo, Fanna Mal, Cuba, 1sis. 190. Ca. Antilles.

Marginella avena Kien., Beau. 6. G. 
Marginella arena Kien., Jahrb., vii, 63.

Margiueila aveua Kien., Jour. de Conch., ii, 54.

Marginella avena Kien., Jour. de Conch., ii, 58.

Marginella avena Kien., Jour. de Conch., ii, 429. G.

Marginella arena Kien., Krebs. 43.

Marginella avena Kieu., Krebs. 45.

Marginella avena Kien., Moll. Cuba, ii, 98.

Marginella avena Kien., Moll. Cnba, ii, 100.

Marginella avena Val., Moll. of Bermudas. 17.

Marginella avena Val., Poulsen. 12.

Marginella avena Val., var. beyerleana Bernardi. Poulsen. 12.

Marginella arenacea Desh., Arango, Fauna Mal. Cuba, 187S. 190. Ca. Antilles.

Marginella avenacea Desh., Krebs. 15. ST. SJ. SB. Bb.

Marginella (aveua Val. var. ?) arenella Dall, Report Blake Moll. 73.

Marginella balteata Rve, Arango, Fauma Mal. Cuba, 1878. 189. Ca. J.

Marginella beyerleana Bern., Jahrb., vii, 63. (J. de C. iv, pl. 5, figs.

15-16. 149).

Marginella beyerieana Bern., Arango, Fauna Mal. Cuba, 1878. 190. Ca. Autilles.

Marginella belangeri Kien., Jahrb., vii, 57. Bz.

Marginella bibalteata Rve., Am. Jour. Couch., i, 276.

Marginella bibalteata Rve., Am. Jour. Conch., v, 94.

Marginella bibalteata Rve., Jahrb., vii, 49. J.

Marginella bifasciata Lam., Moll. Cuba, ii, 102.

Marginella bivaricosa Kien., Moll. Cuba, ii, 96.

Margiuella bivaricosa Lam., Krebs. 44.

Marginella bivarieosa Lam., Moll. Cuba, ii, 96.

Marginella bullata Born, Jahrb., vii, 57. Bz.

Marginella cærulescens Lam., Am. Jour. Conch., v, 90.

Marginella cærulescens Lam., Beau. 6. G.

Marginella cærulescens Lam., Jour. de Conch., iv, 418. G.

Marginella carulescens, Lam. Moll. Cuba, ii, 96.

Marginella calculus Redf., Am. Jour. Conch., vi, 173. SV.

Marginella calculus Redf., Jahrb., vii, 51. SV.

Marginella candida Redf., Krebs. 43. ST. SCx. SB. Ag.

Marginelia candida Sby., Alango, Fauna Mal. Cnba, 1S7s. 18s. Ca. SV.

Marginella camnella Jouss., Jahrb., vii, 58.

Marginellat caribar DOH'b, Arango, Famna Mal. Cuba, 1878. 187. Ha-

vana. Antilles. Flis.

Marginella caribxa D'Orb., Jahrb., rii, 59.

Margivella caribea D'Orb., Jomr. de Conch., iv, 418. G.

Marginella caribea D'Orb., Krebs. 43.

Marginella caribata D'Orb., Moll. Cuba, ii, pl, xx, figs. 24-26. 97. 
Marginella carnea Sby., Arango, Fauna Mal. Cuba, 1876. 186. Ca. Ba. Yucatan.

Marginella carnea Storer, Jahrb., vii, 5S. Fla.

Marginella carnea Storer, Jour. de Conch., ii, 53.

Marginella catenata Mont., Jahrb., vii, 52.

Marginella catenata Mont., Krebs. 43. SM. SB. G.

Marginella catenata Mont., Krebs. 45.

Marginella catenata Mont., Poulsen. 12.

Marginella catenata Sby., Arango, Fauna Mal. Cuba, 1878. 189. Ca. Ba.

Marginellat ehrysomelina Redf., Anu. N. Y. Ljc., iv, 492, pl. 22, fig. 2, 1817. WI.

Marginella chrysomelina Redf., Arango, Fauma Mal. Cuba, 1878. 187. Ca. Ba.

NIarginella chrysomelina Redf., Jahrb., vii, 52.

Marginella ehrysomelina Redf., Jour. de Conch., ii, 55.

Marginella chrysomelina Redf., Krebs. "43.

Marginella chrysomelina Redf., Krebs' Remarks. 396.

Marginella chrysomelina Redf., Poulsen. 12.

Marginella cincta Kien., Ain. Jour. Uonch, v, 90. J., \&c.

Marginella cineta Kien., Jahrb., vii, 60.

Marginella cincta Kien., Krebs' Remarks. 396.

Marginella couoidalis Kien., Araugo, Fauma Mal. Cuba. 1878. 187.

Havana. Antilles. Fla.

Marginella conoidalis Kien., Jahrb., vii, 59.

Marginella conoidalis Kiēn., Jour. de Conch., ii, 45.

Marginella conoidalis Kien., Jour. de Couch., ii, 53. Fla.

Marginella conoiralis Kien., Krebs. 43.

Marginella conoidalis Kien., Krebs' Remarks. 396.

Marginella conoidalis Sby., Arango, Fauna Mal. Cuba, 1875. 187.

Havaua. Antilles. Fla.

Marginella coronata Chem., Poulsen. 11.

Marginelia crassilabrum Sby., Arango, Fauna Mal. Cuba, 1S78. 188.

Ca. J. Coa. Tort. Ba.

Marginella crassilabrum Sby., Jour. de Conch., ii, 53.

Marginella crassilabrum Sby., Krebs. 43.

Marginella crassilabrum Rre., Jahrb., vii, 60.

Marginella cuvieri Desh., Jahrb., vii, 57. Bz.

Marginella delessertiana Recl., Beau. 6. G.

Marginella delessertiana Recl., Jour. de Conch., ii, 54.

Marginella delesseriana Recl., Krebs. 43.

Marginella diaphana Kien., Jahrb., vii, 59. Ca.

Marginella diaphaua Kien,, Jour. de Conch., ii, 53. Ba.

Marginella diaphana Kien., Krebs. 45. ST.

Marginella diaphana Kien.=M. pellucida Pfr, Poulsen. 11. 
Marginella diaphana Kï̌st., Arango, Fatuna Mal. Cuba. 1s7s. 188. Ca. Coa.

Marginella effulgens Rre., Am. Jour. Conch.; i, 276. S'1.

Marginella effulgens Rve., Jahrb., vii, 63.

Marginella exilis Gmel.= M. tritacea, Kien., Poulsen. 12.

Marginella fauna Sby., Am. Jour. Coucb., v, 94. Ca.?

Marginella famna Sby., Arango, Fauna Mal. Cuba, 1878. 188. Ca. Coa.

Marginella fauna Sby., Jour. de Coneh., ii, 54. Coa.

Marginella fauna Sby., Poulsen. 12.

Miarginella thavida Ad., Jour, de Conch., ii, 53. Ba.

Marginelia flavida Redf., Am. X. Y. Lye., 4, 163, pl. x, fig. 4, 1546. Ca. Marginella Havida liedf., Arangro, Fauna Mal. Cuba, 1875. 187. Ha-

rana. Antilles. Fla.

Marginella flavida Redf., Jahrb., vii, 59.

Marginella flavida Rerlf., Krebs. 43.

Marginella fluctuata C. B. Alams., Contr. to Conch. 56. J.

Marginella fluctuata C. B. Adams, Jahrb., vii, 52.

Marginella fluctuata C. B. Adams, Jour. de Conch., ii, 55. J.

Marginella fluctuata C: B. Ariams, Krebs' Remarks. 396.

Marginella frumentum Sby., Joux. de Conch., ii, 55.

Marginella frumentum Sby., Krebs. 44.

Marginella fulmiuata Kien., Jahrb., vii, 59. Bz.

Margiuella fusea Sby., Jahrb., vii, 62.

Marginella finsca Sby., Jour. de Conch., ii, 48.

Marginella fusea Sby., Jour. de Conch., ii, 54.

Marginella fiusea Sby., Krebs. 44.

Marginella fusina Dall, n. s., lieport Blake Moll. 72. Yueatan Strait. Marginella gibberula sby., Alaugo, Fama Mal. Cuba, 1878. 1S8. Ca.

.J. Coa. Tort. Ba.

Marginella glabella Linn., Mal. Bl., xii, 130 .

Marginella glans Mke., Jour. de Conch., i, 53. SD.

Marginella glaus Mke., Krebs. 44.

Marginella gracilis C. B. Adams, Am. Jour. Conch., v, 94. J.

Marginella gracilis C. B. Mrans, drango, Famua Mal. Cuba, 187S. 189.

Ca. J.

Marginella gracilis C. B. Adams, Contr. to Conch. 130. J.

Marginella gracilis Ad., Jahrb., vii, 49. J.

Marginella gracilis Ad., Jour. de Conch., ii, 54. Ba.

Marginella gundlachi Dkr , Jahrb., vii, 59. J.

Marginella guttata Dillw., Am. Jour. Conch., vi, 172.

Marginella guttata Dill»., Am. Marine Conch. 53.

Marginella guttata Dillw., Jahrb., rii, 58.

Marginella guttata Dillw., M. longivaricosá, Lam., Poulsen. 11.

Marginella guttata Tive, Alango, Fauna Hal, Cuha, 1875. 187. Ha-

vana. Ba. Tort. Hd. 
Marginella guttata Swains., Jahrb., vii, 51. SV.

Marginella guttula Rve., Jalırb., vii, 63.

Marginella hæmatita Kieu., Jahrb., vii, 46. PR.

Marginella hæmatita Kien., Poulsen. 11.

Marginella hæmatita Kien., Report Blake Moll. 137.

Marginella hæmatita Kien., Jour. de Conch., ii, こ4. PR.

Marginella houdurasensis Rve., Jour. de Conch., i, 276. Hd.

Marginella (Cryptospira) hondurasensis Rve., Marrat, Argo Exp., 1876.

12. Tucacas.

Marginella houdurensis Rve., Jahrb., vii, 60. Mexico. Hd. WI.

Marginella imbricata Hinds, Krebs' Remarks. 396. CS.

Marginella interrupta Kien., Moll. Cuba, ii, 97.

Marginella interrupta Lam., Am. Jour. Conch., v, 91.

Marginella interrupta Lam., Jahrb., vii, 51. Vz.

Marginella interrupta Lam., Kírebs' Remarks. 396. Porto Cabello.

Marginella interrupta Lam., Jour. de Conch., ii, 55.

Margiuella interrupta Lam., Jour. de Conch., ii, 57.

Marginella interrupta Lam., Krebs. 43. NGr.

Marginella interrupta Lam., Moll. Cuba, ii, 97.

Marginella interrupte-lineata Meg. v. Miihl., Poulseu. 11.

Marginella kieneriana Petit, Jahrb., vii, 51. Vz.

Marginella kieneriana Petit, Mag. de Zool., 1838, pl. 112. Vz.

Marginella labiata Kien., Jahrb, vii, 5s. Vz.

Marginella labiata Kien., Jour. de Conch., ii, 52.

Marginella labiata Val., Am. Jour. Conch., v, 91.

Marginella labiosa Redf., Araugo, Fauna Mal. Cuba, 1878. 188. Ca. J. Coa. Tort. Ba.

Marginella lachrimula Gld., Otia. 238. Gra.

Marginella lachrymula Głld., Am. Marine Concí. 54.

Marginella iactea Kien., Am. Jour. Conch., v, 93.

Marginella lactea Kien., Beau. 6. G.

Marginella lactea Kien., Jahrb., vii, 62.

Marginella lactea Kien., Jour. de Gonch., ii, 54.

Marginella lactea Kien., Jour. de Conch., ii, 429. G.

Marginella lactea Kien., Krebs. 44.

Marginelia lactea Kien., Poulsen. 12.

Marginella lactea Rve, Arango, Fauna Mal. Cuba, 1875. 190. Ca.

Antilles.

Marginella largillierti Kien., Jahrb., vii, 55. Bz.

Marginella laralleaua D’Orb., Arango, Fatua Mal. Cuba, 187s. 189.

Ca. M. ST. G. Ba.

Marginella Livalleana DOOrb., Wall, Hemphill's shells. 324.

Marginella lavalleana $\mathrm{D}^{\prime} \mathrm{Orb}$., Jahrb., vii, 5̃t. J. M.

Marginella lavalleana D’Orb., Krebs. 44. ST.

Marginella lavalleana D’Opb., Moll, Cuba, ii, pl. xx, figs. 36-3s. 101.

Bull. 24-11 
Marginella lavalleana D'Orb. = M. minima Guild., Poulsen. 12.

Marginella limatula Con., Am. Jour. Conch., v, 93.

Marginella livida Hinds, Arango, Fauna Mal. Cuba, 1878. 187.

Havana. Antilles. Fla.

Margmella livida Hinds, Jahrb., vii, 59.

Marginella livida Hinds, Jour. de Cuba, ii, 53. Ca.

Marginella livida Rve., Arango, Fauna Mal. Cuba, 1878. 190. Ca. Autilles.

Marginella lirila Rre., Jahrb., vii, 63.

Marginella lougivaricosa D'Orb., Arango, Fauna Mal. Cuba, 1878. 187.

Ilavana. Ba. Tort. Hd.

Marginella longiraricosa Lam., Am. Jour. Conch., vi, 172.

Marginella longivaricosa Lam., Arango, Fanna Mal. Cnba, 1878. 187.

Havana. Ba. Tort. ПId.

IIarginella longivaricosa Lam., Jahrb., vii, 58.

Uarqmella longivaricosa Lam., Krebs. 44.

Harginella longiraricosa Lam., Moll. Cuba, ii, 95.

Marginella maculosa Kien., Am. Jonr. Couch., vi, 173. SV., \&e.

Marginella maculosa Kien., Arango, Fauna Mal. Cuba, 1878. 188. Ca.

J. Coa. Tort. Ba.

Marginella maculosa Kien., Jahrb., vii, 51.

Marginella maculosa Kien., Krebs. 44.

Mat: ginella maculosa Kien.=M. muralis Hinds, Poulsen. 11.

Marginella maculosa Rre., Jahrb., vii, 51, SV.

Inrminella margarita Kien., Am. Jour. Conch., v, 95. Not WI.?

Iarginella margarita Kien., Arango, Fama Mal. Uuba, 1878. 188. Ca. SV.

Marginella margarita Kien., Moll. Cuba, ii, 98.

Marginella margarita Kien.=M. candida Sby., Poulsen. 11.

Margivella margaritea D’Orb., Moll. Cuba, ii, 101.

Marginella marginata Born, Krebs. 44. SM.

Marginella marginata Born, Krebs' Remarks. 396.

Mirginella (Cryptospira) marginata Boru, Marrat, Argo Exp., 1876.

13. Santa Marta.

Marginella marginata Born, Moll. Cuba, ii, 96.

Marginella marginata Born, Poulsen. 11.

Marginella minima Guild., Arango, Fauna Mal. Cuba, 1878. 188. ST. G. Ca. M.

Marginella minima Guild., Dall, Iemphill's shells. 324. Cedar Keys. Marginella minima Guild., Krebs. 44.

Marginella minuta Pfr., Arango, Fauba Mal. Cuba, 1878. 188. Ber. Ca. II. ST. G.

Marginella miunta Pfr., Jahrb., vii, 54.

Marginella minuta Píeifler, Arehiv fiir naturg, 1840. 259. 'Ca.

Marginella muralis Hiuds, Am. Jour. Conch., vi, 173. SV., \&e. 
Marginella muralis Ilinks, Arango, Famma Mal. Cuba, 1S7s. 1ss. Ca. J. Coa. 'Iort. Ba.

Marginella muralis Hiuds, Jalrb., vii, 51.

Marginella muralis Hinds, Jour. de Conch., ii, 55. J.

Marginella muralis Hinds, Krebs. 45.

Marginella navicella Rre., Am. Jour. Conch, v, 94.

Marginella nitida Hinds., Poulsen. 12.

Marginella nivea C. 13. Alaws, Arango, Fama Mal. Cuba, 1878. 189. Ca. J.

Marginella nirea C. B. Adams, Coutr. to Conch. 56. J.

Marginella nivea C. B. Adams, Julirh., vii, 59. J.

Marginella uivea U. B. Adlams, Jour. de Couch., ii, 53. J.

Marginella nivea C. B. Adams, Krebs. 45.

Marginella nivea C. B. Adams, Krebs' Remarks. 396.

Marginella nirea C. B. Adams, Ponlsen. 11.

Marginella nivea Born, Krebs' Remarks. 396.

Marginella nivosa Hinds, Jabrb, vii, 59. J.

Marwinclla obusa Redf., Am. Jomr. Couch, v, 91.

Marginella obesa Redf., Anи. N. Y. Lyc., 4, pl. x, fig. 5. 164. 1846.

Carthagena.

Marginella obesa Realf., Jahrb.; vii, 51. Bz.

Margiuelia obesa Redf., Krebs. 44. NGr.

Marginella obesa Redt., Krebs. 45.

Marginella obesa Rerlf., Poulsen. 11.

Marginella oblonga Rve., Arango, Fauna MIal. Cuba, 1878. 187. Ca. Ba. Yueatan.

Mrarginella oblonga Rve., Jahrb., vii, 5S. Fla.

Marginella olnlonga Swains., A lango, Fauna Mal. Cuba, 1S7s. 186. Ca. Ba. Yueatan.

Marginella ollunga Swains., Jalurb., vii, 5.S.

Marginella oblonga Swains., Jour. tle Conch., ii, 53.

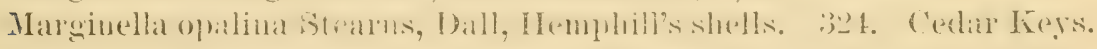
Margiuella (Glabella) opbaliua Stearns, Proc. B. S., N. H., xv, 21, 1572. Tampa. IV. Fli!.

Marginella ovuliformis Jo'b., Arango, Fanna Marl. Cuba, 187s. 188. (a) M. G. S'T.

Margiuella orulitormis D'Orb., Beau. 6. G.

Marginella ovuliformis D'Orb., Jahrb., rï, כ0. M. G. ST.

Marginella ovuliformis $\mathrm{D}^{\prime} \mathrm{Or} b$., Krebs. 45.

Marginella oruliformis D'Orb., Moll. Cuba, ii, pl. xx, figs. 33-35. 101.

Margiuella pallida D'Urb., Noll. Cuba, ï, 100.

Marginella pallida Liun., Jahrb., vii, 61.

Marginella pallida Linn., Jour. de Conch., ir, 418. G.

Marginella pallida Linn., Krebs. 45. S'T. SJ. SB. SMI. Bb.

Marginella pallida Linn., Poulsen. 12.

Marginella pellucida Pfi., Mal. Bl., xxx, „2. 
Marginella pellucida Pfr., Jahrb., vii, 59. Fla.

Marginella pellucida Pfr., Jour. de Conch., ii, 53. Ua.

Marginella pellueida Pfr., Krebs. 45. S'T.

Harginella pellucida Pfeiffer, Arehiv fiur naturg., 1840. 25S. Ca.

Marginella pellucida Schum., Poulsen. 12.

Marginella phrygia Sby., Krebs. 45.

Marginella porcellana G mel., Jahrb., vii, 50. Vz.

Marginella pruinosa Hinds, Am. Jour. Conch., r, 93.

Marginella pruinosa Hinds, Jahrb., vii, 59.

Marginella pruinosa Hinds, Jour. de Conch., ii, 53.

Marginella prunum var.?, Jour. de Conch., ii, 53. SD.

Marginella prunum Gmel., Am. Jour. Conch., v, 90.

Marginella prunum Gmel., Beau., 6. G.

Marginella prunum Gmel., Jour. de Conch., iv, 418. G.

Marginella (Cryptospira) prunum Gmel., Marrat, Argo Exp., 1876.

12. Tucacas.

Margineila prunum Gmel.=M. subcoerulea Mart., Poulsen. 11.

Marginella pudiea Gask., Arango, Fauna Mal. Cuba, 1878. 187. Ca. Ba.

Marginella pulchella Kien., Poulsen. 12.

Marginella pulcherrima Ad., Krebs. 45.

Marginella pulcherrima Gask., Arango, Famna Mal. Cuba, 1878. 189 Ca. Ba.

Marginella pulcherrima Gask., Jour. de Conch., ii, 55.

Marginella pulcherrima Gask., Poulsen. 12.

Marginella pulchra Gray, Jahrb., vii, 60.

Marginellả pulchra Gray, Jour. de Conch., ii, 53. SD.

Marginella pulchra Gray, Krebs. 45.

Narginella pulchra Gray, Poulsen. 11.

Marginella pyrulata Redf., Am. Jour. Conch., v, 91.

Marginella pyrulum Rve., Am. Jour. Conch., i, 277. ST.

Marginella pyrulum Rve., Jahrb., vii, 53. ST.

Marginella quadriplicata Rve., Am. Jour. Conch., r, 93.

Marginella redfieldii Tryon, Dall. Uemphill's shells. 324. Key West.

Margiuella roseida Redf., Am. Jour. Coneh., v, 93.

Marginella roscida Redf., Am. Marine Conch. 53.

Mirroinella roscida Redf., Dall, Hemphill's shells. 324. Cedar Key's.

Sarasota Bay.

Narginella rubella C. B. Adams., Am. Jour. Conch., v, 94. J.

Mirginella rubella C. B. Adams., Krebs. 45. ST.

Marginella rubella C. B. Arlams., Poulsen. 12.

Marginella rubella C. B. Adams, Synops. 1, 1845. J.

Marginella sagittata Hinds, Jahrb., vii, 52. Bz.

Marginella sapotilla Hinds, Am. Jour. Conch., r, 90.

Marginellit sanleyana Petit, Am. Jonr. Conch., v, 94.

Marginella sanleyana Petit, Jahrb., vii, 58. Bz.

Marginelia sauleyaua live., Jahrb., vii, 60. 
Marginella scalaris Jouss, Jahrb., vii, 47. B\%.

Marginella seminula Dall, 14. s., Report Iblake Moll. 72. Yucatan Strait.

Marginella serrata Gaskoin, Am. Jour. Conch., i, 277.

Marginella serrata Gaskoin, Am. Jour. Conch., v, 95. IVL.?

Marginella similis Sby., Am. Jour. Conch., v, 91.

Marginella similis Sby., Jahrb., vii, 51. Vz.

Marginella similis Sby., Krebs. 45.

Marginella smithii Verrill, Bush, Comn. Acarl., vi, 46:. Matt.

Marginella spilota Rav., Kurtz. 9. NO. SC.

Marginella storeria Couthony, Am. Jour. Conch., r, 94.

Marginella storeria Couthous, Bost. Tomru. Nat. Hist., i, 4.10., pl. !), figs. 1,2. 1837. Gulf of Mexico.

Marginella storeria Couthouy, Jour. de Conch., ii, 53.

Marginella storeria Couthouy, Poulsen. 11.

Marginella striata Sby., Arango Fauna Mal. Cuba, 1878. 189. Ca. ST.

Marginella striata Sby., Jahrb., vii, 47.

Marginella striata Sby., Jour. de Concb., ii, 54.

Marginella subcœerulea Mart., Krebs. 45.

Marginella subtriplicata D'Orb., Am. Jour. Conch., v, 93.

Marginella subtriplicata D’Orb., Arango, Fama Mal. Cuba, 1S7S. 190.

Ca. Antilles.

Marginella subtriplicata D’Orb., Beau. 6. G.

Marginella subtriplicata D'Orb., Jahrb., vii, 62.

Marginella subtriplicata D'Orb., Jour. de Conch., ir, 418. G.

Marginella subtriplicata D'Orb., Krebs. 46. G. SM. SJ. Ag.

Marginella subtriplicata DOrb., Moll. Cuba, ii, pl. xx, figs. 30-32. 99. Marginella subtriplicata D'Orb., Poulsen. 11.

Marginella succinea Conrad, Am. Journ. Sci., n. s., ii, 397, $18+6$. Fla.

Marginella succinea Commel, Proc. Acad. Nat. Sci. Phil., iii, 26, pl. i, fig.

16. 1845. 'Tampa.

Marginella sulcata I’Orb., Arango, Fauna M[al. Cuba, 187., 189. M.

Marginella sulcata J'Or'b., Jahrb., vii, 47.

Marginella sulcata D'Orb., Krebs. 45.

Marginella sulcata D’Orb., Moll. Cuba, ii, pl. xxi, figs. 14-16. 102.

Marginella swainsoniana Petit, Jahrb., vii, 51. SV.

Marginella tæniata Sby., Am. Jour. Conch., v, 93.

Marginella tæniata Sby., Mal. Bl., xxx, 28.

Marginella tenera Mke., Jour. de Conch., ii, 54. PR.

Marginella tessellata Lam., Am. Jour. Conch., vi, 173.

Marginella tessellata Lam., Jahrb., vii, 50. Vz.

Marginella tessellata Lam., Krebs. 46.

Marginella tessellata Sby., Jahrb., vii, 52.

Marginella torticula Dall, n. s., Report Blake Moll. 73. 
Marginella triplicata Dơrb., Arango, Famma Mal. Cuba, 1S7S. 190. Ca. Antilles.

Marninella varia Sby., Arango, Fauna Mal. Cuba, 1S7s. 190. Ca., Antilles.

Marginella varia Sby., Beau. 6. 'G.

Marginella varia Sby., Jahrb., vii, 49. Ca.

Marginella raria Sby., Jahrb., vii, 63.

Marginella varia Sloy., Jour. de Conch., ii, 51.

Marqiuella varia Sloy., Jour. de Conch., ii, 5 S.

Marginella varia Sby., Jour. de Conch., ii, 429. G.

Marginella varia Sby., Krebs. 45.

Marginulla virginea Jouss, Jahrb., vii, 59.

Marginella wallacei Jouss., Jahrb., vii, 58.

Marginella watsoni Dall, n. S., Report Blake Moll. 71.

Marginella xauthostoma Mörch, Krebs. 46.

Marginella (var.?) yncatecana Dall, Report Blake Moll. 72. Yucatan Strait.

Marginella zonata Kien., Krebs. 46.

Margiuella zonata Kien., Mal. Bl., xxx, 28.

Marginella zonata Kien., Poulsen. 12.

Marginella zonata Lam., Jour. de Conch., ii, 54.

Marisa cormu-arietis Linn., Marrat, Argo Exp., 1876. 10. Td.

Marisal luteostoma Swaims, Marrat, Mrgo Exp., 1876. 12. Tucacas.

Marsevia rangii Bergh, Mal. Bl., xxiv, 93. ST.

Martesia (see Pholas).

Martesia caribaea Arango, Fauna Mal. Cuba, 1878. 236. Ca. ST. G. Martesia corticaria Ad., Arango, Fauna Mal. Cuba, 1578. 236. Ca. ST. G.

Martesia cuneiformis Say, Am. Marine Conch. 127.

Martesia striata Leach, Krebs. 114.

Martesia striata Linn., Arango, Fannat Mal. Cuba, 1S7S. 236. Ca. G. Mathilda trochlea Mörch, Mal. Bl., xxii, 165. ST.

Mathilda trochlea Mörch, Poulsen. 8.

Mazza (see Turbinella).

Mazza scolymus Guel., Marlat, Argo Exp., 1876. 13. Santa Marta.

Meioceras (see Caccum).

Meioceras cornn-bovis Cpr., Poulsen. 11.

Meioceras deshayesii Cpr., Poulsen. 11.

Meioceras nitirlum Cpr., Poulseu. 11.

Melampus (Melamplus) biclentatus Say, Am. Jour. Conch., iv, pl. 18. fig. 5. s.

Melampus bidentatus Say, Am. Jour. Conch., ir, 16.

Melampus bidentatus Say, Dall, Hemphill's shells. 322. Tampa. Cedar Keys. St. Augustine.

Melampus (Leuconia) bidentatus Mtg., Dall, Proc. U. S. Nat. Mus., 1885. 283. 
Melampus (Detracia) bulloides, Mtg., Dall, Proc. U. S. Nat. Mus., 1855. 285. Iila. WI.

Melampus caffeus Lam., Dall, Hemphill's shells. 322. Key West.

Melampus eingulatus Fér., Jahrb., iv, 360.

Melampus cingulatus Fér., Mal. Bl., i, 170. PR.

Melampus cingulatus Fér., Jahrb., vii, 284. SV.

Melampus cingulatus Pfr., Jahrb., vii, 265. Ca.

Melampus cingulatus Pfr., Jahrb, vii, 275. J.

Melampus cingulatus Pfr., Jahrb., vii, 277. Hy.

Melampus cingulatus Pfr., Jahrb., vii, 279. PR.

Melampus cingulatus Pfr., Mal. Bl., i, 147.

Melampus coffè Linn., Jahrb., iv, 360.

Melampus coffea Linn., Mal. Bl., vii, 129. Cay.

Melampus coffea Linn.,* Am. Jour. Conch., ir, pl. xviii, figs. 7, 8. 8. Fla.

Melampus coffea Linn., Am. Jour. Conch., iv, 17.

Melampus coffea Limn., Beau. 15. G.

Melampus coffea Linn., Jahrb., vii, 275. J.

Melampus coffea Linn., Jahrb., vii, 277. Hd.

Melampus coffea Linu., Jahrb., vii, 284. M.

Melampus coffea Linn., Jabrb., vii, 2s6. Ber.

Melampus coffea Linn., Jour. de Conch., хxii, 166. M.

Melampus cotfea Linn., Jour. de Conch., xxx, 178.

- Melampas coffea Linn., Mal. Bl., i, 147.

Melampus coffea Linn., Mal. Bl., vi, 117. SCx.

Melampus coffens Lam., Jahrb., vii, 279. PR.

Melampus coffens Linn., Jahrb, vii, 265. Ca.

Melampus coffeu- Linn., Marrat, Argo Exp., 1876. 16. Nassau.

Melampus coniformis Brug., Mal. Bl., i, 170. PR.

Melampus (Auricula) coniformis Brus., Beau. 15. G.

Melampus couiformis Fér., Jahrb., vii, 282. G.

Melampus corneus (Desli.) Stm., Am. Jour. Conch., iv, 11

Melampus coronatus C. B. Adams, Jahrb., vii, 275. J.

Melampus coronatus C. B. Adams, Mal. Bl., i, 147. J.

Melampus dominicensis Fér., Jahrb., vii, 282. G.

Melampus flavus Gmel., Jahrb., iv, 360.

Melampus Harus Gmel., Mal. Bl., vii, 129. Cay.

Melimpus flarus Gmel.," Am. Jour. Conch. iv, pl. 18, fig. 6. 8. Fla.

Melampus flavus Gmel., Am. Jour. Conch., iv, 17.

Melampus flavus Gmel., Beaur. 15. G.

Melampus flarus Gmel., Dall, Hemphill's shells. 322. Fernandina,

Fla.

Melampus flarus Gmi.l., Jahrb., vii, 265, Ca.

Melampus flavus Gmel., Jahrb., vii, 275. J.

Melampus flavus Gmel., Jahrb., vii, 277. Hy.

Melampus flarus Gmel., Jahrb., vii, 279. PR. 
Melampus flarus Gmel., Tahbr., vii, 282. G.

Melampus tlavus Gmel., Mal. BI., i, 147.

Melampus flavus Gmel., Mal. Bl., v, 196. Ca.

Melampus flavus Gmel., Moll. of Bermudas. 22.

Melampus floridanus Shuttlew., Mal. Bl., i, 147. Fla.

Melampus globulus Fér., Mal. Bl., i, 147.

Melampus gundlachi Pfr., Mal. Bl., i, 147.

Melampus gundlachi Pfr., Mal. Bl., ii, 85. Ca.

Melampus gundlachi Pfr., Zt. Mal., x, 126. Ca.

Melampus lineatus Say, Dall, Proc. T. S. Nat. Mus., 1885̃. 282. Fla. Tex. Hatt.

Melampus monilis Lam. Mal. Bl., i, 170. PR.

Melampus (Auricula) monilis Lam., Beau. 15. G.

Melampus nitens Lam. Mal. Bl., i, 170. PR.

Melampus (Auricula) nitens Lam., Beau. 15. G.

Melampus obliquus (Auricula Say), Kurtz. 9. SC.

Melampus obliquus Say, Mal. Bl., i, 147. Cl.

Melampus olivula MFor., Mal. B1., i, 147. Bz.

Melampus ovula Pfr., Jahrb., vii, 282. G.

Melampus poeyi Pfr., Mal. Bl., i, 147. Ca.

Melampus poeyi Pfr.,* Zt. Mal., x, 126. Ca.

Melampus pusillns Gmel., Jahrb., iv, 360.

Melampus pusillus Gmel., Mal. Bl., vii, 129. Cay.

Melampus pusillus Gmel., Beau. 15. G.

Melampus pusillus Gmel., Jour. de Conch., xxii, 167. M.

Melámpus pusillus Gmel., Mal. Bl., i, 147.

Melampus pusillus Gmel., Mal. Bl., v, 196. Ca.

Melampus pusillus Gmel., Jahrb., vii, 265. Ca.

Melampus pusillus Gmel., Jahrb., vii, 275. J.

Melampus pusillus Gmel., Jahrb., vii, 277. Нy.

Melampus pusillus Gmel., Jahrb., vii, 279. PR.

Melampus pusillus Gmel., Jahrb., vii, 284. M.

Melampus redfieldi Pfr.,* Mal. Bl., i, 112. Ber.

Melampus redfieldi Pfr., Mal. Bl., i, 14\%. Ber.

Melampus redfieldii Pfr., Moll. of Bermudas. 22.

Melampus (see Leuconia, Detracia, Tifata, Tralia, Sayella).

Melania acus Pfr. (note), Mal. Bl., xxii, 166. Ca.

Melavia attenuata Anth., Arango, Fanna Mal. Cuba, 1S7s. 140. Ca.

Melania brevis Brot, Arango, Famma Mal. Cuba, 1878. 141. Ca.

Melania brevis D'Olb. Arango, Fanna Mal. Cuba, 1878. 141. Ca.

Melania brevis D'Orb., Moll. Cuba, ii, pl. x, fig. 15, 10.

Melania campanellæ Phil., Mal. Bl., xxii, 162. ST.

Melania conica D'Orb., Arango, Fauna Mal. Cuba, 1878. 140. Td. Ca.

Melania conica D'Orb., Moll. Cuba, ii, pl. x, fig. 20, 12.

Melania cubaniana D’Orb., A rango, Famna Mal. Cuba, 1878. 140. Ca. 
Melania enbaniana D'Orb., Moll. Cuba, ii, pl. x, fig. 16. 11.

Melania gemella Rev. Arango, Fauna Mal. Cuba, 1szs. 141. Td. Ca.

Melania nigrata Poes, Arango, Fauna Mal. Cuba, 1578. 140. Ca.Tr.

Melania nigrita (Poey) Rev., Arango, Fama Mall. Cuba, Lsos. 141. Ca.

Melania ormata Poes, Arango, Famna Mal. Cuba, isis. 140. Ca.

Melania pallida Gundl., Arango, Fama Mal. Cuba, 1878. 140. Ca.

Melania spinifera Ad., Arango, Fauna Mal. Cuba, 187s. 140. Ca. Antilles.

Melania turritella Pfr. (note), Mal. Bl., xxii, 166. Ua.

Melania zebra Brot, Arango, Fauna Mal. Cuba, 1878. 141. Ca.

Melanopsis cepnla Gupp5, Geol. Mag., 1874. 442.

Melanopsis lineolata Gray, Arango, Fanma MIal. Cuba, 185s. 142. J.

Melaraphe (see Littorina), Marrat, Argo Exp., 1S76. 9.

Melaraphe carinata D'Orb., Jahrb., ii, 244.

Melaraphe flava Brod., Jahrb., ii, 244.

Melaraphe scabra Linn., vars. Dall, Iemphill's shells. 33t. Key West.

Meleagrina (see Margaritiphora).

Meleagrina longisquamosa D'Ork., Arango, Fauna Mral. Cuba, 1878. 268. Ca.

Meleagrina longisquamosa Dkr., Arango, Fauna híal. Cuba, 1878. 268. Ca.

Meleagrina longisquamosa Dkr., ${ }^{*}$ Zt. Mal., ix, $76 . \quad \mathrm{Vz}$.

Meleagrina pica Phil., Arango, Fama Mal. Cuha, 1878. 268. Ca.

Meleagrina placunoides Rre., Moll. of Bermudas. 24.

Neleagrina radiata? Lam., Arango, Fauna Mal. Cuba, 187s. 268. Ca. Ba.

Meleagrina radiata Lam., Krebs. 132.

Meliua (see Isognomon, Perna).

Melina alata Gmel., Krebs. 132. VgI.

Meliua bicolor Ad., Krebs. 132.

Melina perna Linn., Krebs. 132.

Meliua perniformis Retz., Krebs. 132.

Melina vulsella Lam., Krebs. 132.

Melo reticulatus Kniph., Mal. Bl., xxir, 42.

Melongena (see Hemifnsus, Cassidula).

Melougena belknapi Petit, Mal. B1, iii, 190.

Melongena belknapi Petit, Jonr. de Conch., iii, pl. ii, fig. 5, 65. G.

Melongena belkuapi Petit, Jour de Conch., iii, 145. Fla.

Melongena belknapi Petit, Jour. de Conch,, r, 38. Key West.

Melongena bispinosa Phil., Jour. de Conch., iii, 146.

Melongena bispinosa Phil., Jour. re Conch., iii, pl. viii, fig. 3. $15 \%$.

Melongena bispinosa Phil., Jour. de Conch., v, 39. Fla.

Melongena corona Linn., Jour. de Conch., iii, 146. Fla.

Melongena fasciata Schum., Jour. de Conch., iii, 145. SD.

Melongena morio Lam., Jour. de Conch., iii, 147.

Menestho dubiosa Ads., Jour de Conch., viii, 182. G. 
Merceuaria (see Venus).

Mercenaria cimpechiensis Gmel., Mal. Bl., xii, 136.

Mercenaria fulguraus Tryou, ${ }^{*}$ Am. Jour. Conch., i, pl. xxvi, figs. 1, 2, 3.

- 297. Fla.

Mercenaria mercenaria Linn., Am. Marine Conch. 15s. NC.

Mercenaria mercenaria Linn., var. Venus notata Say, Am. Mariue Conch. 15 S.

Mercenaria mercenaria Linn., Mal. Bl., xii, 135.

Mercenaria mortoni Con., Aní. Marine Conch. 158.

Mercenaria mortoni Con., Mal. Bl., xii, 137.

Mercenaria mortoni Con., Mal. Bl., xiv, 125.

Merope (see Standella), Mariat, Argo Exp., 1876. 12.

Mesalia caribæa D'Orb., Mal. Bl., xxiii, 129.

Mesolesma concentrica Holm., n. s., Post.Pleiocene Fossils S. C., pl. vi, fig. 10.44.

Mica (see Oliva).

Mica marmorata Mart., Krebs. 38.

Microgaza rotella Dall, n. s., Report Blake Moll., 51. Bb.

Miralda babỵlonica C. B. Adams, Mal. Bl., xxii, 165. J.

Mitra albicostata C. B. Adams, Contr. to Concī. 57. J.

Mitra albicostata (. B. Arlams, Dall, Hemphill's shells. 327. Key

West.

Mitra albocincta Ar., Arango, Fama Mal. Cuba, 1578. 197. Ca.J.

Mitra albocincta C. B. Alams, Synops., 2, 1845. J.

Mitra (Turricula) albocincta C. B. Ad., Poulsen. 12.

Mitra alreolata Rve., Krebs. 36.

Mitra barbatensis Fmel., Arango, Fanna Mal. Cuba, 1878. 197. Ca. G. SV. Ba.

Mitra barbadensis Gmel., Beau. 10. G.

Mitra barbadeusis Gmel., Krebs. 36. ST. SJ. SB. SM. Bb.

Mitra barbadensis Gmel., Poulsen. 12.

Mitra caligena Rve., Krebs. 37. ST.

Mitra caeligena live., Póulsen. 12.

Mitra candida R re., Krebs. 37. ST.

Mitra candida Rve., Poulsen. 12.

Mitra carea Rve, Beau. 10. G.

Mitra carea Rve. bis, Krebs. 37.

Mitra cornicularis Lam., Zt. Mal., x, 79. SV.

Mitra dermestina Lam., ?= pulchella Rve., Krebs. 37.

Mitra dermestina Lam., Krebs. 37. ST. SJ. SM. Ag.

Mitra (Turricula) dermestina Lam., Poulsen. 12.

Mitra exigua U. B. Adams, Synops., ㄹ, 1845. J.

Mitra exilis Rve., Arango, Fauna Mal. Cuba, 1878. 197. Ca.

Mitra (Scaphella) florida Gld., Otia. 221. Fla.?

Mitra (Mitromorpha?) floridana Dall, n. s., Dall, Hemplill's shells. 327. Key West. 
Mitra (Scabricola) granulosa Brug., Marrat, Argo Exp., 1S76. 15. VCz. Mitra granulosa Lam., Am. Marine Conch. 53.

Mitra granulosa Lam., Beau. 10. G.

Mitra granulosa Lam., Krebs. 37.

Mitra granulosa Lam., Marrat, Argo Exp., 1876. 18. Abaco.

Mitra granulosa Lam, Moll. Cuba, ii, 127.

Mitra granulosa Linn., Jour, de Conch., iv, 418. G.

Mitra hanleyi Dohru, Mal. Bl. viii, 138. Figure in Dunker, nor. choncol. withont habitat. (Fla.)

Mitra huugariea Klein, Mal. Bl., xxiv, 97.

Mitra (Turricula) interpunctata Cpr., Poulsen. 12.

Mitra microzonias Kieu., Arango, Fanna Mal. Cuba, 1878. 197. Ca.

Mitra microzonias Kien. bis, Krebs. 37. Ba.

Mitra microzonias Lam., Krebs. 37.

Mitra (Turricula) microzonias Lam.= MI. sulcata Gmel., Poulsen. 12.

Mitra microzonias Rve.? Krebs. 37.

Mitra microzonias var. bifasciata bis., Krebs. 37.

Mitra monilifera C. B. Arlams, Contr. to Conch. 57. J.

Mitra norlulosa Gmel., Alango, Fanna Nal. Cuba, 1878. 197. Ca. Antilles.

Mitra nodulosa Gmel., Beau. 10. G.

Mitra nodulosa Gmel., Jour. de Conch., iv, 418. G.

Mitra nodulosa Gmel., Krebs. 37. ST. SJ.

Mitra nodulosa Gmel., Moll. Cuba, ii, 126.

Mitra nodulosa Gmel.=H. granulosa Lam., Poulsen. 12.

Mitra nucleola Rve., Krebs. 37.

Mitra obliquata Lam., P'feiffer, Archiv füir naturg., 1840. 259. Ca.

Mitra parella live., Arango, Fauna Mal. Cuba, 1sis. 197. Ca. Havana.

Mitra puella Rre., Beau. 10. G. ST. Ba.

Mitra puella Rve., Krebs. 3S. ST. SJ. SM. SCx.

Mitra (Terricula) puclla Rve., Poulsen. 12.

Mitra pulchella Rre., Beau. 10. G.

Mitra pulchella Rve., Krebs. 37.

Mitra semicostata Anton, Krebs. 37. SCx. SM. Ag.

Mitra (Turricula) semicostata Anton, Poulsen. 12.

Mitra speciosa Mörch, Krebs. 37.

Mitra striatula Lam., Beau. 10. G.

Mitra striatula Lam., Jour. de Conch., ii, 429. G.

Mitra striatula Lam., Krebs. 36.

Mitra (Nebulania) striatula Latm., Mirrat, Argo Exp., 1876. 6. Aa. Mitra striatula Lam., Moll. Cuba, ii, 126.

Mitra sulcata Grmel., Dall, Hemphill's shells. 327. Key West. Antilles. Mitra sulcata Gmel., Krebs. 37.

Mitra sulcata-bifasciata Krebs. 37.

Mitra tiarella Lam., Krelss. 38.

Mitrella (see Columbella) Marrat, Argo Exp., 1876. 9. 
Mitrularia equestris Lam., Krebs. 69.

Modiola (see Lithophagus, Lithodomus, Morliolaria, Modiolus, Perna, Mytilus).

Modiola americana Fav. d'Herb., Poulsen. 16.

Modiola autillarum Phil., Arango. Fanua Mal. Cuba, 18is. 267. Ca. M. SD. Santa Lucia. G.

Modiola antillarum Phil., Zt. Mal., iv, 116. S'T.

Modiola antillarum Phil., Zt. Mal., iv, 117.

Modiola appendiculata Phil., Jahrb., ii, 252.

Modiola capax Con., Jahrb., ii, 252.

Modiola caribæa Phil.,* Zt. Mal., ir, 116.

Modiola carolinensis Con., Am. Marine Conch. 187. SC.

Modiola carolinensis Comma, Tourn. Acarl. Nat. Sei. Phil., vii, 243, pl. xx, fig. 6, 1837. Charleston, SO.

Nodiola cinnamomea Lam., Moll. Cuba, ii, 333.

Modiola eitrina Bolt., Poulsen. 16.

Modiola citrina Chem., Beau. 22. G.

Modiola citrina Chem., Jour. de Conch., ir, 415. G.

Mudiola demisia Comad, Am. Jomrn. Sci., ii, n. s., 42, 1846. Tampa Bay.

Iorliola divaricata Phil., Arango, Famma MIal. Cuba, 187S. 266. Ca.

Modiola divaricata Phil., Zt. Mal., ir, 115.

Modiola exustus D’Orb., Jour. de Conch., ir, 415. G.

Modiola lithophagus Lam., Moll. Cuba, ii, 331.

Modiola modiolus Linn., Krebs, Catalogne. 430. Ba.

Modiola opifex Say, Jahrb., ii, 252.

Modiola papuana Limn., Mal. Bl., xii, 126.

Modiola paprria Comral, Dall, Hemphill's shells. 341. Cedar Keys. Tampa Bay.

Modiola papyria Comrad, Proc. Acad. Nat. Sei., Phil., iii, 24, pl. 1, fig. 8, 1845. Tampa Baу.

Modiola petagna Scacchi, (Rre.), Dall, Itemphill's shells. 341.

Modiola plicatnla Lam., Am. Marine Conch. 187.

Modiola polita Verrill \& Smith, Report Blake Mroll. 116. CS.

Modiola spinifera Cpr., Jahrb., ii, 252.

Modiola sulcata Lam., Arango, Famna Mal. Cuha, 1878. 265. Ca. Ba.

Modiola sulcata Lam., Beau. 22. G.

Modiola suleata Lam., Jour. de Conch., ir, 415. G.

Modiola tulipa Chem., Arango, Fama Mal. Cuha, 1875. 266. Ca. Ba. Modiola tulipa Lam., Arango, Fanua Mal. Cuba, 1878. 266. Ca. Ba.

Morliola tulipa Lam., Beau. 22. G.

Modiola tulipa Lam., Jour. de Conch., ii, 426. G.

Modiola tulipa Lam., Moll. of Bermudas. 24.

Modiola tulipa Lam., Moll. Cuba, ii, 329.

Morliola tulipa Linn., Am. Marine Conch. 187.

Modiolaria lateralis Say, Am. Jour. Conch., v, 108. Fla. 
Modiolus americana Leach, Krebs. 128.

Modiolus americana Fav., Krebs. 128.

Modiolus appendiculatus Phil., Krebs. 129.

Modiolus barbata Linn., Krebs. 129.

Modiolus castanea Say, Krebs. 129. ST. SCx. Tort.

Modiolus chemnitzii P. \& M., Krebs. 129.

Modiolus cinnamomea Lam., Krebs. 130.

Modiolus citrinus Bolt., Krebs. 129.

Modiolus citriuus polydentalus Chem., Krebs. 129.

Modiolus exustus Gmel., Krebs. 129.

Modiolus faramnii P. \& M., Krebs. 130.

Modiolus litoralis Say, Krebs. 129.

Modiolus modiolus Linn., Krebs. 129.

Modiolus sulcatus Lam., Krebs. 129.

Modiolus tulipa Lam., Krebs. 128.

Modulus (see Monodonta, Trochus).

Modulus angulatus Acl., Aliungo, Irauua Mal. C'uba, 1S7s. 174. Ca. Ba. J.

Modulus canaliculatus Beck, Mral. Bl, xxiii, 131.

Modulus canaliculatus Beck, Poulsen. 9.

Modulus cerodes A. Ad., Mal. Bl., xxiii, 129.

Modulus convexior Beck, Mal. Bl., xxiii, 129, 130.

Modulus convexior Beck, Ponlsen. 9.

Modulus corrugatus Stm., Dall, Hemphill's shells. 335.

Modulus floridauus Con., Am. Jour. Conch. v, 11. xii, fig. 6, 107. Fla. Mordulus floridanus (Yon., Dall, Hemplhill's shells. 3ijo. Sarasota Bay. Cedar Keys.

Modulus floridanus Con., Mal. Bl., xxiii, 131. Fla.

Modulus floridanus Con., Poulsen. 9.

Modulus krebsii Möreh, Mal. Bl., xxiii, 129.

Modulus krebsii Mörch, Poulsen. 9.

Modulus lenticularis Chem., Am. Jour. Conch., v, 107.

Modulus lenticularis Chem., Beau. 13. G.

Morhlus lenticularis Chem., Dall, Hemphill's shells. 333\%. Key West.

Modulus lenticularis Chein., Jour. de Conch., iv, 134.

Modulus lenticularis Chem., Krelıs. 62. ST. SII. SB. G. NGr.

Modulus lenticularis Chem., Mitrat. Argo Lry., 1siti. (j. Aa. Barbuda. Tucacas.

Modulus lenticularis Chem., Moll of Bermudas. 19.

Modulus modulus Linn., Alango, Fanma Mal. Cuba, 187s. 175. Ca. Ba.

Modulus (Trochus) modulus Linu., Beau. 13. G.

Modulus modulus Linn., Mal. Bl., xxiii, 130. SCx,

- Modulus modulus Linn., Poulsen. 9.

Modulus perlatus Krebs. 63. NGr.

Modulus perlatus (Wood?), Krebs' Remarks. 396.

Modulus pisım Beck, Mal. Bl., xxiii, 130. Ber. 
Modulus pisum Beck, Poulsen. 9.

Modulus unidens Chem., Mal. Bl., xx̃ii, 131.

Modulus unidens Chem.= M. carchedonius Lam., Poulsen. . 9.

Modulus unidens var. minor, Mal. Bl., xxiii, 132.

Modulus unidens List., Am. Jour. Conch., v, 107.

Modulus unideus List., Arango, Fauna Mal. Cuba, 157s, 175. Ca.

Moneta (Cspræa) nigritarum Pet., Moll. Cuba, ii, 92.

Monodactylus subfuscus Klein, Mal. Bl., xxiv, 19.

Monodonta (see Trochus, Modulus).

Monodonta augulata C. B. Adams, Mrango, Fama Mal. Cuba, 1878.

174. Ca. Ba. J.

Monodonta angulata C. B. Adams, Mal. Bl., xxiii, 132.

Monodonta angulata C. B. Adams, Syuops., 7, 1845. J.

Mónodonta basilea Guppy, u. s., Geol. Mag., 1874. 442. J.

Monodonta carchedonius Lam., Arango, Famna Mal. Cuba, 1sis. 175. Ca.

Monodonta carchedonius Lam., Mal. Bl., xxiii, 131.

Monodonta carchedonius Lam., Moll. Cuba, ii, 57.

Monodonta carneola? Lam., Jougr. de Conch., ii, 428. G.

Monolonta hotessieriana D'Orb., Jour. de Conch., iv, 417. G.

Monodouta kiawahensis T. \& H., n. s., Jleioeene Iossils, S. C., pl. xxvi, fig. 1. 116.

Monodonta livido-maculata C. B. Adams, Synops., 7, 1845. J.

Monodonta maculata Ads., Jour. de-Conch., ir, 417. G.

Monodouta maculo-striata C. B. Adams, Syuops., 6, 1845. J.

Monodonta modulus Lam., Mal. Bl., xxiii, 130.

Monodonta modulus Lam., Moll. Cuba, ii, 57.

Monodonta modulus Linn., Jour. de Conch., ii, 42S. G.

Monodonta var. striata Beau, Jour. de Gonch., ir, 417. G.

Monodonta unidens Ch. Desh., Mal. Bl., xxiii, 132.

Monopty gma Mörch non Lea, (compure Udostomia, Canceliopsis, Myonia).

Monopt.g gma clathratula Mörch, Mal. Bl., xxii, 169. ST.

Monoptygma clathratula Mörch, Poulsen. S.

Monopt.g gma styliformis Möreh,* Mal. B1., xxii, 169. ST.

Monoptygma stỹliformis Mörch, Ponlsen. S.

Monostigma striosa Ad., Alango, Fanna Mal. Cuba, 157s. 169. Ca. J.

Montacuta bidentata Mont., Bush, Conn. Ac. vi, 479. Hatt.

Montacuta bowmani Holm., n. s., Post-Pleiocene Fossils S. C., pl. vii, fig. 2. 30 .

Montacuta tumidula Jeff., Verrill, List, 1SS4. 279.

Morula tuberculata Blainv, Jahrb., ii, 242. Bz.

Morum (see Cassidaria, Oniscia).

Morum conoideum Scop., Mal. Bl, xxiv, 39.

Morum deunisoni Rve., Mal. Bl., xxir, 41. G.

Morum oniscus Mal. Bl., xxir, 40. SCx.

Morum oniscus Liun., Mal. Bl., xxiv, 40. 
Morum onisens Iimm., Marrat, Argo Exp., 1876. 16. Nassau. Abaco. Morum ouiscus Linn., Ponlsen. 10.

Morum strombiforme Rve, Mal. Bl., xxir, 40.

Morum strombiforme Rre., Poulsen. 10.

Mulinea (see Mactra).

Mulinea donaciformis Gray, Krebs. 105.

Mulinea milesii Holm., u. s., Post-P'leiocene Fossils S. C., pl. vii, fig. 11 42.

Mulinea portoricensis Shuttlew., Mal. Bl., iv, 2S. PR.

Mulinea portoriceusis Shuttlew., Jour. de Conch., v, 174. PR.

Mulleria (Ostrea) arborea Fér., Krebs. 136.

Murehisonella spectrum Mörch,* Mal. Bl, xxii, 184. S'I.

Murehisonia spectrum Mörch, ${ }^{*}$ Hal. Bl., xxii, 1S4. ST.

Horex (see Cerithium), Arango, Fauna Mal. Cuba, 1sis. 207. Ca. G. M. S'T. Ba.

Murex (see Fasciolaria), Arango, Famba Mal. Cuba, 1875. 217. Ca. G M. S'T. Ba.

Murex (see Fusus), Arango, Fanna Mal. Cuba, 1575. 216. Ca. M.

Murex (see Muricilea), Araugo, Famba Mal. Cuba, 1875. 212. Ca. Ba. Murex (see P'urpura), A rango, Fama Mal. Cubat, 15is. 202. Ca. G. Ba. Murex (see Triton), Arango, Fauma Mal. Cuba, 187s. 212, 213, 214. Ca, G. MI. Santa Lucia. Ba. ST.

Murex (see Turbinella), Arango, Fauna Mal. Cuba, 1878. 222. Ca. M. G. Santa Lucia. ST. Ba.

Murex alyssicola C'rosse, Jahrb., iv, 154. G. P.

liurex abyssicula Crosse, * Jour. de Conch. xiii, pl. i, figs. 4, 5, 30. G. Murex aceinctus Born, Arango, Fauma Mal. Cuba, 1878. 204. Ca.

Baracoa. Ba. = Vionnea luswart

Murex accinctus Born, Krebs. 31.

Murex accinctus Born, Moll. Cuba, ii, 146.

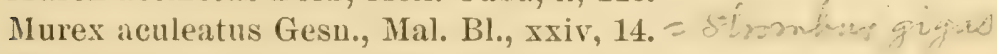

Murex aculeatus Grew., Mal. Bl., xxir, 15.

Murex alamsii Kobelt, Jahrb., ir, 154. M.

Murex alustus Lam., Pfeiffer, Archiv füir naturg., 1840. 25̃7. Ca.

Murex alabaster Ad., Jalnb., ir, 154. = a dar.wi

Murex alveatus Веяu, Jahrb., iv, 244. G.

Murex alveatus Kien., Arango, Fauna Mal. Cuba, 1S7S. 210. Ca.

Havana.

Murex alveatus Kien., Beau. 9. G.

Murex alreatus Kien., Jour. de Conch., iv, 417. G.

Murex alveatus Kien., Krebs. 18. S'T. SUx. SM.

Murex alveatus Kien., Krebs. 21.

Murex alveatus Kien., Poulsen. 11.

Murex antilinum D’Orb., Arangu, Fama Mal. Cuba, 1878. 211. Ca. Murex antillarum Hinls, Krebs' Remarks. 397. SCx. Murex argus Gmel., Mal. Bl., xxir, 32. = Grisicen 
Murex aruanum Limn., Arango, Fauna Mal. Cuba, 1878. 216. Ca. Fla.

Murex aruanus Linn., Jahrb., ii, 360.

Murex asperrimus D'Orb., Moll. Cuba, ii, 158.

Mnrex asperrimus Lam., Araugo, Fauna Mal. Cuba, 1878. 211. Ca. Havina. SV. G. M. ST. Ba.

Murex asperrimus Lam., Beau. 9. G.

Murex asperrimus Lam., Jahrb., iv, 154.

Murex asperrimus Lam., Jour. de Conch., ii, 429. G.

Murex asperrimus Lam., Moll. Ouba, ii, 158.

Murex atratus Born, Mal. Bl., xxiii, 113.

Murex atratus Schröt., Mal. Bl., xxiii, 113.

Murex anritus, \&e., Mal. Bl., xxiv, 17. = Lizondew

I Murex beaui Petit, Jahrb., iv, 147. MG.

Murex beanii Fisch. \& Beru., Beau. 9. MG.

Murex beauii F. \& B., Jour. de Conch. v, pl. viii, fig. 1, 295. M. G.

Murex beauii F. \& B., Krebs. 18.

Murex bellus live., Arango, Famna Mal. Cuba, 1878. 210. Ca. SD.

Murex bellus Rive., Jahrb., iv, 147. = Chrystetoma "zray

Murex bellus live., Krebs. 18. ST.

Murex bellus Rre., Ponlsen. 11.

Murex bilineatus Rve., Beau. 9. Goyave. G.

Murex bilineatus Rve., Krebs. 18.
Murex bilingue Sloane, Mal. Bl., xxiv, 21. L dimuas pujilis.

Murex brasilieusis Sby, Jahrb, iv, 153. Bz. = temcqace ‘...

Murex breviculus Sby., Beau. 9. G. = Letraponus var,

Murex breviculus Sby., Krebs. 22.

Murex brevitions Lam., Arango, Fanna Mal. Cuba, 1878. 210. Ca.

Havana. Matanzas. G. M. S'T. Ba.

Murex brevifrous Lam., Beau. 9. G.

Murex brevifrons Lam., Jahrb., iv, 153.

Murex brevifrous Lam., Jour. de Conch., ii, 429. G.

Murex brevifrons Lam., Krebs. 18, 19.

Murex brevifrons Lam., Krebs' Remarks. 396.

Murex brevifrons Lam., Moll. Cuba, ii, 159.

Murex brevifions Lam., Poulsen. 11.

Murex cabrittii Bern., Jahrb., iv, 147. G.

Murex cailleti Petit, Beau. 9. G.

Murex cailleti Petit, Jahrb., iv, 147. G.

Murex cailleti Petit, Jour. de Conch. v, pl. 2, figs. 1, 2, 87. G.

Murex cailleti Petit, Jour. de Conch., v, 153. G.

Murex cailleti Petit, Krebs. 19.

Murex cailleti Petit, Poulsen. 11.

Murex calcitrapa Lam., Araugo, Fauma Mal. Cuba, 1S7S. 210. Ca.

Havana. Matanzas. G. M. ST. Ba.

Murex calcitrapa Lam., Beau. 9. G. 
Murex calcitrapa Lam., Jahrb., iv, 153.

Murex calcitrapa var. ? Lam., Jour. de Conch., ii, 429. G.

Murex calcitrapa Lam. Krebs. 18, 19.

$\checkmark$ Murex canaliculatus Linn., Jahrb., ii, 361.

Murex cancellatus Sby., Krebs. 19.

Murex (Typhis) cancellatus Sby., Poulsen. 11.

Murex cautraiuei Petit, Beau. 9. G. $=$

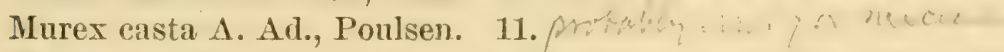

Murex cellulosa Comrad, Am. Jouru. Sei., n. s., ii, 397, 1846. Fla.

Murex cellulosa Conrarl. Proe. Acarl. Nat. Sei. Phil., iii, 25, 1845.

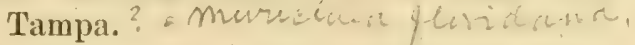

Murex chrysostoma Gray, Jahrb., iv, 147.

Murex chrysostoma Grą, Krebs. 19.

Murex chrysostomus Gray, Poulsen. 11.

Murex coccineus A. Ad., Poulsen. 11.

Murex collatus Guppy, n. s., Geol. Mag., 1874. 441. J.

Murex cormu-cerri Mart., Arango, Fauna Mal. Cuba, 1878. 210. Ca.

Havana. Matanzas. G. MI. ST. Ba. = herefron

Murex cornu-cervi Mart. bis, Krebs. 19.

Murex cornu-cervi Mart., Krebs. 22.

Murex cormu-cervi Mart., Krebs' Remarks. 396.

Murex cornu-cervi Mart., Poulsen. 11.

Murex costatus Bolı, Mal. Bl., xxir, 28. = Whi

Murex costatus Gmel., Jahrb., iv, 153. Bz. = denegnichw

Murex crassa Dillw., Jahrb., iii, 332. = Ranceta 2p,

Murex crassa Dillw., Mal. Bl., xxiv, 23.

Murex erassivaricosa Rve., Krebs. 19. ST. $=$ m. irevifrons:

Murex crassiraricosa Rre., Ponlsen. 11.

Murex cyclostomus Fath., Arango, Fauna Mal. Cuba, 1878. 210. Ca. Ba. Murex decussatus Rire., Arango, Fama Mal. ('uba, 1S78. 210. Ca... Murex dubius Sby., Poulsen. 11. $x$ wew am s.p.

Murex elegans Beck, Arango, Fanna Mal. Cuba, 1878. 210. M. SD.

Murex elegans Beck, Jahrb., iv, 146. Comp. hotivins

Murex elegans Beck, Moll. Cuba, ii, 160.

Murex elegans Beck, c. F., Poulsen. 11.

Murex elegans Kieu., Beau. 9. G.

Murex elegans Kien., Jour. de Conch., ii, 429. G.

Murex elougatus, Lan., Araugo, Fauna Mal. Cuba, 1878. 211. Ca.

Antilles. = brvifrens va

Murex elongatus Lam., Jahrb., iv, 153.

Murex elongatus Lam., Krebs. 20.

Murex (Chicoreus) elongatus Lam., Marrat, Argo Exp., 1876. 11. Pnerto Cabello.

Murex fasciatus Sby., Jabrb., ii, 241. W. Africa?

Murex fastigiatus \&c., Mal. Bl., xxiv, 27. = Sritonum

Bull. 24-12 
Murex femorale Linn., Moll. Cuba, ii, 160. = Fitorium

Murex fucus Gmel., Zt. Mal., x, 73. SV. = Purpura up.

Murex fulvescens Sby., Jahrb., iv, 155.

Murex funiculatus Rie., Jahrb., iv, 147. = recurvirostis Bor?.

Murex funiculatus Rve., Krebs. 20. NGr.

Murex galea Chem., Moll. Cuba, ii, 147. = Y...

Murex gundlachi Dlir., * Mal. Bl., xxxi, 35. Ca.

Murex (Tritonidea) haneti Petit, Jour. de Conch., v, pl. ii, figs. 7, 8, 90.

Rio.

Murex hexagonus Lam., Beau. 9. G.

Murex hexagouus Lam. bis, Krebs. 20. ST. Tort.

Murex hexagouus Lam., Poulsen. 11.

$\checkmark$ Murex hidalgoi Crosse,* Jour. de Conch., xrii, 405.

Murex hidalgoi Crosse, ${ }^{*}$ Jour. de Coneh. xix, pil. i, fig. 4. 68.

Murex (Chicorens) imbricatus Higgius \& Marrat, Marrat, Argo Exp.,

1876. 9. Grenada? = axicornis fide Jryos.

Murex infundibulum Gmel., Arango Fauna Mal. Cuba, 1878. 222. Ca.

Baracoa. G. M. Santa Lucia. = Jusbindla $\Delta p$.

Murex infundibulum Gmel., Krebs. 16. SM. SCx. ST.

Murex infundibulum Gmel., Moll. Cuba, ii, 178.

Murex intermedius C. B. Adams, Contr. to Conch. 60. J. fiven Murex intermedius C. B. Adams, Krebs. 21.

Murex jamaicensis Petiv., Mal. Bl., xxiv, 15. = Strombusop.

Murex labiosus Wood, Mal. Bl., xxir, 3\%. = Awi-...t? of

Murex laceratum Desh., Jour. de Conch. v, pl. iii, figs. 3, 4. 79. (Algeria.)

Murex lacteus Mrus. Gottw., Mal. B1., xxiv, 18. =

Murex lacteus Rond., Mal. Bl., xxiv, 18.

Murex litteratum Born, Moll. Cuba, ii, 153. = Cerivinum efp.

Murex litteratum Gmel., Moll. Cuba, ii, 153.

Murex litteratus Born, Mal. B1., xxiii, 111. = Ceritriem s/s.

Murex litteratus Schröt., Mal. Bl., xxiii, 111.

Murex magis auritus Bon, Mal. Bl., xxiv, 15. = scrombus op

Murex magis auritus, \&c., Mal. B1., xxiv, 19.

Murex marmoreus Aldrov., Mal. Bal., xxiv, 17. = Stronnbw sps,

Murex marmoreus Grew., Mal. Bl., xxiv, 19.

Murex marmoreus Rond., Mal. Bl., xxiv, 14.

Murex marmoreus candidus Aldrov., Mal. Bl., xxiv, 21.

Murex megacerus Sby., Beau. 9. G. = error for trevifornd?

Murex megacerus Sby., Jahrb., iv, 154.

Murex megacerus Sby., Krebs. 21.

Murex (Chicoreus) megacerus Sby., Marrat, Argo Exp., 1876. 13. Santa Marta.

Murex melongena Linn., Arango, Fauna Mal. Cuba, 1878. 216. Ca.

G. M. Cartagena. Fla. = Nucongenn sp.

Murex messorius Sby., Beau. 9. G. Comp. recurasentis.

Murex messorius Sby., Krebs. 21. 
Murex messorins Sby., Moll. Cuba, ii, 159.

Murex messorius Sby., Poulsen, 11.

Murex mexicanus Petit, Arango, Fauna Mal. Cuba, 1878. 211. Ca.

Havana. San Vicente. G. M. ST. Ba. = pomum va.

Murex mexicanus Petit, Jahrb., iv, 154.

Murex mexicanus Petit, Jour, de Conch. iii, pl. ii, f. 9. 51.

Murex microphyllus Lam., Moll. Cuba, ii, 159. = bxuetrome.

Murex minimus Gmel., Mal. Bl., xxiii, 93. Fla. = 8 :200enw of .

Murex minor \&c. Mal. Bl., xxiv, 20. = Skromitus s .

Murex morio Linn., Moll. Cuba, ii, 166. = metonjena sp.

Murex motacilla Chem., Arango, Fauna Mal. Cuba, 1878. 211. Ca.

Murex motacilla Chem., Krebs. 21.

Murex motacilla Chem., Poulsen. 11.

Murex mulus Dillw., Mal. Bl. xxiv, 34. = Aistoreico jo.

Murex musicalis Mart., Arango, Fauna Mal. Cuba, 1878. 196. Ca. Antilles. = Voluta op.

Murex musicalis Mart, Moll. Cuba, ii, 124.

Murex nassa Gmel, Moll. Cuba, ii, 177. = Jurbinella 4\%

Murex neritoideus Linn., Zt. Mal., x, 73. SV. =

Murex niveus Gmel., Mal. Bl., xxiv, $32 .=$ Filtrium sp,

Murex nodatus Rve., Beau. 9. G.

Murex nodatus Rve., Jour. de Conch., iv, 417. G.

Murex nodatus Rve., Krebs. 21.

Murex nodus Liun., Arango, Fauna Mal. Cuba, 1878. 202. Ca. Harana.

$\mathrm{Ba} .=$ Ricisula ej.

Murex nodus Linn., Krebs, 27. ST.

Murex nuceus Mörch, Krebs. 21. SCx. ST. SM.

Murex nuceus Mörch, Poulsen. 11.

Murex oculatus Rve., Jahrb., iv, 154. $=$ m. ponum,

Murex oculatus Rve., Krebs. 21.

Murex (Phyllonotus) oculatus Rve., Marrat, Argo Exp., 1876. 9. SV. Murex olearium Linn., Mal. Bl., xxiv, 29. = Fritonimu $\Delta p_{1}$

Murex orthocentros List., Moll. Cuba, ir, 121. = Sl.mutsw b.ey de

Murex orthocentrus \&c., Mal. Bl., xxiv, 21.

$\checkmark$ Murex ostrearum Coural, Am. Jour. Sci., n. s., ii, 397, 1846. Fla.

Murex ostrearum Conrad, Proc. Acad. Nat. Sci., Phil., iii, 25, 1845.

Tampa.

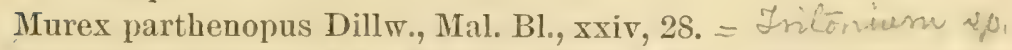

" Murex pauperculus Ad., Arango, Famma Mal. Cuba, 187s. 211. Ca.

Havana. G. Ba. L milne alventwo hienew

Murex panperculus C. B. Adams, Beau. 9. G.

Murex pauperculus C. B. Adams, Contr. to Conch. $60 . \mathrm{J}$.

Murex pauperculus C. B. Adams, Jour. de Conch., v, 156. G.

Murex pauperculus Ad., Krebs. 21.

Murex panperculus Ad., Krebs. 23.

Murex pauperculus C. B. Adams, Mal. Bl., iv, 27. G. 
$\checkmark$ Murex pazi Crosse, Am. Jour. Conch., r, 115.

Murex pazi Crosse, Am. Jour. Conch., vi, 82.

Murex pazi Crosse, Jahrb., iv, 245.

Murex pazi Crosse,* Jour. de Conch., xvii, 183.

Murex pazi Crosse, ${ }^{*}$ Jour. de Conch. xviii, pl. i, fig. 7. 99.

Murex perversus Linn., Am. Jour. Conch., -iii, 146.

Murex perversus Linn., Arango, Fauna Mal. Cuba, 1878. 216. Ca. Fla.

Murex perversus Linn., Moll. Cuba, ii, 167.

Murex pileare Chem., Mal., Bl., xxiv, 28. = Jilönim sp.

Murex pileare Lam., Moll. Cuba, ii, 162.

Murex (Typhis) pinnatus Brod?, Poulsen. 11. a west anv,

Murex pleurotomoides Rre., Krebs. 21. SM.

Murex plicatus Linn., A rango, Fauna Mal. Cuba, 1878. 203. Ca. Ba.

Murex polygonus Grmel., Moll. Cuba, ii, 179.

Murex pomiformis Mart., Arango, Fanna, Mal. Cuba, 1878. 211. Ca.

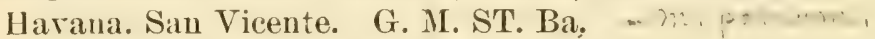

Murex pomiformis Mart., Jahrb., iv, 154.

Murex pomiformis Mart., bis., Krebs. 21.

Murex pomiformis Mart., Poulsen. 11.

Murex pomum Gmel., Arango, Fanna Mal. Cuba, 1878. 211. Ca. Havana. San Vicente. G. M. ST. Ba.

Murex pomum Gmel., Beau., 9. G.

Murex pomum Gmel., Jahrb., iv, 154.

Nurex pomum Gmel., Jour. de Conch., ii, 429. G.

Murex pomum Gmel., Moll. Cuba, ii, 158.

Murex pudicus Rve., Jahrb., iv, 245.

Murex pudicus Rve., Krebs. 22.

Murex pudoricolor Rve., Beau. 9. G. $=C_{r} \ldots$

Murex pudoricolor Rve., Jahrb., iv, 153.

Nurex pudoricolor Rve., Krebs. 22.

Murex pudoricolor Rve, Poulsen. 11.

Murex purpuratus Rve., Krebs. 19.

Murex purpuratus Rve., Krebs. 22.

Murex ranunculus Meusch., Arango, Fauna Mal. Cuba, 1878. 213. Ca. G. Ba.

Murex rarispina Lam., Krebs. 22.

Murex rarispina Lam., Krebs' Remarks. 397. SCx.

Murex recurrirostris Brod., Marrat Argo Exp., 1876. 11. Puerto Cabello. Tucacas. Santa Marta. Havana.

Mi urex reticularis Linn., Jour. de Conch., viii, 359.

Murex reticulatum Lain., Mal. Bl., xxiv, 34.

Murex rosarium Chem., Zt. Mal., x, 78. SV.

Murex rubridentatus Rve., Jour. de Conch., iv, 417. G.

Murex rubridentatus Rve., Krebs. 22.

Mirex rubridentatus Rve., Beau. 9. G.

Murex rubridentatus Rve., Poulsen. 11. 
Murex rufus Lam., Arango, Fauna Mal. Cuba, 1878. 211. Ca. Ba.

Murex rufus Lam., Poulsen. 11.

Murex salleauus A. Ad., Jahrb., iv, 245.

Murex scolywus Gmel., Arango, Fauna Mal. Cuba, 1878. 222. Ca. Ba. = Lustivelia2

Murex senegalensis Gmel., Jahrb., iv, 153. Bz.

Murex senegalensis Gmel., Mal. Bl., x, 225.

Murex senegalensis Linn., Jáhrb., ii, 240.

Murex scruato-spinosus Dkr.,* Mal. Bl., xxxi, 35.

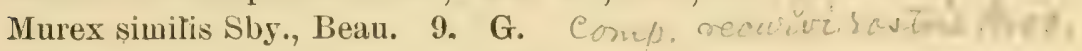

Murex similis Sby., Jahrb., iv, 146.

Murex similis Sby., Krebs. 22.

Murex spectrum Rve., Beau. 9. G.

comp ithelimit axiconus:

Murex spectrum Rve., Krebs. 22.

Murex spectrum Rve., Krebs' Remarks. 396.

Murex spinacosta" Kurtz. 8. NC. SC.

Murex spinicostatus Val., Am. Marine Conch. . 19. SU. NC.

Murex spinicostatus Val, Jahrb., iv, 155.

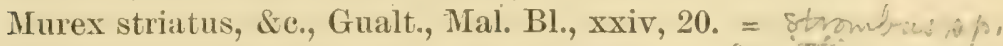

Murex subulatus Mtg., Mal. Bl., xxiii, 98. = Eerickicese $s /$,

Murex subulatus Wood, Mal. Bl., xxiii, 98.

Murex sulcatus Gmel., Zt. Mal., x, 72. SV.

Murex tampaëusis Con., Am. Jour. Conch., v, 106. Fla. =

Murex tampaënsis Con., Proc. Acall. Nat. Sci. Phil., 1545, ii, 25. Tampa.

Murex tectumsinense Desh., Jour. de Conch., v, pl. iii, figs. 1-2. 78.

(Algeria.) = eorallioprith is, car.

Murex tetragonus Brod., Beau. 9. G.

Murex tetragonus Brod., Krebs. 22.

Murex triangularis Aldrov., Mal. Bl., xxiv, 38. = Cow 1 i 2\%,

Murex triangularis Gesn., Mal. Bl., xxir, 38.

Murex triangularis Rond., Mal. Bl., xxiv, 38.

Murex trigounlus Lam., Beau. 9. MG. G. compl, he, theeres,

Murex trigouulus Lam., Jahrb., iv, 147.

Murex trigonulus Lam., Jour. de Conch., iv, 417. G.

Murex trigonulus Lam., Krebs. 22.

Murex trigonulus Lam., Poulsen. 11.

Murex trilineatus Rve., Arango, Fauma Mal. Cuba, 1878. 211. Ca.

Murex trilineatus Rve., Jahrb., iv., 147.

Murex trilineatus Rve., Poulsen. 11.

Murex triqueter Kien., Jahrb., iv, 147. = higomeins,

Murex tulipa Liun., Jahrb., ii, 362.

Murex tulipa Linn., Moll. Cuba, ii, 168.

Murex turbinatus Sby., Jahrb., iv, 155.

Murex variegatus Mart., Jahrb., iv, 147. = Welewo fiesu

Murex vespertilio Mart., Moll. Cuba, ii, 123.

Muricidea (see Murex). 
Muricidea floridana Con., Dall, Hemphill's shells. 326. Sarasota Bay. Muricidea hexagoua Lam., Arango, Fanna Mal. Cuba, 1878. 212. Ca. Ba.

Muscade rare Dav., Moll. Cuba, i, 132.

Musculus radiatus Linn., Krebs, 103.

Musica gallorum Bon., Moll. Cuba, ii, 124.

Musica gallorum Klein, Moll. Cuba, ii, 124.

Mya arenaria Linn., Am. Marine Conch. 140.

Mya arenaria Linn., Kurtz. 3. NC. SC.

Mya nitens Lask., Krebs. 110.

Mya norvegica Gmel., Moll. Cuba, ii, 224.

Mya perna Linn., Jahrb., ii, 250.

Mya simplex Holm., n. s., Post-Pleiocene Fossils, S. C., pl. viii, fig. 16. 55.

Myrtæa (see Lucina) Marrat, Argo Exp., 1876. 7.

Myrtea (see Lueina) Marrat, Argo Exp., 1876. 15.

Myrtea muricata'Speng., Marrat, Argo Exp., 1876. 12. Tucacas.

Myrtea seabra Turt., Krebs. 121.

Mysia punctata Say, Am. Marine Conch. 170.

Mytilopsis (see Dreissena).

Mytilopsis leucophæatus Conrad, Proc. Acad. Nat. Sci. Phil., 1857. 167.

S. coast U. S.

Mytilus (see Modiola, Perna, Lithodomus, Melina).

Mytilus (see Area) Arango, Fauna Mal. Cuba, 1878. 261. Ca. J.

Mytilus americana Leach, Kurtz. 6. NC. SC.

Mytilus americanus D'urb., Araugo, Fauna Mal. Cuba, 187s. 266. Ca.

Ba.

Mytilus americanus Fav., Arango, Fauna Mal. Cuba, 18i8. 266. Ca.

Ba.

Mytilus americanus Fav., Moll. Cuba, ii, 329

Mytilus attenuata Desh., Kurtz. 6.

$\checkmark$ Mytilus barbadensis Pet, Moll. Cuba, ii, 321.

Mytilus bifureatus Con., Jahrb., ii, 251.

Mytilus bilocularis Lam., Pfeiffer, Archir fiir naturg., 1840. 260. Ca. Mytilus brasiliensis Chem., Guppy, Paria Fauna, 1877. 154.

Mytilus brunneus Sol., Arango, Fauna Mal. Cuba, 1875. 266. Ca. J. M.

SD. G.

Mytilus brunneus Sol., Krebs. 130. Botula fusca

Mytilus castanea Say, Kurtz. 6. SC.

Mytilus caudigera Lam., Kurtz. 6. SC.

Mytilus eimnamomeus Chem., Araugo, Fanna Mal. Cuba, 1878. 266.

Ca. J. M. SD. G.

Mytilus einnamomeus Chem., Moll. Cuba, ii, 333.

Mytilus cubitus Say, Arango, Fanna Mal. Cuba, 1878. 265. Ca.

Mytilus cubitus Say, Kurtz. 6. NO. SC.

Mytilus discors Linn., Moll. Cuba, ii, 327. 
Mytilus domingensis Lam., Jour. de Conch., xviii, 178. D.

Mytilus tomingensis Lam., Krebs. 12S.

Mytilus domingensis Lam., Moll. of Bermudas. 24.

Mytilus dominguensis D'Orb., Arango, Fauma Mal. Cuba, 1S78. 265.

Ca. M.

Mytilus dominguensis D'Orb., Moll. Cuba, ii, 328.

Mytilus dominguensis D'Orb., Report Blake Moll. 117.

\& Mytilus dominguensis Lam., Arango, Mal. Cuba, 1878. 265. Ca. M.

$\checkmark$ Mytilus dominguensis Lam., Moll. Cuba, ii, 328.

$\checkmark$ Mytilus edulis Linn., Am. Marine Conch. 186.

Mytilus edulis var. pellucidus, Am. Marine Conch. 186.

Mytilus elongatus Chem., Jahrb., ii, 250.

$\checkmark$ Mytilus exiguus Dkr., Jahrb., ii, 251.

$\checkmark$ Mytilus exustus D’Orb., Alango, Fama Mal. Cuba, 187s. 265. Ca. Ba.

$\checkmark$ Mytilus exustus Gmel., Moll. Cuba, ii, 329.

Mytilus exustus Lam., Am. Marine Conch. 186.

Mytilus exustus Lam., Jour. de Conch., vii, 39.

Mytilus exustus Lam., Alango, Fauna Mal. Cuba, 1878. 265. Ca. M.

Mytilus exustus Lam., Dall, Hemphill's shells. 341. Key West. Autilles.

Mytilus (Aulacomya) exustus Lam., Marrat, Argo Exp., 1876. 8. D. Havana. Nassau. Long Key Island.

$\checkmark$ Mytilus exustus Lam., Moll. Cuba, ii, 328.

Mrtilus exustus Linn., Arango, Fauna Mal. Cuba, 187S. 265. Ca. Ba. Mytilus exustus Linn., Beau. 22. G.

Mytilus exustus Linn., Jour. de Conch., ii, 426. G.

Mytilus exustus Linn., Kirebs. 12S. ST. SJ.

Mytilus exustus Linn., Mal. Bl., x, 231.

Mytilus exustus Linn., Moll. Cuba, ii, 329.

Mytilus exustus Linn., Poulsen. 16.

Mytilus exustus Linn., Report Blake Moll. 117.

Mytilus favanui P. \& M., Arango, Fauna Mal. Cuba, 1878. 266. Ca. J. M. SD. G. Nista

Mytilus fuseus Gmel., Arango, Fauma Mal. Cuba, 1878. 266. Ca. J. M. SD. G. Rothila

Mytilus granulatus Hanl., Zt. Mal., x, 79. SV.

Mytilus hamatus Say, Am. Marine Conch., 186.

Mytilus hamatus Say, Jour. de Conch., xviii, 178. Fla.

Mytilus (Aulacomya) hamatus Say, Marrat, Argo Exp., 1876. 15.

$\mathrm{VCz}$.

Mytilus inflatus T. \& H., n. s., Pleiocene Fossils S. C., pl. xiv, fig. 3. 33. Mytilus lateralis Say, Kurtz. 6. NC. SC.

Mytilus lavalleanus D’Orb., Arango, Fama Mal. Cuba, 1878. 265. Ca. $\checkmark$ Mytilus lavalleanus D'Orb., Krebs. 128.

Mytilus lavalleanus D'Orb., Moll. Cuba, ii, pl. xxviii, figs. 3-5. 328.

Mytilus lavalleanus D'Orb., Poulsen. 16. 
Mytilus lavalleanus D'Orb., Report Blake Moll. 117.

Mytilus lencophæatus Conr., Jour. Acad. Nat. Sci. Phil., vi, 263, pl. x, fig. 13, April, 1831. Southeru coast U. S.

Mytilus leucophratus Conr., Jour. de Conch., xxvi, 103.

Mytilus ligueus Rve., Guppy, Paria Fauna, 1877. 154.

Mytilus lithophaga Linn., Kurtz. 6.

Mytilus luteus Jeffreys, Report Blake Moll. 116.

Mytilus margaritiferus Gmel., Moll. Cuba, ii, 342.

Mytilus modiolus Chem., Arango, Fauna Mal. Cuba, 1878. 266. Ca. Ba.

Mytilus modiolus Chem., Moll. Cuba, ii, 329.

Mytilus modiolus Linn., Moll. Cuba, ii, 330.

$\checkmark$ Mytilus miilleri Dkr., Jahrb., ii, 250.

$\checkmark$ Mytilus ovalis Lam., Marrat, Argo Exp., 1876. 6. Aa.

$\checkmark$ Mytilus perna Linn., Jahrb., ii, 250.

$\checkmark$ Mytilus perna Linn., Poulsen. 16.

$\checkmark$ Mytilus plicatula Lam., Kurtz. 6. NC. SC.

$\checkmark$ Mytilus puniceus Gmel., Mal. Bl., xii, 126.

$\checkmark$ Mytilus pmiceus Gmel., Zt. Mal., x, 79. SV.

Mytilus tulipa Lam., Kurtz. 6. NC. SC.

Mytilus viator D'Orb., Arango, Fauna Mal. Cuba, 187s. 265. Ca.

Mstilus viator D'Orb., Krebs. 128.

$\checkmark$ Mytilus viator D'Orb., Moll. Cuba, ii, 327.

Myurella (see Terebra) Marrat, Argo Exp., 1876. 12.

Nacea canrena Linn., Mal. B1., xxiv, 62.

Naranaio costata Gray, Arango, launa Mral. Cuba, 1878. 248. Ca. Ba.

Nariea aculeata Recluz, Mal. Bl., xxiv, 94. G.

Narica acuta Recluz, Mal. Bl., xxir, 94. G.

Narica (?) anomala C. B. Adams, Contr. to Couch. 109. J.

Narica anomala C. B. Adams, Mal. Bl., xxiv, 96. J.

Narica? anomalus C. 13. Adams, Jour. de Conch., xii, 2巳ō6. J.

Narica granulosa? Reel., Arango, Fauna Nal. Cuba, 1878. 171. Ca. Havalla.

Narica granulosa Reel, Krebs. 68. S'T.

Nariea lamellosa D'Orb., Arango, Fauna 1Ial. Cuba, 187s. 171. Ca. II. G.

Narica lamellosa D'Orb., Beau. 13. G.

Nariea lamellosa D'Orb., Jour. de Conch., iv, 416. G.

Narica lamellosa D'Orb., Krebs. 68.

Nario lamellosa D’Orb., Mag. de Zool., 1s45, pl. 129, fig. ¿. G. Ca.

Nariea lamellosa D'Orb.,* Mal. Bl., xxir, 94. G.

Narica lamellosa 1)'Orb., Moll. ('uba, ii, pll, xvii, figs. 32-34. 41.

Nariea lamellosa Rerluz, Mal. 131., xxir, 94. G.

Narica striata D'Orb., Arango, Fama Mal. Cuba, 1878. 172. Ca.

Nariea striata D'Orb., Krebs. 68.

Nariea striata D'Orb., Mal. Bl., xxiv, 95. 
Narica striata D'Orb., Moll. Cuba, ii, pl. xvii, figs. 29-31. 40.

Narica sulcata D'Orb., Arango, Fauna Mal. Cuba, 1878. 172. Ca. Havana. J. Santa Lucia.

Narica sulcata D'Orb., Jour. de Conch., v, 152. G.

Narica sulcata D'Orb., Jour. de Conch., xii, 256. J.

Narica sulcata D'Orb., Krebs. 68.

Narica sulcata D'Orb., Mag. de Zool., 1545, pl. 126, fig. 2. Ca.

Narica sulcata D'Orb., Mal. Bl., xxiv, 95. Ca.

Narica suleata D'Orb., Moll. Cuba, ii, pl. xrii, figs. 2/-28. 39.

Narica sulcata jun. D'Orb., Mal. Bl., xxiv, 95. G.

Narica sulcata Recluz, Mal. Bl., xxiv, 95. Ca.

Nassa (see Buccinum, Ilyanassa, Neritula).

Nassa (see Phos), Arango, Fauna Mal. Cuba, 1878. 201. Ca. G.

Nassa acuta Say, Am. Marine Conch. 35.

Nassa albescens Dkr., Krebs. 31.

Nassa ambigua Mtg., Moll. Cuba, ii, 143.

Nassa ambigua D'Orb., Moll. Cuba, ii, 142.

Nassa ambigua Mtg., Beau. 11. G.

Nassa (Hima) aubigua Mtg., Marratt, Argo Exp., 1876. 15. VCz.

Nassa ambigua Mtg., Moll. of Bermudas. 16.

Nassa anbigua Mor., Dall, Hemphill's shells. 325. Key West.

Nassa ambigua Pult., Krebs. 31. ST. SCx. SB. Ag.

Nassa (Hima) ambigua Pult., Marrat, Argo Exp., 1876. 6. Aa. D. La

Guayra. Athol Island.

Nassa ambigua Pult., Poulsen. 12.

Nassa (Hima) annellifera Rve, Marlat, Argo Exp., 1876. 8. Barbuda. Nansa antillarum D'Orb., Arango, Fauna Mal. Cuba, 1878. 200. Ca.

Havana. G.

Nassa antillarum D'Orb., Beau. 11. G.

Nassa antillarum D'Urb., Jonr. de Conch., iv, 418. G.

Nassa antillarum D'Orb., Krebs. 31.

Nassa antillarum D'Orb., Moll. Cuba, ii, pl. xxiii, figs. 1-3. 141.

Nassa antillarum Phil., Krebs. 31. S'1'. NGr.

Nassa (Phroutis) antillarum P'hil., Marrat, Argo Exp., 1876. 10. Td.

Nassa (Phos) antillarum Phil., Poulsen. 12.

Nassa beauii F. \& B., Krebs. 32.

Nassa candei D'Orb., Arango, Fauna Mal. Cuba, 1878. 200. Ca.

Havana. Ba.

Nassa candei D'Orb., Beau., 11. G.

Nassa caudei D'Orb., Krebs. 32.

Nassa candei D'Orb., Moll. Cuba, ii, pl. xxiii, figs. 4-6. 142.

Nassa candei D’Orb., Poulsen. 12.

Nassa candidissima $\mathbf{A d}$., Krebs. 32.

Nassa consensa Rav., Am. Mariue Conch. 35. SC.

Nassa consensa Rav., Bush, Conn. Ac., vi, 462. Hatt.

Nassa fretensis Perkins, Am. Marine Conch. 34. 
Nassa guadalupensis Petit, Beau. 11. G.

Nassa guadalupensis Petit, Krebs. 32. ST. NGr.

Nassa guadalupeusis Petit, Poulsen. 12.

Nassa guarlalupensis Petit,* Jour. de Conch., iii, pl.ii, figs. 3, 4. 56. G. Nassa hotessieri D’Orb., Arango, Fauna Mal. Cuba, 1878. 201. J. G. M.

Nassa hotessieri D'Orb., Krebs. 32.

Nassa hotessieri D'Orb., Moll. Cuba, ii, pl. xxi, figs. 40-42. 142.

Nassa hotessieriana D'Orb., Arango, Fauna Mal. Cuba, 1878. 201. J. G. M.

Nassa hotessierii D'Orb., Beau. 11." G.

Nassa inerassata Muill., Guppy, Geol. Mag., 1874. 447. Td. Cumana. J. Нy.

Nassa obsoleta Say, Am. Marine Conch. 33.

Nassa (Hima) obtusata A. Ad., Marrat, Argo Exp., 1876. 16. Nas. sau. At?ol Island.

Nassa (Uzita) obtusata A. Ad., Marrat, Argo Exp., 1876. 17. Long Key Island.

Nassa oliracea Desh, Krebs. 32.

Nassa polygona D'Orb., Moll. Cuba, ii, 140.

Nassa polygona Lam., Aravgo, Fauna Mal. Cuba, 1878. 201. Ca. M. G. Nassa polygona Lam., Beau. 11. G.

Nassa polygona Lam., Krebs. 32.

Nassa septemstriata Mke., Mal. Bl., xxiii, 93. Fla. •

Nassa stercus-muscarum Say, Mal. B1., xxiii, 111.

Nassa sturmil Phil., Jahrb., ii, 241.

Nassa textilina Mörch, Krebs. 33.

Nassa trivittata Say, Am. Marine Conch. 33.

Nassa umbilicata Chem., Moll. Cuba, ii, 113.

Nassa unicincta Say, Am. Marine Conch. 35.

Nassa unicincta Say, Kurtz. 9.

Nassa vibex Say, Am. Marine Conch. 34.

Nassa vibex Say, Beau. 11. G.

Nassa vibex Say, Krebs. 32.

Nassa vibex Say, Poulseu. 12.

Nassa (Phrontis) zonalis Brug., Marrat, Argo Exp., 1876. 12. Tucacas.

Havana.

Nassæ minores Chem., Mal. Bl., xxiii, 125.

Nassæ truncatæ Chem., Ma. Bl., xxiii, 124.

Natica (See Lumatia, Neverita, Nerita, Naticina, Mamma, Stigmaulax, Mamilla, Pollinices, Nacea, Cochlis).

Natica affinis V. d. Busch, Mal. Bl., xxiv, 64.

Natica alba Loven, Jour. de Conch., iv, 416. G.

Natica arachinoidea Mke., Mal. Bl., xxiv, 66. M.

Natica arachnoidea Mke. Zt. Mal. viii, 46. PR.

Natica avellana? Phil., Arango, Fauna Mal. Cuba, 1878. 170. Ca. 
Natica bahiensis Recl., Mal. Bl., xxir, 59 .

Natica beruardii Recl., * Jour. de Conch. ii, pl. 5, f. 1. 197.

Natica bernardii Recl., Mal. Bl., xxiv, 58.

Natica broderipiana Recl., Marrat, Argo Exp., 1876. 12. Tucacas.

Natica brunnea Link, Krebs, p. 66.

Natica brunnea Link, Mal. Bl., xxiv, 59.

Natica campechiensis (Recl.) Melvill, Dall, Hemphill's shells. 342.

Natica campechiensis Recl., Mal. Bl., xxiv, 57.

Natica campechiensis Rve., Mal. Bl., xxir, 57.

Natica cancellata Gmel., Krebs. 67.

Natica cancellata Herrm., Mal. Bl., xxiv, 61.

Natica cancellata Herrm., Poulsen. 10.

Natica cancellata Lam., Beau. 17. G.

Natica cancellata Lam., Krebs' Remarks. 397.

Natica cancellata Lam., Mal. Bl. xxiv, 62.

Natica eancellata Lam., Moll. Cuba, ii, 33.

Natica cancellata Lea., Krebs' Remarks. 397.

Natica candidissima Recl., Mal. Bl. xxiv, 59. Bz.

Natica canrena D'Orb., Arango, Fauna Mal. Cuba, 1878. 170. Antilles.

Natica canrena D'Orb., Moll. Cuba, ii, 30.

Natica canrena Lam., Moll. Cuba, ii, 30.

Natica caurena Linn., Arango, Fama Mal. Cuba, 1878. 170. Antilles.

Natica canrena Linn., Beau. 17. G.

Natica canreua Liun., Dall, Ilemphill's Shells. 335. Sarasota Bay.

Natica canrena Linn., Jour. de Conch, iv, 416. G.

Natica canrena Linn., Krebs. 66. SB. ST. SJ. A. \&c.

Natica canrena Linn., Krebs. 67.

Natica canrena Linm., Marrat, Argo Exp., 1876. 13. Santa Marta.

Natica canrena Linn., Moll. of Bermudas. 18.

Natica canrena Linn.,* Mal. Bl., xxiv, 62.

Natica canrena Linn., Mal. Bl., xxiv, 63.

Natica canrena Linn., Poulsen. 10.

Natica canrena Payraud., Moll. Cuba, ii, 30.

Natica canrena P. aud M., Mal. Bl., xxiv, 63.

Natica canrena Phil., Arango, Fauna Mal. Cuba, 1878. 170.0 Antilles.

Natica canrena Sby., Moll. Cuba, ii, 30.

Natica caribæa Phil., Mal. Bl., xxiv, 61. G.

Natica caribæa Phil., Poulsen. 10.

Natica cayennensis Recl., Mal. Bl., xxiv, 64.

Natica chemnitzii Pfr., Mal. Bl., xxir, 64.

Natica costata Mke., Krebs. 67.

Natica costata Mke., Krebs' Remarks. 397.

Natica costata Mke., Mal. Bl., xxiv, 62.

Natica cumingiana Recl., Arango, Fauna Mal. Cuba, 1878. 170. Ca.

Santa Lucia. Baracoa.

Natica depressa Gray, Mal. Bl., xxiv, 66. M. 
Natica draparnaudi Recl., Mal. Bl., xxiv, 59.

Natica draparnaudii Recl.,* Jonr. de Conch. ii, pl. 5, f. 11. 198.

Natica duplicata Gld., Mal. Bl., xxiv, 58.

Natica duplicata Phil., Mal. Bl., xxiv, 58.

Natica duplicata Say, Mal. Bl., xxiv, 58.

Natica duplicatum Say, Zt. Mal., r, 159.

Natica filosa Phil., Mal. Bl., xxiv, 66. Ca.

Natica fossata Gld., Mal. Bl., xxiv, 57. Fla.

Natica fossata Gld., Otia. 202. Fla.

Natica fringilla Dall, n. s., Report Blake Moll. 93. Cape San Antonio. Yucatan Strait.

Natica fucata Recl., Arango, Fauna Mal. Cuba, 1878. 170. Ca. Santa Lucia. Baracoa.

Natica fuscata Chem., Krebs. 66.

Natica fuscata Chem., Mal. Bl., xxiv, 59.

Natica fuscata Humph., Mal. Bl., xxiv, 58.

Natica fuscata Humph.=N. mamillaris Lam., Poulsen. 10.

Natica haneti Recl., Jour. de Conch., v, 32. Bahia.

Natica haneti Recl., Mal. Bl., xxiv, 62. Bz.

Natica jamaicensis C. B. Adams, Arango, Fauna Mal. Cuba, 1878. 171.

Havana. Ba.

Natiea jamaicensis C. B. Adams., Contr. to Conch. 111. J.

Natica jamaicensis Ad., Krebs. 67.

Natica jamaiceusis Ad., Mal. Bl. xxir, 65. Ca.

Natica lacernula D'Orb., Arango, Fauna Mal. Cuba, 1878. 170. Ca.

Natica lacernula D'Orb., Krebs. 66.

Natica lacernula D'Orb., Mal. Bl. xxiv, 65, 66. M.

Natica lacernula D’Orb., Moll. Cuba, ii, pl. xvii, figs. 23-25. 35.

Natica lacernula D'Orb., Poulsen. 10.

Natica lactea Guild., Mal. Bl., xxiv, 60.

Natica lactea Guild., Poulsen. 10.

Natica lactea Phil., Arango, Fauna Mal. Cuba, 1878. 170. Ca. Havaua.

Natica lactea Phil., bis, Krebs. 67. ST.

Natica leptalea Watson, Lin. Soc. Journ., 1880, xr, 261. Sombrero.

Natica leptalea Watson, Report Blake Moll. 93. Yucatan Strait. WI.

Natica limacina Jouss., Mal. Bl., xxiv, 64. SCx.

Natica limacina Jouss, Poulsen. 10.

Natica lineolata Phil., Jour. de Conch., iii, 271.

Natica livida Pfr., Hal. BI., xxiv, 64. J.

Natica livida Pfeiffer, Archiv fuir naturg., 1840. 254. Ca.

Natica livida Pfr. =N. jamaicensis C. B. Adams, Poulsen. 10.

Natica lurida Phil., Arango, Fauna Mal. Cuba, 1878. 170. Ca.

Natica mamilla Lam., Moll. Cuba, ii, 31.

Natica mamilla Lam., Mal. Bl., xxiv, 61. G.

Natica mamilla Linn., Krebs. 67. 
Natica (Mamma) mamilla Limn., Narrat, Argo Exp., 1876. 8. Barbuda. Natica mamillaris Krebs. $66 . \quad$ Td. SJ. ST. PPI. J.

Natica mamillaris D'Orb., Arango, Fanna Mal. Cuba, 1878. 170. Ca. Santa Lucia. Baracoa.

Natica mamillaris Lam., Bear. 17. G.

Nática mamillaris Lam., Mal. Bl., xxiv, 59.

Natica mamillaris Lam., Moll. Cuba, ii, 32 .

Natica mamillaris Linn., Jour. de Conch., iv, 416. G.

Natiea mamillaris Rve., Mal. Bl., xxir, 59.

Natica mamillaris Schr., Arango, Fauna Mal. Cuba. 1878. 170. Ca.

Santa Lucia. Baracoa.

Natica maroccana Chem., Guppy, Paria Fauna, 1877. 138.

Natica marochiensis? Lam., Beau. 17. G.

Natica marochiensis Lam., Krebs. 67.

Natica marochiensis Lam., Moll. of Bermudas. 18.

Natica marochiensis Recl., Marrat, Argo Exp., 1876. 16. Nassau.

Natica menkeana Mke., Mal. Bl., xxiv, 66. M.

Natica menkeana Pfr., Krebs. 67.

Natica menkeana Phil., Mal. Bl., xxiv, 66. Ca.

Natica menkeana Phil.,* Zt. Mal. viii, 45. PR.

Natica, var. nigrescens, Ad., Mal. Bl., xxiv, 65. Ca.

Natica nitila Don., Arango, Fanna Mal. Cuba, 1878. 171. Ca. M. G.

Santa Lucia. Ba.

Natica nitida Don., Krebs. 67. ST. Tort. Bb.

Natica nitida Don., Mal. Bl., xxiv, 60. Ca.

Natica nitida Don., Moll. Cuba, ii, 32.

Natica ochrostoma Recl., Krebs. 67.

Natica ochrostoma Recl., Mal. Bl., xxiv, 60.

Natica ochrostoma Recl., Poulsen. 10.

Natica pennata Chem., Mal. Bl., xxir, 64.

Natica pes-elephantis Pfr., Mal. Bl., xxiv, 60. Ca.

Natica pes-elephantis Pfr., Zt. Mal., viii, 45 .

Natica p feifferi Phil., Arango, Fauma Mal. Cuba, 1878. 171. Oa.

Natica pfeifferi Phil., Krebs. 67. ST.?

Natica pfeifferi Phil., Mal. Bl., xxiv, 60. Ca.

Natica pfeifferi Phil.,* Zt. Mal., viii, 45.

Natica porcellana D’Orb., Mal. Bl., xxiv, 60. Bz.

Natica proxima C. B. Adams, Contr. to Conch. 111. J.

Natica proxima Ad., Krebs. 67. ST. SJ. PPI. Bb.

Natica proxima C. B. Adlams, Mal. Bl., xxiv, 65.

Natica proxima C. B. Adams, Poulsen. 10.

Natica proxima Reeve, Mal. Bl., xxiv, 65. Ca.

Natica pulchella Pfr., Arango, Fauna Mal. Cuba, 1878. 171. Havana. Ba.

Natica pulchella Pfr., Krebs. 67. ST. Ag. Td.

Natica pulchella Pfr., Mal. Bl., vi, 232. 
Natica pulchella Pfr., Mal. Bl., xxiv, 66. Ca.

Natica pulchella Pfr., Mal. Bl., xxiv, 66. M.

Natica pulchella Pfeifter, Archiv für naturg., 1840. 254. Ca.

Natica pusilla Say, Am. Marine Conch. 58. NC.

Natica pusilla Say, Beau. 17. G.

Natica pusilla Say, Jour. de Conch., iv, 416. G.

Natica pusilla Say, Krebs. 67.

Natica radiata Watson, Liu. Soc. Journ. 1850, xv, 258. Ber.

Natica rufilabris Reeve, Mal. Bl., xxir, 65.

Natica rugosa Chem., Krebs, 67, 68. SJ. PPl. SCx. Td.

Natica rugosa Chem., Krebs' Remarks. 397.

Natica rugosa Chem., Mal. Bl., xxiv, 62.

Natica rugosa Gmel., Krebs. 67.

Natica rugosa Gmel., Krebs' Remarks. 397.

Natica sagraiana D’Orb., Arango, Fauna Mal. Cuba, 1878. 171. Havana, Ba.

Natica sagraiana D'Orb., Jour. de Conch., iii, 271. Ca.

Natica sagraiana D'Orb., Krebs. 67.

Natica sagraiana D'Orb., Mal. Bl., vi, 232.

Natica sagraiaua D’Orb., Mal. Bl., xxiv, 66. Ca.

Natica sagraiana D’Orb., Moll. Cuba, ii, pl. xvii, figs. 20-22. 34.

Natica sagraiana Weink., Mal. Bl., xxiv, 66. Ca.

Natica semisulcata Gray, Mal. Bl., xxiv, 57. PR.

Natiea semisulcata Rve., Mal. Bl., xxir, 57. PR.

Natica sulcata Born, Araugro, Fauna Mal. Cuba, 1878. 171. Ca.

Natica suleata Boru, Krebs. 67.

Natica sulcata Born, Krebs' Remarks. 397.

Natica sulcata Born,* Mal. Bl., xxiv, 62. G.

Natica (Stigmaulax) sulcata Boru, Marrat, Argo Exp.,1876. 18. Abaco.

Natica sulcata Born, Moll. Cuba, ii, 33.

Natica sulcata, Born, Poulsen. 10.

Natica suleata Dillw., Moll. Cuba, ii, 33.

Natica sulcata D'Orb., Arango, Fauna Mal. Cuba, 1878. 171. Ca.

Natica sulcata Mörch, Mal. Bl., xxir, 62.

Natica suleata Rre., Mal. Bl., xxiv, 62.

Natica treniata Mke., Mal. Bl., xxiv, 64 .

Natica texasiana Phil, Mal. Bl., xxiv, 57. Fla.

Natica texasiaua Philipli, Roemer's Texas. 457. 1849. Texas.

Natica texasiana Phil., Z Zt. Mal., v, 15S. Tex.

Natica texasiana Rïm. (=fossata Gld.), Mal. Bl., xxir, 57. Fla.

Natica uberina DOOrb., Arango, Fauna Mal. Cuba, 1878. 171. Ca. M.

G. Santa Lucia.

Natica uberina D'Orb., Mal. Bl., xxiv, 60. Ca.

Natica uberina D'Orb., Moll. Cuba, ii, pl. xvii, fig. 19. 31.

Náticina (see Natica, Sigaretus).

Naticina lactea Guild., Krebs. 67. 
Naticina lactea Guild., Mal. Bl., xxiv, 60. Ca.

Naticina regia Guppy, n. s., Geol. Mag., 1874. 436. J.

Naticina semisulcata Gray, Mal. Bl., xxiv, 57. PR.

Nautilus (see Spirula).

Nantilus exiguus Lister, Arango, Fanna Mal. Cuba, 1878. 149. Ca. Ba.

Nautilus exiguus Lister, Moll. Cuba, i, 64.

Nautilus papiraceus Mart., Moll. Cuba, i, 24.

Nautilus spirula Schröt., Arango, Fauna Mal. Cuba, 1878. 149. Ca. Ba.

Nautilus spirula Schröt., Moll. Cuba, i, 64.

Naricula (Arca) oceidentalis Blr., Krebs. 125.

Neæra alternata D'Orb., Krebs. 111.

Neæra alternata D'Orb., Report Blake Moll. 110. CS.

Neæra arcuata Dall, n. s., Report Blake Moll. 113. CS.

Nesera claviculata Dall, n. s., Report Blake Moll. 112. CS.

Nesera cleryana D’Orb., Krebs. 111.

Neæra costata Bush, Conu. Ac., vi, pl. xlv, fig. 21 472. Hatt.

Neiera costellata (Deshayes) Phil., lieport Blake Moll. 110. CS.

Neæra gigantea Verrill, List., 1884. 277. Chesapeake Bay.

Neæra granulata Dall, n.s., Report Blake Moll. 11. CS.

Neæra jeffreysi Dall, n. s., Report Blake Moll. 111. CS.

Neæra lamellifera. Dall, n. s., Report Blake Moll. 113. CS.

Nerera limatula Dall, n. s., Report Blake Moll. 112. CS.

Nexera multicostata Var. curta (J.), Verrill, List., 1884. 277. Ber. CS.

Neæra oruatissima D'Orb., Jahrb., ii, 248. G.

Neara ornatissima D'Orb., Krebs. 111. S'T.

Neæra ornatissima D’Orb., Poulsen. 15.

Nerera oruatissima D'Urb., Report Blake Moll. 110. CS.

Neæra pancistriata Dall, Bush. Conn. Ac., vi, 473. Hatt.

Neæra rostrata (Speng.) Lovén, Verrill, List., 1S84. 277. CS.

Neæra rostrata Speng., Report Blake Moll. 111. CS.

Neæra undata Verrill, List., 1584. 277. Chesapeake Bay.

Nebularia (see Mitra), Marrat, Argo Exp., 1876. 6.

Neilo dilatata Seguenza, Report Blake Moll. 125. CS.

Nemafera compressa Stm., n. g. and s., Kurtz. 8. SC.

Nerita (see Natica) Arango, Fama Mal. Cuba, 1878. 170. Antilles.

Nerita (see Natica) Arango, Fauna Mal. Cuba, 1878. 171. Ca.

Nerita (see Neritina) Arango, Fauna Mal. Cuba, 1878., 141, 173, 174, 279.

Ca. G. Antilles.

Nerita ala-papilionis Chem., Moll. Cuba, ii, 30.

Nerita albipunctata Rve., Marrat, Argo Exp., 1876. 9. SV.

Nerita ampullacea Müll., Moll. Cuba, ii, 3 .

Nerita antillarum Dillw., Moll. Cuba, ii, 52.

Nerita antillarum Desh., Moll. Cuba, ii, 52.

Yerita antillarum D'Orb., Arango, Fauna Mal. Cuba, 1878. 173. Ha. vana. Ca. 
Nerita antillarum Gmel., Arango, Fauna Mal. Cuba, 1878. 172. Havana. Ca.

Nerita antillarum Gmel., Jour. de Conch., i, 282.

Nerita antillarum Gmel., Krebs. 76. ST. NGr.

Nerita antillarum Gmel., Moll. Cuba, ii, 52.

Nerita cancellata Chem., Moll. Cuba, ii, 40.

Nerita cancellata Dillw., Moll. Cuba, ii, 33.

Nerita cancellata Gmel., Mal. Bl., xxiv, 61.

Nerita cancellata Gmel., Moll. Cuba, ii, 33 .

Nerita cancellata Herrm., Mal. Bl., xxiv, 61.

Nerita canrena Linn., Jour. de Conch., ii, 251.

Nerita canrena Born, Moll. Uuba, ii, 30.

Nerita canrena Dillw., Mal. Bl., xxir, 64.

Nerita canrena Gmel., Mal. Bl., xxiv, 64.

Nerita canrena Gmel., Moll. Cuba, ii, 30.

Nerita canrena var. Gmel., Moll. Cuba, ii, 33.

Nerita canrena Linn., Moll. Cuba, ii, 30.

Nerita duplicata Wood, Mal. Bl., xxiv, 57.

Nerita erythrodon Recl., Beau. 17. G.

Nerita erythrodon Recl., Jour. de Conch., i, 283.

Nerita erythrodon Recl., Krebs. 76.

Nerita exarata Pfr., Arango, Fauna Mal. Cuba, 1878. 173. Ca.

Nerita exarata Pfr., Archiv für naturg., 1840. 255. Ca.

Nerita flammea Gmel., Moll. Cuba, ii, 50.

Nerita listeri Pfr., A rehiv für naturg., 1840, 255. Ca.

Nerita magdalenæ Gmel., Jour. de Conch., i, 282.

Nerita mamilla Gmel., Mal. Bl., xxiv, 59.

Nerita mamilla Gmel., Moll. Cuba, ii, 32.

Nerita mamilla Schröt., Mal. Bl., xxiv, 59.

Nerita mamillaris Schröt., Arango, Fauna Mal. Cuba, 1878. 170. Ca.

Santa Lucia. Baracoa.

Nerita mamillaris Schröt., Moll. Cuba, ii, 32 .

Nerita maroccana Chem., Mal. Bl., xxiv, 64.

Nerita marochiensis Gmel., Mal. Bl., xxiv, 64.

Nerita nigerrima var., Chem., Arango, Fauna Mal. Cuba, 1878. 172. Havana. Ca.

Nerita nigerrima Chem, Krebs. $76 . \mathrm{PPI}$.

Nerita nigerrima Chem., Moll. Cuba, ii, 52.

Nerita nigerrima Chem., Poulsen. 13.

Nerita nitida Mtg., Mal. Bl., xxiv, 61. G.

Nerita nitida Phil., Mal. Bl., xxiv, 61. G.

Nerita peloronta Blv., Moll. Cuba, ii, 50.

Nerita peloronta Born., Moll. Cuba, ii, 49.

Nerita peloronta D’Orb., Arango, Fauna Mal. Cuba, 1878. 173. Ca. Antilles.

Nerita peloronta Gmel., Moll. Cuba, ii, 49. 
Nerita peloronta Lam., Moll. Cuba, ii, 49.

Nerita peloronta Lam., Jour. de Conch., vii, 27. G.

Nerita peloronta Lam., Jour. de Conch., vii, 28. G.

Nerita peloronta Liun., Arango, Fanna Mal. Cuba, 1878. 173. Ca. Autilles.

Nerita peloronta Liun., Beau. 17. G.

Nerita peloronta Linn., Jour. de Conch., ii, 427. G.

Nerita peloronta Linn., Krebs. 76. SM. ST. SJ. G. Bb.

Nerita (Pila) pelorontal Linu., Marrat, Argo Exp., 1876. 7. Aa. Barbuda. D. SV. Tucacas. Havana. Point Savanilla. Nassau. Long Key Island. Abaco.

Nerita pelorouta Linn., Moll. of Bermudas. 20.

Nerita peloronta Linn., Moll. Cuba, ii, 49.

Nerita peleronta Linu., Poulsen. 13.

Nerita peloronta Sby., Moll. Cuba, ii, 50 .

Nerita peloronta Wood, Moll. Cuba, ii, 49.

Nerita pennata Schröt., Mal. Bl., xxiv, 64.

Nerita pica.Chem., Moll. Cuba, ii, 51.

Nerita præcoguita C. B. Adams, Beau. 17. G.

Nerita pracognita C. B. Adams, Jour. de Conch., v, 152. G.

Nerita præcognita C. B. Adams, Krebs. 76. ST. SCx. SJ.

Nerita præcognita C. B. Adams, Poulsen. 13.

Nerita præcognita C. B. Adams, Synops, 7, 1845. J.

Nerita pupa Gmel., Moll. Cuba, ii, 45.

Nerita pupa Linn., Pfeiffer, Arehiv für naturg., 1840. 255. Ca.

Nerita pupa Wood, Moll. Cuba, ii, 45.

Nerita rufa Brown, Mal. Bl., xxiv, 65. J.

Nerita rufa Mtg., Mal. Bl., xxiv, 65. J.

Nerita rugosa Chem., Moll. Cuba, ii, 33.

Nerita rugosa Gmel., Mal. Bl., xxiv, 62.

Nerita (Pila) striata Chem., Marrat, Argo Exp., 1876. 6. Aa. Havalla.

Nerita striata Chem., Moll. Onba, ii, 50.

Nerita (I'ila) striata Mart., Marrat, Argo Exp., 1876. 8. D. Tueacas. SM. VC\%. Nassau. Long Key Island.

Nerita sulcata Born, Mal. Bl., xxiv, 62.

Nerita sulcata Born, Moll. Cuba, ii, 33.

Nerita sulcata Gmel., Moll. Cuba, ii, 33.

Nerita tessellata Gmel., Jour. de Conch., vii, 28. G.

Nerita tessellata var., Marrat, Argo Exp., 1876. 13. SM. Nassau.

Nerita tessellata D'Orb., Arango, Fauna Mal. Cuba, 1878. 173. Ca.

Nerita tessellata Gimel., Arango, Famma Mal. Cuba, 1878. 173. Ca.

Nerita tessellata Gmel., Beau. 17. G.

Nerita tessellata Gmel., Jour. de Conch., ii, 427. G.

Nerita tessellata Gmel., Krebs. 76.

Bull. 24-13 
Nerita tessellata Gmel., Mal. Bl., xii, 131.

Nerita (Pila) tessellata Gmel., Marrat, Argo Exp., 1876. 6. Aa. Barbuda. D. Tucacas. Point Savanilla. Long Key Island. Abaco.

Nerita tessellata Gmel., Moll. of Bermudas. 20.

Nerita tessellata Gmel., Moll. Cuba, ii, 51.

Nerita tessellata Lam., Moll. Cuba, ii, 51.

Nerita tessellata Wood, Moll. Cuba, ii, 52.

Nerita varia Meusch., Krebs. 76. ST. SJ. NGr.

Nerita varia Meusch., N. tessellata Gmel., Poulsen. 13.

Nerita variegata Chem., Araugo, Fauna Nal. Cuba, 1878. 173. Ca.

Nerita variegata Chem., Krebs. 77. ST. SJ. PPl. NGr.

Nerita variegata Chem., Poulsen. 13.

Nerita versicolor Desh., Moll. Cuba, ii, 50.

Nerita versicolor Gmel., Jour. de Conch., vii, 27. G.

Nerita versicolor Gmel., Jour. de Conch., vii, 28. G.

Nerita versicolor Gmel., Araugo, Famna Mal. Cuba, 1878. 173. Ca.

Nerita versicolor Ginel., Beau. 17. G.

Nerita versicolor Gmel., Jour. de Conch., i, 2S4. Ca. M.

Nerita versicolor Gmel., Jour. de Conch., ii, 427. G.

Nerita versicolor Gmel., Krebs. 77.

Nerita versicolor Gmel., Moll. Cuba, ii, 50.

Nerita versicolor Lam., Moll. of Bermudas. 20.

Nerita versicolor Lam., Moll. Cuba, ii, 50.

Nerita versicolor Wood, Moll. Cuba, ii, 50.

Nerita virginea Chem., Moll. Cuba, ii, 44.

Nerita virginea Dillw., Moll. Cuba, ii, 44.

Nerita virginea D'Orl), Araugo, Fauna Mal. Cuba, 1878. 142. Ca. Antilles.

Nerita virginea Gmel., Moll. Cuba, ii, 44.

Nerita virginea Linn., Moll. Cuba, ii, 44.

Nerita virginea Sby., Arango, Famna Mal. Cuba, 1878. 142. Ca. Antilles.

Nerita virginea Wood, Moll. Cuba, ii, 44.

Nerita riridis Born, Moll. Cuba, ii, 46.

Nerita viridis Chem., Moll. Cuba, ii, 46.

Nerita viridis Gmel., Moll. Cuba, ii, 46.

Nerita viridis Linn., Moll. Cuba, ii, 46.

Nerita viridis Sby., Arango, Fauna Mal. Cuba, 1878. 174. Ca. Antilles.

Nerita virjđis Schröt., Moll. Cuba, ii, 46.

Nerita viridis Wood, Moll. Cuba, ii, 46.

Neritæformis fusca Meusch., Mal. Bl., xxiv, 63.

Neritæformis pressa Meusch., Mal. Bl. xxiv, 57.

Neritella (see Neritina) Araugo, Fauna Mal. Cuba, 1878. 141. Ca. Havana.

Neritella meleagris Lam., Jahrb., ii, 245. 
Neritella (Vitta) meleagris Lam., Marrat, Argo Exp., 1876. 8. D. Ha. vana.

Neritella (Vitta) mertoniana Recl., Marrat, Argo Exp., 1876.10. Td. Tueacas. Athol Island.

Neritella (Vitta) mertoniana Risso, Marrät, Argo Exp., 1876. 13. Santa Marta.

Neritella (Vitta) pupa Lam., Marrat, Argo Exp., 1876. 14. Havaua. Neritella (Vitta) pupa Linn., Marrat, Argo Exp., 1876. 17. Long Key Island. Abaco.

Neritina brasiliana Recl., Beau. 17. G.

Neritina brasiliana Recl., Jour. de Conch., ii, 427. G.

Neritina brasiliana Recl., Krebs. 77.

Neritina brasiliana Recl., Poulsen. 13.

Neritina cassicula Sby., Krebs. 77.

Neritina eassiculum Sby., Beau. 17. G.

Neritina chlorina Link, Krebs. 77. ST. NGr.

Neritina chlorina Link, Krebs' Remarks. 397.

Neritina chlorina Link $=$ N. meleagris, Lam., Poulsen. 13.

Neritina clandestina Mke., Jour. de Conch., i, 152. Fla.

Neritina delineata Boubée, Jour. de Conch., i, 152. Ca.

Neritina domingensis Lam., Krebs. 77.

Neritina fasciolaris Chris., Jour. de Conch., i, 151.

Neritina floridana Shuttler., Arango, Fauna Mal. Cuba, 1878. 141. Ca. Havana.

Neritina fluviatilis Krebs. 77, 78.

Neritina jamaicensis Ad., Krebs. 77.

Neritina jamaicensis C. B. Adams, Krebs' Remarks. 397.

Neritina jayana Recl.,* Jour. de Conch., i, pl. vii, fig. 13. 157.

Neritina lineolata Lam., Jour. de Conch., i, 152. Ca.

Neritina litturata Sehultz, Jour. de Conch., i, 152. Ca.

Neritina meleagris D'Orb., Araugo, Fauna Mal. Cuba, 1878. 279. Ca. G.

Neritina meleagris D'Orb., Moll. Cuba, ii, 43.

Neritina meleagris Law., Arango, Fauna Mal. Cuba, 1878. 279. Ca. G. Neritina meleagris Lam., Beau. 17. G.

Neritina meleagris Lam., Jahrb. ii, 245.

Neritina meleagris Lam., Jour. de Conch., i, 152.

Neritina meleagris Lam., Krebs. 77.

Neritina meleagris Lam., Moll. Cuba, ii, 43.

Neritina meleagris Sby., Moll. Cuba, ii, 43.

Neritina microstoma D'Orb., Arango, Fauna Mal. Cuba, 1878. 141.

Ca. Havana.

Neritina microstoma D'Orb., Jour. de Conch., i, 152.

Neritina microstoma D'Orb., Krebs. 77.

Neritina microstoma D'Orb., Moll. Cuba, ii, pl. xrii, fig. 36. 48.

Neritina microstoma D'Orb., Poulsen. 13. 
Neritina mutabilis Ziegl., Jour. de Conch., i, 152.

Neritina nigra Sby., Jour. de Conch., i, 153. Ca.

Neritina ornata C. B. Adams, Contr. to Conch. 112. J.

Neritina ornata Ad., Krebs. 77.

Neritina ornata C. B. Adams, Krebs' Remarks. 397.

Neritina pulchella Gray, Krebs. 77.

Neritina punctulata Lam., Jour. de Conch., vii, 26. M. G.

Neritina punctulata Lam., Jour. de Conch., vii, 32.

Neritina punetulata Desh., Moll. Cuba, ii, 47.

Neritina punctulata D'Orb., Arango, Fauna Mal. Cuba, 1878. 141. J. G.

Neritina punctulata Lam., Arango, Fauna Mal. Cuba, 1878. 141. J. G.

Neritina punctulata Lam., Beau. 17. G.

Neritina punctulata Lam., Jour. de Conch., iv, 416. G.

Neritina punctulata Lam., Moll. Cuba, ii, 47.

Neritina punctulata Lam., Poulsen. 13.

Neritina punctulata Sby., Arango, Fauna Mal. Cuba, 1878. 141. J. G.

Neritina punctulata Sby., Moll. Cuba, ii, 47.

Neritina punctulata Lam., Krebs. 77.

Neritina pupa D'Orb., Arango, Fauna Mal. Cuba, 1878. 173. Ca. Antilles.

Neritina pupa Linn., Arango, Fauna Mal. Cuba, 1878. 173. Ca. Antilles.

Neritina pupa Linn., Beau. 17. G.

Neritina pupa Linn., Jour. de Conch., i, 152. Ca.

Neritina pupa Linn., Jour. de Conch., ii, 427. G.

Neritina yupa Linn., Krebs. 78.

Neritina pupa Linn., Krebs. 78. PPl.

Neritina pupa Linn., Poulsen. 13.

Neritina pupa Sby., Moll. Cuba, ii, 45.

Neritina pupa Sby., Moll. Cuba, ii, 48.

Neritina (?) pusilla C. B. Adams, Contr. to Conch. 112. J.

Neritina pusilla Ad., Krebs. 78.

Neritina pygmæa Ad., Krebs. 78.

Neritina pygmæa C. B. Adams, Synops., 7, 1845. J.

Neritina reclivata Say, Arango, Fauna Mal. Cuba, 1878. 141. Ca. Havana.

Neritina reclivata Sar, Dall, Hemphill's shells. 335. Tampa.

Neritina reclivata Say, Jour. de Conch., i, 152. Fla.

Neritina reclivata Say, Poulsen. 13.

Neritina roseolineata (Sby.) Recl., Jour. de Conch., i, 150. G.

Neritina striata Chem., Krebs. 77.

Neritina succinea Recl., Beau. 17. G.

Neritina succinea Recluz., Jour. de Conch., i, 150. G.

Neritina succinea Recl., Jour. de Conch., ii, 427. G. 
Neritina succinea Recl., Krebs. 78. SJ.

Neritina tenebrosa Ad., Krebs. 78.

Neritina tigris Jan., Jour. de Conch., i, 151.

Neritina trabalis Meusch., Krebs. 78.

Neritina tristis D'Orb., Arango, Fauna Mal. Cuba, 1878. 173. Ca. Antilles.

Neritina tristis D'Orb., Beau. 17. G.

Neritina tristis D’Orb., Jour. de Conch., i, 153. Ca.

Neritina tristis D'Orb., Jour. de Conch., iv, 416. G.

Neritina tristis D’Orb., Krebs. 78.

Neritina tristis D'Orb., Moll. Cuba, ii, pl. xvii, fig. 35. 47.

Neritina tristis D'Urb., Poulsen. 13.

Neritina turriculata Mke., Krebs. 78.

Neritina venosa Mke., Jour. de Conch., i, 152. Ca.

Neritina renosa Míke., Jour. de Conch., ii, 427. G.

Neritina virginea D'Orb., Moll. Cuba, ii, 44.

Neritina virginea Lam., Arango, Fauna Mal. Cuba, 1878. 141. Ca. Antilles.

Neritina virginea Lam., Beau. . 17. G.

Neritina virginea Lam., Jour. de Conch., i, 151.

Neritina virginea Lam., Krebs. 77, 78.

Neritina virginea Lam., Moll. Cuba, ii, 43, 44. Fla. WI.

Neritina rirginea (rar.) Linn., Jour. de Conch., ii, 427. G.

Neritina virginea Linn., Krebs. 7S. ST. SCx.

Neritina virginea Linn., Krebs' Remarks. 397.

Neritina virginea Liun., Poulsen. ' 13.

Neritina virginea Sby., Jour. de Conch., i, 152.

Neritina virginea Sby., Moll. Cuba, ii, 44.

Neritina viridis D’Orb., Arango, Fauua Mal. Cuba, 1878. 174. Ca. Antilles.

Neritina viridis Lam., Moll. of Bermudas. 20.

Neritina viridis Lam., Moll. Cuba, ii, 46. Fla. WI.

Neritina viridis Linn., Arango, Fauna Mal. Cuba, 1878. 174. Ca. Antilles.

Neritina viridis Linn., Bean. 17. G.

Neritina viridis Linn., Jour. de Conch, i, 150.

Neritina viridis Linn., Jour. de Conch., ii, 427. G.

Neritina riridis Limn., Krebs. 78. ST. SCx. SJ. SM. Ag. Td.

Neritina viridis Linn., Poulsen. 13.

Neritina viridis Sby., Moll. Cuba, ii, 46.

Neritina zigzag Sby., Jour. de Conch., i, 152. Fla.

Neritula (see Nassa).

Neritula neritea Linn., Arango, Fauna Mal. Cuba, 18is. 201. Ca.

Neverita (sce Natica, Nerita).

Neverita campechieusis Recl., Mal. Bl., xxir, 57.

Neverita duplicata Dall, Hemphill's shells. 342. 
Neverita duplicata Say, Am. Marine Couch. 60.

Neverita duplicata Say, Dall, Hemphill's shells. 335. Cedar Keys.

Neverita duplicata Say, Mal. Bl., xxiv, 58.

Neverita fossata Gld., Mal. Bl., xxiv, 57. Fla.

Nigritarum moneta Lister, Moll. Cuba, ii, 92. (Суpræa.)

Niua tuberculata Gray, Mal. Bl., xxiii, 132.

Nina tuberculata Gray $=$ Littorina antoni Phil., Poulsen. 9.

Niso ægleës Bush, Conn. Ac. vi, pl. xlv, fig. 10. 465. Hatt.

Nitidella (see Columbella) Marrat, Argo Exp., 1876. 8.

Nitidella cribraria Lam., Dall, Hemphill's shells. 326. Key West.

Nitidella filosa Stearns, Proc. Phil. Acad., 1873. 345. Tampa. W. Fla.

Nitidella nitidula Sby., Krebs. 29. Ag. ST. SJ. SCx. SM. SB.

Nitidella Stearusii Tryon (filosa St.), Man., v, 1883. 179. Fla.

Noetia (Arca) martinii Recl., Jahrb., ii, 253.

Noetia ponderosa Say, Am. Jour. Conch., i, 77. Fla.

Notarchus laciniatus Riipp., Guppy, Paria Fauna, 1877. 137.

Notarchus lacinulatus Couth., Mal. Bl., xxii, 176. Bz.

Notarchus pleii D’Orb., Arango, Fauna Mal. Cuba, 187s. 156. Antilles. ST. SCx.

Notarchus pleii Rang, Arango, Fauna Mal. Cuba, 1878. 156. Antilles.

Notarchus pleii Rang, Jour. de Conch., xi, 25. ST.

Notarchus pleii Rang, Mal. Bl. xxii, 176. ST.

Notarchus pleii Rang, Moll. Cuba, i, 118.

Notarchus poījomma Mörch, Jour. de Conch., xi, 25. ST.

Notarchus polyomma Mörch, Mal. Bl., xxii, 176. ST.

Nucula acuta Con., Kurtz. 5. NC. SC.

Nucula baccata Guppy, Geol. Mag., 1874. 451. Td.

Nucula crenifera Sby., Jahrb., ii, 253.

Nucula crenulata A. Adams, Report Blake Moll. 123.

Nucula crenulata Hanley, Report Blake Moll. 123.

Nucula cytherea Dall, n. s., Report Blake Moll. 123.

Nucula dilatata Phil., Report Blake Moll. 125.

Nucula eborea Conrad, Proc. Acad. Nat. Sci. Phil., iii, 24, pl. i, fig. 4.

1845. Tampa Bay.

Nucula proxima Say, Am. Marine Conch. 180. NC.

Yucula recurva Conrad, Journ. Acad. Nat. Sei. Phil., vi, 262, pl. xi, fig. 21, 1831. WI.

Nucula tenuis Forbes \& Hanles, Report Blake Mioll. 123.

Nucula tenuis Mtg, Report Blake Moll. 123.

Nucula vieta Guppy, Geol. Mag., 1874. 451. Td.

Nuculocardia (see Crenella).

Nuculocardia divaricata D’Orb., Arango, Fauna Mal. Cuba, 1878. 260.

Antilles.

Nuculocardia divaricata D'Orb., Moll. Cuba, ii, pl. xxvii, figs. 56-59. 311.

Nuculocardia divaricata D'Orb., Report Blake Moll. 116.

Nux marina major Gualt., Moll. Cuba, i, 121. 
Nux marina oblonga Gualt., Moll. Cuba, i, 122.

Nux marina oblonga spiralis Gualt, Moll. Cuba, i, 131.

Obeliscus (see Pyramidella).

Obeliscus candidus Meusch., Mal. Bl., xxii, 158. S'T.

Obeliscus (Longchaeus) candidus Meusch., Poulsen. 7.

Obeliscus crenulatus Holmes, Am. Mariue Conch. 64.

Obeliseus erenulatus Holmes, Dall, Hemphill's shells. 330.

Obeliscus crenulatus Holmes, n. s., Post-Pleiocene Fossils, S. C., pl. xiii, figs. 14,14 a. 88 .

Obeliscus dolabratus (= terebellum) Dall, Hentphill's shells. 330. Antilles.

Obeliscus dolabratus Linn., Mal. Bl., xxii, 157. ST.

Obeliscus dolabratus Linn., c. v. subdolabratus Poulsen. 7.

Obeliscus floridanus Morch,* Mal. Bl., xxii, 15S. ST.

Ubeliscus hastatus A. Ad., Mal. Bl., xxii, 158. ST.

Obeliseus niveus Morch, Mal. Bl., xxii, 159. ST. V.

Obeliscus (Triptychus) niveus Morch, Poulsen. 7.

Obeliscus perforatus A. Ad., Mal. Bl., xxii, 157.

Obeliscus subdolabratus Morch, Mal. Bl., xxii, 157. ST.

Obeliscus terebellum Lam., Mal. Bl., xxii, 157. ST.

Ubeliscus terebellum Mull., Marrat Argo Exp., 1876. 16. Nassau. Abaco.

Obeliseus terebellum Sby. \& Mel., Dall, \#emphill's shells. 330.

Obeliscus tesselatus Ad., Dall, Hemphill's shells. 330. Cedar Keys.

Ocinebra (see Muricidea, Murex).

Ocinebra cantrainei Recl., Jahrb., iv, 244. G.

Ocinebra coccineus A. Ad., Jahrb., iv, 244. ST.

Ocinebra cyclostoma Sby., Dall, Hemphill's shells. 325.

Ocinebra despectus A. Ad., Jahrb., iv, 244.

Ocinebra fasciatus Sby., Jahrb., ii, 241.

Ocinebra fasciatus Sby., Jahrb., iv, 244. Bz.

Ocinebra haneti Petit, Jahrb., iv, 244. Bz.

Ocinebra hexagonus Lam., Jahrb., iv, 244.

Ocinebra hidalgoi Crosse, Jahrb., iv, 244.

Ocinebra nuceus Morch, Jahrb., ir, 245.

Ocinebra paupercula C. B. Adams, Jahrb., iv, 244. G.

Octopus americauus Blr., Arango, Fauna Mal. Cuba, 187S. 145. Antilles. M. G. Ca.

Octopus americanus Blv., Moll. Cuba, i, 18.

Octopus americanus D'Orb., Guply, Paria Fanna, 1877. 136.

Octopus antiquorum Blv., Moll. Cuba, i, 24.

Octopus appendiculatus Blv., Arango, Fauna Mal. Cuba, 1878. 146. Ca.

Octopus appendiculatus Blv., Moll. Cuba, i, 11 .

()etopus barkeri (Fér.) D’Orb., Arango, Fauna Mal. Cuba, 1878. .145. Autilles. M. G. Ca. 
Octopus barkeri Fér., Moll. Cuba, i, 18.

Octopus carolinensis Verrill, List, 1SS4. 265. Cape Hatteras.

Octopus graunlatus Blv., Moll. Cuba, i, 18.

Octopus granulatus Fér., Moll. Cuba, i, 18.

Octopus granulatus Lam., Arango, Fauna Mal. Cuba, 1878. 145. Antilles. M. G. Ca.

Octopus grauulatus Law., Moll. Cuba, i, 18.

Octopus mas Barker, Moll. Cuba, i, 18.

Octopus mas Brug., Moll. Cuba, i, 18.

Octopus ruber Rat.? Arango, Fauna Mal. Cuba, 1878. 146. Ca.

Octopus ruber Raf., Moll. Cuba, i, 15.

()etopus rugosus Blv., Moll. Cuba, i, 19.

Octopus rugosus Bose, Arano, Famma Mal. Cuba, 187S. 145. Antilles. M. G. Ca.

Octopus rugosus D’Orb., Arango, Fauna Mal. Cuba, 1878. 145. Antilles. M. G. Ca.

Octopus tuberculatus Blv., Moll. Cuba, i, 21.

Octopus tuberculatus Blv., Arango, Fanna Mal. Cuba, 1878. 146. Ca. Uctopus tubereulatus Blv., Moll. Cuba, i, 15, 16.

Octopus tubereulatus D'Orb., Arango, Fauma Mal. Cuba, 1878. 146. Ca. Octopus tuberculatus Raf.? Arango, Fauna Mal. Cuba, 1878. 146. Ca. Octopus vulgaris Blv., Moll. Cuba, i, 11.

Octopus vulgaris Carus, Moll. Uuba, i, 11.

Octopus vulgaris D’Orb., Araugo, Fauna Mal. Cuba, 1878. 146 Ca.

Octopus vulgaris D'Orb., Moll. Cuba, i, 11.

Octopus vulgaris Lam., Moll. Cuba, i, 11.

Octopus vulgaris Limn., Arango, Fauna Mal. Cuba, 1878. 146. Ca.

Octopus vulgaris Limn., Moll. Cuba, i, 11.

Ocythoe antiquorum Blv., Moll. Cuba, i, 24.

Ocythoe antiqnorum Leach, Arango, Fauna Mal. Cuba, 1878. 147. Antilles.

Ocythoe antiquorum Leach, Moll. Cuba, i, 24.

Ocythoe tuberculata Raf., Arango, Famna Mal. Cuba, 1878. 146. Antilles.

Ocythoe tuberculata Raf., Moll. Cuba, i, 24.

Odontostoma (see Odostomia).

Odontostoma depressa D'Orb., Krebs. 71.

Odontostoma depressa, D'Orb., Moll. Cuba, i, 238.

Odontostoma globosa D'Orb., Krebs. 71.

Odontostoma globulosa D'Orb., Moll. Cuba, i, 239.

Odontostoma pusilla' Pfr., Krebs. 71.

Odostomia (see Monoptygina, Odontostoma).

Odostomia acutidens n. s., Dall, Hemphill's shells. 331. Cedar Keys. Odostomia alba Callins, Dall, Hemphill's shells. 341. Cedar Keys. Orlostomia canaliculata C. B. Ariams, Contr. to Conch. 109. J. Odostomia canaliculata Ad., Krebs. 53.

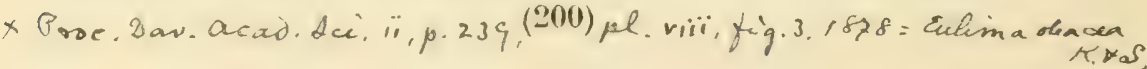


Odostomia canaliculata C. B. Adams, Krebs' Remarks. 397.

Odostomia canaliculata C. B. Adams, Mál. Bl., xxii, 167. J.

Odostomia canaliculata C. B. Adams, Poulsen. 8.

Odostomia cancellata I)Orb., Guppy, Geol. Mag., 1874. 445. Td.

Odostomia cubensis Pfr.; Mal. Bl., ii, 85. Ca.

Odostomia disparilis Verrill, List, 1584. 273. Cape Hatteras.

Odostomia engonia Bush, Conn. Ac., vi, 466. Hatt.

Odostomia gemmulosa ? Acl., Arango, Fauna Mal. Cuba, 1878. 161.

Odostomia gemmulosa C. B. Adams, Contr. to Conch. 109. J.

Odostomia gemmulosa C. B. Adams, Krebs. 54.

Odostomia gemmulosa C. B. Adams, Mal. Bl., xxii, 159. V. ST.

Odostomia impressa Say, Am. Marine Conch. 68.

Orlostomia impressa Say, Dill, Hemphill's shells. 331. Tampa. Cedar Keys. Saint Augustine.

Odostomia impressa Say, var. granatina Dall, Hemphill's shells. 331. Cedar Keys.

Odostomia lavigata D’Orb., Mal. Bl., xxii, 166. J.

Odostomia lævigata I'Orb., Poulsen. S.

Odostomia nitida Elder, Arango, Fauna Mal. Cuba, 1878. 162. Ca.

Odostomia ovuloides C. B. Adams, Contr. to Conch. 109. J.

Odostomia ovuloides Ad., Krebs. 55.

Odostomia pusilla Linn., Mal. Bl., ii, 85. Ca.

Odostomia pusilla Linn., Mal. Bl., xxii, 167. Ca. ST.

Odostomia pusilla Linn., Pfr., Poulsen. 8.

Odostomia pusilla Pfr., Arango, Fauna Mal. Cuba, 1878. 161.

Odostomia pusilla Pfr., Mal. Bl., xxii, 167. Ca.

Odostomia pusilla Pfr., ${ }^{*}$ Zt. Mal., viii, 88. Ca.

Odostomia seminuda O. B. Adams, Am. Marine Conch. 68.

Odostomia solida Ad., Krebs. 55.

Odostomia solida C. B. Adams, Krebs'.Remarks. 397.

Odostomia solidula C. B. Adams, Contr. to Conch. $110 . \quad J$.

Odostomia solidula C. B. Adams, Mal. Bl., xxii, 166. J.

Odostomia (var.) teres Bush, Comn. Ac. vi, pl. xly, fig. 9. 467. Hatt.

Odostomia toreula Mörch,*. Jour. de Conch., xxiv, 372. ST.

Odostomia tornata Verrill, List, 18St. 273. Cape Hatteras.

Oliva (see Olivella), Aringo, Fauna Mal. Cuba, 1878. 193. Ca. Ba.

Oliva aldinia Duclos, Jahrb., $\mathbf{r}, 116$.

Oliva ancillaria D'Orb., Krebs. 39.

Uliva angulata Lam., Krebs. 38.

Oliva araneosa Lam., Beau. 6. G.

Oliva araneosa Lam., Krebs. 40.

Oliva attenuata Rve., Krebs. 38.

Oliva bewleyi Marr, Jahrb., v, 117.

Oliva brasiliana Lam., Jahrb., ii, 242.

Oliva brasiliana Rre., Jabrb., v, 120. Bz.

Oliva brasiliana Weink, Jahrb., v, 120. Bz. 
Oliva brasiliensis Chem., Jahrb., v, 120. Bz.

Oliva brasiliensis Chem., Poulsen. 12.

Oliva bullata Marr., Jahrb., v, 116.

Oliva bullula Rve., Beau. 6. G.

Oliva bullula Rre., Krebs. 38. ST. SB.

Oliva bullula Rve., Poulsen. 12.

Oliva bullula Sby., Moll. of Bermudas. 17.

Oliva circinata Marr., Jahrb., v, 117.

Oliva conoidalis Duclos, Moll. Cuba, ii, 106.

Oliva conoidalis Lam., Arango, Fauna Mal. Cuba, 1878. 192. Ca. Coa.

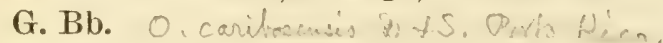

Oliva conoidalis Lam., Beau. 6. G.

Oliva conoidalis Lam., Jour. de Conch., ii, 430. G.

Oliva conoidalis Lam., Krebs. 38.

Oliva conoidalis Lam., Moll. Cuba, ii, 105.

Oliva conoidalis Lam., Poulsen. 12.

Oliva conoidalis Lam., v. mygdonia Ducl., Poulsen. 12.

Oliva dealbata Rre., Beau. 6. G.

Oliva dealbata Rve., Jour. de Conch., v, 153. G.

Oliva dealbata Rve., Krebs. 38.

Oliva deshayesiana Duclos, Jahrb., v, 120. Bz.

Oliva eburnea Duclos, Moll. Cuba, ii, 106.

Oliva eburnea Lam., Arango, Fauna Mal. Cuba, 1878. 193. Ca. Ba.

Oliva eburnea Lam., Beau. 6. G.

Oliva eburnea Lam., Jour. de Conch., ii, 430. G.

Oliva eburnea Lam., Krebs. 40.

Oliva eburnea Lam., Moll. Cuba, ii, 106.

Oliṿa esther Duclos, Krebs. 38.

Oliva exigua Mart., Arango, Fauna Mal. Cuba, 1878. 192. Ca. Ba.

Oliva exigua Mart., Krebs. 38; ST. Bb. C.

Oliva fimbriata Rve. ? Arango, Fauna Mal. Cuba, 1878. 192. Ca. G.

Oliva fimbriata Rve., Beau. 6. G.

Oliva fimbriata Rve., Jour. de Conch., v, 153. G.

Oliva fimbriata Rve., Krebs. 38.

Oliva fimbriata Rve., Poulsen. 12.

Oliva flammulata Lam., Krebs. 38.

Oliva flammulata Lam., Mal. Bl., x, 225.

Oliva fulgida Rve., Bean. 6. G.

Oliva fulgida Rre. Krebs. 38. ST. SB. Ag.

Oliva fulgurina Bory St. Vincent, Poulsen. 12.

Oliva fusiformis Lam., Beau. 6. G.

Oliva fusiformis Lam., Jahrb., v, 116. Fla.

Oliva fusiformis Lam., Jour. de Conch., iv, 419. G.

Oliva fusiformis Lam., Krebs. 39.

Oliva guildingii Rve., Krebs. 38.

Oliva ispida Link, Krebs. 39. ST. 
Oliva ispida Link, $=$ O. fusiformis Lam., Poulsen. 12.

Oliva ispida Weink., Jahrb., v, 116. Fla.

Oliva jamaicensis Marrat, Am. Jour. Conch., iv, 48. J.

Oliva jaspidea Gmel., Beau. 6. G.

Oliva jaspidea Gmel., Jour. de Conch., ii, 430. G.

Oliva lanceolata Rve., Krebs. 40.

Oliva leucostoma Duclos, Krebs. 39.

Oliva leucostoma Duclos, Moll. Cuba, ii, 111.

Oliva ligneola Rve., Krebs. 39.

Oliva litterata Lam., Jour. de Conch., ir, 419. - G.

Oliva litterata Lam., Am. Marine Conch. 46.

Oliva litterata Lam., Arango, Fauna Mal. Cuba, 1878. 191. Сa. Наvana.

Oliva litterata Lam., Jahrb., v, 117.

Oliva litterata Lam., Jour. de Conch., ii, 430. G.

Uliva litterata Lam., Krebs. 39. VCz.

Oliva litterata Lam., Poulsen. 12.

Oliva mercatoria Marr., Jahrb., v, 116.

Oliva mica Duclos, Araugo, Fauna Mal. Cuba, 1878. 192. J.

Oliva mica Duclos, Krebs. 39.

Oliva mica Duclos, Moll. Cuba, ii, 107.

Oliva mica Duclos, Poulsen. 12.

Oliva micans Sol., Krebs. 39.

Oliva miliola D'Orb., Krebs. 39.

Oliva millepunctata Duclos, Krebs. 39.

Oliva minuta Link, Krebs. 39.

Oliva mitreola Duclos, Poulsen. 12.

Oliva mitrula Duelos, Krebs. 39.

Oliva mutica Say, Poulsen. 12.

Oliva mutica Rve., Araugo, Fauma Mal. Cuba, 187S. 192. Ca. G.

Oliva mutica Rve., Beau. 6. G.

Oliva mutica Rve, Krebs. 39. ST. SB. Ber.

Oliva mutica, Say, Am. Marine Conch. 46.

Oliva mutica Say, Jour. de Conch., iv, 419. G.

Oliva myriadiua Duclos, Arango, Fauna Mal. Cuba, 1878. 192. Ca.

Oliva myriadina Duclos, Krebs. 39.

Oliva myriadina Duclos, Moll. Cuba, ii, 108.

Oliva myriadina Duclos, Poulsen. 12.

Oliva nana Lam., Krebs. 39.

Oliva nana Lam., Krebs. 40.

Oliva nana Lam., Mal. Bl., x, 225.

Oliva nitidula Dillw., Guppy, Paria Fauna, 1877. 145.

Oliva nitidula Link, Krebs. 39.

Oliva nitidula Liuk, Krebs. 41.

Oliva nitidula Sol., Arango, Fauna Mal. Cuba, 1878. 192. Ca. G.

Oliva nivea D’Orb., Krebs. 39. 
Oliva nivea Gmel., Beau. 6. G.

Oliva nivea Gmel., Jour. de Conch., ii, 430. G.

Oliva nivea Gmel., Poulsen. 12.

Oliva obesina Duclos, Beau. 6. G.

Oliva obesina Duclos, Jahrb., $\vee$, 116. Fla.

Oliva obesina Duclos, Krebs. 40.

Oliva olivaceus Mensch., Krebs. 40. Tort. Ag. ST. SB. SCx. SJ. Td.

Oliva onisea Duclos, Jahrb. v, 116.

Olira oryza Lam., Arango, Fauna Mal. Cuba, 1878. 192. Ca. Ba.

Oliva oryza Lam., Beau. 6. G.

Oliva oryza Lam., Jonr. de Conch., ii, 430. G.

Oliva oryza Lam., Krebs. 40. SB. SM.

Oliva oryza Lam., c. v. xauthospira, Poulsen. 12.

Oliva parrula Mart., Krebs. 40. S'T. SCx. SB. Ag. Bb.

Oliva pindarina Duclos, Beau. 6. .G.

Oliva pindarina Duclos, Krebs. 40.

Oliva polita Marr., Jahrb., v, 117.

Oliva pulchella Duclos, Krebs. 40. ST.

Oliva pulchella Reeve, Poulsen. 12.

Oliva pura Rve., Krebs. 40. SM. ?

Oliva pura Reeve, Poulsen. 12.

Oliva reticularis D'Orb., Arango, Fauna Mal. Cuba, 1878. 191. Ca. G. Ba.

Oiiva reticularis Duclos, Moll. Cuba, ii, 109.

Oliva reticularis Lam., Am. Marine Conch. 46. WI.

Oliva reticularis Lam., Arango, Fauna Mal. Cuba, 1878. 191. Ca. G. Ba.

Oliva reticularis Lam., Beau. 6. G.

Oliva reticularis Lam., Jour. de Conch., iv, 419. G.

Oliva reticularis Lam., Krebs. . 40.

Oliva reticularis Lam., Moll. Cuba, ii, 109.

Oliva reticularis Lam., c. . fasciata Ducl., unicolor, alba et castanea, Poulsen. 12.

Oliva reticulata Lam., Moll. of Bermudas. 17.

Oliva rosalina Duclos, Arango, Fanna Mal. Cuba, 1878. 193. Ca.

Oliva rosalina Duclos, Krebs. 41.

Oliva rosalina Duclos, Poulsen. 12.

Oliva rufifasciata Rve., Krebs. 41. ST.

Oliva sayana Rav., Ravenel. 16. SC.

Oliva scripta D'Orb., Arango, Fauna Mal. Cuba, 1878. 192. Ca. G. Santa Lucia. Fla.

Olira scripta Duclos, Moll. Cuba, ii, 110.

Oliva seripta Lam., Arango, Fauna Mal. Cuba, 18̈8, 192. Ca. G. Santa Lucia. Fla.

Oliva scripta Lam., Beau. 6. G.

Oliva scripta Lam., Jour. de Conch., iv, 419. G. 
Oliva scripta Lam., Krebs. 41.

Oliva scripta Lam., Moll. Cuba, ii, 110.

Oliva scripta Lam., Poulsen. 12.

Uliva strigata Rve., Jahrb., v, 123.

Oliva strigata Rve., Krebs. 39.

Oliva strigata Rive., Krebs. 41.

Oliva tigrina Lam., Poulsen. 12.

Oliva timoria Duclos, Beau. 6. G.

Oliva timoria Duclos, Krebs. 40.

Oliva venulata Duclos, Beau. 6. G.

Oliva venulata Duclos, Krebs. 40.

Oliva zigzac Duclos, Krebs. 39.

Olivancillaria brasiliensis Chem., Jahrb., ii, 242. Bz.

Olivancillaria leucostoma D'Orb., Moll. Cuba, ii, 111.

Clivaris (see Columbella), Arango, Fauna Mal. Cuba, 1878. 198. Antilles.

Olivaris barbadensis Pet., Moll. Cuba, ii, 133.

Olivella (see Oliva, Olivina).

Olivella alba Marr, Jahrb., v, 127.

Olivella bullula Rve., Jahrb., v, 129.

Olivella conoidalis Lam., Jahrb., $\nabla, 128$. G.

Olivella cuneata Marr., Jahrb., $\nabla, 127$.

Olivella dealbata Rve., Jahrb., v, 127.

Olivella eburnea Lam., Jahrb., $\mathbf{v}, 126$.

Olivella elongata Marr., Jahrb., v, 127.

Olivella exigua Marr., Jahrb., v. 128. G.

Olirella exigua Mart., Araugo, Fauna, Mal. Cuba, 1878. 192. Ca. Ba. Olivella fimbriata Rve., Arango, Fauna Mal. Cuba, 1878. 192. Ca. G.

Olivella fimbriata Rre., Jahrb., v, 125.

Olirella floralia Duclos, Jahrb, v, 127.

Olivella fulgida Rve., Jahrb., v, 127.

Olivella gracilis Brod. \& Sby., Marrat, Argo Exp., 1876. 11. La Guayra.

Olivella guildingi Rve., Jahrb., v, 127. SV.

Olivella guillingii Rve., Marrat, Argo Exp., 1876. 8. Barbuda.

Olivella jaspidea Gmel., Arango, Fauma Mal. Cuba, 1878. 192. Ca.

Coa. G. Bb.

Olivella jaspidea Gmel., Jahrb., v, 128. G.

Olivella lepta Duclos, Jahrb.,, 127.

Olivella mica Duclos, Arango, Fauna Mal. Cuba, 1878. $192 . \quad$ J.

Olivella mica Duclos, Jahrb., v, 125.

Olivella micans Dillw., Jahrb., v, 125.

Olivella miliacea Marr., Jahrb., v, 129.

Olivella miliola D'Orb., Arango, Fauna Mal. Cuba, 1878. 192. J. M.

Olivella millepunctata Duclos, Jahrb., v, 125.

Olivella miriadina Duclos; Jahrb., v, 129.

Olivella monilifera Rve., Jahrb., v, 126. 
Olivella mutica Rve., Jahrb., v, 125. G.

Olivella mutica Say, Dall, Hemphill's shells. 324. Cedar Keys.

Olivella mutica Say, Jahrb., v, 125.

Olivella mygdonia Duclos, Jahrb., r, 12S. G.

Olivella myriarlina Duclos, Arango, Fauna Mal. Cuba, 1878. 192. Ca.

Olivella nana Lam., Jahrb., $\nabla, 125$.

Olivella nitidula Dillw., Jahrb., v, 125.

Olivella nivea Gmel., Jahrb.,.v, 126.

Olivella oryza Duclos, Jahrb., v, 127.

Olivella oryza Lam., Arango, Fauua Mal. Cuba, 1878. 192. Ca. Ba.

Olivella oryza Lam., Dall, Hemphill's shells. 324. Sarasota Bay.

Olivella oryza Lam., Jahrb., v, 126.

Olivella oryza Lam., Marrat, Argo Exp., 1876. 18. Abaco.

Olivella oryza Sol., Jahrb., ii, 242.

Olivella parvula Marr., Jahrb., v, 126.

Olivella parrula Mart., Arango, Fauna Mal. Cuba, 187s. 193. Ca. Ba.

Olivella parvula Mart., Marrat, Argo Exp., 1876. 11. La Guayra. Abaco.

Olivella pellucida Rve., Jahrb., v, 127.

Olivella pellucida Rve., Marrat, Argo Exp., 1876. 16. Nassau.

Olivella plana Marr., Jahrb., v, 129.

Olivella pulchella Rve., Jahrb., v, 127.

Olivella pulchella Rre., Marrat, Argo Exp., 1876. S. Barbuda.

Olivella pulla Marr., Jahrb., r, 127.

Olivella pusilla Marr., Jahıb., v, 125.

Olivella reevei Duclos, Jahrb., v, 127.

Olivella rosalina Duelos, Arango, Fauma Mal. Cuba, 1878. 193. Ca.

Olivella rosalina Duclos, Jahrb., v, 130.

Olivella rubra Marr., Jahrb., $\nabla, 127$.

Olivella rufifasciata Rve., Jahrb., v, 125.

Olivella sowerbyi Duelos, Jahrb., v, 130.

Olivella verreauxi Duclos, Jahrb., v, 125. G.

Olivella vitilia Watson, Lin. Soc. Jomrn., 1S52, xvi, 342. Cul.

Olirella volutelloides Marr., Jahrb., r, 130.

Olivella zigzac Duclos, Jahrb., v, 125.

Olivella zonalis Lam., Dall, Hemphill's shells. 324. Sarasota Bay.

Olivina (see Olivella), Araugo, Fauna Mal. Caba, 187s. 192. J. M.

Olivina conoidalis D'Orb.; Moll. Cuba, ii, 105.

Olivina mica D'Orb., Arango, Fauna Mal. Cuba, 187S. 192. J.

Olivina miliola D’Orb., Moll. Cuba, ii, pl. xxi, figs. 20-22. 108.

Olivina mica D'Orb., Moll. Cuba, ii, 107.

Olirina myriadina D'Orb., Arango, Fauna Mal. Cnba, 1878. 192. Ca.

Olivina myriadina D’Orb., Moll. Cuba, ii, 108.

Olivina nivea D'Orb., Moll. Cuba, ii, 106.

Ommastrephes bartramii D'Orb., Arango, Fauna Mal. Cuba, 1878.

279. Antilles.

Ommastrephes bartramii D'Orb., Moll. Cuba, i, 59. 
Ommastrephes bartramii Lesueur, Arango, Fauna Mal. Cuba, 1878. 279. Antilles.

Ommastrephes todarus Raf., Moll. Cuba, i, 60-62.

Omphalius (see Trochus, Chlorostoma.)

Omphalius excavatus Lam., Arango, Fauna Mal. Cuba, 1878. 175. Baracoa. M. G.

Omphalius excaratus Lam., Marrat, Argo Exp., 1876. 9. SV. Tucacas. Abaco.

Omphalius fasciatus Born, Arango, Fauna Mal. Cuba, 1878. 175. Ca. G. M. Santa Lucia. Ba.

Omphalius gundlachi Phil., Arango, Fanna Mal. Cuba, 1878. 175. Ca. Omphalius hotessierianus D'Orb., Arango, Fauna Mal. Cuba, 1878. 176. Ca. Antilles. G. Fla. Ba.

Omphalius indusii Chem., Arango, Fauna Mal. Cuba, 1878. 176. Ca. Ba.

Omphalius indusii Phil., Krebs. 84.

Omphalius maculo-striatus C. B. Adams, Marrat, Argo Exp., 1876. 18. Abaco.

Omphalius sealaris Anton, Marrat, Argo Exp., 1876. 16. Nassau. Abaco.

Omphalius viridulus Gmel., Jahrb., ii, 246.

Omphalius viridulus Gmel., Jahrb., ii, 253.

Omphaloclathrnm reticulatum Linn., Arango. Fauna Mal. Cuba, 1878.

$$
\text { 249. Ca. = lisloni } 819-
$$

Onchidium floridanum Dall, Proe. U. S. Nat. Mus., 18s5. 288. Knight's Key, Fla.

Onchis (Peronella) armadilla Mörch, * Jour. de Conch., xi, 43. ST.

Oniscia (see Cassidaria, Cassidea, Morum).

Oniscia dennisoni Desh., Măl. Bl., xxiv, 41. G.

Oniscia dennisoni Rve, Beau. 8. G.

Oniscia dennisoni Rve., Jahrb., $\nabla, 239 . \quad$ G.

Oniscia dennisoni Rre., Mal. Bl., xxiv; 41. G.

Oniscia dennisonii Rve., Krebs. 35.

Oniscia lamarcki Desh., Jahrb., v, 238.

Oniscia lamarckii Desh., Mal, Bl., xxiv, 40.

Oniscia oniscus Lam., Jahrb., v, 238.

Oniscia oniscus Linn., Arango, Fanna Mal. Cuba, 1878. 224. Ba. Ca. Havana. G. Santa Lucia.

Oniscia oniscus Linn., Bean. 8. G.

Uniscia oniscus Linu., Jahrb., $\nabla, 238$.

Oniscia oniscus Linn., Krebs. 35. ST. Tort. SB. Ag. SM. Td.

Oniscia oniscus Sby., Jour. de Conch., iv, 418. G.

Oniseia oniscus Sby., Mal. Bl., xxir, 40.

Oniscia oniscus Sby., Moll. Cuba, ii, 130, 131.

Oniseia quadriseriata Mke., Jahrb., 5,239 . Bb.

Oniscia quadriseriata Mke., Mal. Bl., xxir, 41. 
Oniscia strombiformis Rre., Jahrb., v, 238.

Oniscia strombiformis Rve., Mal. Bl., xxiv, 40.

Oniscia triseriata Mke., Jahrb., v, 238.

Oniscia triseriata Mke., Mal. Bl., xxiv, 40.

Onustus (see Phorus, Trochus, Xenophora).

Onustus caribæus Petit, Mal. Bl., xxiv, 108. MG.

Onustus maximus Humph., Mal. Bl., xxiv, 108.

Onustus trochiformis Born, Arango, Fama Mal. Cuba, 1878. 181. Antilles. M.

Onustus trochiformis Humph., Krebs. 68.

Onustus trocbiformis Mörch, Mal. Bl., xxir, 109. G.

Onychia (see Onykia).

Onychia augulata Lesueur, Arango, Fauna Mal. Cuba, 1878. 278. Antilles.

Onychia caraibæa Lesueur, Moll. Cuba, i, 57.

Onyehia caribea Lesueur, Arango, Fanna Mal. Cuba, 1878. 279. Gulf of Mexico.

Onychoteuthis angulata D'Orb., Moll. Cuba, i, 46.

Onychoteuthis angulata Guér., Moll. Cuba, i, 46.

Onychotêuthis angulata Lesueur, Jour. de Conch., vi, 143. G.

Onychoteuthis angulata Lesueur, Moll. Cuba, i, 46.

Onyehoteuthis bergii D'Orb., Arango, Fauna Mal. Cuba, 1878. 278. Antilles.

Onychotenthis bergii Licht., Arango, Fama Mal. Cuba, 1878. 278. Antilles.

Onjchotenthis bergii Licht., Moll. Cuba, i, 46.

Onychoteuthis caraibæa D'Orb., Moll. Cuba, i, 57.

Onychoteuthis caraibæa Lesueur, Moll. Cuba, i, 56, 57.

Onychoteuthis cardioptera D’Orb., Arango Fauna Mal. Cuba, 1878.

279. Gulf of Mexico.

Onychoteuthis cardioptera D'Orb., Moll. Cuba, i, 53.

Ouychoteuthis carlioptera Peron, Arango, Fauna Mal. Cuba, 1878.

278. Gulf of Mexico.

Onychotenthis caribaea D'Orb., Arango, Fauna Mal. Cuba, 1878. 279. Gulf of Mexico.

Onychoteuthis caribaa Lesneur, Arango, Fauna Mal. Cnba, 1878. 279. Gulf of Mexico.

Onychotenthis feliua B|v., Moll. Cuba, i, 46.

Onychoteuthis fleuryi Reynaud, Arango, Fauma Mal. Cuba, 1878. 278. Antilles.

Onychoteuthis fleuryi Rey., Moll., Cuba, i, 46.

Onyehotenthis lessonii (Fér.) D’Orb., Arango, Famma Mal. Cuba, 1878. 278. Antilles.

Oychoteuthis lessonii Fér., Moll. Cuba, i, 46.

Onychoteuthis uncinata Q. \& G., Moll. Cuba, i, 46.

Onykia angulata Lesueur, Moll. Cuba, i, 46. 
Onylia carriboen Lesueur, Ac. Nat. Sci. Phil., Journ., ii, 98.

Oöcorys (see Benthodolium).

Operculatum (Umbrella) bermudense Mörch, Mal. Bl., xxii, 179. Ber.

Orbicula (see Discina).

Orbicula antillarum D’Orb., Arango, Fauna Mal. Cuba, 1878. 275. Ca. Orbicula antillarum D'Orb., Beau. 27. G.

Orbicula antillarum D'Orb., Jour. de Conch., xiv, 273. Ca. M. J.

Orbicula antillarum D’Orb., Moll. Cuba, ii, pl. xxviii, figs. 34-36. 368. Ca.

Orbicula cumingii Brod., Jahrb., ii, 254.

Orbicula striata Brod., Jahrb., ii, 254.

Orbicula strigata Brod., Jahrb., ii, 254.

Orthocera trachea Arango, Famna Mal. Cuba, 1878. 233. Ca.

Osteodesma (see Lyonsia).

Osteodesma beauiana D'Orb., Beau. 26. G.

Osteodesma beaniana D'Orb., Jour. de Conch., vi, 383. G.

Osteodesma hyalina Couthouy, Kurtz. 3. NC.

Osteorlesua orbignyi Fisch., *Jour. de Conch. vi, pl. xi, figs. 7, 8. 382. M.

Ostriea (see Isognomon), Arango, Fauna Mal. Cuba, 1875. 269. Ca. M. Ва.

Ostrea (see Ostrea, Pecten, Lima, Avicula, Spondylus, Radula, etc.). Ostrea (see Lima), Arango, Fama Mal. Cuba, 187S. 267. Ca. M. G. Santa Lucia. Ba.

Ostrea (see Vola), Arango, Fauna Mal. Cuba, 1878: 270. Ca. G. M. Santa Lncia. SD. S'T. Ba.

Ostrea alata Gmel., Arango, Fauna Mal. Cuba, 1878. 269. Ca. M. Ba.

Ostrea alata Gmel., Krebs. 132.

Ostrea arborea Chem., Krebs. 136.

Ostrea arborea Ohem. = O. parasitica Gmel., Poulsen. 16.

Ostrea aspera Chem., Arango, Fauna Mal. Cuba. 1878. 267. Ca. M.

G. Sauta Lucia. Ba.

Ostrea cauteriata Meusch., Krebs. 132.

Ostrea cristata Born, Jour. de Conch., vii, 30.

Ostrea cristata Born, Beau. 21. G.

Ostrea cristata Born, Krebs. 136.

Ostrea crucella Lam., Arango, Famua Mal. Cuba, 1578. 273. Ca. Ba. Ostrea cucullata Born, Krebs. 136.

Ostrea echinata Martyu, Arango, Fauna Mal. Cuba, 1878. 271. Ca. II. G.

Ostrea echinata Mart., Moll. Cuba, ii, 359.

Ostrea equestris Say, Am. Marine Conch. 193.

Ostrea erucella Lam., Krebs. 136.

Ostrea excarata Lam., Pfeiffer, A rchir fiir naturg., 1810. 260. Ca.

Ostrea foliacea Linn., Marrat, Argo Lxp., 1876. 19. Abaco.

Bull. $24-14$ 
Ostrea folium Born, Moll. Cuba, ii, 365.

Ostrea folium Chem., Moll. Cuba, ii, 365.

Ostrea folium D'Orb., Arango, Fauma Mal. Cuba, 1878. 273. Ca. Ba.

Ostrea folium D'Orb., Krebs. 136.

Ostrea folium Lam., Moll. Cuba, ii, 365.

Ostrea folium Liun., Arango, Fanna Mal. Cuba, 1878. 273. Ca. Ba.

Ostrea folium Linn., Moll. Cuba, ii, 365.

Ustrea frons Limu., Arango, Fauna Mal. Cuba, 1878. 273. Ca. Ba.

Ostrea frons Linn., Krebs. 136. VgI.

Ostrea frons Linn. $=$ O. limacella Lam., Poulsen. 16.

Ostrea fundata Say, Kurtz. 6. SC.

Ostrea gibba Linn., A rango, Fauna Mal. Cuba, 1878. 270. Ca.

Ostrea gibba Linn., Moll. Cuba, ii, 352.

Ostrea glacialis Gmel., Moll. Cuba, ii, 336.

Ostrea glacialis Solis, Krebs. 133.

Ostrea imbricata Gmel., Krebs. 134.

Ostrea limacella Lam., Arango, Fanna Mal. Cuba, 1878. 273. Ca. Ba.

Ostrea limacella Lam., Krebs. 136.

Ostrea limacella Lam; Mal. Bl., xxiv, 122.

Ostrea limacella Lam., Moll. Cuba, ii, 365.

Ostrea limacina Lam., Moll. Cuba, ii, 366.

Ostrea nodosa Born, Moll. Cuba, ii, 353.

Ostrea nodosa Limn., Arango, Famna Mal. Cuba, 1878. 270. Ca. Havana. G. M. Ba.

Ostrea nodosa Linn,, Moll. Cuba, ii, 353.

Ostrea parasitica Gmel., Jour. de Conch., vii, 26. M. G.

Ostrea parasitica Gmel., Jour. de Conch., vii, 33.

Ostrea parasitica Gmel., Jour. de Conch., vii, 39.

Ostrea parasitica Gmel., Arango, Fauna Mal. Cuba, 1878. 273. Ca.

Ostrea parasitica Gmel., Beau. 21. G.

Ostrea parasitica Gmel., Jour. de Conch., iv, 416. G.

Ostrea parasitica Gmel., Krebs. 136.

Ostrea puelchana D'Orb., Jahrb., ii, 254.

Ostrea radicum Chem., Krebs. 136.

Ostrea rareneliana T. \& H., n. s., Pleiocene Fossils S. C., pl. vi, figs. 1, $2,3.21$.

Ostrea rhizophoræ Chem., Krebs. 136.

Ostrea rostralis Lam., Beau. 21. G.

Ustrea rostralis Lam., Jour. de Conch., ii, 426. G.

Ostrea rostralis Lam., Krebs. 136.

Ostrea rostralis Lam., Poulsen. $\mathbf{1 6 .}$

Ostrea rubella Lam., Araugo, Fanua Mal. Cuba, 1878. 273. Ca. Ba.

Ostrea rubella Lam., Beau. 21. G.

Ostrea rubella Lam., Jour. de Conch., v, 151. G.

Ostrea rubella Lam., Krebs. 136.

Ostrea rubella Lam., Moll. Cuba, ii, 365, 66. 
Ostrea scabra Born, Moll. Cuba, ii, 336.

Ostrea semiauricula Linn., Moll. Cuba, ii, 347.

Ostrea,semicylindrica Say, Am. Marine Conch. 194.

Ostrea spondyloidea Meusch., Krebs. 135.

Ustrea spondyloides Gmel., Krebs. 135.

Ostrea spreta D’Orb., Arango, Fauna Mal. Cuba, 1878. 273. Ca.

Ostrea spreta D'Orb., Krebs. 136.

Ostrea spreta D'Orb., Moll. Cuba, ii, pl. xxviii, fig. 30. 365.

Ostrea transtra Meusch., Krebs. 136.

Ostrea triangularis Holmes, Am. Marine Conch. 193. SC.

Ostrea turgida Gmel., Arango, Fauna Mal. Cuba, 1878. 270. Ca.

Ostrea turgida Gmel., Moll. Cuba, ii, 352.

Ostrea virginiana Lister, Am. Marine Oonch. 192.

Ostrea virginica Gmel., Mal. Bl., x, 229.

Ostrea virginica Lam., Pfeiffer, Archiv für vaturg., 1840. 259. Ca.

Ostrea virginica List., Kurtz. 6. NC. SC.

Ostrea zigzag Born, Moll. Cuba, ii, 356.

Ostrea zigzag Linn., Moll. Cuba, ii, 356.

Ovula (see Ovulum, Rhizorus).

Ovula (see Cyphoma), Arango, Fauna Mal. Cuba, 1878. 183. Ca. Ha. vana.

Orula (see Volva), Arango, Fauna Mal. Cuba, 1878. 183. Ca. Ba.

Ovula acicularis Lam., Beau. 7. G.

Orula acicularis Lam., Jahrb., ix, 177.

Ovula acicularis Lam., Krebs. 43. SM.

Ovula acicularis Lam., Kurtz. 9. NC. SC.

Ovula acicularis Lam., Mal. Bl., xxiv, 54. SM. Antilles.

Ovula acicularis Lam., Moll. Cuba, ii, 87.

Uvula acicularis Lam., Poulsen. 10.

Ovula antillarum Rve., Jahrb., ix, 179. = umplecotá

Ovula antillarum Sby., Mal. Bl., xxiv, 55. Antilles.

Ovula birostris Lam., Pfeiffer, Archiv fuir naturg., 1840. 25̃9. Ca.

Ovula carolinensis Mörch, Mal. Bl. xxiv, j4. SC.

'Ovula gibbosa D'Orb., Araugo, Fauna Mal. Cuba, 1878. 183. Havana.

G. M. Santa Lucia. Ba.

Orula gibbosa Lam., Krebs. 43. ST. SJ. Ag. Td.

Ovula gibbosa Lam., Jour. de Conch., vi, 142.

Ovula gibbosa Lam., Moll. Cuba, ii, 86.

Ovula gibbosa Lam., Moll. Cuba, ii, 87.

Orula gibbosa Linn., Beau. 7. G.

Ovula gibbosa Linn., Jour. de Conch., ii, 429. G.

Ovula gibbosa Linn., Mörch, Mal. Bl., xxiv, 53. Fla. Antilles. CS. Cumana.

Orula gibbosa Linn., Poulsen. 10.

Qvula intermedia, Kurtz, 9. NC. SO. 
Orula intermedia Sby., Jahrb., ix, 175.

Orula intermedia Sby., Mal. Bl. xxiv, 53. Bz.

Ovula pharetra Perry, Mal. Bl.,.53. Ca. = giliva a d

Ovula subrostrata Sby., Jahrb., ix, 179. Hd. = miplieatr

Ovula subrostrata Sby., Mal. Bl., xxiv, 54. Hd. Bz.

()vula uniplicata Sby., Jahrb., ix, 178.

Ovula uniplicata Sby., Mal. Bl., xxir, 54. Bz.

Ovulum requale Sby., Mal. Bl., xxiv, 55. Fla. Paneina.

$\checkmark$ Orulum aciculare Sby., Jahrb., ix, 177.

Orulum acieulare Sby., Mal. Bl., xxiv, 54.

Orulum antillarum Rve., Am. Jour. Conch., ii, 161. = uniplieata

$\checkmark$ Ovulum gibbosum Linn., Moll. of Bermudas. 18.

Orulum gibbosum Sby., Mal. Bl., xxiv, 53. G.

Orulum immunitum Guppy, n. s., Geol. Mag., 1874. 439. . J.

Orulum intermedium Sby., Mal. B1., xxiv, 53, 54.

Ovum vanille peregrinum Seba, Moll. Cuba, i, 132.

Oxynoё (see Lophocercus, Sigaretus).

()хупоё antillarum Mörch, Jour. de Conch., xi, 27. ST.

Oxynoë autillaruın Mörch, Mal. Bl., xxii, 179. ST.

Oxynoё antillarum Oersted, Jour. de Conch., xi, 46.

Pachybathron (see Erato).

P'achybathron cassidiforme Gask., ${ }^{*}$ Mal. Bl., xxiv, 51.

Pachybathron eassidiformis Gask., Mal. Bl., xxiv, 52.

P'achybathron cassidiforme Gask., Poulsen. 10.

Pachybathron marginelloideum Chenu, Mal. Bl., xxiv, 52.

I'achybathron marginelloideum Gask., Mal. Bl., xxiv, 52.

Pachybathron marginelloideum Gask., Poulsen. 10.

Pachspoma (see Trochus).

P’achypoma cirlata Ḱlein, Arango, Fanua Mal, Cuba, 1878. 179. Ca.

M. G. Santa Lucia. Ba.

P'achypoma cubaua Phil., Arango, Fauna Mal. Cuba, 1878. 180. Ca. Paludestriua (see Amnicola).

l'aludestrina aftinis D’Orb., Arango, Fauna Mal. Cuba, 1878. 140. Ca. Antilles.

Paludestrina affinis D’Orb., Moll. Cuba, ii, pl. x, fig. 8. 8 .

P'aludestrina auberiaua D’Orb., Arango, Fauna Mal. Cuba, 1878. 140. Ca. Antilles.

I'aludestrina auberiana D'Orb., Moll. Cuba, ii, pl. 10, figs. 6-7. 8.

Paludestriua cancleana D'Orb., Arango, Fauna Mal. Cuba, 1878. 140. Ca. Antilles.

I'alurlestrina candeana D'Orb., Moll. Ouba, ii, pl. x, figs. 13-14. 49.

Paludina (see Paludestrina).

Paludina anthracina Migh., Arango, Fauna Mal. Cuba, 1878. 140.

Ca. Antilles.

Paludiua bermondiana D'Orb., Arango, Fauna Mal. Cuba, 1878, 139. Ca. 
Paludina bermondiana D'Orb., Moll. Cuba, ii, pl. x, fig. 5. †.

Paludina cisteruicola Mor., Arango, Fauna Mal. Cuba, 1878. 140. Ca. Antilles.

Paludina desnoyersii Payr., Moll. Cuba, ii, 5.

Paludina jamaicensis Ad., Arango, Fauna Mal. Cuba, 187s. 140. Ca. Antilles.

Paludina truncata Payr., Moll. Cuba, ii, 5.

Paludinella helicoides Gundl., Arango, Fauna Mal. Cuba, 187s. 139. Ca.

Paludinella succinea Pfr., Arango, Fauna Mal. Cuba, 1875. 139. Ca.

Pandora carolininensis Bush, Coun. Ac. vi, 474. Hatt.

Pandora oblonga Sby., Jahrb., ii, 248.

Pandora oblonga Sby.(?), Report Blake Moll. 109.

Pandora trilineata Say, Am. Marine Conch. 136.

Pandora ziczac Meg., Krebs. 134.

Panopea bitruncata Con., Am. Marine Conch. 138. NC. Fla.?

Papyridea (see Cardium).

Papyridea (lulvia) bullata Linu., Marrat, Argo Exp., 1876. 17. Nassau.

Papyridea spiuosum Sws., Krebs. 116.

Parastarte triquetra Conrad, Dall, Hemphill's shells. 339. = Astarte triquetra Conrad. Cedar Keys. Sarasota Bay. Tampa Bay.

Parthenia (see Chemnitzia) Arango, Fauna Mal. Cuba, 1s7s. 279. Ca. Parthenia cedrosa Dall, 11. s., Hemphill's shells. 331. Cerlar Kieys.

Patella (see Acmrea, Capulus, Tectura, Lottia, Pectinodonta, Coceulina, Addisonia, Hipponyx).

Patella (see Calyptræa) Araugo, Fauna Mal. Cuba, 1878. 226. Ca. Bh. Patella (see Cochlolepas) Arango, Fauna Mal. Cnba, 187s. 226. Ca.

Patella (see Crepidula) Arango, Fanua Mal. Cuba, 1sis. 220. Ca. Ba. Patella (see Emarginula) Arango, Fauna Mal. Cuba, 1s7s. 22.?). Ca.

Patella (see Fissurella) Arango, Fauna Mal. Cula, 1575. 227, 228, 229.

Ca. G. Ba. Fla.

Patella aculeata Gmel., Mal. Bl., xxiv, 102. Fla.

Patella aculeata Gmel., Moll. Cuba, ii, 191.

Patella albicosta Ad., Arango, Fanna Mal. Cuba, 1S78. 2:9. Ca. G.

Patella albicosta C. B. Adams, Beau. 19. G.

Patella albicosta C. B. Adams, Jour. de Conch., v, 151. G.

Patella albicosta C. B. Adams, Krebs. 88.

Patella albicosta C. B. Adams, Synops., 8, 184วั. I.

Patella albistriata List., Moll. Cuba, ii, 189.

Patella angusta Gmel., Krebs. 86.

Patella antillarum Sowerby, Krebs' Catalogne, 431. Ba. ST.

Patella antiquata Linu., Aringo, Fauna Mal. Cuba, 1sis. 225. Ca. G. Ba.

Patella antiquata Linn., Jour. de Conch., x, 20.

Patella antiquata Linn., Mal. Bl., xxiv, 98. 
Patella antiquata Linn., Zt. Mal., x, 79. SV.

Patella auricula Gmel., Mal. Bl., xxiv, 101.

Patella auricula Humph., Mal. Bl., xxiv, 102.

Patella auriculata Chem., Mal. Bl., xxiv, 101.

Patella auriculata Gmel., Krebs. 69.

Patella barbadensis Gmel.. Jahrb., ii, 246.

Patella barbadensis Gmel., Moll. Cuba, ii, 196.

Patella candeana D'Orb., Arango, Fauna Mal. Cuba, 1878. 229. Ca. G. M. Santa Lucia.

Patella candeana D'Orb., Beau. 19. G.

Patella candeana D'Orb., Jour. de Conch., v, 155. G.

Patella candeana D'Orb., Krebs. 89.

Patella eandeana D'Orb., Mal. Bl., iv, 27. G.

Patella candeana D'Orb., Moll. Cuba, ii, pl. xxr, figs. 1-3. 199.

Patella cernua Gmel., Mal. Bl., xxiv, 98.

Patella cernua Spengl., Krebs. 70.

Patella concamerata Grew., Mal. Bl., xxiv, 103.

Patella confusa Guild., Beau. 19. G.

Patella confusa Guild., Jour. de Conch., ii, 426. G.

Patella confusa Guild., Krebs. 88.

Patella cubaniana D'Orb., Arango, Fauna Mal. Cuba, 1878. 229. Ca. Fla.

Patella cubaniana D'Orb., Krebs. 88.

Patella cubaniana D'Orb., Moll. Cuba, ii, pl. xxv, figs. 4-6. 199.

Patella cubensis Rre., Beau. 19. G.

Patella cubensis Rve.. Krebs. 88.

Patella elegans Pliil., Krebs. 88.

Patella equestris Dillw., Mal. Bl., xxiv, 100. Hd.

Patella equestris Gmel., Moll. Cuba, ii, 189.

Patella equestris' $\beta$ Linn., Mal. Bl., xxiv, 100.

Patella equestris Linn., Moll. Cuba, ii, 189.

Patella extinctorium Sby., Krebs. 75.

Patella fungoides Bolt., Krebs. 75.

Patella incurva Gmel., Mal. Bl., xxiv, 97.

Patella jamaicensis Gmel., Moll. Cuba, ii, 197.

Patella laqueare Gray, Krebs. 86.

Patella lateraria Meusch., Mal. Bl., xxiv, 97.

Patella leucoplenra Gmel., Arango, Fauna Mal. Cuba, 1878. 230. Ca.

M. Santa Lucia. G. Ba.

Patella leucopleura Gmel., Moll. Cuba, ii, 198.

Patella leucopleura Lam.. Moll. Cuba, ii, 198.

Patella manipula Humph., Krebs. 85.

Patella militaris Pult., Mal. Bl., xxiv, 97.

Patella militaris Linn., Krebs. 70.

Patella militaris Wood, Moll. Cuba, ii, 187.

Patella mitrula Gmel., Krebs. 70. 
Patella mitrula Gmel., Mal. Bl., xxiv, 98. G.

Patella mitrula Gmel., Moll. Cuba, ii, 186.

Patella mitrula Gmel., Zt. Mal., x, 79. SV.

Patella mulleri Dkr.,* Jahrb., ii, 246.

Patella neptuni Dillw., Mal. Bl., xxiv, 100. Hd.

Patella nimbosa Gmel., Moll. Cuba, ii, 195.

Patella noachina Linn., Report Blake Moll, 74.

Patella nodosa Born, Moll. Cuba, ii, 197.

Patella notata Chem., Krebs. 88.

Patella notata Lam., Beau. 19. G.

Patella notata Lam., Jour. de Conch., ii, 426. G.

Patella notata Lam., Krebs. 88.

Patella notata Linn., Arango, Fauna Mal. Cuba, 18is. 230. Ca. Ba.

Patella octoradiata var., Gmel., Krebs. 75.

Patella pallescens Phil., Krebs. 75.

Patella porphyrozonias Gmel., Krebs. 86.

Patella porphyrozonias Gmel., Moll. Cuba, ii, 196.

Patella pulcherrima Guild., Mal. Bl., ir, 27. G.

Patella pulcherrima Guild., Bean, 19. G.

Patella pulcherrima Guild., Jour. de Conch., ii, 426. G.

Patella pulcherrima Guild., Jour. de Conch., v, 155. G.

Patella pulcherrima Guild., Krebs. 89.

Patella punctulata Gmel., Arango, Fauna Mal. Cuba, 1878. 230. Ca.

Havana. Ba.

Patella punctulata Gmel., Beau. 19. G.

Patella punctulata Gmel., Krebs. 75.

Patella puncturata Lam., Krebs. 75.

Patella puncturata Lam., Arango, Fauna Mal. Cuba, 187s. 230. Ca. Havana. Ba.

Patella puncturata Lam., Dall, Hemphill's shells. 337. Key West.

Patella puncturata Lam., Jour. de Conch., ii, 426. G.

Patella pustula Chem., Moll. Cuba, ii, 195.

Patella pustula D'Orb., Arango, Fauma Mal. Cuba, 1878. 229. Ca.

Fla. G. Ba.

Patella pustula Gmel., Moll. Cuba, ii, 195.

Patella pustula Wood, Moll. Cuba, ii, 195.

Patella pustulata Helb., Arango, Fauna Mal. Cuba, 1878. 230. Ca.

Havana. Ba.

Patella rosea Gmel., Moll. Cuba, ii, 196.

Patella rugosa Lesson, Krebs. 75.

Patella spinosa Gmel., Arango, Fanna Mal. Cuba, 1878. 228. Ca.

Havana. Ba.

Pateila spirata Helb., Mal. Bl., xxir, 97.

Patella subrufa List., Arango, Fauna Mal. Cuba, 1878. 226. Ca.

Patella subrufa List., Moll. Cuba, ii, 187.

Patella tenera C. B. Adams, Synops., 8, 1845. J. 
Patella trigona Gmel., Mal. Bl., xxiv, 98. ST.

Pecten (see Amussium, Janira, Ostrea, Pleuronectia).

Pecten antillarum D'Orb., Krebs. 133. Dit?

Pecten antillarum Recl., Arango, Fauna Mal. Cuba, 187s. 269. Ca. G. Ba.

Pecten antillarum Recl., Beau. 21. G.

Pecten antillarum Recl., * Jour. de Conch. iv, pl. v, fig. 1. 153. G.

Pecten antillarum Recl., Jour. de Conch., iv, 416. G.

Pecten antillarum Recl., Krebs. 134.

Pecten antillarum Recl., Poulsen. 16.

$\checkmark$ Pecten (Pseudamussium) argenteus Rve., Marrat, Argo Exp. 1876. 7.

Aa. = autillarum jo, not argenteus Rve (China)

Pecten circularis (?) Sby., Guppy, Paria Fauna, 1877. 15̃. = gith-i

Pecten clintonius Say, Bush, Conn. Ac., vi, 479. Hatt. $=$ magelyram as

Pecten comparilis T. \& H., n. s., Pleiocene Fossils S. C., pl xi, figs. 6 to

10. 29.

Pecten corallinus Chem., Beau. 21. G. = nodosus

Pecten corallinus Chem., Moll. Cuba, ii, 353.

$\sqrt{ }$ Pecten dislocatus Say, Am. Marine Conch. 196.

Pecten exasperatus Sby., Arango, Fauna Mal. Cuba, 1878. 270. Ca.

Ba.

Pecten exasperatus Sby., Krebs. 134.

Pecten exasperatus, Sby., Mal. Bl., x, 231.

Pecten exasperatus Sby., Marrat, Argo Exp., 1876. 7. Aa.

Pecten exasperatus Sby., Poulsen. 16.

Pecten fragosus Conrad, Journ. Acad. Nat. Sci. Phil., n. s., i, 214. pl.

39, fig. 11, 1849. WI. = nodoses var.

$\checkmark$ Pecten fucatus Rve., Krebs. 134. = antillarum Red.

Pecten fuscopurpureus Conrad, Journ. Acad. Nat. Sei. Phil., n. s. i, 209, 28v. pl. 39, fig. 10, 1849. Tampa. = ck spen otres fin.

. Pecteu gibbus D'Orb., Arango, Fauna Mal. Cuba, 1878, 270. Ca.

Pecten gibbus Lam., Krebs. 134.

Pecten gibbus Lam., Moll. Cuba, ii, 352.

Pecten gibbus Linn., Arango, Fauna Mal. Cuba, 1878. 270. Ca.

Pecten gibbus Linn., Moll. Cuba, ii, 352.

Pecten gibbus Sby., Moll. Cuba, ii, 352.

Pecten gruneri Rve., Moll. of Bermulas. 24.1864 (ubi?)

Pecten imbricatus Gmel., Krebs. 134. SCx. Td. Tort.

Pecten imbricatus Gmel., Poulsen. 16.

Pecten irradians Lam., Am. Marine Couch. 196. T connes thi ens Soy

Pecten magellanicus Gmel., Am. Marine Conch. 195.

Pecten multisquamatus Dkr., Moll. Mar., pl. xxiii, fig.1-3. 67. Havana.

Pecten multisquamatus Dkr., Am. Jour. Conch, ii, 177. Ca.

Pecten multisquamatus Dkr., Arango, Fauna Mal. Cuba, 1878. 270.

Ca. Havana.

Pecten multisquamatus Dkr.,* Mal. B1., xi, 100. Ca. 1864 
Pecten nodosus Kurtz. 6. NC.

Pecten nodosus D'Orb., Arango, Fauna Mal. Cuba, 1878. 270. Ca. Havana. G. M. Ba.

Pecten nodosus Lam., Moll. Cuba, ii, 353.

Pecten nodosus Linu., Am. Marine Conch. 197.

Pecten nodosus, Linu., Arango, Fauna Mal. Cuba, 1878. 270. Ca.

Havana. G. M. Ba.

Pecten nodosus Linn., Beau. 21. G. .

Pecten nodosus Linn., Jour. de Conch., iv, 416. G.

Pecten nodosus Linn., Krebs. 134. VgI.

Pecten nodosus Linn., Mal. B1., x, 231.

Pecten nodosus Linn., Poulsen. 16.

Pecten nodosus Sby., Moll. Cuba, ii, 353.

'Pecten nucleus Born, Beau. 21. G.

Pecten nucleus Born, Jour. de Conch., ii, 426. G.

Pecten nucleus Born, Krebs. 134.

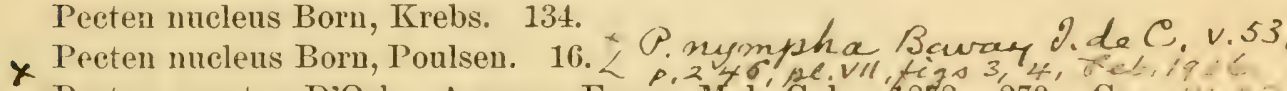

$\checkmark$ Pecten ornatus D'Orb., Arango, Fauna Mal. Cuba, $18 \% 8.270$. Ca. ${ }_{1}$,

Santa Lucia. Fla. Ba.

Pecten ornatus Lam., Am. Marine Conch. 197.

Preten ornatus Lam., Arango, Fauna Mal. Cuba, 1878. 270. Ca. Santa

Lucia. Fla. Ba.

Pecten ornatus Lam., Beau. 21. G.

Pecten ornatus Lam., Jour. de Conch., ii, 426. G.

Pecten ornatus Lam., Krebs. 134. S'T. Tort.

Pecten (Chlamys) ornatus Lam., Marrat. Argo Exp., 1876. 19. Abaco.

Pecten ornatus Lam., Moll. Cuba, ii, 353.

Pecten ornatus Lam., Poulsen. 16.

Pecten ornatus Rve., Arango, Fauna Mal. Cuba, 1878. 270. Ca. Sauta

Lucia. Fla. Ba.

Pecten ornatus Sby., Moll. Cuba, ii, 353.

$\checkmark$ Pecten peedeensis T. \& H., n. s., Pleiocene Fossils S. C., pl. xii, figs.

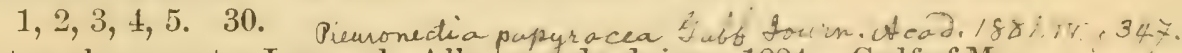
$\checkmark$ Pecten pleuronectes Lamarck, Albatross dredgings, 1SSt. Gulf of Mex. $\checkmark$ Pecten purpuratus Lam., Conrad, Am. Laur. Conch., 10, pl. ii, fig. 1,

1831. Fla. = disfocatus S...

$\checkmark$ Pecten sauciatus Lam., Pfeiffer, Archiv für naturg., 1840. 260. Ca. = "- trieats

$\checkmark$ Pecten schrammi Fisch., Jour. de Conch. viii, pl. xii, fig. 3, 330. G.

$\checkmark$ Pecten sowerbii Guild., Krebs. $134 .=$ dis Lo catus

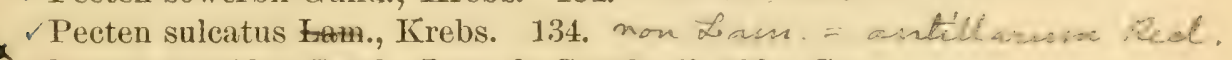

r Pecten turgidus Gmel., Jour. de Conch., ii, 426. G.

Pecten turgidus Lam., Krebs. 134.

Pecten turgidus Lam., Moll. Cuba, ii, 352.

$\checkmark$ Pecten ziczac Linn., Beau. 21. G.

Pecten ziczac Linn., Jour. de Conch., ii, 426. G.

Pecten ziczac Linn., Krebs. 134. VgI.

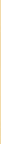


Pecten ziczac Linn., Poulsen. 16.

Pecten zigzag Chem., Moll. Cuba, ii, 356.

Pecten zigzag Linn., Moll. of Bermudas. 23.

Pecten zigzag Sby., Moll. Cuba, ii, 356.

Pectinium ornatum Link, Krebs. 134.

Pectinodonta areuata Dall, Proc. U. S. Nat. Mus., April, 1882. 410. ST. Santa Lucia.

Pectunculatus oculatus Rre., Krebs. 127. T.

Pectunculus (see Axinæa), Marrat. Argo Exp., 1876. 17.

Pectunculus n. s., Kurtz. 6. NC. SC.

Pectunculus angulatus Lam., Krebs. 126.

Pectunculus carolnensis Holm., n. s., Post-Pliocene Fossils S. C., pl. iii, fig. 4. 15.

Pectunculus castaneus Lam., Arango, Fauna Mal. Cuba, 1878. 261. Ca.

Pectunculus castaneus Lam., Beau. 22. G.

Pectunculus eastaneus Lam., Krebs. 126.

Pectunculus castaneus Lam., Moll. Cuba, ii, 314.

Pectuneulus castaneus Rve., Krebs. 126. Tort.

Pectunculus castaneus Rre., Moll. Cuba, ii, 314.

Pectunculus castaneus Rve., Poulsen. 16.

Pectunculus charlestonensis Holm., n. s., Post-Pleiocene Fossils S. C. pl. iii, fig. 5. 16.

Pectunculus costatus Meusch., Arango, Fauna Mal. Cuba, 1878. 261. Ca.

Pectunculus decussatus Linn., Arango, Fauna Mal. Cuba, 1878. 260. Ca. Santa Lucia. G. Ba.

Pectuneulus decussatus Limn., Krebs. 126. Tort.

Pectunculus decussatus Linn., Poulsen. 16.

Pectunculus hirtus Phil., Krebs. 127.

Pectunculus isocardia Mart., Krebs. 115.

Pectunculus lineatus Rre., Arango, Fauna Mal. Cuba, 1878. 260. Ca. Santa Lucia. G. Ba.

Pectunculus lineatus Rre., Beau. 22. G.

Pectunculus lineatus Rve., Krebs. 127.

Pectunculus lineatus Rve., Moll. Cuba, ii, 314.

Pectunculus marmoratus Lam., Pfeiffer, Archiv fuir naturg., 1840. 260. Ca.

Pectunculus minimus Gmel., Krebs. 127.

Pectunculus minor D'Orb., Krebs. 127.

Pectunculus minutus Phil., Report Blake Moll. 119.

Pectunculus morum Rve., Jour. de Conch., iv, 415. G.

Pectunculus oculatas Rve., Arango, Fauna Mal.Cuba, 1878. 261. M. G.

Pectunculus oculatus Rve, Beau. 22. G.

Pectuneulus oculatus Rre., Moll. Cuba, ii, 313.

Pectunculus oculatus Rre., Poulsen. 16. 
Pectunculus pectinoides Desh., Krebs. 127.

Pectunculus pectinatus Gmel., Krebs. 127. ST.

Pectunculus pectinatus Gmel., Poulsen. 16.

Pectunculus pectinatus Lam., Arango, Fauna Mal. Cuba, 1878. 261. Ca.

Pectunculus pectinatus Lam., Beau. 22. G.

Pectunculus pectiniformis D'Orb., Arango, Fauna, Mal. Cuba, 1878. 261. M. G.

Pectunculus pectiniformis D'Orb., Jour. de Conch., iv, 415. G.

Pectunculus pectiniformis Lam., Arango, Fauna Mal. Cuba, 1878. 261. M. G.

Pectunculus pectiniformis Lam., Krebs. 127.

Pectunculus pectiniformis Lam., Moll. Cuba, ii, 313.

Pectunculus pectiniformis Linn., Arango, Fauna Mal. Cuba, 1878. 261. M. G.

Pectunculus pennaceus Arango, Fanna Nal. Cuba, 1878. 260. Ca. Santa Lucia. G. Ba.

Pectunculus pennaceus Lam., Am. Marine Conch. 180.

Pectunculus pennaceus Lam., Bear. 22. G.

Pectunculus pennaceus Lam., Jour. de Conch., iv, 415. G.

Pectunculus pennaceus Lam., Krebs. 126.

Pectunculus pennaceus Lam., Moll. Cuba, ii, 314.

Pectunculus pennaceus Rre., Moll. Cuba, ii, 314.

Pectunculus scriptus Lam., Krebs. 127.

Pectunculus sericatus D'Orb., Arango, Fauna Mal. Cuba, 1878. 261. Tortola.

Pectunculus sericatus Rre., Arango, Fauma Mal. Cuba, 1878. 261. Tortola.

Pertunculus sericatus Rve, Beau. 22. G.

Pectunculus sericatus Rve., Krebs. 127. Tort.

Pectunculus sericatus Rre., Moll. Cuba, ii, 313.

Pectunculus sericatus Rve., Poulsen. 16.

Pectunculus spadiceus Rve., Krebs. 127.

Pectunculus spadiceus Rve., Poulsen. 16.

Pectunculus tricenarius Conr., Busu, Conn. Ac. vi, 479. Hatt.

Pectunculus undatus D’Orb., Arango, Fauna Mal. Cuba, 1878. 261. Ca.

Santa Lucia. G. Ba.

Pectunculus undatus D'Orb., Moll. Cuba, ii, 314.

Pectunculus undatus Linn., Krebs. 127. ST. Tort.

Pectunculus undatus Linn., Poulsen. 16.

Pectunculus undulatus Lam., Arango, Fauna Mal. Cuba, 1878. 260. Ca. Santa Lucia. G. Ba.

Pectunculus undulatus Lam., Krebs. 127.

Pectunculus undulatus Lam., Moll. Cuba, ii, 314.

Pectunculus variegatus Chem., Arango, Fauna Mal. Cuba, 1878. 261. Ca. 
Pectunculus variegatus Chem., Beau. 22. G.

Pectuneulus variegatus Chem., Krebs. 126.

Pectunculus variegatus D'Orb., Arango, Fauna Mal. Cuba, 1878. 261. Ca.

Peotunculus variegatus D'Orb., Moll. Cuba, ii, 314.

Pectunculus variegatus (?) D’Orb., Report Blake Moll. 118.

Pedicularia albida Dall, n. s., Report Blake Moll. 39. Bb.

Pedicularia decussata Gld., Am. Marine Conch. 37.

Pedicularia decussata Gld., Jour. de Conch., xvii, 114. Ca.

Pedicularia decussata Gld., Jour. de Conch., xxvi, 102.

Pedicularia decussata Gld., Mal. Bl., xxiv, 55. Fla.

Pedicularia decussata Gld., Otia, 215. Ga.

Pedipes elougatus Dall, Proc. U. S. Nat. Mus. 1885, pl. 18, fig. 4; 279. Fla.

Pedipes globulosus C. B. Adams, Synops. 12. 1845.=P. quadridens Pfr.? J.

Pedipes mirabilis Miihlf., Jahrb., ir, 360 .

Pedipes mirabilis Muilllf., Beau. 16. Pointe-à-Pître.

Pedipes mirabilis Mïhlf., Jahrb., vii, 265. Ca.

Pedipes mirabilis Muihlf, Jahrb., vii, 275. J.

Pedipes mirabilis Mühlf., Jahrb., vii, 277. Ну.

Pedipes mirabilis Miihlf., Jahrb., vii, 279. PR.

Pedipes mirabilis Miillf., Jahrb., vii, 281. ST.

Pedipes mirabilis Miihlf., Jahrb., vii, 282. G.

Pedipes mirabilis Mühlff, Jabrb., vii, 284. M.

Pedipes mirabilis Miihlf., Jour. de Conch., xxii, 167. M.

Pedipes mirabilis Mühlf., Mal. Bl, i, 148. G.

Pedipes mirabilis Miihlf,, Mal. B1., ii, 85. Ca.

Pedipes mirabilis Mühlf,, Mal. Bl., $\vee, 195$. Ca.

Pedipes naticoides Stearns, Am. Jour. Conch., v, 220. Fla.

Pedipes naticoides Stearns, Dall, Hemphill's shells. 323. Key West. Pedipes naticoides Stearus, Proc. B. S. N. H., xiii, 1869. 108. Tampa. W. Fla.

Pedipes ovalis C. B. Adlams, Contr. Coneh, 41, 1849. Jamaica.

Pedipes ovalis C. B. Adams, Jahrb., vii, 275. J.

Pedipes ovalis C. B. Adams, Mal. Bl., i, 148. J.

Pedipes quadridens Pfr., Mal. Bl., i, 170, PR.

Pedipes quadridens Pfr., Mal. Bl., r, 195. Ca.

Pedipes quadrideus Pfeiffer, Archiv fuir naturg., 1839, 1. 357. 1840, 251. Ca.

Pedipes quadridens Pfr, Beau. 16. G.

Pedipes quadridens Pfr, Jour. de Conch., v, 152. G.

Pedipes quadridens Pfr., Mal. Bl., i, 148.

Pedipes quadridens Pfr., Mal. Bl., ii, 85. Ca.

Pedipes tridens Pfr, P. Z. S., 1854. 122. Ca.

Pedipes tridens Pfr., Mal. Bl., v, 195. Ca. 
Pedipes tridens Pfr., Mal. Bl., i, 148. B. Ca.

Pedipes tridens Pfr., Mal. Bl., ii, 85. Ca.

Pelagella sp., Mal. Bl., xxii, 177. SCx.

Pelta prasiua Mörch, ${ }^{*}$ Jour. de Conch., xi, 42. SCx.

Pelta prasina Mörch, Mal. Bl., xxii, 1S0. SCx.

Pennaria muricata Browne, Krebs. 131.

Pentadactylus (Sistrum) anaxares Duclos, Marrat, Argo Exp., 1876.

14. Havaua.

Perdix (see Dolium).

Perdix jamaicensis, \&c., Mal. Bl., xxiv, 42.

Perdix reticulatus Mtf., Mal. Bl., xxiv, 42.

Periploma anguliferum Phil.,* Zt. Mal., iv, 73.

Periploma inrequivalvis Schum., Beau. 25. MG. Pointe-à-Pître.

Periploma inæquivalvis Schum., Krebs. 108.

Periploma inaquivalvis Schum., Marrat, Argo Exp.,1876. 12. Tucacas.

Periploma leana Con., Am. Marine Conch. 133.

Periploma papyracea Say, Am. Marine Conch. 133.

Periploma trapezoides Lam., Bean. 25. G.

Periploma trapezoides var. Lam., Jour. de Couch., iv, 414. G.

Peristernia luculenta H. \& A. Ad., Jahrb., iii, 26. WI.

Perna (see Avicula, Modiola, Mytilus).

Perna alata Gmel., Beau. 22. G.

Perna americana Adans., Krebs. 128.

Perna bicolor C. B. Adams, Synops., 9, 1845. J.

Perua chemnitziana I'Orb., Arango, Fauna Mal. Cuba, 1S78. 269.

Ca. M. SCx.

Perna ehemnitziana D'Orb., Krebs. 132.

Perna chemnitziana D’Orb., Moll. Cuba, ii, 346.

Perna ephippium Lam., Dall, Hemphill's shells. 341. Key West.

Perna ephippium Lam., Pfeiffer, Archiv fuir naturg,, 1840. 260. Ca.

Perua ephippium Liun., Moll. of Bermudas. 24.

Perna lamarckiana D’Orb., Arango, Fauma Mal. Cuba, 1878. 269. Ca.

M. Ba.

Perna lamarckiana D'Orb., Krebs. 132.

Perna lamarckiana D'Orb., Moll. Cuba, ii, 347.

Perna linnaei Pfr., Araugo, Fauna Mal. Cuba, 1878. 269. Ca. M. Ba.

Perna linnæi Pfr., Krebs. 132.

Perna obliqua D'Orb., Jour. de Conch., vii, 39.

Perna obliqua D'Orb., Arango, Fauma Mal. Cuba, 1878. 269. Ca. M.

Ba.

Perua obliqua Lam., Arango, Fauna Mal. Cuba, 187S. 269. Ca. M.

$\mathrm{Ba}$.

Perna obliqua Lam., Beau. 22. G.

Perna obliqua Lam., Jour. de Conch., ii, 426. G.

Perna obliqua Lam., Krebs. 132. VgI.

Perua obliqua Lam., Moll. Cuba, ii, 346. 
Perna pernula Chem., Jour. de Conch., iv, 215. G.

Perna semiaurita Chem., Beau. 22. G.

Perna semiaurita Chem., Jour. de Conch., ii, 426. G.

Perna seminuda Lam., Jour. de Conch, iv, 415. G.

Perna (Brachydontes) subramosa Haul., Marrat, Argo Exp., 1876. 19. Abaco.

Perna sulcata Lam., Arango, Fauna Mal. Cuba, 1878. 269. Ca. M. Ba.

Perna sulcata Lam., Krebs. 132.

Perna tulipa Lam., Marrat, Argo Exp., 1876. 13. Santa Marta. VCz. Nassau.

Perna varicosa Lam., Jour. de Conch., iv, 415. G.

Perna vulsella Lam., Krebs. 132.

Perna vulsella Lam., Moll. Cuba, ii, 347.

Peronæoderma (see Tellina).

Peronæoderma punicea Born, Jahrb., ii, 248.

Peronæoderma punicea Mörch, Krebs. 103.

Peronella armadilla IIörch, ${ }^{*}$ Jour. de Conch., xi, 43. ST.

Persicula (see Marginella).

Persicula carnea Storer, Arango, Fauna Mal. Cuba, 1878. 186. Ca.

Persicula guttata Link, Marrat, Argo Exp., 1876. 16. Nassau. Long Key Island.

Persicula interruptolineata Mühilf., Marrat, Argo Exp., 1876. 12. Tucacas. Santa Marta.

Persicula kieneriana Petit, Marrat, Argo Exp., 1876. 10. La Guayra.

Persicula oblonga Swains., Arango, Fauna Mal. Cuba, 1878. 186. Ca. Ba. Yucatan.

Persicula parva Mart., Krebs. 44.

Persicula vesiculata Mart., Arango, Fauna Mal. Cuba, 1878. 187. Havana. Ba. Tort. Hol.

Persona (see Distorsio, Tritou).

Persona cancellina Roissy, Jahrb., v, 370.

Persona reticularis Linn., Guppy, Paria Fauna, 1877. 142.

Persona reticulata Linn., Jahrb., v, 370.

Petaloconchus (see Vermetus).

Petaloconchus domingensis Sby., Jour. de Conch. viii, 41. SD.

Petaloconchus renisectus Carp., Jour. de Conch., vii, 357. ST.

Petaloconchus seulpturatus Lea, Jour. de Conch., viii, 41. SD.

Petaloconchus varians D'Orb., Jour. de Conch., viii, 41. SV.

Petaloconchus varians D'Orb., Mal. Bl., xxiv, 117.

Petaloconchus varians Mörch, Mal. B1, xxiv, 116. Bz.

Petricola (see Choristodon, Naranaio).

Petricola costata Lam., Arango, Fauna Mal. Cuba. 1878. 248. Ca. M.

SD. Ba.

Petricola costata Gray \& Lam., Krebs. 108.

Petricola dactylus Sby., Kurtz. 4. SC. 
Petricola divaricata D’Orb., Arango, Fauna Mal. Cuba, 1878. 248. Ca. M. SD. Ba.

Petricola divaricata D'Orb., Moll. Cuba, ii, 265.

Petricola divaricata Chem., Krebs. 108.

Petricola gracilis Desh., Guppy, Paria Fauna, 1877. 151.

Petricola lapicida Chem., Arango, Fauna Mal. Cuba, 1878. 248. Ca. M. SD. Ba.

Petricola lapicida Chem., Beau. 24. G.

Petricola lapicida Chem., Krebs. 108.

Petricola lapicida Chem $=\mathrm{P}$. costata Lam., Poulsen. 15.

Petricola lithophaga Brown, Arango, Fauna Mal. Cuba, 1878. 248. Ca. Ba.

Petricola lucinalis Lam., Krebs. 108.

Petricola pholadiformis D'Orb., Arango, Fauna Mal. Cuba, 1878. 248. Ca.

Petricola pholadiformis Lam., Am. Marine Conch. 162.

Petricola pholadiformis Lam., Arango, Fauna Mal. Cuba, 1878. 248. Ca.

Petricola pholadiformis Lam., Mal. Bl., x, 226.

Petricola pholadiformis Lam., Moll. Cuba, ii, 265.

Petricola pholadiformis Lam., Poulsen. 15.

Petricola pholadiformis Lam., Krebs. 108.

Petricola pholadiformis Linn., Mal. Bl. $x, 228$.

Petricola pholadiformis Sby., Moll. Cuba, ii, 265.

Petricola robusta Sby., Mal. BI., iv., 27. G.

Petricola robusta Sby., Beau. 24. G.

Petricola robusta var. Sby., Jour. de Conch., iv, 414. G.

Petricola robusta Sby., Jour. de Conch., v, 155. G.

Petricola rocellaria Lam., Krebs. 108.

Petricola simulans C. B. Adams, Mal. Bl., x, 228.

Petricola typica Jonas, Krebs. 108.

Petricola (Choristodon) typica Jonas, Poulsen. 15.

Petricola typica Sby., Mal. Bl., $x, 229$.

Petricola ventricosa Desh., Mal. Bl., х, 228.

Phakellopleura (see Chiton), Jour. de Conch., $\nabla, 169$.

Phakellopleura spiculosa Gray, Jour. de Conch., r, 151. G.

Phasianella (see Littorina).

Phasianella affinis C. B. Adams, Contr. to Conch. 67. J.

Phasianella affinis C. B. Adams, Dall, Hemphill's shells. 335.

Phasianella affinis Ad., Krebs. 79. ST. SB.

Phasianella angulifera Lam., Araugo, Fauna Mal. Cuba, 1878. 160. Ca. M. G. J.

Phasianella angulifera Lam., Mal. Bl., xxiii, 137.

Phasianella biearinata D'Orb., Poulsen. 13.

Phasianella brevis C. B. Adams, Contr. to Conch. 67. J.

Phasianella brevis Ad., Krebs. 79. 
Phasianella brevis D'Orb., Arango, Fauna Mal, Cuba, 1878. 181. Ca.M. Phasianella brevis D'Orb., Krebs. 79.

Phasianella brevis D'Orb., Moll. Cuba, ii, 79.

Phasianella concinna C. B. Adams, Contr. to Conch. 69. J.

Phasianella concinna Ad., Krebs. 79. ST. SM. SB.

Phasianella concolor C. B. Adams, Contr. to Conch. 68. J.

Phasianella concolor Ad., Krebs. 79.

Phasianella lineata Deless., Mal. Bl. xxiii, 137.

Phasianella lineata Dkr., Krebs. 79.

Phasianella lineata Lam., Arango, Fauna Mal. Cuba, 1878. 159. Ca. M. Ba.

Phasianella lineata Lam., Mal. Bl. xxiii, 137.

Phasianella lineata Lam., Mal. Bl., xxiii, 138.

Phasianella lineata Lam., Moll. Cuba, i, 208.

Phasianella minuta Ant., Krebs. 79.

Phasianella nebulosa Deless., Mal. Bl., xxiii, 136.

Phasianella nebulosa Lam., Mal. Bl., xxiii, 136.

Phasianella pulla Linn., Jour. de Conch., ii, 428 . G.

Phasianella punctata Pfr., Mal. Bl., xxiii, 141.

Phasianella punctata Pfeiffer, Archiv für naturg., 1840. 255. Ca.

Phasianella sulcata Deless., Mal. Bl., xxiii, 135.

Phasianella sulcata Lam., Mal. Bl., xxiii, 135.

Phasianella tessellata C. B. Adams, Beau. 12. G.

Phasianella tessellata C. B. Adams, Contr. to Conch. 67. J.

Phasianella tessellata C. B. Adams, Jour. de Conch., v, 153. G.

Phasianella tessellata Ad., Krebs. 79. SJ. Ag. Td.

Phasianella umbilicata D'Orb., Arango, Fauna Mal. Cuba, 1878. 182. J. G.

Phasianella umbilicata D'Orb., Beau. 12. G.

Phasianella umbilicata D'Orb., Dall, Hemphill's shells. 335. Key West. Ca.

Phasianella umbilicata D'Orb., Krebs. 79. ST.

Phasianella umbilicata D’Orb., Moll. Cuba, ii, pl. xix, figs. 32-34. 77.

Phasianella umbilicata D'Orb., Poulsen. 13.

Phasianella vieuxii Payr., Pfeiffer, Archiv für naturg., 1840. 255. Ca.

Phasianella zebrina D'Orb., Arango, Fauna Mal. Cuba, 1878. 182. Ca. G.

Phasianella zebrina D'Orb., Beau. 12. G.

Phasianella zebrina D'Orb., Moll. Cuba, ii, pl. xix, figs. 35-37. 78. Phidiana lynceus Bergh, Mal. Bl., xxii, 178. ST. G.

Philine (see Bulla).

Philine candeana D'Orb., Krebs. 94.

Philine candeana D'Orb., Mal. Bl., xxii, 175. G.

Philine sagra D'Orb., Mal. Bl., xxii, 175. M. Ca.

Philine sagra D'Oro., Poulsen. S.

Philippia (see Solarium). 
Philippia krebsii Mörch, Mal. Bl., xxii, 155. PPl.

Philonexis quoyanus D'Orb., Arango, Fauna Mal. Cuba, 1878. 146. Antilles.

Philonexis quoyanus D'Orb., Moll. Cuba, i, 21, 29.

Pholadomya candida Sby., Beau. 26. Pointe-à-Pitre. Tort.

Pholadomya candida Sby., Krebs. 111. ST.

Pholadomya candida Sby., Poulsen. 14.

Pholas (see Cyrtopleura, Diplothyra, Xylophaga).

Pholas (see Lithodomus), Arango, Fauna Mal. Cuba, 1878. 267. Ca. M. SD. Santa Lucia. G.

Pholas (see Nartesia), Arango, Fauna Mal. Cuba, 1878. 236. Ca. G.

Pholas americanus Klein (Modiola), Krebs. ' 128.

Pholas beauiana Petit, Jour. de Conch., iv, 413. G.

Pholas beauiana Recl., Arango, Fauma Mal. Cuba, 1878. 236. Ca. ST. G.

Pholas beauiana Recl., Beau. 27. G.

Pholas beaniana Recl.,* Jour. de Couch., iv, pl. ii, figs. 1-3. 49. G.

Pholas beauiana Recl., Krebs. 113.

Pholas beauiana Recl. = P. hornbeckii D’Orb., Poulsen. 14.

Pholas campechensis Gmel., Krebs. 113. NGrr.

Pholas eampechensis Gmel., Poulsen. 14.

Pholas candeaua D’Orb., Araugo, Fuuna Mal. Cuba, 1878. 236. Ca.

Pholas candeana D:Orb., Krebs. 113.

Pholas candeana D'Orb., Moll. Cuba, ii, pl. xxv, figs. 18-19. 215.

Pholas caribaea D’Orb., Araugo, Fauna Mal. Cuba, 1878. 236. Ca. ST. G.

Pholas caribæa D'Orb., Krebs. 113. ST.

Pholas caribaea D'Orb., Moll. Cuba, ii, pl. xxv, figs. 20-22. 216.

Pholas clavata Lam., Arango, Fauma Mal. Cuba, 1878. 236. Ca. G.

Pholas clavata Lam., Beau. 27. G.

Pholas clavata Lam., Jahrb., ii, 247.

Pholas clavata Lam., Krebs. 114.

Pholas clavata Lam., Moll., Cuba, ii, 214.

Pholas conoides Fleming, Arango, Fauna Mal. Cuba, 187s. 236. Ca.

G. Great Britain. Philippines.

Pholas corticaria Sby., Contr. to Conch. 75. J.

Pholas corticaria Sby., Krebs. 113.

Pholas costata Chem., Moll. Cuba, ii, 213.

Pholas costata U'Orb., Arango, Fauna Mal. Cuba, 1878. 235. Havana.

Pholas costata D'Orb., Moll. Cuba, ii, 213.

Pholas costata Gray, Krebs. 113.

Pholas costata Lam., Moll. Cuba, ii, 213.

Pholas costata Linn., Am. Marine Conch. 125.

Pholas costata Liun., Arango, Fauna Mal. Cuba, 1878. 235. Havana. Pholas costata Linn., Jahrb., ii, 247.

Bull. $24-15$ 
Pholas costata Linu., Moll. Cuba, ii, 213.

Pholas costata Liun., Poulsen. 14.

Pholas (Martesia) cuneitormis Say, Dall, Hemphill's shells, 337. Cedar Keys, Fla.

Pholas (Xylophaga) cuneiformis Say, Kurtz, 3. NC. SC.

Pholas falcata IVood, Arango, Fanna Mal. Cuba, 1878. 236. Ca. G. Pholas hiaus Chem., Arango, Fauna Mal. Cuba, 1878. 237. Ca. G.

Pholas hians Chem., Krebs. 114.

Pholas hians Chem., Moll. Cuba, ii, 228, 229.

Pholas hornbeckii D'Orb., Krebs. 113.

Pholas hormbeckii D’orb., Arango, Fauma Mal. Cuba, 1S78. 236. Ca. ST. G.

Pholas horubeckii I'Orb., Moll. Cuba, ii, pl. xxv, figs. 23-25. 217.

Pholas krebsii Ad., Krebs. 113. ST.

Pholas lignornu Spengl., Arango, Famna Mal. Cuba, 1878. $236 . \quad$ Ca.G.

Pholas lignorum Sp «ngl., Jahrb., ii, 247.

Pholas memmingeri T. \& H. n. s., Pleiocene Fossils S. C., pl. xxir, fig. 6. 104.

Pholas nana P’ult., Arango, Fauna Mal. Cuba, 1878. 236. Ca. G.

Pholas naua Pult., Jahrb., ii, 247.

Pholas niger List., Krebs. 130.

Pholas niger List., Moll. Cuba, ii, 331.

Pholas oblongata Say, Arango, Famua Mal. Cuba, 1878. 236. Ca.

Pholas oblongata Say, Kurtz. 3. NC. SC.

Pholas pusilla Lim., Araugo, Fanna Mal. Cuba, 1878. 236. Ca. G.

Pholas pusilla Linu., Jahrb., ii, 247.

Pholas pusilla Linn., Krebs. 113.

Pholas pusilia Linn., Krebs. 114.

Pholas pusilla Limn., Poulsen. 14.

Pholas pusillus Chem., Moll. Cuba, ii, 214.

Pholas pusillus D'Orb., Noll. Cuba, ii, 214-216.

Pholas pusillus Limn., Moll. Cuba, ii, 214.

Pholas semicostata II. C. Lea, Boston Jour. Nat. Hist. v, 1844, pl. xxiv, fig. 1. 285. SC.

Pholas semicostata Leal, Arango, Fauna Mal. Cuba, 1S78. 236. G. Ca.

Pholas striata Linn., Beau. 27. G.

- Pholas striata Linn., Jour. de Conch., iv, 413. G

Pholas striata Linn., Krebs. 113.

Pholas striata Linn., Krebs. 114. ST. NGr.

Pholas striata Linu., Moll. of Bermudas. 26.

Pholas striata Liun., Poulsen. 14.

Pholas terediformis Sby., Arango, Fauna Mal. Cuba, 1878. 236. Ca. G. Pholas (Cyrtopleura) truncata Say, Am. Marine Conch. 126.

Pholas truncata Say, Kurtz. 3. SC.

Phorcus (see Trochus).

Phoreus canus Koch,* Zt. Mal., v, 102. WI., in sponges, 
Phoreus gruneri Phil., Zt. Mal., v, 107. WI.

Phorcus gundlachi Phil.,* Zt. Mal., v, 108. Ca.

Phorcus scabriculus V. d. Busch,* Zt. Mal., v, 125. WI.

Phorus (see Onustus, Xenophora, Trochus).

Phorus agglutinans Linn., Moll. of Bermudas. 19.

Phorus agglutinans Mtf., Mal. Bl., xxiv, 109. ST.

Phorus conchyliophorus Mtf., Mal. Bl., xxiv, 109. G.

Phorus conchyliophorus Mtf., Moll. Cuba, ii, pl. xix, fig. 31. 70, 71.

Phorus onustus Rve., Arango, Fauna Mal. Cuba, 1878. 181. Antilles. M.

Phorus trochiformis Mtf., Krebs. 68.

Phos antillarum Petit, Beau. 10. G. = candei, or

Phos antillarum Petit, Jour. de Conch., iv, 235. La Guayra.

Phos antillarum Petit," Jour. de Conch. is, pl. viii, fig. 9. 242. La Guayra.

Phos antillarum Petit, Jour. de Conch., iv, 41S. G.

Phos antillarum Phil., Krebs. 31. = canbi.

Phos beauii Fisch. \& Bern., Beau. 10. MG.

Phos beauii F. \& B., Jour. de Conch. v, pl. xii, figs. 8, 9, 35̃. M. G.

Phos beauii F. \& B., Krebs. 32.

Phos candei D’Orb., Arango, Fauna Mal. Cuba, 187s. ‘01. Ca. M.

Phos erectus Guppy, n. s., Guppy, Geol. Mag., 1874. 440. J.

Phos guadalupensis Petit, Arango, Fauna Mal. Cuba, 1878. 201. Ca.

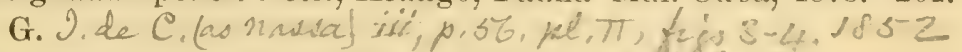

Phos guadelupensis Petit, Krebs. 32. = unicincta śon.

Phos intricatus Dall, n. s. Dall, Hemphill's shells. 320̃. Key West.

Phosinella (see Rissoina).

Phosinella bicollaris Schwartz, * Mal. BI. xxiii, 52. Ca.

Phosinella cancellata Phil. Mal. B1. xxiii, 52. Ca.

Phosinella fenestriata Schwartz, * Mal. Bl. xxiii, 52. Ca.

Phosinella labrosa Sehwariz, Mal. Bl. xxiii, 51. Ca.

Phosinella privati Folin, Mal. Bl. xxiii, 52.

Phosinella pulchra C. B. Adams, Mal. Bl., xxiii, 51. J.

Phosinella sagraiana D'Orb., Mal. B1., xxiii, 51. ST. M.

Phrontis (see Nassa), Marrat, Argo. Exp., 1876. 10.

Phyllobranchus viridis Desh., Mal. Bl., xxii, 180. ST.

Phyllonotus (see Murex), Marrat, Argo Exp., 1876. 9.

Physa acuta D'Orb., Beau. 15. G.

Physa acuta D'Orb., Moll. Cuba, i, 191. Ca. J. G. M.

Physa cubensis Pfr., Arango, Fauna Mal. Cuba, 1878. 135. Ca.

Physa cubensis Pfr., Marrat, Argo Exp., 1876. S. St. Kitt's.

Physa guadalupensis Fisch., Beau. 15. G.

Physa jamaicensis Ad., Arango, Fauna Mal. Cuba, 1578. 136. Ca. J. Physa sow erbyana D’Orb., Moll. Cuba, i, pl. xiii, figs. 11-13. 190. Ca. M. Physa sowerbyaua 1)'Orb., Arango, Fauna Mal. Cuba, 1S78. 136. J. Physa sowerbyana D'Orb., Beau. 15. G. 
Physa striata D’Orb., Moll. Cuba, i, pl. xiii, figs. 14-16. 192. M. or Ca. Physa striata D'Orb., Arango, Fauna Mal. Cuba, 1878. 136. Ca. Pila (see Nerita), Marrat, Argo Exp., 1876. 6.

Pila peloronta Klein, Krebs. 76 .

Pileopsis (see Capulus, Hipponyx).

Pileopsis intorta Blainv., Moll. Cuba, ii, 186.

Pileopsis iutorta Lam., Arango, Fauna Mal. Cuba, 1878. $226 . \quad$ Ca.

Pileopsis intorta Lam., Beau. 19. G.

Pileopsis intorta Lam., Jour. de Conch., iv, 416. G.

Pileopsis intorta Lam., Krebs., 70.

Pileopsis intorta Lam., Mal. Bl., xxiv, 97. G.

Pileopsis intorta Lam., Moll. Cuba, ii, 186.

Pileopsis militaris Mtg., Beau. 19. G.

Pileopsis militaris Mtg., Jour. de Conch., iv, 416. G.

Pileopsis mitrula Gmel., Jour. de Conch., ii, 426. G.

Pileopsis mitrula Lam., Beau. 19. G.

Pileopsis mitrula Lam., Mal. Bl., xxiv, 98.

Pileopsis mitrula Lam., Moll. Cuba, ii, 186.

Pileopsis mitrula Lam., Zt. Mal., x, 79. SV.

Pileopsis subrufa Lam., Beau. 19. G.

Pileopsis subrufa Lam., Mal. Bl., xxiv, 98.

Pileopsis subrufa Lam., Moll. Cuba, ii, 187.

Piliscus Mörch, non Lovén = Anisomyon Meek.

Piliscus (Allerya) krebsii Mörch, n. s., Jour. de Conch. xxv, 210. ST. $\mathrm{Bb}$.

Piliscus krebsii Mörch, Mal. Bl., xxiv, 100. ST.

Piliscus krebsii Mörch, Poulsen. 10.

Pinna curnea Gmel., Arango, Fauna Mal. Cuba, 1878. 264. Ca. Ba.

Pinna carnea Gmel., Krebs. 131.

Pinna degenera Lam., Arango, Fauna Mal. Cuba, 1878. 264. Ca. Ba. Pinna degenera Link, Krebs. 131.

Pinna d'orbignyi Hanl., Marrat, Argo Exp., 1876. 6. Aa.

Pinna flabellum Lam., Arango, Fauna Mal. Cuba, 1878. 264. Ca. Ba. Pinna flabellum Lam., Krebs. 131.

Pinna muricata Linn., Moll. Cuba, ii, 325.

Piuna muricata Linn., Am. Marine Conch. - 191.

Piuna muricata Lim., Arango, Fauna Mal. Cuba, 1878. 264. Ca. Ba. Pinna muricata Linn., Krebs. 130. ST.

Piuna muricata Linn., Poulsen. 16.

Pinna nobilis Chem., Arango, Fauna Mal. Cuba, 1878. 264. Ca. M. G. Pinna nobilis Chem., Krebs. 130.

Pinna nobilis Chem., Moll. Cuba, ii, 325.

Pinna pectiuata Linu., Pfeiffer, Archiv für naturg., 1840. 260. Ca. Pinua pernula Chem., Araugo, Fauna Mal. Cuba, 1878. 264. Ca. Ba. Pinna pernula Chem., Krebs. 131.

Pinna pernula Chem., Marrat, Argo Exp., 1876. 13. Santa Marta. 
Pinna pernüla Chem., Moll. Cuba, ii, 325.

Pinna pernula Chem., Poulsen. 16.

Pinna pernula D'Orb., Arango, Fanna Mal. Cuba, 1878. 264. Ca. Ba.

Pinna ramulosa Rve., Guppy, Paria Fauna, 1877. 153.

Pinna rigida Chem., Arango, Fauna Mal. Cuba, 1878. 264. Ca. M. G.

Pinna rigida D'Orb., Arango, Fauna Mal. Cuba, 1878. 264. Ca. M. G.

Pinna rigida Sol., Arango, Fauna Mal. Cuba, 1878. 264. Ca. M. G.

Pinna rigida Sol., Krebs. 131.

Pinna rigida Sol., Moll. Cuba, ii, 325.

Pinna rigida Sol. \& Dillw., Moll. Cuba, ii, 325.

Pinna rigida Wood, Moll. Cuba, ii, 325.

Pinna rudis Linn., Moll. of Bermudas. 24.

Pinna seminuda Lam., Am. Marine Conch. 191.

Pinna seminuda Lam., Arango, Fauna Mal. Cuba, 1878. 264. Ca. M. G.

Pinna seminuda Lam., Beau. 22. G.

Pinna seminuda Lam., Krebs. 130.

Pinna seminuda Lam., Moll. Cuba, ii, 325.

Pinna squamosissima Phil., Roemer's Texas, 454, 1849. Tex.

Pinna squamosissima Phil., *Zt. Mal., v, 164. Tex., \&c.

Pinna raricosa (Lam.) D’Orb., Arango, Fauna Mal. Cuba, 1878. 264.

Ca. Ba.

Pinna varicosa Lam., Beau. 22. G.

Pinna raricosa Lam., Krebs. 131.

Pinna varicosa Lam., Moll. Cuba, ii, 325.

Pirena (see Cerithidea).

Pirena scalariformis De Kay, Mal. Bl., xxiii, 87.

Pirena scalariformis Say, Mal. Bl., xxiui, 87.

Pisania (see Cautharus, Tritonidea).

Pisania bilivatum Rve., Moll. of Bermudas. 16.

Pisania crenilabrum A. Ad., Jahrb., ix, 18.

Pisania janeirensis Phil, Jahrb., ix, 19. Bz.

Pisania nephela Rav., n. s., Ravenel. 8. SC.

Pisania peunata Brown, Krebs. 31.

Pisania plumatum Gmel., Jahrb., ix, 19.

Pisania pusio Linn, Jahrb., ix, 19.

Pisania pusio Linn., Marrat, Argo Exp., 1876. 9. SV.

Pisania tritonis Rve., Krebs. 31.

Pisidium consanguineum Prime, Arango, Fauna Mal. Cuba, 1878. 143.

$\mathrm{Ca}$.

Pisidiuin gundlachi Arango, Fauna Mal. Cuba, 1878. 143. Ca.

Pitar (see Cytherea).

Pitar munda Röm., Mal. Bl., vii, 150. ST.

Placunanomia abdominalis Gray, Beau. 21. Pointe-à-Pître.

Placunanomia abdominalis Gray, Krebs. 136.

Placunanomia echinata Brod., Krebs. 136. 
Placunanomia echinata Brod., Poulsen. 16.

Placunanomia foliata Brod., Mal. Bl., x, 229.

Placunanomia plicata T. \& H., n. s., Pleiocene Fossils S. C. 19.

Placunanomia rudis Brod., Krebs. 137. VgI.

Placunanomia rudis Brod., Poulsen. 16.

Planaxis buccinea A. Ad.,* Mal. Bl., xxiii, 123.

Planaxis buccineus E. Smith, Mal. Bl., xxiii, 123. WI.

Planaxis crassilabrum Guppy, n. s., Geol. Mag., 1874. 441. Td.

Planaxis eborea Mörch,* Mal. Bl., xxiii, 124. S'T.

Planaxis eboreus Smith, Mal, Bl., xxiii, 124. SV.

Planaxis lineata Da Costa,* Mal. Bl., xxiii, 124. J.

Planaxis liueata Da Costa, Marrat, Argo Exp., 1876. 9. SV. Abaco.

Planaxis lineata Da Costa, Poulsen. 9.

Planaxis lineata Duval., Mal. Bl., xxiii, 124.

Planaxis lineata Jay, Zt. Mal., x, 70. SV. WI.

Planaxis lineata Lam., Dall, Hemphill's shells. 335. Key West.

Planaxis lineatus Da Costa, Arango, Fanna Mal. Cuba, 1878. 205.

Ca. Ba.

Planaxis lineatus Da Costa, Krebs. 52. SJ. SM. ST.

Planaxis nucleola Mörch, Mal. Bl., xxiii, 126. SCx.

Planaxis nucleus Desh., Moll. Cuba, ii, 151.

Planaxis nucleus D'Orb., Arango, Fauna Mal. Cuba, 1878. 205. Ca. J. $\mathrm{Bb}$.

Planaxis nucleus D'Orb., Mal. Bl., xxiii, 126.

Planaxis nucleus Lam., Krebs. 52. ST. SM. SJ. Ag.

Planaxis nuclens Wooul, Arango, Fauna Mal. Cuba, 1878. 205. Ca.

J. $\mathrm{Bb}$.

Planaxis nucleus Wood, Beau. 12. G.

Planaxis nucleus Wood, Moll. Cuba, ii, 151.

Planaxis pedicularis Lam., Krebs. 52.

Planaxis pedicularis Linn., Bean. 12. G.

Planaxis pedicularis Linn., Jour. de Conch., ii, 428. G.

Planaxis semisulcata Sby., Mal. Bl., xxiii, 125.

Planaxis semisuleata Sby., Mal. Bl., xxiii, 126.

Planaxis semisuleata Sby., Marrat, Argo Exp., 1876. S. D. SV.

Planaxis semisulcata Sby., Poulsen. 9.

Planaxis semisuleatus Sby., Arango, Fauna MIal. Cuba, 1878. 205. Ca.

Planaxis suceineta A. Ad., Mal. Bl., xxiii, 125.

Planaxis succincta E. Smith, Mal. Bl., xxiii, 125.

Planaxis sulcatus Lam., Arango, Fauna Mal. Cuba, 1878. 205. Ca. J. Bb.

Planaxis sulcatus Lam., Beau. 12. G.

Planaxis sulcatus Lam., Jour. de Conch., ii, 428. G.

Planorbis (see Segmentina), Arango, Fanna Mal. Cuba, 1878. 137. Ca. Planorbis affinis Ad., Arango, Fauna Mal. Cuba, 1878. 136. Ca. J. 
Planorbis aracasensis Gundl., Arango, Fama Mal. Cuba, 1878. 136. Ca. Td.

Planorbis caribæus D'Orb., Beau. 15. G.

Planorbis caribæus D’Orb., Arango, Fanua Mal. Cuba, 187S. 136. Ca. Havana. PR.

Planorbis caribæus D'Orb., Moll. Cuba, i, pl. xiii, figs.17-19. 193. Наvana. Vera Cruz.

Planorbis cultratus, D'Orb., Moll. Cuba, i, pl. xiv, figs. 5-8. 196. MI. or Ca.

Planorbis cultratus D’Orb., Arango, Fauna Mal. Cuba, 18\%8. 142. M. Planorbis dentatus Gld., Arango, Fauna Mal. Cuba, 1878. 137. Ca. Planorbis dentiferus Ad., Arango, Fauma Mal. Cuba, 1S7S. 137. Ca. Planorbis edentatus Ad., Araugo, Fauna Mal. Cuba, 1878. 137. Ca. Planorbis guadalupensis Sby., Beau. 15. G.

Planorbis haranensis Pfr., Arango, Fauna Mal. Cuba, 187s. 136. Ca. Havana.

Planorbis lanierianus D'Orb., Moll. Cuba, i, pl. xiv, figs. 1-4. 195. Ca. Planorbis lanierianus D’Orb., Arango, Fauna Mal. Cuba, 1878, 136. J. G. PR. Ua. Havana.

Planorbis lucidus Pfr., Arango, Famna Mal. Cuba, 187s. 136. Ca. Havana. J. G. PR.

Planorbis lucidus Pfr., Beau. 15. G.

Planorbis relfieldi Ad., Arango, Fauna Mal. Cuba, 187S. 136. J. G. PR. Ca. Havana.

Planorbis stagnicola Mor., Araugo, Fauna Mal. Cuba, 1878. 137. Ca. Planorbis tæniatus Mor., Arango, Fanna Mal. Cuba, 187S, 136. J.G. PR. Ca. Havana.

Planorbis terverianus D'Orb., Moll. Cuba, i, pl. xiii, figs. 20-23. 194. Ca.

Planorbis terverianus D'Orb., Arango, Famna Hal. Cuba, 1878. 136. Ca. Havana.

Planorbis tumidus Pfr., Arango, Fauna Nal. Cuba, 187S. 136. Ca., Havana, PR.

Platidia anomioides Costa, Report Blake Moll. 104. CS.

Platidia anomioides Scacchi, Report Blake Moll. 104. CS.

Platygyra (see Bittium).

Platygyra albastrulum Mörch, * Mal. Bl., xxiii, 101. ST.

Platygyra albovittatum C. B. Arlams, Mal. Bl., xxiii, 101. J.

Platygyra bicolor C. B. Adams, ${ }^{*}$ Mal. Bl., xxiii, 102. J.

Platygyra cinereoflavum Mörch, Mal. Bl., xxiii, 102. ST.

Platygyra emersoni C. B. Adams, Mal. Bl., xxiii, 9S. SC.

Platygyra exile Adams, Mal. Bl., xxiii, 104. J.

Platygyra flavum C. B. Aclams, Mal. Bl., xxiii, 99. J.

Platygyra gemmulosum C. B. Adlams, ${ }^{*}$ Mal. Bl., xxiii, 102. J.

Platygyra latum C. B. Adams, ${ }^{*}$ Mal. Bl., xxiii, 103. J. G. 
Platygyra rugulosum C. B. Adams, Mal. Bl., xxiii, 100.

Platygyra subulatum Mtg., Mal. Bl., xxiii, 98.

Platygyra terebellum C. B. Adams, Mal. Bl., xxiii, 99. J.

Platygyra terebrale C. B. Adams,* Mal. Bl., xxiii, 99.

Platygyra vicinum Adams, Mal. Bl., xxiii, 101.

Platyodoris angustipes Mörch, Jahrb., iv, 73.

Platystoma (see Natica).

Platystoma litterata Klein, Mal. Bl., xxiv, 63.

Plecotrema cubensis Pfr., Mal. Bl., ii, 85. Ca.

Plecotrema cubensis Pfr., Mal. Bl., i, 150. Ca.

Plecotrema cubensis Pfr.,* Mal. Bl., i, 153. Ca.

Pleurobranchus areolatus Mörch, Jour. de Conch., xi, 28. ST.

Pleurobranchus areolatus Mörch, Mal. Bl., xxii, 178. ST.

Pleuronectia (see Pecten).

Pleuronectia lucida Jeffreys, Report Blake Moll. 11\%. CS.

Pleurophyllidia cuvieri Meckel, Bush, Conu. Ac. vi, 469. Hatt.

Pleurotoma (see Clathurella, Cithara, Drillia, Ancistrosyrinx, Pleurotomella, Bela, Mangelia, Surcula, Turris, Daphnella).

Pleurotoma (Mangelia) acanthodes Watson, Lin. Soc. Journ., 1881, xv, No. 87, 433. Ber.

Pleurotoma affinis Gray, Beau. 8. G.

Pleurotoma affinis Gray, Krebs. 7.

Pleurotoma (Drillia) aglaophanes, Watson, Lin. Soc. Journ., 1882, xvi, 250. Cul.

Pleurotoma alabaster Rre., Krebs. 7.

Pleurotoma albella Ad., Am. Marine Conch. 131. J.

Pleurotoma albella C. B. Adams, Contr. to Conch. $63 . \quad J$.

Pleurotoma albella Ad., Contr. to Conch. 131.

Pleurotoma albella Ad., Krebs. 7.

Pleurotoma albida Ad., Krebs. 7.

Pleurotoma albida C. B. Adams, Krebs' Remarks. 397.

Pleurotoma albida C. B. Adams, Synops. 3, 1845. J.

Pleurotoma albocincta Ad., Arango, Fauna Mal. Cuba, 1878. 219.

Ca. Havana. J. Ba.

Pleurotoma albocincta Adams, Krebs. 7. ST. SM.

Pleurotoma albocincta C. B. Adams, Synops. 3, $1845 . \mathrm{J}$.

Pleurotoma albomaculata Ad., Jour. de Conch., ir, 417. G.

Pleurotoma albomaculata Ad., Krebs. 7.

Pleurotoma albomaculata C. B. Adams, Srnops :3, 1S45. J.

Pleurotoma albomaculata D'Orb., Arango, Fauna Mal. Cuba, 1878. 217. Ca. G.

Pleurotoma albomaculata D’Orb., Beau. 8. G.

Pleurotoma albomaculata D'Orb., Krebs. 7.

Pleurotoma albomaculata D'Orb., Moll. Cuba, ii, pl. xxiv, figs. 16-18. 176. Ca. 
Pleurotoma albovittata C. B. Adams, Contr. to Conch. 55. J.

Pleurotoma alborittata C. B. Adams, Synops. 4, 1845. J.

Pleurotoma (Drillia) amblia Watson, Lin. Soc. Jour., 1882, xri, 249 Cul.

Pleurotoma angulifera Rve., Krebs. 8.

Pleurotoma angustæ Ad., Krebs. 8.

Pleurotoma antillarum Crosse, Jour. de Conch., xviii, 372. G.

Pleurotoma antillarum Crosse, Jahrb., iv, 4.

Pleurotoma antillarum Crosse, * Jour. de Conch., xiii, pl. i, fig. 8. 34. G. Pleurotoma antillarum D'Orb., Arango, Fauna Mal. Cuba. 1878. 217. Ca. G.

Pleurotoma antillarum D'Orb., Beau. 8. G.

Pleurotoma antillarum D'Orb., Krebs. 8.

Pleurotoma antillarum D'Orb., Moll. Cuba, ii, pl. xxir, figs. 1-3. 173.

Ca. M.

Pleurotoma (Mangilia) antonia Dall, n. s., Report Blake Moll. 59. CS. Pleurotoma (Defrancia) araneosa Watson, Lin. Soc. Journ. 1881, xv, 462. Cul.

Pleurotoma auberiana D'Orb., Arango, Fauna Mal. Cuba, 1878. 217. Ca.

Pleurotoma auberiana D'Orb., Beau. 8. G.

Pleurotoma auberiana D'Orb., Jour. de Conch., ir, 417. G.

Pleurotoma auberiana D'Orb., Krebs. 8.

Pleurotoma auberiana D'Orb., Moll. Cuba, ii, pl. xxiv, figs. 4-6. 174. Ca. M.

Pleurotoma augustæ C. B. Adams, Contr. to Conch. 61. J.

Pleurotoma augustæ C. B. Adams, Krebs' Remarks. 397.

Pleurotoma badia Rve., Arango, Fauna Mal. Cuba, 1878. 217. Ca. J. Ba.

Pleurotoma badia Rve., Krebs' Remarks. 396.

Pleurotoma balteata Rre., Krebs. 8.

Pleurotoma (Mangilia) bandella Dall, n. s., Report Blake Moll. 59. CS. Pleurotoma bicolor Sby., Poulsen. 13.

Pleurotoma biconica C. B. Adams, Contr., Conch. 65. Ca. J. Pleurotoma biconica Ad., Arango, Fauna Mal. Cuba, 1878. 217. Ca. J. Pleurotoma (Bela) blakeana Dall, n. s., Report Blake Moll. 54. WI. Pleurotoma (Bela) blakeana rar. extensa, Report Blake MIoll. 55. WI. Pleurotoma (Bela) blakeana var. normalis, Report Blake MIoll. 54. WI. Pleurotoma cancellata Gray, Krebs. 8. ST.

Pleurotoma caudeana D'Orb., Arango, Fauna Mal. Cuba, 1878. 218. M. G.

Pleurotoma candeana D'Orb., Beau. 8. G.

Pleurotoma candeana D'Orb., Krebs. 8.

Pleurotoma candeana D'Orb., Moll. Cuba, ii., pl. xxiv, figs. 10-12. 175. G. $M$. 
Pleurotoma candidissima C. B. Adams, Arango, Fauna Mal. Cuba, 1878. 217 Ca. J. Ba.

Pleurotoma candidissima C. B. Adams, Synops. 4. 1845. J.

Pleurotoma candidula Rve., Krebs. 8.

Pleurotoma caribæa D'Orb., Arango, Fanna Mal. Cuba, 1878. 218. Ca. M. G.

Pleurotoma caribæa D'Orb., Bean 8. G.

Pleurotoma caribæa D'Orb., Krebs. S.

Pleurotoma caribea D'Orb., Moll. Cuba, ii, pl. xxiii, figs. 32-34. 172.

M. Ca. G.

Pleurotoma cerina Kurtz \& Stm., Am. Marine Conch. 48.

Pleurotoma cerinum Kurtz \& Stm., Kurtz. 9. NC. SC.

Pleurotoma cerinum Kurtz \& Stm., Ponlsen. 13.

Pleurotoma cerinum Kurtz \& Stm., Proe. Bost. Soc. Nat. Hist., iv. 115. 1851. SC.

Pleurotoma (Borsonia) ceroplasta Watson, Lin., Soc. Journ., 1881, xv, 473. Cul.

Pleurotoma (Defrancia) chariessa Watson, Lin. Soc. Journ., 1881, xv, 458. Cul.

Pleurotoma cinctellum Pfeiffer, Archiv fiir naturg., 1840. 258. Ca.

Pleurotoma (Defrancia) circumroluta Watson, Lin. Soc. Journ., 1881, xr, 465. Cul.

Pleurotoma clathratus Rve., Krebs. 8.

Plenrotoma coccinata Rre., Arango, Fauna Mal. Cuba, 1878, 218. Ca.

Pleurotoma coceinata Rre., Krebs. 8. ST. SJ. SB. SM. Ag.

Pleurotoma coccinata Rve., Krebs' Remarks. 397.

Pleurotoma coccinata Rre., Poulsen. 13.

Pleurotoma collaris Rve., Beau. 7. G.

Pleurotoma collaris Rve., Jour. de Conch., v, 153. G.

Pleurotoma collaris Rive., Krebs. 8.

Pleurotoma (Mangilia) columbella Dall. n. s., Report Blake Moll. 60. CS.

Pleurotoma (Mangilia) comatotropis Dall, n. s., Report Blake Moll. 58. CS.

Pleurotoma (Mangelia) corallina Watson, Lin. Soc. Journ., 1881, xv, 435. Cul.

Plenrotoma costata D'Orb., Arango, Fauna Mal. Cuba, 1878. 218. San Vicente. G. M.

Pleurotoma costata Gray, Arango, Fama Mal. Cuba, 1878. 218. San Vicente. G. M.

Pleurotoma costata Gray, Beau 7. G.

Pleurotoma costata Gray, Krebs, 8. ST. SJ. SB. SM. Ag.

Pleurotoma costata Gray, bis, Krebs. 12.

Pleurotoma costata Gray, Moll. Cuba, ii, 169, 174. G. SV. M.

Pleurotoma decorata C. B. Adlams, Contr. to Conch. 62. J. 
Pleurotoma decorata C. B. Adams, Jour. le Conch., iv, 417. G.

Pleurotoma densestriata C. B. Adans, Arango, Fanna Mal. Cuba, 1878. 217. Ca. J. Ba.

Pleurotoma (Drillia) detecta Dall, u. s., Report Blake Moll. 65. CS. Pleurotoma (Genota) didyma Watson, Lin. Soc. Journ., 1881, xv, 404. Sombrero.

Pleurotoma diminuta C. B. Aclams, Contr. to Conch. 62. J. Pleurotoma diminuta C. B. Adams, Krebs 9, 14.

Pleurotoma d'orbignii Rve., Krebs. 9. ST. Lj.

Pleurotoma dorvilliae Gray, Arango, Fauna Mal. Cuba, 1878. 218. Ca. $\mathrm{Ba}$.

Pleurotoma dorvilliæ Gray, Beau. 8. G.

Pleurotoma dorvilliæ Gray, Krebs. 9.

Pleurotoma dorvillix Gray, Moll. Cuba, ii, 171. SV.

Pleurotoma dubia C. B. Adams, Synops., 4, 1845. J.

Pleurotoma (Thesbia) drscrita Watson, Lin. Soc. Journ., 18s1, xv, 448. Sombrero.

Pleurotoma dysoni Rve., Arango, Fauma Mal. Cuba, 1878. 218. Ca.

Pleurotoma elatior Ad., Krebs. 9.

Pleurotoma elatior C. B. Arlams, Synops, 4, 1S45. J.

Pleurotoma elatior D'Orb., Moll. Cuba, ii, pl. xxiii, figs. 35-37. 173. ST. Pleurotoma elatior D'Orb., Arango, Fauna Mal. Cuba, 1878. 218. ST. Pleurotoma elatior D'Orb., Beau. 8. G.

Pleurotoma elatior D’Orb., Krebs. 9.

Pleurotoma elongata Ant., Pfeifter, Archir für naturg., 1840. 258. Ca.

Pleurotoma (Drillia) elusira Dall, n. s., Report Blake Moll. 69. Yucatan Strait.

Pleurotoma emblema Rav., Ravenel. 19. SC.

Pleurotoma (Drillia) exasperata Dall, n. s., Report Blake Moll. 63. CS.

Pleurotoma (Drillia) exsculpta Wratson, Lin. Soc. Journ., 1852, xvi, 247. Cul.

Pleurotoma (Bela) filifera Dall, n. s., Report Blake Moll. 56. CS.

Pleurotoma flarocincta C. B. Adams, Contr. to Conch. 63. J.

Pleurotoma flavocincta Ad., Krebs. 9.

Pleurotoma fucata Rve., Arango, Fanua Mal. Cuba, 1878. 218. Ca. Ba. Pleurotoma fucata Rve., Krebs. 9. ST. SB. SM. Ag.

Pleurotoma fucata Rve., Poulsen. 13.

Pleurotoma fusca C. B. Adams, Synops., 4, 1845. J.

Pleurotoma fuscescens Gray, Arango, Fauna Mal. Cuba, 1878. 220. Ca. Havana.

Pleurotoma fuscescens Gray, Beau. 7. G.

Pleurotoma fuscescens Gray, Krebs. 9, 10. ST. SCx.

Pleurotoma fuscescens Gray, bis., Krebs. 11.

Pleurotoma fuscescens Gray, Poulsen. 13.

Pleurotoma fuscocincta C. B. Adams, Contr. to Conch. 62. J. 
Pleurotoma fuscocincta Ad., Krebs. 9.

Pleurotoma fuscolineata C. B. Adams, Arango, Fauna Mal. Cuba, 1878. 219. Ca. J.

Pleurotoma fuscolineata C. B. Adams, Contr. to Conch. 54. WI.

Pleurotoma fuscolineata C. B. Adams, Synops., 4. 1845. J.

Pleurotoma fusiformis C. B. Adams, Contr. to Conch. 64. J.

Pleurotoma fusiformis Ad., Krebs. 9.

Pleurotoma fusiformis C. B. Adams, Krebs' Remarks. 397.

Pleurotowa (Drillia) gratula Dall, n. s., Report Blake Moll. 64. OS.

Pleurotoma gruneri Phil., Jahrb., ir, 5.

Pleurotoma gruneri Phil., Poulsen. 13.

Pleurotoma gruneri Phil.,* Zt. Mal., v, 12. ST.

Pleurotoma guildingii D’Orb., Arango, Fauna Mal. Cuba, 1878. 219.

Ca. San Vicente.

Pleurotoma guildingii Rve., Arango, Fauna Mal. Cuba, 1878. 219. Ca.

San Vicente.

Pleurotoma guildingii Rve., Beau. 7. G.

Pleurotoma guildingii Rve., Krebs. 9. ST.?

Pleurotoma guildingii Rve., Moll. Cuba, ii, 170. Ca. SV.

Pleurotoma harpula Val., Krebs. 10. SCx.

Pleurotoma harpula Val. bis, Krebs. 11.

Pleurotoma hastula Rre., Krebs. 10. ST.

Pleurotoma (Drillia) havanensis Dall, n. s., Report Blake Moll. 67. CS. Pleurotoma hexagonum Pfeiffer, Archiv fiir naturg., 1840. 258. Ca.

Pleurotoma (Defrancia) hormophora Watson, Lin. Soc. Journ., 1881, xv, 457. Sombrero. Cul.

Pleurotoma (Drillia) incilis Watson, Lin. Soc. Journ., 1881, xr, No. 87. 425. Cul.

Pleurotoma insculpta Mighels, Proc. Bost. Soc. Nat. Hist., 1844, i, 189. Key West.

Pleurotoma (Mangilia?) ipara Dall, n. s., Report Blake Moll. 57. Yucatan Strait.

Pleurotoma jayana C. B. Adams, Contr. to Conch. 61. J.

Pleurotoma jayana Ad., Krebs. 10. ST.

Pleurotoma jelskii Crosse, ${ }^{*}$ Jour. de Conch. xiii, pl. i, figs. 6, 7. 33. G. Pleurotoma jelskii Crosse, Jahrb., iv, 4.

Pleurotoma laqueata Rve., Krebs. 10.

Pleurotoma laqueata Rve., Arango, Fauna Mal. Cuba, 1878. 217. Ca. J. Ba.

Pleurotoma lavalleana D'Orb., Arango, Fauna Mal. Cuba, 1878. 219. Ca. G. J. M.

Pleurotoma lavalleana D'Urb., Beau. 8. G.

Pleurotoma lavalleana D'Orb., Krebs. 10.

Pleurotoma lavalleana D'Orb., Moll. Cuba, ii, pl.xxiv, figs. 7-9. 174. Pleurotoma (Drillia) leucomata Dall, n. s., Report Blake Moll. 63. 
Pleurotoma (Daphnella ?) leucophlegma Dall, n. s., Report Blake Moll. 70. CS.

Pleurotoma (Bela) limacina Dall, n. s., Report Blake Moll. 55. CS.

Pleurotoma limnæiformis Kien., Beau. 7. G.

Pleurotoma (Daphnella) limnæiformis Kien., Poulsen. 13.

Pleurotoma limnæiformis? Rve., Jour. de Conch., iv, 417. G.

Pleurotoma (Raphitoma) lincta Watson, Lin. Soc. Journ., 1881, xv, 442. Cul.

Pleurotoma lineolata ? Rre., Arango, Fauna Mal. Cuba, 1878. 219. Ca. Pleurotoma (Mangilia) lissotropis Dall, n. s., Report Blake Moll. 59. CS. Pleurotoma (Raphitoma) lithocolleta Watson, Lin. Soc. Journ., 1881, xv, 441. Sombrero.

Pleurotoma luctuosa D’Orb., Arango, Fauna Mal. Cuba, 1878. 219. Ca. Pleurotoma luctuosa D'Orb., Bear. 8. G.

Pleurotóna luctuosa D'Orb., Krebs. 10.

Pleurotoma luctuosa D'Orb., Moll. Cuba, ii, pl. xxiii, figs. 29-31. 172. Ca.

Pleurotoma luteofasciata Rìe, Contr. to Conch. 55.

Pleurotoma maculata C. B. Adams, Contr. to Conch. 62. J. Pleurotoma maculata Ad., Krebs. 10.

Pleurotoma minor C. B. Adams, Contr. to Couch. 54. WI.

Pleurotoma minor Ad., Krebs. 10.

Pleurotoma minor C. B. Adams, Krebs' Remarks. 397.

Pleurotoma minor C. B. Adams, Synops., 4. 1845. J.

Pleurotoma (Genota) mitrelli, Dall, n. s., Report Blake Moll. 56. Yucatan Strait.

Pleurotoma monilifera C. B. Adams, Krebs' Remarks. 397.

Pleurotoma monilifera Sbj., Arango, Fauna Mal. Cuba. 1878. 219. Ca. J.

Pleurotoma monilifera (Columbella) Sby, Contr. to Conch. 55.

Pleurotoma monilifera Sby., Krebs. 10.

Pleurotoma (Drillia) morra Dall, n. s., Report Blake Moll. 69. Havana.

Pleurotoma multilineata C. B. Adams, Synops., 3. 1845. J.

Plenrotoma nigrescens C. B. Adams, Contr. to Conch. 54. J.

Pleurotoma nigrescens Ad., Krebs. 10.

Pleurotoma nigrescens C. B. Adams, Synops., 3. 1845. J.

Pleurotoma nigrescens D'Orb., Arango, Fauna Mal. Cuba, 1878. 219.

Ba. San Vicente.

Pleurotoma nigrescens Gray, Arango, Fauna, Mal. Cuba, 1878. 219.

Ba. San Vicente.

Pleurotoma nigrescens Gray, Beau. 8. G.

Pleurotoma nigrescens Gray (Ad.?), Contr. to Conch. 54.,

Pleurotoma nigrescens Gray, Krebs. 9, 10.

Pleurotoma nigrescens Gray, bis, Krebs. 10. ST. SCx. Ag. SM.

Pleurotoma nigrescens Gray, Moll. Cuba, ii, 170. SV. 
Pleurotoma nodata C. B. Adams, Contr. to Conch. $64 . \quad J$.

Pleurotoma nodata Ad., Krebs. 11.

Pleurotoma (Drillia) nucleata Dall, n. s., Report Blake Moll. 62.

Pleurotoma obesicostata Rve., Arango, Fauna Mal. Cuba, 1878. 219. Ca.

Pleurotoma obesicostata Rve., Krebs. 11.

Pleurotoma occidentalis Rve., Krebs. 14. ST.

Pleurotoma (Drillia) oleacina Dall, n. s., Report Blake Moll. 66. CS. Pleurotoma ornata, Moll Cuba, ii, 176.

Pleurotoma oruata D'Orb., Arango, Fauna Mal. Cuba, 1878: 219. Ca. Havana. J. Ba.

Pleurotoma ornata D’Orb., Krebs. 11.

Pleurotoma ornata D'Orb., Moll Cuba, ii, pl. xxiii, figs. 26-28. 171. Ca.

Pleurotoma (Defrancia) pachia Watson, Lin. Soc. Journ., 1881, xv, 460. Cul.

Pleurotoma pagodus Rve., Krebs. 11. ST.

Pleurotoma paria Rve., Krebs. 11.

Pleurotoma parvula Rre., Arango, Fauna Mal. Cuba, 1878. 220. Ca. Pleurotoma paxillus Rve., Krebs. 11. ST.

Pleurotoma (Mangilia) pelagia Dall, n. s., Report Blake Moll. 61. WI. Pleurotoma pellis-phocæ Rve., Krebs. 11.

Pleurotoma pellis phocæ Rve., Poulsen. 13.

Pleurotoma pentagonalis Gray, Araugo, Fauna Mal. Cuba, 1878. 220.

Ca. San Vicente.

Pleurotoma pentagonalis Gray, Beau. 7. G.

Pleurotoma pentagonalis Gray, Krebs. 11.

Pleurotoma pentagonalis Gray, Moll. Cuba, ii, 171.

Pleurotoma pentagonalis Gray, Moll. Cuba, ii, 174. SV.

Pleurotoma (Drillia) peripla Dall, n. s., Report Blake Moll. 68. Yucatan Strait.

Pleurotoma (Defrancia) perpauxilla Watson, Lin. Soc. Journ., 1881, xv, 468. Cul.

Pleurotoma planilira Rav., Ravenel. 19.

Pleurotoma plicatum Ad., Kurtz. 9. NC. SC.

Pleurotoma plicata C. B. Adams, Bost. Journ. Nat. Hist. iii, pl. 3, fig. 6. 318,1840 . N. Engl. Fla.

Pleurotoma plicosa C. B. Adams (plicata Adams, non Lam., P. brunnea Perkins), Contr. to Conch., 54.

Pleurotoma (Drillia) polytorta Dall, n. s., Report Blake Moll. 61. CS. Pleurotoma (Mangilia) pourtalesii Dall, n. s., Report Blake Moll. 60. WI.

Pleurotoma (Defrancia) pudens Watson, Lin. Soc. Journ., 1881, xv, 461. Cul.

Pleurotoma pulchella Gray, Araugo, Fauna Mal. Cuba, 1878. 220. Ca.

Pleurotoma pulchella Rve, Krebs. 11. 
Pleurotoma pulchra Gray, Krebs. 11. ST.

Pleurotoma pura Rve., Arango, Fauna Mal. Cuba, 1878. 220. Ca.

Pleurotoma pura Rve., Krebs. 11. SM.

Pleurotoma pusilla Ad., Arango, Fauna Mal. Cuba, 1878. 220. Ca.

Pleurotoma pygmxa C. B. Adams, Contr. to Conch. 63. J.

Pleurotoma pygman Ad., Krebs. 11.

Pleurotoma pygmaa C. B. Adams, Krebs' Remarks. 397.

Pleurotoma quadrata Rve., Contr. to Conch. 62. J.

Pleurotoma quadrifasciata Rve., Krebs. 11.

Pleurotoma quadrilineata C. B. Adams, Contr. to Conch. 64. J.

Pleurotoma quadrilineata Ad., Krebs. 11.

Pleurotoma rubricata Rve., Krebs. 12.

Pleurotoma rustica Sby., Arango, Faura Mal. Cuba, 1S78. 220. Ca. Havana.

Pleurotoma sacra Rve, Arango, Fauna Mal. Cuba, 1878. 220. Ca. Pleurotoma scalpta Rve., Contr. to Conch. 54. J.

Pleurotoma semigranosa Rve., Krebs. 10.

Pleurotoma semigranosa Rve., Krebs. 11.

Pleurotoma semigranosa Rve, Krebs. 12. ST.

Pleurotoma (Drillia) serga Dall, n. s., Report Blake Moll. 65. WI.

Pleurotoma (Pleurotomella?) sigsbei Dall, n. s., Report Blake Moll. 57.

Pleurotoma sinuata Rve., Arango, Fauma Mal. Cuba, 1S78. 220. Ca. Pleurotoma slatior D'Orb., Moll. Cuba, ii, 173.

Pleurotoma (Drillia) smirna Dall, n. s., Report Blake MIoll. 66. WI.

Pleurotoma solida C. B. Adams, Contr. to Conch. 61. J.

Pleurotoma solida Ad., Krebs. 12.

Pleurotoma (Drillia) subsida Dall, u. s., Report Blake Moll. 63.

Pleurotoma (Surcula) syugenes Watson, Lin. Soc. Journ., 1S81, xv, 396. Sombrero. Cul.

Pleurotoma thiarella Val., Arango, Fauna Mal. Cuba, 1878. 220. Ca. Havana.

Pleurotoma (Mangelia) tiara, Watson, Lin. Soc. Jouru., 1s81, xv, 440. Cul.

Pleurotoma trifasciata Gray, Arango, Fauna Mal. Cuba, 1878. 220. Ca.

Pleurotoma trifasciata Gray, Contr. to Conch. 55.

Pleurotoma trifasciata Gray, Krebs. 9.

Pleurotoma trifasciata Gray, bis, Krebs. 12.

Pleurotoma trilineata C. B. Adams, Contr. to Conch. 55. J.

Pleurotoma trilineata O. B. Adams, Synops., 3, 1845. J.

Pleurotoma triticea Rve., Krebs. 15. Ag.

Pleurotoma turbinelloides Rve., Krebs. 12.

Pleurotoma urnula Rve., Krebs. 12.

Pleurotoma (Drillia) verrillii Dall, n. s., Report Blake Moll. 68. WI. 
Pleurotoma respuciana D'Orb., Arango, Fauna Mal. Cuba, 1878. 221. Ca. M. G.

Pleurotoma vespuciana D'Orb., Beau. 8. G.

Pleurotoma vespuciana D'Orb., Krebs. 12.

Pleurotoma vespuciana D’Orb., Moll. Cuba, ii, pl. xxiv, figs. 13-15. 175. M. Ca. G.

Pleurotoma villiersii Mich., Pfeiffer, Archiv für naturg., 1840. 258. Ca. Pleurotoma vir go Lam., Jahrb., iv, 4.

Pleurotoma virgo Lam., Krebs. 12.

Pleurotoma zebra Kien., Krebs. 10. ST.

Pleurotoma zebra Lam., Krebs. 12. ST. SJ. W.

Pleurotoma zebra Lam., Poulsen. 13.

Pleutomaria adausoniana C. \& F., Jour. de Conch. ix, pl. v, figs. 1-3. 163. G.

Pleurotomaria adausoniana C. \& F., Jour. de Conch. $x x x, 6,12$. G. Bb.

Pleurotomaria adansoniana C. \& F., Report Blake Moll. 78 . Bb.

Pleurotomaria quoyana F. \& B., Krebs. 85.

Pleurotomaria quoyana F. \& B., Jour. de Conch., xxii, 66.

Pleurotomaria quoyana Fisch. \& Bern., Beau. 13. MG. G.

Pleurotomaria qnoyaua F. \& B., Jour. de Conch. v, pl. v, figs. 1-3. 165. G.

Pleurotomaria quosana F. \& B., Jour. de Conch., xxx, 6. Bb. D.

Pleurotomaria quoyana F. \& B., Jour. de Conch., xxx, 14. Bb. D.

Pleurotomaria quoyana Fiseh. \& Bernardi, Report Blake Moll. 78. Mg. CS.

Pleurotomella (see Pleurotoma).

Pleurotomella bandella Dall, Verrill, List, 1884. 266. CS. WI.

Pleurotomella emertoni Verrill \& Sm., Verrill, List, 1884. 266. CS. WI.

Pleurotomella verrillii Dall, n. s., Report Blake Moll. 57. CS.

Plicatella (see Latirus, Turbinella).

Plicatella attenuata Rve., Jahrb., iii, 20.

Plicatella contempta A. Ad., Jahrb., iii, 22.

Plicatella filamentosa Koch, Jahrb., iii, 22.

Plicatella infundibulum Gmel., Jahrb., iii, 20.

Plicatella trochlearis Kob., Jahrb., iii, 20.

Plicatula (see Ostrea, Spondylus).

Plicatula barbadensis D'Orb., Arango, Fauna Mal. Cuba, 1878. 271. Ca. Ba.

Plicatula barbadensis D'Orb., Moll. Cuba, ii, 361.

Plicatula barbadensis Pet., Krebs. 135.

Plicatula barbadensis Pet., Moll. Cuba, ii, 360.

Plicatula cristata Lam., Arango, Fauna Mal. Cuba, 1878. 271. Ca. Ba.

Plicatula cristata Lam., Beau., 21. G.

Plicatula cristata Lam., Jour. de Conch., iv, 416. G.

Plicatula cristata Lam., Krebs. 135. 
Plicatula cristata Lam., Kurtz. 6. SC.

Plicatula cristata Lam., Moll. Cuba, ii, 360.

Plicatula depressa Lam., Arango, Fauna Mal. Cuba, 1878. 271. Ca. Ba.

Plicatula depressa Lam., Krebs. 135.

Plicatula depressa Lam., Moll. Cuba, ii, 360.

Plicatula plicata Chem., Beau., 21. G.

Plicatula ramosa Lam., Am. Marine Conch. 198.

Plicatula ramosa Lam., Arango, Fauna Mal. Cuba, 1878. 271. Ca. Ba. Plicatula ramosa Lam., Beau., 21. G.

Plicatula ramosa Lam., Krebs. 135.

Plicatula ramosa Lam., Moll. Cuba, ii, 360.

Plicatula reniformis Lam., Arango, Fauna Mal. Cuba, 1878. 271. Ca. Ba.

Plicatula reniformis Lam., Krebs. 135.

Plicatula reniformis Lam., Moll. Cuba, ii, 360.

Plicatula spoudyloidea Meusch., Arango, Fauma Mal. Cuba, 187S. 271. Ca. Ba.

Plicatula spondyloidea Meusch., Poulsen. 16.

Plicatula spondyloides Meusch., Krebs. 135.

Plicatula vexillata Guppy, 1. s., Geol. Mag., 1874. 444. J.

Poegia gundlachioides Bourg., Arango, Fauna Mal. C'uba, 187s. 138. Ca. Pollia (see Cantharus, Tritoniclea, Pisania).

Pollia cancellaria Conrad, Jahrb., ix, 21. Fla.

Pollia cancellaria Comrad, Proc. Acad. Tat. Sci. Phil., iii, 25, pl. i, fig: 12, 1S45. Ship Island. Gulf of Mexico.

Pollia caribæum D'Orb., Jour. de Conch., v, 155. G.

Pollia coromandeliana Lam., Jahrb., ix, 21.

Pollia lauta Rre., Jahrb., ix, 23.

Pollia limbata Phil., Jahrb., ix, 23.

Pollia tincta Conıad, Jahrb., ix, 25.

Pollia tincta Conrad, Krebs. 32.

Pollia tiucta Comrad, Proc. Acad. Nat. Sci. Phil., iii, 25̃, pl. 1, fig. 9, 1845. Tampa.

Pollia variegata Gray, Zt. Mal., x, 73. SV.

Pollinices (see Natica, Lunatia).

Pollinices candidissima Recluz, Mal. Bl., xxir, 59. Bz.

Pollinices caribæa Phil., Mal. Bl., xxiv, 61. G.

Pollinices draparuaudi Recluz, Mal. B1., xxiv, 59.

Pollinices fuscata Chem., Mal. Bl., xxiv, 58.

- Pollinices lactea Guild., Mal. Bl., xxir, 60. Ca.

Pollinices ochrostoma Recluz, Mal. Bl., xxiv, 60.

Pollinices porcellaua D'Orb., Mal. Bl.; xxiv, 60. Bz.

Polypus mas Seba, Moll. Cuba, i, 18.

Polypus octopodia Leach, Moll. Cuba, i, 11.

Polypus octopus Rond., Moll. Cuba, i, 11.

Bull, 24- 16 
Pomus (sce Ampullaria).

P’omus depressus say, Dall, Proc. U. S. Nat. Mus. 1855, pl. xrii, figgs, 4, 5. ร55. Fla.

Pomus caliginosa Rive, Dall, Proc. U. S. Nat. Mus. 1855. 255. Fla.

Nicaragua. Teluantepec.

Pomus urceus Mull., Marrat, Argo Exp., 1876. 10. Td.

Porcellana (see Cypraea).

Porcellana achates D'Arg., Moll. Cuba, ii, 89.

Porcellana carthageniensis Mart., Mal. Bl., xxir, 46.

Porcellana exanthema Linn., Am. Marine Conch. 55.

Porcellana girisea Meusch., Mal. Bl., xxir, 47. J.

Porcellana integra Gualt., Mal. Bl., xxiv, 53.

Porcellana longa Klein, Moll. Cuba, ii, 88.

Porcellana maculosa Heb., Moll. Cuba, ii, 89.

Porcellana oliviformis T.\& H., n. s., Pleincene Fossils S. C., pl. xxvii, figs. 12. 13. 131.

Porcellana simplex Mart., Moll. Ouba, ii, 89.

Porcellana spiralis Gualt., Mal. Bl., xxiv, 45.

Porcellana thoracica Gualt., Moll. Cuba, ii, 92.

Poromya (see Corbula).

Poromya (?) granatiua Dall, u. s., Report Blake Kioll. 109. WI.

Poromya granulata Nyst \& West., Report Blake Moll. 10S. WI.

Porompa quadrata Con., Arango, Fauna Mal. Cuba, 187S. 25̃3. Autilles. G.

Poronya sublævis Verrill, List, 1854. 277. Chesapeake Bay.

Porphyria (see Dactylus), Marrat, Argo Exp., 1876. 12.

Portlandia intermedia M. Sars, Report Blake .Moll. 127.

Posterobranchra gemmatum Mörch, Mal. Bl., xxii, 175.

Posterobranchiea gemmulatum Mürch, Jour. de Conch., xi, 25. ST.

Potamides (see Cerithidea), Arango, Fama Mal. Cuba, 1878. 209. Ca.

Potamides fragilis Defr., Pfeiffer, Archiv fuir naturg., 18t0. 257. Ca.

Potamides hegewischii Phil., Mal. Bl., xxiii, 89.

Potamides iostoma Pfr., Mal. Bl., xxiii, 89.

Potamides minutus Dkr., Mal. Bl, xxiii, 92. Ca.

Potamides tenuis Pfr., Arango, Fauma Mal. Cuba, 1878. 209. Ca.

Po:amides tenuis Pfr., Mal. Bl., xxiii, S8.

Propilidium elegans Verrill, List, 1S84. 271. Chesapeake Bas.

Psammobia (see Tellina), Arango, Fauna Mal. Cuba, 1878. 242. Ca. Harana.

Psammobia aftinis C. B. Adams, Arango, Famna Mal. Cuba, 1878. 246. Ca. Antilles. Fla.

Psammobia affinis, C. B. Adams, Synops., 10, 1S45. J.

Psammobia biradiata C. B. Adams, Arango, Fauna Mal. Cula, 1878. 246. Ca. Antilles. Fla.

Psammobia biradiata C. B. Adams, Synops., 10, 1845. J.

Psammobia cayeunensis, Jahrb., ii, 248 . = macrmes 
$\checkmark$ Psammobia cajennensis Lam., Moll. Cuba, ii, 244. = Mna $c^{0}$ wa

Psammobia cayennensis Lam., Krebs., 100.

r Psammobia cerina C. B. Aclams, Synops., 10, 18t5. J. = Machma c:

Psammobia circe Möıch,* Jour. de Conch., xxiv, 373. 'Tort.

Psammobia constricta Lim., Krelos., 101. $=$ n..........

Psammobia jurpureo-maculata C. B. Adims, Arango, Fauna Mal.

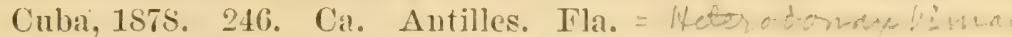

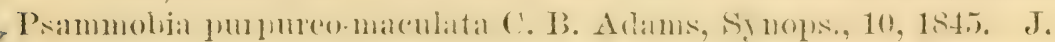

Psammobia rosea Desh., Moll. Cuba, ii, 247. wa Jangminctarie or

Astmmobia rosea Lam., Jour. de Conch., ii, 425. G. " " "

Psammobia rugosa Sby., Moll. Cuba, zi, 25s. = as $2 / \lambda_{1}$

Psammobia squamosa Lam., Krebs. 104.

Psammocola pleiocena T. \& II., n. s., I'leioune Fossils. C., pl. xxii, fig.

S. 91. $=$ axtes

Psammocola rugosa Blr., Moll. Cuba, ii, 25S. = wa $a, 6$

Psammosoleu (see Macha).

Psammosolena sancta martha Chem., Krebs. 113. ST. Tort.

Psendamussium (sec P'ecten), Marrat, Arge Exp), 1876. †.

Psencioneptunea multangula Phil., Jahrb., ix, 17.

Psendorotella (see Gilobulus, Etbaliat).

Psendurotella anomala D'Orb., Jour. de Conch., ri, 174. ST.

Psend rotella curinata D'Orb., Jour, de Conch., vi, 174. S'1.

Psendorotella pusillat Pfr., Jomr. the Conch., ri, 173. Sl'.

Psemblotella semistriata D'Orb., Jom: de Conch., vi, 173. Ca.

Pseudorotella strinta D'Orb., Jomr. de Conch, vi, 17t. J.

Pugil (see Stromlus).

Pugil cinereus Hlb., Mal. Bl., xxir, 19.

Pugil costoso-muricatus, \&c., Mal. Bl., xxir, 19

Pugilina (see Hemifusus, Cassidulus, Melongena).

Pugiliua coromata Lam., Jabrb., ii, 357. Bz.

Pugilina morio Linn., Jalnb., ii, 357. Bz.

Puucturella (ne Fissurella, Cemoria).

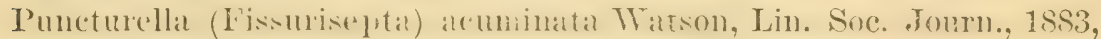
xrii, 3S. Cul.

Puncturella acuta Watson, Lin. Soc. Journ., 1S\$3, xrii, 35. Cul.

Puncturella agger Watson, Joux. cle Couch., xxxi, So.

Puncturella agger Watson, Lin. Soc. Jomrn., 1SS3, xvii, 32. Cnl.

Puncturella (Cranopsis) asturiaua Fischer, Watson, Liu. Soc. Journ. 1s83, xvii, 29. Cul.

Puncturella circularis Dall, n. S., Report Blake Moll. 75. WI.

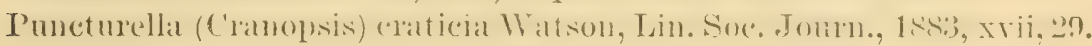
Cul.

Puncturella (c'runopis) gramulata Seguenza, Watson, Lin. Soc. Jompu., 18ะ3, xvii, 31. Cul.

Puncturella oxia Watson, Jour. de Conch., xxxi, S0.

Puncturella oxia Wạtson, Lin. Soc. Jouru., 1ss3, xvii, 36. Cul, 
Puncturella plecta Watson, Jour. cle Conch., xxxi, So.

Puncturella plecta Watson, Lin. Soc. Journ., 1\$83, xvii, 34. Cul.

Puncturella profundi Jeffreys, Watson, Lin. Soe. Journ., 1883, xrii, 35. Cul.

Puncturella (Fissurisepta) rostrata Seguenza, Watson, Li ı. Soc. Journ,, 1883, xvii, 38. Cul.

Puncturella sportella Watson, Jour. de Conch., xxxi, 80 .

Puncturella sportella Watson, Lin. Soc. Journ., 1853, xvii, 37. Cul.

Puncturella trifolium Dall, n. s., Report Blake Moll. 76. Yueatan Strait.

Puncturella (Cranopsis) tuberculata Watson, Lin. Soc. Jouru., 1ss3, x rii, 31. Cul.

Purpura (sce Murex), Arango, Famma Mal. Cuba, 1878. 210. Ca. IIavana. Matanzas. G. M. ST. Ba.

Purpura (see Riciuula,) Araugo, Fauna Mal. Cuba, 1878. 203. Ca. Ha. vana. G. Ba.

Purpura abreviata Blv., Krebs. 26. ST. SJ.

Purpura abbreviata Lam., Beau. 10. G.

Purpura aberrans C. B. Adams, Contr. to Conch. 58. J.

Purpura aberrans Ad., Krebs. 25.

Purpura accincta Born, Moll. Cuba, ii, 146.

Purpura aceincta D'Orb., Arango, Fauna Mal. Cuba, 187S. 204. Ca. Baracoa. Ba.

Purpura accincta D'Orb., Krebs. 31. 'Tort.

Purpura auriculata Lam., Guppy, Paria Fauna, 1877. 144.

Purpura bicostalis Lam., Moll. of Bermudas. 17.

Purpura bicostrlis Lam., Krebs. 25. ST.

Purpura bicostalis Lam., Poulseu. 11.

Purpura cateracta Chem., Kuirtz. 8. NC. SC.

Purpura clathrata A. Ad., Arango, Fauna Mal. Cuba, 185s. 201. Ca. P'urpura deltoidea Lam., Arango, Fama Mal. Cuba, 187S. 202. Ca. Havana. Ba.

Purpura deltoidea Lam., Bean. 10. G.

Purpura deltoidea Lam., Jour. de Conch., ii, 429. G.

Purnura deltoidea Lam., Krebs. 27. ST.

Papura (Thalessa) deltoidea Lain., Marrat. Argo Exp., 1876. 5. Madeira. Aa. SV. Puerto Cabello. Tueacas. Santa Marta. Poiat Saranilla. VCz. Nassau. Loug Key Island. Athol Island. Abaco.

Purpura deltoidea Lam., Moll. of Bermudas. 16.

Purpura dentata Mike., Zt. Mal., x, 74. SV.

Purpura dubia Stimpson, 11. s., Kurtz. S. NC. SC.

Purpura fisciata Rve., Jour. de Coneh., iii, 163.

Purpura fitsciata Rre., Bean. 10. G.

Purpura fisciata Rve., Jour. de Conch., ii, 429. G.

Purpura fasciata live, inebs. 25. NGr. 


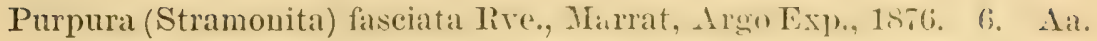
D. SV. Puerto Cabello. Tucacas. Santa Marta. YCz. Nassan. Long Key Island.

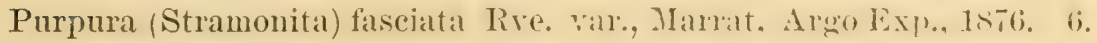
Point Savanilla.

Purpura floridana Con., Am. Marine Conch., 36.

Purpura floridana Con., Arango, Famua Mal. ('uba, 1sirs. 20)1. ('a. Havana. Fla. Ba.

Purpura floridana Con., Jahrb. ii, 242. Fla.

Purpura floridana Con., Jour. de Conch., $\div, 40$. Fla.

Purpura tlorillana Con., Journ. Acarl. Nat. Seic, Phil, vii, 26.5. pl. xx, fig. 21, 18:37. Fla. Ala.

Purpura floridana Con., Krebs. 26.

Purpura (Stramonita) florieliana Con., Marrat, Argo Exp., 1sit6. 12. Tucacas.

Pupura gilea Chem., Arango, Fama MaI. Cuba, 1878. 202. Ca. G. Ba.

Purpura galea Chem., Beau. 10. G.

Purpura galea Chem., Jour. de Conch., r, 153. G.

Purpura galea Chem., Krebs. 26.

Purpura galea Chem., P. abbreviata Blr., Poulsen. 11.

Purpura galea Dorb., Arango, Fauna Hal. Cuba, 1S7S. 202. Ca. G. Ba.

Purpura galea D'Orb., Moll. Cuba, ii, 147.

Purpura gibbosa Rve., Krebs. 26.

Purpura gigantea Rre., Guppy, Paria Fauna, 1877. 144.

Purpura gundlachi Dlir., Araugo, Famar Mal. Cuba, 1sis. 202. Ca. Harama. Fla. Ba.

Purpura hremastoma D'Orb., Moll. Cuba, ii, 144.

Purpura hæmastoma Lam., Krebs. 26. ST.

Purpura hæmastoma Lam., Moll. Cuba, ii, 144.

Purpura hamastoma Liun., Jahrb., ii, 241. Bz.

Purpura (Stramonita) hatuastoma Linn., Marrat, Argo Exp., 1876. 5. Madeira.

Purpura hæmastoma Linn., Poulsen. 11.

Purpura kieneri Desh., Krebs. 26.

Purpura lapillus Linn., Am. Marine Conch. 36.

Purpura lineata Lam., Krebs. 26.

Purpura marginalba Blr., Arango, Famna Mal. Cuba, 1S78. 203.

Purpura miocenica Gupps, n. s., Guppr, Geol. Mag., 1874. 410. J.

Purpura neritoides Lam., Zt. Mal., x, 73. SV.

Purpura nodulosa C. B. Arlams, Sjnops., 2, 1845. J.

Purpura nucleus Lam., Mal. Bl., xxiii, 126.

Purpura orbignyana Petit, Jour. de Conch., $\nabla, 38$.

Purpura patula D'Orb., Jour. de Conch., vii, 27. G. 
Purpura patula D’Orb, Arango, Fauna Mal. Cuba, 18тs. 202. Ca.Mr. G. Ba.

Purpura patula Lam., Jour. de Conch., vii, 60.

Purpura patula Lam., Moll. Cuba, ii, 144.

Purpura patula Linu., Arango, Fauna Mal. Cuba, 18is. 202. Ca. M. G. Ba.

Purpura patula Linu., Bean. 10. G.

Purpura patula Linn., Jour, de Conch., ii, 429. G.

Purpura patula Linn., Krebs. 26. ST. SMI. NGr.

Purpura patula Linn., Marrat, Argo Exp., 1876. 6. Aa. Barbuda. D.

SV. P'nerto Cabello. Santa Marta. Nassau. Long Kes Island.

Purpura patula Linn., Ponlsen. 11.

Purpura patulum Linn., Grippy, Paria Fauna, 1877. 144.

Purpura picta Turt., Pfeiffer, Arehiv für naturg., 1840. 257. Ca.

Purpura plicata Mart., Krebs. 26. ST. SJ. Ag.

Purpura plicata Mart., Cantharus erosus Bolt., Poulsen. 11.

Purpura rustica Lam., Krebs. 27. SM.

Purpura rustica Lam., Ponlsen. 11.

Purpura subeleltoidea Blv., Arango, Fauna MIal. Cuba, 18is. 202. Ca. M.

Purpura subdeltoidea Blr., Krebs. 27.

Purpura subdeltoidea Blv., Moll. Cuba, ii, 145.

Purnura subeleltoidea D’Orb., Arango, Fanua Mal. Cuba, 1878. 202. Ca. MI.

Purpura trapa Bult., Arango, Fanna Mal. Cuba, 18is. 202. Ca. Нarana. Bi.

Purpura trapa Bolt., Krebs. 27. ST.

Purpura trapa Bolt, Ponlsen.- 11.

Pupura trilentata T. \& II., n. s., Pleiocene Fossils S. C., pl. xxviii, fig.

9. 137. Ann.maq. M.H. Xv.1875 p.50" "like mancinella"

Pupura trinitatensis Guppy, Paria Fauna,1877. 144.

Purpura triserialis Stm., Moll, of Bermudas. 16.

Purpura turbinella Lam., Pfeifter, Archir für naturg., 1840. 257. Ca. Parpura undata DoOrb., Jour. de Conch., vii, 61.

Purpurat undatat DOObb, Arango, Fatuma Mal. Cuba, 187s. 203. Ca.

Purpura undata D'Orb., Moll. Cuba, ii, 145.

Purpura mulata Lam., Arango, Fama MIal. Cuba, 1878. 203. Ca.

Purpura undata Lam., Bean. 10. G.

Purpura undata Lam., Krebs. 27.

Purpura undata Lam., Moll of Bermudas. 17.

Purpura mulata Lam., Moll. Cuba, ii, 145.

Purpura rexillum Lam., Krebs. 27.

Purpura vexillum Lam.,Moll. Cuba, ii, 147.

Pusio (see Fusus).

Pusio articulatus Lam., Guppy, Paria Fauna, 1877. 143. 
P’unio penuatus Mart, Arango, Fanna Mal. Cuba, 1875. 204. Ca. Batacoa. Ba.

Pyramea (see Strombus).

Pyramea luciter Link, Mal. Bl., xxiv, 16.

Pyramidella (see Obeliscus, Longchaens).

Pyramidella alreata Conrad, Am. Jonr. Sci., 1846, n. s., ii, 39s. Fla. Pyramidella dolabrata D’Orb., Arango, Fauna Mal. Cuba, 1878. 161.

Ca. G. Bi.

Pyránidella dolabrata Lam., Beau. 13. G.

Prramidella dolabrata Lam., Moll. Cuba, i, 228.

Pyramidella dolabrata Lam., Moll. Cuba, i, 229.

Pyramidella dolabrata Liuw., Araugo, Fauna Mal. Cuba, 1878. 161. Ca. G. Ba.

Pyramidella dolabrata Linn., Jour. de Conch., iv, 417. G.

Pyramidella dolabrata Linn., Krebs. 71. ST. PP. SM. Ag.

Pyramidella dolabrata Woodw., Mal. Bl., xxii, 157.

Pyramidella gundlachi Dkr., Arango, Fauma Mal. Cuba, 1878. 161.

Ca.

Pyramidella pusilla Pfr., Krebs. 71.

Psramidella pusilla Pfr., Mal. Bl., xxii, 167. Ca. $=0$

Pyramidella pusilla Pfr., Zt. Mal., viii, 88. Ca.

Pyramidella terebella Lam., Moll. Cuba, i, 229.

Pyramidella terebellum Lam., Krebs. 71.

Pyramidella tessellata Ad, Arango, Fauna Mal. Cuba, 1S7S. 161. Ca. Pyamidella ? vincta Dall, n. s., Dall, Hemphill's shells. 330. Key West.

Pyramis (see Rissoina).

Pyramis nitens, Ainno, Famna Mal. Cuba, 187S. 167. Ca. Maurice. Pyrazus (see Cerithium, Lampanella).

Pyrazus albivitatum Ad., Moll. of Bermudas. 18.

Pyrazus eriensis Val., Mal. Bl., xxiii, 94. ST. G.

P.razus eriensis Val., Ponlsen. 8.

Pyrazus minimus Gmel., Nal. Bl., xxiii, 93. Fla.

Pyrazus minimus Gmel., var. Cerithiclea clathratum Mlie., Poulsen. S. Pyrazus nigrescens Mke., Mal. Bl., xxiii, 94. Ca.

Pyrazus nigrescens Mke., Poulsen. 8.

Pyrazus zonalis Brug., Ponlsen. 8.

Pyrazus zonalis Brug., Mal. Bl., xxiii, 94. J.

Pyrula (see Busyeon, Fulgur, Sycotypus, Ficus, Melongena, Cassidulus,

Hemifusus. Fusus).

Pyrula abbreviata Lam., Arango, Fauna Mal. Cuba, 1878. 202. Ca. G. Ba.

Pyrula abbreviata Lam, Moll. Cuba, ii, 147.

Pyrula aruama Linụ., Krebs. 18.

Pyrula belknapi Petit, Jahrb., ii, 355. Fla.

Pyrula canaliculata Brug., Kurtz. 9. NO. SC. 
Pyrula canaliculata Say, Jour. de Conch., iii, 142. Fla.

Pyrula canaliculata Say, Jour. de Conch., iii, 144. Fla.

Pyrula caudelabrum Lam., Jahrb., ii, 360. Fla.

Pyrula candelabrum Lam., Jour. de Conch., iii, 145.

Pyrula carica Brug., Kurtz. 9. NC. SC.

Pyrula carica Gmel., Krebs. 18.

Pyrula clathrata Rous., Mal. Bl., xxiv, 43.

Pyrula coarctata Sby.. Am. Jour. Conch., iii, 146. Fla.

Pyrula coarctata Sby., Jour. de Conch., iii, 145. Fla.

Pyrula coarctata Sby., *Jour. de Conch. iii, pl. xiii, fig. 3. 155.

Pyrula corona Gmel., Jahrb., ii, 355. Fla.

Pyrula corona Pfeiffer, Archiv fiir naturg, 1840. 258. Ca.

Pyrula coronatus Lam., Beau. 8. G.

Pyrula dussumieri Kien., Mal. Bl., xxiv, 43. Fla.

Pyrula ficus Lam., Zt. Mal., i, 30.

Pyrula ficus Lam., Zt. Mal., r, 97.

Pyrula fortior Mörch, Mal. Bl., xxir, 43.

Pyrula kieneri Phil., Jour. de Conch., iii, 145.

Pyrula kieneri Phil., *Zt. Mal., v, 98.

Pyrula melongena Lam., Mal. Bl., iii, 190.

Pyrula melongena D'Orb., Arango, Fauna Mal., Cuba, 1878. 216.

Ca. G. M. Caitagena. Fla.

Pyrula melongena Lam., Jour. de Couch., iii,64, 65. G.

Pyrula melongena Lam., Jour. de Conch., iii, 141.

Pyrula melongena Lam., Jour. de Conch., vii, 60.

Pyrula melongena Lam., Moll. Cuba, ii, 167.

Pyrula melongena Linn., Arango, Famna Mal. Cuba, 1878. 216. Ca. G.

II. Cartagena. Fla.

Pyrula melongena Linn., Beau. 8. G.

Pyrula melongena Linn., Krebs. 18. Tort.

Pyrula morio Linn., Jour. de Conch., vii, 28. G.

Pyrula morio Linn., Beau. S. G.

Pyrula morio Linn:, Krebs. 18.

Pyrula nebula Humph., Mal. Bl, xxiv, 43. Fla.

Pyrula papyracea Say, Kurtz. 9. NC. SC.

Pyrula papyratia Say, Mal. Bl., xxiv, 43. Fla.

Pyrula papyratia Say, Poulsen. 10.

Prrula perversa D'Orb., Arango, Fauna Mal. Cuba, 1878. 216. Ca.

Fla.

Pyrula perversa var. Kien., Zt. Mal.,,, 98.

Pyrula perversa Lam., Am. Jour. Conch., iii, 146.

Pyrula perrersa Lam., Jour. de Conch., iii, 141.

Psrula perversa Lam., Krebs. 18.

Pyrula perversa Lam., Moll. Cuba, ii, 167.

Pyrula perversa Linn., Jour. de Conch., iii, 144. Fla.

Pyrula (Fulgur) pyruloides Say, Kurtz. 9. NC. SC. 
Pyrula reticnlata Kien., Mial. Bl., xxir, 43.

Pyrula reticulata Lam., Mal. Bl., xxir, 43.

Pyrula reticulata Sby., Mal. Bl., xxir, 44. Fla.

Pyrula spirata Kieu., Jahrb., ii, 361.

Pyrula spirata Lam., Am. Jour. Conch., iii, 150.

Pyrula spirata Lam., Arango, Fauna Mal. Cuba, 1878. 216. Ca. Fla. Pyrula spirata Lam., Jour. de Conch., iii, 145. Fla.

Pyrula rentricosa Sby., Mal. Bl., xxir, 44.

Pyrum triangulare \&c., Mal. Bl., xxiv, 31.

Pyxipoma (see Tenagodus, Vermetus).

Pyxipoma anguillæ Mörch, Mal. Bl., xxiv, 110. PR.

Radula (see Lima).

Radula caribrea Klein, Krebs. 133.

Radula lima Desh., IIarrat, Irgo Exp., 1876. 17. Nassau. Abaco.

Radula (Ctenoides) scabra Born, Marrat, Argo Exp., 18i6, 6. Ma. Puerto Cabello.

Raëta (see Lutraria, Mactra).

Raëta canaliculata Say, Am. Narine Conch. 146.

Raëta lineata Say, Am.. Marine Conch. 146.

Rana crassa Humph., Mal. Bl., гxir, 23.

Ranella (see Euplenra. Triton, Biplex, Lampas, Apollon; Gyrinea, Aspella, Bufo).

Ranella anceps Chenu., Mal., Bl., xxir, 25.

Ranella anceps Kien., Mal. Bl., xxiv, 25.

Runella anceps Lam., Mal. Bl., xxir, 24.

Ranella anceps Lam., Poulsen. 9.

Ranella caudata Sas, Am. Marine Conch. 20.

Ranella caudata Say, var., Arango, Fauna Mal. Cuba, 1878. 214. Ca.

Ranella corruga Perrs, Mal. Bl., xxir, 24.

Ranella corrugata Perry, Poulsen. 9.

Ranella crassa Desh., Beau. 8. G.

Ranella crassa Desh., Krebs. 25.

Ranella crassa Dillw., Jabrb., iii, 332.

Ranella crassa D'Orb., Mal. Bl., xxir, 23. G.

Ranella crassa Rve., Moll. Cuba, ii, 164.

Rauella cubaniaua D’Orb., Alango, Fama Mal. Cuba, 1878. 215. Ca. Harana. Santa Lucia. Ba.

Ranella cubaniaua D'Orb., Beau. 8. G.

Ranella cukaniana D’Orb., Jahrb., iii, 328.

Ranella cubaniana D'Orb., Krebs. 25.

Ranella cubaniana D'Orb., Mal. Bl., xxir, 24.

Ranella cubaniana D'Orb., Noll. Cuba, ii, pl. xxiii, fig. 24. 165.

Ranella cubaniana D'Orb., Poulsen. 9.

Ranella granifera Lam., Mal. Bl., xxiv, 24.

Ranella granulata Lam., Beau. S. G.

Rauella granulata Lam., Jahrb., iii, 332. 
Ranella granulata Lam., Krebs. 25.

Rawella granulata Lam., Mal. Bl., xxiv, 23. G.

Ranella granulata Lam., Moll. Cuba, ii, 164.

Ranclla grayaua Dkr., Arango, Fauna Mal. Cuba, 1878. 215. Ca. Havana.

Rane la lanceolata Mke., Mal. Bl., xxir, 25.

Ranella livida Rve., Krebs. 25.

Ranella ponderosa Rve., Arango, Fauna Mal. Cuba, 1878. 215. Ca. Baracoa.

Ranella ponderosa Rve., Krebs. 25. SCx.

Rauella ponderosa Rve., Mal. Bl., xxiv, 24.

Ramella ponderosa Rve., Zt. Mal., x, 78. SV.

Ranella rhodostoma Beck, Krebs. 25.

Ranella rhodostoma Beck, Mal. Bl., xxis, 24.

Ramella semigranosa Lam., Mal. Bl., xxir, 24.

Ravella spadicea Mtf., Mal. Bl., xxiv, 23.

Ranella tampaensis Con., Am. Jonr. Conch., v, 106. Fla.

Ranella thome D’Orb., Arango, Fauna Mal. Cuba, 1878. 215. Ca. Havana. ST.

Ranella thomæ D'Orb., Beau. S. G.

Rawella thomæ D'Orb., Jalırb., iii, 327.

Ranella thomx D'Orb., Krebs. 25.

Rameila thoma WOrb., Moll. Cuba, ii, pl. xxiii, fig. 23. 164. Ca.

Ravella thomæ D'Orb., Poulsen. 9.

Ranella thoma D'Orb., Mal. Bl., xxir, 24. Ca.

Ranella vexillum Cum., Mil. Bl., xxiv, 32.

Raugia (sce Gnathodou).

Rangia clathrodonta Con. Jour. de Conch., ix, 212.

Rangia cyrenoides Desm., Jour. de Conch., i, 73. Fla.

Raugia cyrenoides Desm., Jour. de Conch., iv, 164. La.

Rangia cyrenoides Desm., Jour. de Conch., ix, 212.

Rangia flexuosa Con., Jour. de Conch., ix, 212.

Rangia lecontii Con., Jour. de Conch., ix, 212.

Rangia mendiea Gould, Jour. de Conch., ix, 212.

Rangia minor Con., Jour. de Conch., ix, 212.

Rangia rostrata Con., Jour. de Conch., ix, 212.

Retusa (see Bulla).

Retusa omphalis Hörch, * Mal. Bl., xxii, 172. ST.

Retusa sulcata D'Orb., Mal. Bl., xxii, 172. G.

Retusa sulcata D'Orb., Poulsen. 8.

Rhacodoris krebsii, Mörch, Jahrb., vi,44. ST.

Rhacodoris krebsii Mörch, Jour. de Conch., xi, 34. ST.

Rhacodoris krebsii Möreh, Mal. Bl., xxii, 177. ST. SCx.

Rhizochilus (see Coralliophila).

Rhizochilus (Coralliophila) abbreviatus Kien., Marrat, Argo Exp., 1876.

6. Aa. 
Rhomboides nodosus Petiv., Mal. Bl., xxir, 40.

Rihoubus isee niscia).

Rhombus dentatus List., Moll. Cuba, ii, 130.

Rhumbas parvus List., Moll. Cuba, ii, 106.

Ricinula ferrugiuosa Rve., Araugo, Fauna Mal. Cuba, 1878. 203. Ca. Ba.

Ricinula ferrugiuosa Rve., Krebs. 27.

Riciuula ferruginosa Rve., Krebs' Remarks. 397.

Ricinula lugubris Adams, Gupps, Paria Fauna, 1877. 144.

Ricinula nodulosa Ad., Arango, Fauna Mal. Cuba, 187S. 203. Ca. IIarana. G. Ba.

Riciuula nodulosa C. B. Adams, Beau. 9. G.

Riciuula nodulosa Ads., Jour. de Conch., iv, 418. G.

Riciuula noclulosa Ad. bis, Frelss. 27. Agr. ST. SJ. SII. SB. NGr.

Ricimula nodulosa C. B. Aclams, Krebs' Remarks. 397.

Ricinula rosea Rre., Krebs. 27. ST.

Ricinula tubereulata Blainv, Jahrb., ii, 242. Bz.

Riciunla turbiuata Kieu., Beau. 9. G.

Riciunla turbiuata Kieu., Jour. de Conch., iv, 418. G.

Ricinula turbinella Kien., Arango, Fauna Mal. Cuba, 1878. 203. J. Ba. Bb. G.

Ricinula turbinella Kien., Krebs. 27. ST. SB. Ag. SM.

Rimula (see Cranopsis).

Rimula asturiaua Fisch., Jour. de Conch., xxx, 277, 278. ST.

Riugicula nitida Verrill, Report Blake Moll. 97. CS.

Ringicula peracuta Matson, Lin. Soc. Jomrn., 1853, xvii, 292. Cul.

Ringl(oula semistriata 1)Orb., Arango, Fanna Mal. Cuba, 1878. 191. J. Ringicula semistriata D'Orb.,* Jour, de Conch, xxvi, 127. J.

Ringiculat semi-striata I'Orb., Mholl. Cuba, ii, pl. xxi, figs. 17-19. 103. J. Ringicula semistriata D'Orb., Report Blake Moll. 97. Ca.

Ringicula trislentata Guppy, n. s., Guply, Geol. Mag., 1874. 436.

Riparia isogoalata Mensch. (Meliua), Krebs. 132.

Rissoa (see Ciugula, Alvania, Littorinella).

Rissoa (see lisisoina), Alango, Fanna, Mal. Cuba, 1875. 168. Ca. J. Rissoa aberrans C. B. Adams, Contr. to Conch. 113. J.

Rissoa aberrans C. B. Adams, Krebs. 53.

Rissoa aberrans C. B. Adams, Mal. Bl., xxiii, 53.

Rissoa aberians C. B. Adams, Mal. Bl., xxiii, 120. J.

Rissoa attinis C. B. Adlaus, Krebs. 53.

Rissoa attinis C. B. Adaus, Synops., 6, 1845. J.

Tissoa alata Mlke., Mrango, Fauna Mal. Cuba, 1S78. 16S. Ca. Panama.

Antilles.

Rissoa alata Mke., Jour. de Conch., ix, 308.

Rissoa alata Mke., Mal. Bl., xxiii, 47.

Rissoa albida Ad., Krebs. 53.

Rissoa albida Ad., Krebs. 55. 
Rissoa albida C. B. Adams, Srnops, 6, 1S45. J.

Rissoa auberiana D'Orb. Arango, Fauna Mal. Cuba, 1878. 166. Ca. J. S'T.

Rissoa auberiana D'Orb., Krebs. 53. S'T.

Rissoa auberiana D'Orb., Mal. Bl., xxiii. 54. Ca. J.

Rissoa auberiana D’Orb., Moll. Cuba, ii, pl. xi, figs 34-36. 22. Ca. J. Rissoa auberiana D'Orb., Poulsen. S.

Rissoa canaliculata Ad., Krebs. 53.

Rissoa cancellata Phil., Krebs. 55.

Rissoa candida Brown, Arango, Fauna Mal. Cuba, 1878. 167. Ca.

G. Antilles.

Rissoa caribæa D'Orb., Arango, Fauna Mal. Cuba, 1878. 167.

Rissoa caribaea D'Orb., Krebs. 53.

Rissoa caribrea D'Urb., Mal. Bl., xxiii, 54. Ca.

Rissoa caribea D'Orb., Moll. Cuba, ii, pl. xi, figs. 31-33. 21. WI.

Rissoa caribea D'Orb., Poulsen. S.

Rissoa carinata Recl., Krebs. 53.

Rissoa chesnelii Mich., Krebs. 53.

Rissoa chesnelii Mich., Moll. Cuba, ii, 24.

Rissoa concinna Ad., Krebs. 53.

Rissoa conica Ad.; Krebs. 53.

Rissoal costulina Sismonta, Arango, Fauna Mal. Cuba, 1878. 168. Ca.

Panama. Antilles.

Rissoa costulina Sismouda, Jour. de Conch., ix, 308.

Rissoa crassicosta C. B. Adams, Krebs. 53.

Ríssoa crassicosta C. B. Adams, Mal. Bl., xxii, 153. G.

Rissoa crassicosta C. B. Adams, Syuops. . 6, 1845. J.

Rissoa crassicosta C. B. Adams, Alango, Fauni Mar. Cuba, 1878. 167. Ca.

Rissoa cretacea Stimpson, n. s., Kurtz. 7. SC.

Rissoa dubiosa C. B. Adams, Contr. to Conch., 114. J.

Rissoa dubiosa C. B. Adams, Krebs. 54.

Rissoa dunkeri P'r., Krebs. 57.

Rissoa dunkeri Pfr., Mal. Bl., xxiii, 49. ST.

Rissoa duplicata D'Orb., Mal. Bl., xxiii, 48.

Rissoa emaciata Mörch, Mal. B1. xxiii, 54. ST.

Rissoa emaciata Mörch, Poulsen. S.

Rissoa eulimoides C. B. Adams, Contr. to Conch. 115. J.

Rissoa eulimoides C. B. Adams, Krebs. 54.

Rissoa fragilis Mich., Marrat, Argo Exp., 1876. 8. Barbuda.

Rissoa gemmulosa Ad., Krebs. 54.

Rissoat gradata DOrb., Arango, Fama Mal. Cuba, 1S78. 167. J.

Rissoa gradata D'Orb., Krebs. 54.

Rissoa gradata D’Orb., Mal. B1., xxiii, 53. J.

Rissoa gradata D'Orb., Moll. Cuba, ii, pl. xi, figs. 37-39. 23.

Rissoa gradata D'Orb., Poulsen. 8. 
Rissoa grarlatula Mö̀ch, Mal. Bl., xxiii, 53. ST.

Rissoa gradatula Mörch, Poulsen. S.

Rissoa incompta Gld., Am. Marine Conch. 85. Fla.

Rissoa iucompta Gld., Otia. 238. Fla.

Rissoa jaums C. B. Adams, Araugo, Fauna Mal. Cuba, 1878. 168. Ca.

Pauama. Antilles.

Rissoa janus C. B. Adams, Jour. de Conch., ix, 308. J.

Rissoa lactea Brown, Arango, Famna Mal. Cuba, 1878. 167. Ca.

Rissoa lavigata C. B. Adams, Contr, to Conch. 114. J.

Rissoa lævigata Arl., Krebs. 54.

Rissoa lævigata C. B. Adams, Mal. B1., xxii, 46. J.

Rissoa laerissima C. B. Arlans, Alango, Fauna Mal. Cuba, 1878. 167.

Hy. M. ST.

Rissoa lævissima C. B. Adams, C'ontr. to Conch. 115̃. J.

Rissoa lavissima Ad., Krebs. 51.

Rissoa lævissima C. B. Adams, Mal. Bl., xxiii, 46. Ca.

Rissoa melanura C. B. Aclams, Alango, Fauna Mal. Cubat, 1878. 168. J.

Rissoa melanura C. B. Adams, Contr. to Conch. 116. J.

Rissoa melaunra C. B. Alams, Krebs. 54. G. Tr. ST. SMI. SCx.

Rissoa melanura C. 13. Adams, Krebs. 56.

Rissoa melanura C. B. Adams, Krebs' Remarks. 397.

Rissoa melanura C. B. Adams, Mal. I3l., xxiii, 57. J.

Rissoa minor O. B. Adams, Krebs' Remarks. 397.

Rissoa modesta Stimpson, Kurtz. 7. SO.

Rissoa mortoni Kurtz, n. s., Kurtz. 7. SC.

Rissoa multicostata C. B. Adams, Contr. to Conch. 114. J.

Rissoa multicostata Ad., Krebs. 55.

Rissoa multicostata C. B. Adams, Krebs' Remarks. 397.

Rissoa ovuloides Ad., Krebs. 55.

Rissoa patens Gld., Am. Marime Conch. S5.

Rissoa patens Gld., Otia. 238. SC.

Rissoa philippiana Pfr., Jour. đe Conch., ix, 309. Ca.J.

Rissoa philippiana Pfr., Mal. Bl., xxiii, 52.

Rissoa princeps C. B. Adams, Arango, Famma Vial. Cuba, 18б8. 169.

Ca. Ba.

Rissoa princeps C. B. Adams, Contr. to Conch. 116. J.

Rissoa princeps C. B. Adams, Krebs. 55.

Rissoa pulchra C. B. Adams, Arango, Famua Mal. Cuba, 1s7s. 167.

Ca. J.

Rissoa pulchra C. B. Adams, Contr. to Conch. 114. J.

Rissoa pulchra C. B. Adams. Jour. de Conch., ix, 309. Ca. J.

Rissoa pulchra C. B. Adlams, Krebs. 55. SB.

Rissoa pulchra C. B. Adams, Krebs. 58.

Rissoa pulchra C. B. Adlams, Mal. Bl., xxiii, 52. J.

Rissoa pupoidea Kurt\% \& stimpnon, Proe Iost. Sor. Nat. Iist, 1S51, iv, 114. $\mathrm{SC}$. 
Rissoa pusilla Desh., Krebs. 55.

Rissoa pyramidella Brown, Arango, Fauna Mal. Cuba, 1S7S. 168. Ca.

Panama. Antilles.

Rissoa pyramidella Brown, Jour. de Conch., ix, 30 .

Rissoa pyramidella Brown, Mal. Bl., sxißi, 47.

Rissoa scalarella C. B. Adams, Arango, Fauna Mal. Cuba, 1:-7S. 167.

Ca. G. Antilles.

Rissoa sealarella C. B. Adams, Coutr. to Couch. 113. J.

Rissoa scalarella C. B. Adams, Krebs. 56.

Rissoa scalarella C. B. Adams, Krebs' Remarlis. 397.

Rissoa scalarella C. B. Adams, Mal. Bl., xxiii, 49. ST.

Rissma scalarella C. B. Adams, Syuops., 6, 1S45. J.

Rissoa scalaris Mich., Mal. Bl., iii, 119. Ca.

Rissoa sealaris Mich., Mal. Bl., ix, 129. Ca.

Rissoa scalaris Mich., Zt. Mal., iii, 18s. Ca. J.

Rissoa scalaroides C. B. Adams, Contr. to Conch. $113 . \mathrm{J}$.

Rissoa scalaroides C. B. Adauns, Krebs. 56, 57.

Rissoa scalaroides C. B. Adams, Krebs. 58.

Rissoa scalaroides O. B. Adams, Krebs' Remarks. 397.

Rissoa sealaroides (minor), C. B. Adams, Krebs. 56, 57.

Rissoa solida C. B. Adams, Krebs. 55.

Rissoa striato-costata DOHb., Arango, Fauna Mal. Cuba, 18\%s. 165.

Ca. Panama. Antilles.

Rissoa striatula Andr\%, Arango, Famna Mal. Cuba, 187s. 168. Ca.

Panama. Antilles.

Rissoa striatula Andrz., Jour. de Conch., ix, 305.

Rissoa striosa C. B. Allams, Araugo, Fanna Mal. Cuba, 1878. 168. Ca.

Panama. Antilles.

Rissoa striosa C. B. Adams, Arango, Famna Mal. Cuba, 1878. 169. Ca. J.

Rissoa striosa C. B. Adams, Contr. to Conch. 116. J.

Rissoa striosa C. B. Adams, Jour. de Conch., is, 308. J.

Rissoa striosa O. B. Adams, Krebs. 56.

Rissoa striosa C. B. Adlams, Mal. Bl., xxiii, 47.

Rissoa subangulata C. B. Arlams, Contr. to Conch. 112. J.

Rissoa subangulata C. B. Arlams, Krebs. 56.

Rissoa subcochlearella DOHb., Mrango, Fauma Mal. Cnba, 187s. 168.

Ca. Panama. Antilles.

Rissoa terraricosa C. B. Adams, Arango, Fauna Nal. Cuba, $18 ; 3$. 168. J.

Rissoa tervaricosa C. B. Adams, Krebs' Remarks. 397.

Rissoa tervaricosa C. B. A., Mal. Bl., xxiii, 5.7. J.

Rissoa tervaricosa C. B. Allams, Synops., 6, 1845. J.

Rissoa tervaricosa C. B. Adams, Krebs. 56.

Rissoa truncata Phil., Moll. Cuba, ii, 6.

Rissoa turricula H. C. Lea, Am. Marine Conch. S3. SC. 
Rissoa umbiliçata Phil., Krebs. 56.

Rissoa umbilicata P'hil., Mal. Bl., xxiii, 56.

Rissoa vitrea U. B. Adams, Contr. to Conch. 115. J.

Rissoa ritrea C. B. Adams, Krebs. 56.

Rissoa ritrea C. B. Adams, Mal. Bl., xxiii, 45. J.

Rissoina (Wee Phomella, Zebina, Zebinella, Rissoa, Scala).

Rissoina aftinis C. B. Adame, Jour. de Conch., ix, 310.

Rissoina affinis C. B. Adams., Mal. Bl., xxiii, 4S. J.

Rissoina albida C. B. Adams, Jour. de Conch., ix, 310.

Rissoina albida C. B. Arlams, Krebs. 5i.

Rissoina albida C. B. Adams, Mal. Bl., xxiii, 4S. J.

Rissoina albida C. B. Adams, Ponlsen. S.

Rissoina albida Schwartz, Mal. B1., xxiii, 48. J.

Rissoina bicollaris Schwartz, Jour. de Conch., ix, 309.

Rissoina bicollaris Schwartz, Mal. Bl., xxiii, 52. Ca.

lissoina browniana D'(O)b., Arango, Famna Mal. Cuba, 18is. 167.

Пу. M. ST.

Rissoina browniana D'Orb., Jour. de Conch., ix, 310.

Rissoina browniana D'Orb., Krelıs. 57. ST. SJ.

Rissoina browniana D'Orb., Nal. Bl., xxiii, 46. Ca.

Jissoina bromuiana D'Orb., Moll. Cuba, ii, pl. xii, figs. 33-35. 28.

Rissoina browniana D'Orb., Poulsen. 8.

Rissoina browniana Schwartz, Nal. Bl., xxiii, 46. Ca.

Rissoina brugierei Payr., Jour. de Conch., ix, 305. ST.

Rissoina bryerea Mtg., Arango, Famna Mal. Cuba, 156s. 167. Ca.

lissoina bryerea Mtg., Jour. de Conch., ix, 30s. Ca.

Rissoiua bryerea Mtg., Mal. Bl., xxiii, 49. ST.

Rissoina bryerea Mtg., Poulsen. $\therefore$.

Rissoina bryerea Schwartz, Mal. Bl, xxiii, 49. ST.

Rissoina bryerii Mtg., Marrat, Argo Exp., 1876. 16. Nassau.

Rissoina cancellata Phil., Arango, Fanna Mal. Cuba, 187s. 167. Ca.J.

Rissoina cancellata Phil., Jour. de Conch., ix, 309. Ca. J.

Rissoina cancellata Phil., Krebs. 57.

Rissoiua cancellata Phil. bis, Mal. Bl., xxiii, 52. Ca.

Rissoina cancellata Phil., Poulsen. 8.

Rissoina cancellata Phil., Z Zt. Mal., ir, 127. Ca.

Rissoiua caucellata Schwartz, Mal. B1., xxiii, 52.

Rissoina cateshya D’Orb., Krebs. 57. PPl. ST. SCx. SJ. SM.

Rissoina catesbya D'Orb., Krebs. 58.

Rissoina catesbya D'Orb., Krebs' Remarks. 397.

Rissoina catesbyana D`Orb., Alango, Famma Mal. Cuba, 1878. 167. Ua.

G. Antilles.

Rissoina catesbyana D'Orb., Arango, Fanna Mal. Cuba, 1878. 169.

Rissoiua cat esbyaua D'Orh., Beau. 16. G.

Rissoiua catesbyana D'Urb., Jomr, de Conch., r, 152. G.

Rissoina catesbyana D'Orb., Mal. Bl., xxiii, 48, 49. S'T, 
Rissoina catesbyana D’Orb., Moll., Cuba, ii, pl. xii, tigs. 1-3. 24.

Rissoina catesbyana D'Orb., Poulseu. 8.

Rissoina chesneli Michaud, Arango, Fanua Mal. Cuba, 1S7s. 167. Ca. G. Autilles.

Rissoina chesneli Mich., Dall, Hemphill's shells. 334. Cedar Keys.

Rissoina chesneli Mich., Jour. de Conch., ix, 308.

Rissoina chesneli Mörch, Mal. Bl., xxiii, 49. ST.

Rissoina decussata Mtg., Arango, Fauna Mal. Cuba, 18is. 16s. Ca.

Panama. Antilles.

Rissoina decussata MItg., Jour. de Conch., ix, 308.

Rissoina decussata Mtg., Krebs. 57.

Rissoina decussata MIg., Mal. Bl., xxiii, 47. Ca. J.

Rissoina decussata Mtg., Poulsen. S.

Rissoina decussata Schwartz, Mal. Bl., xxiii, 47.

Rissoina dubiosa C. B. Adams, Arango, Fauna Mal. Cuba, 1878. 168. Ca. J.

Rissoina dubiosa C. B. Adams, Dall, Hemplill's shells. 334. Key West.

Rissoina dubiosa C. B. Adams, Jour. de Conch., ix, 308. Ca. J.

Rissoina dubiosa U. B. Adams, Krebs. 57.

Rissoina dubiosa C. B. Adams, * Mal. Bl., xxiii, 49. J.

Rissoina rlubiosa C. B. Adams, bis, Mal. Bl., xxiii, 50. J.

Rissoina dunkeri Pfr., Arango, Fanna Mal. Cuba, 187s. 168. Ca. G. Antilles.

Rissoina dunkeri Pfr., Mal. Bl., xxiii, 49, 50. S'T.

Rissoina elegantissima D`Orb., Arango, Fama Mal. Cuba, 1878. 168.

Hy.

Rissoina elegantissima D'Orb., Jour. de Conch., ix, 306. J.

Rissoina elegantissina D'Orb., Krebs. 5 s.

Rissoina elegantissima D’Orb., Krebs. 57. SM. Ag.

Rissoina elegańtissima D’Orb., Mal. Bl., xxiii, 50. J.

Rissoina elegantissima D’Orb., Mal. Bl., xxii, 51. Ca.

Rissoina elegantissima D'Orb., Moll. Cuba, ii, pl. xii, figs. 27-29. 26, 27.

Rissoina elegantissima D'Orb., Poulsen. 8.

Rissoina fenestrata Schmartz, Jour. de Conch., ix, 309. Ca.

Rissoina fenestrata Schwart\%, Arango, Fanna Mal. Cuba, 187S. 168. Ca.

Rissoina fenestrata Schwartz,* Mal. B1., xxiii, 52. Ca.

Rissoina firmata C. B. Adams, Mal. Bl., xxiii, 50. Ca.

Rissoina firmata C. B. Adams, Poulsen. 8.

Rissoina floridana Mörch, Mal. Bl., xxiii, 49. Fla.

Rissoina incerta D'Orb., Mrango, Fauna Mal. Cuba, 1878. 168. J.

Rissoina krebsii Mörch, Mal. Bl., xxiii, 50. Ca.

Rissoiua krebsii Mörch, Poulseu. 8.

Rissoina labiosa Schwartz, Arango, Fauna Mal. Cuba, 1878. 169. Ca,

Rissoina labrosa Schwartz, Jour. de Conch., ix, 309. Ca. 
Rissoina labrosa Schwartz, Mal. Bl., xxii, 51. Ca.

Rissoina lrevigata C. B. Adams, Jour. de Conch., ix, 310. J.

Rissoina lavigata O. B. Adams, Krebs. 54.

Rissoina lævigata C. B. Adams, Mal. Bl., xxiii, 45. J.

Rissoina laevigata C. B. Arlams, P'oulsen. S.

Rissoina lavigata Schwartz, Mal. Bl., xxiii, 46. J.

Rissoina lavissima C. B. Adams, Jour. de Conch., ix, 310.

Rissoina lævissima C. B. Adams, Krebs. う̌t.

Rissoina micans C. B. Adams, Moll. of Bermudas. 19.

Rissoina minor U. B. Adans, Arango, launa Mal. Cuba, 1878. 168.

Ca. G. Antilles.

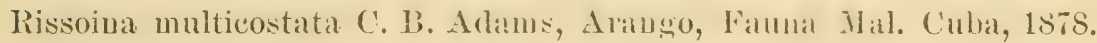
169. Ca. G.

Rissoina multicostata C. B. Adams, Jour. de Conch., ix, 309.

Rissoina multicostata C. B. Adams, Mal. B1., xxii, כ0. J. Cil.

Rissoina multicostata C. B. Adams, Ponlsen. S.

Rissoina philippiana Pfr., Arango, Famna Mal. Cuba, 187s. 167. Ca. J.

Rissoma philippiana Pfṛ., Krebs. 50ّ.

Rissoina princeps C. B. Adams, Mal. Bl., xxiii, 47. J. Ca.

Rissoina princeps C. B. Arlams, Poulsen. S.

Rissoina privati Folin, Mal. Bl., xxiii, 5̃2.

Rissoma pulchra C. B. Adams, Tall, Hemplaills shells. 3i3t. Key West.

Rissoina pulchra C. B. Adams, Krebs. 55̃. SB.

Rissoina pulchra C. B. Adans, Mal. Bl., sxiii, 51. J.

Rissoiua pulchra C. B. Adams, Poulseu. S.

Rissoina reticulata Sby., Arango, Fanna Mal. Cuba, 1878. 169. Ca. Ba.

Rissoina reticulata Sby., Jour. de Conch., ix, 308. Ca.

Rissoina reticulata Sby:, Krebs. js.

Rissoina reticulata Sby. Mal. Bl, xxiii, 47. J. Ca.

Rissoina sagra D'Or!)., Arango, Fama Mal. Cuba, 1878. 169.

Rissoina sagra D'Urb., Moll. Cuba, pl. xii, figs. 4-5. (Not on page 25.) 11. \$ $\mathrm{T}$.

Rissoina sagraiaua D'Orb., Alango, Fauna Mal, Cuba, 1878.169.

Riśsoina sagraiana D'urb., Jour. de Conch., ix, 309.

Rissoina sagraliaua D’Orb., Krebs. 55.

Rissoina sagraiana $\mathrm{D}^{9} \mathrm{O}$ b. , Krebs. 58.

Rissoina saegraiana DOOrb, Mal. Bl, xxiii, 51. ST. M.

Rissoina sagraiana W'Orb., Moll. Cuba, ii, 25. (Not on plate xii).

Rissoina sagraiana D'Orb., I'oulsen. S.

Rissoina scalarella Ad., Krebs. 56.

Rissoina scalarella Ad., Krebs. 57.

Rissoina scalariformis Ad., Krebs. 58.

Bull. 24-17 
Rissoina scalaroides C. B. Adams, Aringo, Fauna Mal. Cuba, 1878. 167. Ca.

Rissoina scalaroides C. B. Adams, Arango, Fauna Mal. Cuba, 1878. 168.

Ca. G. Antilles.

Rissoina scalaroides C. B. Adlams, Krebs. 56.

Rissoina scalaroides C. B. Adams, Krebs. 58.

Rissoina scalarioides C. B. Adams, Mal. Bl., xxiii, 49. ST.

Rissoina scalarioides C. B. Adams, * Zt. Mal., v, 13. Ca. J.

Rissoina semiglabrata Ad., Arango, Fauna Mal. Cuba, 1878. 169. Ca. Rissoina semiglabrata Ad., Jour. de Conch., ix, 310. Ca.

Rissoina signifer Mörch,* Mal. Bl., xxiii, 48. NP.

Rissoina signifer Mörch, Poulsen. 8.

Rissoina sloaniana D'Orb., Krebs. 58.

Rissoina sloaniana D'Orb., Arango, Fama Mal. Cuba, 1878. 169. J. Rissoina sloaniana D'Orb., Jour. de Conch., ix, 310.

Rissoina sloaniana D'Orb., Mal. Bl., xxiii, 46. J.

Rissoiua sloaniana D'Orb., Moll. Cuba, ii, 28, 29.

Rissoina sloaniana D'Orb., Ponlsen. 8.

Rissoina sloaniana Schwartz, Mal. Bl., xxiii, 46. J.

Rissoina striato-costata D'Orb., Jour. de Conch., ix, 308.

Rissoina striato-costata D'Orb., Krebs. 58. SB. ST. PPl.

Rissoina striato costata D'Orb., Mal. Bl., xxiii, 47.

Rissoina striato-costata Dorh., Moll. Cuba, ii, pl. xii, figs. 30-32. 27.

Rissoina subangulata C. B. Adams, Jour. de Conch., ix, 307. J.

Rissoina subangulata C. B. Adams, Mal. Bl., xxiii, 49. J.

Rissoina subangulata C. B. Adams, Poulsen. 8.

Rissoina subcochlearella D'Urb., Jour. de Conch., ix, 308.

Rissoina tæeniata Mörch, Mal. Bl., xxiii, 49. Fla.

Rissoina vitrea C. B. Adams, Jomr. de Conch., ix, 311. J.

Rissoina vitrea C. B. Adams, Mal. B1., xxiii, 45. ST. J.

Rissoina vitrea C. B. Adams, Poulsen. S.

Rissoina vitrinella Mörch, Mal. Bl, xxiii, 45. ST.

Rissoina vitrinella Möreh, Ponlsen. 8.

Rocellaria (seè Gastrochæna, Spengleria).

Rocellaria cuneiformis Spengl., Arango, Fauna Mal. Cuba, 187S. 237. ('a. G.

Rocellaria ovata Sly., Am. Marine Conch. 128. SC.

Rocellaria rostrata Spengl., Krebs. 115.

Rocellaria stimpsonii Tryon, Am. Marine Conch. 128. NC.

Rotella (see Globulus, Ethalia, Psendorotella, Umbonium).

Rotella anomala D'Orb., Alango, Fauna Mal. Cuba, 1878. 177. ST.

Rotella anomala D'Orh., Moll. C'uba, ii, 11. xviii, figs. 32-34. 63.

Rotella cariuata D'Orb., Arango, Fauna Mal. Cuba, 1S7s. 177. ST.

Rotella carinata D'Orb., Moll. Cuba, ii, pl. xviii, tigs. 26-’3. 61.

Rotella cryptospira Verrill, List, 18st. 270. Capo Hatteras. 
Rotella diaphana Dorb., Arango, Famua Mal. Cuba, 1sis. 177. Ca. S'T.

Rotella diaphana D'Orb., Moll. Cuba, ii, pl. xviii, tigs. 23-25. 61.

Rotella parva Stimpson, n. s., Kurtz. S. NC. SC.

Rotella pusilla Pfeiffer, Archiv fïr naturg., 1840. 255. Ca.

Rotella semistriata Dơrb., Arango, Fama Mal. ('uba, 1sis. 177. Ca. S'l.

Rotella semistriata D’Orb., Moll. Cuba, ii, pl. xviri, figs. 20-22. 61, 62.

Rotellat striata D'Orb., Alango, Fanna Mal. Cuba, 1853. 17\%. Ca. S'T. J.

Rotella striata D'Orb., Moll. Cuba, ii, pl. xriii, figs. 20-31. 62, 64.

Ruma (see Natica).

Ruma mamillaris Born, Marrat, Argo Exp., 1576. 15. VCz. Long Key Island.

Sandalium (see Crepidula, Crypta).

Sanclalium aculeatum Schum., Mal. Bl., xxiv, 102. Fla.

Sanguinolaria fusca Cou., Kurtz. 4. NO. SC.

Sanguinolaria lusoria Say, Kurtz. 4. NC. SC.

Sanguinolaria nivea Chem., Krebs. 104.

Sanguinolaria nivea Chem., Mal. Bl., x, 230.

Sanguinolaria nirea Chem., Ps. rosea Lam., Poulsen. 14.

Sanguinolaria rosea Lam., Beau. 24. $11 \mathrm{~g}$.

Sanguinolaria rosea Lam., Krels. 104. ST. SCx. Tort. Td. NGr.

Sanguinolaria rosea Lam., Moll. Cuba, ii, 246.

Sanguiuolaria rugosa Lam., Arango, Fanna Mal. Cuba, 187s. 241. Ca.

Sanguinolaria rugosa Lam., Jour. de Conch., ii, 424. G.

Sanguinolaria rugosa Lam., Jour. do Conch., vii, 135.

Sanguinolaria rugosa Blv., Jour. de Conch, vii, 140.

Sanguinolaria rugosa Blv., Krebs. 105.

Sanguinolaria rugosa Lam., Moll. Cuba, ii, 258.

Saxicava arctica Desh., Kurtz. 4. NC. SC.

Saxicara arctica Linn., Am. Mariue Couch. 137.

Saxicava azaria Dall, 1. s., Report Blake Moll. 116.

Saxicara distorta Say, Kurtz. 4.

Saxicava fragilis Holm., 11. s., Post-Pleiocene Fossils S. C., pl. viii, fig. 18. 57.

Saxicava minuta Guild., Krebs. 107. ST.

Saxicava rugosa Linn., Jalurb., ii, 248.

Sajella (sec Leuconia, Melampus).

Sayella crosseana Dall, J'roc. U. S. Nat. Mus. 1855, pl. xviii, fig. 10. 286. Fla.

Sayella hemphillii Dall, Proe. U. S. Nat. Mus. 1Ss,j, pl. rviii, fig. 11. 286. Fla.

Scabricola (see Mitra) MIarrat, Argo Exp., 1S76. 15.

Scala (see Scalaria, Clathrus, Cirsotrema).

Scala xospila Mörch, Jour. de Conch., xxvi, 394. 
Scala seospila Möreh,* Mal. Bl., xxii, 151. SCx.

Scala reospila Mï̈reh, Poulsen. 7.

Scala albida D'Orb., Poulsen. 7.

Scala blandii Möreh, Mal. Bl., xxii, 145. S'1.

Scala candeana D'Orb., Mal. Bl., xxii, 150. Ca.

Scala candeana D'Orb., Poulsen. 7.

Scala centiquadra Mörch, Jour de Conch., xxvi, 393.

Scala centiquadra M[örch, MIal: BI., xxiị, 145̃. ST. et al.

Scala centiquadra Möreh, S. denticulata, Sby., Poulsen. 7.

Scala (Clathrus) clathrus Limu., Marrat, Argo Exp., 1876. 13. Santa

Marta. Nassan. Abaco.

Scala cochlea Sby., Mal. Bl., xxii, 154. S'. et al.

Scala cochlea Sby., Poulsen. 7.

Seala coronata Lam., Mal. BJ., xxii, 149.

Scala cransicosta Sby., Mal. B1., xxii, 153. G.

Seala crenulata Kieu., Mal. Bl., xxii, 152. ST.

Scala creuulata Kien., Poulsen. 7.

Scala ducalis Möreh, Mal. BI., xxii, 143.

Scala eburnear P. \& M., Mal. Bl., xxii, 149. Ca. et al.

Scala eburnea Pot. \& Mich., Poulsen. 7.

Scala eburnea var. augusta Poulsen. 7.

Scala echinaticosta D'Orb., Mal. Bl., xxii, 145. ST.

Scala echinaticosta var. blandii D'Orb., Poulsen. 7.

Scala erectispina Mörch, Jour. de Conch., xxvi, 393.

Scala erectispiua Möreh, Mal. Bl., xxii, 146. ST.

Scala filaris Möreh, Jomr. de Conch., xxri, 394.

Scala filaris Mörch, Mal. BI., xxii, 152. SM.

Scala filaris Mörch, Poulsen. 7.

Scala foliaceicosta D'Orb., Mial. Bl., xxii, 145. ST. et al.

Scala foliaceicosta D'Orb., Poulsen. 7.

Scala gradatella Mörch, Jour. de Conch., xxri, 393.

Seala gradatella Mörch, Mal. Bl., xxii, 148. ST.

Scala gradatella Möreh, Ponlsen. 7.

Scala hotessieriaua D'Orb., Mal. B1., xxii, 153. G.

Scala hotessieriana D'Orb., Poulseu. 7.

Scala inconspicua Sby., Mal. Bl, xxii, 152. SCx.

Scala incouspicua Sby., Poulsen. $T$.

Seala krebsii Mörch, Jour. đle Conch., xxvi, 393.

Scala krebsii Mörch,* Mal. B1., xxii, 142. ST.

Scala krebsii Mörch, Poulsen. 7.

Seala lamellosa Lam., Mal. Bl., xxii, 149.

Scala lamellosa Lam., S. clathrus, Sby., Poulsen. 7.

Seala mieromphala Möreh, Jour. de Conch., xxvi, 393.

Scala micromphalą Mörch, Mal. Bl, xxii, 147. V.

Scala modesta C. B. Adams, Mal. Bl., xxii, 152. J.

Scala modesta C. B. Adams, Poulsen. 7. 
Scala nantlae Mörch, Jour. de Conch., xxvi, 394.

Scala nautlæ Mörch, Mal. Bl., xxii, 151. VUz.

Seala noremcostata Mörch, Jour. de Conch., xxvi, 393.

Seala noremeostata Mörch,* Ma!. Bl., xxii, 144. ST.

Seala occidentalis Nyst, Mal. Bl., xxii, 146\% ST.

Seala oceidentalis Nyst, S. tenuis Shy., Poulsen. 7 .

Scala octocostata Möreh, Jour. de Conch., xxri, 394.

Scala octocostata Mörch, Mal. Bl., xxii, 14S. ST.

Seala octocostata Möreb, Poulsen. 7.

Seala orbignyi Nyst, Mal. Bl., xxii, 147. ST.

Scala orbignyi Nyst, S. elegans D’Orb. Poulsen. 7.

Scala peruobilis Fiseh. \& Bern., MaI. I31, xxii, 146. G.

Scata pretiosula Mörch, Jonr. de Conch., xxri, 393.

Seala pretiosula Mörch, Mal. B1., xxii, 143. SM.

Seala pretiosula Mörch, Poulseñ. T.

Scala principalis Pallas, Mal. Bl., xxii, 143. Fla.

Seala quindecimeostata Hörch, Jour. de Conch, xxvi, 393.

Scala quindecimcostata Mörch, Mal. Bl., xxii, 147. ST.

Scala scæera hörch, Jour. de Conch., xxri, 394.

Scala screva Mörch, Mal. Bl., xxii, 153. ST.

Scala scera Mörch, l’onlsen. 7.

Scala soluta Dkr., Jour. de Conch., xxvi, 393.

Scala soluta Dkr., Mal. Bl., xxii, 145. ST.

Seala soluta Dkr., Poulsen. 7.

Scala spinæ-rosæ Mörch, Jom, de Couch., xxvi, 393.

Seala spinæ rosæ Möreh, * Mal. Bl., xxii, 145. ST.

Scala spina-rosre Mörch, Poulsen. 7 .

Seala spuria Mörch, Jour, de Conch., xxvi, 393.

Scala spuria Mörch,* Mal. Bl, xxii, 144. Sl. et al.

Seala spuria Mörch, l’oulsen. T.

Scala subraricosa Dkr., dour. de Conch., xxri, 394.

Scala subvaricosa Dki., Mal. Bl., xxii, 153. S'T.

Scala subvaricosa Dkr., Poulsen. 7 .

Scala swiftii Mörch, Jour, fle Conch., xxvi, 393.

Scala swiftii Mörch,* Mal Bl., xxii, 143. ST.

Seala turricula Sby., H1al. B1., xxii, 150. S'T.

Scala turricula Sby., Poulsen. 7.

Seala turitelula Mörch, Jour. de Conch., xxvi, 394.

Scala țuritellula Mörch, Mal. Bl., xxii, 151. SM.

Scala turritellula Mörch, Poulsen. S.

Scala uncinaticosta D'Orb., R'oulser. 7.

Seala undecimeostata Mörch, Jour. de Conch., xxvi, 393.

Scala undecimcostata Mörch, Mal. Bl., xxii, 14S. ST.J.

Seala undecincostata Mörch, I’onlsen. 7.

Scala mifasciata Sby., Mal. Bl., xxii, 148. SMI, et al.

Scala unifasciata Sby., Poulsen. 7. 
Scala renosa Sby., Mal. Bl., xxii, 147. G.

Scala venosa Sby., Poulsen. 7.

Scala volnbilis Mörch, Jour. de Conch., xxri, 393.

Seala rolubilis Mörch,* Mal. Bl., xxii, 146. ST.

Seala rolubilis Mörch, Poulsen. 7.

Sealaria (see Clathrus, Scala, Uirsotrema).

Scalaria aculeata Sby., Mal. Bl., xxii, 141.

Sealaria acuta Pfr., Mal. Bl., xxii, 150.

Scalaria acuta Pfeifler, Archiv fïr naturg., 1840. 256. Ca.

Scalaria albida DOOrb., Arango, Fanna Mal. Cuba, 1878. 166. Ca. G.

Sealaria albida D’Orb., Beau. 12. G.

Scalaria albida D' rb., Jour. de Couch., ir, 417. G.

Scalaria albida D'Orb., Krebs. 63.

Scalaria albida D'Orb., Mal. Bl., xxii, 149. Ca.

Scalaria albida D'Orb., Moll. Cuba, ii, 17.

Scalaria angulata Say, Am. Marine Conch. 77.

Sealari، angulata Say, Dall, Hemplinl's shells. 333. Sarasota Bay.

Scalaria altenuata Helb., Mal. Bl., xxii, 154. SD.

Scalaria candeana D'Orb., Arango, Fauna Mal. Cuba, 1878. 165. Ca. J. ST.

Scalaria candeana D'Orb., Krebs. 63.

Scalaria candeana D'Orb., Moll. Cuba, ii, 20.

Sealaria clathrus Auct., Kurtz. 7. NY. SC.

Scalaria clathrus Lam., Guppy, Geol. Mag., 1874. 445. Td.

Scalaria clathrus Linn., Beau. 12. G.

Scalaria clathrus Linn., Jour. de Conch., ii, 427. G.

Scalaria clathrus Linn., Krebs. 64.

Scalaria clathrus Linn., Mal. BI., xxii, 149.

Scalaria coronata Lam., Beau. 12. G.

Scalaria coronata Lam.. Jour. de Conch., ii, 427. G.

Sealaria coronata Lam., Krebs. 63.

Scalaria coronata Lam., Mal. Bl., xxii, 149.

Scalaria coronata Lam., Mioll. of Bermudas. 18.

Scalaria crenata Linn., Beau. 12. G.

Scalaria crenata Linn., Jour. de Conch., ii, 427. G.

Scalaria crenata Limn., Krebs. 63, 64. ST. SM. Ag.

Scalaria crenata Linn., Krebs' Remarks. 397.

Scalaria crenata Linn., Mal. Bl., xxii, 152. S'T.

Scalaria denticulata Sby., Beau. 12. G.

Scalaria denticulata Sby., Jour de Conch., ii, 428. G.

Scalaria denticulata Sby., Krebs. 64.

Scalaria denticulata Sby., Mal. Bl., xxii, 145.

Scalaria ebünea P. \& MI., Mal. B1., xxii, 149.

Scalaria echinatieosta Dorb., Arango, Fauna Mal. Cuba, 1878. 166. Ca. ST.

Scalaria echinaticosta D'Orb., Krebs. 64. ST. 
Sealaria echinaticosta D'Orb., Moll. Cuba, ii, 15.

Scalaria foliaceicosta D'Orb., Arango, Fama Mal. Cuba, 1S\%s. 166. Ca. G. M. S'T. Ba.

Scalaria foliatceicosta D'Orb., Beau. 12. G.

Scalaria foliaceicosta D'Orb., Jour. de Conch., iv, 417. G.

Scalaria foliaceicosta D'Orb., Krehs. 64. M. G. ST. Ag. PPl.

Scalaria foliaceicosta D'Orb., Mal. Bl., xxii, 144.

Sealaria foliaceicosta D'Orb., Moll. Cuba, ii, 17.

Scalaria fragilis Hanl, Arango, Fanna Mal. Cuba, 1878. 166. Ca. G. Scalaria fragilis Haul., Beau. 12: G.

Scalaria fragilis Haul., Jour. de Conch., iv, 417. G.

Scalaria fragilis Hanl., Krebs. 63.

Scalaria fragilis Hanl., Krebs, 64.

Scalaria fragilis Gray, Mal. Bl., xxii, 149.

Sealaria grossicostata Nyst, Mal. Bl., xxii, 153. G.

Sealaria hotessieriana D'Orb., Arango, Fama Mal. Cuba, 187s, 160. Ca. Scalaria hotessieriana D'Orb., Beau. 12. G.

Scalaria hotessieriana D'Orb., Krebs. 64. SM. PPI.

Scalaria hotessieriana D'Orb., Moll. Cuba, ii, 16.

Scalaria humphreysii Kien., Kurtz. 7. NC. SC.

Scalaria lamellosa Isam., Arango, Fanna Mal. Cuba, 187s. 166. Ca. Antilles.

Scalaria lamellosa Lam., Beau. 12. G.

Scalaria lamellosa var. Lam., Jour. de Conch., ii, 427. G.

Scalaria lamellosa Lam., Krebs. 64. ST. SJ. SM. Ag. G.

Scalaria lamellosa Lam., Moll. Cuba, ii, 15.

Scalaria leptalea Bush, Conn. Ac. vi, 465̃. Hatt.

Scalaria leroyi Guppy, Geol. Mag., 1874. 435. Td.J.

Scaląria ligata C. B. Adams, Contr. to Conch. 67. J.

Scalaria ligata C. B. Adams, Krebs. $6 \pm$.

Scalaria ligata C. B. Adlams, Mal. Bl., xxii, 154.

Sealaria lineata Say, Am. Marine Conch. 76. NC.

Sealaria lineata Say, Beau. 12. G.

Scalaria lineata Say, Dall, Hemphill's shells. 333. Cedar Keys.

Scalaria lineata Say, Jour. de Couch., ii, 427. G.

Scalaria lineata Say, Krebs. 64.

Scalaria martini Wood, Krebs. 65. SM. SB.

Scalaria modesta Ad., Krebs. 65.

Scalaria modesta C. B. Adams, Synops., 1845. 4. J.

Sealaria multistriata Say, Am. Marine Conch. 77.

Scaluria muricata Kur. (? Sbs.), Krebs. 65.

Scalaria pernobilis Fisch. \& Bern., Веau. 12. MG.

Scalaria pernobilis Fisch. \& Bern., Jour. de Conch. r, pi. riii, figs. 2, 3. 293. M. G.

Sealaria pernobilis Fisch. \& Bern., Krebs. 65.

Scalaria pernobilis Fiseh. \& Beru., Mal. Bl., xxii, 146. G. 
Scalaria pseudoscalaris Brocchi, Arango, Fauna Mal. Cuba, 187S. 166. Ca. Antilles.

Scalaria pseudoscalaris D'Orb., Arango, Twma Mal. Cuba, 187o. 166. Ca. Antilles.

Scalaria pseudoscalaris D'Orb., Mal. Bl., xxii, 149. Ca.

Sealaria pseudoscalaris D'Orb., Moll. Cuba, ii, 15.

Scalaria pseudoscalaris Phil., Moll. Cuba, ii, 15.

Scalaria psendoscalaris Risso, Moll, Cuba, ii, 15.

Scalaria rupicola Kurtz, n. s., Kurtz. 7. SC.

Scalaria tenuis Sby., Reau, 12. G.

Scalaria tenuis Sby., Jour. de Conch., ii, 427. G.

Scalaria tenuis Sby., Krebs. 63.

Sealaria tenuis Sby., Krebs. 65. Td. S'T. SM. SJ. SCx. SM.

Scalaria tenuis Sby., Mal. Bl., xxii, 146. ST.

Scalaria teres Bush, Conn. Ac. vi, 465. Hatt.

Scalaria tortilis Watson, Jour. de Conch., xxxi, 79. Danish Antilles.

Scalaria tortilis Watson, Lin. Soc. Journ, 1S83, xvi, 607. Cul.

Scalaria turbinata Con., Am. Marine Conch. 77. NC.

Scalaria turbinata Con., Journ. Arirl. Nat. Sıi. Phil., vii, 263, pl. xx, fig. 26, 1837. Beaufort, NC.

Sealaria turricula Sby., Jahrb., ii, 243.

Scalaria turrita Nyst, Mal. BI., xxii, 150. ST.

Scalaria uncinaticosta D'Orb., Arango, Fauna Mal. Cuba, 187s. 166. K.

Scalaria uncinaticosta D'Orb., Beau. 12. G.

Scalaria uncinaticosta D'Orb., Krebs. 65.

Scalaria uncinaticosta 1'Orb., Moll. Cuba, ii, 19.

Scalaria var. unicolor Kien, Mal. Bl., xxii, 149 .

Scalaria varicosa Lam., Krebs. 65. SCx.

Sealaria varicosa Lam., Mal. Bl., xxii, 154. S'T.

Scalaria venosa Sby., Krebs. 65.

Sealaria webbii D’Orb., Moll. Cuba, ii, 20.

Scaphander (see Bulla).

Scaphander (?) watsoni Dall, n. s., Report Blake Moll. 99.

Scapharca (see Arca).

Scajharea brasiliana Gray, Krebs. 124.

Scapharca chemnitzii Phil., Krebs. 125.

Seapharea compacta Rre., Harrat, Argo Exp., 1siog. 13. Tucacas.

Scapharca mux Sby., Maxrat, Areco Exp., 1876. 13. Tucacas.

Scapharca ovata Rve., Marrat, Argo Exp., 1876. 13. Tueacas.

Scapharca rhombea Born, Jahrb., ii, 253. Ca.

Scintilla eburnea Mörch,* Jour. de Conch., xxir, 373. ST.

Scintilla eburnea Möich, Poulsen. 15.

Sconsia (see Cassis).

Sconsia barbutensis Higgins \& Marmat, Malrat, Argo Exp., 1876. 7. Barbucla.

Sconsia striata Lam., Mal. Bl., xxiv, 41. J. 
Scutellina (sce Aeniæa, Patella, Tectura).

Scutellina antillarum Shuttlew, Poulseu. 14.

Scutigerulus (see Chiton).

Scutigerulus angulatus Meusch., Krebs. 90.

Scyllea edwardsii Verrill, List., 188t. 274. Cape Hatteras.

Seyllea margaritacea Bory St. Vine, Mol. Cuba, i, 110.

Scyllara margaritacea Buse, Arango, Famia Mal. ('uba, 1878. 155.

Ca. Antilles.

Seyllæa margaritacea Bose, MIoll. Cuba, i, 110.

Scyllea margaritacea Roissy, Moll. Cuba, i, 110 .

Segmentina (see Planorbis).

Segmentina allieans Pfr., Arango, Fanna Mal. Cuba, 187s. 137. (a. Segnenzia delicatula Dall, n. s., Report Blake Moll. 48. WI.

Seguenzia formosa Jeffreys, Report Blake Moll. $4 \pi$.

Seguenza formosa Jeffreys, Watson, Lin. Sor. Jonrn., 1879, xir, 5s7.

Cul. Ber.

Seguenzia ionica Watson, Report Blake Noll. 48.

Seguenza ionica Watson, Lin. Soc. Journ., 1879, xir, 589. Cul.

Semele (sce Amphidesma).

$\checkmark$ Semele caucellata D'Urb., Araugo, Fanna Mill. Cuba, 187S. 246. MI.

G. Ba. = heblashade Cone.

Semele cancellata D'Orb., Krebs. 106. ST.

Semele cancellata D'Orb., Poulsen. 14.

Semele deenssata Gray, (inply, Paria Famma, 187т. 148. =

Semele decussata.Wood, Krebs. 106.

Semele formosum Sby., Krebs. 106. tormet-entic Shy is Ps.

Semele lata U. B. Adams, Busb, Conn. Ac. vi, 466. Hatt.

$\checkmark$ Semele nexilis Gld., Am. Marine Conch. 155. = be/n.s/s i-l

* Semele nexilis Gld., Dall, Hemphill's shells. 3iss. Cedar Keys.

Semele nexilis Gld., Otia. 238. Ga.

Semele erbiculata Say, Am. Marine Conch. 154. =

Semele ornata Gld., Am. Marine Conch. 155. = o

-Semele oruata Gld., Otia. 239. Ga.

Semele purpurascens Gimel., Aranga, Famal Mal. Cuba, 1858. 246.

Ca. Ba. = ohlicina "1

$\checkmark$ Semele purpurascens Gmel., Krebs. 106. ST.

$\checkmark$ Semele purpurascens Gmel., Ponlsen. 14.

Semele matiata Say, Am. Harine Coneh. 1.s. =

Semele retienlata Lam., Krebs. 106.

Semele reticulata Linn., Arango, Famna Mal. Cuba, 187s. 247. Ca. G.

II. Ba.

'Semele reticulata Linn., Mal. BI., x, 226.

Semele reticulata Lim., Poulseu. 14.

Semcle reticulata Sby., Jahrb., ii, 249.

Semele subtruncata Sby., Krebs. 106.

Semele variegata Lam., Guppy, Paria Fama, 1876. 149. 
Semicassis (see Cassidea, Cassis, Sconsia).

Semicassis inflata Shaw, Marrat, Argo Exp., 1876. 12. Tucacas.

Semicassis inflatus Shaw, Marrat, Argo Exp., 1876. 15. VCz.

Semicassis recurvirostrum Woor, Arango, Fauna Mal. Cuba, 1878. 223. Ca.

Semicassis rentricosa Mart., Araugo, Fauma Mal. Cuba, 1878. 223. Ca. G. Ba.

Sencetus (see Turbo).

Senectus molticanus Chem.?, Marrat, Argo Exp., 1876. 18. Athol Island.

Sencetus molticanus Chem. ?, Marrat, Argo Exp., 1876. 16. Nassan.

Sepia affinis D’Orb., Alango, Famna Mal. Cuba, 137s. 148. Ca. M.

Sepia affinis Fer., Moll. Cuba, i, 35.

Sepia antillarmm Dơ(Oh., Alango, Fanna Mal. Cuba, 1878. 147. Antilles.

Sepia antillarum D’Orb., Moll. Cuba, i, 33.

Sepia biserialis Bly., Arango, Famma Mal. Cuba, 1878. 148. Ca. M.

Sepia biserialis Blv., Moll. Cuba, i, 35.

Sepia cardioptera Uken, Moll. Cuba, i, 53.

Sepia granulosa Bose, Arango, Fauna Míal. Cuba, 1878. 145. Antilles. M. G. Ca.

Sepia gramulosa Bose, Moll. Cuba, i, 18.

Sepia octopodia Linn., Moll. Cuba, i, 11.

Sepia octopus Gmel., Moll. Cuba, i, 11.

Sepra ofticinalis Lam., Arango, Fanna Mal. Cuba, 1878. 148. Ca. M. Sepia offeinalis Lam., Moll. Cuba, i, 34, 35.

Sepia orbigniana l'ér., Moll. Cuba, i, 33.

Sepia rugosa Bose, Arango, Fatuna Mal. Cuba, 1878. 145. Antilles. M. Gr. Ca.

Sepia rugosa Bose, Moll. Cuba, i, 18.

Sepiola carlioptera Les., Moll. Cuba, i, 53.

Sepioteuthis biangulata Rang, Moll. Cuba, i, 38.

Sepiotentbis biangulata Rang, Arango, Fauna Mal. Cuba, 1S78. 148. Oa. M.

Sepioteuthis biangulata Rang, Mag. de Zool., 1837, pl. 98. M.

Sepioteuthis biaugulata Rang, Moll. Cuba, i, 35 .

Sepioteuthis blainvilliana Fér., Moll. Cuba, i, 37.

Sepiotenthis blainvillianus D’Orb., Arango, Fauna Mal. Cuba, 1878. 148. Ca. M.

Sepioteuthis blainvilliana Fér., Moll. Cuba, i, 35.

Sepioteuthis ovata Gabb., Am. Jour. Couch. ir, pl. 17. 193. SCz.

Sepiotenthis sepiodea Bly., Arango, Famma Mal. Cuba, 187S. 148. Ca. MI.

Sepiotenthis sepioidea Blv., Noll. Cuba, i, 34.

Sepioteuthis sepioidea D'Orb., Arango, Fuma Mal. Guba, 187S. 148. Ca. $M$. 
Serpula (see Vermetus), Arango, Fanna Mal. Cuba, 18is, 164. Ca. Harana. Ba.

Serpula anguina Mawe, Mal. Bl., xxiv, 109.'Ber.

Serpula bicarinata Sby., Jour. de Conch., rii, 357. ST.

Serpula bicarinata Sby., Mal. Bl., xxir, 114.

Serpula conica Dillw., Jour. de Conch., viii, 38. ST.

Serpula conica Wood, Mal. Bl., xxiv, 121.

Serpula decussata Gmel., Jour. de Conch., viii, 34. Bb. ST.

Serpula decussata Lam., Nal. Bl., xxiv, 120. ST.

Serpula dentifera Lam., Mal. Bl., xxir, 113. Hy.

Serpula dentifera Sby., Mal. Bl., xxir, 114.

Serpula glomerata Brooke, Mal. Bl., xxiv, 116.

Serpula imbricata Mörch, Mal. Bl., xxir, 114.

Serpula lumbricalis Grmel., Mal. Bl., xxiv, 119.

Serpula lumbricalis Gmel., Moll. Cuba, i, 234.

Serpula lnmbricalis Limn., Jour. de Conch., riii, 28. M.

Serpula lumbricalis Linn., Moll. Cuba, i, $2: 34$.

Serpula nebulosa Dillw., Jour. de Conch, vii, 357. ST.

Serpula nebulosa Dillw., Mal. Bl., xxiv, 113. Hy.

Serpuloides (see Vermetus).

Serpuloides varians Gray, Jour. de Conch., viii, 41. SV.

Serpuloides varians Gray, Mal. Bl., xхiv, 116. Bz.

Serpulorbis varians $\Lambda$ d., Jour. de Conch., viii, 41. SV.

Serpulorbis variaus D'Orb., Mal. Bl., xxir, 116. Bz.

Sigaretus (see Catinus, Stomatia, Naticina).

Sigaretus antillarum Phil., Mal. Bl., iv, 27. G. = de,2uewa

Sigaretus antillarum Phil., Jour. de Conch., v, 156. G.

Sigaretus antillarum Recl., Bean. 18. G.

Sigaretus antillarum Recl., Jour. de Conch., ii, 185.

Sigaretus antillarum Recl., Krebs. 68.

Sigaretus antillarum Recl., Mal. Bl., xxiv, 56.

Sigaretus eancellatus Lam., Moll. Cuba, ii, 39.

Sigaretus carolinum Cuv., Jour. de Conch., ii, 185. SC. = dentent

Sigaretus concavus Pot., Beau. 18. G.

Sigaretus concavns Pot., Jour. de Conch., ii, 184.

Sigaretus depressus Phil., Krebs. 68. ST. Tort.

Sigaretus depressus Phil, Mal. Bl, xxir, 55.

Sigaretus haliotideus, Lam., Krebs. 68. ㄷ ar.

Sigaretus haliotideus Lam., Moll. Cuba, ii, 37, 38.

Sigaretus halioticleus Limn., Moll. Cuba, ii, 37 .

Sigaretus baliotidens Sby., Moll. Cuba, ii, 38. C.. Santa Lncia.

Sigaretus haliotideus JPOrls., Arango, Fanna Mal. Cuba, 1878. 172.

Ca. Santa Lucia.

Sigaretus haliotidens Lam., Bean. 18. G.

Sigaretus hatiotidens Lam., Jour. de Conch., ii, 185. ST. 
Simaretus halioticus Linn., Arango, Famma, Mial. Cuba, 1875. 172. Ca. Santa Lncia. $=$ depusoa

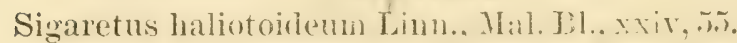

Sigaretus haliotoicleus $D^{\prime} \mathrm{Or}$. ., Mal. I3\}., xxir, 56.

Sigaretus haliotoideus Linu., Jour. de Concl., ir, 41\%. G.

Sigaretus incisus Rre, (note) Mal. BI. xxir, 55. exolie.

Sigaretus junior Reel., Jonr. de Conch., ii, 18.

Sigaretus leachii Bly., Mal. B1., xxir, 56. $=$ deperssa

Sigaretus leachii Sby., Moll. Cuba., ii, 37.

Sigaretus maculatus Phil., Mal. Bl., xxir, 57.

Sigaretus maculatus Say, Beau. 18. G.

Sigaretus maculatus Say, Jour. de Couch., ii, 165. Ca.

Sigaretus maculatus Say, Jour. de Conch., ii, 184. SD.

Sigaretus maculatus Say, Jour. de Conch., ir, 417. - G.

Sigaretus maculatus Say, Krebs. 6.

Sigaretus maculatus Say, Mal. B1., xwiv, 56.

Sigaretus macnlatus Say, Mal. Bl., xxiv, 57. Hatt.

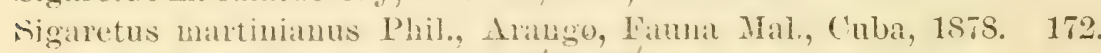

Ua. Antilles. G. Ba. = अaña G. b.

Sigaretus martinianus Phil., Beau. 18. G.

Sigaretus martinianus Phil., Jour. de Conch., ii, 1S4. Ca. M.

Sigaretus martinianus Phil., Krebs. 6s.

Sigaretus martinianus Phil., Nal. Bl., xxiv, 56.

Sigaretus perspectivus De Kay, Mal. Bl., xxir, $56 .=$ depecsia

Sigaretus perspectivus Sir, Jour. le Conch., ii, 168.

Sigaretus perspectivus Say, Jonr, de Conch., î, 185.

Sigaretus perspectivus Say, Mill. Bl., xxiv, 56.

Sigaretus rangii Bergh, Mal. Bl., xxir, 93. ST. = Maver ina sp

Sigaretus rungii Bergh, Poulsen. 10.

Sigaretus zonatus Dơnb, drango, Fannal Mal. Cuba, 1Sis. 172. Ca.

Antilles. G. Ba. = sfomotis:

Siguretus zonatus D'Orb., Bean. 1S. G.

Sigaretus zonatus D'Orb., Jour. de Conch., ii, 184. G.

Sigaretus zonatus D'Orb., Jour. de Conch., ir, 418. G.

Sigaretus zonatus D'Orb., Jour. de Conch., v, 156. G.

Sigaretus zonatus D'Orb., Krebs. 68. ST. SM.

Sigaretus zonatus D'(1,b., Mal. Bl.. ir, 27. G.

Sigaretus zonatus DOOrb., Mal. Bl., xxir, כ6.

Sigaretus zonatus D’O1\%., Moll. Cnba, ii, 38.

Siliqua (sce Solen, Machaera).

Siliqua costaț Gld., Bush, Conn. Ae, ri, 472. Hatt.

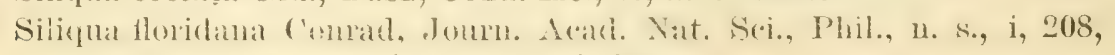

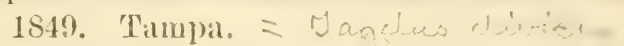

Siliqua lucida Meg., Krebs. 112.

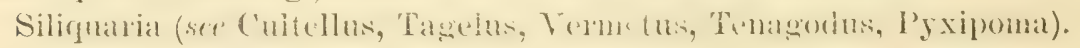

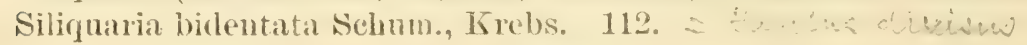




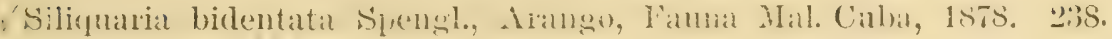
Ca.

Siliquaria centralis Con., Am. Jour. Conch., r, 191. Ga. = 2urách

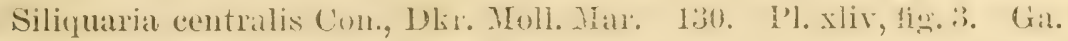
Siliquaria disisa Spengler, Am. Marine Conch. 143. - Teceded Siliquaria echinata Gmel., Krebs. 75. WVI. (Vermetider.) Siliquaria gibba Spengler, Am. Marine Conch. 143. = Gai Siliquaria gibba Spengler, Am. Jour. Conch., r, 191. Ga.

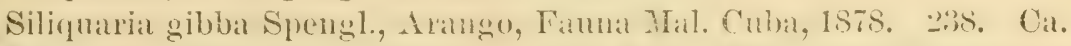
Siliquaria gibba Spengl., Marrat, Argo Exp., 1sis. 12. Tucacas. Siliquaria modesta Dall, n. s., Report Blake Moll. 39. CS. (Verm.) Siliquaria muricata Born, Krelos. 7j. (Verm.)

- Siliquaria muricata Lam., Mal. Bl., xxiv, 109. (Verm.) Siliquaria notata Schum., Krebs. 112. 'a cele Siliquaria rosea Blr., Mal. Bl., xxir, 109. Ber. (Verm.) Siliquaria squamata Blץ., Mal. Bl., xxir, 110. (Verm.) Siliquaria squamata Chenu, Mal. Bl., xxir, 110. (Verm.) Siliquaria squamata Mörch, Mal. Bl., xxir, 110. I'R. (Verm.) Siliquaria suleata Gray, Mal. Bl., xxir, 1.09. Ber. (Verm.) Siupulum (sec Tritonium) Marrat, Argo Exp., 1876. $\quad$ (i. Simpulum americanus D'Orb., Jahrb., v, 244.

Sinusigera cancellata Dorb., Arango, Fanna Mal. ("uba, 187s. 204. J. Sinusigera cancellata D'Orb., Krebs. 15.

Sinusigera cancellata D’(irb., Moll. Cuba, ii, pl, xxiii, figs. 7-9. 150.

Sipho (see Fusus, Neptumea, Chrysodomns).

Sipho benzoni Mörch, Jahrb., viii, 316. Bz.

Sipho obesus Verrill, Verrill, List., 1884. 267. Cape Hatteras.

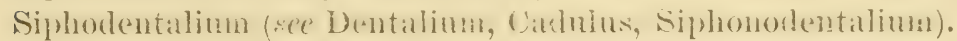

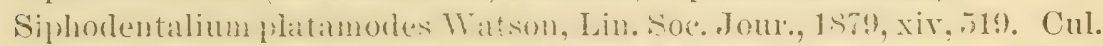
Siphodentalium tytthum Watsu, Lin. Soc. Jomrn., 1879, xiv, 520. Cul. Siphonaria (see Allerya, Piliseus).

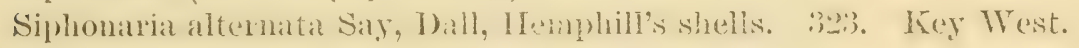
Siphonaria brunnea Haul., Moll. of Bermulas. 21.

Siphouaria cochleariform is Rro., Marrat, Argo Exp., 1876. 16. Nassau. Abaco.

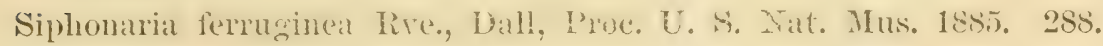
$\mathrm{VCz}$.

Siphonaria lessonii Blv., Moll. Cuba, i, 233.

Siphonaria lineata D'Orb., Beau. 1S. G.

Siphonaria lineata D'Orb., Jour. de Conch., r, 151. G.

Siphonaria lineata I'Or'b., Krebs. 7\%̃.

Siphouaria lineolata D'Orb., A rango, Fanna Mial. Onin, 1875. :30. Qi. MI. Santa Lncia. G. Ba. Fla.

Siphonaria lineolata D'Orb., Moll. Cuba, i, pl. xvii, lins. 13-15. 232.

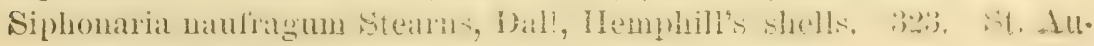
gustine, Fla. 
Siphonaria nanfracnm St Islaud E. Fla.

Siphonaria palpebrum Rive, Marrat, Argo Iixp., 1876. 14. Point Savanilla.

Siphonaria picta D'Orb., Arango, Fauma Mal. Cuba, 187s. 230. Ca. Siphonaria picta D'Urb., Krebs. 75.

Siphonaria picta D'Orb., Moll. Cuba, i, 231.

Siphonaria placentula Mke.,* Zt. Mal., x, 69. SV.

Siphonaria radicata $\mathrm{Bl}$. ., Moll. Cuba, i, 232.

Siphonaria umbonata Mle., * Zt. Mal., x, 69. SV.

Siphonium (see Serpula, Vermetus).

Siphoniun anaulax Mörch,* Mal. B1., xxiv, 114.

Siphonium decussatum Gmel., Guppy, Geol. Mag., 1874. 446. Tr.

Siphonium fissurata Mörch, ${ }^{*}$ Mal. Bl., dxiv, 114.

Siphonium nebulosum Dillw., Jour. de Conch., vii, 357. ST.

Siphonium nebulosum 1Dillw., Mal. Bl., xxiv, 112. Hd.

Siphonium uebulosum Dillw., Poulsen. 10.

Siphonium nebnlosum Mörch, Mal. Bl., xxiv., 113. Hd.

Siphonium rugosa Mörch,* Mal. Bl., xxir, 114.

Siphonium turboides Mörch, Mal. Bl., xxiv, 113.

Siphonolentalium (see Siphorentalium, Dentalium, Carlulus).

Siphonolentalium quadrjentatum 1)all, n. s., liepurt Blalie Moll. 36. Fla.

Siphouodentalium tetraschistum Watson, Report Blake Moll. 36. Fernando de Noronha.

Siphouata (see Aplysia).

Siphowota fasciata Poiret, Jour. de Conch., xi, 22.

Siphonota keraudrenii, Rang, Jour, de Conch., xi, 22.

Siphonota lurida D'Orb., Jour. de Conch., xi, 22.

Siphonota ocellata Rang, Jour. de Conch., xi, 22.

Sistrum (see Peutadactylus) Marrat, Argo Exp., 1876. 14.

Skenea (see Adeorbis, Vitrinella).

Skenea adamsii Fisch., Beau. 13. G.

Skenea beanii Fisch., Beau. 13. G.

Skenea corvea Stimpson, n. s.. Kurtz. 7. NC. SC.

Skenea lirata Verrill, Bush, Conn. Ac. vi, 464. Hatt.

Slienea megastoma C. B. Adams, Beau. 13. G.

Skenea megastoma (Vitrinella) C. B. Adams, Beau. 13. G.

Skenea petitii Fisch., Bean. 13. G.

Skenea petitii Fisch., Jour. de Conch., vi, 173. G.

Skenea petitii Fisch., Jomr, de Couch., ri, 28s. G.

Skenea schrammii Fisch., Beau. 13. G.

Skenea trilix Busb, Conu. Ac. vi, 461. Hatt.

Shoenia, Bean (see Skenea).

Solarimn (sec T'minia, I'hilipuia, Insina, Architectouica, Helacus).

Solarium æethiops Kust., Mal. Bl., xxii, 156. 
Soluium athiops Mke., Beau. 13. G.

Solurium athiops Mke., Jour. de Coneh., iv, 417. G.

Solirium athiops Mke., Krebs. 62.

Solarium athiops Mlie., Zt. Mal., v, 167. PR.

Solarium bicarinatum D'Orb., Mal. Bl., xxii, 156. ST.

Solarium bisulatum. WOrb., Arango, Fauna Mal. Cuba, 1878. 178. Ilarana. J. M.

Solarium bisulcatum D'Orb., Krebs. 62.

Solarim bisulcatum D'Orb., Mal. Bl., xxii, 157.

Solarium bisulcatum D'Orb., Moll. Cuba, ii, pl. xix, figs. 17-20. 66.

Solurium chemnitzii Kien., Arango, Famna Mal. C'uba, 187S. 17S. Ca. Ba.

Solarium chemnitzii Kien., Jonr. de Conch., ii, 428. G.

Solarium chemnitzii Kien., Mal. Bl., xxii, 155. PPl.

Solarium cyclostoma Mlie, Mal. Bl., xxii, 156. ST.

Solarium eyclostomm Mke., Mal. Bl., sxii, 156.

Solarium delphinuloides J)'Orb., Arango, Fauna Mal. Cuba, 1S78. 17 S. Ca.

Solarium delphinuloides D'Orb., Krebs. 62.

Solarium delphinuloides D'Orb., Mal. Bl., sxii, 157. J.

Solarium delphinuloides D'Orb., Moll. Cuba, ii, pl. xix, figs. 20-21. 67.

Solarium dorsuosum Hanl., Mal. Bl., xxii, 156.

Solarium granulatum Lam, Arango, Fauna Mal, Cuba, 1S7s. 178. Havana. G. Ba.

Solarium granulatum Lam., Beau. 13. G.

Solarium granulatum Lam., Krebs. 63. NGr. PPl.

Solarium granulatum Lam., Moll. Cuba, ii, 11. xix, tigs. 25-27. 65.

Solarium granulatum Linn., Mal. B1., xxii, 154. ST.

Solarium heberti Desh, Arango, Fauna Mal. Cuba, 1S7s. 17s. Ca. G.

Solarium heberti Desh., Mal. Bl., xxii, 156. ST.

Solarium heberti D'Orb., Moll. Cuba, ii, 68.

Solarium hebertii Desh., Beau. 13. G.

Solarium hebertii Desh., Krebs. 63. ST.

Solarium infundibuliforme Chem., Jour. de Conch., ii, 42S. G.

Solarium infundibuliformis Chem., Krebs. 63. PPl. S'T. SM.

Solarium infundibuliformis Guel., Arango, Fauna Mal. Cuba, 1STS. 17s. Ca. Ba.

Solarium inornatum DoOrb., Araugo, Fauna Mal. Cuba, 1S7S. 178. ST.

Solarium inornatum D'Orb., Jour. de Conch., vi, 173. ST.

Solurium inornatum D'Orb., Krebs. 63.

Solarium inornatum 1'Orb., Mal. Bl., xxii, 155. ST.

Solarium inornatum D'Orb., Moll. Cuba, ii, 67.

Solarium nubilum Mlie., Krebs. 63.

Solarium unbilum Mlie, Mal, Bl., xxii, 157. 
Solarium semidecussatum Guply, Geol. 19ag., 1874. 438. 'Id.

Solarium tesselatum Desh., Guppy, Paria Fama, 1877. 140.

Solarium rerrucosum Phil., Mal. Bl., vi, 122. VCz.

Solecurtoides lneidus Dest., Krebs. 112. Wsit Am.

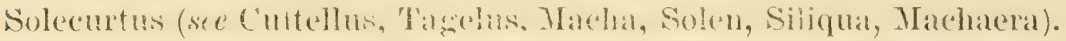

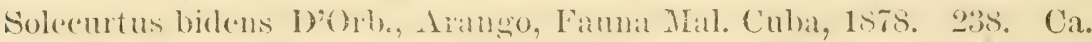

Solecurtus bidens D'Orb, Moll. Cubit, ii, 23L. = divíant 5 pgl,

Solecurtus birleus F. \& H.. Kurtz. 3. NC. SC.

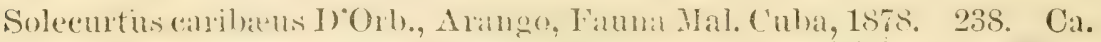
Solecurtus aribrus D'Orb., Moll. Cuba, ii, 231. = ét-buxsogl,

Solecurtus caribaus Lam.. Guply, Paria Fauna, 1577. 147.

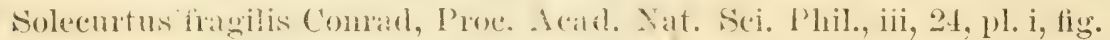
24. 1845. Tampa. = divedua 3 pas.

Solecurtus fragilis Mtg., Juar. de Conch., iv, 414. G. = derizes

Solecurtus gibbus F. \& H., Kurt\%. 3. NC. SC.

$\checkmark$ Solecurtus platensis D'Orb., Moll. Cuba, ii, 231.

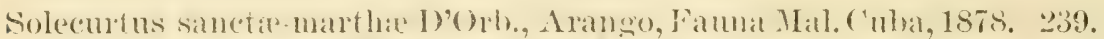

Antilles. Ca. G.

Solecurtus sanctre-marthe D'Orb., Beau. 26. G.

Solceurtus sanctie-martha D'Orb., Jour. de Conch., ir, 414.

Solecurtus sanctie-marthe Dorb., MIoll. Cuba, it, pl. xxy, ligs. 31-32. 232.

Solemya (see Solenomya).

Solemya occidentalis Desh., Jour. de Conch., vi, 139. G.

Solemya occidentalis Desh., Krebs. 123. ST'.

Solemya parrula Sby., Jour. de Conch., vi, 139.

Solen fse Ensis, Cultellus, Solecurtus, Tagelus, Siliquania, Siliqua, Machaerai).

Solen (see Macha), Araugo, Nanna Mal. Cuba, 1875. „̈ü. Antilles. Ca. G.

Solen (sce Siliquaria), Arango, Fauna Mal. Cuba, 1878. 238. Ca.

Solen (see Solena), Arango, Fama Mal. Cuba, 1878. 239. Ua.

Solen ambigums D०Orb, Arango, Fama Mal. Cuba, 1878. 239. Ca.

Solen ambiguus Lam., Arango, Fauma Mal. Cuba, 1878. 239. Ca.

Solen aubiguus Lam., Bean. 26. G. oblinuw 3:. $C_{1}$

Solen ambiguus Lam., Jonr. de Conch., ii, 424. G.

Soleu ambiguus Lain., Krebs. 11:.

Solen ambiguns Lam., Moll. Cuba, ii, 220.

Solen an ericantis Gonll, Da!l, Menuhills shells. 337. Cedar Keys.

Solen bideus Chem., Mringo, Fanma Mal. Cuba, 1sis. „238. Ca.

Solen bidens Chem., Krebs. 112. = Varclew diusisud SBgh,

Solen bidens Chem., Moll. Cuba, ii, 231.

Solen bidentatus Spgl., Arango, Fauna Mal. Cuba, 187s. 238. Ca.

Soleu bidentatus Spgl., Krels. 11:. NGr. S'T. SCx. SJ. Td.

Solen bidentatus Spgl.=S. fragilis Sol., Poulsen. 14.

Solen bullatus Chem., Krebs, $116 .=$ Caridiam 
Solen bullatus Chem., Moll. Cuba, ii, 308.

Solen bullatus Linu., Arango, Fanna Mal. ('uba, 1878. 259. Ca. M. G. Santa Lucia. Ba.

Solen bullatus Linn., Moll. Cuba, ii, 308.

Solen caribaus Iam., Arango, Fauna Mal. Cnba, 1878. 238. Ca.

Solen caribøus Lam., Krebs. 112.

Solen caribrus Lam., Moll. Cuba, ii, 231.

Soleu caribans Lim., Pfeifler, Arehiv für naturg., 1840. 261. Ca.

Solen centralis say, Alango, Fauma Mal. Cuba, 1878. 23s. Ca.

Solen centralis Say, Kurtz. 3. = divided fól.

Solen constricta Brug., Krebs. 101. = Mabe,

Solen constrictus Brug., Arango, Fauna Mal. Cuba, 1878. 242. Ca. Havaua.

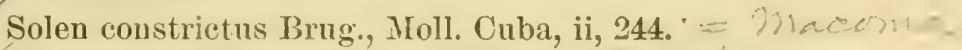

Solen divisus Fabr., Krebs. 112. M Mg L Lat

Solen ensis Dall, Hemphill's shells. 337.

$\checkmark$ Solen ensis Limn., Kurtz. 3. NC. SC.

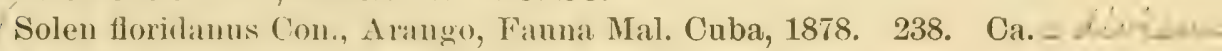
Solen fragilis Glrl., Kurtz. 3.

- Solen fragilis I’ult, Arango, Fauna Mal. Cuba, 1S7S. 238. Ca. - Levidus

Solen fragilis Sol., Krebs. 112. = distas

Solen fueatus Spgl., liaugo, Fanna Mal. ('uba, 1878. 244. Ca. M.G.

Solen fucatus Spgl., Krebs. 104. = xathe? cerit ate aryed a

Solen gibbus Sligl., Krebs. 11:. ST., NGr.

Solen gibbus Spgl.=S. caribaens Iam., Poulsen. 14.

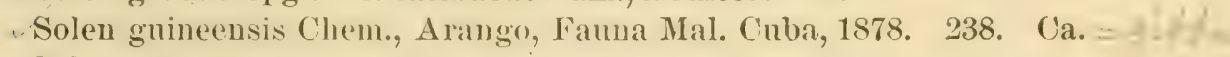
Solen guineensis Chem., Moll. Cuba, ii, 231.

Solen lappeanus Dlir., Moll. Mar., pl. xliv, fig. 1. 129. (Antilles.,

Solen lineatus Spgl., ['oulsen. 14. = : vese zathe.

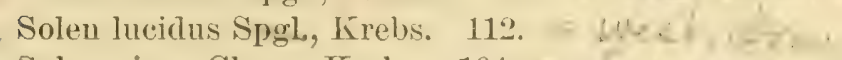

Solen nivea Chem., Krebs. 104.

Solen niveus Hanley, Guppy, Paria Fauna, 1877. 147.

Solen notata Schum., Arango, Fauna Mal., Cuba, 1878. 238. Ca.

Solen obliquus Șpgl., Krebs. 112. ST. PR.

Solen obliquus Spgl. = S. ambiguus Lam., Poulsen. 14.

Solen radiatus Limn., Pfeiffer, Archiv fiir naturg, 1840. 261. Ca.

: Solen sanctie-martha Chem., Krebs. 113. -

Solen sunctie-martha Chem., Moll. Cuba, ii, pl. xxv, figs. 31-32. 232. WI.

$\checkmark$ Solen sanctæ-marthæ Chem., Poulsen. 14.

- Solen sanguinolentus Dillw., Moll. Cuba, ii, 246. =

Solen sanguinolentus Gmel., Arango, Fauna Mal. Cuba, 1878. 244. Ca.

M. G.

Solen sanguinolentus Gmel., Jour, de Conch., ii, 425. G.

Solen sanguinolentris Gmel., Krebs. 104.

Solen sanguinolentus Gmel., Moll. Cuba, ii, 246.

Bull. 24-18 
Solen tagal Adan., Moll. Cuba, ii, 231. = gibtus fp.e.

Solen teunis Wood, Krebs. 112.

Solen riridis Say, Am. Mariue Conch. 142. SC. Fla.

Solena obliqua Browne, Krebs. 112.

Solena obliqua Spgl., Arango, Fauna Mal. Cuba, 1878. 239. Ca.

Solenomya (see Solemya).

Solenomya occilentalis Desh., *Jour. de Conch. vii, pl. vii, fig. 6. 186. G.

Solenomya occidentalis Desh., Poulsen. 16.

Spengleria (see Rocellaria, Gastrochæua).

Spengleria rostrata Spgl., Arango, Fauna Mal, Cuba, 1878. 237. Ca. ST.

Spengleria truncata Sby., Arango, Fauma Mal. Cuba, 1878. 237. Ca. Sphærium cubense Prime, Arango, Fanna Mal. Cuba, 1s78. 143. Ca. Sphena (see Nerra).

Sphena alternata D'Orb., Arango, Fauna Mal. Cuba, 1878. 253. M. Sphena alternata D’Orb.,* Moll. Cuba, ii, pl. xxvii, tigs. 17-29. 286. M.

Sphena alternata D'Orb., Report Blake Moll. 110.

Sphena cleryana D’Orb., Araugo, Fauna Mal. Cuba, 1878. 253. Ca. J. ST. G.

Sphena cleryana D'Orb., Moll. Cuba, ii, 285. Ca. Bz. J. ST. G.

Sphena ornatissima D’Orb., Arango, Fauna Mal. Cuba, 1878. 254. Ca. J. ST. G.

Sphena ormatissiua D'Orb., Moll. Cuba, ii, pl. xxrii, figs. 13-16. 286. Ca.

Sphena ornatissima D'Orb., Report Blake Moll. 110.

Spirialis rostrata Ey. \& Sonl., Arango, Fauna Mal. Cuba, 1878. 153. Ca.

Spiroclimax scalaris Mörch,* Mal. Bl., xxiï, 168. ST.

Spiroglyphus (see Vermetus).

Spiroglyphus anuulatus Blv., Mal. Bl., xxiv, 115.

Spiroglyphus annulatus Bose, Mal. Bl., xxiv, 115.

Spiroglyphus annulatus Daud., Mal. Bl., xxiv, 114.

Spiroglyphus anmulatus Daudin, Poulsen. 10.

Spiroglyphus ammulatus Mörch, Mal. Bl., xxiv, 115.

Spiroglyphus corrodeus Gray, Mal. Bl, xxiv, 115.

Spiroglyphus corrodeus Shuttlew., Mal. Bl., xxiv, 115.

Spiroglephus corrodens D'Orb.. Jour. de Conch., viii, $44 . \quad$ Ca.

Spiroglyphus dentifera Mörch, Mal. Bl., xxiv, 115.

Spiroglyphus glomerata Mörch, Mal. Bl., xxiv, 116.

Spiroglyphus irregularis D'Orb., Jour. de Conch., viii, 45. Ca.

Spirula (see Nautilus).

Spirula fragilis Bose, Moll. Cuba, i, 64.

Spirula fragilis DOOrb., Lrango, Fauma Mal. Cuba, 1878. 149. Ca. Ba. Spirula fragilis Lam., Arango, Fauna Mal. Cuba, 1878. 149. Ca. Ba. 
Spirula fragilis Lam., Beau. 6. G.

Spirula fragilis Lam., Moll. Cuba, i, 64.

Spirula fragilis Roissy, Moll. Cuba, i, 64 .

Spirula perouii Lam., Moll. of Bermudas. 15.

Spirula peronii Lam., Arango, Fauna Mal. Cuba, 1878. 149. Ca. Ba. Spirula peronii Lam., Kurtz. 9.

Spirula peronii Lam., Mal. Bl., xii, 133.

Spirula peronii Lam., Moll. Cuba, i, 64.

Spirula prototypus Peron, Arango, Fauna Mal. Cuba, 1878. 149. Ca. Ba.

Spirula prototypus Peron, Moll. Cuba, i, 64.

Spisula (see Mactra).

Spisula corbulvides Desh., Marrat, Argo Exp., 1876. 12. Tucacas.

Spisula fragilis Gray, Jahrb., ii, 248.

Spondylus (see Ostrea, Hinnites, Plicatula).

Spondylus americanus Lam., Arango, Fauma Mal. Cuba, 1878. 271. Ca. SD.M.

Spondylus americanuś Lam., Beau. 21. G.

Spondylus americanus Lam., Jour. de Conch., iv, 416. G.

Spondylus americanus Lam., Krebs. 135.

Spondylus americanus Lam., Moll. Cuba, ii, 358.

Spondylus americanus Lam., Poulsen. 16.

Spondylus harbadeusis Pet., Araugo, Fauma Mal. Cuba, 1878. 271.

Ca. Ba.

Spondylus barbadensis Pet., Moll. Cuba, ii, 360 .

Spondylus eoceineus Lam., Beau. 21. G.

Spondylus coccineus Lam., Krebs. 135.

Spondylus croceus Chem., Arango, Fanna Mal. Cuba, 1878. 271. Ca. Havana.

Spondylus croceus Rve., Poulsen. 16.

Spondylus divaricatus Schröt., Krebs. 135.

Spondylus echiuatus D'Orb., Arango, Fauna Mal. Cuba, 1878. 271.

Ca. M. G.

Spondylus echinatus D'Orb., Krebs. 135.

Spondylus echinatus D'Orb., Moll., Cuba, ii, 359.

Spondylus echinatus Martyn, Araugo, Fanna Mal. Cuba, 1878. 271.

Ca. M. G.

Spondylus ericinus Rve., Moll. of Bermudas. 24.

Spondylus fimbriatus Meusch., Krebs. 135.

Spondflus fimbriatus Meusch. = S. spathuliferus Lam., Poulsen. 16. Sponclylus folia-brassicae Chem., Arango, Fauna Mal. Cuba, 1878.

271. Ca. SD.M.

Spondylus folia-brassica Chem., Moll. Cuba, ii, 358.

Spondylus folia-brassicae D’Orb., Arango, Fauna Mal. Cuba, 1878.

271. Ca. SD. M.

Spondylus gæderopus Chem., Moll. Cuba, ii, 355. 
Spondylus gæderopus Chem., Krebs. 135.

Spondrlus gæderopus Chem., Moll. Cuba, ii, 358, 359.

Spondylus longitudinalis Lam., Arango, Fauna Mal. Cuba, 1878. 271. Ca. M. G.

Spondylus longitudinalis Lam., Moll. Cuba, ii, 359.

Spondylus plicatus Chem., Krebs. 135.

Spondylus plicatus Chem., Moll. Cuba, ii, 360.

Spondylus plicatus var. Linn., Arango, Fauna Mal. Cuba, 1878. 271.

Ca. Ba.

Spondylus radians Lam., Krebs. 135.

Spondylus spathuliferus Lam., Krebs. 135.

Spondylus striato-spinosus Chem., Krebs. 135.

Spondylus ustulans Rve., Moll. of Bermudas. 24.

Standella (see Mactra).

Standella fragilis Chem., Jahrb., ii, 248.

Standella fragilis Gray, Jahrb., ii, 248.

Standella (Merope) senegalensis Phil., Marrat, Argo Exp., 1876. 12. Tucacas.

Stephanoconus (see Conus) Marrat, Argo Exp., 1876. 9.

Sthenoteuthis bartramii Verrill, List, 1884. 264.

Stigmaulax (see Natica) Marrat, Argo Exp., 1876. 18.

Stigmaulax cancellata Herrm., Mal. Bl., xxiv, 61.

Stigmaulax haneti Recl., Mal. Bl., xxiv, 62. Bz.

Stigmaulax rugosa Chem., Mal. Bl., xxiv, 62.

Stigmaulax sulcata Born,* Mal. Bl., xxiv, 62. G.

Stigmaulax suleata Mörch, Mal. Bl., xxiv, 62.

Stomatella (see Stomatia).

Stomatella coccinea A. Ad., Krebs. 85. ST. SCx.

Stomatella coccinea A. Ad., Poulsen. 13.

Stomatella picta D'Orb., Beau. 14. SM.

Stomatella picta D'Orb., Poulsen. 13.

Stomatia (see Sigaretus, Catinus).

Stomatia depressa Phil., Mal. Bl., xxiv, 55.

Stomatia depressa Phil., Poulsen. 10.

Stomatia maculata Say, Mal. Bl., xxiv, 57.

Stomatia perspectiva Say, Mal. B1., xxiv, 56. SC.

Stomatia perspectiva Say, Poulsen. 10.

Stomatia picta Dorb., Arango, Fauna Mal. Cuba, 1878. 225. Ca.

- Stomatia picta D'Orb., Krebs. 85. ST. SB. PPl. Ag.

Stomatia picta D'Orb., Moll. Cuba, ii, pl. xxiv, figs. 19-21. 184.

Stomatia zonata D'Orb., Mal. Bl., xxiv, 56.

Stomatia zonata D’Orb.=Sigaretus martinianus Phil., Poulseu. 10.

Stramonita (see Purpura), Marrat, Argo Exp., 1876. 5.

Stramonita Horidana Con., Jahrb., ii, 242. Bz.

Stramonita hamastoma Limn., Jahrb., ii, 241. Bz.

Strephona (see Dactylus), Marrat, Argo Exp., 1876. 8. 
Strigilla (see Tellina, Lneina).

Strigilla areolata Mke, Arango, Fauna Mal. Cuba, 1878. 242. Ca. Ba.

Strigilla carnaria Linn., Am. Marine Conch. 151.

Strigilla earnaria Linn., Mal. Bl., x, 228. Ca.

Strigilla carnaria Linn., Poulsen, 14.

Strigilla carnaria Turt., Krebs. 100.

Strigilla Hexuosa Say, Am. Marine Conch. 151.

Strigilla mera Say, Am. Marine Conch. 151. SC.

Strigilla pisiformis Limn., sp.? Marrat, Argo Exp., 1876. 12. Tucacas.

Strigilla pisiformis Linn., Mal. Bl., x, 230.

Strigilla pisiformis Linn., Am. Jour. Conch., vi, 24.

Strigilla pisiformis Limn., Poulsen. 14.

Strigilla producta Tryon, ${ }^{*}$ Am. Jour. Conch. vi, pl. 1, fig. 4. 24. J. Strigilla quadrisuleata Gray, Krebs. 120.

Strigilla rombergii Möreh, Poulsen. 14.

Strombiformis einctus Da Costa, Mal. Bl., xxiii, 128.

Strombiformis costatus Da Costa, Mal. Bl., xxiii, 91.

Strombus (see Oniscia), Arango, Fauna Mal. Cuba, 1878. 224. Ca.

Havana. G. Santa Lucia. Ba.

Strombus accipiter Dillw., Mal. Bl., xxir, 18.

Strombus aceipitrinus Lam., Jour. de Conch, vii, 27. G.

Strombus accipitrinus D'Orb., Arango, Fauna Mal. Cuba, 1878. 195. Ca. Hy. G.

Strombus accipitrinus Lam., Beau. 7. G.

Strombus accipitrinus Lam., Jahrb., ii, 256.

Strombus accipitrinus Lam., Jour, de Conch, iv, 418. G.

Strombus accipitrinus Lam., Moll. Cuba, ii, 119.

Strombus accipitrinus Mart., Araugo, Fauna Mal. Cuba, 1878. 195. Ca. Hy. G.

Strombus accipitrinus Mart., Krebs. 1. Tort. ST. SCx.

Strombus accipitrinus Mart., Krebs. 6.

Strombus aceipitrinus Mart., Krebs' Remarks. 398. 'Fla.

Strombus accipitriuus Mart., Marrat, Argo Exp., 1876. 16. Nassau.

Strombus aceipitrinus Lam., Mal. Bl., xxiv, 18.

Strombus aecipitrinus var. Rve., Mal. Bl., xxiv, 18.

Strombus affinis Gmel., Krebs. 1.

Strombus alatus Gmel., Am. Marine Conch. 17.

Strombus alatus Gmel., Araugo, Famna Mal. Cuba, 1878. 196. Ca. G.

Santa Lucia. Ba.

Strombus alatus Gmel., Jahrb., ii, 255. Fla.

Strombus alatus Gmel., Krebs. 1.

Strombus alatus Gmel., Krebs' Remarks. 398.

Strombus alatus Gmel., Mal. Bl., xxiv, 22.

Strombus ảlatus Gmel., Mal. Bl., xxiv, 23.

Strombus alatus Gmel., Poulsen. 9.

Strombus alatus Sby., Mal. Bl., xxiv, 22. 
Strombus bitubercularis Lam., Beau. 7. G.

Strombus bitubercularis Lam., Krebs. 1.

Strombus bituberculatus Lam., Jour, de Conch., vii, 27. G.

Strombus bituberculatus D'Orb., Arango, Fauna Mal. Cuba, 1878. 195.

Ca. M. G. Santa Lncia. J. Ba.

Strombus bituberculatus Lam., Jour. de Conch., ii, 429. G.

Strombus bituberculatus Lam., Mal. Bl., xii, 128.

Strombus bituberculatus Lam., Mal. Bl., xxiv, 20.

Strombus bituberculatus Lam., Moll. Cuba, ii, 120.

Strombus bituberculatus Swaius., Jahrb., ii, 259.

Strombus bubonius Linn., Mal. Bl., xii, 128.

Strombus coecyx Klein, Mal. Bl., xxiii, 128.

Strombus coìmella Meusch., Mal. Bl., xxii, 157.

Strombus cornutus Perry, Mal. Bl., xxiv, 21.

Strombus costatus Don., Mal. Bl., xxiii, 91.

Strombus costatus Gmel., Arango, Fauna Mal. Cuba, 1878. 195. Ca. Hy. G.

Strombus costatus Gmel., Beau. 7. G.

Strombus costatus Gmel., Jahrb., ii, 256.

Strombus costatus Gmel., Krebs. 1.

Strombus costatus Gmel., Mal. Bl., xxiv, 18.

Strombus costatus Gmel., Moll. Cuba, ii, 119.

Strombus costatus Gmel., Poulsen. 9.

Strombus costatus Mtg., Mal. Bl., xxiii, 91.

Strombus costatus Pult., Mal. Bl., xxiii, 91.

Strombus costatus Wood, Mal. Bl., xxiii, 91.

Strombus costoso-muricatus Mart., Arango, Fauna Mal.Cuba, 1878. 195.

Ca. M. G. Santa Lucia. J. Ba.

Strombus costoso-muricatus Mart., Krebs. 1. NGr. Vz. Bb. Ag. SB. SM. S'T. Td. Tort.

Strombus curruca Bolt., Krebs. 1.

Strombus dilatatus Lam., Jahrb., ii, 259.

Strombus dubius Sby., Arango, Fauna Mal. Cuba, 1878. 196. Ca. G. Sauta Lucia. Ba.

Strombus dubius Sby., Krebs. 2.

Strombus dubius Sby., Krebs' Remarks. 398.

Strombus dubius Sby., Mal. Bl., xxiv, 23.

Strombus fragilis D'Orb., Moll. Cuba, ii, 121.

Strombus gallus D’Orb., Arango, Fauna Mal. Cuba, 187S. 195. Ca.

M. Santa Lucia. G. Ba.

Strombus gallus Gmel., Moll. Cuba, ii, 120.

Strombus gallus Lam., Moll. Cuba, ii, 120.

Strombus gallus Limn., Arango, Fauna Mal. Cuba, 1878. 195. Ca. M.

Santa Lucia. G. Ba.

Strombus gallus Linu., Beau. 7. G.

Strombus gallus Linn., Jour. de Conch., vii, 27. G. 
Strombus gallus Linu., Jahrb., ii, 257.

Strombus gallus Liun., Jour. de Conch., ir, 418. G.

Strombus gallus Linu., Krebs. 2. SJ. Tort.

Strombus gallus Linn., Krebs. 23.

Strombus gallus Linn., Mal. Bl., xxiv, 20.

Strombus gallus Linn., Moll. Cuba, ii, 119.

Strombus gallus Linn., Poulsen. 9.

Strombus gigas Linn., Jour. de Conch., vii, 28, 29. G.

Strombus gigas Linn., Jour. de Conch., vii, 33.

Strombas gigas Linn., Moll. of Bermudas. 16.

Strombus gigas D'Orb., Arango, Fanua Mal. Cuba, 1878. 195. Ca.

Ba. Antilles.

Strombus gigas Gmel., Moll. Cuba, ii, 118.

Strombus gigas Lam., Jahrb. Nachblt., 1877. 59.

Strombus gigas Lam, Moll. Cuba, ii, 118.

Strombus gigas Limn., Am. Marine Conch., 17. WI.

Strombus gigas Linn., Alango, Fanna Mal. Cuba, 187s. 195. Ca. Ba. Antilles.

Strombus gigas Linn., Beau. 7. G.

Strombus gigas Linn., Jahrb., ii, 258.

Strombus gigas Linn., Jour. de Conch., iv, 418. G.

Strombus gigas Linn., Jour. de Conch., vii, 26. M. G.

Strombus gigas Linn., Jour. de Conch., vii, 357. ST.

Strombus gigas Linn., Jour. de Conch., ix, 215.

Strombus gigas Linn., Krebs. "2. NGr. Tort. ST. SJ. SCx. SB.

Strombus gigas Linn., Mal. Bl., xxiv, 14.

Strombus gigas Linn., Marrat, Argo Exp., 1876. 12. Tucacas.

Strombus gigas Linn., Marrat, Argo Exp., 1876. 14. Cartagena.

Strombus gigas Limn., Moll. Cuba, ii, 118.

Strombus gigas Linn., Poulsen. 9.

Strombus goliath Chem., Beau. 7. G.

Strombus goliath Chem., Jahrb., ii, 258.

Strombus goliath Chem., Nachblt., 1877. 59.

Strombus goliath Chem., Krebs. 2. T.

Strombus goliath Chem., Krebs' Remarks. 398.

Strombus goliath Chem., Mal. Bl., xxiv, 16.

Strombas goliath Chenu, MaI. Bl., xxiv, 17.

Strombus goliath Ducl., Mal. Bl., xxiv, 17.

Strombus goliath Rve., Mal. Bl., xxiv, 17.

Strombus goliath Wood, Mal. Bl., xxiv, 16.

Strombus gracilis Siby., Arango, Fauna Mal. Cuba, 1878. 196. Ca.

Strombus granulatus Sby., Krebs. 2.

Strombus haitensis Sby., Mal. Bl., xxiv, 18. Fla.

Strombus inermis Desh., Mal. Bl., хxir, 18.

Strombus inermis Ducl., Mal. Bl., xxiv, 18.

Strombus inermis Sby., Krebs. 1. 
Strombus inermis Sby., Krebs' Remarks. 398. Ba. Fla.

Strombus inermis Sby., Mal. Bl., xxiv, 18.

Strombus inermis Swains., Jahrb., ii, 256.

Strombus inermis Swains., Mal. Bl., xxiv, 18.

Strombus inermis Swains., Poulsen. 9.

Strombus integer Swains., Mal. Bl., xxir, 17. Fla.

Strombus latus Gmel., Jahrb., ii, 259.

Strombus lentiginosus Gmel., Moll. Cuba, ii, 122.

Strombus lentiginosus Lam., Moll. Cuba, ii, 122.

Strombus lentiginosus Linn., Krebs. 2.

Strombus lentiginosus Linn., Moll. Cuba, ii, 122.

Strombus listeri Gray, Mal. B1., xxiv, 22.

Strombus listeri Gray, Mal. Bl., xxiv, 23.

Strombus lobatus Sby., Beau. 7. G.

Strombus lobatus Sby., Mal. Bl., xxiv, 20.

Strombus lobatus Swains, Jahrb., ii, 259.

Strombus lobatus Swains., Jour. de Conch., ii, 429. G.

Strombus lobatus Swains., Krebs. 1.

Strombus lobatus Swains, Mal. Bl., xxir, 20.

Strombus lueifer Linn., Mal. Bl., xxiii, 16.

Strombus magnus Aldrov., Mal. Bl., xxiv, 27. J.

Strombus oniscus Chem., Moll. Cuba, ii, 131.

Strombus oniscus Gmel., Moll. Cuba, ii, 131.

Strombus onisens Linn., Jahrb., v, 238.

Strombus oniscus Linn., Mal. Bl., xxiv, 40.

Strombus oniscus Linn., Moll. Cuba, ii, 130.

Strombus pugilis, Krebs. 70.

Strombus pugilis Kurtz. 9. NC.

Strombus pugilis Dillw., Krebs. 1.

Strombus pugilis IOOrb., Arango, Fatual Mal. Cuba, 1878. 196. Ca.

G. Santa Lucia. Ba.

Strombus pugilis D'Orb., Mal. Bl., xxiv, 21.

Strombus pugilis Gmel., Moll. Cuba, ii, 121.

Strombus pugilis Lam., Jour. de Conch., vii, 27. G.

Strombus pugilis Lam., Moll. Cuba, ii, 121.

Strombus pugilis Linn., Arango, Fauna Mal. Cuba, 1878. 196. Ca.G.

Santa Lucia. Ba.

Strombus pugilis Linn., Beau. 7. G.

Strombus pugilis Linn., Jahrb., ii, 261.

Strombus pugilis Linn., Jour. de Conch., ii, 429. G.

Strombus pugilis Limn., Krebs. 2. ST. SCx. Td. NGr. VCz.

Strombus pugilis Linu., Krebs' Remarks. 398.

Strombus pugilis Linn., Mal. Bl., xxir, 21.

Strombus pugilis Limu., Moll. Cuba, ii, 121.

Strombus pugilis Linn., Poulsen. 9.

Strombus flugiloides Guply, u. s., (reol. Mag., 1874. 441. Ј. Нy. Ag. 
Strombus pumilio Mörch, Mal. Bl, xxiv, 20. VCz.

Strombus pyrulatus Kien., Mal. Bl., xxir, 22.

S roubus pyrulatus Lam., Arango, Fauna Mal. Cuba, 1S78, 196. Ca. Strombus pyrulatus Lam., Jahrb., ii, 255. Fla.

Strombus pyrulatus Lam., Krebs. 1.

Strombus pyrulatus Lam., Mal. Bl., xxiv, 22.

Strombus quadratus Perry, Mal. Bl., xxiv, 20.

Strombus ranipus Gmel., Krebs. 1.

Strombus rauinus Gmel., Mal. Bl., xxir, 19.

Strombus raninus Gmel., Poulsen. 9.

Strombus reticulatus Miihlf, Mal. Bl., xxiii, 96.

Strombus sloanii Lieach, Mal. Bl., xxiv, 21.

Strombus suleatus Anton, Mal. Bl., xxir, 22.

Strombus tubereularis Lam., Marrat Argo Exp., 1876. 13. Santa

Marta.

Strombus turboformis Mtg., Mal. Bl., xxiii, 92.

Strombus undulatus Chem., Mal. Bl., xxiv, 22.

Strombus vexillum Chem., Moll. Cuba, ii, 147.

Strombus vexillum Gmel., Moll. Cuba, ii, 147.

Strombus xylohelix Klein, Mal. Bl., xxiii, 127.

Strongylocera (Nassa) textilina Mörch, Krebs. 32.

Stylifer var. abbreviata Mörch, Mal. Bl., xxii, 183.

Stylifer corallinus Chem., Arango, Fauma Mal. Cuba, 1878. 164. Ca.

Havana.

Stylifer corallinus (Helix) Cben., Arango, Fauna Mal. Cuba, 1878.

164. Ca. Havana.

Stylifer corallinus Chem., Jour. de Conch., xii, 100.

Stylifer corallinus Chem., Poulsen. S.

Stylifer corallium Chem., Mal. Bl., xxii, 184. S'l.

Stylifer subangulatus A. Ar., Alango, Fama Mal. Cuba, 187s. 164.

Ca. Havana.

Stylifer subangulatus A. Ad., Jour. de Conch., xii, 102.

Stylifer subaugulatus C. B. Adams, Mal. Bl., xxii, 183. ST.

Stylifer subangulatus ('. I). Aclams, Poulseu. S.

Stylifer subulatus Brod., Arango, Fama Mal. Cuba, 1878. 164. Ca.

Havana.

Stylifer subulatus Brod. \& Sby., Jour. de Conch., xii, 100.

Stylifer subulatus Brod., Mal. Bl., xxii, 184. Bb.

Stylifer subulatus Brod, Poulsen. S.

Stylina subulata Brod., Krebs. 66. ST. SCx. SM.

Styliola acus Dkr., Jahrb., ii, 240. Bz.

Styliola subulata Q. \& G., Bush, Coun. Ac., vi, 470. Hatt.

Styliola virgula Rang, Bush, Conn. Ac., vi, 470. Hatt.

Subemarginula (see Emarginula,) Marrat Argo Exp., 1876. 6.

Subemarginula clausa D'Orb., Bean. 19. G.

Subemarginula depressa Blainv., Beau. 19. G. 
Sycotypus (see Pyrula, Ficus, Fulgur, Busycon).

Sycotypus canaliculatum Lam., Jahrb., ii, 361.

Sycotypus canalieulatus Linu., Am. Marine Conch. 23.

Sycotypus canaliculatus Linn., Am. Marine Conch. 24.

Sycotypus caricus Gmel., Am. Marine Conch. 23.

Sycotypus coarctata Sby., Jahrb., ii, 361. Fla.

Sycotypus papyratia Stm., Mal. Bl., xxiv, 43. Fla.

Sycotypus perversus Linn., Am. Marine Conch. 24.

Sycotypus plagosum Con., Jahrb., ii, 361.

Sycotypus pyrum Dillw., Am. Jour. Conch., iii, 150.

Sycotypus pyrum Dillw., Am. Marine Conch. 23.

Sycotypus pyrum Dillw., Jahrb., ii, 361.

Syndosmya (see Abra).

Syudosmya iequalis Say, Dall, Hemphill's shells. 338. Cedar Keys.

Syudosmpa lioica Dall, n. s., Report Blake Moll. 133. CS. Ca.

Syndosmya Iongicallis Scacchi, Report Blake Moll. 133.

Syrnola pulchella A. Ad., Mal Bl., xxii, 159. ST.?

Tagelus (see Cultellus, Solen, Siliquaria).

Tagelus bidentatus Gray, Krebs. 112.

Tagelus gibbus Spengler, Dall., Hemphill's shells. 337.

Tamarindiformis striatus Meusch., Krebs. 129.

Tapes (see Venus).

Tapes grus Holn., Post. Pliocene Fossils S. C. pl. vii, fig. 5. 37.

Taranis mörchii Malm., Report Blake Moll. 70.

'Tectaria dilatata D'Orb., Jahrb., x, 225.

Tectarius (see Littorina).

Tectarius antonii Phil., Marrat, Aigo Exp., 1876. 16. Nassau. Long Key Island.

Tectarius dilatata D'Orb., Marrat, Argo Exp., 1876. 14. Havana. Loug Key Island.

'Tectarius dilatatus D'Orb., Marrat, Argo Exp., 1876. 9. SV. Nassau. Athol Island.

Tectarius muricatns Born, Dall, Hemphill's shells. 335. Key West.

Tectarius muricatus Linu., Marrat, Argo Exp., 1S76. 6. Aa. SV. Tu-

cacas. Nassau. Long Key Island. Athol Island.

Tectarius pyramidalis Quoy., Marrat, Argo Exp., 1876. 12. Tucacas. Tectura (see Acmæa, Patella, Scutellina).

Tectura aspera Lam., Marrat, Argo Exp., 1876. 13. Santa Marta.

Tectura candeaua D'Orb., var., Marrat, Argo Exp., 1876. 9. SV.

Tectura cimelita Rve, Marrat, Argo Exp., 1876. 16. Nassan. Long

Key Island. Abaco.

Tectura eumingii Rve, Marrat, Argo Exp., 1876. 8. D.

Tectura fungus Meusch., Poulsen. 14.

Tectura lencopleura Gmel., Marrat, Argo Exp., 1876. 14. Havana.

Nassau. Long Key Island.

Tectura leucopleura Gmel., Poulsen. 14.

Tectura melanoleuca Gmel., Marrat, Argo Exp., 1876. 19. Abaco. 
Tectura melanoleuca Gmel., Poulsen. 14.

Tectura melanosticta Gmel., L. antillarum Slsy., Poulsen. 14.

Tectura pustula Helb. = P. puncturata Lam., Poulsen. 14.

Tectura striata Quoy \& Gaim., Marrat, Argo Exp., 1876. 8. D.

Tectus (see Tectarius).

Tectus gothicus Bolt., Krebs. 60 .

Tectus muricatus Chem., Krebs. 60.

Teinostoma (see Ethalia, Rotella).

Teinostoma pusilla Ptr., Poulsen. 13.

Tellidora (see Lucina, Tellina).

Tellidora cristata Reel., Mag. de Zool., 1843, pl. 60. Y. Fla.

Tellidora lunulata Holmes, Am. Marine Conch. 153.

Tellidora schrammi Recl., Guppy, Paria Fauna, 1577. 149.

Tellina (see Macoma, Strigilla, Tellidora, Tellinella, Aungulus, Peronæoderma).

Tellina (see Arcopagia), Arango, Famma Mal. Cuba, 187S. 246. Ca. Antilles. Fla.

Tellina (sce Semele), Arango, Fauna Mal. Cuba, 1876. 246. Ca. Bä. Tellina (see Semele), Arango, Fauna Mal. Cuba, 1878. 247. Ca. G. M. Ba.

Tellina acuta Woorl, Arango, Fauna Mal. Cuba, 1878. 243. Ca. Tellina acuta Wood, Krebs. 102.

Tellina agilis Stimpson, Dall, Hemphill's shells. 338. Sarasota Bay. Cedar Keys.

Tellina alternata Say, Am. Marine Conch. 148. SC. Fla.

Tellina alternata Say \& Sby., Krebs. 103.

Tellina alternata Say, Krebs. 104.

Tellina alternata Say, Moll. Cuba, ii, 243.

Tellina alteruata Sby., Moll. Cuba, ii, 243.

Tellina angulosa Gmel., Krebs. 103.

Tellina anomala Born, Arango, Fauna Mal. Cuba, 187S. 241. Ca.

Tellina anomala Born, Jour. de Conch., vii, 140.

Tellina anomala Born, Moll. Cuba, ii, 258.

Tellina anomala Chem., Krebs. 105.

Telliua antillarum D’Orb., Arango, Fauna Mal. Cuba, 18is. 241. Ca. Tellina antillarum D’Orb., Beau. 25. G.

Tellina antillarum D’Orb., Krebs. 99.

Tellina antillarum D'Orb., Mull. Cuba, ii, pl. xxx, figs. 45, 46. 250.

Tellina antoni Phil., Krebs. 100.

Tellina antoni Phil., Poulsen. 14.

Tellina antoni Phil., Dall, Report Blake Moll. 134.

Telliua antonii Hanl., Beau. 25. G.

Tellina autonii Phil., Arango, Fauna Mal. Cuba, 1878. 241. Ca. G. Tellina (Tellinella) antonii P'hil., Marrat, Argo Exp., 1876. 17. Nassau. Telliua arcuata Sby., Am. Jour. Conch., iii, 247. J.

Tellina athroa Rav., Ravenel. 64. SC. 
Tellina bimaculata Chem., Moll. Cuba, ii, 257.

Tellina bimaculata Lam., Moll. Cuba, ii, 257.

Tellina bimaculata Linn., Krebs. 100. Tort. SCx. ST. Td. Coa.

Tellina bimaculata Linn., Moll. Cuba, ii, 257.

Tellina brasiliaua Lam., Arango, Fauua Mal. Cuba, 1878. 243. Ca. M. Tellina brasiliana Lam., Jahrb., ıi, 248.

Tellina brasiliana Lam., Krebs. 102.

Tellina brasiliana Lam., Moll. Cuba, ii, 245.

Tellina brevifrons Say, Am. Marine Conch. 149.

'Tellina brevifrons Say, Krebs. 100.

Tellina candeaua D’Orb., Arango, Fauna Mal. Cuba, 1878. 241. M. 'Tellina candeana D'Orb., Beau. 25. G.

Tellina candeana D'Orb., Krebs. 100.

Tellina candeana D'Orb., Moll. Cuba, ii, pl. xxv, figs. 50-52. 251. Tellina caribea D’Orb., Arango, Fauna Mal. Cuba, 1878. 242. Ca. M. G. Ba.

Tellina caribæa D'Orb., Beau. 25. G.

Tellina caribæa D'Orb., Jour. de Conch., iv, 414. G.

Tellina caribæa D'Orb., Krebs. 100.

Tellina caribæa D'Orb., Moll. Cuba, ii, pl. xxr, figs. 47-49. 251.

Tellina caribæa D'Orb., Poulsen. 14.

Telliua carnaria Chem., Moll. Cuba, ii, 249.

Tellina carnaria Chem., Moll. Cuba, ii, 244.

Telliua carnaria D’Orb., Arango, Fauna Mal. Cuba, 1878. 242. Ca. Ba.

Tellina carnaria D'Orb., Moll. Cuba, ii, 244.

Tellina carnaria Limn., Araugo, Fauma Mal. Cuba, 1878. 242. Ca. Ba. Tellina carnaria Linn., Beau. 25. G.

Tellina carnaria Linn., Jour. de Conch., ii, 425. G.

Tellina carnaria Linu., Krebs. 100. Td. ST. SeT. Tort. SB. SCx.

Tellina carnaria Linn., Moll. Cuba, ii, 244.

Tellina cayenneusis Lam., Arango, Fauna Mal. Cuba, 1878. 242. Ca. Havana.

Tellina cayennensis Lam., Krebs. 100.

Telliua cayennensis Lam., Sby., Thes. Conchyl., 312, pl. 62, fig. 190. 1847. Fla.

Tellina cerina Ad., Krebs. 100.

Tellina cleryana D'Orb., Moll. Cuba, ii, 250.

Tellina consobrina D'Orb., Arango, Fauna Mal. Cuba, 1878. 242. M. 'Tellina consobrina D'Orb., Krebs. 100.

Telliua consobrina D'Orb., Moll. Cuba, ii, pl. xxvi, figs. 9-11. 254. M. Tellina consobrina D'Orb., Poulsen. 14.

Tellina constricta Brug., Jahrb., ii, 248.

Tellina coustricta Brug.=Ps. cayennensis Lam., Poulsen. 14.

Tellina constricta D'Orb., Arango, Fauna Mal. Cuba, 187S. Ca. Havana.

Tellina constricta D'Orb., Moll. Cuba, ii, 245.

Telliua constricta Phil., Krebs. 100. 
Tellina constricta Phil., Krebs. 101.

Tellina constricta Phil., Moll. Cnba, ii, 244.

Tellina crystallina Chem., Beau. 25. G.

Tellina crystallina Chem., Krebs. 101. NGr.

Tellina erystallina Chem., Poulsen. 14.

Tellina cubana D'Orb., Jour. de Conch., iv., 414. G.

Tellina cuneata D'Orb., Arango, Fauna Mal. Cuba, 1878. 242. Ca. Fla.

Tellina cuneata D’Orb., Beau. 25. G.

Tellina cuneata D'Orb., Krebs. 101.

Telliua cuneata D'Orb., Moll. Cuba, ii, 256. Ca. Fla.

Tellina cuneata D'Orb.=T. gouldii Hauley, Poulsen. 14.

Telliua cuneata D'Orb., Report Blake Moll. 134.

Tellina decora Say, Am. Marine Conch. 150.

Tellina decussata Ad., Krebs. 101. NGr.

Tellịa decussata Wood, Krebs. 106.

Tellina decussatula C. B. Adams, Synops., 10, 1845. J. L henta. of

Tellina digitalina Lam., Krebs. 101.

Tellina digitalina Linn., Bean. 25. G.

Tellina divaricata Chem., Krebs. 120.

Tellina elevata Sby., Am. Jour. Conch.; $\nabla, 55$.

Tellina elliptica Lam., Arango, Fauna Mal. Cuba, 1878. 243. Ca.

Tellina elliptica Lam., Krebs. 102.

Tellina eupareia Rav., Kavenel. 64. SC.

Telliua exilis D'Orb., Arango, Famna Mal. Cuba, 187S. 242. G.

Tellina exilis Lam., Arango, Fauna Mal. Cuba, 1878. 242. G.

Tellina exilis Lam., Bean. 25. G.

'Tellina exilis Lam., Jour. de Conch., ii, 425. G.

Tellina exilis Lam., Krebs. 101.

Tellina exilis Lam., Moll. Cuba, ii, 252.

Tellina exilis Sby., Arango, Fauna Mal. Cuba, 1878. 242. G.

Tellina exilis Sby., Moll. Cuba, ii, 252.

Tellina fausta Dillw., Arango, Fauna Mal. Cuba, 1878. 242. Ca. G.

M. Santa Lucia. Ba.

Telliua fausta Dillw., Moll. Cuba, ii, 246.

Tellina fausta D'Orb., Arango, Fauna Mal. Cuba, 1878. 242. Ca. G.

II. Santa Lncia. Ba.

Tellina fausta Sol., Beau. 25. G.

Tellina fausta Sol., Krebs. 101. ST. T. NGr.

Tellina fansta Sol., Poulsen. 14.

Tellina flexuosa D'Orb., Arango, Fauna Mal. Cuba, 1878. 243. J. G. Fla.

Tellina flexuosa Say, Arango, Fauna Mal. Cuba, 1878. 243. J. G. Fla.

Tellina flexuosa Sas, Beau. 25. G.

Tellina flexuosa Say, Krebs. 101. 
Tellina flexuosa Say, Moll. Cuba, ii, 248.

Tellina flexuosa Sby, Moll. Cuba, ii, 248.

Tellina gouldii Hanl., Report Blake Moll. 134. WI.

Tellina grïneri Phil., Jour. de Conch., iv, 414. G. •

Tellina grüneri Phil., Krebs. 101. ST. SJ.

Tellina grimeri Phil., Poulsen. 14.

Tellina grüneri Phil.,* Zt. Mal., ii, 150.

Tellina grünerii Phil., Beau. 25. G.

Tellina guadalupensis D’Orb., Arango, Fauna Mal. Cuba, 1878. 243. G.

Tellina guadalupensis D'Orb., Beau. 25. G.

Tellina guadalupeusis D'Orb., Jour. de Conch., iv, 414. G.

Tellina guadalupensis D’Orb., Krebs. 102.

Tellina guadalupeusịs D'Orb., Moll. Cuba, ii, pl.xxvi, figs.1-3. 252. G. Tellina guadalupeusis D'Orb., Poulsen. 14.

Tellina imbricata Chem., Krebs. 121.

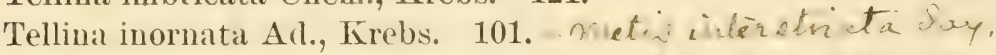

Tellina interrupta D’Orb., Arango, Fauma Mal. Cuba, 1878. 243. Ca. Antilles.

Tellina interrupta Sol., Krebs. 102. ST. SJ. Tort.

Tellina interrupta Sol., Poulsen. 14.

Tellina interrupta Wood, Arango, Fauma Mal. Cuba, 1878. 243. Ca. Antilles.

Tellina interrupta Wood, Beau. 25. G.

Tellina interrupta Wood, Jour. de Conch., ii, 425. G.

Tellina interrupta Wood, Moll. of Bermudas. 26.

Tellina interrupta Wood, Moll. Cuba, ii, 247.

Tellina interstriata Say, Krebs. 101.

Tellina intrastriata Say, Jahrb., ii, 248.

Telliua iris Say, Moll. Cuba., ii, 251.

Tellina iris Say, Am. Marine Conch. 149.

Tellina iris Say, Poulsen. 14.

Tellina lævigata Lam., Beau. 25. G.

Fellina levigata Linu., Dall, Hemphill's shells. 338. Sarasota Bay.

Tampa.

Tellina lævigata Linn., Krebs. 102.

Tellina lavigata Linu., Moll. of Bermudas. 25.

Tellina læris Chem., Krebs. 102.

Tellina læris Chem. = T. lævigata Linn., Poulsen. 14.

Tellina lævis Wood, Beau. 25. G.

Tellina lavis Wood, Jour. de Conch., ii, 425. G.

Tellina lavis Wood, Krebs. 101.

Tellina lateralis Say, Am. Marine Conch. 150. - … wetret whe : Tellina lineata D'Orb., Arango, Fanna Mal. Cuba, 1878. 243. Ca. M. Telliua lineata D'Orb., Moll. Cuba, ii, 245.

Telliua lineata Hanl., Beau. 25. G. 
Tellina lineata var. Hanl., Jour. de Conch., iv, 414. G.

Tellina lineata Hanl., Krebs. 102.

Tellina lineata Sby, Moll. Cuba, ii, 245.

Tellina lineata Turt., Arango, Fauna Mal. Cuba, 1878. 243. Ca. M.

Tellina lineata Turt., Jahrb., ii, 248.

Tellina lineata Turt., Krebs. 102.

Tellina (Tellinella) lineata Turt., Marrat, Argo Exp., 1876. 15. Havana.

Tellina lineata Turt., Moll. Cuba, ii, 245.

Tellina lineata Turt., Poulsen. 14.

Tellina lineata Turt., Thesaurus ('onchyl. 233, pl. 56, figs. 35, 36, 1847. Fla.

Tellina lintea Conrad, Jour. Acad. Nat. Sci. Phil., vii, 25̃9, pl. xx, fig. 3, 1837. Mobile, Ala.

Tellina lintea Conrad, Bush, Conn. Ac., vi, 476. Hatt.

Tellina listeri Bolt., Arango, Famua Mal. Cuba, 1878. 243. Ca. Antilles.

Tellina listeri Bolt., Krebs. 102.

Tellina longicallis Scacchi, Report Blake Moll. 133.

Tellina lusoria Say, Am. Marine Conch. 150.

'Tellina maculosa Lfm., Arango, Fauna Mal. Cuba, 187S. 243. Ca. Antilles.

Tellina maculosa Lam., Beau. 25. G.

Tellina maculosa Lam., Jour. de Conch., ii, 425. G.

Tellina macnlosa Lam., Krebs. 102.

Tellina maculosa Lam., Moll. Cuba, ii, 247.

Tellina magna Spengl., Araugo, Fauna Mal. Cuba, 1878. 243. Ca.

Tellina magna Spengl., Krebs. 102. VgI.

Tellina magna Spengl., Moll. of Bermudas. 26.

Tellina magna Spengl., = T. elliptica Lam., Poulsen. 14.

Tellina martinicensis D'Orb., Arango, Fanna Mal. Cuba, 1878. 244. G. M.

Tellina martinicensis D’Orb., Beau. 25. G.

Tellina martinicensis D'Orb., Krebs. 102.

Tellina martinicensis D'Orb., Moll. Cuba, ii, pl. xxvi, figs. 6-8. 253.

Tellina martinicensis D'Orb., Poulsen. 14.

Tellina mera Say, Dall, Hemphill's shells. 338. Key West.

Tellina mexicana Rre., Arango, Fauma Mal. Cuba, 1878. 243. Ca. Antilles.

Tellina mirabilis Phil., Arango, Fanna Mal. Cuba, 1878. 243. J. G. Fla.

Tellina mirabilis Phil., Krebs. 101.

Tellina mirabilis Phil., Moll. Cuba, ii, 248.

Tellina muricata Chem., Moll. Cuba, ii, 298.

Tellina muricata Spengl., Krebs. 121.

Tellina nitens Ad., Krebs. 102. 
Tellina nitens C. B. Adams, Synops., 10. 1845. J.

Tellina nirea Wood, Arango, Fauna Mal. Cuba, 1878. 244. Ca. Antilles.

Tellina nivea Wood, Krebs. 103. ST. SCx. SJ. Tort. Td. NGr.

Tellina nivea Wood, Moll. Cuba, ii, 248.

Tellina obliqua Wood, Krebs. 106.

Tellina obtusa Sby., Am. Jour. Conch., v, 55. ST.

Tellina occidentalis Mörch, Am. Jour. Conch., iii, 247. WI.

Tellina omoia Rav., Ravenel. 64. SC.

'Tellina oviformis Mus. Berol., Pfeifler, Archiv für naturg., 1840. 261.

$\mathrm{Ca.}$

Tellina pauperata D'Orb., Arango, Fauna Mal. Cuba, 1878. 244. M. G.

Tellina pauperata D'Orb., Beau. 25. G.

Tellina pauperata D’Orb., Krebs. 102.

Tellina pauperata D'Orb., Moll. Cuba, ii, pl. xxvi, figs. 18-20. 255. G. Tellina pauperata D'Orb., Poulsen. 14.

Tellina pellucida Phil., Beau. 25. G.

Tellina pellucida Phil., Jour. de Conch., v, 150. G.

'Tellina pellucida Phil., Krebs. 103.

Tellina pisiformis Linn., Jour. de Conch., iv, 414. G.

Tellina pisiformis D'Orb., Arango, Fanna Mal. Cuba, 1878. 244. Antilles. PR. G.

Tellina pisiformis D'Orb., Beau. 25. G.

Tellina pisiformis Gmel., Moll. Cuba, ii, 249.

Tellina pisiformis Linn., Arango, Fanna Mal. Cuba, 1878. 244. Antilles. PR. G.

Tellina pisiformis Linn., Krebs. 103, 119.

Tellina pisiformis Linn., Moll. Cuba, ii, 249.

Tellina pisiformis Sby., Moll. Cuba, ii, 249.

Tellina plectrum Hanl., Dall, Report Blake Moll. 134. WI.

Tellina polita Say, Am. Marine Conch. 148.

Tellina pudica Hanl., Beau. 25. G.

Tellina pudica Hanl., Krebs. 103. J.

Tellina (Tellinella) pulchella Lam., Marrat, Argo Exp., 1876. 12. Tucacas.

Tellina pulchella Lam., Poulsen. 14.

Tellina punicea Born, Arango, Fauna Mal. Cuba, 1878. 244. Ca. Havana. M. G.

Tellina punicea Born, Jahrb., ii, 248.

Tellina punicea Born, Jour. de Conch., ii, 425. G.

Tellina punicea Born, Jour. de Conch., iv, 414. G.

Tellina punicea Boru, Krebs. 99.

Telliua punicea Born, Krebs. 103, 104.

Tellina punicea Born, Mal. Bl., x, 228.

Tellina punicea Born, Moll. Cuba, ii, 243.

Yellina punicea Boru, Poulsen. 14. 
'Tellina punicea D'Orb., Arango, Fauna Mal. Cuba, 1878. 244. Ca. J. M. G.

Tellina punicea D'Orb., Moll. Cuba, ii, 243.

Tellina punicea Gmel., Moll. Cuba, ii, 243.

Tellina punicea Lam., Moll. Cuba, ii, 246.

Tellina punicea Sby., MIoll. Cuba, ii, 243.

Tellina radiata Chern., Moll. Cuba, ii, 253, 254.

Tellina radiata Chem., Moll. Cuba, ii, 248.

Tellina radiata D'Orb., Arango, Fauna Mal. Cuba, 1878. 244. Ca. Antilles.

Tellina radiata Lam., Moll. Cuba, ii, 248.

Tellina radiata Linn., Arango, Fauna Mal. Cuba, 1878. 244. Ca. Antilles.

Tellina radiata Linn., Beau. 25. Ga.

Tellina radiata Linn., Jour. de Conch., ii, 425. G.

Tellina radiata Linn., Krebs. 103.

Tellina radiata Linn., Mal. Bl., $x, 226$.

Tellina radiata Limn., Harrat, Argo Exp., 1876. 19. Abaco.

Tellina radiata Linn., Moll. of Bermudas. 26.

Tellina radiata Linu., Moll. Cuba, ii, 248.

Tellina radiata Linn., c. v., Poulsen. 14.

Tellina radiata Wood, Moll. Cuba, ii, 248.

Tellina remies Born, Krebs. 101.

Tellina remies Chem., Moll. Cuba, ii, 246.

Tellina remies Lam., Beau. 25. G.

Tellina remies Lam., Jour. de Conch., ii, 425. G.

Tellina remies Lam., Moll. Cuba, ii, 246.

Tellina remies Linn., Arango, Famna Mral. Cuba, 1878. 242. Ca. G. M. Santa Lucia. Ba.

Tellina remies Linn, Moll. Cuba, ii, 246.

Tellina reticulata Chem., Moll. Cuba, ii, 240.

Tellina reticulata Lam., Krebs. 106.

Tellina reticulata Linn., Moll. Cuba, ii, 240.

Tellina rosea D'Orl., Arango, Fannia Mal. Cuba, 1878. 244. Ca. II. G.

Telliva rosea Gmel, Arango, Famna Mal. Cnba, 1878. 244. Ca. M. G. Tellina rosea Gmel., Beau. 25. G.

Tellina rosea Gmel., Krebs. 104.

Tellina rosea Gmel., Moll. Cuba, ii, 246.

Tellina rubieunda Bolt., Krebs. 104.

Tellina rufescens Chem., Poulsen. 14.

Tellina schrammi Petit, Mal. Bl., x, 230. G.

Tellina schrammi Recl.," Jour. de Conch. iv, pl. vi, figs. 7, S. 152. G.

Tellina schrammi Recl., Jour. de Conch., iv, 414. G.

Tellina schrammii Recl., Beau. 25. G.

Tellina schrammii Recl., Krebs. 101.

Bull. 24-19 
Tellina sexratliata Lam., Arango, Fauna Mal. Cuba, 1878. 246. Ca., Antilles. Fla.

Tellina sexradiata Lam., Krebs. 100.

Tellina similis D'Orb., Moll. Cuba, ii, 251.

Tellina similis D'Orb., Arango, Fauna MIal. Cuba, 1878. 245. M.

Tellina similis Sby., Arango, Famna Mal. Cuba, 1878. 245. M.

Telliua similis Sby., Krebs. 103.

Tellina similis Sby., Mal. BI., x, 230.

Tellina (Angulus) similis Sby., Marrat, Argo Exp., 1876. 12. Tucacas. Harana.

Tellina similis Sby., Moll. Cuba, ii, 249.

Tellina simplex D'Crb., Arango, Fama Mal. Cuba, 1878. 245. G.

Tellina simplex D'Orb., Beau. 25. G.

'Tellina simplex D'Orb., Moll. Cuba, ii, pl, xxvi, figs. 15-17. 255. G.

Tellina sincera Hanl., Beau. 25. G.

Tellina sincera Hanl., Krebs. 103.

Tellina souleyetiana Recl., Beau. 25. G.

Tellina sonleyetiana Recl.,* Jour. de Conch. iii, pl. x, figs. 5, 51 . 253. G.

Tellina souleyetiana Recl., Jour. de Conch., iv, 414. G.

Tellina souleyetiana Recl., Krebs. 104. ST.

Tellina souleyetiana Recl., Poulsen. 14.

Tellina striata Chem., Krebs. 103.

Tellina striata Hanl., Beau. 25. G.

Tellina striata Hanl., Jour. de Conch., iv, 414. G.

Tellina striata Haul., Krebs. 103, 104.

Tellina striata Mont., Arango, Fauma Mal. Cuba, 1878. 243. Ca. M.

Tellina striata Mont., Jahrb., ii, 248.

Tellina subradiata Sehum., Arango, Fauna Mal. Cuba, 1878. 244. Ca. J. M. G.

Tellina subradiata Schum., Krebs. 101.

Tellina subradiata Schum., Krebs. 104. S'T.

Tellina subradiata Schum., Poulsen. 14.

Tellina sybaritica Dall, n. s., Report Blake Moll. 134.

Tellina tampaensis Con., ${ }^{*}$ Am. Jour. Conch. ii, pl. 15, fig. 8. 281. Fla. Tellina taylori Sby., Am. Jour. Conch., iii, 327. Mexico.

Tellina tenera Say, Am. Marine Conch. 148. New England to S. C.

Tellina tenera Say, Araugo, Fauna Mal. Cuba, 1878. 245. Ca.

Tellina tenera Say, Poulsen. 14.

Tellina tenera Sby., Arango, Fauna Mal. Cuba, 1878. 245. Ca.

Tellina tenta Say, Am. Marine Conch. 149. New England to S. C.

Tellina tumida Sby., Am. Jour. Conch., iii,247. J.

Tellina unimaculata Lam., Arango, Fama Mal. Cuba, 1878. 244. Ca. Antilles.

Tellina unimaculata Lam., Beau. 25. G.

Tellina unimaculata Lam., Jour. de Conch., ii, 425. G.

Tellina unimaculata Lam., Krebs. 103. 
Tellina unimaculata Lam., Moll. Cuba, ii, 248.

Tellina respuciana D'Orb., Arango, Famna Mal. Cuba, 1878. 245. J. M.

Tellina vespuciana D'Orb., Krebs. 104.

Tellina vespuciana D’Orb., Moll. Cuba, ii, pl. xxvi, figs. 12-14. 254. M. Tellina vitraea D’Orb., Moll. Cuba, ii, pl. xxvi, figs. 4-5. 253. G.

Tellina vitrea D'Orb., Fauna Mal. Cuba, 1878. 245. G.

Tellina vitrea D'Orb., Beau. 25. G.

Tellina vitrea D'Orb., Jour. de Conch., iv, 414. G.

Tellina ritrea D'Crb., Krebs. 104.

Tellina vitrea D'Orb., Poulsen. 14.

Tellinella (see Tellina), Marrat, Argo Exp., 1876. 12.

Tellinella antoni Gray, Krebs. 100.

Tellinella lineata Turton, Jahrb., ii, 248.

Tenagodus (see Siliquaria, Vermetus).

Tenagodus anguillæ Mörch, Mal. Bl., xxiv, 110. PR.

Tenagodus anguillæ Mörch, Poulseu. 10.

Tenagodus roseus Blainv., Poulsen. 10.

Tenagodus ruber Mörch, Mal. Bl., xxiv, 110. Ber.

Tenagodus ruber Schum., Mal. Bl., xxiv, 109.

Tenagodus squamatus Blainv., Mal. Bl., xxiv, 110. PR.

Terebellum burdenii T. \& H., n. s., Pleiocene Fossils S. C., pl. xxvi, fig. 11. 122.

Terebellum etiwanensis T. \& H., n. s., Pleiocene Fossils S. C., pl. xxvi, figs. $9,10.122$.

Terebellum striatum T. \& II., n. s., Pleiocene Fossils, S. C., pl. xxvi, fig. 7. 120 .

Terebellum subcinereum, \&c., Mal. Bl., xxiii, 127.

Terebra (see Acus, Myurella).

Terebra acicularis Lam., Krebs. 33.

Terebra aciculiua Lam., drango, Fanna Mal. Cuba, 1878. 205. Ca. G. Santa Lucia.

Terebra aciculina Lam., Mfoll. Cuba, ii, 152.

Terebra (Mrurella) armillata Hiukls, Marrat, Argo Exp., 1876. 12. Tucacas.

Terebra castanea Kien., Beau. 11. G.

Terebra castanea Kien., Krebs. - 33.

Terebra cinerea Born, Arango, Fauna Mal. Cuba, 1878. 205. Ca. G. Santa Lucia.

Terebra cinerea Born, Krebs. 33.

Terebra cinerea Born, Poulsen. 12.

Terebra cinerea D’Orb., Arango, Fauna Mal. Cuba, 1878. 205. Ca. G.

Santa Lucia.

Terebra cinerea D’Orb., Moll. Cuba, ii, 152.

Terebra clavata Rav., Ravenel. 11. SC.

Terebra concava Say, Kurtz. 8. NC. SC. 
Terebra costata C. B. Adams, Krebs. 33.

Terebra dislocata Say, Kurtz. 8. NC. SO.

Terebra hastata Gmel., Arango, Fauna Mal. Cuba, 1878. 205. Ca. G. Ba.

Terebra hastata Gmel., Jour. de Conch., iv, 418. G.

Terebra hastata Gmel., Krebs. . 33. A.

Terebra liastata Gmel., Poulsen. 12.

Terebra hastata Kien., Beau. 11. G.

Terebra jamaicensis C. B. Adams, Arango, Fauna Mal. Cuba, 1878. 205.

Ca. Antilles.

Terebra jamaicensis C. B. Adams, Beau. 11. G.

Terebra jamaicensis C. B. Adams, Contr. to Conch. 58. J.

Terebra jamaicensis C. B. Adams, * Jour. de Conch. vi, pl. r, fig. 9. 81. J. Terebra jamaicensis C. B. Adams, Krebs. 33. ST.

Terebra lacinula Gmel., Krebs. 33. Ag.

Terebra (Myurella) larvæformis Hinds, Marrat, Argo Exp., 1876. 16.

Nassau.

Terebra obesa Hinds, Jour. de Conch., v, 152. G.

Terebra (Truncaria) obesa Hinds, Poulsen. 13.

Terebra petitii Kien., Arango, Fauna Mal. Cuba, 1878. 206. Ca. Ba.

Terebra petiveriana Desh., T. aspera Hinds, Poulsen. 12.

Terebra rudis Gray, Arango, Fauna Mal. Cuba, 1878. 206. Ca. Ba.

Terebra rudis Gray, Krebs. 33.

Terebra rudis Gray, Moll. Cuba, ii, 152.

Terebra rudis Gray, Poulsen. 12.

Terebra stylina Adams, Jour. de Conch., iv, 418. G.

Terebratula anomioides Scacchi, Report Blake Moll. 104. WI.

Terebratula cubensis Pourt, Jour. de Conch., xvii, 116. Ca.

Terebratula cubensis Pourt., Am. Jourt. Conch., vi, 166. Ca.

Terebratula cubensis Pourt., Arango, Fauna Mal. Cuba, 1878. 274.

Ca. compare Isppass Is carneoides, Escene.

Terebratula enbensis Pourt., Jour. de Conch., xvii, 114. Ca.

Terebratula cubensis Pourt., Jour. de Conch., xrii, 115. Ca.

Terebratula cubensis Pourt., Report Blake Moll. 103.

Terebratulina cailleti Crosse, Arango, Fauna Mal. Cuba, 1878. 274.

Ca. Havana, G.

Terebratulina cailleti Crosse, ${ }^{*}$ Jour. de Conch. xiii, pl. i, figs. 1-3, 27. G.

Terebratulina cailleti Crosse, Jour. de Conch., xiv, 267, 271. G.

Terebratulina cailleti Crosse, Jour. de Conch., xvii, 114, 116. G.

Terebratulina cailleti Crosse, Report Blake Moll. 103.

Terebratulina cailleti Pourt., Araugo, Fauna Mal. Cuba, 1878. 274.

Ca. Havana. G.

Terebratulina caput-serpentis Linn., Jour. de Conch., xiv, 266.

Terebratulina cubensis Pourt., Jour. de Conch., xxii, 76. Ca.

Terebratulina septentrioualis Couth., Jour. de Conch., xiv, 266. 
Teredo bisiphites Less., Poulsen. 14.

Teredo (Lyrodus) chlorotica Gould, Dall, Hemphill's shells. 337. Cedar Keys.

Teredo dilatata Stimpson, Am. Marine Conch. 129.

Teredo dilatata Stimpson, Kurtz. 3. NC. SC.

Teredo megotara Hanl., Dall, Hemplill's shells. 337. Cedar Kejs.

Teredo navalis Lam., Moll. Cuba, ii, 211.

Teredo navalis Limu., Arango, Fana Mal. Cuba, 1878. 238. Ca.

Teredo navalis Linn., Krebs. 114. ST.

Teredo navalis Linn., Moll. Cuba, ii, 211.

Teredo norvagica Spengl., Beau. 27. G.

Teredo norvegica Spengl., Krebs. 114.

Thalessa (see Purpura), Marrat, Argo Exp., 1876. 5.

Thecidıum barretti Woodw., Jour. de Conch., xvii, 116.

Thecidium barretti Woodw., Am. Jour. Conch., vi, 151. J.

Thecidium barretti Woodw., Jour. de Conch., xir, 268.

Thecidium barretti Woodw., Jour. de Conch., xiv, 272. J.

Thecidium barretti Woodw., Report Blake Moll. 104.

Thecidium barretti Woodw., Am. Jour. Conch., iii, 91. J.

Thecidium mediterranean Sby, Jour. de Conch., xvii, 116.

Thecidium mediterraneum Sby., Am. Jour. Conch., vi, 151. J.

Thecidium mediterraneum Sby., lieport Blake Moll. 104.

Theliostyla (see Nerita).

Theliostyla varia Mörch, Krebs. 76.

Theodoxus (see Neritina, Neritella).

Theodoxus recliratus Say, Arango, Fauna Mal. Cuba, 1S78. 141. Ca.

Havana.

Thetis (see Gouldia, Crassatella, Poromya).

Thetis cerina C. B. Adams, Report Blake Moll. $130 .=$

Thetis cerina C. B. Adams, Synops. 9. 1845. J.

Thetis granulata Nyst \& Westendorp, Report Blake Moll. 108.

Thetis parva O. B. Adams, Report Blake Moll. 131.

Thetis parva C. B. Adams, Synops. 9. 1845. J.

Thracia dissimilis Guppy, Paria Fauna, 1877. 147. Am

Thracia inæequalis Adims, Am. Jour. Sci., o. s., xliii, 145, figs. 1-3, 1842.

Gulf of Mexico.

Thracia magnifica Jonas, Poulsen. 15.

Thracia nitida Verrill, List, 1584. 278. Chesapeake Bay.

Thracia papyracea Jeftreys, Report Blake Moll. 110.

Thracia phaseolina Kiener, Report Blake Moll. 110.

Thracia phaseolina Phil., Report Blake Moll. 110.

Thracia plicata Desh., Krebs. 109. ST.T.

Thracia plicata Desh., Marrat, Argo Exp., 1876. 12. Tucacas.

Thracia rugosa Con., Arango, Fauna Mal. Cuba, 1878. 240. Ca.

Thracia rugosa Con., Krebs. 108.

Thracia rugosa Con., Moll. Cuba, ii, 226.

$$
\text { * has ivo long separate sipturns fide Suppy. }
$$


Thracia rugosa D’Orb., Arango, Fauna Mal. Cuba, 187S. 240. Ca.

Thracia rugosa D'Orb., Moll. Cuba, ii, 226.

Thracia undata Cuming, Krebs. 109.

Thylacodus (see Vermetus).

Thylacodus brasiliensis Rouss.,* Mal. Bl., xxiv, 122. Bz.

Thylacodus decussatus Linn., Poulsen. 10.

Thylacodus limacella Mörch,* Mal. Bl., xxir, 122.

Thylacodus mühlenpfordtii Mörch, * Mal. Bl., xxiv, 122.

Thylacodus personata Mörch, Mal. Bl., xxiv, 119. SCx.

Thylacodus riisei Mörch,* Mal. Bl., xxiv, 121. ST.

Thylacodus riisei Mörch, Poulsen. 10.

Thylacodus thylacodus Mörch, Mal. Bl., xxir, 119. SCx.

Thylacodus costata Mörch,* Mal. Bl., xxiv, 119. 'J.

Thylacodus electrina Mörch, Mal. Bl., xxiv, 118. ST.

Thylacodus monile Mörch, Mal. Bl., xxir, 117. Ca.

Thylacodus occlusa Mörch,* Mal. Bl., xxiv, 117.

Thylacodus perlata Mörch,* Mal. Bl., xxiv, 119. ST.

Thylacodus proboscis Mörch,* Mal. Bl., xxiv, 120. ST.

Tichogonia domingensis Recl., Zt. Mal., x, 92. SD.

Tichogonia pfeifferi Dkr., * Zt. Mal., x, 88. Ca.

Tichogonia riisei Dkr.,* Zt. Mal., x, 91. ST.

Tigris barbadensis Klein., Mal. Cuba, ii, 54.

Timoclea (see Chione), Marrat, Argo Exp., 1876. 12. Tucacas.

$\checkmark$ Tivela (see Venus, Chione, Trigona).

(Tivela dillwyni Desh., Mal. Bl., viii, 25. =

Tivela mactroides Born, Mal. Bl., viii, 23.

Tivela mactroides Born, Marrat, Argo Exp., 1876. 12. Tucacas.

Tivela trigonella Lam., Mal. Bl., viii, 26.

Torcula (see Turritella).

Torcula exoleta Linn., Mal. Bl., xxiii, 127.

Toreulum sulco, \&e., Mal. Bl., xxiii, 128.

Torculuın sulco Fav, Mal. Bl., xxiii, 128.

Torinia (see Philippia, Heliacus, Solarium).

Torinia bisulcata D'Orb., Mal. B1., xxii, 157. J.

Torinia eyclostoma bis, Mke., Mal. Bl., xxii, 156.

Torinia cyclostoma Mke., Poulsen. 7.

Torinia cylindracea Chem., Mal. Bl., xxii, 156. ST.

Torinia cylindracea Chem., Poulsen. 7.

Torinia infundibuliformis Chem., Mal. B1., xxii, 155. ST.

Torinia infundibuliformis Chem., Poulsen. 7.

Torinia riisei Mörch, Mal. B1., xxii, 156. ST.

Tornatella (see Actæon).

Tornatella bullata Kieu., Mal. Bl., xxii, 171. Ca.

Tornatella bullata Kien., Krebs. 94.

Tornatella cubensis Pfr., Mal. B1., i, 152. Ca.

Tornatella cumingii Reeve, Mal. Bl., xxii, 169. PR. 
Tornatella fasciatus Lam., Report Blake Moll. 94.

Tornatella ovulum Pfr., Mal. Bl., xxii, 170. Ca.

Tornatella orulum Pfeiffer, Arehir fiir naturg., 1840. 256. Ca.

Tornatella punctata D'Orb., Araugo, Fauna Mal. Cuba, 1878. 162.

Ca. ST. J. M. G.

Tornatella punctata D'Orb., Bèu.- 18. G.

Tornatella punctata D'Orb., Mal. Bl., xxii, 170. Ca.

Tornatella punctata D'Orb., Moll. Cuba, i, pl. xvii, figs. 10-12. 230.

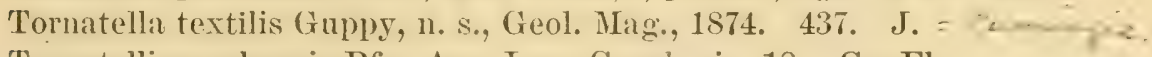

Tornatellina cubensis Pfr., Am. Jour. Conch., iv, 18. Ca. Fla.

Tornatina (see Bulla, Cylichna).

Tornatina bullata Kien., Mal. Bl., xxii, 171. Ca.

Toruatina bullata Kien., Ponlsen. 8.

Tornatina caualiculata D’Orb., Grupy, Geol. NIag., 1874. 445. Td. = Lellat A

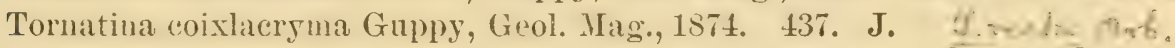

Tornatina liratispira Ed. Smith, Mal. Bl., xxii, 171. Bz.

Tornatina olivula A. Ad., Mal. Bl, xxii, 171. Ca. = bullata Kien.

Tornatina pusilla Pfr., Mal. Bl., xxii, 171. Ca. ? = marginella jor.

Tornatina pusilla Pfr., Poulsen. 8.

Tornatina recta D'Orb., Mal. Bl., xxii, 171. ST. G. Antilles.

Tornatina recta D'Orb., Poulsen. 8.

Trachyeardium (see Cardium), Mirrrat, Argo Exp., 1876. 6.

Trachycardium isocardia Mörch, Krebs. 115.

Trachyeardium muricatum Linn., Jahrb., ii, 249.

Tralia (see Melampus, Detracia, Tifata, Auricula).

Tralia eingulata Pfr., * Am. Jour. Conch. ir, pl. 18, fig. 10. 9, 17. Fla.

Tralia cingulata I'fr., Dall, Hemphill's shells. 323. Key West.

Tralia floridana Shuttlew., Am. Jour. Conch. ir, pl. xviii, fig. 11. 9,17. Fla.

Tralia pusilla Gmel.,* Am. Jour. Couch. ir, pl. xriii, fig. 9. 9, 17. Fla.

Tralia pusilla Gmel., Mrarrat, Argo Exp., 1876. 16. Nassau.

Trapezium (see Cardita, Cypricardia).

Trapezium dactylus Brug., Krebs. 123. VgI.

Trapezium gracilis Shuttlew., Krebs. 123.

Trapezium hians Blv., Krebs. 114.

Trichotropis migrans Dall, n. s., Report Blake Moll. 71.

Trichotropis orbignyanum Petit, Jour. de Conch., $v, 37$.

Tricolia (see Eutropia), Marrat, Argo Exp., 1876. 11.

Tridachia (see Elysia).

Tridachia crispata Bergh, Mal. Bl., xxii, 180.

Tridachia erispata Oersted, Jour. de Conch., xi, 40. SCx.

Tridachia crispata Oersted, Mal. Bl., xxii, 180.

Tridachia schrammi Desh., Jour. de Conch., xi, 41.

Triforis (see Triphoris, Cerithium).

Triforis (bigemma Watson, rar.?) abruptus Dall, n. s., Report Blake

Moll. 84. Cape San Antonio. Yucatan Strait. 
Triforis bigemma Watson, Report Blake Moll. 81. Yucatan Strait.

Triforis bigemma Watson, Lin. Soc. Jouru., 1S80, xv, 101. Cul.

Triforis colon Dall, n. s., Report Blake Moll. 86. Havana. Cape San Antonio.

Triforis cylindrellus Dall, 11. s., Report Blake Moll. 83. Cape San Antonio.

Triforis dealbatus Ad., Krebs. 51.

Triforis decoratus Ad., Krebs. 51.

Triforis exiguus Ad., Krebs. 51.

Triforis guttata Guppy, Geol. Mag., 1874. 438. Td.

Triforis hircus Dall, u. s., Report Blake Moll. 83. Yucatan Strait.

Triforis ibex Dall, n. s., Report Blake Moll. 86.

Triforis inflatum Watson, Report Blake Moll. 81. Yucatan Strait.

Triforis inflatum Watson, Lin. Soc. Journ., 1880, xv, 103. Cul.

Triforis intermerius Ad., Krebs. 51.

Triforis intermedius Dall, n. s., Report Blake Moll. 85.

'Triforis (Ino) longissimus Dall, n. s., Report Blake Moll. 80.

Triforis melanura Ad., Krebs. 51.

Triforis mirabilis Ad., Krebs. 51.

Triforis modestus Ad., Krebs. 51.

Triforis nauus Ad., Krebs. 51.

Triforis nigrocinctus Adams, Dall, Hemphill's shells. 333. Cedar Keys. Key West.

Triforis ornatus Desh., Krebs. 51. ST. SCx.

Triforis pulchellus Ad., Krebs. 52.

Triforis torticulus Dall, n. s., Report Blake Moll. 82. Yucatan Strait. Triforis triserialis Dall, n. s., Report Blake Moll. 84. Yucatan Strait. Triforis turris thomæ Chem., Arango, Fauna Mal. Cuba, 1878. 209. Ca. G.

Triforis turris-thomæ D'Orb., Krebs. 52.

Triforis turris-thomæ D'Orb., Report Blake Moll. 81. Ca. G.

Triforis turris-thomæ D’Orb., Bush, Conn. Ac., vi, 463. Hatt.

'Trigona (see Tivela).

Trigona angulifera Phil., Mal. Bl., viii, 226.

Trigona incerta Röm., Mal. Bl., viii, 26. = J. Lrgonectea w au

Trigona mactroides Born, (̇uply, Paria Fauna, 1877. 152.

Trigona trigonella Lam., (xuplyy, Paria Fauna, 1877. 152.

'Trigonituna ornata Chemu, Report Blake. Moll. 105.

'Trigonulina (see Verticordia).

'Trigounliua oruatal W'Orb., Arango, Fanna Mal. Cuba, 1878. 254. J.

Trigonulina ornata D'Ork.. Jour. de Conch., viii, 295, 299. J.

'Trigounlina ornata W'illb., "Noll. Cubs, ii, pl. xxvii, tigs. 30-33, 292.

Trigonulina ornata D'Orb., Report Blake Moll. 105.

'Triphoris (see Triforis).

Triphoris dealbatus C. B. Adaus, * Mal. Bl., xxiii, 110. J.

Triphoris lecoratus C. B. Adams, ${ }^{*}$ Mal. Bl., xxiii. 109. J. 
Triphoris decoratus C. B. Adams, Poulsen. 9.

Triphoris exiguus C. B. Adams, * Mal. Bl., xxiii, $106 . \quad J$.

Tripboris intermedius C. B. Adaws, * Mal. Bl., xxiii, 108. J.

Triphoris intermedius C. B. Adams, Poulsen. 9.

Triphoris melanura C. B. Adams, * Mal. Bl. xxiii, 108. J.

Triphoris melanura U. B. Adams, Poulsen. 9.

Triphoris modestus C. B. Adaus, * Mal. Bl, xxiii, 107. J.

Triphoris molestus C. B. Adams, Ponisen. 9.

Triphoris nana Sby., Mal. Bl., xxiii, 107. J.

'Triphoris nanus C. B. Adams,* Mal. Bl., xxiii, 107. J.

'Triphoris nanus C. B. Adams, Poulsen. 9.

Triphoris nigrocinetum Gld., Mal. Bl., xxiii, 106.

Triphoris nigrocinetum Stimp., Mal. Bl., xxiii, 106.

Triphoris nigrocinetus C. B. Adams, Am. Marine Conch. 72. NC.

Triphoris nigrocinetus C. B. Adams,* Mal. Bl., xxiii, 106.

Triphoris ornatus Desh., Mal. Bl., xxiii, 109. ST.

Triphoris o: natus Mö.ch, Mal. Bl., xxiii, 109.

Triphoris ornatus Desh., Poulsen. 9.

Triphoris ornatus Hinds, Beau. 11. G.

Triphoris ornatus Hinds, Jour. de Conch., ii, 4:8. G.

Triphoris turris-thoma Chem., Beau. 11. G.

Triphoris turris-thomæ Chem., Jour. de Conch., iv, 417. G.

Triphoris turris thom Chem., Mal. Bl., xxiii, 110. ST.

Triphoris turris thomæ Chem., Poulsen. 9.

Triphoris turris-thomæ Mörch, Mal. Bl., xxiii, 110.

Triphoris variegatus A. Ad.,* Mal. Bl., xxiii, 110. ST.

Triphoris variegatus A. Ad., Poulsen. 9.

Triptychus (see Obeliscus).

'Triptychus niveus Mörch, Mal. Bl., xxii, 159. ST. V.

Triton (see Tritonium).

Triton americanum D’Orb., Arango, Fanna Mal. Cuba, 1878. 213. Ca.

G. Santa Lncia. ST. Ba.

Triton americanum D'Orb., Mal. Bl., xxiv, 28.

Triton americanum D'Orb., Moll. Cuba, ii, pl. xxiii, tig. 22. 163.

Triton antillarum D'Orb., Arango, Famna Mal. Cuba, 1878. 213. Ca.

G. Ba.

Triton antillarum W'Orb., Beau. 8. G.

Triton antillarum D'Orb., Jahrb., v, 361.

Triton antillarum D'Orb., Nachblt., 1877. 59.

Triton antillarum D'Orb., Jour. de Conch., iv, 417. G.

Triton antillarum D'Orb., Mal. Bl., xxiv, 31, 32. G.

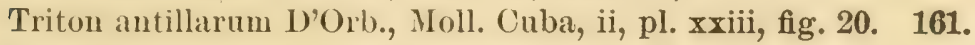

Triton antillarum D'Orb., Poulsen. 10.

'Triton aquatile Rre., Mal. Bl., xxiv, 29.

Triton aquatile Rie., Poulsen. 10. 
Triton aquatilis Rve., Araugo, Fauma Mal. Cuba, 1878. 213. Ca. G. Santa Lucia. ST. Ba.

Triton articulare Mke., Zt. Mal., x, 72. SV.

Triton balteatus Beck, Mal. Bl., xxiv, 32 .

Triton bituberosum Lam., Jour. de Conch., ii, 429. G.

Triton brazilianum Gould, Mal. Bl., xxiv, 28.

Triton cancellinum Roissy, Jour. de Conch., viii, 359.

Triton cantrainei Recl., Mal. Bl., iv, 27. G.

Triton cantrainei Recl., Arango, Fauna Mal. Cuba, 1878. 211. Ca. Havana. G. Ba.

Triton cantrainei Recl., Jahrb., ir, 244. G.

Triton cantrainei Recl., ${ }^{*}$ Jour. de Conch. iv, pl. viii, fig. 10. 246. G. Triton cantrainei Recl., Jour. de Conch., iv, 418. G.

Triton cantrainei Jiecl. (= Murex pamperculus Ad.), Jour. de Conch. v, 156. G.

Triton cantrainei Recl., Krebs. 21. S'T. SJ.

Triton caribxorum D'Orb., Jour. de Conch., v, 155. G.

Triton caribæum D'Orb., Arango, Fauna Mạl. Cuba, 1878. 214. Ca. Matanzas.

Triton caribæüm D'Orb., Cuba, ii, 162.

Triton caudatus Gray, Jahrb., v, 247.

Triton chemnitzii Gray, Mal. Bl., xxiv, 32.

Triton chlorostoma D’Orb.; Arango, Fauna Mal. Cuba, 1878. 212. Ca.

Havana. G. M. Santa Lucia. Ba.

Triton chlorostoma Lam., Arango, Fauna Mal. Cuba, 1878. 212. Ca.

Havana. G. M. Santa Lucia. Ba.

Triton chlorostoma Lam., Moll. of Bermudas. 16.

Triton chlorostoma Lam., Noll. Cuba, ii, 161.

Triton chlorostomum Kien., Mal. Bl., xxiv, 29. Ca.

Triton chlorostomum Lam., Arango, Fauna Mal. Cuba, 1878. 212. Ca.

Havana. G. M. Santa Lucia. Ba.

Triton chlorostomum Lam., Beau. S. G.

Triton chlorostomum var. Lam., Jour. de Conch., ii, 429. G.

Triton chlorostomum Lam., Krebs' Remarks. 398.

Triton chlorostomum Lam., Mal. Bl., xxiv, 29. Ca.

Triton chlorostomum Lam., Poulsen. 10.

Triton cingulatus Lam., Jahrb., v, 247.

'I'riton clathratum Lam., Beau. 8. G.

Triton clathratum Lam., Jahrb., v, 370.

Triton clathratum Lam., Jour. de Conch., iv, 417. G.

'Triton clathratum Lam., Jour. de Conch., viii, 359.

Triton elathratum Lam., Mal. Bl., xxiv, 34.

Triton clathratum Lam., Mal. Bl., xxir, 34. G.

Triton commutatum Dlir., Arango, Fauna Mal. Cuba, 1878. 212. Ca. T'riton commutatum Dkr., Jahrb., v, 243. G. Ba.

Triton commutatum Dkr., Mal. Bl., xxir, 34 . 
Triton costatum Born, Arango, Fauna Mal. Cuba, 1878. 212. Ca. G. Ba.

Triton costatum Born, Beau. 8. G.

Triton costatum Born, Mal. Bl., xxiv, 27.

Triton costatum Born, Poulsen. 9.

'Triton cynocephalum Kien., Mal. Bl., xxiv, 32, 33.

Triton cynocephalum Lam., Arango, Fauna Mal. Cuba, 1878. 212. Ca. G. Ba.

Tritón cynocephalum Lam., Beau. 8. G.

Triton cynocephalum Lam., Jour. de Conch., iv, 417. G.

Triton cynocephalum Lam., Mal. Bl. xxiv, 32 .

Triton cynocephalum Lam., Poulsen. 10.

Triton cynocephalum Lam., Jahr., v, 250.

Triton femorale D’Orb., Arango, Fauna Mal. Cuba, 1878. 212. Ca.

G. M. Santa Lucia. Ba.

Triton femorale Lam., Moll. Cuba, ii, 160.

Triton femorale Linn., Araugo, Fauna Mal. Cuba, 1878. 212. G. M.

Santa Lucia. Ba.

Triton femorale Linn., Beau. S. G.

Triton femorale Linn., Jahrb. Nachblt., 1877. 59.

Triton femorale Linn., Jour. de Conch., ii, 429. G.

Triton femorale Linn., Mal. Bl., xxiv, 31.

Triton femorale Linn., Poulsen. 10.

Triton gibbosus Rve., Jour. de Conch., $\nabla, 38$.

Triton gracile Rve., Mal. Bl., xxiv, 30 .

Triton gracilis Rve., Jahrb., v, 363.

Triton krebsii Mörch, Jahrb., v, 246.

Triton krebsii Mörch, Mal. Bl., xxiv, 30.

Triton krebsii Mörch, Poulsen. 10.

Triton labiosum Wood, Arango, Fauna Mal. Cuba, 1878. 213. Ca.

Havana. Matanzas. G. Ba.

Triton labiosum Wood, Mol. Bl., xxiv, 34.

Triton labiosum Wood, Poulsen. 10.

Triton labiosus Wood, Jahrb., v, 249.

Triton labiosus Wood, Mal. Bl., xxiv, 34.

Triton lauceolatum Desh., Mal. Bl., xxiv, 25.

Triton lanceolatum Kien., Jour. de Conch., v, 153. G.

'Triton lanceolatum Mlie., Arango, Fauna Mal. Cuba, 1878. 213. Ca.

J. PR. G. Ba.

'Triton lanceolatum Mke, Beau. 8. G.

'Triton lanceolatum Mke., Mal. BI., xxiv, 25. G.

Triton lanceolatum Mke., Poulsen. 9.

Triton lauceolatum Mörch, Mal. Bl., xxiг, 25.

Triton lanceolatum Rve., Mal. Bl., xxiv, 25.

Triton lanceolatus Kien., Moll. of Bermudas. IB. 
Triton lanceolatus Mke., Jahrb., v, 366.

Triton latior Mörch, Mal. Bl., xxiv, 29. Ca. G.

Triton liweolatum Conrad, Am. Journ. Sci., n. s., ii, 1846. 398. Fla.

Triton lineolatum Conrad, Proc. Acad. Nat. Sci. Phil., n. s., i, 26, pl. 1, fig. 35, 1845. Tampa.

Triton loroisi Petit, Arango, Fauma Mal. Cuba, 1878. 213. Сa. Наvana. Matanzas. G. Ba.

Triton loroisi Petit, Jahrb., v, 249.

Triton loroisi Petit, Jour. de Conch., iv, 418. G.

Triton loroisi Petit, Jour. de Conch., v, 38.

Triton loroisi Petit, Mal. Bl., xxiv, 34.

Triton loroisi Petit,* Jour. de Conch., iii, pl. ii, fig. 8. 53. G.

Triton loroisii Petit, Beau. 8. G.

Triton maculosum Gmel., Jour. de Conch., iv, 418. G.

Triton marmoratum Con., Nachblt, 1877. 58.

Triton marmoratum Link, Jahrb., v, 243.

Triton marmoratum Link, Mal. Bl., xxiv, 26.

Triton marmoratum Link $=\mathrm{T}$. variegatum Lam., Poulsen. 9.

Triton martini D’Orb., Nachblt., 1877. 59.

Triton martinianum D'Orb., Arango, Fauna Mal.Cuba, 1878. 213. Ca.

G. Santa Lucia. ST. Ba.

Triton martinianum D’Orb., Beau. 8. G.

Triton martinianum D'Orb., Mal. Bl., xxiv, 28.

'Triton martinianum D'Orb., Moll. Cuba, ii, 162.

Triton martinianum D'Orb., Poulsen. 10.

Triton martiuianus D'Orb., Jahrb., v, 245.

Triton minor Mörch, Nachblt., 1877. 59.

Triton nobile Uon., Nachblt., 1877. 58.

Triton nolsile Con.,* Mal. Bl., xxiv, 27. J.

Triton nobile Con., Poulsen. 9.

Triton nobilis Con., Jahrb., v, 242.

Triton nobilis Cou., Proc. Acad. Nat. Sci. Phil., iv, 121. 1848. WI. Triton uodulus Mart., Arango, Fauna Mal. Cuba, 1878. 213. Ca. G. Ba.

Triton nodulus Mörch, Jahrb., $\nabla, 361$.

Triton nodulus Mörch, Mal. Bl., xxiv, 31. G.

Triton obscurum Rre, Beau. 8. G.

'Triton obscurus Mörch, Jahrb., v, 365.

Triton obscurus Reeve, Mal. Bl., xxiv, 25.

Triton olearium Linn., Mal. Bl, xxiv, 28.

Triton parvus Ad., Arango, Fauna Mal. Cuba, 1878. 213. Ca. Havana. 'J.

Triton parvus C. B. Aciams, Contr. to Conch. 59. J.

Triton pileare D'Orb., Mal. Bl., xxiv, 28.

Triton pileare D'Orb., Moll. Cuba, ii, 163.

Triton pileare Lam., Mal. B1., xxir, 22. 
Triton pileare Iam., Beau. 8. G.

'Triton pileare Lam., Mal.Bl., xxiv, 28.

Triton pileare Lam., Moll. Cuba, ii, 162.

Triton pileare Linn., Jour. de Couch., iv, 418. G.

Triton pileare Linn., Jour. de Conch., viii, 358.

Triţon pileare Mörch, Mal. Bl., xxiv, 29.

Triton pilearis Lam., Arango, Fauna Mal. Cuba, 1878. 213. Ca. G. Santa Lucia. ST. Ba.

Triton pılearis Lam., Moll. of Bermudas. 16.

Triton pilearis Linn., Arango, Fauna Mal. Cuba, 1878. 213. Ca. G.

Santa Lucia. S'T. Ba.

Triton poulsenii Mörch, Jahrb, v. 247.

Triton ponlsenii Mörch,* Mal. Bl., xxir, 33. ST.

Triton poulsenii Mörch, Poulsen. 10.

Triton pulchellus C. B. Arlams, Arango, Fanna Mal. Cuba, 1878. 212.

Ca. Havana. G. M. Santa Lucia. Ba.

Tritou pulchellus C. B. Adams, Contr. to Conch. $60 . \mathrm{J}$.

Triton pulchellus C. B. Adams, Jahrb., v, 245.

Triton pulchellus C. B. Adams, Krebs' Remarks. 398.

Triton pulchellus C. B. Adams, Mal. Bl., xxiv, 30. J.

Triton var. pumilio Mörch, Mal. Bl. xxiv, 29.

'Triton pyriformis Con., Ardngo, Fauna Mal. Cuba, 1878. 213. Ca. G. Ba.

Triton pyriformis Con., Jahrb., v, 361.

Triton pyriformis Con., Mal. B1., xxiv, 32. G.

Triton reticulare Linn., Beau. 8. G.

Triton rostratum Mart., Mal. Bl., xxiv, 33.

Triton rostratus Mörch, Jahrb., v, 247.

Triton rubecula Linn., Arango, Fauna Mal. Cuba, 1878. 214. Ca.

Triton rubecula Linn., Jahrb. v, 245. ST.

Triton rubecula Linn., Mal. Bl., xxiv, 29. ST.

Triton succinctum Lam., Arango, Fauna Mal. Cuba, 1878. 212. Ca. G: Ba.

Triton succinctum Lam.. Beau. 8. G.

Triton suecinetum Lam., Mal. Bl., xxiv, 28.

Triton succinctum Lam., Moll. Cuba, ii, 163.

Triton tesselatus Rive, Krrebs Remarks. 398. Ber. SCx. ST.

Triton testaceum Mörch, Nachblt., 1877. 58.

Triton testaceum Mörch, Mal. Bl., xxiv, 25.

Triton testaceum Mörcn, Poulsen. 9.

Triton testaceum Mörch, Alango, Fauma Mal. Cuba, 1878. 214. Ca. Ba.

Triton testaceus Mörch, Jahrb., v, 365.

Triton testaceus Mörch, Mal. Bl. xxir, 25.

Triton thersites Rve., Jahrb., . , 361.

Triton thersites Rve., Mal. Bl., xxiv, 30. SCx.

Triton thersites Rve.; Poulsen. 10. 
Triton tigrinum Brod., Beau. 8. G.

Triton tranquebaricum Lam., Mal. Bl., xxiv, 32.

Triton tranquebaricum Lam., Poulsen. 10.

Triton tranquebaricum Rve., Mal. Bl., xxiv, 32.

Triton tuberosum Lam., Jour. de Conch., iv, 417.

Triton tuberosum Lam., Moll. Cuba, ii, 162.

Triton tuberosum Gld., Jahrb., v, 361.

Triton tuberosum Gld., Mal. Bl., xxir, 31. M.

Triton tuberosum Lam., Mal. Bl., xxiv, 31. G.

Triton tuberosum Rve., Beau. 8. G.

Triton tuberosum Rve., Mal. Bl., xxiv, 31. G.

Triton tuberosus Lam., Araugo, Fauna Mal. Cuba, 1878. 213. Ca. G. Ba.

Triton undosum Kien., Jahrb., v, 247.

Triton undosum Kien., Mal. Bl., xxiv, 33. ST.

Triton variegatum Blainv., Mal. Bl., xxiv, 27. J.

Triton variegatum Con., Mal. Bl., xxiv, 27.

Triton variegatum Kien., Mal. Bl., хxis, 26.

Triton variegatum Lam., Beau. 8. G.

Triton variegatum Lam., Jahrb., v, 243.

Triton variegatum Lam., Jour. de Conch., iv, 417. G.

T'riton variegatum Lam., Mal. Bl., xxiv, 26.

Triton variegatum Rre., Mal. Bl., xxiv., 27. J.

Triton variegatus Lam., Moll. of Bermudas. 16.

Tritou veliei Calkins, Dall, Hemphill's shells. 341. Key West.

Triton vespaceum Lam., Mal. Bl., xxiv, 30.

Triton respaceus Lam., Araugo, Fauna Mal. Cuba, 1878. 214. Ca. Matanzas.

Triton vestitum Hinds, Beau. 8. G.

Triton vestitum Hinds, Mal. Bl., xxiv, 29.

Tritonidea (see Cantharus, Pisania, Pollia).

Tritonidea (see Cantharus), Marrat, Argo Exp., 1876. 6.

Tritonidea autillarum Dkr., Arango, Fauma Mal. Cuba, 1878. 214. Ca. Havana.

Tritonidea auricula Lam., Arango, Fauna Mal. Cuba, 1878. 214. Ca. Matanzas.

Tritonidea cancellaria Con., Dall, Hemphill's shells. 327. Cedar Keys, Gulf of Mexico.

Tritonidea floridana Petit, Dall, Hemphill's shells. 327.

Tritonidea tincta Con., Dall, Hemphill's shells. 327. Cedar Kejs. Florida Keys.

Tritonium (see Triton), Arango, Fauma Mal. Cuba, 1878. 213. Ca. G. Ba.

Tritonium aequale Rve., Krebs. 22.

Tritonium americanum D'Orb., Krebs. 22.

Tritonium antillarum D'Orb., Krebs. 22.

* Proc. Daw Acad. Sei. ii, p. $235(302)$ prii-figp 1, 2, 1878 . 
Tritonium (Gutturnium) antillarum D'Orb., Marrat, Argo Exp., 1876. 16. Nassau.

Tritopium cantrainii Recl., Krebs. 23.

Tritonium caribæum D'Orb., Krebs. 23.

Tritonium chlorostomum Lam., Krebs. 23. ST.

Tritonium clathratum Lam., Krebs. 24. Tort.

Tritonium commutatum Dkr., Arango, Fauna Mal. Cuba, 1878. 212. Ca. G. Ba.

Tritonium costatum Born, Krebs. 23.

Tritonium cutaceum Linn., Mal. Bl., xii, 128.

Tritonium cynocephalum Lam., Krebs. 23. SCx. SM. Td.

Tritonium (Gutturnium) cynocephalum Lam., Marrat, Argo Exp., 1876.

9. SV. Tucacas.

Tritonium femorale Linn., Krebs. 23. Tort. ST. SCx. SH. Td.

Tritonium labiosun Wood, Krebs. 23. ST. SCx. SM. Tort.

Tritonium lauecolatum Mlke, Krebs. 23. ST. SCx. Td. Ag. SM.

Tritonium loroisii Petit, Krebs. 23.

Tritonium marmoratum Lam., Krebs. 24. VCz. ST.

Tritonium marmoratum Link, Mal. Bl., xxiv, 26.

Tritonium martinianum D'Orb., bis, Krebs. 24.

Tritonium nobile Con., Krebs. 23.

Tritonium obscurum Rve., Krebs. 23. SOx.

Tritonium parvum Ad., Krebs. 24. ST. SB. Ag.

Tritonium (Simpulum) pileare Linn., Marrat, Argo Exp., 1876. 15. $\mathrm{VCz}$.

Tritonium pileare Liun. \& Lam., bis, Krebs. 24. ST. Tort. SM. Bb.

Tritonium pulchellum Ad., Krebs. 24.

Tritonium reticulare Linn., Krebs. 24.

Tritonium rostratum Mart., Krebs. 24.

Tritonium succinctum Lam., Krebs. 23. Tort.

Tritonium testaceum Mörch, Krebs. 24. ST. SCx. SM.

Tritonium tigrinum Brod., Krebs. 24.

Tritonium tritonis Linn., Marrat, Argo Exp., 1876. 9. SV. Puerto Cabello. Tucacas.

Tritonium (Gutturnium) tnberosum Lam., Narrat, Argo Exp., 1876. 6. Aa. Havana. Long Key Island.

Tritonium tuberosum Rve., Krebs. 22. ST. Tort.

Tritonium undosum Kien., Krebs. 24.

Tritonium variegatum Lam., Krebs. 24.

Tritonium variegatum Linn., Marrat, Argo Exp., 1876. 16. Nassau.

Tritonium vestitum Hinds. Krebs. 24.

Tritonium (Simpulum) restitum Hiuds. Marrat, Argo Exp., 1876. 6. Aa.

Trivia (see Cypræa), Jour. de Conch., v, 153. G.

Trivia (see Cypræa), Marrat, Argo Exp., 1876. 6. Aa.

Trivia affiuis Marrat, Am. Jour. Conch., iv, 48. 
Trivia affinis Marrat, Jahrb., viii, 155.

Trivia armandina Ducl., Jahrb., viii, 154. G.

Trivia candidula Gask.; Mal. Bl., xxiv, 50.

Trivia candidula Gask., Poulsen. 10.

Triria candidula Gask., Report Blake Moll. 137.

Trivia globosit Gray, Jahrb., viii, 156.

Trivia globosa Gray, Mal. Bl., xxiv, 50. Ca.

Trivia globosa Gray, Poulsen. 10.

Trivia globosa Gray, Report “ Blake” Moll. 137.

Trivia labiosa Gask., Jahrb., viii, 154.

Trivia nirea Gray, Arango, Fauna Mal. Cuba, 1878. 185. Ca. G. Ba.

Trivia nivea Gray, Jahrb., viii, 155. Fla.

Trivia nivea Gray, Mal. Bl., xxiv, 50.

Trivia nivea Gray, Poulson. 10.

Trivia oryza Gray, Mal. Bl., xxiv, 50.

Trivia oryza Lam., Arango, Fauna Mal. Cuba, 1878. 185. Ca.

Trivia oryza Lam., Report Blake Moll. 137.

Trivia pediculus Linn., A rango, Fauna Mal. Cuba, 1878. 185. Ca. Antilles.

Trivia pediculus Linn., Jahrb., viii, 154.

Trivia pediculus Lion., Mal. Bl., xxiv, 48. Fla.

Trivia pediculus Linu., Marrat, Argo Exp., 1876. 7. Barbuda. SV. Abaco.

Tricia pediculus Linn., dark red var., Marrat, Argo Exp., 1876. 16. Nassau.

Trivia pediculus Linn., Poulsen. 10.

Trivia pediculus Rumphius, Report Blake Moll. 137.

Trivia quadripuuctata Gray, Am. Marine Conch. 55.

Trivia quadripunctata Gray, Jahrb., viii, 156.

Trivia quadripunctata Gray, Mal. Bl., xxiv, 49. Ca.

Trivia quadripunetata Gray, Marrat, Argo Exp., 1876. 18. Abaco.

Trivia quadripunetata Gray, Poulsen. 10.

Trivia quadripunctata Gray, Report Blake Moll. 137.

Trivia rotunda Kien., Arango, Fauna Mal. Cuba, 1875. 185. Ca. Antilles.

Trivia rotunda Kien., Jahrb., viii, 156.

- Trivia scabriuscula Kien., Jahrb., viii, 155. Fla.

Trivia subrostrata Gray, Arango, Fauna Mal. Cuba, 18is. 185. Ca.G. Ba.

Trivia subrostrata Gray, Jahrb., viii, 156.

Trivia subrostrata Gray, Mal. Bl., xxir, 50. G.

Trivia subrostrata Gray, Poulsen. 10.

Trivia subrostrata Gray, Report Blake Moll. 137.

Trivia suffusa Desh., Mal. Bl., xxiv, 49.

Trivia suffusa Gray, Arango, Fauna Mal. Cuba, 1878. 186. Ca. G. Ba. 
Trivia suffusa Gray, Jahrb., viii, 154.

Trivia suffusa Gray, Mal. Bl., xxiv, 49.

Trivia suffusa Gray, Poulsen. 10.

Trivia sulcata Dillw., Jahrb., viii, 154.

Trochilus (see Moduius).

Trochilus unidens List., Moll. Cuba, ii, 57.

Trochilus unidens listeri Chem., Mal. Bl., xxiii, 131.

Trochilus unidens striatus List, Mal. Bl., xxiii, 131.

Trochita (see Galerus, Infundibulum).

Trochita candeana D'Orb., Krebs. 69. PPl. ST. SM. Ag.

Trochita occidentalis-Ad., Mal. Bl., xxiv, 107. SV.

Trochita occidentalis Gray, Mal. Bl., xxiv, 106. SV.

Trochocochlea integra uuidens Klein, Mal. Bl., xxiii, 131.

Trochus (see Calliostoma, Gibbula, Chlorostoma, Margarita, Callopoma, Gaza, Callogaza, Microgaza, Phorus, Onustus, Xenophora).

Trochus (see Astralium), Arango, Fauna Mal. Cuba, 1878. 180. Ca.

Trochus (see Calcar), Arungo, Fauna Mal. Cuba, 1878. 180. Ca. Ba.

Trochus (see Lithopoma), Arango, Mal. Cuba, 1878. 180. Ca. M. SOx. Ba.

Trochus (see Modulus), Arango, Fauua Mal. Cuba, 1878. 175. Ca. Ba.

Trochus (see Omphalius), Arango, Fauna Mal. Cuba, 1878. 176. Oa. Antilles. G. Fla. Ba.

Trochus (see Solarium), Arango, Fauna Mal. Cuba, 1878. 178. Ca. Ba.

Trochus (see Uvanilla), Arango, Fauna Mal. Cuba, 1878. 180. Ca. M.

Bb. San Juan.

Trochus agglutinans Bowd., Mal. Bl., xxiv, 109. M.

Trochus agglutinans Lam., Arango, Fauna Mal. Cuba, 1878. 181. Antilles. M.

Trochus agglutinaus Lam., Mal. Bl., xxiv, 109. SCx.

'Irochus agglutinans Lam., Moll. Cuba, ii, 71.

Trochus angulatus C. B. Adams, Krebs' Remarks. 396.

Trochus antiquarius Meusch., Mal. Bl., xxiv, 108.

Trochus armillatus T. \& H., n. s., Pleiocene Fossils S. C., pl. xxvi, fig. 3. 118.

Trochus asperulus Lam., Krebs. 83.

Trochus aster Phil., Arango, Fauna Mal. Cuba, 1878. 181. Oa. Ba.

Trochus aster Phil., Beau. 13. G.

Trochus aster Phil., Krebs. 83.

Trochus ater Less., Krebs. 83. WI.

Trochus bidentatus Mke., Krebs. 85.

Trochus brasilianus Mke., Poulsen. 13.

Trochus brevispina Lam., Moll. Cuba, ii, 73.

Trochus brevispinus Lam., Jour. de Conch., ii, 428. G.

Trochus byronianus Gray, Guppy, Paria Fauna, 1877. 145.

Trochus calatus Chem., Jour. de Conch., vii, 27. G.

Bull. 24-20 
Trochus cælatus Chem., Arango, Fauna Mal. Cuba, 1878. 179. Ca. M. G. Santa Lucia. Ba.

T'rochus cælatus Chem., Beau. 13. G.

Trochus cælatus Chem., Jour. de Conch., ii, 428. G.

Trochus cælatus Chem., Moll. Cuba, ii, 74.

Trochus cælatus Gmel., Moll. Cuba, ii, 74.

'Trochus cælatus Lam., Moll. Cuba, ii, 74 .

Trochus calcar Argenv., P feiffer, Archiv fuir naturg., 1840. 256. Ca.

Trochus calcar Gmel., Arango, Fauna Mal. Cuba, 1878. 181. Ca. Ba.

Trochus calcar Gmel., Moll. of Bermudas. 20.

Trochus calcar Gmel., Moll. Cuba, ii, 73.

Trochus calvus Mke., * Zt. Mal., x, 70. SV.

Trochus canaliculatus D'Orb., Arango, Fauna Mal. Cuba, 1878. 176. Ca. Ba.

'Trochus canaliculatus D’Orb., Krebs. 84.

Trochus canaliculatus D'Orb., Moll. Cuba, ii, pl. xviii, figs. 18-19. 60. Trochus canus Koch,* Zt. Mal., r, 102.

Trochus carneolus Lam., Araugo, Fauna Mal. Cuba, 1878. 175. Ca.G.

M. Santa Lucia. Ba.

Trochus carneolus Lam., Beau. 13. G.

Trochus carneolus Lam., Mfoll. Cuba, ii, 58.

Trochus carneus Gmel., Krebs. 84.

Trochus castaneus Gmel, Arango, Fauna Mal. Cuba, 1878. 179. Ca. G. Ba.

Trochus catenulatus Phil, Arango, Fauna Mal. Cuba, 1878. 174. Ca. Ba. J.

Trochus chemnitzii Kien., Krebs. 63.

Trochus concavus Gmel., Krebs. 83.

Trochus concavus Gmel., Moll. Cuba, ii, 55.

Trochus concarus Lam., Moll. Cuba, ii, 55.

Trochus concavus Wood, Moll. Cuba, ii, 55.

Trochus conchsliophorus Born, Arango, Fauna Mal. Cuba, 1878. 181. Antilles. M.

Trochus conchyliophorus Born, Mal. Bl., xxiv, 108.

Trochus conchyliophorus Born, Moll. Cuba, ii, 70 .

Trochus conchyliophorus Gmel., Moll. Cuba, ii, 70.

Trochus conchyliophorus Roissy, Moll. Cuba, ii, 70.

Trochus costulatus Lam., Jour. de Conch., ii, 428. G.

Trochus erenulatus Bolt., Krebs. 84.

Trochus crenulatus Wood, Krebs. 83. NGr.

Trochus cubanus Phil., Beat. 13. G.

Trochus eubanus Phil., Jour. de Conch., xiii, 37, 38. Ca.G.

Trochus cubanus Phil., Krebs. 83.

Trochus cubanus Phil.,* Zt. Mal., v, 104. Ca.

Troehus eylindraceus Chem., Araugo, Fauna Mal. Cnba, 1878. 178. Ca. G. 
Trochus eylindraceus Chem., Krebs. 63.

Trochus cylindraceus Chem., Mal. Bl., sxii, 156. ST.

Trochus eylindraceus Gmel., Araugo, Fauna Mal. Cuba, 1878. 178. Ca. G.

Trochus cyliudricus Gmel., Krebs. 63.

Trochus eylindriens Gmel., Mal. Bl., xxii, 156. ST.

Trochus decipiens Guppy, Geol. Mag., 1874. 443. Td. J.

Trochus decipiens var. laticariuatus Guppy, Geol. Mag., 1874. 443. Td.

Trochus dentatus Gmel., Arango, Famna Mal. Cuba, 1878. 175. Ca.

G. M. Santa Lucia. Ba.

Trochus dolabratus Born, Moll. Cuba, i, 228.

Trochus dolabratus Chem., Moll. Cuba, i, 228.

Trochus dolabratus Gmel., Moll. Cuba, i, 228.

Trochus dolabratus Linn., Araugo, Fauna Mal. Cuba, 1878. 161. Ca. G. Ba.

Trochus dolabratus Linu., Moll. Cuba, i, 228.

Trochus dolabratus Wood, Moll. Cuba, i, 229.

Trochus duplici \&c., Mal. Bl., xxiii, 133.

Trochus excavatus D'Orb., Araugo, Fauna Mal. Cuba, 1878. 175. Baracoa. M. G.

Trochus excavatus Lam., Araugo, Fauma Mal. Cuba, 1878. 175. Baracoa. M. G.

Trochus excavatus Lam., Beau. 13. G.

Trochus excavatus Lam., Jour. de Conch., ii, 428. G.

Trochus excavatus Lam., Krebs. \$4. ST. SCx. SJ. PPl.

Trochus excavatus Lam., Moll. Cuba, ii, 56.

Trochus excavatus Lam., Poulsen. 13.

Trochus fasciatus Born, Arango, Fauna Mal. Cuba, 1878. 175. Ca. G.

M. Santa Lucia. Ba.

Trochus fasciatus Born., Ḱrebs. 84. PPI.ST. SJ. SM. Ag. Td. Bb.

Trochus fasciatus Born=T. carneolus Lam., Poulsen. 13.

Trochus filosus Helbl., Mal. Bl., xxiii, 130.

Trochus flammulatus Lam., Krebs. 84.

Trochus gemma T. \&. П., u. s., Pleiocene Fossils S. C., pl. xxvi, fig. 4. 118.

Trochus globulus Phil., Zt. Mal., r, 107.

Trochus granulatus Meusch., Mal. Bl., xxiii, 134.

Trochus gruneri Phil.,* Zt. Mal., r, 107.

Trochus gumdlachi Chemu, Arango, Famna Mal. Cuba, 1878. 176. Ca. Trochus gumllachi I'hil., Araugo, Fauna Mal. Cuba, 1878. 175. Ca.

'Trochus gundlachii Phil., Z Zt. Mal., r, 108. Ca.

Trochus heliacus Phil., Beau. 13. G.

Trochus heliacus Phil., Krebs. 84.

Trochus botessierianus D'Orb., Beau. 13. G.

Trochus hotessierianus D'Orb., Jour. de Conch., v, 152. G.

Trochus hotessierianus D'Orb., Krebs. $84 . \quad$ ST. SII. PPI. 
Trochus hotessierianus D'Orb., Moll. Cuba, ii, pl. xviii, figs. 15-17. 59. Trochus hotesserianus D'Orb. $=$ S. occultus Phil., Poulsen. 13.

Trochus imbricatus Gmel., Jour. de Conch., ii, 428. G.

Trochus imbricatus Gmel., Krebs. 81.

Trochus indusii Chem., Arango, Fauna Mal. Cuba, 1878. 176. Ca. Ba. Trochus indusii Chem., Krebs. 84. VgI.

Trochus indusii Chem. = T. canaliculatus D'Orb., Poulsen. 13.

Trochus inermis Gmel., Arango, Fauna Mal. Cuba, 1878. 181. Ca. Ba. Trochus inermis Lam., Moll. Cuba, ii, 73.

Trochus infundibuliformis Chem., Mal. Bl., xxii, 155. ST.

Trochus interrupto-striatus U. B. Adams, Krebs. 84.

Trochus janthinus Chem., Moll. Cuba, ii, 81.

Trochus javanicus Lam., Arango, Fauna Mal. Cuba, 1878. 176. Ca.

Trochus javanicus Lam., Krebs. 84.

Trochus javanicus Lam., Poulsen. 13.

Trochus jujubinus Gmel., Araugo, Fauna Mal. Cuba, 1878. 176. Ca. G. Ba.

Trochus jujubinus Gmel., Jour. de Conch., iv, 417. G.

Trochus jujubinus Gmel., Krebs. 85. VgI.

Trochus jujubinus Gmel., Poulsen. 13.

Trochus jujubinus Phil., Arango, Fauna Mal. Cuba, 1878. 176. Ca. G. Ba.

Trochus lavis Chem., Arango, Fauna Mal. Cuba, 1878. 175. Ca. G. M. Santa Lucia. Ba.

Trochus lenticularis Chem., Jour. de Conch., iv, 134.

Trochus lenticularis Chem., Arango, Fauna Mal. Cuba, 1878. 175. Ca. Ba.

Trochus lenticularis Chem., Mal. Bl., xxiii, 130.

Trochus lenticularis Chem., Moll. Cuba, ii, 57.

Trochus lithophorus Chem., Mal. Bl., xxiv, 108.

Trochus lithophorus Chem., Moll. Cuba, ii, 70.

Trochus livido-maculatus Ad., Arango, Fauna Mal. Cuba, 1878. 176. Ca. Ba.

Trochus livido-maculatus Ad., Krebs. 84.

Trochus lividus Phil., Krebs' Remarks. 396. Tobago.

Trochus lividus Phil. (vide Modulus), Krebs' Remarks. 398.

Trochus livon Adan., Moll. Cuba, ii, 54.

Trochus longispina Anton, Arango, Fauna Mal. Cuba, 1878. 181. Ca. Trochus longispina Lam., Arango, Fauna Mal. Cuba, 1878. 181. Ca. Ba.

Trochus longispina Lam., Krebs, 82. ST. SCx. Tort. NGr. Trochus longispina Lam., Moll. Cuba, ii, 72.

Trochus lunatus Bolt., Krebs. 85.

Trochus magus, Moll. Cuba, ii, 60 .

Trochus microstomus D'Orb., Krebs. 85 .

Trochus modulus Gmel., Mal. B1., xxiii, 130. 
Trochus modulus Gmel., Mal. Bl., xxiii, 131.

Trochus modulus Gmel., Moll. Cuba, ii, 57.

Trochus modulus Linn., Jour. de Conch., iv, 133.

Trochus modulus Linu., Jour de Conch., iv, 134.

'Trochus modulus Linn., Mal. Bl., xxiii, 130.

Trochus modulus Linn., Moll. Cuba, ii, 57.

Trochus modulus Schröt., Mal. Bl., xxiii, 130.

Trochus muricatus Schröt., Mal. Bl., xxiii, 134.

Trochus nassariensis Chem., Arnngo, Fauua Mal. Cuba, 1878. 176. Ca. Antilles. G. F'la. Ba.

Trochus nassaviensis Chem., Krebs. 84.

Trochus nodulosa Gmel., Arango, Fauma Mal. Cuba, 1878. 160. Ca. M. J. Santa Lucia. G. Ba.

Trochus nodulosus Gmel., Moll. Cubx, i, 205.

Trochus ocenltus Phil., Arango, Fania Mal. Cuba, 1878. 176. Ca. Antilles. G. Fla. Ba.

Trochus occultus Phil., Beau. 13. G.

Trochus occultus Phil., Krebs. 84.

Trochus onustus Sol., Mal. Bl., xxiv, 108.

Trochus pagodus Chem., Mal. Bl., xxiii, 133.

Trochus perlatus Dillw., Mal. Bl., xxiii, 132.

Trochus perlatus Wood, Arango, Fauna Mal. Cuba, 1878. 174. Ca. Ba. J.

Trochus perlatus Wood, Mal. Bl., xxiii, 132.

Trochus perlatus Wood, Moll. Cuba, ii, 57.

Trochus perspectivus Chem., Moll. Cuba, ii, 65.

Trochus perspectivus Koch, Poulsen. 13.

'Trochus perspectivus Linn., Moll. Cuba, ii, 65.

Trochus pica D'Orb., Arango, Fauna Mal. Cuba, 1878. 174. Oa. Antilles, $\mathrm{Ba}$.

Trochus pica D'Orb., Moll. of Bermudas. 20.

Trochus pica D'Orb., Moll. Cuba, ii, 54.

'Trochus pica Linn., Beau. '13. G.

Trochus pica Linn., Poulsen. 13.

Trochus pica Pfeiffer, Archir für naturg., 1840. 256. Oa.

Trochus pisum Phil.,* Zt. Mal., $\nabla, 111$.

Trochus plicomphalus Guply, Geol. Mag., 1874. 443. Td.

Trochus pulcher U. B. Adams, Contr. to Conch. 69. J.

Trochus pulcher C. B. Adams, Krebs. 85. ST. SCx. PPl.

Trochus pulcher C. B. Adams, Poulsen. i3.

Trochus pyramidalis Seba, Arango, Famua Mal. Cuba, 1878. 179. Ca. M. G. Santa Lucia. Ba.

Trochus pyramidalis Seba, Moll. Cuba, ii, 74.

Trochus scabriculus V. d. Busch, * Zt. Mal., v, 125.

Trochus scalaris Anton, Arango, Famma Mal. Cuba, 1878. 176. Ca. Ba. 
Trochus scalaris Anton, Krebs. 84.

Trochus schrammii Fisch., Beau. 13. G.

Trochus schrammii Fisch., Krebs. 80.

Trochus schrammii Fisch., Krebs. 85.

Trochus senegalensis Mke., Zt. Mal., x, 71. SV.

Trochus solaris Chem., Arango, Fauna Mal. Cuba, 1878. 181. Ca. Ba.

Trochus solaris Chem., Moll. Cuba, ii, 72.

Trochus spinulosus Lam., Arango, Fauna Mal. Cuba, 1878. 181. Ca. Ba.

Trochus spinulosus Lam., Beau. 13. G.

Trochus spinulosus Lam., Jour. de Conch., v, 152. G.

Trochus stellat Lam., Pfeiffer, Archir für naturg., 1840. 256. Ca.

Trochus striatellus Dillw., Mal. B1., xxiii, 93. Fla.

Trochus tampaënsis Conrad, Am. Journ. Sci., n. s., ii, 1846. 398. Fli. Trochus tampaënsis Courad, Proc. Acad. Nat. Sci. Phil., 1845, iii, pl. „2, fig. 35, 26. Tampa.

Trochus tectum Gmel., Mal. Bl., xxiii, 131.

Trochus tectum Gmel., Míoll. Cuba, ii, 57.

Trochus tectum Wood, Moll. Cuba, ii, 57.

Trochus terebellus Wood, Moll. Cuba, i, 229.

Trochus (Ziziphiuus) tiara Watson, Report Blake Moll. 45. Ca.

Trochus tuber Born, Moll. Cuba, ii, 75 .

Trochus tuber Gmel, Moll. Cuba, ii, 75 .

Trochus (Lithopoma) tuber Gray, Marrat, Argo Exp., 1876. 6. Aa.

Trochus tuber Lam., Moll. Cuba, ii, 75 .

Trochus tuber Linn., Bean. 13. G.

Trochus tuber Linn., Jour. de Couch., ii, 428. G.

Trochus tuber Linn., Jour. de Conch., vii, 27. G.

Trochus tuber Linu., Moll. Cuba, ii, 75.

Trochus tuber Wood, Moll. Cuba, ii, 75.

Trochus tuberculatus Chem., Moll. Cuba, ii, 75.

Trochus (Delphinula) tuberculosa D'Orb., Beau. 13. G.

Trochns umbilicaris Chem., Arango, Fanna Mal. Cuba, 1878. 175.

Baracoa. M. G.

Trochus umbilicaris Chem., Krebs. 84.

Trochus unidens Chem., Arango, Fauna Mal. Cuba, 1878. 175. (Vir.

Trochus unidens Chem., D'Orb., Mal. Bl., xxiii, 130.

Trochus unidens Chem., Moll. Cuba, ii, 57.

Trochus unidens D'Urb., Mal. B1., xxiii, 131. Fla.

'Trochus unidens D'Orb., Mal. Bl., xxiii, 132.

'Trochus unideus List., Aıango, Fauna Mal. Cuba, 1878. 175. Ca.

Trochus unidens List., Krebs. 62.

Trochus unidens List., Mal. Bl., xxiii, 130.

Trochus unidens List., Moll. Cuba, ii, 57.

Trochus venustus Phil.,* Zt. Mal., v, 126.

Trochus vidua D'Arg., Moll. Cuba, ii, 54. 
Trochus viridis Chem., Moll. Cuba, ii, 75.

Trochus viridulus Gmel., Jahrb., ii, 246.

Trochus ziczac Cliem., Mal. Bl., xxiii, 138.

Trochus ziczac Gmel., Mal Bl., xxiii, 138.

Trochus ziczac, Gmel., Moll. Cuba, i, 210.

Trochus ziczac Wood, Moll. Cuba, i, 210.

Trochus zicezali Chem., Arango, Fauna Mal. Cuba, 1878. 160. Ca. M. Ba.

Trochus ziczak Chem., Moll. Cuba, i, 210.

Trochus zigzac Chem., Mal. Bl., xxiii, 138.

Trochus zigzag Chem., Moll. Cuba, i, 208.

Trochus zigzag Gmel., Moll. Cuba, i, 208.

Truncatella adamsi Pfr., Jahrb., vii, 272. J.

Truncatella adamsi Pfr., Zt. Mal, iii, 1S9. J.

Truncatella barbatensis Pfr., Jahrb., rii, 284. Bb.

Truncatella beardsleana O. B. Adams, Jahrb., vii, 272. J.

Truncatella bilabiata Pfr., $1 \mathrm{~m}$. Jour. Conch. ir, pl. 18, figs. $32,33$.

14. Fla.

Truncatella bilabiata Pfr., Am. Jour. Conch., iv, 19,

Truncatella bilabiata Pfr., Jahrb., vii, 260. Fla.

Truncatella bilabiata Pf., Jahrb., vii, 265. Ca.

Truncatella bilabiata Pfi., Jabrb., vii, 277. Hy.

Truncatella bilabiata Pfr., Mal. Bl., iii, 119. Ca.

Truncatella bilabiata Pfr., Mal. Bl., ix, 122. Ca.

Truncatella bilabiata Pfr., Mal. Bl., ix, 1:9. Ca.

Truncatella bilabiata Pfr., Zt. Mal., iii, 187. Ca.

Truncatella capillacea Gundl., Jahrb., vii, 265. Ca.

Truucatella capillacea Gundl., Mal: Bl., vi, 77. Ca.

Truncatella capillacea Gundl.* Mal. Bl., ix, 128. Ca.

Truncatella caribæensis Küst., Mal. Bl., iii. 119. Ca.

Truncatella caribrensis Küst., Mal. Bl., ix, 122. Ca.

Truncatella caribreusis Sby., Am. Jour. Conch. iv, pl. 18, figs. 27, 28.

14. Fla.

Truncatella caribrensis Sby., Am. Jour. Conch., iv, 19.

Truncatella caribæensis Sby., Beau. 16. G.

Truncatella caribæensis Sby., Jahrb., vii, 260. Fla.

Truncatella caribæensis Sby., Jahrb., vii, 265. Ca.

Truncatella caribæensis Sb5.. Jahrb., vii, 272. J.

Truncatella caribæensis Sby., Jahrb., vii, 277. Нy.

Truncatella caribæensis Sby., Jahrb., rii, 283. G.

Truncatella caribæensis Sby., Jahrb., rii, 284. M.

Truncatella caribæensis Sby., .Ial. Bl., iii, 119. Ca.

Truacatella caribæensis Sby., Mal. Bl., v, 195. Ca.

Truncatella caribæensis Sby., Mal. Bl., ix, 12s. Ca.

Truucatella caribæensis Sbr.,* Zt. Mal., iii, $18 \%$.

Truncatella caribæus Sby., Jour. de Conch., v, 152. G. 
Truncatella caribbeёnsis Sby., Dall, Hemphill's shells. 323. Key West.

Truncatella clathrus Lau., Jour. le Conch., v, 152. G.

Truncatella clathrus Lowe, Bean. 16. G.

Truncatella clathrus Lowe, Jahrb., vii, 279. PR.

Truncatella clathrus Lowe, Jahrb., vii, 281. ST.

Truncatella clathrus Lowe, Jahrb., vii, 283. G.

Truncatelía costata Pfr., Bean. 16. G.

Truncatella costata Pfr., Mal. Bl., iii, 119. Ca.

Truncatella costata Pfr., Mal. Bl., ix, 129. Ca.

Truncatella costata Pfr.; Zt Mil., iii, 188. Ca. J.

Truncatella costulata Risso, Moll. Cuba, ii, 5.

Truncatella cumingii C. B. Adams, Beau. 16. G.

Truncatella cumingii C. B. Adams, Mal. Bl., iii, 119. Ca.

Truncatella cumingii C. B. Adams, Synops., 12. 1845. J.

Truncatella cumingii O. B. Adams, Zt. Mal, iii, 188. Ca. J.

Truncatella dubiosa C. B. Adams, Beau. 16. G.

Truncatella dubiosa C. B. Adams, Jahrb., vii, 283. G.

Truncatella dubiosa C. B. Arlams, Jour. de Couch., viii, 181. G.

Truncatella elongata Poey, Jahrb., vii, 265. Ca.

Truncatella elongata Poes, Mal. Bl., iii, 119. Ca.

Truncatella elongata Poer, Mal. Bl., vi, 76. Ca.

Truncatell: elongata Poey, Mal. Bl., ix, 123, 124. Ca.

Truncatella elongata Poey, Mal. Bl., ix, 126, $127 . \quad$ Ca.

Truncatella filicosta Gundl., Jahrb., vii, 265. Ca.

Truncatella filicosta Gundl., Mal. Bl, ix, 123-125. Ca.

Truncatella filicosta Gundl.,* Mal. Bl., ix, 127. Ca.

Truncatella filicostata Guudl., Mal. Bl., vi, 77. Ca.

Truncatella gouldii C. B. Adams, Mal. Bl., iii, 119. Ca.

Truncatella gouldii C. B. Adams, Zt. Mal., iii, 183.

Truncatella grayana C. B. Adams, Jahrb., vii, 272. J.

Truncatella gnerinii Parr., Mal. Bl., iii, 119. Ca.

Truncatella lævigata Risso, Moll. Cuba, ii, 5.

Truncatella lirata Pfr., Mal. Bl., ix, 123, 124. Ca.

Truncatella lirata Pfr., Mal. B1., ix, 126, 127. Ca.

Truncatella lirata Poey, Jahrb., vii, 265. Ca.

Truucatella lirata Poey, Mal. Bl., iii, 119. Ca.

Truncatella lirata Poey, Mal. B3., vi, 76. Ca.

Truncatella modesta C. B. Arlams, Beau. 16. G.

Truncatella modesta C. B. Adlams, Contr. to Conch. 132. J.

Truncatella modesta C. B. Adams, Jahrb., vii, 272. J.

Truncatella modesta C. B. Adams, Jahro., vii, 283. G.

Truncatella modesta C. B. Allams, Jahrb., vii, 284. M.

Truncatella pulchella Pfr., Mal. Bl., ix, 122. Ca.

Truncatella pulchella Pfr.,* Am. Jour. Conch. iv, pl. xviii, figs. 34-36.

15. Fla.

Truncatella pulchella Pfr., Am. Jour. Conch. iv, 19. 
Truncatella pulchella, Pfr., Dall, Hemphill's shells. 323. Koy West.

Truncatella pulchella Pfr., Jahrb., vii, 260. Fla.

Truncatella pulchella Pfr., Jahrb., vii,265. Ca.

Truncatella pulchella Pfr., Jahrb., vii, 272. J.

Truncatella pulchella Pfr., Jabrb.; vii, 277. Hy.

Truncatella pulchella Pfr., Jahrb., vii, 279. PR.

Truncatella pulchella Pfr., Jahrb., vii, 281. ST.

Truncatella pulchella Pfr., Mal. B1., iii, 119. Ca.

Truncatella pulchella Pfr., Mal. Bl., ix, 129. Ca.

Truncatella pulchella Pfr., Zt. Mal., iii, 186. Ca.

Truncatella pygmæa C. B. Adams, Jahrb., vii, 272. J.

Truncatella scalariformis C. B. Adams, Synops., 12. 1845. J.

Truncatella scalariformis C. B. Adams, Zt., Mal., iii, 189. J.

Truncatella scalaris Mich., Beau. 16. G.

Truncatella scalaris Mich., Jahrb., rii, 265. Ca.

Truncatella scalaris Mich., Jahrb., vii, 272. J.

Truncatella scalaris Mich., Jahrb., vii, 283. G.

Truncatella scalaris Mich., Mal. Bl., iii, 119. Ca.

Truncatella scalaris Mich., Mal. Bl., v, 195. Ca.

Truncatella scalaris Mich., Mal. Bl., ix, 122. Ca.

Truncatella scalaris Mich., Mal. Bl., ix, 129. Ca.

Truncatella scalaris Mich., Zt. Mal. iii, 188. Ca. J.

Truncatella subcylindrica D’Orb., Moll. Cuba, ii, 5.

Truncatella subeylindrica Glay, * A m. Jour. Conch. iv, pl. 18, figs. 29-31.

14. Fla.

Truncatella subcylindrica Gray, Jahrb., vii, 260. Fla.

Truncatella subcylindrica Gray, Jabrb., vii, 265. Ca.

Truncatella subçlindrica Gray, Jahrb., vii, 272. J.

Truucatella subcylindrica Gray, Jahrb., vii, 277. Ну.

Truncatella subcylindrica Gray, Jahrb., vii, 279. PR.

Truncatella subcylindrica Gray, Jahrb., vii, 281. S'T.

Truncatella subcylindrica Gray, Jahrb., vii, 286. Ber.

Truncatella subcylindrica Gray, Mal. Bl., iii, 119. Ca.

Truncatella subcylindrica Gray, Mal. Bl., v, 195. Ca.

Truncatella subeylindrica Gray, Mal. Bl., vi, 76. Ca.

Truncatella subcyliudrica Gray, Mal. Bl., ix, 122. Ca.

Truncatella subeylindrica Gray, Mal. Bl., ix, 128, 129. Ca.

Truncatella subcylindrica Pult., Am. Jour. Conch., iv, 19.

Truncatella succinea C. B. Adams, Am. Jour. Couch., iv, 19.

Truncatella succinea C. B. Adams, Beau. 16. G.

Truncatella succinea C. B. Atlams, Mal. Bl., iii, 119. Ca.

Truncatella succinea C. B. Adams, Moll. of Bermudas. 19.

Truncatella succinea C. B. Adams, Synops., 12. 1845. J.

Truncatella succinea C. B. Adams, Zt. Mal., iii, 183.

Truncatella truncatula Drap., Mal. Bl., v, 195. Ca.

Truncatella truncatula Drap., Mal. Bl., ix, 122. Ca. 
Truncatella truncatula Lowe, Moll. Cuba, ii, 5.

Truncatella truncatula Lowe, Zt. Mal., iii, 183.

Truncatella turrita Pfr., Zt. Mal., iii, 186. Ca.

Truncatella turritz Pfr., Zt. Mal, iii, 187. Ca.

Truncatella variabilis Pfr., Mal. Bl., iii, 119. Ca.

Truncatella variabilis Pfr., Zt. Mal., iii, 183.

Truncatella wrighti Pfr., Jahrb., vii, 265. Ca.

Truncatella wrighti Pfr., * Mal. Bl., ix, 127. Ca.

Tuber (see Calcar, Trochus).

Tuber aculeatus Gmel., Krebs. 81.

Tuber inermis Gmel., Krebs. 82.

Tuber spinulosa Lam., Krebs. 82.

'Tubulus anguinus Mart., Jour. de Conch., viii, 23. Ca.

Turbinella (see Latirus, Plicatella, Lencozonia, Mazza, Pyrula, Vasum, Voluta).

Turbinella ananas Chem., Beau. .9. G.

Turbinella ananas Chem., Krebs. 16.

Turbinella ananas Chem., Krebs. 20.

Turbinella auanas Chem., Poulsen. 11.

Turbinella angularis Rve., Krebs. 16.

Turbinella annulata Bolt, Krebs. 16. SJ. VD.

Turbinella annulata Bolt., Poulsen. 11.

Turbinella attenuata Rre., Araugo, Fauna Mal. Cuba, 1878. 221. Ca. Turbinella attenuata Rre., Krebs. 16.

'Turbinella barelayi live., Arango, Fauna Mal. Cuba, 1878. 221. Ca.

'Turbinella brasiliana D'Orb., Jahrb., ii, 242.

'Turbinella brasiliana D'Orb., Poulsen. 11.

Turbinella brevicaudata Rve., Arango, Fauna Mal. Cuba, 1878. 221. Ca. Ba.

Turbinelia brevicaudata Rve., Krebs. 16.

Turbinella capitellum D'Orb., Araugo, Fauna Mal. Cuba,1878. 221. G. II. Santa Lucia.

Turbinella capitellum Lam., Moll. Cuba, ii, 178.

Turbinella capitellum Liun., Arango, Fauma Mal. Cuba, 1878. 221. G.

M. Santa Lueia.

Turbinella capitellum Linu., Beau. 9. G.

Turbinella capitellum Linn., Jour. de Conch., ii, 428. G.

Turbinella capitellum Linn., Krebs. 16. ST.

Turbinella capitellum Linn., Poulsen. 11.

Turbinella carinifera Lam., Krebs. 16. ST.

Turbinella cassidiformis Kien., Jahrb., iii, 16.

Turbinella ciugulifera Lam., Araugo, Fauna Mal. Cuba, 1878. 222.

Ca. M. G. Santa Lucia. ST. Ba.

Turbinella cingulifera Lam., Beau. 9. G.

Turbinella cingulifera Lam., Jahrb., ii, 242.

Turbinella cingulifera Lam., Krebs. 16. 
Turbinella cingulifera Lam., Moll. Cuba, ii, 177.

Turbinella cingulifera List., Jour. de Conch., ii, 428. G.

Turbinella distinctia A. Ad., Arango, Fauna Mal. Cuba, 1878. 222. Ca.

Turbinella dubia Petit,* Jour, de Conch. ir, pl. 2, figs. 9, 10. 75.

Turbinella filamentosa Koch, Krebs. 16. ST. Tort.

Turbiuella filamentosa Koch, Poulsen. 11.

Turbinella filosus Lam., Krebs. 17.

Turbinella fuscata Gmel., Krebs. 16.

Turbinella fuscata Gmel., T. lencozonalis Lam., c. v., Poulsen. 11.

Turbiuella gibbula Gmel., Krebs. 17.

Turbinella globulus Lam., Krebs. 17. Aa.

Turbinella globulus Lam., Poulseu. 11.

Turbinella gracilis Rre., Krebs. 17. ST.

T'urbinella infuudibulum D’Orb., Alango, Fauna Mal. Cuba, 1878. 222.

Ca. Baracoa. G. M. Santa Lucia.

Turbinella infundibulum Gmel., Arango, Fauna Cuba, 1878. 222. Ca.

Baracoa. G. M. Santa Lucia.

Turbinella infundibulum Gmel., Beau. 9. G.

Turbinella infundibulum Gmel., Jour. de Couch., iv, 417. G.

Turbinella infundibulum Lam., Moll. Cuba, ii, 178.

Turbinella leucozomalis Lam., Krebs. 17. ST. SCx. Tort.

Turbinella lineata Lam., Krebs. 17. ST.

Turbinella muricata Boru, Beau. 9. G.

Turbinella muricata Boru, Jahrb., iii, 16.

Turbinella muricata Born, Krebs. 17. Tort.

Turbinella muricata Boru. Poulsen. 11.

Turbinella nana Rve., Krebs. 17. SCx.

Turbinella nana Rve., Poulsen. 11.

Turbiuella nassa D'Orb., Araugo, Fauna Mal. Cuba, 1878. 222. Oa. M.

G. Santa Lucia. ST. Ba.

Turbinella nassa D'Orb., Moll. Cuba, ii, 177.

Turbinella nassa Gmel., Arango, Fauna Mak. Cuba, 1878. 222. Ca. M.

G. Santa Lucia. S'T. Ba.

Turbinella nassa Gmel., Jour. de Conch., ii, 428. G.

Turbinella nassa Gmel., Krebs. 17.

Turbinella nassa Gmel., Poulsen. 11.

Turbinella nigella Chem., Arango, Fauna Mal. Cuba, 1878. 222. Ca. Ba.

Turbinella nigella Chem., Beau. 9. G.

Turbinella nigella Chem., Krebs. 17.

Turbinella nigella Chem., Poulsen. 11.

Turbinella ocellata Gmel., Arango, Fauna Mal. Cuba, 1878. 222. Ca.

Ba.

Turbinella ocellata Gmel., Beau. 9. G.

Turbinella ocellata Lam., Jour. de Conch., ii, 428. G.

Turbinella (Pyrula) ocellata Gmel., Krebs. 17. ST. Ag. SM. G.

Turbinella ovoidea Kien., Jahrb., iii, 16. Bz. 
Turbinella polygona Lam., Krebs. 17.

Turbinella polygona Lam., Moll. Cuba, ii, 179.

Turbinella pugillaris D'Orb., Arango, Fauna Mal. Cuba, 1878. 222. Ca. Baracoa.

Turbinella pugillaris Lam., A rango, Fauna Mal. Uuba, 1878. 222. Ca. Baracoa.

Turbinella pugillaris Lam., Beau. 9. G.

Turbinella pugillaris Lam., Jahrb., iii, 16.

Turbinella pugillaris Lam., Jour. de Conch., iv, 417. G.

Turbinella pugillaris Lam., Krebs. 17.

Turbinella pugillaris Lam., Moll. Cuba, ii, 117.

Turbinella recurvirostra Wagn., Krebs. 17.

Turbinella recurvirostra Wagn., Poulsen. 11.

Turbinella rhinoceros Lam., Krebs. 17.

Turbinella rudis Rve. Krebs. 18. ST. SJ. G.

Turbinella scolymus Gmel., Arango, Fauna Mal. Cuba, 18i8. 222. Ca. Ba.

Turbinella scolymus Gmel., Poulsen. 11.

Turbinella scolymus Lam., Jahrb., iii, 16.

Turbinella triserialis Lam., Zt. Mal., x, 78. SV.

Turbinellus infundibulum Gmel., Guppy, Paria Fauna, 1877. 142.

Turbinellus nassa Gimel., Guppy, Paria Fauna, 1877. 142.

Turbo (see Leptothyra, Lirona, Leptopoma, Callopoma).

Turbo (see Triforis), Arango, Fauna Mal. Cuba, 1878. 209. Ca. G.

Turbo acuta, \&c., Mal. Bl., xxiii, 128.

Turbo albus, \&c., Mal. Bl., xxiii, 127.

Turbo apertus Gault., Mal. Bl., xxiii, 111.

Turbo arenarius M. \& R., Mal. Bl., xxiii, 47.

Turbo brevispina Lam., Moll. Cuba, ii, 73.

Turbo brevispina Lam., Poulsen. 13.

Turbo (Liotia) briareus Dall, n. s., Report Blake Moll. 52. Havana.

Turbo bryereus Mtg., Mal. Bl., xxiii, 49. ST.

Turbo cælatus Chem., Poulsen. 13.

Turbo cælatus Klein., Moll. Cuba, ii, 74 .

Turbo cailletii Fisch. \& Bern., Beau. 12. La Basse-Terre.

Turbo cailletii F. \& B., Jour. de Conch. v, pl. 9, figs. 10, 11. 294. G.

Turbo cailletii F. \& B., Krebs. 79.

Turbo calcar Linn., Krebs' Remarks. 398.

Turbo calcar Linn., Poulsen. 13.

Turbo carinatus Mühlf., Mal. Bl., xxiii, 92.

Turbo carneolus Lam., Krebs. 84.

Turbo castanea Gmel., Arango, Fauna Mal. Cuba, 1878. 179. Ca. G.

$\mathrm{Ba}$.

Turbo castanea Gmel., Moll. Cuba, ii, 76.

Turbo castaneus Chem., Krebs. Su. ST. Tort. SM. G. Td. PPl.

Turbo eastaneus Chem.=T. hippocastanum Lam., Poulsen. 13. 
Turbo castaneus Gmel., Beau. 12. G.

Turbo castaneus Gmel., Jour. de Conch., v, 152. G.

Turbo castaneus Gmel., Moll. Cuba, ii, 76.

Turbo castaneus Wood, Moll. Cuba, ii, 76.

Turbo elathrus Linn., Mal. Bl., xxii, 149.

Turbo coniferus Mtg., Mal. Bl., xxii, 153. G.

Turbo costatus Don., Arango, Fauna Mal. Cuba, 1878. 167. Oa

Turbo costatus Don., Mal. Bl., xxiii, 49. ST.

Turbo crenulatus Chem., Jour. de Conch., vii, 27. G.

Turbo crenulatus Chem., Jour. de Conch., vii, 35. G.

Turbo crenulatus Chem., Krebs. 80.

Turbo crenulatus Gmel., Am. Marine Conch. 87.

Turbo crenulatus Gmel., Beau. 12. G.

Turbo crenulatus Gmel., Jour. de Conch., iv, 417. G.

Turbo crenulatus Gmel., Poulsen. 13.

Turbo cubanianum Phil., Poulsen. 13.

Turbo decussatus Dillw., Mal. Bl., xxiii, 47.

Turbo dentatus Gmel., Krebs. 84.

Turbo dispar Linn., Mal. Bl., xxiii, 143.

Turbo exoletus Born, Mal. Bl., xxiii, 128. G.

Turbo exoletus Linn., Arango, Fauna Mal. Cuba, 1878. 165. Oa. J.G.

Ba.

Turbo exoletus Linn.; Mal. Bl., xxiii, 128.

Turbo hippocastanum Lam., Arango, Fauna Mal. Cuba, 1878. 179. Ca.

G. Ba.

Turbo hippocastanum Lam., Krebs. 80.

Turbo hippocastanum Lam., Moll. Cuba, ii, 76.

Turbo imbricatus Gmel., Moll. Cuba, ii, 13.

Turbo imbricatus Hanl., Mal. Bl., xxiii, 127. J.

Turbo imbricatus Linn., Arango, Fauna Mal. Cuba, 1878. 165. Ca. J.

Santa Lucia.

Turbo imbricatus Linn., Mal. Bl., xxiii, 127. J.

Turbo imbricatus Linn., Moll. Ouba, ii, 13.

Turbo imbricatus Mart., Moll. Cuba, ii, 13.

Turbo imbricatus Wood, Moll. Cuba, ii, 13.

Turbo indutus Watson, Jour. de Conch., xxvii, 365. Cal.

Turbo inermis D'Orb., Moll. of Bermudas. 20.

Turbo inermis Gmel., Moll. Cuba, ii, 72.

Turbo integer, \&c., Mal. Bl., xxiii, 127.

Turbo irroratus Say, Kurtz. 7. NC. SC.

Turbo longispina D'Orb., Moll. Cuba, ii, 72.

Turbo marmoratus Chem., Mal. Bl., xxiii, 127.

Turbo minima Wood, Jahrb., x, 221. G.

Turbo minimus Gray, Mal. Bl., xxiii, 141. G.

Turbo mirabilis Mlf., Mal. Bl., ii, 85. Ca.

Turbo muricata Linn., Jahrb., x, 226. 
'Turbo muricatus Boru, Mal. Bl., xxiii, 134.

Turbo muricatus Born, Moll. Cuba, i, 204.

Turbo muricatus Born, Mal. Bl., xxiii, 134.

Turbo muricatus Chem., Mal. Bl., xxiii, 134.

Turbo muricatus Cihem., Moll. Cuba, i, 204.

Turbo muricatus Gmel., Moll. Cuba, i, 204.

Turbo muricatus Herbst., Mal. Bl., xxiii, 134.

Turbo muricatus Lam., Mal. Bl., xxiii, 134. J.

Turbo muricatus Lam., Moll. Cuba, i, 204.

Turbo muricatus Linn., Arango, Fauma Mal. Cuba, 1878. 159. Ca. Ba.

Turbo muricatus Linn., Mal. Bl., xxiii, 134.

Turbo neritoideus muricatus Linu., Moll. Cuba, i, 204.

Turbo niveus Chem., Moll. Cuba, ii, 41.

Turbo nodulosa Boru, Moll. Cuba, ii, 54.

Turbo nodulosa Chem., Moll. Cuba, ii, 54.

Turbo nodulosus Wood, Moll. Cuba, ii, 55.

Turbo ohsoletus Gmel., Mal. Bl., xxiii, 128. G.

Turbo phoebia, Bolt.-T. longispina Lam., Poulsen. 13.

Turbo pica Born, Jour. de Conch., vii, 31.

Turbo pica Born, Moll. Cuba, ii, 54.

Turbo pica Chem., Moll. Cuba, ii, 54.

Turbo pica Gmel., Arango, Fauma Mal. Cuba, 1878. 174. Ca. Antilles. Ba.

Turbo pica Gmel., Moll. Cuba, ii, 54.

Turbo pica Lam., Moll. Cuba, ii, 55.

Turbo pica Linu., Arango, Fauna Mal. Cüa, 1878. 174. Ca. Antilles. Ba.

Turbo pica Linn., Jour, de Conch., ii, 428. G.

Turbo pica Linn., Jour. de Conch., vii, 26. M. G.

Turbo pica Linn., Krebs. 80. ST. Ber. SCx. ST. PPl.

Turbo (Livona) pica Linu., Marrat, Argo Exp., 1876. 6. Aa.

Turbo pica Linn., Moll. of Bermudas. 20.

Turbo pica Linn., Moll. Cuba, ii, 54.

Turbo pica Wood, Moll. Cuba, ii, 55.

Turbo principalis Pallas, Mal. Bl., xxii, 143. Tort.

Turbo psendo-scalar.s Brocchi, Arango, Fauna Mal. Cuba, 1878. 166. Ca. Antilles.

Turbo pseudosealaris Brocchi, Moll. Cuba, ii, 15.

Turbo pulchellus C. B. Adams, Mal. Bl., xxiii, 142.

Turbo pulchellus C. B. Adams, Synops., 7, 1845. J.

Turbo ramosus Meusch.=T. imbricatus Gmel., Poulsen. 13.

Turbo rhodostomus Lam., Poulsen. 13.

Turbo spenglerianus Chem., Poulsen. 13:

Turbo spenglerianus Gmel., Beau. 12. G.

Turbo terebra Don., Mal. Bl., xxiii, 127.

Turbo torculosus Born, Mal. Bl., xxiii, 128. 
Turbo torculosus Dillw., Mal. Bl., xxiii, 128.

Turbo trochiformis Boru, Mal. Bl., xxiv, 108.

Turbo trochiformis Dillw., Arango, Fauna Mal. Cuba, 1878. 160. Ca. M. J. Santa Lucia. G. Ba.

Turbo tuber Arango, Fauna Mal. Cuba, 1878. 164. Ca. M.

Turbo tuber Linn., Poulsen. 13.

Turbo tuber Linn., Moll. Cuba, ii, 75.

Turbo tuberculatus Wood, Arango, Fauma Mal. Cuba, 1878. 159. Ca. G.

Turbo tuberculatus Wood, Moll. Cuba, i, 206.

Turbo tuberculatus Gray, Mal. Bl., xxiii, 132.

Turbo turris-thomæ Chem., Mal. Bl., xxiii, 110. ST.

Turbo turris-thomæe Chem., Moll. Cuba, ii, 155.

Turbo variegatus Linn., Mal. Bl., xxiii, 126.

'Tubo vidua D’Arg., Aranğo, Famna Mal. Cuba, 1878. 174. Ca. Antilles. Ba.

'Turbo ziczac M. \& R., Mal. B1., xxiii, 143.

Turbonilla (see Chemuitzia, Dunkeria, Elusa, Mumiola).

Turbonilla acicula Holm., n. s., Post-Pleiocene Fossils, S. C., pl. xiii, figs. $10,10 a, 10 b .86$.

'Turbonilla babylonia Ad., Krebs. 72.

Turbonilla cancellata D'Orb., Krebs. 72.

Turbonilla caucellata Holn., n. s., Post-Pleiocene Fossils S. C., pl. xiii, figs. $6,6 a, 6 b . \quad 84$.

Turbonilla caroliniana Holm., 1. s., Post.Pleiocene Fossils S. O., pl. xiii, figs. $9,9 a, 9 b . \quad$ S6.

Turbonilla curtiua Gld., Am. Marine Conch. 65. SO.

'Turbonilla dnbia D'Orb., Krebs. 72.

'Turbonilla elegans D’Orb., Krebs. 72.

'Turbonilla exilis Ad., Krebs. 72.

Turbouilla flavocineta . Id., Krebs. 72.

'Turbonilla interupta Totten, Am.' Marine Conch. 64. NO.

Turbonilla lavigata D’Orb., Krebs. 72.

Turbonilla lævis Ad., Krebs. 72.

Turbonilla latior Ad., Krebs. 72.

Tubonilla lineata Holm., n. s., Post-Pleiocene Fossils S. C., pl. xiii, figs. 7, 7 $a, 7 \iota . \quad 8 \tilde{\text { o. }}$

Turbonilla modesta D'Orb., Krebs. 72.

Turbonilla multicostata Ad., Krebs. 72.

Turbonilla obeliscus Ad.. Krebs. 72.

Turbonilla oriata D'Orb., Krebs. 72.

Turbonilla pulehella D'Orb, Krebs. 73.

Turbonilla punctata Ad., Krebs. 73.

Turbonilla (viridaria var. ?) punicea Dall, n. s., Hemphill's shells. 332.

Cerlar Keys.

Turbonilla pupoides D’Orb., Krebs. 73.

Turbonilla pusiila Ad., Krebs. 73. 
Turbonilla quinque-striata Holm., n. s., Post-Pleiocene Fossils S. C., pl. xiii, figs. $5,5 a, 5 b$. 85 .

'Turbonilla rathbuni Verrill, Dall, Hemphill's shells. 332.

Turbonilla reticulata Ad., Krebs. 73. ST.

Turbonilla simplex D’Orb., Krebs. 73.

Turbonilla spirata Kurtz \& Stm., Am. Marine Conch. 65. NO.

'Turbonilla subcarinata D'Orb., Guppy, Geol. Mag., 1874. 445. Td.

Turbonilla subcoronata Holm., n. s., Post-Pleiocene Fossils S. C., pl. xiii, figs. $12,12 a, 12 b .87$.

Turbonilla substriata $\Lambda$ d., Krebs. 73. ST.

Turbonilla subulata Ad., Krobs. 73. ST.

Turbonilla subulata Holı., n. s., Post-Pleiocene Fossils S. C., pl. xiii, figs. $8,8 a, 8 b$. 85 .

Turbonilla suturalis Gld., Am. Marine Conch. 66. SC.

Turbonilla textilis Kurtz, Am. Marine Conch. 65. SC.

Turbonilla turris D’Orb., Guppy, Geol. Mag., 1874. 437. Td.

Turbonilla turris D'Orb., Jahrb., ii, 243.

Turbonilla turris D'Orb., Krebs. 73.

Turbonilla (viridaria var.?) virga Dall, n. s., Hemphill's shells. 332. Cedar Keys.

Turbonilla viridaria Dall, n. s., Hemphill's shells. 332. Cedar Keys.

Turricula rawsoni Mörch,* Jour. de Conch., xxir, 373 . Bb.

Turritella (see Toreula, Haustator, Terebellum).

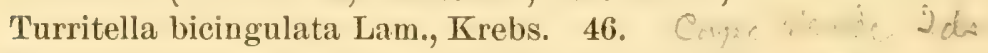

Turritella bicingulata Lam., Zt. Mal., x, 70. SV.

Turritella caribæa D’Orb., Arango, Fauna Mal. Cuba, 1878. 165. Oa.

Turritella caribaa D'Orb., Krebs. $46 .=$ vis a

Turritella caribæa D'Orb., Mal. Bl., xxiii, 129.

Turritella caribæa D'Orb., Moll. Cuba, ii, pl. x, fig. 21. 14.

$\sqrt{ }$ Turritella exoleta Kien., Mal. Bl., xxiii, 128. G.

Turritella exoleta Lam., Mal. Bl., xxili, 128.

Turritella exoleta Linn., Arango, Fauna Mal. Cuba, 1878. 165. Oa. J. G. Ba.

Turritella exoleta Linn., Beau. 11. G.

Turritella exoleta Linn., Jour. de Conch., iv, 417. G.

Turritella exoleta Linn., Krebs. 46. SM. Td.

'Turritella exoleta Linn., Krebs' Remarks. 398. J.

Turritella exoleta Linn., Mal. Bl., xxiii, 128.

Turritella exoleta Linn., Poulsen. 9.

Turritella exoleta Sby., Mal. Bl., xxiii, 128.

Turritella exoleta, var. gracilior $=$ Turbo obsoletus Gmel., Poulsen. 9.

Turritella imbricata D'Orb., Arango, Fauna Mal. Cuba, 1878. 165. Oa.

J. Santa Lucia. $=$ veme

Turritella imbricata D'Orb., Mal. Bl., xxiii, 127. J.

Turritella imbricata Kien., Mal. Bl., xxiii, 127. J.

'Turritella imbricata Lam., Mal. Bl., xxiii, 127. 
Turritella imbricata Lam., Mal. Bl., xxiii, 127. J.

Turritella imbricata Lam., Moll. Cuba, ii, 13.

Turritella imbricata Linn., A rango, Fauna Mal. Cuba, 1878. 165. Ca. J. Santa Lucia.

Turritella imbricata Linn., Krebs. 46, 47.

'Turritella imbricata Linn., Krebs' Remarks. 398. WI.

Turritella imbricata Linn., Mal. Bl., xxiii, 127.

Turritella imbricata Liun., Poulsen. 9.

Turritella imbricata Rre., Mal. B!., xxiii, 127. J.

Turritella marmoratus Chem., Krebs. 47.

Turritella meta Cpr., Mal. Bl., xxiii, 127. = vaseigata

Turritella meta Krebs, Mal. Bl. xxiii, 127.

Turritella meta Rve., Krebs. 46. NGr.

Turritella meta Rve., Mal. Bl., xxiii, 127.

Turritella planigyrata Gupps, Geol. Mag., 1874. 438. Td.

Turritella torcularis Born, Bean. 11. G. = exoleta 2

Turritella torcularis Born, Krebs. 46.

Turritella umbricata Lam., Moll. Cuba, ii, 13. =

$\checkmark$ Turritella varia Kien., Mal. Bl., xxiii, 129. = 7hes.acs

$\sqrt{ }$ Turritella variegata Linn., Krebs. 47. ST.

Turritella variegata, Krebs' Remarlis. 398. Carthagena. Cumana.

Turritella variegata Linu., Mal. Bl., xxiii, 126.

Turritella (Haustator) variegata Limn., Marrat, Argo Exp., 1876. 12.

Tucacas. Santa Marta.

Turritella variegata Linn., c. . meta Rre., Poulsen. 9.

Turritella yucatecanum Dall, n. s., Report Blake Moll. 93. Yucatar Strait.

Typhis (see Murex, Muricidea).

Typhis alatus Sby., Guppy, Geol, Mag., 1874. 442. Td. J. Ну.

Uber (see Natica).

Uber albatum Humph., Mal. Bl., xxiv, 60. Ca.

Ultimus (see Orulum, Cyphoma, Volra).

Ultimus gibbosus Mtf., Mal. B1., xxir, 53. Ca.

Unicorum nevicensis, \&c., Mal. Bl., xxiii, 128.

Unio gundlachi Dkr., Araugo, Fauma Mal. Cuba, 187s. 144. Ca.

Unio scamnatus Mor., Arango, Famna Mal. Cuba, 1878. 144. Ca.

Urosalpinx caroliuensis Verrill, List, 1884. 268. Cape Hatteras.

Urosalpinx cinerea Say, Am. Jour. Conch., i, 58. Fla.

Urosalpinx cinereus Say, Am. Marine Conch. 19.

Urosalpinx cinereus Say, Dall, Hemphill's shells. 326. Cedar Keps.

St. Augustine.

Urosalpinx cinereus Say, Jahrb., vi, 173.

Urosalpinx floridana Con., * Am. Jour. Conch., r, pl.12, fig. 4. 106. Fla.

Urosalpinx mexicanus Rve, Jabrb., ri, 173.

Urosalpinx tampaënsis Con., Dall, Hemphill's shells. 326. Sarasota Bay. Cedar Keys.

Bull. 24-21 
Utriculus (see Bulla, Cylichna).

Utriculus bidentatus Chem., Mal. B1., xxii, 171. Ca.

Utrieulus canaliculatus Say, Am. Marine Conch. 103. SC.

Utriculus cavaliculatus Say, Dall, Hemphill's shells. 324. Sarasota

Bay. Cedar Keys.

Utriculus frielei Dall, n. s., Report Blake Moll. 101.

Utriculus spatha Watson, Lin. Soc. Jour., 1883, xrii, 330. Cul.

Utriculus vortex Dall, n. s., Report Blake Moll. 100.

Uvanilla (see Trochus).

Uranilla brevispina Lam., Arango, Fauna Mal. Cuba, 1878. 180. Ca.

M. Bb. Sau Juan.

Uvanilla brevispina Lam., Marrat, Argo Exp., 1876. 13. Santa Marta. Uzita (see Nassa) Marrat, Argo Exp., 1876. 17. Long Key Island.

Vanikoro (see Narica).

Vanikoro lamellosa D'Orb., Mal. Bl., xxir, 94. G.

Vanikoro lamellosa D'Orb., Poulsen. 10.

Tauikoro osyclone Mörch,* Mal. Bl., xxir, 94. ST.

Vanikoro oxychone Mörch, Poulsen. 10.

Vanikoro striata D'Orb., 1Ial. Bl., xxiv, 94. Ca.

Vanikoro sulcata D'Orb., ${ }^{*}$ Mal. Bl., xxir, 95. Ca. J.

Vanikoro suleata D'Orb., Poulsen. 10.

Vanikoro ritrinæformis Mörch, Mal. Bl., xxir, 93.

Vanikoro vitrinæformis Mörch, Poulsen. 10.

Vasum (see Turbinella).

Vasum globulus Rve., Marrat, Argo Exp., 1876. 7. Barbuda.

Tasum muricatum Born, Arango, Fauna Mal. Cuba, 1878. 222. Ca.

Baracoa.

Velutina cane-llata Q. \& G., Moll. Cuba, ii, 40.

Venerea brasiliensis Kirch., Mal. Bl., xxir, 53.

Venericardia (see Cardita).

Venericardia grauulata Say, Bush, Coun. Ac. vi, 477. Hatt.

Venericardia obliqua Bush, Conn. Ac. vi, 478. Hatt.

Veneroides barbadensis Petiv., Moll. Cuba, i, 121.

Veneroides barbadensis Petiv., Moll. Cuba, i, 122.

Venus (see Chione, Callista, Dione, Mercenaria, etc.)

Venus (see Asaphis) Araugo, Fanua Mal. Cuba, 1878. 241. Ca.

Venus (see Cytherea) Arango, Fauna Mal. Cuba, 1878. 251. Ca.

Venus (see Dosinia) Arango, Fauna Mal. Cuba, 1878. 251. Ca.

Venus (see Lucina) Arango, Fauna Mal. Cuba, 1878. 255. Ca. ST. M. Ba.

Venus (see Lueina) Arango, Fauma Mal. Cuba, 1878. 256. Ca. M. G. ST. Ba.

Venus (see Petricola) Arango, Fauna Mal. Cuba, 1878. 248. Ca. M. $\mathrm{SD} . \mathrm{Ba}$.

Venus affinis Grel., Krebs. 95.

Venus albida Gmel., Jour. de Conch, rii, 26. M. G. 
$\checkmark$ Venus albida Gmel., Jour. de Conch, vii, 37.

Venus albida Gmel., Beau. 24. G.

Venus albida Gmel., Krebs. 95.

Venus (Chione) alveata Cour., Bush, Conn. Ac. vi, 477. Hatt. =

Venus antillarum D’Orb., Arango, Fauna Mal. Cuba, 1878. 249. Oa. M. ST. J.

Venus antillarum D'Orb., Krebs. 95.

Venus autillarum D'Orb.,* Moll. Cuba, ii, pl. xxvi, figs. 41-43. 278. ST. J.

Venus asperrima Sby., Jahrb., ii, 249.

Venus auberiuna D’Orb., Alaugo, Fauna Mal. Cuba, 1878. 249. Ca. Ba.

Venus auberiana D'Orb., Krebs. 95.

Venus auberiaua D'Orb., Moll. Cuba, ii, 11. xxri, figs. 35-37. 277. Ca.

Venus beaui Reel., Jour. de Conch, iv, 415. G.

Venus beauii Recl., Jour. de Conch, vii, 26. M. G.

Venus beanii Recl., Jour. de Conch, vii, 37.

Venus beauii Recl., Beau. 24. G.

Venus beauii Recl., Jour. de Conch. iii, pl. xii, fig. 15a, 15b. 412. G.

Venus beauii Recl., Krebs. 95.

Venus beauii Recl., Krebs. 98.

Venus blandiana Guppy, n. s., Geol. Mag., 1874. 444. J.

Venus cancellata Chem., Jour. de Conch, vii, 26. M. G.

Veuus cancellata Chem., Jour. de Conch, vii, 37.

Venus cancellata Chem., Mal. Bl., xiv, 45.

Venus cancellata Chem., Jour. de Conch, ii, 425. G.

Venus cancellata Chem., Krebs. 97.

Venus cancellata Gron., Guply, Geol. Mag., 1874. 450. Td. Oumana. Ну.

Venus cancellata Lam., Mal. Bl., x, 231.

Venus cancellata Lam., Moll. Uuba, ii, 271.

Venus cancellata Linn., Arango, Fauna Mal. Cuba, 1878. 251. Ca. Ba.

Venus cancellata Linu., Beau. 24. G.

Venus cancellata Linn., Krebs. 98.

Tenus cancellata Limn., Marrat, Argo Exp., 1876. 15. Havana.

Venus cancellata Linn., Moll. of Bermudas. 25.

Venus cardioides Lam., Krebs. 95.

Venus cincta Chem., Mal. Bl., xiv, 116.

Venus cingenda Bose, Mal. Bl., xir. 45.

Venus cingenda Dillw., Jour. de Conch., ii, 425. G.

Venus cingenda Dillw., Krebs. 98.

Venus cingulata Lam., Am. Jour. Couch., i, 297.

Venus circinata Born, Arango, Fauna Mal. (Yuba, 1878. 249. Oa

Venus circinata Born, Krebs. 95. ST. Td. NGr.

Venus circinata Born, Moll. Cuba, ii, 275.

Venus circiuata Chem., Moll. Cuba, ii, 275. 
Venus circinata D’Orb., Arango, Fauna Mal. Cuba, 1878. 249. Ca.

Venus concentrica Moll. Cuba, ii, 268, 270.

Venus concentrica Born, Moll. Cuba, ii, 274.

Venus concentrica Chem., Moll. Cuba, ii, 274.

Venus corbicula Gmel., Jour. de Conch., ir, 415. G.

Venus corbicula Gmel., Arango, Fauna Mal. Cuba, 1878. 250. Ca. G. Ba.

Venus corbicula Gmel., Moll. Cuba, ii, 276.

Venus crenata Dillw., Mal. Bl., xiv, 41. =

Venus crenata Gray, Krebs. 95.

Venus erenifera Sby., Moll. of Bermudas. 25. Ex - Le

Venus crenulata Chem., Krebs. 98.

Venus crenulata Chem., Krebs. 95.

Venus crenulata Chem., Marratt, Argo Exp., 1876. 13. Santa Marta.

Venus crenulata Chem., Poulsen. 25.

- Venus cubaniana D'Orb., Arango, Fauna Mal. Cuba, 1878. 249. Ca. M. Fla. I

Venus cubaniana D'Orb., Krebs. 96.

Venus cubaniaua D'Orb., Moll. Cuba, ii, pl.xxvi, figs.44-46. 278. M. Fla.

Venus cubaniana D'Orb., Report Blake Moll. 130.

Venus cuneimeris Courad, Pro. Acad. Nat. Sci., Phila., iii, 24, pl. 1, fig.

13. 1845. Tampa Bay.

- Venus deflorata Gmel., Jour. de Conch, ii, 425. G. $=C_{1} t_{-}$;

Venus deflorata Linn., Jour. de Conch., vii, 140.

Venus deflorata Limn., Moll. Cuba, ii, 258.

Venus dione Chem., Moll. Cuba, ii, 269.

Venus dione Chem., Moll. Cuba, ii, 274.

Veuus dione D'Orb., Arango, Fauna Mal. Cuba, 1878, 249. Ca.Td.

Venus dione Linn., Arango, Fanna Mal. Cuba, 1878. 249. Ca. Td.

Venus dione Linn., Krebs. 96.

Venus dione Linn., Moll. Cuba, ii, 274, 275.

Venus divaricata Chemn., Arango, Fauna Mal. Cuba, 1878. 248. Ca.

M. SD. Ba.

Venus divaricata Chem., Krebs. 108.

Venus divaricata Chem., Moll. Cuba, ii, 265.

Venus divergens Gmel., Arango, Fauna Mal. Cuba, 1878. 248. Ca. M. SD. Ba.

Venus divergens Gmel., Krebs. 108.

Venus divergens Gmel., Moll. Cuba, ii, 265.

Venus dysera Chem., Krebs. 96.

Venus dysera Chem., Moll. Cuba, ii, 271.

Venus dysera D'Orb., Arango, Fauna Mal. Cuba, 1878. 251. Ca. Ba.

Venus dysera D'Orb., Krebs. 98.

Venus dysera D'Orb., Moll. Cuba, ii, 271.

Venus dysera Linn., Arango, Fauna Mal. Cuba, 1878. 251. Ca. Ba. 
Venus dysera Linn., Mal. Bl., x, 231. =

Venus dysera Linn., Moll. Cuba, ii, 271.

$\checkmark$ Venus edentula Chem., * Zt. Mal., ii, 180.

$\checkmark$ Venus edentula Chem., Krebs. 119.

Venus edentula Chem., Moll. Cuba, ii, 299.

Venus edentula Linn., Moll. Cuba, ii, 299.

Venus edentula Linn., Zt. Mal., ii, 179.

Venus elegans Gray, Krebs. 98. $=c$

Venus eximia Chem., Mal. Bl., xiv, 41.

Venus eximia Phil., Beau. 24. G.

Venus (crenulata jr. ?) eximia Phil., Jour. de Conch., v, 150. G.

Venus eximia Phil., Krebs. 95.

$\checkmark$ Venus eximia Phil., * Zt. Mal., iv, 90.

Venus exoleta var. Gray, Mal. Bl., ix, 67.

Venus fimbriata Linn., Krebs. 122.

Venus flexuosa Chem., Moll. Cuba, ii, 270.

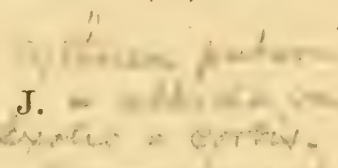

Venus flexuosa D'Orb., Arango, Fauma Mal. Cuba, 1878. 249. Ca.

Venus flexuosa D'Orb., Moll. Cuba, ii, 271.

Venus flexuosa Liun., Arango, Fauna Mal. Cuba, 1878. 249. Ca.

Venus flexuosa Linn., Jahrb., ii, 249.

Venus flexuosa Linn., Krebs. 96. NGr. J. ST.

Venus flexuosa Linn., Moll. Cuba, ii, 270.

Venus fulminata Mke., Arango, Fauna Mal. Cuba, 1878. 251. Ca. M. G.=

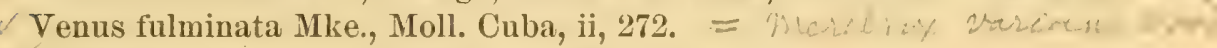

$\checkmark$ Venus gemma Totteu, Kurtz. 5. NO. SC.

Venus granulata Gmel., Jour. de Couch., vii, 26. M. G.

Venus granulata Gmel., Jour. de Couch., vii, 37.

Venus grauuata D'Orb., Alaugo, Fauna Mal. Cuba, 1878. 250. Ca. II. G. Ba.

Venus granulata Gmel., Arango, Fauna Mal. Cuba, 1878. 250. Ca.

M. G. Ba.

Venus granulata Gmel., Beau. 24. G.

Venus granulata var. Gmel., Jour. de Conch., ii, 425. G.

Venus graulata Gmel., Krebs. 96. S'T. SJ. Tort. SCx. NGr.

Venus granulata Gmel., Moll. Cuba, ii, 276.

Venus granulata Gmel., Poulseu. 15.

Venus granulata Lam., Moll. Cuba, ii, 276.

Venus guineensis Gmel., Arango, Fauna Mal. Cuba, 1878. 249. Ca. Venus guineensis Gmel., Moll. Cuba, ii, 275.

Tenus hebræa Lam., Krebs. 97. ST.

Venus inequalis Say, Kurtz. 5.

Venus inæquivalvis DOrb., Arango, Fauna Mal. Cuba, 1878. 250.

Ca. M. Fla.

Venus inæquivalvis D'Orb., Krebs. 97. $=p y$;

Venus iuæquivalvis D'Orb., ${ }^{*}$ Moll. Cuba, ii, 1)l. xxvi, figs. 38-40. 277.

Ca. 
Venus inæquivalvis D'Orb., Poulsen. 15.

Venus intapurpurea Conrad, Journ. Acad. Nat. Sci. Phil., n. s., i, 209, 280, pl. 39, fig. 9, 1849. Tampa.

Venus jamaicensis Chem., Moll. Cuba, ii, 294.

Venus lamarckii Gray, Mal. B1., iv, 27. G.

Venus lamarckii Gray, Beau. 24. G.

Venus lamarckii Gray, Jour. de Conch., ii, 425. G.

Venus lamarckii Gray, Jour. de Conch, iv, 415. G.

Venus lamarckii Gray, Krebs. 98.

Venus lamarckii Petit, Jour. de Conch., v, 155. G.

Venus lanceata Say, Conrad., Am. Jouru. Sci,, n. s., i, 404, 1846. S. coast U.S.

Venus lapicida Chem., Krebs. 108.

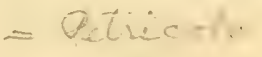

Venus lapicida Chem., Moll. Cuba, ii, 265.

Venus lapicida Gmel., Moll. Cuba, ii, 265.

Venus listeri Gray, Krebs. 97.

Venus listeri Gray, Poulsen. 15.

Venus lucinalis Lam., Krebs. 97.

Venus mactroides Born, Jour, de Conch., vii, 26. MG.

Venus mactroides Born, Jour. de Conch., vii, 37.

Venus mactroides Born, Arango, Fanna Mal. Cuba, 1878. 250. Ca. G. Ba.

Venus mactroides Born, Jour. de Conch., iv, 415. G.

Venus mactroides Born, Krebs. 97. NGr.

Venus mactroides Born, Moll. Cuba, ii, 276.

Venus mactroides Chem., Moll. Cuba, ii, 276.

Venus mactroides I'Orb., Arango, Fauna Mal. Cuba, 1878. 250. Ca. G. Ba.

Venus mactroides Wood, Moll. Cuba, ii, 276.

Venus maculata Chem., Moll. Cuba, ii, 269.

Venus maculata D'Orb., Arango, Fauna Mal. Cuba, 1878. 250. Ca. G. M. J. Santa Lucia. Ba.

Venus maculata D’Orb., Moll. Cuba, ii, 269.

Veuus maculata Linn., Arango, Fauna Mal. Cuba, 1878. 250. Ca. G. M. J. Santa Lucia. Ba.

Venus maculata Linn., Jour. de Conch., iv, 415. G.

Venus maculata Linn., Krebs. 97. ST.

Venus maculata Linn., Moll. Cuba, ii, 269.

Venus maculosa Brug., Mal. Bl., xiv, 45.

Venus marica Born, Moll. Cuba, ii, 278.

Venus marica Born, Arango, Fanna Mal. Cuba, 18r8. 250. Ca. M. G. Ba.

Venus marica Born, Moll. Cuba, ii, 276.

Venus marica Chem., Krebs. 96.

Venus marica Sclröter, Mal. Bl., xiv, 93.

Venus marica-spuria Brug., Mal. Bl., xiv, 93. 
Venus mercenaria Linn., Kurtz. 5. NC. SC. Fla. to N. Eugland.

Venus metastriata Say, Conrad, Am. Journ. Sci., i, 404. 1846. Fla.

Venus minuta Koch, Krebs. 97.

Venus mortoni Conral, Jomru. Acad. Nat. Sei., Phil., vii, 25̃. 1837. Charleston, SC.

Venus notata Sas, Kurtz. 5. NC. SC.

Venus orbicularis Kurtz, u. s., Kurtz. 5. SC. :

Venus paphia D'Orb., Arango, Fauna Mal. Cnba, 1878. 250. Ca. G. Ba.

Venus paphia D’Orb., Moll. Cuba, ii, 273.

Venus paphia Linu., Krebs. 98.

Venus paphia Linn, Alango, Fauna Mal. Cuba, 1878. 250. Ca. G Ba.

Venus paphia Linu., Beau. 24. G.

Venus paphia Linn., Jour. de Couch., iv, 415. G.

Venus paphia Linn., Krebs. 97. ST. Tort. SJ. SCx. NGr.

Venus paphia Liuu., Mal. Bl., $\mathbf{~}, 231$.

Venus paphia Linn., Moll. Cuba, ii, 273.

Venus paphia Liun., Poulsen. 15.

Venus pectorina Lam., Krebs. 98.

Veuus pectorina Lam., Poulsen. 15.

Venus pennsylvanica Born, Moll. Cuba, ii, 300. = Codakia

Venus pennsylvanica Chem., Krebs. 118.

Venus pennsylvanica Chem., Moll. Cuba, ii, 300.

Venus pennsylvanica Linn., Krebs. 120.

Venus pennsylvauica Linn., Moll. Cuba, ii, 300.

Venus philippi D’Orb, Arango, Fauna Mal. Cuba, 1878, 252. Ca.

Venus philippi D'Orb., Krebs. 98.

Venus philippi D'Orb., Moll. Cuba, ii, 270. =

Venus phillipsii D'Orb., Moll. Cuba, ii, 274. =

Venus plicata Gmel., Mal. Bl., xiv, 45.

Venus porteriaua D’Orb., Mal. Bl., x, 231. “Ca.

Venus præparca Say, Kurtz. 5. NC. SO.

Venus puerpera var. Lam., Krebs. 97. = Listeri

Venus pulicaria Brod., Mal. Bl., xiv, 119.

Venus pullastra, Moll. Cuba, ii, 268.

Venus punctata Linn., Krebs. 120.

Venus punctifera Gray, Krebs. 96.

Venus purpurascens Gmel., Krebs. 106. = Lumle

Venus purpurata Gmel., Arango, Fauna Mal. Cuba, 1878. 241. Ca.

Veuus pygmæa Lam., Arango, Fanna Mal. Cuba, 1878. 251. Ca. Ba.

Venus pygmæa Lam., Beau. 24. G.

Venus pygmæa Lam., Jour. de Conch., ii, 425. G.

Venus pygmra Lam., Krebs. 98. SJ. ST. Tort. SUx. SB.

Venus pygmæa Lam., Poulsen. 15.

Venus reticulata Linn., Arango, Fauna Mal. Cuba, 1878. 249. Ca. = 
Venus rigida Gray, Mal. Bl., xir, 116:

Venus rigida Sol., Krebs. 98.

Venus rostrata Sowerby, Thes. Conchyl., 787, pl. 156, fig. 91, 1847. Fla.

Venus rubiginosa D'Orb., Arango, Fauna Mal. Cuba, 1878. 251. Ca.

M. G.

Venus rubiginosa D’Orb., Moll. Cuba, ii, 272.

Venus rubiginosa Mke., Araugo, Fauna Mal. Cuba, 1878. 251. Ca. M. $\mathrm{G}$.

Venus rubiginosa Phil., Jour. de Conch., iv, 415. G.

Venus rugosa Chem., Moll. Cuba, ii, 268.

Venus rugosa Chem., Krebs. 98. SCx. Tort. SJ.

Venus rugosa Chem., Moll. Cuba, ii, 273.

Venus rugosa Chem., Poulsen. 15.

Jenus rugosa D'Orb., Arango, Fauma Mal. Cuba, 1878. 251. Ca. M. G. Ba.

Venus rugosa D'Orb., Moll. Cuba, ii, 273.

Venus rugosa Gmel., Araugo, Fauna Mal. Cuba, 1878. 251. Ca. M. G. Ba.

Venus rugosa Gmel., Beau. 24. G.

Venus rugosa Gmel., Jour. de Conch., iv, 415. G.

Venus rugosa Gmel., Moll. Cuba, ii, 273.

Venus rugosa Lam., Moll. Cuba, ii, 273.

Venus rugosa orientalis, Mal. Bl., xir, 116.

Venus subrostrata Lam., Krebs. 95.

- Venus subrostrata Lam, Krebs. 98.

Venus tigerina Born, Moll. Cuba, ii, 297.

Venus tigerina Chem., Moll. Cuba, ii, 297.

Venus tigerina Linn., Mol. Cuba, ii, 297.

Venus trapezoidalis Kurtz. n. s. Kurtz. 5. NC. SC.

Venus trapezoidalis Kurtz, Mal. Bl., xiv, 60.

Venus trigouella Lam., Arango, Fauna Mal. Cuba, 1878. 251. Ca.

Venus trigonella Lam., Krebs. 98.

Venus tripla Linu., Krebs. 95. NGr.

/ Venus rerrucosa Linn., Moll. Quba, ii, 267, 268.

Vencos or vivecenses Sby

Venus verrucosa Linn., Mal. Bl., xii, 126.

Venus rerrucosa Limn., Zt. Mal., x, 81. SV.

Veuus rersicolor Gmel., Arango, Fauna Mal. Cuba, 1878. 241. Ca.

Venus violacea Gmel., Arango, Fauna Mal. Cuba, 1878. 250. Ca. M.

G. Ba.

Venus violacea Gmel., Moll. Cuba, ii, 276.

Venus violacea Lister, Mal. Bl., xiv, 93.

Venus ziczac Linn., Arango, Fauna Mal. Cuba, 1878. 251. Ca. Ba.

Venus ziczac Linn., Krebs. 98. SCx. SJ. ST. Tort. PR.

Venus ziczac Linn., Poulsen. 15. 
Vermetus (see Serpula, Serpuloides, Serpulorbis, Siliquaria, Petaloconchus, Bironia, Thylacodus, Anguinaria, Tenagodus, Pyxipoma, Vermicularia, Siphonium, Spiroglyphus).

Vermetes conicus Dillw., Poulsen. 10.

Vermetes varians D'Orb., Poulsen. 10.

Vermetus badia Mörch.,** Mal. Bl., xxiv, 118. ST.

Vermetus bicarinatus Desh., Jour. de Conch., viii, 29. Ca.

Vermetus bicarinatus Desh., Mal. Bl., xxiv, 112.

Vermetus brasiliensis Mörch, Mal. Bl., xxiv, 123. Bz.

Vermetus brasiliensis Rouss., Mal. Bl., xxiv, 123. Bz.

Vermetus candidissima Mörch,* Mal. Bl, xxir, 118. ST.

Vermetus carpenteri Mörch, Mal. Bl., xxiv, 117. SV.

Vermetus conicus Dillw., Jour. de. Conch., viii, 3S. ST.

Vermetus conicus Dillw., Mal. Bl., xxiv, 119.

Vermetus conicus Dillw., Mal. Bl., xxiv, 121.

Vermetus conieus Mörch, Mal. Bl., xxiv, 119.

Vermetus corrodens D'Orb., Arango, Fauna Mal. Cuba, 1878. 164. Ca. M.

Vermetus corrodens D'Orb., Mal. Bl., xxiv; 115.

Vermetus corrodeus D'Orb., Moll. Cuba, i, pl. xriii, figs. 1-3. 235.

Vermetus corrodens D'Orb., Jour. de Consh., viii, 44. Ca.

Vermetus corrorlees D'Orb., Krebs. 74.

Vermetus decussata Gmel., Krebs. 74.

Vermetus decussatus Blainv., Mal. Bl., xxiv, 120. ST.

Vermetus decussatus Desh., Mal. Bl., xxir, 120. ST.

Vermetus decussatus Gmel., Jour. de Conch, viii, 3t. Bb. ST.

Vermetus decussatus Lam., Jour. de Conch., viii, 38. ST.

Vermetus dentifera Gray, Mal. Bl., xxiv, 114.

Vermetus dentiferus Sby, Jour. de Conch.., vii, 357. ST.

Vermetus electrinus Mörch, Mal. Bl., xxir, $1^{\top}$ s.

Vermetus glomeratus, Sby., Mal. Bl., xxiv, 117.

Vermetus gordialis Mörch, Mal. Bl., xxiv, 120. ST.

Vermetus indicus Rouss., Mal. Bl., xxiv, 111. Ca.

Vermetus irregularis D'Orb., Arango, Fama Mal. Cuba, 1878. 164. M.

Vermetus irregularis D'Orb., Jour. de Conch., viii, 45. Ca.

Vermetus irregularis D'Orb., Krebs. 74.

Vermetus irregularis D'Orb., Mal. B1., xxiv, 116.

Vermetus irregularis D'Orb., Mal. Bl. хxir, 118. Ca.

Vermetus irregularis D'Orb., Moll. Cuba, i, pl. xvii, figs. 16-18. 235.

Vermetus irregularis Mörch,* Moll. Bl., xxiv, 117. Ca.

Vermetus knorrii Desh., Krebs. 74. ST.

Vermetus knorrii Desh., Mal. Bl., xxiv, 111.

Vermetus knorrii Say, Moll. of Bermudas. 18.

Vermetus lumbricalis Bowd., Mal. B1., xxiv, 111. Ca.

Vermetus lumbricalis Linn., Zt. Mal., v, 17. 
Vermetus lumbricalis D'Orb., Arango, Famna Mal. Cuba, 1878. 164. Ca. Havana. Ba.

Vermetus lumbricalis D'Orb., Mal. Bl., xxiv, 111.

Vermetus lumbricalis Gld., Mal. Bl., xxiv, 111.

Vermetus lumbricalis Linu., A rango, Fauna Mal. Cuba, 1878. 164. Ca. Havana. Ba.

Vermetus lumbricalis 'Linn., Krebs. 74.

Vermetus lumbricalis Linu., Marrat, Argo Exp., 1876. 6. Aa. Nassin. Abaco.

Vermetus lumbricalis Linn., Moll. Cuba, i, 234.

Vermetus lumbricalis Linu., var. nigricans, Dall, Hemphill's shells. 334. Sarasota Bay.

Vermetus lumbricalis Sby., Mal. Bl., xxiv, 111. Ca.

Vermetus lumbricalis Wood, Mal. Bl., xxir, 112.

Vermetus personata Mörch, Mal. BI., xxiv, 119. SOx.

Vermetus planorboides Mörch, Mal. BI., xxir, 114.

Vermetus quadrangularis Phil., Mal. Bl., xxiv, 111. Bb.

Vermetus quadrangulus Phil., Jour. de Conch., viii, 29. Yucatan.

Vermetus quadrangulus Phil.,* Zt. Mal., v, 17. Y.

Vermetus radicula Gray, Mal. Bl., xxiv, 111.

Vermetus radicula Stm., Am. Marine Conch. 74. NC.

Vermetus radicula Stm., Mal. Bl., xxir, 111.

Vermetus scalaris Möreh,* Mal. Bl., xxiv, 112. H.

Vermetus spiratus Phil., Zt., Mal., i, 23. Ca.

Vermetus spiratus Phil., Arango, Fauua Mal. Cuba, 1878. 165. Ma. tanzas.

Vermetus spirat us Phil., Jour. de Conch., viii, 29. Ca.

Vermetus spiratus Phil., Mal. Bl., xxiv, 111.

Vermetus tricarinatus Desh., Zt. Mal., i, 23. Ca.

Vermetus trilineatus Guppy, Geol. Mag., 1874. 438. Td.

Vermetus trochicola Möreh, Mal. Bl., xxir, 116. ST.

Vermetus varians Cpr., Mal. Bl., xxir, 117. Bz.

Vermetus varians D’Orb., Jour. de Conch., viii, 41. SV.

Vermetus varians D'Orb., Mal. Bl., xxiv, 116. Bz.

Vermetus varians Gray, Mal. Bl., xxiv, 117. Bz.

Vermetus varians var. Mörcb, Mal. Bl., xxiv, 118.

Vermicularia (see Vermetus).

Vermicularia arenaria Grav., Mal. Bl., xxiv, 114.

Vermicularia glomerata Grav., Mal. Bl., xxir, 112.

Vermicularia glomerata Grav., Mal. Bl., xxiv, 120. ST.

Vermicularia spirata Phil., Mal. Bl., xxiv, 111. Bb.

Vermicularia spirata Phil., Poulsen. 10.

Vermicularia spirata Phil, var. scalaris Mörch, Poulsen. 10.

Vermicularia spirata Phil,, var. unguliua Mörch, Ponlsen. 10.

Vermiculus lnmbricalis Linn., Jour. de Conch., viii, 28. M.

Vermiculus spiratus Phil., Jour, de Conch., viii, 29. Ca. 
Veronicella floridana Binney, Terr. Moll. U. S., ii, pl. Ixvii, 17, 1851. Fla.

Veronicella occidentalis Guild., Beau. 14. G.

Vertagus (see Cerithiun).

Vertagus stercus-muscarum Say, Mal. Bl., xxiii, 111. Fla.

Verticordia acuticostata Phil., Report Blake Moll. 105.

Verticordia deshayesiana Fiseher, Teport Blake IIoll. 105.

Verticordia elegautissima Dall, 1. s., Report Blalie .Ioll. 106.

Verticordia fischeriana Dall, n. s.. Report Blake Mfoll. 106.

Verticordia japonica A. Adams, Report Blake Moll. 105.

Verticordia oruata D'Orb., Jour. de Conch., viii, 299. J.

Verticordia ornata D'Orb., Report Blake Moll. 105.

Verticordia oruata Fiseher, Report Blake Moll. 105.

Vestis attalica Mart., Moll. Bl., xxiv, 39.

Vitrinella (see Skenea).

Vitrinella adamsii Fisch., Krebs. S0.

Vitrinella beauii Fisch., Krebs. 81.

Vitrinella helicoidea C. B. Adams, Jour. de Conch., vi, 173. J.

Vitrinella helicoidea C. B. Adams, Krebs. 81.

Vitrinella helicoidea C. B. Adams, Mon. Vitr., 1850. 9. J.

Vitrinella hyalina C. B. Adams, Jour. de Conch., vi, 173. J.

Vitrinella hyaliua C. B. Adams, Krebs. 81.

Vitrinella hyalina O. B. Adams, Mon. Vitr., 1850. 5. J.

Vitrinella interrupta C. B. Adams, Jour. de Conch., vi, 173. J.

Vitrinella interrupta C. B. Adams, Krebs. 81.

Vitrinella interrupta C. B. Adams, Mon. Vitr., 1850. 6. J.

Vitrinella marginata Guppy, Geol. Mag., 1874. 443. Td.

Vitrinella rnegastoma C. B. Adams, Jour. de Conch., vi, 173. J.

Vitrinella megastoma C. B. Arlams, Jour. de Conch., vi, 288. J.

Vitrinella megastoma C. B. Adams, Krebs. 81.

Vitriuella megastoma C. B. Adams, Mon. Vitr., 1850. 7. J.

Vitrinella petitii Fisch., Krebs. 81.

Vitrinella schrammii Fisch., Krebs. 81.

Vitrinella tincta C. B. Adams, Jour. de Conch., vi, 173. J.

Vitrinella tineta C. B. Adams, Krebs. 81.

Vitrinella tincta C. B. Adams, Mon. Vitr., 1850. 8. J.

Vitta (see Neritella), Marrat, Argo Exp., 1876. 8. D. Havana.

Vola (see Pecten).

Vola aspersa Sby., Marrat, Argo Exp., 1876. 12. Tucacas. Nassau.

Vola ziczac Klein., Krebs. 134.

Vola zigzag Linn., Arango, Fauna Mal. Cuba, 1878. 270. Ca. G. M.

Santa Lucia. SD. ST. Ba.

Volsella (see Modiola).

Volsella americana Favart, Mal. Bl., x, 230.

Volsella americana Seop., Krebs. 128.

Voluta (see Ljria). 
Voluta (see Cancellaria) Arango, Fauna Mal. Cuba, 1878. 197. Ca. G. Aa.

Voluta (see Columbella), Arango, Fanna Mal. Cuba, 1878. 199. Ca. Antilles.

Voluta (see Mitra), Arango, Fauna Mal. Cuba, 1878. 197. Ca. G. SV. Ba.

Voluta (see Olivella), Arango, Fauna Mal. Cuba, 1878. 192. Ca. Coa. G. $\mathrm{Bb}$.

Voluta (see Turbinelia), Arango, Fauna Mal. Cuba, 1878. 221. G. M., Santa Lucia.

Voluta americana Rre., Jahrb., ir, 309. Bz. P. zs 51856 , pl $33,2 \mathrm{iq}, 1,2$

Voluta archeri Angas, Jour. de Conch., siv, 113.

Voluta beaui Fisch., Jour. de Conch., xiv, 107. MG.

Voluta beani Fiseh., Jour. de Conch., xiv, 112. MG.

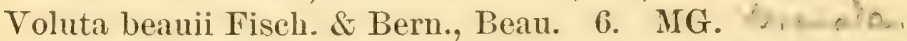

Voluta beauii Fisch. \& Bern., Jour. de Couch., r, pl. ix, figs. 1, 2. 296. MG.

Voluta beauii Fisch. \& Bern., Krebs. 36.

Voluta capitellum Linn., Moll. Cuba, ii, 178.

- Voluta carneolata Lam., Beau. 6. G.

Voluta carneolata Lam., Jahrb., iv, 301.

Voluta carneolata Lam., Jour. de Conch., xix, 271.

Voluta chlorosina Lam., Beau. 6. G.

Voluta clersana Petit, Jahrb., iv, 309. Bz. Cep or. xheme, y

Voluta cleryana Petit, Jour. de Conch., v, 182. Bz.

Voluta cœrulescens Lam., Krebs. $4 \check{.}$

Voluta coffea Linn., Mal. Bl., vi, 117. SCx.

ctuin Voluta corticata Mart., Krebs. 38.

Voluta cumingi Brod., Jour. de Coneh., xiv, 114. ST.

Voluta cylleniformis Sby., Poulsen. 12. it
Voluta dubia Brod.* Jahrb., vi, 153. Fla.

-Voluta granulosa Lam., Arango, Fauna Mal. Cuba, 1878. 197. Ca. Antilles.

Voluta guildingi Sby., Jour. de Conch., siv, 115. SV.

Voluta guildingii Sby, Krebs. 36.

Voluta guinaica Lam., Beau. 6. G.

Voluta guinaica Lam., Jahrb., iv, 301.

Voluta guinaica Lam., Jour de Concb., xix, 271.

- Voluta guttata Dillw.; Arango, Fauna Mal. Cuba, 1878. 187. Havana. Ba. Tort. Hd.

Voluta heteroclita Mont., Mal. Bl., ir, 27. G.

$\checkmark$ Voluta incrassata Sol., Krebs. 38.

Voluta jaspidea Gmel., Krebs. 38.

$\checkmark$ Voluta junonia Chem., Jour. de Conch., xxii, 64, 65.

$\checkmark$ Voluta junonia Chem., Am. Marine Conch. 53.

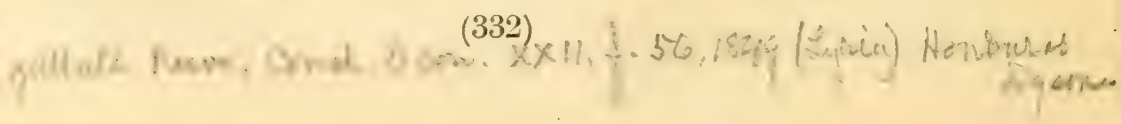


$\checkmark$ Toluta junonia Chem., Arango, Fauna Mal. Cuba, 1878. 196. Matanzas.

$\checkmark$ Voluta junonia Chem., Jahrb., iv, 306.

Voluta junonia Chem., Jahrb., vi, 152. Fla.

Voluta junonia Chem., Jour. de Conch., xix, 266.

Voluta junonia Chem., Jour. de Conch., xix, 285. Fla.

$\checkmark$ Voluta junouia Chem., Jour de Conch., xxiv, 397.

$\checkmark$ Voluta junonia Chem, Jour. de Conch., xxvi, 102.

voluta junovia Lam., Thesaurus Conchyl., 1847, 197, pl. 99, fig. 44. Gulf of Mexico.

$\checkmark$ Voluta lærigata Lam., Beau. 6. G.

Voluta lærigata Lam., Jahrb., iv, 301.

$\checkmark$ Voluta lævigata Lam., Jour. de Coneh., xis, 271.

$\checkmark$ Voluta marginata Born, Moll. Cuba, ii, 96. Margmella

$\checkmark$ Voluta marginata Chem., Moll. Cuba, ii, 96.

Voluta marginata Gmel., Moll. Cuba, ii, 96.

Volnta mereatoria Gmel., Moll. Cuba, ii, 132.

Voluta mercatoria Linn., Moll. Cuba, ii, 132.

Toluta minuta Gmel., Am. Jour. Conch., ir, 17. , 14! - pred

$\checkmark$ Voluta musica Chem., Moll. Cuba, ii, 124.

8. Voluta musica D'Orb., Arango, Fauna Mal. Cuba, 1878. 196. Ca. An. tilles.

- Voluta musica Gmel., Moll. Cuba, ii, 124.

$\checkmark$ Voluta musica Lam., Moll. Cuba, ii, 125.

Voluta musica Linn., Arango, Fauna Mal. Cuba, 1878, 196. Oa. Antilles.

$\checkmark$ Voluta musica Linn., Beau. 6. Iles des Saintes.

Voluta musica Linn., Jahrb., iv, 301.

- Volnta musica Linn., Jour. de Conch., iv, 419. G.

$\sim$ Voluta musica Linn., Jour. de Conch., xix, 266.

Voluta musica Linn., Jour. de Conch., xix, 270.

- Voluta musica Liun., Krebs. 36. Bb.

- Voluta musica Linn., Moll. Cuba, ii, 124.

- Voluta musica Linn., Poulsen. 12.

Voluta musica Wood, Moll. Cuba, ii, 125.

Voluta musica var., ${ }^{*}$ Jour. de Couch., xxir, pl. v, fig. 6. 163.

$\checkmark$ Voluta nasuta Gmel., Mal. Bl., xxiv, 27. Shitri $2 \mu_{1}$

Voluta nirea Gmel., Arango, Fauna Mal. Cuba, 1878. 193. Ca. Ba.

$\checkmark$ Voluta nivea Gmel., Krebs. 40. cévelea.,

Voluta nivea Gmel., Moll. Cuba, ii, 106.

Voluta nodulosa D'Orb., Arango, Fauna Mal. Cuba, 1878. 197. Ca. Antilles. mitra

Voluta nodulosa Gmel., Arango, Fauna Mal. Cuba, 1878. 197. Ca. Antilles.

Voluta nodulosa Gmel., Moll. Cuba, ii, 126. Inilra

$\checkmark$ Voluta nodulosa Lam., Beau. 6. G. 
$\checkmark$ Voluta nodulosa Wood, Moll. Cuba, ii, 127. mitres

Voluta ocellata Gmel., Arango, Fauna Mal. Cuba, 1878. 198. Ua. Ba. Voluta ocellata Gmel., Moll. Cuba, ii, 134.

Voluta ocellata Gmel., Zt. Mal., x, 75. SV.

voluta pinguis Dillw., Jahrb., ii, 242.

$\checkmark$ Voluta plicata Dillw., Jahrb., iv., 301. = mukica ves.

Voluta plicata Dillw., Jour, de Conch., xix, 271.

$\checkmark$ Volut polypleura Crosse, Jahrb., ir, 301.

Voluta polyzonalis Kien., Jahrb., ir, 301.

inederVoluta prunum Gmel., Krebs. 45.

ieularivoluta reticulata Grmel., Moll. Cuba, ii, 128.

Voluta reticulata Linu., Moll. Cuba, ii, 128.

Voluta reticulata Wood, Moll. Cuba, ii, 128.

Voluta striatula Mart., Krebs. 38.

Voluta sulcata Chem., Jahrb., iv, 301.

$\checkmark$ Voluta sulcata Chem., Jour. de Conch., xix, 271.

$\checkmark$ Voluta sulcata Lum., Beau. 6. G.

$\checkmark$ Voluta thiarella Lam., Araugo, Fama Mal. Cuba, 1878. 196. Ca. Antilles.

$\checkmark$ Voluta thiarella Lam., Beau. 6. G.

Voluta thiarella Lam., Jahrb., iv, 301.

Voluta thiarella Lam., Jour. de Conch., xix, 271.

$\checkmark$ Voluta thiarella Lam., Moll. Cuba, ii, 125.

, Voluta trenholmii T. \& H., n. s., Pleiocene Fossils, S. O., pl. xxvii, figs. 7, 9.128.

Voluta triplicata Don., Am. Jour. Conch., iv, 17.

Voluta verruculata Karsten, Mal. Bl., xxiv, 40.

Voluta respertilio Chem., Moll. Cuba, ii, 123.

Voluta vespertilio Gmel., Moll. Cuba, ii, 123.

Voluta vespertilio Lam., Moll. Cuba, ii, 123.

Voluta respertilio Linn., Moll. Cuba, ii, 123.

Voluta respertilio Rumph., Arango, Fauna Mal. Cuba, 1878. 196. Antilles.

Voluta respertilio Rumpl., Krebs. 36.

Voluta respertilio Rumph., Moll. Cuba, ii, 123.

Voluta respertilio Wood, Moll. Cuba, ii, 124.

Volutella (see Marginella, Persicula).

Volutella lacrimula Gld., Dall, Hemphill's shells. 324. Ga. Cedar Keys.

Volutella lachrimula Glk., Verrill, List, 18S4. 267. Cape Hatteras.

Volutomitra Wandoensis Holm., 1. s., Post-Pleiocene Fossils, S. O. pl. xii, figs. $10,10 a$. 77.

Volva (see Ovulum, Radius).

Volva acicularis Chem., Jahrb., ix, 177.

Volra acicularis Lam., Arango, Fauna Mal. Cuba, 1878. 183. Ca. Ba.

Volra subrostrata Sby., Marrat, Argo Exp., 1876. 16. Nassau. Abaco. 
Volva uniplicata Sby., Am. Marine Conch. 56.

Volvaria (see Marginella, Volvariua).

Volvaria avena Val., Pfeiffer, Archiv fuir naturg., 1840. 259. Ca.

Volraria pallida Lam., Arango, Fauna Mal. Cuba, 1878. 190. Ca. Ber.

G. Ba.

Volvaria pallida Lam., Beau. 6. G.

Volvaria pallida Lam., Krebs. 46. ST. SJ. SB. Ag. SM.

Tolvaria pallida Lam., Moll. Cuba, ii, 100.

Tolvaria pellucida Schum., Arango, Fauna Mal. Cuba, 1878. 190. Ca. Ber. G. Ba.

Volvaria pellucida Schum., Krebs. 46.

Volraria (Volrarina) subtriplicata D'Orb., var., Marrat, Argo Exp., 1876. 18. Abaco.

Volvaria triticea Lam., Pfeiffer, Archiv fuir naturg., 1840. 259. Ca.

Volvarina (see Volvaria), Marrat, Argo Exp., 1876. 18. Abaco.

Tolvarina catenata Mont., Gupuy, Geol. Mag., 1874. 448. Ta.

Volvarina fusca Sby., Jahrb., vii, 62.

Volvarina nitida Hinds, Dall, Hemphill's shells. 324.

Volvarina pallida Lam., Cuppy, Geol. Mag., 1874. 448. Td.

Tolvarina subtriplicata D'Orb., Hemphill's shells. 324. Key West.

Volvarina succinea Courad, Dall, Hemplill's shells. 324. Cedar Keys.

Tampa.

Volvula (see Bulla).

Volrula minuta Bush, Comn. Ac., vi, 469. Hatt.

Volvula oxytata Bush, Conn. Ac., vi, 468. Hatt.

Volvula persimilis Mörch, Poulsen. 8 .

Volvula persimilis Mörch, Mal. Bl., xxii, 179.

Volrula recta D'Orb., Mal. Bl., xxii, 179. ST.

Taldheimia floridana Pourt., Am. Jour. Conch., vi, 112. Fla.

Waldheimia floridana I'ourt., Arango, Fauna Mal. Cuba, 1878. 275. Ca.

Waldheimia floridana L'ourtalès, Report Blake Moll. 103. Fla.

Xenophora (see Onustus, Phorus, Trochus).

Xenophora agglutinans Lam., Beau. 12. G.

Xenophora agglutinans Montf., Jour. de Conch., $\nabla, 250$.

Xenophora caperata Phil., Mal. Bl., ir, 218.

Xenophora caribæa Petit, Beau. 12. MG.

Xenophora caribaea Petit, Jour. de Couch. r, pl. 10, figs. 1, 2. 284-51. MIG.

Xenophora caribaa Petit, Jour. de Conch., vı, 25.

Xenophora caribaa Petit, Krebs. 69.

Xenophora caribaa Petit, Mal. B1., iv, $21 \mathrm{~S}$.

Xenophora caribæa Petit, Mal. Bl., хxiг, 108. MG.

Xenophora conchyliophora Born, Jour. de Conch., $\nabla, 250$.

Xenophora conchyliophorus Boru, Beau. 12. G.

Xenophora onusta Rve., Beau. 12. G. SM.

Xenophora lævigata Fisch., Mal. Bl., xxir, 109. 
Xenophora meandrina Fisch., Mal. Bl., xxiv, 109. SD.

Xenophora onusta Rre., Jour. de Conch., r, 250.

Xenophora tricostata Fisch., Mal. Bl., xxiv, 109.

Xenophora trochiformis Born, Krebs. 68. ST. Tort. PPl. SCx. SJ.

Xenophora trochiformis Born, Mal. Bl., xxiv, 10s. G.

Xenophora trochiformis Born, Poulsen. 10.

Xenophora vulcanica Fisch., Mal. Bl., xxiv, 109. G.

Xenophora fimbriata Jeffreys, Am. Marine Conch., 132. NC.

Xylotrya (see Teredo).

Xylotrya fimbriata Jeffreys, var. subæqualis Dall, Hemphill's shells. 337. Cedar Keys.

Yoldia jeffreysi (Hidalgo) Verrill, List, 18s4. 279. CS.

Yoldia liorhina Dall, n. s., Report Blake Moll. 127.

Yoldia messanensis (Seguenza) var. Verrill, List, 1884. 280. CS.

Yoldia solenoides Dall, n. s., Report Blake Moll. 127.

Zebina (see Rissoina).

Zebina browniana D'Orb., Mal. Bl., xxiii, 46. Ca.

Zebina lavigata C. B. Adams., Mal. Bl., xxiii, 45. J.

Zebina sloaniana D'Orb., Mal. Bl, xxiii, 46. J.

Zebina vitrea C. B. Adlams, Mal. Bl., xxiii, 45. J.

Zebina vitrinella Mörch, Mal. Bl., xxiii, 45. ST.

Zebinella (see Rissoina).

Zebinella albida C. B. Adams, ${ }^{*}$ Mal. Bl., xxiii, 48. J.

Zebinella decussata Mtg., Mal. Bl., xxiii, 47.

Zebinella princeps C. B. Arlams, Mal. Bl., xxiii, 47. J. Ca.

Zebinella signifer Mörch, Mal. Bl., xxiii, 48. NP.

Zeidora (see Emarginula).

Zeidora naufraga Watson, Jour. de Conch., xxxi, 80.

Zeidora naufraga Watson, Lin. Soc. Journ., 1883, xvii, 27. Cul.

Zizyphinus (see Calliostoma, Trochus).

Zizyphinus javanicus Chem., Arango, Fauna Mal. Cuba, 1878. 176. Ca. Zizyphinus javanicus Lam., Arango, Fauna Mal. Cuba, 1878. 176. Ca. Zizyphinus jujubinus Gmel., Arango, Fama Mal. Cuba, 1878. 176. Ca. G. Ba.

Ziziphinus leanus C. B. Adams, Marrat, Argo Exp., 1876. 10. La Guayra.

Ziziphinus stirophorus Watson, Lin. Soc. Journ., 1879, хiv, 695. Cul. Ziziphinus tiara Watson, Lin. Soc. Jouru., 1879, xir, 696. Cul. Ber.

AUGUST 20, 1885 


\title{
A DVETTIS F M E N T.
}

\author{
[Bulletin No. 24.]
}

The publications of the United States Geological Survey are issued in accordance with the statute, approrod March 3, 1879, which declares that-

"The publications of the Geological Survey shall consist of the annual report of operations, geological and economic maps illustrating the resources and classification of the lands, and reportsupon general and economic geology and paleontologs. The annual report of operations of the Geological Survey shall accompany the annual report of the Secretary of the Interior. All special memoirs and reports of said Survey shall be issued in uniform quarto series if deemed necessary by the Director, but otherwise in ordinary octaros. Three thousand copies of each shall be published for seientific exchanges and for sale at the price of publication; and all literary and cartographic materials receired in exchange shall be the property of the United States and form a part of the library of the organ. ization: And the money resulting from the sale of such publications shall be covered into the Treasury of the United States."

On July 7, 1882, the following joint resolution, referriug to all Government publications, was passed ljy Congress:

"That whenever any document or report shall be ordered printed br Congress, there shall be printed, in addition to the number in each case stated, the 'nsual number' $(1,900)$ of copies for binding and distribution among those entitled to receive them."

Under these general laws it will be seen that none of tho Surrey publications are furnished to it for gratuitous distribution. The 3,000 copies of the Annual Report are distributed through the cocument rooms of Congress. The 1,900 copies of each of the publications are distributed to the ofticers of the legislative and executive departments and to stated depositories throughout the United States.

Except, therefore, in those cases where an extra number of any publication is supplied to this oftice by special resolution of Congress, as has been done in the case of the Second, Third, Fourth, and Fitth $\Delta \mathrm{n}$. nual leports, or where a number has been ordered for its use by the Secretary of the Interior, as in the case of Mineral Pesources and Dictionary of Altitudes, the Survey has no copies of any of its publications for gratuitous distribution.

\section{ANNUAL REPORTS.}

Of the Annual Reports there hare been already jublished:

I. First Annual Report to the Hon. Carl Schurz, by Clarenco King. 1880. $8^{\circ}$ - 79 pp. 1 mal,$-\mathbf{A}$ preliminary report describing plan of organization and publications.

II. Report of the Director of the Jnited States Geological Survey for 1880-'81, by J. WV. Powell. 1882. $8^{\circ} .15,588 \mathrm{pp} .61 \mathrm{pl} .1$ map.

IIX. Third Annual Report of the United States Geological Survey, 1881-'82, by J. W. Powell. 1883. $8^{\circ}$. xviii, $564 \mathrm{pp} .67 \mathrm{pl}$. and maps.

IV. Fourth Annual Report of the United States Geological Survey, 1882-83, by J. W. Powell. 1884. $8^{\circ}$. xii, $473 \mathrm{pp}$. $85 \mathrm{pl}$. and maps.

The Fifth and Sixth Annual Reports are in press.

MONOGRAPHS.

Of the Monographs, Nos. II, IU, IV, V, VI, VII, and VIII are now published, viz:

II. Tertiary History of the Grand Cañon District, with atlas, by Clarence E. Dutton, Capt. U. S. A. 1882. $4^{\circ}$. xiv, $264 \mathrm{pp}$. $42 \mathrm{pl}$. and atlas of 24 sheets folio. Prico $\$ 10.12$.

III. Geology of the Comstock Lode and the Washoe District, with atlas, by George F. Becker. 1882. 40. $x \nabla, 422 \mathrm{pp} .7 \mathrm{pl}$. aud atlas of 21 sheets folio. Price $\$ 1 \mathrm{~J}$.

IV. Comstock Mining and Miners, by Eliot Lort. 1883. 40. xiv, 451 pp. 3 pl. Price $\$ 1.50$.

V. Copper-bearing Rocks of Lake Superior, by Roland D. Irring. 1883. 40. xvi, 464 pp. 151. 29 pl. Price $\$ 1.85$.

VI. Contributions to the Knowledge of the Older Mesozoic Flora of Virginia, by $\Pi \mathrm{m}$. M. Fontaine. 1883. 40. xi, $144 \mathrm{pp}, 5 \$ 1.54 \mathrm{pl}$. Price $\$ 1.05$.

VII. Silver-lead Deposits of Eureka, Nevada, by Joseph S. Curtis. 1881. 40. xiii, 200 pp. 16 pl. Price \$1.20.

VIII. Paleontology of the Eureka District, by Charles D. Walcott. 1884. 40: xiii, 298 pp. 211. 24 pl. Prico $\$ 1.10$. 
The following are in press, viz:

1X. Brachiopoda and Lamellibranchiata of the Raritan Clays and Greensand Marls of New Jersey, by loovert P. Whitfield. 1885. 4․ ix, $338 \mathrm{pp} .35 \mathrm{pl}$.

x. Divocerata. A Monograph of an Extinct Order of Gigantic Mammals, by Othniel Charles Marsh. 1885. 4०.,-- pp. $56 \mathrm{pl}$.

XI. Geological History of Lake Lahontan, a Quaternary Lake of Northwestern Nevada, by Iarael Cook Russell. 1885. 40, xiv, 288 pp. $46 \mathrm{pl}$.

The following are in preparation, viz:

I. The Precious Metals, by Clarence King.

-Geology and Mining Industry of Leadville, with atlas, by S. F. Emmons.

-Geology of the Eureka Mining District, Nevada, with atlas, by Arnold Hague.

-Lake Bonneville, by G. K. Gilbert.

-Sauropoda, by Prof. O. C. Marsh.

-Stegosauria, by Prof. O. C. Marsh.

\section{BULLFTINS.}

The Bulletins of the Survey will contain such papers relating to the general purpose of its work as do not properly come under the heads of ANNUAL Ripolis or MIONOGRapHs.

Each of these Bulletins will contain but one paper, and will be cotoplete in itself. They will, how. ever, be numbered in a continuous series, and will in timo be united into volumes of convenient size. To facilitate this, each Bulletin will hare two paginations, one proper to itself and another which belongs to it as part of the volume.

Of this series of Bulletins Nos. 1 to 24 are alrearly published, viz:

1. On Hypersthene-Andesite and on Triclinic Pyroxene in Augitio Rocks, by Whitman Cross, with a Geological Sketch of Buttalo P'eaks, Colorado, by S. F. Emmons. 1383. $8^{\circ} .42 \mathrm{pp} .2 \mathrm{pl}$. Price 10 cents.

2. Gold and Silver Conversion Tables, giving the coining value of troy ounces of fine metal, etc., by Albert Williams, jr, 1883.80 . ii, 8 pp. Price 5 cents.

3. On the Fossil Faunas of the Upper Devonian along the meridian of $76^{\circ} 30^{\prime}$, from Tompkins County, New York, to Bidifond Count 5 , Pennsylvania, by Henry S. Williams. 1884. $8^{\circ}$. 36 pp. Price 5 cents.

4. On Mesozoic Fossils, by Charles A. White. 1884. 8 . 36 pp. 9 pl. P'rice 5 cents.

5. A Dictionary of Altitudes in the United States, compiled by Henry Gannett. 1884. $8^{\circ}$. 325 pp. Price 20 cents.

6. Elevations in the Dominion of Canada, by J. W. Spencer. 1884. $8^{\circ}, 43$ pp. Price 5 cents.

7. Mapoteca Geologica Americana. A catalogue of geological maps of America (North and South), 1752-1881, by Jules Narcou and John Belknap Marcou. 1884. 8०. 184 pp. Priceß 10 cents.

8. Un Secondary Enlargements of Miseral Fragments in Certain Rocks, by R. D. Irring and C. R. Van Hise. 1884. $8^{\circ}, 56 \mathrm{pp} .6 \mathrm{pl}$. Price 10 cents.

9. A Report of work done in the Washington Laboratory during the flacal year 1883-'84. F. W. Clarke, chief chemist; T. MI. Chatard, assistant. 1884. $80.40, \mathrm{pp}$. Price 5 cents.

10. On the Cambrian Faunas of North America. Preliminary studies by Charles Doolittle Walcott. 1884. $80.74 \mathrm{pp} .10 \mathrm{pl}$. Price 5 cents.

11. On the Quaternary and Recent Mollusca of the Great Basin; with Descriptions of New Forms, by R. Ellsworth Call; introduced by a sketch of the Quaternary Lakes of the Great Basin, by G. $\mathbf{K}$. Gilbert. 1884. $80.66 \mathrm{pp}$. $6 \mathrm{pl}$. Price 5 cents.

12. A Crystallographie Study of the Thinolite of Lake Lahontan, by Edward S. Dana. 1884. 80. $24 \mathrm{pp} .3 \mathrm{pl}$. Price 5 cents.

13. Boundaries of the United States and of the several States and Territories, by Henry Gannett. 1885. 80. $135 \mathrm{pp}$. Price 10 cents.

14. The Electrical and Magnetic Properties of the Iron-Carburets, by Carl Barus and Vincent Strouhal. 1885. $8^{\circ}$. $238 \mathrm{pp}$. Price 15 cents.

15. On the Mesozoic and Cenozoic Paleantology of California, by Dr. C. A. White. $1885.80,33 \mathrm{pp}$. Price 5 cents.

16. On the ligher Devonian Faunas of Ontario County, Now York, by J. M. Clarke. 1885. $8^{\circ}$. $86 \mathrm{pp} .3 \mathrm{pl}$. Price 10 cents.

17. On the Derelopment of Crystallization, ete., by Arnold Hague and J. P. Iddings. 1885. $8^{\circ}$. 44 pp. Price 5 cents.

18. On Marine Eocene, Fresh-water Miocene, and other Fossil Mollasca of Western North America, by Dr. C. A. White. 1885. $8 \circ .26 \mathrm{pp}, “ 3 \mathrm{pl}$. Price 5 cents.

19. Notes on the Stratigraphy of California, by George F. Becker. 1885. $8 \circ .28$ pp. Price 5 cents.

20. Contributions to the Mineralogy of the Rocky Mountains, by Whitman Cross and W. F. Hillebrand. 1885. $8^{\circ} .114 \mathrm{pp} .1 \mathrm{pl}$. Price 10 cent.

21. The Lignites of the Great Sioux Reservation, by Bailey Willis. 1885. $88^{\circ}$ - 16 pp. 5 pl. Price 5 cents.

22. On New Cretaceous Fossils from California, by Charles A. White, M. D. 1885. 80. 25 pp. 5 pl. Price 5 cents. 


\section{ADVERTISEMENT:}

23. The Junction between the Eastern Sandstone and the Keweenaw Series on Keweenaw Point, by R. D. Irring and T. C. Chamberlin. 1885. $8^{\circ}$. - pp. $17 \mathrm{pl}$. Price - cents.

24. List of Marine Llollusca, comprising the Quaternary Fossils and recent forms, from American localities between Cape Hatteras and Cape Roque, incluling the Bermudas, by W. II. Dall. 1885. $8^{\circ}$. 336 pp. Price 25 cents.

Numbers 1 to 6 of the Bulletins form Volume I; Numbers 7 to 14, Volume II; and Numbers 15 to 23, Volurne III. Volume IV is not yet complete.

The following are in press, viz:

25. The Present Technical Condition of the Steel Industry of the United States, by Phineas Barnes. 1885. $8^{\circ} .82 \mathrm{pp}$. Price 10 cents.

20. Copper Smelting, by H. M. Howe. 1885. $8^{\circ}$. - pp. Price - cents.

STATISTICAL PAPERS.

A fourth series of publications, having special reference to the mineral resources of the United States, is contemplated.

Of that series the first has been published, riz:

Mineral liesources of the United States [1882], b5 Albert Williams, jr. 1883. $8^{\circ}$. xrii, 813 pp. Price 50 cents.

The second volume of this series, Mineral Resources of the United States, 1883 and 1884, is in press. Correspondence relating to the publications of the Survey, and all remittances, which must be by POSTAL NOTE OR SHONEY ORDER, should be addressed

To THE DIRECTOR OF THE

Unithd Stateb Grological Subver,

W Ashunaton, D. C., August 28, 1885.

Washington, D. U. 
7521 




